\title{
Chemical Concepts and X-ray Technologies challenged by Charge Density
}

\section{Dissertation}

zur Erlangung des mathematisch-naturwissenschaftlichen D oktorgrades

"Doctor rerum naturalium"

der Georg-A ugust-Universität Göttingen

im Promotionsprogramm Chemie

der Georg-August University School of Science (GAUSS)

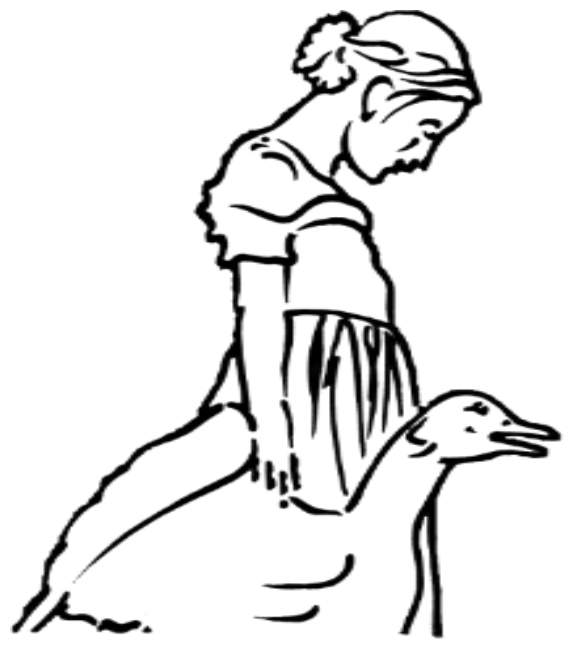

vorgelegt von

Christian Joseph Schürmann

aus V echta

Göttingen, 2018 

Betreuungsausschuss

Prof. Dr. Dietmar Stalke

Prof. Dr. Ricardo A. Mata

Mitglieder der Prüfungskommission

Referent: Prof. Dr. Dietmar Stalke

Prof. Dr. Ricardo A. M ata

weitere Mitglieder der Prüfungskommission

Prof. Dr. Götz Eckold

Prof. Dr. Franc M eyer

Prof. Dr. Manuel Alcarazo

Dr. Heidrun Sowa

Tag der mündlichen Prüfung: 16.01.2019 

If you base medicine on science you cure people.

If you base the design of planes on science they fly.

If you base the design of rockets on science they reach themoon.

It works - bitches.

- Richard Dawkins 





\section{Table of Contents}

CHAPTER 1 INTRODUCTION AND THEORETICAL BACKGROUND

1.1 INTRODUCTION _ 1

1.2 THE BASICS OF X-RAY DIFFRACTION __ 2

1.3 The INDEPENDENT ATOM MODEL __ 3

1.4 Phase Problem _ 5

1.5 DATA PROCESSING _ 5

1.5.1 Data Collection _ 5

1.5.2 Data Integration _ 6

1.5.3 Data Scaling___ 6

1.6 STRUCTURE REFINEMENT _

Chapter 2 EXPERIM ENTAL ChARge Density INVESTIGATIONS

2.1 INTRODUCTION__ 8

2.2 The Multipole Model___ 8

2.3 RefinementSTRATEGY _ 9

2.4 EVAlUATION IN THE QUANTUM TheORY OF ATOMS IN MOLECULES ___ 11

2.5 The CoMbination of TheORETICAL AND EXPERIMENTAL CHARGE DeNSITY 12

2.5.1 AIMALL 13

2.5.2 Crystal Explorer 13

ChAPTER 3 ASSESSMENT OF M ODEL- AND DATA QUALITY _-_-_-_-_-_-_-_-_-_-_-_-_-_-_-_-_-_-_-_ 15

$3.1 \quad$ INTRODUCTION

3.2 FIGURES OF MERT: DATA___ 15

3.3 FIGURES OF MERIT: MODEL __ 17

3.3.1 Residual Density Analysis __ 18

3.3.2 DRKplot__ 19

3.3.3 Cross Validation 19

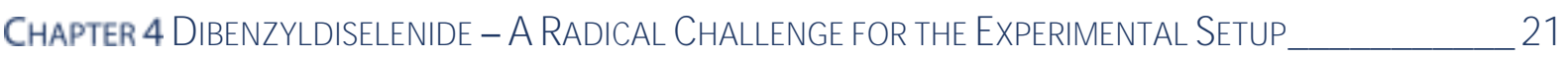

4.1 INTRODUCTION _ 21

4.1.1 Selenium and Radiation __ 21

4.1.2 Selenium in Macromolecular XRD _ 22

4.1.3 Reactivity of $(\mathrm{BzSe})_{2}$

4.1.4 Structural Features _ 24

4.1.5 Experimental Challenges __ 26

4.2 Data Collection AND PROCESSING __ 27

4.2.1 Mo TXS and Apex2 _ 27

4.2.2 Ag IuS2 and Apex2 28 
4.2.3 Ag $1 \mu \mathrm{S} 2$ and Pilatus3 _ 29

4.2.4 Ag 007 and Pilatus3 30

4.2.5 Ag I $\mu \mathrm{S} 3$ and Photon2 _ 30

4.2.6 In MetalJet and Photon2

4.2.7 Ag $\mathrm{l} \mu \mathrm{S} 3$ and Photon3 _ـ 32

4.3 Charge Density Refinement ___ 32

4.3.1 Charge Density in XD__ 33

4.3.2 Charge Density in MoPro _ـ 36

4.4 Residual Density PeAK ANALysis __ 37

$4.5 \quad$ DFT OPTIMIZATIONS __ 39

4.6 EPRINVESTIGATION _ 42

4.7 CONCLUSION __ 44

Chapter 5 Data Quality Assessment of New X-ray AREA DeteCtOrS _-_-_-_-_-_-_-_-_-_-_-_ 45

\begin{tabular}{lll}
5.1 & INTRODUCTION \\
\hline
\end{tabular}

5.1.1 Precision and Accuracy___ 45

5.1.2 The Latest Generation of X-ray Area Detectors ___ 46

5.2 BeNCHMARKSTRUCTURES __ 50

5.3 EXPERIMENTALDETAILS _

5.4 ASSESSMENT OF PRECISION___ 52

5.5 ASSESSMENT OF ACCURACY _ـ 53

5.6 CONCLUSION _ـ 55

Chapter 6 Charge Density InVESTIgATIONS On Frustrated LeWIS PAIRS __-_-_-_-_-_-_-_-_-_- 57

$\begin{array}{lll}6.1 & \text { INTRODUCTION } & 57\end{array}$

6.2 EXPERIMENTAL__ 60

6.2.1 Charge Density Refinement ___ 60

6.2.2 Refinement of Disordered Hydrogen ___ 61

6.2.3 Determination of Bad Data___ 61

6.3 EVALUATION__

6.4 CONCLUSION _ـ 66

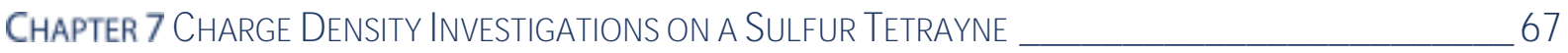

$\begin{array}{lll}7.1 & \text { INTRODUCTION } \\ \end{array}$

7.2 THEORETICAL INVESTIGATION __

7.3 EXPERIMENTAL _

\begin{tabular}{ll}
7.4 & EVALUATION \\
\hline
\end{tabular}

7.5 CONCLUSION 
8.1 CRYSTALSTRUCTURES IN COLLABORATION WITH RAJENDRA GHADWAL___ 76

8.1.1 Unprecedented Borylene Insertion into a C-N Bond___ 76

8.1.2 Mono- and Di-Cationic Hydrido Boron Compounds __ 80

8.1.3 Abnormal-NHC Palladium(II) Complexes: Rational Synthesis, Structural Elucidation, and Catalytic Activity

8.1.4 Normal-to-Abnormal Rearrangement of an N-heterocyclic Carbene with a Silylene Transition Metal Complex

8.1.5 Abnormal-NHC-Cobalt(II) Complexes___ 90

8.1.6 Normal- and Abnormal- N-Heterocyclic Carbene (NHC) Magnesium Compounds ___ 93

8.1.7 Unpublished Crystal Structures __ 94

8.2 CRystalStructures in Collaboration With Sudipta RoY $\quad 104$

8.2.1 Activation of Elemental Sulfur at a Two-Coordinate Platinum(0) Center _ 104

8.2.2 Two Structurally Characterized Conformational Isomers with Different C-P Bonds__ 107

8.3 Crystal Structures in Collaboration with Chandrajeet Mohapatra

CHAPTER 9 APPENDIX 115

SUPPLEMENTARY INFORMATION TO CHAPTER 4 _ 115

SUPPLEMENTARY INFORMATION TO CHAPTER 5

SUPPLEMENTARY INFORMATION TO CHAPTER 6 _ 158

SUPPLEMENTARY INFORMATION TO CHAPTER $7 \_169$

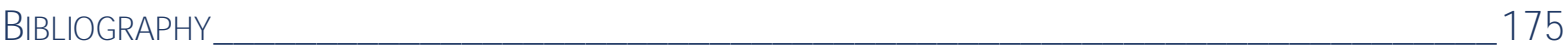





\section{Chapter 1 INTRODUCTION AND THEORETICAL BACKGROUND}

\subsection{Introduction}

X-ray structure analysis has come a long way since the first diffraction experiments by Laue, Friederich, and Knipping in 1912 to prove the electromagnetic nature of X-rays (Friedrich et al.) and the first structure determinations of Bragg in 1913. W ith the emergence of new, more powerful radiation sources, better $\mathrm{X}$-ray detectors, improved crystal handling methods and raising computational power ever more crystal structures are accessible with great precision in short time with lower costs. Thereby, crystal structure determination has become a standard analytical method for many scientists in recent years. Still it is fascinating to achieve an image of the otherwise somewhat abstract molecular structure. Even more fascinating is the fact that by $X$-ray diffraction, not only the arrangement of atoms can be observed, but also the electron (or charge) density between them. And the electronic interactions of atoms are what chemistry is all about. So with X-ray diffraction, we can have a glimpse at the heart of chemistry itself.

This thesis focusses on the thorough evaluation of high-resolution X-ray diffraction data. Thereby, we can have a close look at the charge density distribution in some molecules, achieve a better understanding of the interatomic interactions, and test our current chemical concepts. M ost noticeably, metastable radical states induced and detected by $X$-rays during the diffraction experiment were found. Furthermore, this thesis endeavors to push the frontiers of $X$-ray diffraction by testing the latest generation of $\mathrm{X}$-ray detector technology.

Parts of this thesis have been published separately and are reproduced herein:

[1] C. J. Schürmann, R. H erbst-Irmer, T. L. Teuteberg, D. Kratzert, G. Erker, R. A. M ata, D. Stalke, Experimental charge density study on FLPs and a FLP reaction product, zkri 2018, 233, 723. 


\subsection{The Basics of X-ray Diffraction}

Whenever an electromagnetic wave passes through a lattice with a lattice constant $d$ in the same order of magnitude as the wavelengths $\lambda$, it is diffracted according to Bragg's law (Bragg $\&$ Bragg, 1913) with the scattering angle $\theta$.

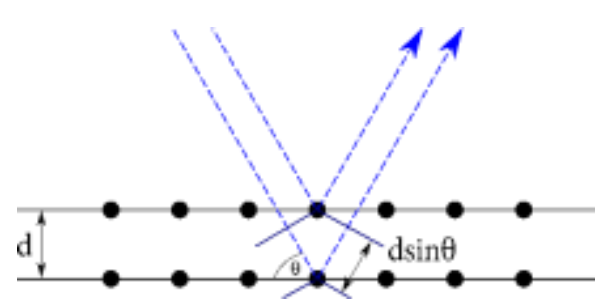

Figure 1.1: V isualization of Bragg's law.

$$
2 d \sin (\theta)=n \lambda \quad n \in \mathbb{Z}
$$

This can also be expressed with the vector of the incident wave $\mathbf{k}$ and the lattice vector $\mathbf{k}_{d}$ as

$$
\boldsymbol{k}_{i}-\boldsymbol{k}_{d}=\mathbf{H}=n \lambda \quad n \in \mathbb{Z}
$$

Interatomic distances are in the $10^{-10} \mathrm{~m}$ range therefore $X$-rays whose wavelengths are in the same range are diffracted at atomic and molecular lattices. M olecular or atomic lattices are almost always three-dimensional and are called crystals. The one-dimensional Bragg's law has therefore to be applied to three dimensions. Only if Bragg's law is fulfilled for the three lattice dimensions of the crystal, a diffraction maximum is observable. This is called Laue condition and is expressed as

$$
\begin{array}{ll}
\boldsymbol{a} \cdot \mathbf{H}=h & h \in \mathbb{Z} \\
\boldsymbol{b} \cdot \mathbf{H}=k & k \in \mathbb{Z} \\
\boldsymbol{c} \cdot \mathbf{H}=l & l \in \mathbb{Z}
\end{array}
$$

with $\mathbf{a}$, band $\mathbf{c}$ being the three lattice vectors, defining the crystal and h, $k, I$ the order of the maxima, called Miller Indices (Miller, 1839). So the position of diffraction maxima or reflections is solely dependent on the crystal lattice.

The crystal lattice is chosen according to distinct rules that simplify the lattice to one most simple description of the unit cell with a maximum of applicable symmetry (Hahn, 2005). The intensity of the reflections on the other hand depends on the content of the unit cell. This is described by the scattering factor $F(\mathbf{H})$. The intensity I is proportional to the absolute $F^{2}(\mathbf{H})$.

$$
I \propto\left|F^{2}(\mathbf{H})\right|
$$

The diffraction vector $\mathbf{H}$ is defined as

$$
\mathbf{H}=\mathrm{h} \boldsymbol{a}^{*}+k \boldsymbol{b}^{*}+l \boldsymbol{c}^{*}
$$

with the reciprocal lattice vectors $\mathbf{a}^{*}, \mathbf{b}^{*}$ and $\mathbf{c}^{*}$ as 


$$
\mathbf{a}^{*}=\frac{b \times \boldsymbol{c}}{V}, \quad \mathbf{b}^{*}=\frac{a \times c}{V}, \quad \mathbf{c}^{*}=\frac{a \times b}{V}, \quad V=(\boldsymbol{a} \times \boldsymbol{b}) \cdot \boldsymbol{c}
$$

Each diffraction maximum is a superposition of all elemental waves reflected from any location in the crystal. The diffraction strength of each location in the crystal is dependent on the charge density. The structure factor $F(\mathbf{H})$ is therefore expressed as an integral function of the mean charge density distribution within the unit cell $\rho(\mathbf{r})$ with $\mathbf{r}$ being the fractional coordinates within the unit cell.

$$
F(\mathbf{H})=\int_{V} \rho(\boldsymbol{r}) e^{2 \pi i \mathbf{H} r} d \boldsymbol{r}
$$

This operation is basically a Fourier transformation (Fourier, 1822) of the charge density distribution, therefore the inverse Fourier transformation is also true.

$$
\rho(\boldsymbol{r})=\int_{V} F(\mathbf{H}) e^{-2 \pi i \mathbf{H} r} d \boldsymbol{r}
$$

This expression is periodic. However, in the diffraction pattern, only the diffraction maxima as defined by the Laue conditions are visible. The expression can therefore be simplified to a Fourier summation.

$$
\rho(\boldsymbol{r})=\frac{1}{V} \sum_{h} \sum_{k} \sum_{l} F(\mathbf{H}) e^{-2 \pi i \mathbf{H} r}
$$

By the Fourier summation of all observed scattering factors, an approximation of the charge density distribution within the unit cell can beachieved. V ice versa, the Fourier summation of the charge density distribution within the crystal would yield the scattering factors. But in order to do so, the charge density has to be described by a periodic model.

\subsection{The Independent Atom Model}

The independent atom model (IAM) gives a sufficient approximation of the charge density for most applications. The charge density is distributed around atom positions. The Fourier transformation of an atom's charge density is the atomic scattering or atomic form factor $\mathrm{f}$.

$$
f^{0}=\int \rho(\boldsymbol{r}) e^{2 \pi i s r} d \boldsymbol{r}
$$

Here, s denotes the phase difference between the electrons within the atomic density. Photons scattered at different points within the atomic density experience a relative phase change and subsequently interfere with each other. The more spread out the atomic density, the more noticeable are the effects of interference. Therefore, the diffuse valence density is strongest in the low-resolution range, while the compact core density is strong in all data. This is well observable in the progression of the resolution dependent scattering factor in Figure 1.2. 


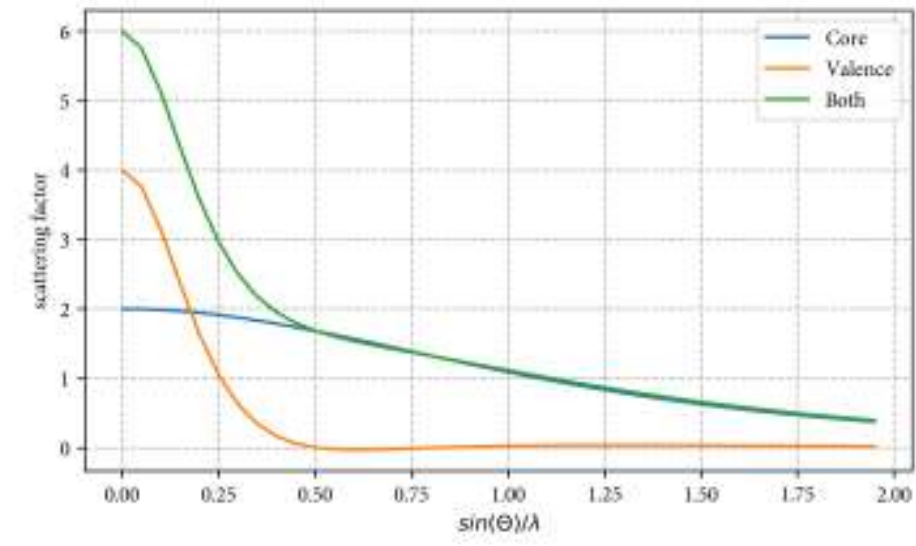

Figure 1.2: Resolution dependence of the atomic scattering factor build up by valence- and core density for carbon, based on Su \& Coppens, 1998.

A tomic scattering factors have been determined for virtually every atom and most ions, based on theoretical calculations and are tabulated angular-dependent for the use in X-ray diffraction. The scattering power mainly relies on the number of electrons as illustrated in Figure 1.3.

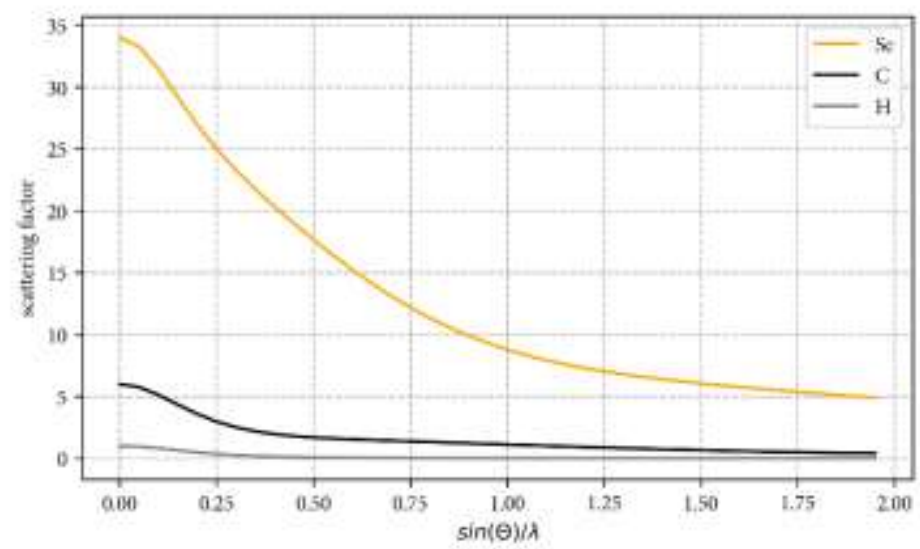

Figure 1.3: Resolution dependence of the atomic scattering factors, based on Su \& Coppens, 1998.

Furthermore, inelastic scattering may occur and weaken the intensity. Both effects are accounted for by the expression of the atomic scattering factor $f(\theta, \lambda)$ being comprised of a theoretically determined atomic part $f^{0}$ plus a real part $\Delta f^{\prime}$ and imaginary part $\Delta f^{\prime \prime}$ for the consideration of inelastic or anharmonic scattering.

$$
f(\theta, \lambda)=f^{0}(\theta)+\Delta f^{\prime}(\lambda)+i \Delta f^{\prime \prime}(\lambda)
$$

The anharmonic scattering factors are tabulated for various wavelengths and elements. As diffraction experiments are performed at temperatures above zero Kelvin, the thermal motion of an atom also has to be considered. This is achieved with a thermal motion factor $U$ that describes either an isotropic motion $\mathrm{U}_{\mathrm{iso}}$ or a six-parameter displacement tensor $\mathrm{U}_{\mathrm{ij}}$.

$$
U=U_{11} h^{2} \boldsymbol{a}^{* 2}+U_{22} k^{2} \boldsymbol{b}^{* 2}+U_{33} l^{2} \boldsymbol{c}^{* 2}+2 U_{23} k l \boldsymbol{b}^{*} \boldsymbol{c}^{*}+2 U_{13} h l \boldsymbol{a}^{*} \boldsymbol{c}^{*}+2 U_{12} h k \boldsymbol{a}^{*} \boldsymbol{b}^{*}
$$


With this model of the atomic density, the Fourier summation over all atoms $j$ at the fractional coordinates $x, y, z$ within the unit cell, vice versa to the Fourier summation of all scattering factors in Eq. 1.11 is feasible.

$$
F(\mathbf{H})=\sum_{j} f_{j} e^{2 \pi i\left(h x_{j}+k y_{j}+l z_{j}\right)} e^{-2 \pi^{2} U_{j}(\theta)}
$$

\subsection{Phase Problem}

If the structure factors $F$ were experimentally accessible, theFourier summation in Eq. 1.11 and the determination of the charge density distribution would be easy. But with $\mathrm{F}$ being a complex number and only its amplitude $|F|$ being accessible by the experimentally determined intensity as $\sqrt{I}$, the charge density distribution cannot be determined directly. This fundamental problem has become known as the crystallographic phase problem. Only by the application of a periodic model, the phase problem can be overcome. When atom positions are known, calculated structure factors $F_{\text {calc }}$ can be determined and compared to the observed structure factors $F_{\text {obs }}$. By the adjustment of the model parameters, the fit of $F_{\text {calc }}$ and $F_{\text {obs }}$ is optimized until the model sufficiently describes the charge density. This process is called refinement.

However, in order to refine the model, some atom positions have to be known. The determination of starting positions is still a huge problem for macromolecular crystallography, such as protein crystallography. For small molecule crystallography, this problem has been overcome with clever software and computational power. The recently released program SHELXT (Sheldrick, 2014b) uses a combination of Patterson map superposition and iterative dual-space recycling and was used for the structure solution of all structures in this thesis.

\subsection{Data Processing}

For the determination of a crystal structure, diffraction data have to be collected, reduced, scaled, sometimes corrected, and subsequently refined with an appropriate model.

\subsubsection{Data Collection}

All datasets in this thesis have been collected on in-house diffractometers, equipped with 3- or 4-circle goniometers, radiation sources with monochromatic $\mathrm{Cu}, \mathrm{M} \mathrm{O}, \mathrm{Ag}$ or $\operatorname{In~} \mathrm{K}_{\alpha}$ radiation and area detectors. All diffractometers were operated, using the APEX 2 software suite (Bruker AXS Inc., 2012). M ost important for the collection of excellent diffraction data is the selection of a perfect single crystal. Furthermore, it is crucial to collect a full dataset up to a resolution, matching the requirements of the refined model ( $<0.83 \AA$ for IAM,$<0.50 \AA$ for multipole model) with full completeness, good multiplicity ( $>3$ for all data) and significant intensity $(>3 \sigma)$. In order to do so, a data collection strategy has to be determined, considering the crystal orientation and symmetry. If the aspherical density should be refined, the low-resolution reflections are of utmost importance, as they hold all information about the diffuse valence density (see also Figure 1.2). It is therefore crucial to determine these intensities with 
greatest precision and accuracy. An often-encountered problem is that the strong low-resolution reflections exceed the dynamic range of the detector. Therefore, the exposure time has to be adapted, so that these strong reflections are collected with highest intensity, but without exceeding the dynamic range.

\subsubsection{Data Integration}

In order to reduce the amount of data from a sometimes gigabyte large series of images to a better manageable table of reflections, the intensities have to be extracted from the frames. All diffraction data in this thesis were integrated using the Bruker software SAINT (Bruker AXS Inc., 2016), which is a heavily modified version of XDS (Kabsch, 2010), therefore the working principles of this software are outlined here briefly.

First, the reflections on the frames have to be indexed according to the unit cell size, symmetry and the crystal orientation. Then, an integration box is calculated around the area of the reflection on the detector and for the progression of the reflection on consecutive frames. N ext, a background noise or error $\sigma$ is determined by the intensity on the edge of the integration box. If the intensity of the reflection is above a certain threshold (usually $>5 \sigma$ ), the intensity of all pixels within the integration box is added up. If, however, theintensity is below this threshold, a peak profile, determined for thestrongest reflections (usually $>15 \sigma$ ) in the same detector area, is applied to the reflection. The integration results in tables of raw intensities featuring the M iller indices $h, k, I$, the intensity I as the energy flux per second and rotational degree $\left[\mathrm{e} / \mathrm{s}^{\circ}\right]$, the error $\sigma(\mathrm{l})$, as well as the spot position and the direction cosines that relate the peak position to the crystal orientation.

\subsubsection{Data Scaling}

Crystals are rarely spherical and therefore the effective scattering volume of the crystal in the Xray beam varies as it rotates. Furthermore, in many cases the data are affected by absorption of the crystal. In order to correct the data for these effects, they were scaled using SADABS (Krause et al., 2015b). O utliers are rejected and the intensities of the same reflections at different crystal orientations and exposure times are scaled to match each other. Furthermore, SADABS offers the option to scale the errors $\sigma(I)$ by the application of an error model.

$$
\sigma^{2}(I)_{\text {corr }}=\left[K \sigma(I)_{\text {raw }}\right]^{2}+(g\langle I\rangle)^{2}
$$

Thus, the errors can be scaled to match the standard deviations of the reflections. For standard resolution data for the refinement of an IAM model, the parameter $K$ was adapted for each single run and $g$ was optimized for all runs combined. However, for high-resolution data, no scaling $(K=1, g=0)$ was applied, so the experimental errors were retained. The reason for this is that the errors of the reflections serve as a weighting factor for that reflection in the consecutive structure refinement. The IAM is not able to describe the aspherical density sufficiently, as only atom positions and vibrational parameters are described (Eq. 1.16). By the application of an error model, the assigned error usually rises 
for the strongest reflections and drops for the weak. While this is sensible for IAM refinements, it is not advisable for charge density refinements, as will be pointed out in the following.

\subsection{Structure Refinement}

The data scaling is followed by the structure solution as outlined in 1.4 and the structure refinement in the IAM as outlined in 1.3 or in the multipole formalism for the refinement of the aspherical density. During refinement, the model structure is adjusted by an optimization algorithm to fit the data. This is done via the calculation of the models structure factors $F_{\text {calc. The optimization }}$ algorithm used in the refinement programs for this thesis is a least-squares al gorithm (Pratt, 1987).

$$
\sum_{\mathbf{H}} w \Delta^{2}=\sum_{\mathbf{H}} w_{\mathbf{H}}\left(s F_{o b s}^{2}-F_{\text {calc }}^{2}\right)^{2}
$$

It minimizes the squared divergence of $F_{o b s}^{2}$ and $F_{c a l c}^{2}$. In order to bring observed and calculated structure factors into scale, one overall scaling factor (OSF) s is refined. Furthermore, for each intensity a weighting factor $w$ is applied.

$$
\begin{gathered}
w=1 /\left[\sigma^{2}\left(F_{o}^{2}\right)+(a P)^{2}+b P\right] \\
P=\left[\frac{2}{3} \cdot F_{c}^{2}+\frac{1}{3} \cdot \max \left(F_{o}^{2}, 0\right)\right]
\end{gathered}
$$

Here, the factors $a$ and $b$ can be customized. This is usually done for refinements in the IAM, as the weighting scheme generally lowers the weight of all strong reflections. These strong reflections are mainly found in the low-resolution range, which holds most information about the diffuse valence density as illustrated in Figure 1.2. As the IAM is incapable of describing the valence density correctly, it is beneficial to down-weigh the corresponding data. The given weighting scheme is applicable in all used refinement programs.

For a charge-density refinement, the strong low-resolution reflections are of greatest importance and it is not advisable to weigh them down artificially. Therefore the parameters of the weighting scheme $a$ and $b$ were set to 0 for all charge density refinements within this thesis.

$$
w=1 /\left[\sigma^{2}\left(F_{o}^{2}\right)\right]
$$

The same principal problem arises for the error model during data scaling. Therefore, the experimentally determined errors were retained also in that step and were applied as weights throughout all refinements. 


\section{Chapter 2 EXPERIMENTAL ChARGe Density INVESTIGATIONS}

\subsection{Introduction}

The IAM assumes a spherical charge density distribution around each atom and is therefore incapable of describing the aspherical valence density. The only atomic parameters refined in this model are the position and its vibration. N evertheless, the interatomic interactions are foremost established by the interaction of the valence shell. The charge density $\rho(\mathbf{r})$ is a fundamental property that characterizes the ground sate of a system (H ohenberg $\&$ Kohn, 1964). Therefore, the analysis of $\rho(\mathbf{r})$ can yield a diverse range of properties.

\subsection{The Multipole Model}

An aspherical description of the atomic charge density is achieved by the application of spherical harmonics (Figure 2.1) (Stewart, 1972, 1973) and was implemented in refinement software by $\mathrm{H}$ ansen $\&$ Coppens (1978; Volkov \& Coppens, 2001).

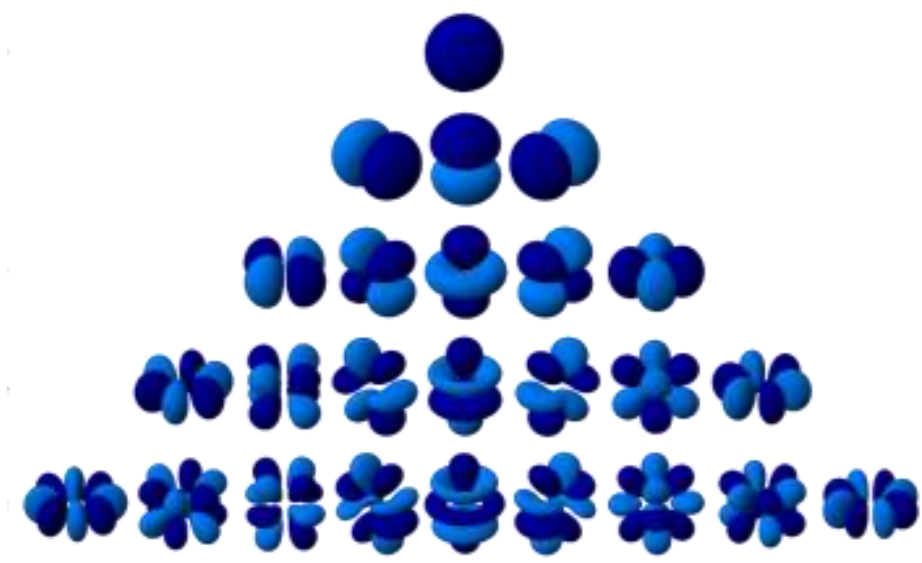

Figure 2.1: Representation of the spherical harmonics $d_{\operatorname{lm}}$ up to hexadecapoles $d_{4 \mathrm{~m} \pm}$

In the multipole approach, the IAM is extended by spherical harmonics (or multipoles). Hence, the atomic density $\rho_{\text {atom }}(\mathbf{r})$ is described as the sum of the core density $\rho_{c}(\mathbf{r})$ with the population factor $P_{c}$, the spherical valence density $\rho_{v}(\mathbf{r})$ with the population factor $P_{v}$ and the dimensionless expansion coefficient $\kappa$ and an expression for the aspherical valence density. This expression is comprised of a radial function $\mathrm{R}_{l}(\kappa, \mathbf{r})$ with a dimensionless expansion coefficient $\kappa$ ' that is combined with spherical harmonics $\mathrm{d}_{\mathrm{Im}}(\theta, \phi)$ and their population $\mathrm{P}_{\mathrm{Im}}$. 


$$
\rho_{\text {atom }}(\boldsymbol{r})=P_{c} \rho_{c}(\boldsymbol{r})+P_{v} \kappa^{3} \rho_{v}(\boldsymbol{r})+\sum_{l=0}^{l_{\max }} \kappa^{\prime 3} R_{l}(\boldsymbol{r}) \sum_{m=0}^{l} P_{l m} d_{l m}(\theta, \phi)
$$

The population of the monopole $(I=0)$ gives the number of an atom's aspherical valence electrons. This isotropic function is then deformed by the population of dipoles $(I=1)$, quadrupoles $(I=2)$, octupoles $(I=3)$, hexadecapoles $(I=4)$, 32-poles $(I=5)$, 64-poles $(I=6)$ and a theoretically possible further expansion to infinite multipoles. The spherical harmonics or multipole functions (Figure 2.1) are defined as

$$
\begin{aligned}
\int_{\phi=0}^{2 \pi} \int_{\theta=0}^{\pi}\left|d_{l m}\right| d \Omega & =2, \quad l>0,-l \leq m \leq l \\
& =1, \quad l=0, m=0
\end{aligned}
$$

The radial functions $R_{l}(\mathbf{r})$ are Slater-type functions:

$$
R_{l}(\boldsymbol{r})=\frac{\alpha_{l}^{n(l)+3}}{[n(l)+2] !} e^{-\alpha_{i} \boldsymbol{r}}
$$

with $\mathrm{n}(\mathrm{I})>$ I to obey Poisson's equation (Stewart, 1977) and $\alpha_{l}$ calculated for single-zeta wave functions. Following the M ultipole expansion, the corresponding atomic form factor is given by

$$
f_{\text {atom }}(\mathbf{H})=f_{c}(\quad)+P_{v} f_{v}\left(\frac{H}{\kappa}\right)+\sum_{l}\left\langle J\left(\frac{H}{\kappa^{\prime}}\right)_{l}\right\rangle \sum_{m=-l}^{l} P_{l m} d_{l m}\left(\frac{\mathbf{H}}{H}\right)
$$

where $\left\langle J_{l}\right\rangle$ is the I-th order Fourier-Bressel transformation of $\mathrm{R}_{l}$

$$
\left\langle J_{l}\right\rangle=4 \pi i^{l} \int j_{l}(2 \pi H r) R_{l}(\boldsymbol{r}) \boldsymbol{r}^{2}
$$

and $f_{c}$ and $f_{v}$ are the Fourier transformations of $\rho_{c}$ and $\rho_{v}$ respectively. (Volkov et al., 2016b)

In many cases, an improved description of the atomic vibration is required, in order to describe the structure correctly in addition to the elaborated description of the atomic scattering factor. Therefore, the vibration is described by Gram-Charlier expansions Uj (Johnson \& Levy, 1974). While $\mathrm{U} 1$ corresponds to an isotropic motion and $\mathrm{U} 2$ to an anisotropic motion as introduced in the IAM (Eq. 1.14), $U 3$ and $U 4$ describe an anharmonic distribution or probability density function (PDF) around the atom position.

\subsection{Refinement Strategy}

In total, the multipole formalism offers a drastically increased number of potential parameters to refine for each atom (see Table 2.1). Therefore, it has to be car efully selected which of those parameters are to be refined. Only the refinement of meaningful parameters that significantly improve the model is desired. Even with perfect, high-resolution datasets, the number of data restricts the refineable number 
of parameters severely. Furthermore, all multipole parameters describe the diffuse valence density, while al most all information of the valence density is comprised in only relatively few data up to a resolution of $d=2 \AA$ (or $\sin (\theta) / \lambda=0.25 \AA^{-1}$ ). Some parameters highly correlate with each other, like $\kappa$ and $\mathrm{P}_{00}$ or $\kappa^{\prime}$ and $U 2$, as these functions describe very similar shapes.

For the refinement of multipoles, a local coordinate system has to be defined for each atom, according to the orientation of the multipoles and the local symmetry of the chemical surroundings of the atom. As a starting point, highest local symmetry is assumed for the atom and all multipoles that contradict that symmetry are kept at zero $\left(P_{I m}=0\right)$. Furthermore, chemically equivalent atoms are constrained to have the same multipolepopulations and the order of multipoles is restricted to a reasonable level, corresponding to the valence shell.
Table 2.1: Possible parameters in IAM and Multipole M odel

\begin{tabular}{|c|c|c|}
\hline & IAM & Multipole \\
\hline Occupancy & 1 occ & $1 P_{v}$ \\
\hline Positions & $3 x y z$ & $3 x y z$ \\
\hline Thermal & $1 U_{\text {iso }}$ & \\
\hline motion & $5 U_{i j}$ & $6 U_{i j}$ \\
\hline & & $10 \mathrm{GC} 3^{\text {rd }}$ \\
\hline & & $15 \mathrm{GC} 4^{\text {th }}$ \\
\hline \multirow[t]{8}{*}{ Multipoles } & & $2 \kappa, \kappa$ \\
\hline & & $1 P_{00}$ \\
\hline & & $3 \quad P_{|m|} \mid=1,-1 \leq m \leq 1$ \\
\hline & & $5 \quad P_{\mid m} \mid=2,-2 \leq m \leq 2$ \\
\hline & & $7 \quad P_{|m|} \mid=3,-3 \leq m \leq 3$ \\
\hline & & $9 \quad P_{\mid m} \mid=4,-4 \leq m \leq 4$ \\
\hline & & $11 \mathrm{P}_{\mid \mathrm{m}} \mid=5,-5 \leq \mathrm{m} \leq 5$ \\
\hline & & $13 P_{\mid m} \mid=6,-6 \leq m \leq 6$ \\
\hline Total & 10 & 86 \\
\hline
\end{tabular}

The refinement strategy has to start with a highly restricted model and the complexity is increased gradually (Volkov et al., 2016b). Therefore, the multipole refinement always follows a refinement in the IAM . The refinement strategy prioritizes parameters according to their improvement of the model. As theIAM refinement provides a reasonably good estimation of the atoms' position and vibration, the first step after scaling would be the introduction of multipoles (usually $1 \leq l \leq 4$ ) for an assumed highest possible molecular and atomic symmetry. This is then usually followed by the successive introduction of monopoles $(I=0)$, vibrational (U2), positional ( $x y z)$ and expansional $(\kappa)$ parameters and the refinement of hydrogen positions $(\mathrm{H} x y z)$. The lowering of molecular or atomic symmetry and the introduction of further multipoles or the refinement of anharmonic vibration (U3, U 4) might follow this procedure.

The objective of this procedure is the successful dissection of vibrational (U1-U4) and multipolar density $\left(\kappa, \kappa^{\prime}, P_{I m}\right)$ around the atom positions, because only the multipolar density $\left(\kappa, \kappa, P_{I m}\right)$ is included in the following evaluation of the charge density. This is al ready considered during the evaluation of a starting model. In order to prevent the vibrational parameters (U1, U2) from describing the anisotropic density, they are refined only against high-resolution data that hold little information about the valence density as illustrated in Figure 1.2. The samefigure shows that hydrogen can only be detected in the lowresolution range and therefore hydrogen positions are located in the difference Fourier map of the lowresolution data. Subsequently, $\mathrm{H}$ ydrogen positions ale al ways only optimized for the low-resolution data up to $d=1 \AA$ (or $\left.\sin (\theta) / \lambda=0.5 \AA^{-1}\right)$. 


\subsection{Evaluation in the Q uantum Theory of Atoms in Molecules}

The quantum theory of atoms in molecules (QTAIM) was developed by Richard Bader in the early 1970s (Bader et al., 1971; Bader \& Beddall, 1972, 1973; Bader, 1975, 1991). It allows for a detailed and unique analysis of the charge density (or electron density). Theanalysis of the densities' topology directly leads to chemical concepts, such as atoms, bonds, molecules, or charges. H owever, it has to be stated that the QTAIM only offers a concept of a bonding, based on the only observable, the charge density distribution. The bond itself is not a quantum mechanical observable (H aaland et al., 2004), but within the QTAIM framework, the concepts of atoms and bond are uniquely defined. The properties can be easily extracted by the analysis of the charge density topology in the form of a simple curve sketching.

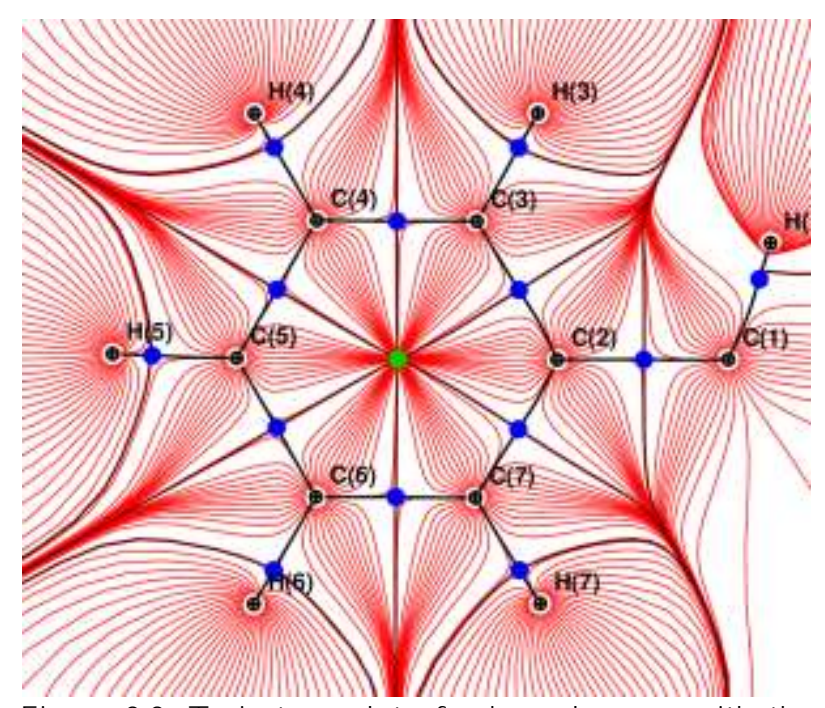

Figure 2.2: Trajectory plot of a benzyl group with the density gradients (red), atom positions (black dots), bond paths (black lines), BCPs (blue), RCP (green) and atomic basins (violet paths).

The first derivative of the charge density $\nabla \rho(\boldsymbol{r})$ is called the density gradient and the progression of the gradient trajectory is shown in Figure 2.2. Critical points are found in locations where the density gradient reaches zero $(\nabla \rho(\boldsymbol{r})=0)$. The critical point is further defined by the second derivatives of the density. This three dimensional derivation is given by the Hessian M atrix of $\rho(\boldsymbol{r})$

$$
H(\boldsymbol{r})=\left(\begin{array}{ccc}
\frac{\partial^{2} \rho}{\partial x^{2}} & \frac{\partial^{2} \rho}{\partial x \partial y} & \frac{\partial^{2} \rho}{\partial x \partial z} \\
\frac{\partial^{2} \rho}{\partial y \partial x} & \frac{\partial^{2} \rho}{\partial y^{2}} & \frac{\partial^{2} \rho}{\partial y \partial z} \\
\frac{\partial^{2} \rho}{\partial z \partial x} & \frac{\partial^{2} \rho}{\partial z \partial y} & \frac{\partial^{2} \rho}{\partial z^{2}}
\end{array}\right)
$$

Diagonalization of the Hessian matrix yields the principal axis and its eigenvalues $\lambda_{n}$, which also denote the curvatures of the matrix. The Laplacian of the density is given by the sum of eigenvalues.

$$
\nabla^{2} \rho(\boldsymbol{r})=L(\boldsymbol{r})=\frac{\partial^{2} \rho}{\partial x^{2}}+\frac{\partial^{2} \rho}{\partial y^{2}}+\frac{\partial^{2} \rho}{\partial z^{2}}=\lambda_{1}+\lambda_{2}+\lambda_{3}
$$

The sign of a dimensions' eigenvalue determines whether the extremum is a minimum or maximum in that direction. The rank of the critical point is given by thenumber of non-zero eigenvalues (usually 3), and is further classified by the algebraic sum of the eigenvalues' signs. This allows for four different types of critical points. An atom position is a maximum in the density, therefore all eigenvalues are negative $(3,-3)$. A t a bond-critical point (BCP), two eigenvalues are negative (3,-1). The BCP denotes a local minimum on a path of maximum density, connecting two atom positions. While BP and $B C P$ are indicativefor a bonded interaction, they are neither necessary nor the only true evidence for the presence 
of a chemical bond (Bader, 2009; Farrugia et al., 2006; Shahbazian, 2018; M acchi et al., 2002). In a ring critical point $(R C P)(3,+1)$, the curvature is positive in two directions, while negative in the third, and at a cage critical point $(C C P)$, the density rises in all directions $(3,+3)$.

For a molecule, the critical points have to fulfill the Poincaré-Hopf relationship (Hopf, 1927; Collard \& Hall, 1977) in order to be complete. The expression has its origin in the field of differential topology and is defined as:

$$
n_{a p}-n_{B C P}+n_{R C P}-n_{C C P}=1
$$

The electron density at a BCP is utilized to distinguish between various types of atomic interactions (Koritsanszky \& Coppens, 2001; Gatti, 2005; Gatti \& M acchi, 2012). The absolute value of the electron density at the BCP $\rho\left(\mathbf{r}_{\mathrm{BCP}}\right)$ corresponds generally to the strengths of the interaction. The Laplacian at the BCP $\nabla^{2} \rho\left(\boldsymbol{r}_{B C P}\right)$ provides information about the nature of the interaction. While a negative Laplacian corresponds to charge concentration and a covalent interaction, values near zero imply a dispersive or strongly polarized, and positive values an ionic interaction.

The ellipticity at the BCP $\epsilon\left(\boldsymbol{r}_{B C P}\right)$ is the maximum eigenvalue perpendicular to the bond $\lambda_{1}$ divided by its orthogonal value $\lambda_{2}$ as depicted in Figure 2.3. High values of the ellipticity generally correspond to an elliptic bond shape, like a double bond, while a low value corresponds to a spherical bond, i.e. a single or triple bond.

A tomic charges can be calculated by the integration of the electron density within the atomic basins, confined by thezero flux

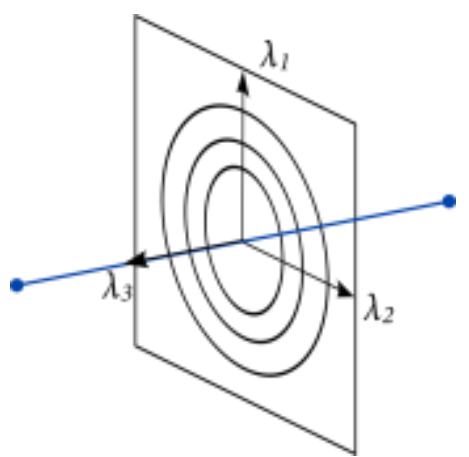

Figure 2.3: Spatial orientation of the eigenvalues at a BCP boundaries

$$
\nabla \rho(\boldsymbol{r}) \cdot \boldsymbol{n}(\boldsymbol{r})=0, \quad \boldsymbol{r} \in S(\boldsymbol{r})
$$

where $\mathbf{n}(\mathbf{r})$ is the normal to the boundary $S(\mathbf{r})$.

Further information about the charge distribution can be achieved by the analysis of the Laplacian $\nabla^{2} \rho(\boldsymbol{r})$. The quality of $\nabla^{2} \rho(\boldsymbol{r})$ determines, whether charge is locally concentrated, $\nabla^{2} \rho(\boldsymbol{r})<0$, or depleted, $\nabla^{2} \rho(\boldsymbol{r})>0$. V alence Shell Charge Concentrations (VSCCs), apparent as $(3,-3)$ critical points in $\nabla^{2} \rho(\boldsymbol{r})$, can be located around atoms and lead to valuable information about the hybridization state.

\subsection{The Combination of Theoretical and Experimental Charge Density}

The definitions of atoms in molecules are only valid and useful because the associated properties coincide with properties predicted by quantum mechanics (Bader, 1990). The charge density is quantum-mechanic observable that may be obtained from diffraction experiments or ab initio calculations likewise and is ther efore particularly comparable and comprehensible for both branches of science. Software like TONTO (Jayatilaka \& Grimwood, 2003) or DenProp (Volkov et al., 2016a) are 
capable of computing structure factors for theoretical models, so that theoretical models are applicable for experimental methods.

\subsubsection{AIMALL}

The AIM ALL suite (Keith, 2017) is a tool for the interpretation of DFT optimized wave functions within the QTAIM framework. It is partly derived from AIM PAC (Cheeseman et al., 1992) which was developed and maintained by members of Richard F.W. Bader's research group, and also the author of AIM ALL was also part of this group. AIM ALL is capable of reading wave function files from programs for DFT optimization e.g. Gaussian, calculating the charge density distribution of the molecule and subsequently interpreting the charge density within the QTAIM framework. The program offers easy access to all fundamental values via a graphical user interface (GUI). The scope of functions and results of AIM ALL are very well comparable with the results of tools for the QTAIM analysis of experimental charge densities, such as XD 2006 (V olkov et al., 2006) and M oPro (Guillot et al., 2001; J elsch et al., 2001; Jelsch et al., 2018).

\subsubsection{Crystal Explorer}

CrystalExplorer (Turner et al., 2018) is a program for the visualization of crystal structures and molecular properties. It offers an easy access to quantum mechanical calculations via the built-in TONTO framework (Jayatilaka \& Grimwood, 2003) and is therefore a unique tool for the elegant and comprehensive depiction of molecular properties and intermolecular interactions.

One function of CrystalExplorer, used in this thesis is the depiction of Hirshfeld Surfaces (Spackman \& Byrom, 1997;

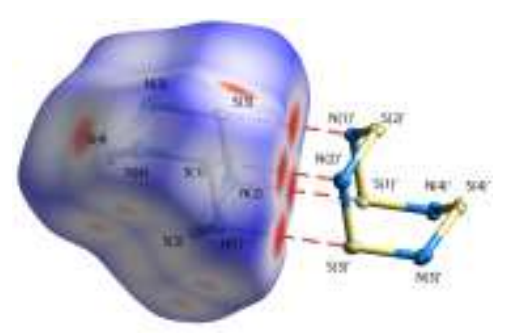

Figure 2.4: Hirshfeld surface of the intermolecular interactions in $\mathrm{S}_{4} \mathrm{~N}_{4}$. McKinnon et al., 2004). The structure is partitioned according to H irshfeld (1977), and a Hirshfeldsurface is calculated for a chosen fragment according to M cKinnon et al. (1998). The surface is then colored by different properties. Most importantly in this context, it is colored according to the interatomic distances: red, if the distance is smaller than the sum $V$ an der W aals radii, white if they are equal and red for distances smaller than the sum of $V$ an der $W$ aals radii (see Figure 2.4).

Furthermore, the energy of intermolecular interactions is calculated and plotted, corresponding to the molecules' symmetry equivalents. The energy of interaction between molecules is expressed in terms of four key components: electronic, polarization, dispersion, and exchange repulsion.

$$
\mathrm{E}_{\mathrm{tot}}=k_{\text {ele }} E_{\text {ele }}+k_{\text {pol }} E_{\text {pol }}+k_{\text {dis }} E_{\text {dis }}+k_{\text {rep }} E_{\text {rep }}
$$

Based on the experimental geometry, the monomer wave function is optimized. Different levels of theory are accessible for the optimizations (e.g. B3LYP/6-31G(d,p) or HF/3-21G). The interaction energies of pairs or clusters of molecules are calculated and can be depicted in color-schemed plots or as energy frameworks (Turner et al., 2015; M ackenzie et al., 2017). 



\section{Chapter 3 Assessment OF MOdel-AND DATA QUALITY}

\subsection{Introduction}

This thesis and experimental charge density in general is in many ways concerned with the correct assessment of model- and data quality. Which data are "good" and which parameters are sensible to refine? - These questions have to be answered for every refinement of a crystal structure model. Especially when it comes to the comparison of different datasets, strict standards in data treatment and refined parameters need to be applied (H erbst-Irmer \& Stalke, 2017).

\subsection{Figures of Merit: Data}

Several statistical tools are used to test the reasonability of the data and refined model. M ost of them are formulated as Residual- or R-values. These figures of merit (FOM ) serve as data- and modelquality indicators throughout the data processing, model refinement, and ultimately publication. But it has to be noted that the crystallographic R-values are neither the only, nor the single most important indicators for good structures (D estro et al., 2004). O ne widely accepted way to assess the quality of data is to check the deviation from a mean value of equivalent reflections.

$$
R_{\text {merge }}, R_{\text {int }}=\frac{\sum_{h} \sum_{i}\left|F_{o, i}^{2}(h)-\left\langle F_{o}^{2}(h)\right\rangle\right|}{\sum_{h} F_{o}^{2}(h)}
$$

Here the exact definition of equivalent reflections leads to different $R$-values. For $R_{\text {int }}$ the summation involves all input reflections for which more than one symmetry equivalent is averaged, while for $\mathrm{R}_{\text {merge }}$ (also known as $\mathrm{R}_{\text {sym }}$ or $\mathrm{R}_{\text {linear }}$ ), the summation involves all reflections with more than one absolute equivalent. The data precision is given by their $I / \sigma$ or the $R_{\text {sigma, }}$ which averages over the experimental errors, divided by the reflections' intensity.

$$
R_{\text {sigma }}=\frac{\sum_{h} \sigma\left(F_{o}^{2}(h)\right)}{\sum_{h} F_{o}^{2}(h)}
$$

However, all of those R-values are prone to misinterpretation, as they scale with the multiplicity (also called redundancy) of collected data. Ideally, during data collection all reflections are collected multiple times in order to obtain an accurate mean intensity and exclude the influence of systematical 
errors. To this means, the multiplicity-independent merging $R$-value $\left(R_{\text {rim }}\right.$ or $\left.R_{\text {meas }}\right)$ was introduced (Diederichs \& Karplus, 1997; W eiss, 2001).

$$
R_{\text {rim }}=\frac{\sum_{h} \sqrt{\frac{n}{n-1}} \sum_{i=1}^{n}\left|F_{o, i}^{2}(h)-\left\langle F_{o}^{2}(h)\right\rangle\right|}{\sum_{h} F_{o}^{2}(h)}
$$

It gives a correct, multiplicity-independent estimation for the mean precision of individual reflections. The precision indicating merging $\mathrm{R}$-value $\mathrm{R}_{\text {pim }}$ on the other hand gives a measure for the precision of the mean intensity of a reflection (W eiss, 2001).

$$
R_{\text {pim }}=\frac{\sum_{h} \sqrt{\frac{1}{n-1}} \sum_{i=1}^{n}\left|F_{o, i}^{2}(h)-\left\langle F_{o}^{2}(h)\right\rangle\right|}{\sum_{h} F_{o}^{2}(h)}
$$

Multiplicity-dependent R-values will inevitably increase with raising multiplicity and indicate worse data. However, high multiplicity denotes a more careful screening of the crystal and should therefore not affect the R-valuenegatively. It is therefore argued that the $R_{\text {rim }}$ and $R_{\text {pim }}$ are better assessors for the data quality, than the more commonly used $R_{\text {merge. }}$
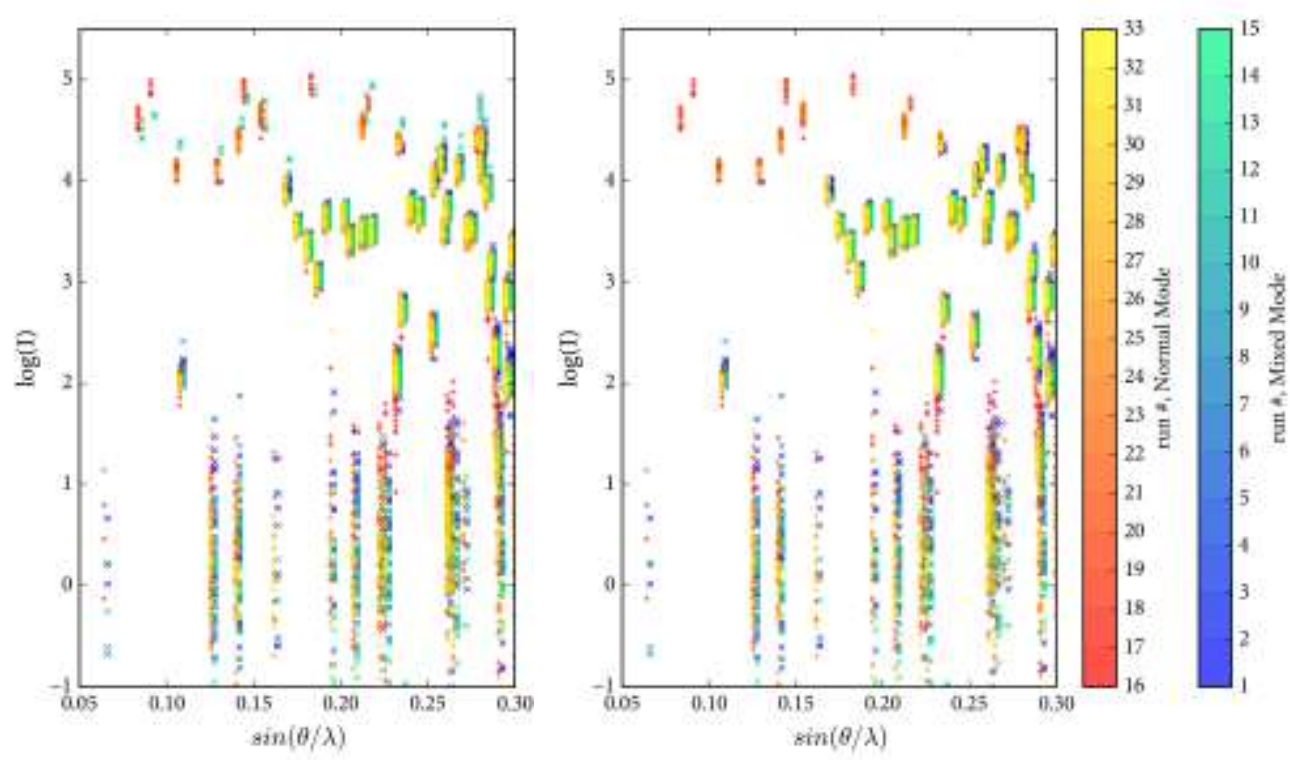

Figure 3.1: Resolution-dependent evaluation of observed intensities, coloured by the run number. In the right graph, run 12, which does not accord to all other collected runs, was omitted.

Simply looking at the data can unveil specific effects within it. In Figure 3.1, the reflection intensity from various runs is plotted against resolution, yielding the repetitive measurement of the same reflection as accumulations of points at the same resolution. In the left image, run 12 (turquoise) stands out, as it is the only run yielding high-intensity reflections from the "mixed mode" subset (shades of blue) that differ significantly from the other intensities of the corresponding reflection. It could therefore be determined that run 12 is affected by some sort of systematic error. 
Kay Diederichs (2010) suggested to plot the significance $I / \sigma$ versus the logarithm of the intensity in order to highlight features within the collected data and assess the data quality. The highest absolute significance is well observableas criterion for the data quality. Furthermore, it can be monitored, whether parts of the data do not concur with the rest. The plot was originally designed for macromolecular crystallography, but has been adopted to SADABS and therefore also became a standard tool in small molecule crystallography. For the use in this thesis, the Diederichsplot was extended by the application of a run-dependent coloration, highlighting the

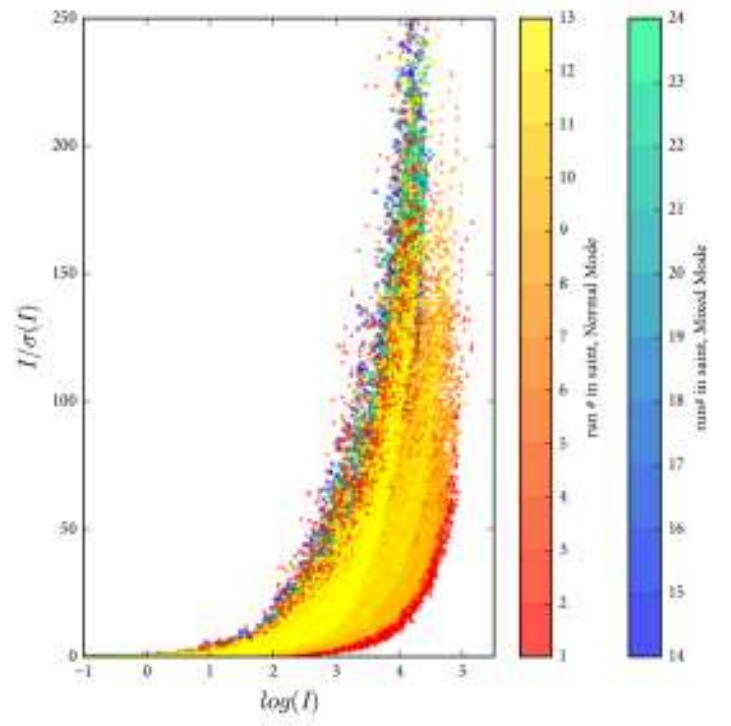

Figure 3.2: Diederichsplot coloured by run number. (Diederichs, 2010) course of significance for each run and allowing a better detection of outliers. For the given examplein Figure 3.2, the differences between the two subsets "Normal Mode" (shades of blue) and "Mixed Mode" (shades of orange) are clearly visible. While the "Normal Mode" shows reflections of higher absolute intensity ( $X$-axis), the "Mixed Mode" yields higher significance (y-axis).

\subsection{Figures of Merit: Model}

The resulting values from the structure refinement as outlined in 1.6 are also commonly displayed as R-values, showing the agreement of model and data. R1 and R2 give the unweighted agreement.

$$
\begin{aligned}
R 1 & =\frac{\sum_{h k l}|| F_{o}(h k l)|-| F_{c}(h k l)||}{\sum_{h k l}\left|F_{o}(h k l)\right|} \\
R 2 & =\sqrt{\frac{\sum_{h k l}\left\{F_{o}^{2}(h k l)-F_{c}^{2}(h k l)\right\}^{2}}{\sum_{h k l}\left\{F_{o}^{2}(h k l)\right\}^{2}}}
\end{aligned}
$$

$w R 1$ and $w R 2$ also incorporate the weighting factor $w$ and are therefore subjected to the weighting scheme, outlined in 1.6.

$$
w R 2=\sqrt{\frac{\sum_{h k l} w\left\{F_{o}^{2}(h k l)-F_{c}^{2}(h k l)\right\}^{2}}{\sum_{h k l} w\left\{F_{o}^{2}(h k l)\right\}^{2}}}
$$

The goodness of fit (GOF) yields a measure of accordance between the divergences between calculated and observed intensities and their expected error, given by the reflections' weight.

$$
\mathrm{GOF}=\sqrt{\frac{\sum_{h k l} w\left\{F_{o}^{2}(h k l)-F_{c}^{2}(h k l)\right\}^{2}}{n_{o b s}-n_{\text {par }}}}
$$


The GOF is normalized by the number of data $n_{\text {obs }}$ and the number of parameter $n_{\text {obs. }}$. If the divergence is within the expected range, the GOF yields unity. However, the GOF is highly dependent on the weighting scheme, which is usually optimized for the GOF to reach unity. If no weighting scheme is applied, the GOF yields the accordance of models' deviation from the data and the experimental error.

These indicators deliver an estimate of the model quality, but they can only provide information about the agreement of model and data and do not judge the reliability of the derived model. Artifacts or systematic errors cannot be determined.

\subsubsection{Residual D ensity Analysis}

Even after a refinement of the aspherical density around atom positions in the multipole approach, the observed and calculated structure factors do not correspond perfectly. The differences of observed and calculated structure factors can be analyzed as the residual in a difference Fourier map. This is established by the Fourier transformation of the difference $\left|F_{o}^{2}(h k l)\right|-\left|F_{c}^{2}(h k l)\right|$ with the phases of the model. The residual density can be analysed for the maximal values and is superimposed with the structure for the highlighting of features.

In order to further qualify the residual density, M eindl \& Henn (2008) suggested several quality indicators for the residual density and the residual density distribution. The Residual density is calculated for a number of grid points in $x, y, z$ of the unit cell and the distribution of the residual density $\rho_{0}$ for the fractal dimension $\mathrm{d}^{f}$ are plotted. The height of the fractal dimension plot in Figure $3.3 \mathrm{~d}^{\mathrm{f}}(0)$ corresponds with the number of grid points with a residual density of zero and subsequently the featurelessness of the residual density. The broadness of the plot $p(d=2)$ corresponds to the distribution of nonzero residual density and therefore the flatness of the residual density. The total broadness of the plot corresponds to minima and maxima of the residual density. It can therefore be shown in one simple plot whether the residual density is flat and featureless. Furthermore, Meindl \& Henn established a measure for the total divergence of model and data egross. The factor is half of the integrated absolute residual density.

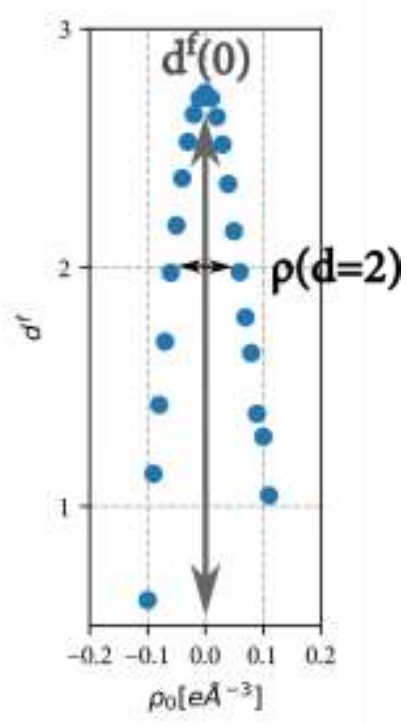

Figure 3.3: Residual density distribution plot (Meindl \& Henn, 2008).

$$
e_{\text {gross }}=\frac{1}{2} \int_{V}\left|\rho_{0}(\boldsymbol{r})\right| d^{3} \boldsymbol{r}
$$

The residual density is highly dependent on the grid size for which it is calculated, therefore only residual densities that were calculated with the same resolution are comparable. 


\subsubsection{DRKplot}

Systematic errors can often be highlighted by the resolution dependent divergence of observed and calculated intensities. The calculation of such plots is established in the DRK plot tool (Zavodnik et al., 1999; Zhurov et al., 2008). For the given example in Figure 3.4, the divergence $\sum F_{o}^{2} / \sum F_{c}^{2}$ is plotted for resolution shells in $0.5 \AA^{-1}$ steps. Thereby global, resolutiondependent systematic errors can be identified. In the given example, the progression of the divergence shows a characteristic "W"-shape that was associated with the effects of thermal diffuse scattering (TDS) (Willis, 2001) by Niepötter et al., 2015. The data were therefore corrected for TDS according to $\mathrm{N}$ iepötter et al.. It is widely accepted, that divergence must not be larger than $5 \%$.

Another interesting plot from the DRK plot tool is the normal probability plot. It is complementary to the GOF and tests the normal distribution of the divergence of observed and calculated structure factors. The plot shows the distribution of the experimental divergence against the
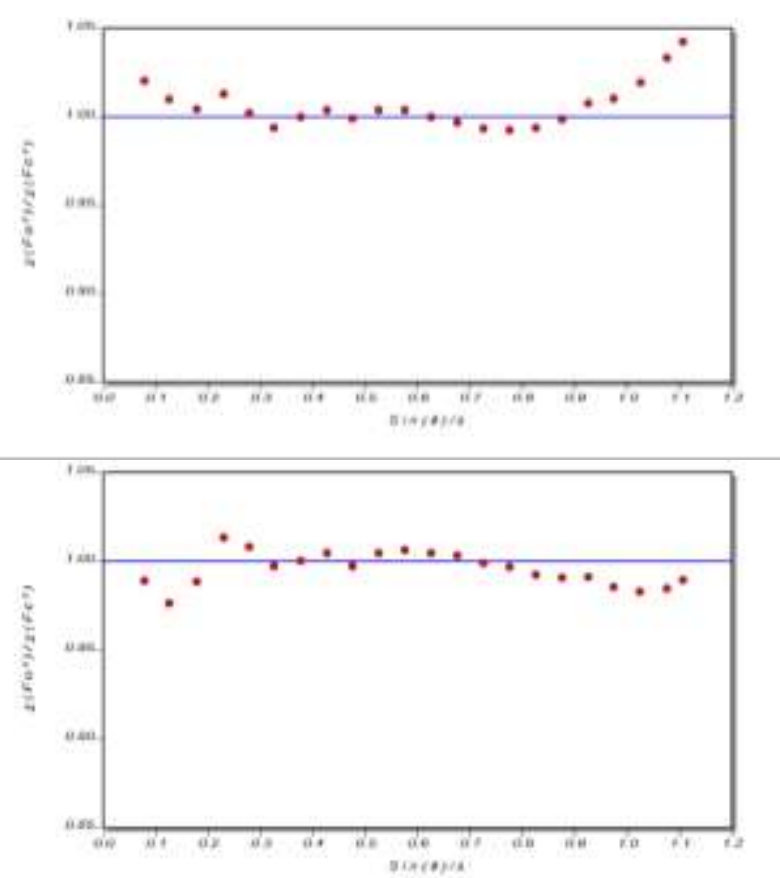

Figure 3.4: Divergence plots before (top) and after (bottom) TDS correction (Zavodnik et al., 1999; Zhurov et al., 2008)
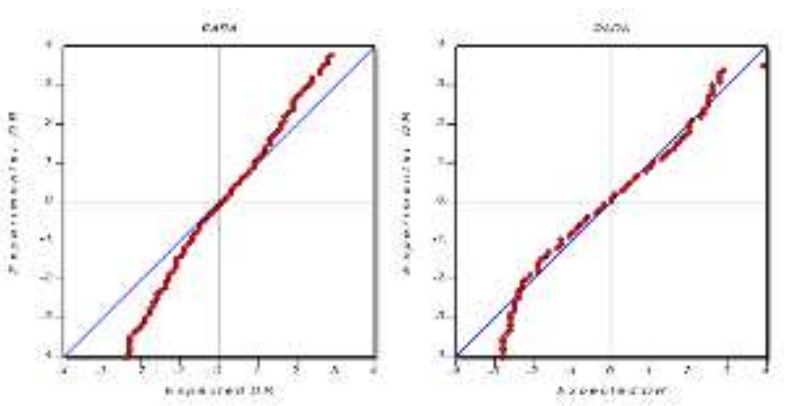

Figure 3.5: Normal probability plots without (left) and with (right) adaptation of a weighting scheme. expected normal (Gaussian) distribution and is globally better known as Quantile-Quantile (or Q-Q) plot. If the divergences are distributed normally, the plot shows unity. For the given example in Figure 3.5 , the divergence distribution is brought closer to a normal distribution by the application of a weighting scheme.

\subsubsection{Cross Validation}

One of the perils of the experimental charge density is the option to refine dozens of additional parameters per atom. By the introduction of many additional parameters to the model, the agreement with the data will inevitably increase. H owever, at a certain point, the parameters no longer fit the overall trends of the data, but the individual errors of the data points. This phenomenon is called overfitting. The question that needs to be answered is how many and which parameters areallowed to be refined for the current dataset. It is widely accepted that in any case, the ratio of data to parameter should never fall 
below ten. However, it is not necessarily true that this will prevent overfitting. In macromolecular chemistry, the statistical technique of cross-validation is therefore a viable tool to prevent overfitting. (Brünger, 1992, 1997; Zarychta et al., 2011). This technique has been adapted for small molecule (Lübben \& Grüne, 2015) and charge density refinements (Paul et al., 2011; Krause et al., 2017). As single reflections can have huge influence on the model in a charge-density refinement, a k-fold cross validation is employed, in contrast to macromolecular crystallography, where usually only one "free" set is used.

In a k-fold cross validation, the dataset is divided into $k$ sets of equal size as indicated in Figure 3.6. One part of the dataset is excluded from the refinement as free set and a model refinement is
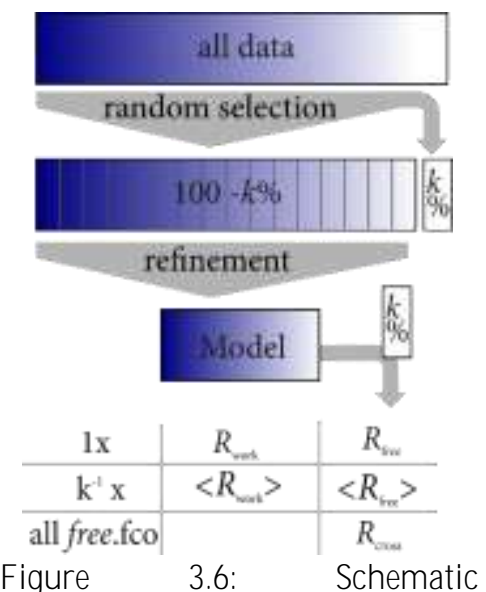
Figure representation of the $\mathrm{k}$ fold cross validation technique. (Krause et al., 2017) performed for the residual work set. After refinement, R-values are calculated for the accordance of the model with the work and free sets as $R_{\text {free }}$ and $R_{\text {work. }}$. This process is permutated $k$-fold for each of the $k$ subsets and mean values for $R_{\text {free }}$ and $R_{\text {work }}$ are accessible. Furthermore, the cross-validation $R$-factor $R_{\text {cross }}$ is calculated for the $F_{d} / F_{0}$-data from all free sets that combined yield a full dataset. $R_{\text {cross }}$ and $\left\langle R_{\text {free }}\right\rangle$ usually yield similar values but in praxis, $R_{\text {cross }}$ has been shown to be more reliable. For each step in the refinement, the progression of $R_{\text {cross }}$ and $R_{\text {work }}$ is observed. In order to not over-fit the data, the progression of $R_{\text {cross }}$ and $R_{\text {work }}$ should be similar. If a drop in $R_{\text {work }}$ is accompanied by a rise in $R_{\text {cross, }}$ the model was obviously fitted to the errors of the dataset and no longer fits its general trends. 


\section{Chapter 4 Dibenzyldiselenide - A RADICAL Challenge fOR the EXPERIMENTALSETUP}

\subsection{Introduction}

Dibenzyldiselenide (BzSe) ${ }_{2}$, first described by Jackson in 1875 , is an interdisciplinary fascinating compound. The interplay of selenium with radiation is physically interesting. In life sciences, selenium is introduced in biomolecules in order to conquer the phase problem in macromolecular crystallography. (BzSe) ${ }_{2}$ can be seen as a model compound for organoselenium compounds and diselenide bridges in such compounds. Chemically, (BzSe) ${ }_{2}$ and organoselenium compounds in general show a vivid photochemistry and are used as photocatalysts (D evillanova, 2007; Ortgies \& Breder, 2017). From the viewpoint of experimental charge density, the molecule in its solid state structure is interesting, because of the chalcogen-typical gauche-formation (Holleman et al., 2007) and chalcogen-chalcogen interactions that play an important role in the buildup of the crystal. The whole scope of $(\mathrm{BzSe})_{2}$ properties is of great importance for the findings in this work.

\subsubsection{Selenium and Radiation}

Selenium's relationship with radiation, especially regarding the interplay with X-rays, shows some interesting features. Selenium exhibits a relatively high $X$-ray absorbance, at least for the context of experimental charge density investigations, and with its $\mathrm{K}$-absorption-edge at $12.7 \mathrm{keV}$ selenium also emits $\mathrm{K}_{\alpha}$ radiation at $11.2 \mathrm{keV}$ - in the same range as radiation used for $\mathrm{X}$-ray diffraction (see Figure 4.1). This feature is called $X$-ray fluorescence and occurs with a fluorescence yield of 0.567 (Lederer et al., 1978). X-ray fluorescence can lead to problems in the collection of diffraction data (Alexandropoulos et al., 2006; Shmueli, 2010).

Amorphous selenium is used as a semiconductor in an X-ray imaging technique called xeroradiography (Boag, 1973). Here, charge-separations are induced within the semiconductor layer and read out upon X-irradiation. Classical "X-rays" are created this way. Selenium-based large area Xray detectors have been developed since the early 2000 years (e. g. by marXperts), but were technologically not longer pursued any further (e. g. Sultana et al., 2008). This, however, would not rule out any options based on amorphous selenium in the future. So it can be noted, that electronic shifts can be introduced in selenium by $\mathrm{X}$-rays. 


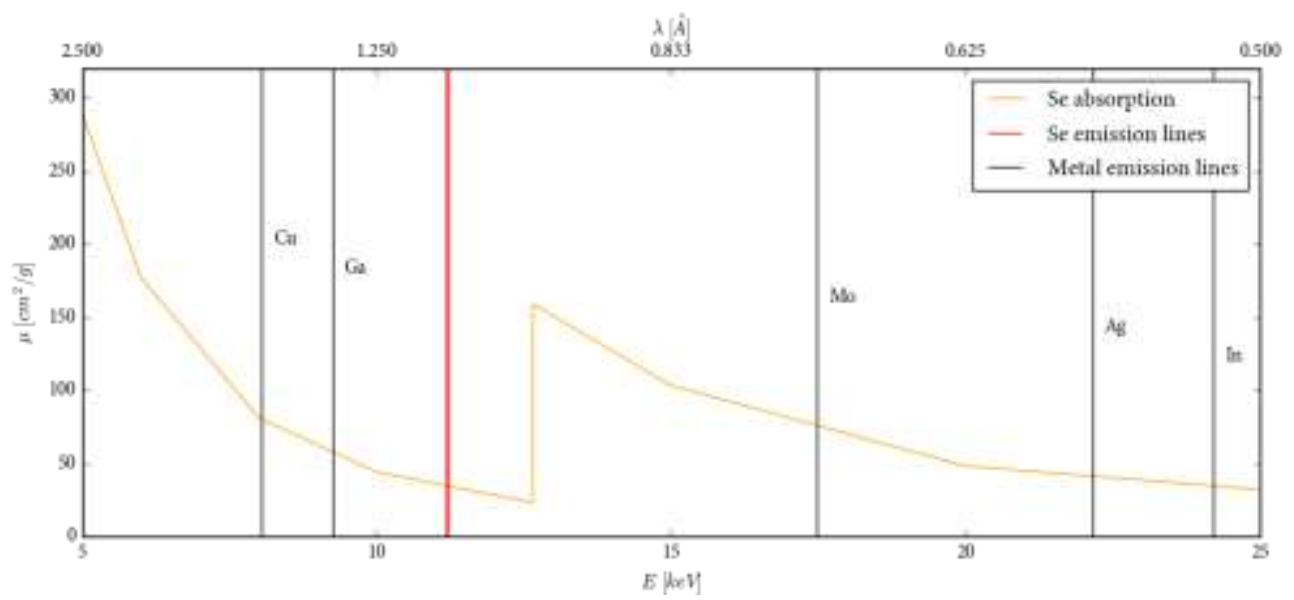

Figure 4.1: Selenium X-ray absorption spectra, $\mathrm{Se}_{\mathrm{a}_{1} 1}$ and $\mathrm{K}_{\mathrm{a} 2}$ lines and emission lines of metals, used in X-ray sources (Bearden, 1967).

\subsubsection{Selenium in Macromolecular XRD}

In macromolecular crystallography, solving of the phase problem is often a crucial and demanding step in the structure determination. One of the most important tools to tackle this problem is multiwavelengths anomalous diffraction (M AD) phasing (Hendrickson, 1991; Smith et al., 2006). In M AD, single crystal diffraction data are collected for multiple wavelengths. Then, a Fourier transformation is performed for the differences of the structure factors at two different wavelengths. As only the anomalous signal is dependent on the wavelengths, in this difference Fourier map only the maxima of anomalous signal can be observed. M oreover, as biomolecules contain mainly light elements with weak anomalous signal, only the positions of heavy atoms with strong anomalous signals are obtained. For these heavy atom positions, the phase problem can be resolved and, starting from the heavy atom positions, the structure can be refined. Naturally occurring biomolecules only rarely contain heavy atoms, therefore sulfur and oxygen in specific positions are frequently substituted for selenium (H endrickson et al., 1990; W alden, 2010; Lin et al., 2011; M etanis \& Hilvert, 2014; Pike et al., 2016). One typical example of this technique is the introduction of selenomethionine in proteins.

However, this approach also causes problems. On the one hand, it has to be tested whether the structures of selenated and wild type biomolecules are the same. On the other hand, biomolecules suffer from radiation damage. Especially the high brilliance and intensity of synchrotron beams leads to the decomposition of the crystal (Banumathi et al., 2004; Nave \& Garman, 2005). Moreover, the predominant sites of radiation damage are heavy atoms, like selenium. In the diffraction pattern, radiation damage is observable in the weakening of all reflections and in the crystal structure: the heavy atoms seem to vanish over time. In fact, electronic changes are induced, bonds are broken, and the atoms react with the surrounding crystal water. This feature again can be used for phasing. Heavy atom sites are more susceptible to radiation damage. The Fourier map of the difference in the data collected early and late during the diffraction experiment thus shows the sites of the heavy atoms. This technique is called radiation-damage induced phasing (RIP) (Zwart et al., 2004; Sanctis et al., 2016). By irradiation 
with intense UV -light, such radiation damage can be induced intentionally and is exploited in the UV RIP technique (Leiros et al., 2006; Sanctis et al., 2011).

\subsubsection{Reactivity of (BzSe) 2}

Dibenzyldiselenide (1) is widely used as a synthetic agent in selenium chemistry and therefore readily available. It shows a rather interesting photochemistry. By the irradiation with UV -light, $(\mathrm{BzSe})_{2}$ decomposes to dibenzylselenide and elemental selenium (a) (Stanley et al., 1974). If air-exposed, it decomposes to benzaldehyde and selenium (b) and under an atmosphere of tetrachlorocarbon, it decomposes to benzlychloride and selenium (Chu et al., 1975) (c).
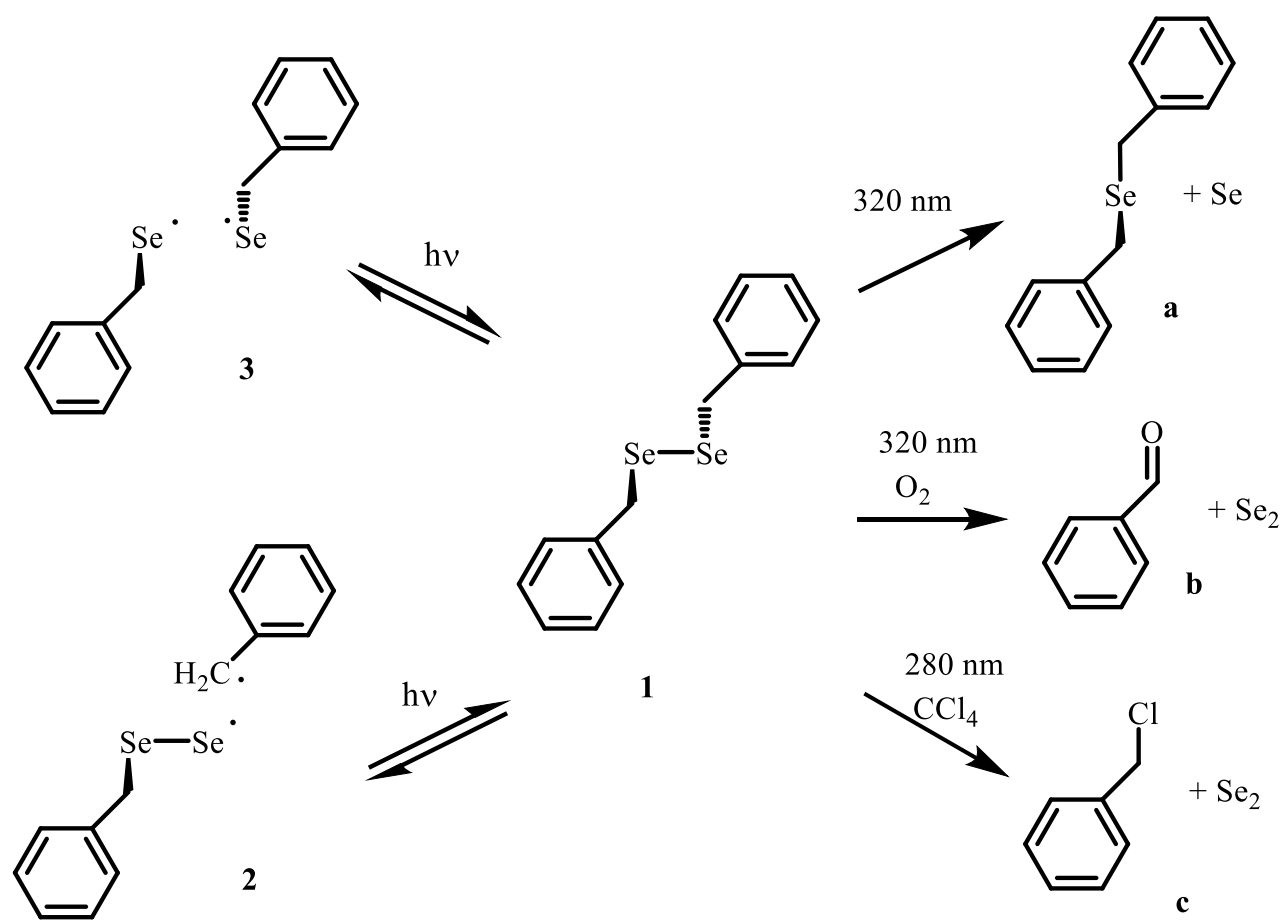

Figure 4.2: Reactivity of (BzSe) $)_{2}$.

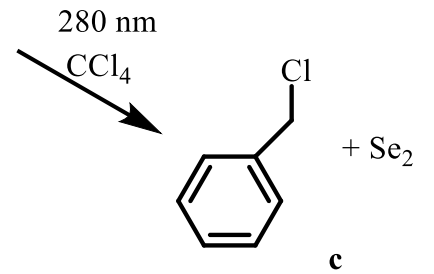

All three reactions $(\mathbf{a}-\mathbf{c})$ proceed via a radical pathway, starting either with the hemolytic photolytic cleavage of the $\mathrm{Se}$ Se or $\mathrm{Se}-\mathrm{C}$ bond. The reactions according $\mathbf{a}$ and $\mathbf{b}$ are observable in organic solvents as well in the solid state (BzSe) 2 . Because of this reactivity it is argued that the dissociation of the Se-C bond (3) is preferred in contrast to $(\mathrm{PhSe})_{2}$, were the Se-Se dissociation is preferred (Deryagina et al., 1993). An investigation of UV -irradiated (BzSe) ${ }_{2}$ by electron paramagnetic resonance (EPR) found a broad signal at $\mathrm{g}=2.003$, that was tentatively assigned to the benzyl-radical (Windle et al., 1964). 


\subsubsection{Structural Features}

(BzSe) ${ }_{2}$ crystallizes in the centrosymmetric scape group $\mathrm{C} 2 / \mathrm{c}$. The solid-state structure is shown in Figure 4.4. The molecule itself is in gaucheformation with a dihedral angle close to $90^{\circ}$. The gauche-formation is generally argued to bethe result of the least possible overlap of the two double-populated orbitals and is common for all di- and polychalcogens (Steudel,

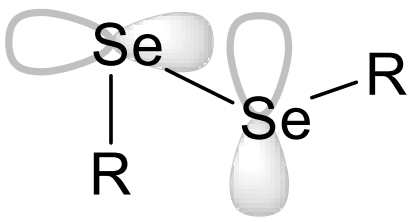

Figure 4.3: V isualization of the fullpopulated Se p-orbital. 1975a, 1975b; Zaccaria et al., 2016). In the case of oxygen the gauche-formation results from the overlap of sp-orbitals, while for sulfur and heavier chalcogens, the p-orbitals are responsible as illustrated in Figure 4.3 (Aida \& Nagata, 1986).

An experimental charge density determination might model these orbitals. The sum of three singularly populated $p$-orbitals would result in a close to isotropic density around selenium and only the double-populated p-orbital as two ED maxima perpendicular to the Se-Se-R plane might be visible in the anisotropic density.

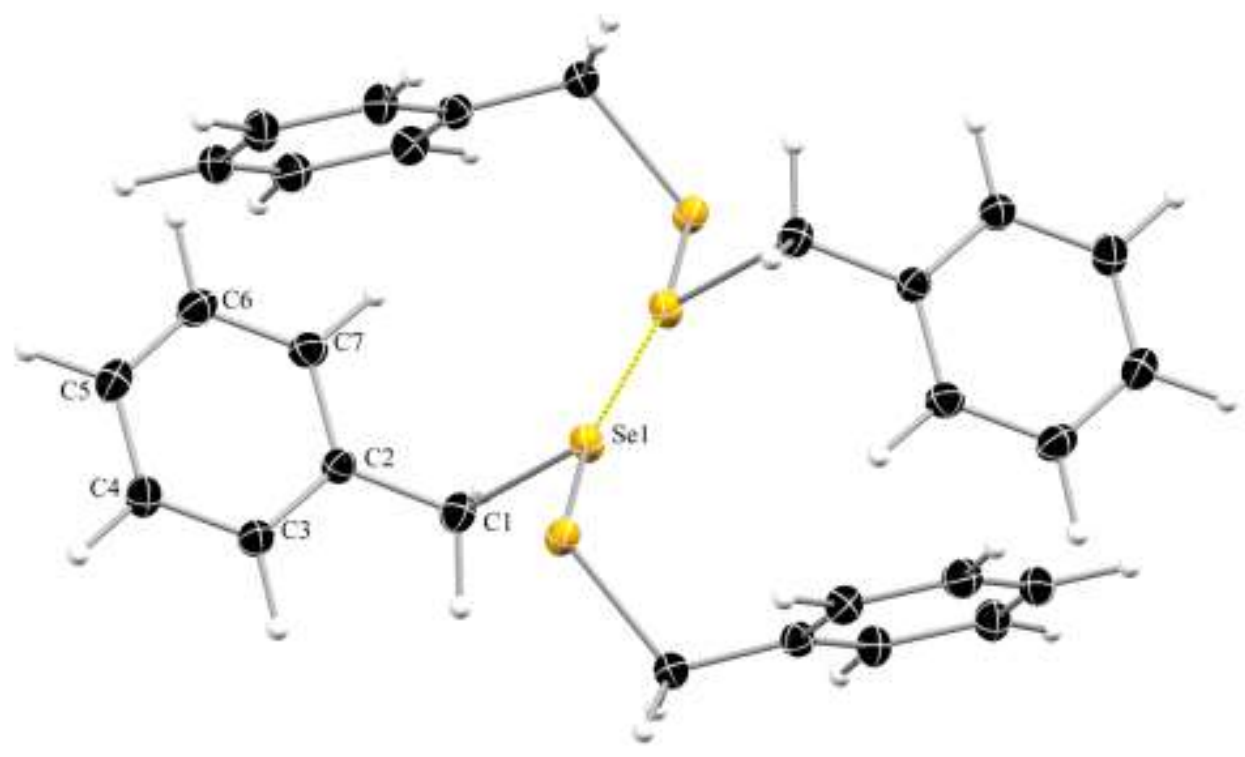

Figure 4.4: Crystal structure of $(\mathrm{BzSe})_{2}$ based on dataset $\mathrm{C}$. The structure contains half a molecule per asymmetric unit. Selected bond lengths $[\AA]$ ] and angles [ ${ }^{\circ}$ ]: Sel-Sel 2.31532(10), Sel-C1 1.9841(4), intermolecular Se1-Se1 3.4430(1), C1-Se1-Se1 101.11(1), C1-Se1-Se1-C1 93.66(1)

The second interesting structural feature of $(\mathrm{BzSe})_{2}$ is the very short intermolecular Se...Se distance of $3.44 \AA$, well below the sum of $V$ an der $W$ aals radii (3.8 $\AA$ ). The short distance implies strong chalcogen-chalcogen interactions. Intermolecular strands of selenium are thus formed throughout the crystal. 

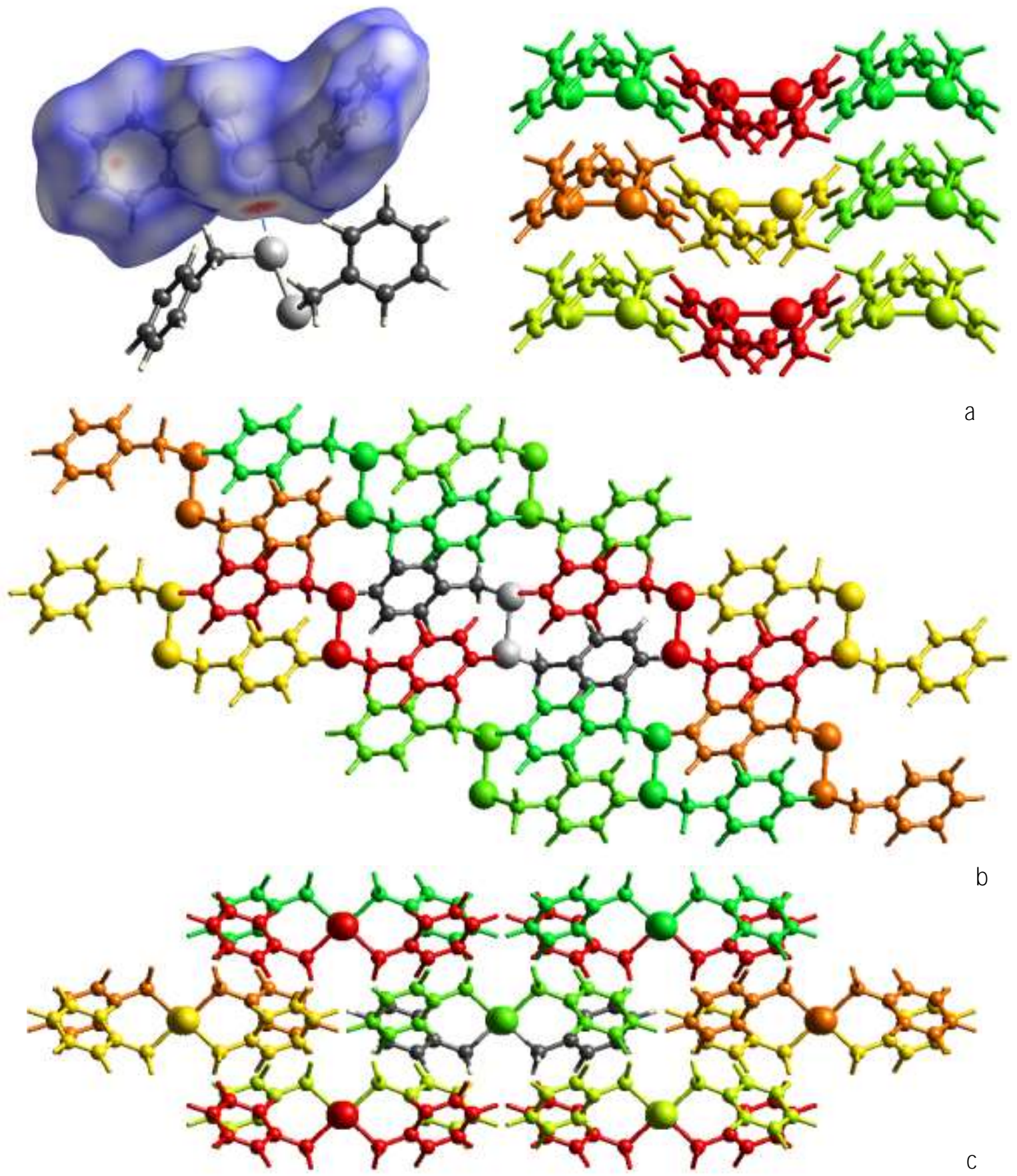

b C

Figure 4.5: Hirshfeld-Surface and crystal lattice along viewing axes $a, b$ and c, determined with CrystalExplorer (Turner et al., 2017). Interaction energies are determined for the black/grey central molecule and its colour-schemed symmetry equivalents. 
Table 4.1: interaction energies in $\mathrm{kJ} / \mathrm{mol}$ at the HF/3-21G theory level, calculated by CrystalExplorer (Turner et al., 2017). $R$ is the distance of molecular centroids. $E_{\text {ele: }}$ electrostatic, $E_{\text {pol: }}$ polarization, $E_{\text {dis: }}$ : dispersive and $E_{\text {tot: }}$ total interaction energy.

\begin{tabular}{|l|l|l|l|l|l|l|l|}
\hline & Symmetry operation & $R$ & $E_{\text {ele }}$ & $E_{\text {pol }}$ & $E_{\text {dis }}$ & $E_{\text {rep }}$ & $E_{\text {tot }}$ \\
\hline & $x+1 / 2, y+1 / 2, z$ & 7.94 & -7.8 & -2.5 & -36.4 & 24.6 & -22.4 \\
\hline & $-x,-y,-z$ & 14.00 & -4.5 & -0.6 & -11.5 & 7.9 & -8.9 \\
\hline & $x, y, z$ & 13.7 & -1.4 & -0.3 & -8 & 2.6 & -6.7 \\
\hline & $-x+1 / 2,-y+1 / 2,-z$ & 8.89 & -11.9 & -3.3 & -35.6 & 20.1 & -30.1 \\
\hline & $-x,-y,-z$ & 5.77 & -13.7 & -2.8 & -33.1 & 31.6 & -20 \\
\hline & $-x+1 / 2,-y+1 / 2,-z$ & 9.40 & -9.6 & -1.8 & -16.4 & 9.9 & -17.6 \\
\hline
\end{tabular}

A theoretical investigation in CrystalExplorer (Turner et al., 2018) at the HF/3-21G level of theory discloses strong dispersive interactions for the selenium strands along the crystallographic c-axis $([-x,-y,-z]$ symmetry equivalent, green). Furthermore, strong dispersive interactions to the $[x+1 / 2$, $y+1 / 2, z]$ equivalent (red) and $[-x+1 / 2,-y+1 / 2,-z]$ equivalent (light green) are found. As the dispersive interactions are strong and the phenyl groups of the symmetry equivalents are coplanar, $\pi$ stacking is probably the most important intermolecular interaction in the crystal.

An experimental charge density determination, it would be advantageous to characterize these interactions. Especially the chalcogen-chalcogen interactions should be accessible in the form of an intermolecular bond critical point (BCP) between the selenium atoms. It is expected to find a BCP with modest electron density and a low, positive Laplacian, corresponding to a rather strong, non-covalent interaction.

\subsubsection{Experimental Challenges}

In the context of experimental charge density, selenium is a rather heavy element with high X-ray absorption (see Figure 4.1). X-ray absorption leads to reduced diffraction intensities and possible errors during data scaling and structure refinement (M urray et al., 2004; M aslen, 2006). In order to minimize this effect, shorter $X$-ray wavelengths from silver or indium radiation sources can be used. However, harder radiation brings some challenges with it. On the one hand, these radiation sources are generally weaker and on the other hand, the radiation is generally harder to detect.

The second problematic feature of selenium is the X-ray fluorescence. The fluorescence leads to an isotropic glow of the irradiated crystal, noticeable as an elevated background during data collection. The correct determination of the reflection intensities over this elevated background is challenging for the detector setup and the integration procedure. Because of these experimental challenges, $(\mathrm{BzSe})_{2}$ was selected as a benchmark structure for a comparison of the latest generation of $X$-ray sources and detectors. High-intensity radiation sources as rotating anodes or M etall et sources combined with Dectris Pilatus3 300K CdTe or Bruker Photon2 detectors were applied. 


\subsection{Data Collection and Processing}

A series of datasets was collected from the same or similar crystals at a range of in-house single crystal diffractometers, equipped with different detectors and radiation sources. The employed detectors were the Bruker SM ART APEX II (short A pex2), the Dectris PILATUS 3 300K CdTe (short Pilatus3) the Bruker Photon 2 (short Photon2) and the Bruker Photon 3 (short photon 3).

As the used detectors are the subject of an extended data quality assessment, they are described and discussed in detail in Chapter 5. The used X-ray sources were a Bruker SRA TXS rotating anode (1.2 kW) with molybdenum as anode material (short M o TXS) and high brilliance Incoatec M icrofocus Sources $(I \mu S)$ of the second $(30 \mathrm{~W})$ and third $(70 \mathrm{~W})$ generation with silver as anode material (short $\mathrm{Ag}$ $\mathrm{I} \mu \mathrm{S} 2$ and $\mathrm{Ag} \mid \mu \mathrm{S} 3$ ). Furthermore, a Rigaku M icroM ax 007 rotating anode with silver as anode material and an Excillum MealJet, equipped with optics for indium radiation were used. All datasets were collected from crystals of similar size and at the same temperature of $100 \mathrm{~K}$

All data were integrated in SAINT v8.37A (Bruker AXSInc., 2016). Therefore all images collected with Pilatus3 detectors had to be converted into the Bruker frame format via a Python script (Ruth, 2017). In order to cope with the high background and in order to adjust the integration defaults to the specific datasets, series of integrations have been iterated for each dataset, testing different SA INT input values. Parameters that had a potentially positive effect on the determined intensities were the integration box-size (SPOTSIZE, YSPOTSIZE, SPREAD, short: box size) and the number of points for the peak profile fit (PROFXHALF, PROFYHALF, PROFZHALF, short: peak profile). Furthermore, variations in the default values of the strong and weak reflection limits (STRONGTHRESH and LS_IOVS_MAX, short: StrT and LSIOS), the background correlation lengths (BGCORSCALE, short $\mathrm{BgCS})$, and the background determination procedure (PLANEBG) were customized.

For the iteration over integration input values, the whole process of integration, scaling, and refinement was automated via a Python (Guido van Rossum) script. Thus, it was possible to iterate dozens of different integrations and refinements for each dataset.

In order to retain comparability, all datasets were integrated to a resolution of $0.45 \AA$ and scaled in SA DABS (Krause et al., 2015b). No weighting scheme was applied, so that the experimental weights were retained. All datasets meet the requirements for an experimental charge density investigation with full completeness, high multiplicity and with significant reflection intensities up to a very high resolution. Data quality statistics of all datasets are shown in Tables S4.1 to S4.7 in the A ppendix and are partly discussed in Chapter 5.

\subsubsection{Mo TXS and Apex2}

Dataset $\mathbf{A}$ was collected on a Bruker D 8 3-circle goniometer, equipped with a $1.2 \mathrm{~kW}$ TXS rotating anode $\left(\mathrm{M} \mathrm{OK}_{\mathrm{a}}\right.$ radiation), M ontel M irror optics and a Bruker APEX II detector. The dataset was collected at the Institut für Anorganische Chemie in Göttingen and represents the standard setup for charge density datasets in the Stalke working group with a high intensity $X$-ray source and the well-established 
Table 4.2: Overview of collected (BzSe) ${ }_{2}$ datasets.

\begin{tabular}{|c|c|c|c|c|c|c|c|}
\hline Dataset & A & B & C & D & E & $\mathbf{F}$ & G \\
\hline Detector & Apex2 & A pex2 & Pilatus 3 & Pilatus 3 & Photon2 & Photon2 & Photon3 \\
\hline X-ray source & TXS & $\mathrm{I} \mu \mathrm{S}$ & $\mathrm{I} \mu \mathrm{S}$ & 007 & $\mathrm{I} \mu \mathrm{S} 3$ & M etaljet & $\mathrm{I} \mu \mathrm{S} 3$ \\
\hline Power [W ] & 1200 & 30 & 30 & 1200 & 70 & 140 & 70 \\
\hline$\lambda[\AA]$ & 0.71073 & 0.56086 & 0.56086 & 0.56086 & 0.56086 & 0.5136 & 0.56086 \\
\hline $\mathrm{a}[\AA]$ & $13.7033(10)$ & $13.717(2)$ & $13.7123(18)$ & $13.7199(11)$ & $13.7247(7)$ & $13.7299(6)$ & 13.7185(3) \\
\hline $\mathrm{b}[\AA]$ & $8.0012(6)$ & $8.0127(13)$ & $8.0051(11)$ & $8.0119(6)$ & $8.0053(4)$ & $8.0135(4)$ & 7.99970(11 \\
\hline$c[\AA]$ & $11.4769(8)$ & $11.4944(18)$ & $11.4849(15)$ & $11.4766(9)$ & $11.4831(6)$ & $11.4875(5)$ & 11.4813(2: \\
\hline$\beta\left[^{\circ}\right]$ & $99.293(2)$ & $99.303(7)$ & $99.260(5)$ & $99.301(2)$ & $99.2776(16)$ & $99.2957(15)$ & $99.2494(6)$ \\
\hline$V\left[\AA^{-3}\right]$ & $1241.84(16)$ & $1246.8(3)$ & $1244.3(3)$ & $1244.95(17)$ & $1245.15(11)$ & $1247.31(16)$ & $1243.63(5$ \\
\hline $\begin{array}{c}\text { Crystal size } \\
\max [\mathrm{mm}]\end{array}$ & 0.420 & 0.330 & 0.330 & 0.4 & 0.4 & 0.4 & 0.359 \\
\hline $\operatorname{mid}[\mathrm{mm}]$ & 0.259 & 0.255 & 0.255 & 0.3 & 0.3 & 0.3 & 0.163 \\
\hline $\min [\mathrm{mm}]$ & 0.136 & 0.178 & 0.178 & 0.2 & 0.2 & 0.2 & 0.129 \\
\hline$\mu\left[\mathrm{mm}^{-1}\right]$ & 5.923 & 3.129 & 3.135 & 3.134 & 3.133 & 2.463 & 3.137 \\
\hline $\mathrm{d} \min .[\AA]$ & 6.886 & 6.901 & 6.889 & 6.118 & 6.892 & 5.689 & 6.770 \\
\hline $\max .[\AA]$ & 0.449 & 0.450 & 0.449 & 0.450 & 0.450 & 0.450 & 0.450 \\
\hline Collected ref. & 124453 & 124280 & 96429 & 212148 & 125335 & 121883 & 95273 \\
\hline Independent ref. & 7165 & 7200 & 7123 & 7201 & 7171 & 7177 & 7149 \\
\hline $\mathrm{R}_{\text {int }}[\%]$ & 4.57 & 2.46 & 3.23 & 2.21 & 2.59 & 3.51 & 2.60 \\
\hline
\end{tabular}

A pex2 detector. The detector has been in use for over ten years and therefore, the integration, scaling, and refinement software has been adapted to this detector to a maximum extent. Comparing the used detectors, the A pex 2 forms the gold standard for all newly developed X-ray detectors. As the crystals absorption coefficient $\mu$ for the molybdenum wavelengths is relatively high, increased absorption and subsequently X-ray fluorescence were expected for this dataset (see al so Figure 4.1). For the integration, using the PLANEBG option showed to be beneficial to the data quality. By this option, the background is determined via the best fit plane algorithm (Pratt, 1987).

\subsubsection{AgluS2 and A pex2}

In order to circumvent the effects of high absorption and X-ray fluorescence, dataset B was collected using silver radiation (Figure 4.1) on a Bruker D8 3-circle goniometer, equipped with a $50 \mathrm{~W}$ Incoatec M icrofocus Source (I $\mu S)$ ( g K $_{\alpha}$ radiation) with QUAZAR mirror optics (Schulz et al., 2009) and a Bruker SMART APEX 2 detector. The dataset was collected at the Institut für Anorganische Chemie in Göttingen. It was integrated, using a fixed integration box size $(0.8,0.8,0.5)$ and a reduced background correlation lengths ( $\mathrm{BgCS}=-2$ ).

As datasets have been collected for all detectors with the same or a similarly intense silver I $\mu \mathrm{S}$ radiation source, these datasets are comparable and differences should occur mostly due to the used detector. In this context, the A pex 2 dataset is the baseline for all newly developed detectors. 


\subsubsection{AgI $\mathrm{AS2}$ and Pilatus3}

Dataset C was collected on a Bruker D8 3-circle goniometer, equipped with a $50 \mathrm{~W}$ Incoatec Microfocus Source (I $\mu \mathrm{S})\left(\mathrm{Ag} \mathrm{K}_{\alpha}\right.$ radiation) with QUAZAR mirror optics (Schulz et al., 2009) and a Dectris Pilatus3 300K CdTe pixel detector (DECTRIS Ltd., 2015) at the Institut für Anorganische Chemie in Göttingen. Dectris kindly lent the detector to the working group and $F$. Engelhardt incorporated it into the X-ray housing (Engelhardt, 2017).

The Pilatus3 detector provides an adaptable energy threshold for the detection of photons. By the discrimination of low-energy events, the noise is extremely low as illustrated in Figure 4.6. Furthermore, the threshold can be adapted to a value above the selenium $\mathrm{K}_{\alpha}$ line at $12.7 \mathrm{keV}$, effectively omitting the elevated background by $\mathrm{X}$-ray fluorescence.

For the collection of this dataset, the energy threshold was set to $15.5 \mathrm{keV}$. The Dataset was integrated with an increased number of profile fitting points (PROFXHALF $=12$, PROFYHALF $=12$, PROFZHALF $=12$ ) and the refined integration box size resulted in relatively large mean values of SPOTSIZE $=1.280$, YSPOTSIZE $=1.471$, SPREAD $=0.754$ ). The strong and weak reflection limits were adapted (STRONGTHRESH $=10$ and LS_IOVS_MAX =15). It is assumed that by the extremely low background, the peak broadening by thermally diffuse scattering (TDS) is detected and effectively separated from the background. TDS leads to a very broad peak-shape, insufficiently described by the standard amount of peak profile points.
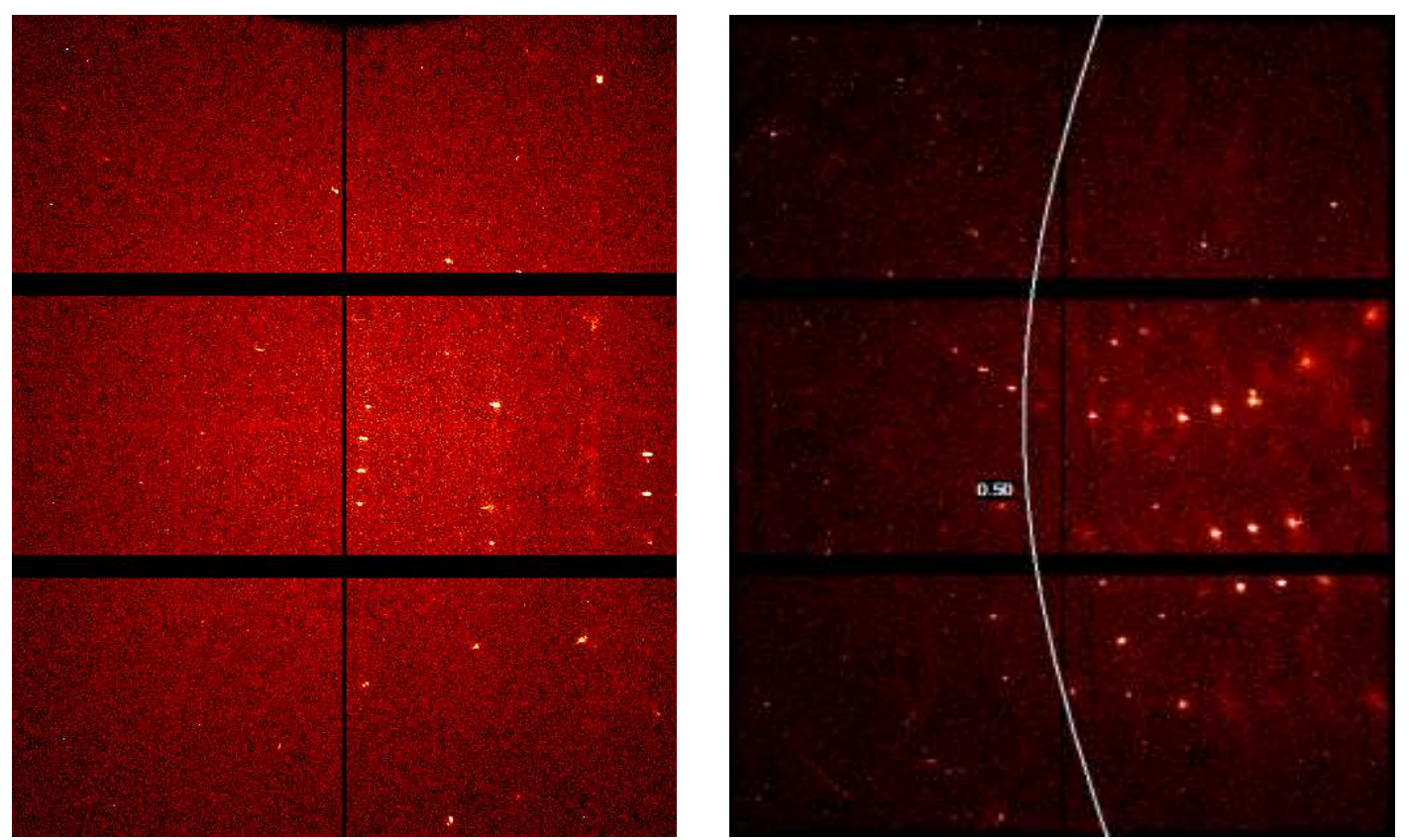

Figure 4.6: Frames of the Pilatus $3300 \mathrm{~K}$ CdTe pixel detector without (left) and with adapted energy discrimination (right). Black pixel resemble an intensity of 0. 


\subsubsection{Ag 007 and Pilatus3}

Dataset D was collected on a Rigaku kappa goniometer, equipped with a $1.2 \mathrm{~kW}$ Rigaku M icroM ax 007 (A g K ${ }_{a}$ radiation) and a Dectris Pilatus3 300K CdTe pixel detector (DECTRISLtd., 2015). The energy threshold was set to $13.0 \mathrm{keV}$. It was integrated with a reduced background correlation length (BGCORSCALE =-2). Dr. J. Ferrara at the Rigaku A mericas Corporation in The W oodlands, Huston, Texas, kindly performed and facilitated the collection of this dataset.

Silver rotating anodes are quite scarce and uncommon for single crystal XRD. Remarkably, the intensity of the 007 is superior to any other used radiation source with silver or indium radiation so far. The high intensity was expected to be beneficial to the data quality. But it also brought problems, as the dataset was collected with frames of $50 \mathrm{~s}$ exposure time only, that lead to overexposure, despite the Pilatus3 detector featuring the highest count rate and memory well depth of all compared detectors the high intensity at long exposure time brought it to its limitations.

\subsubsection{Agl $\mu \mathrm{S} 3$ and Photon2}

Dataset E was collected on a Bruker V enture kappa goniometer, equipped with a $70 \mathrm{~W}$ Incoatec M icrofocus Source (I $\mathrm{SS})\left(\mathrm{Ag} \mathrm{K}_{\mathrm{\alpha}}\right.$ radiation) and a Bruker Photon 2 detector by Dr. Holger Ott at the Bruker AXS facilities in Karlsruhe, Germany. The I $\mu \mathrm{S} 3$ has an improved intensity to the older model, present at the Institut für Anorganische Chemie. Still, the radiation intensity is in the same order of magnitude, therefore the datasets of I $\mu \mathrm{S}$ and I $\mu \mathrm{S} 3$ are well comparable. The Photon 2 detector has been introduced in 2015 and is the current top-shelf Bruker detector. The dataset was integrated using the SAINT defaults.

\subsubsection{In MetalJ et and Photon2}

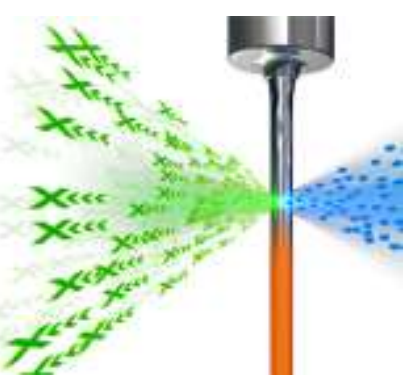

Figure 4.7: Functional principle of a M etall et X-ray source.

the indium radiation of a MetalJet source.

The Excillum Metaljet is a new groundbreaking radiation source, as instead of a solid metal a liquid metal alloy jet is used as anode material. The alloy is pressed through a nozzle to a jet
Dataset $\mathbf{F}$ was collected on a Bruker Venture kappa goniometer, equipped with a $140 \mathrm{~W}$ Excillum M etalJet X-ray source (In $\mathrm{K}_{\alpha}$ radiation), Incoatec mirror optics, and a Bruker Photon 2 detector at the Bruker AXS facilities in Karlsruhe, Germany. The dataset was one of the first datasets, collected with

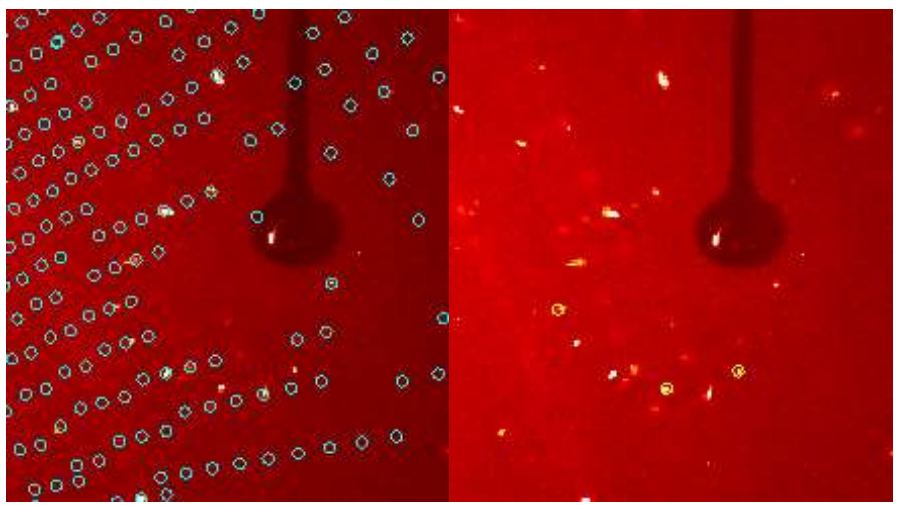

Figure 4.8: Frame section from the first attempt of a M etaljet dataset with the overlay of expected reflections of indium (blue) and gallium (yellow) radiation. 
that is irradiated by an electron beam from an electron canon, normally employed in electron microscopy. Upon the electronic excitation, $\mathrm{X}$-rays are emitted. Because of the improved heat dissipation, more focused electron beams can be used in the radiation source, resulting in $\mathrm{X}$-ray beams of very high brilliance. With a full mean half width (FMHW) of only $60 \mu \mathrm{m}$, the beam is more brilliant than any other in-house radiation source and is therefore comparable to a secondgeneration synchrotron.

The alloy of the metaljet contains gallium and indium and is liquid at

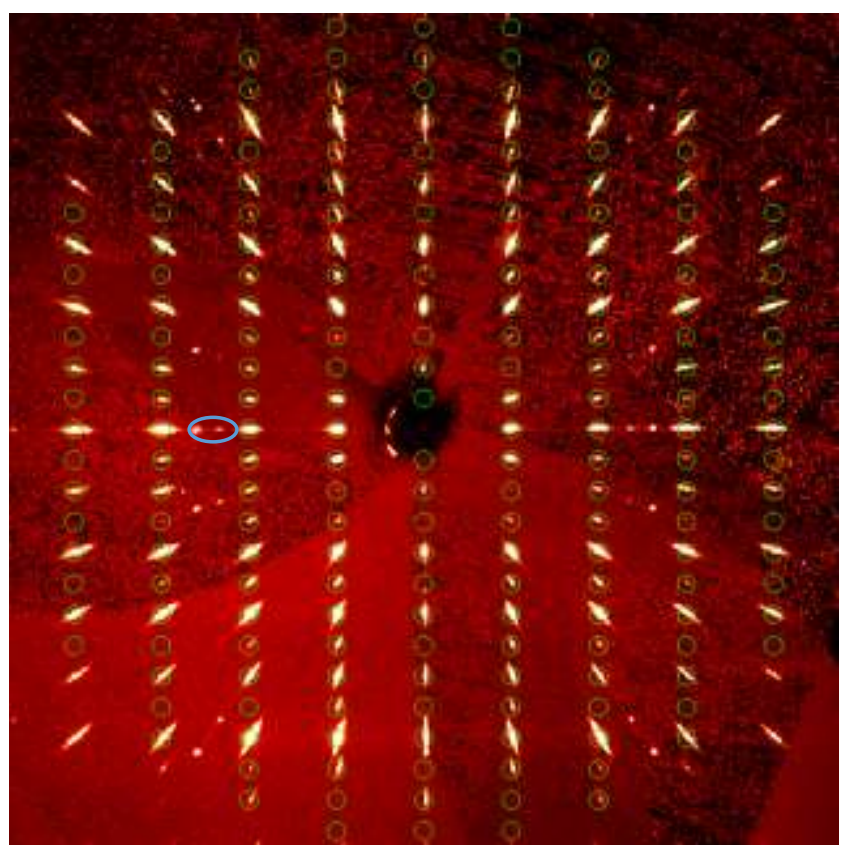

Figure 4.9: Precession image from the first attempt of a $M$ etaljet dataset, featuring gallium contamination peaks with an overlay of expected In reflections (green). room temperature. The Metallet has been established for gallium radiation over the last years and the relatively soft radiation of $9.251 \mathrm{keV}$ is used mainly for imaging and macromolecular crystallography (Romell et al., 2018; Nam et al., 2017; Töpperwien et al., 2018). For small molecule crystallography and experimental charge density investigations, the harder indium radiation of $24.209 \mathrm{keV}$ is more interesting.

The used alloy in this particular radiation source was optimized for gallium so it contained less than $20 \%$ indium. The exact composition remained Excillums trade secret. Special prototype mirror optics by Incoatec were fitted to this machine in order to extract the indium content of the beam. A first collection of the dataset resulted in corrupted data, because a significant amount of indium radiation passed the mirror and contaminated the experiment. In a second attempt, a thicker aluminum attenuator was used, effectively eliminating the gallium radiation, but also weakening the indium radiation beam.

W ith the brilliant beam, the M etaljet is optimized for very small crystals within the range of the beams' FM H W. H owever, because of the weakened intensity, a significantly larger crystal was selected for the data collection. Only with the increased scattering volume, it was possible to collect sufficient data. The collection frames resulted in a good dataset, which was integrated using the SAINT defaults. 


\subsubsection{Agl $\mathrm{AS} 3$ and Photon3}

Dataset G was collected on a Bruker Venture kappa goniometer, equipped with a 70W Incoatec M icrofocus Source (I $\mu \mathrm{S})\left(\mathrm{Ag} \mathrm{K}_{\alpha}\right.$ radiation) and a Bruker Photon 3 detector by Dr. H. Ott at the Bruker AXS facilities in Karlsruhe, Germany. The dataset was one of the first ever-collected datasets with the Photon3 detector and processing pipeline.

A detailed description of the Photon3's principally function and the data processing is given in Chapter 5. Basically, the detector is capable to collect data in a normal mode and a "single photon counting" or "mixed" mode that leads to an improved noise and background cancellation at the cost of a reduced dynamic range and low accuracy of strong reflections.

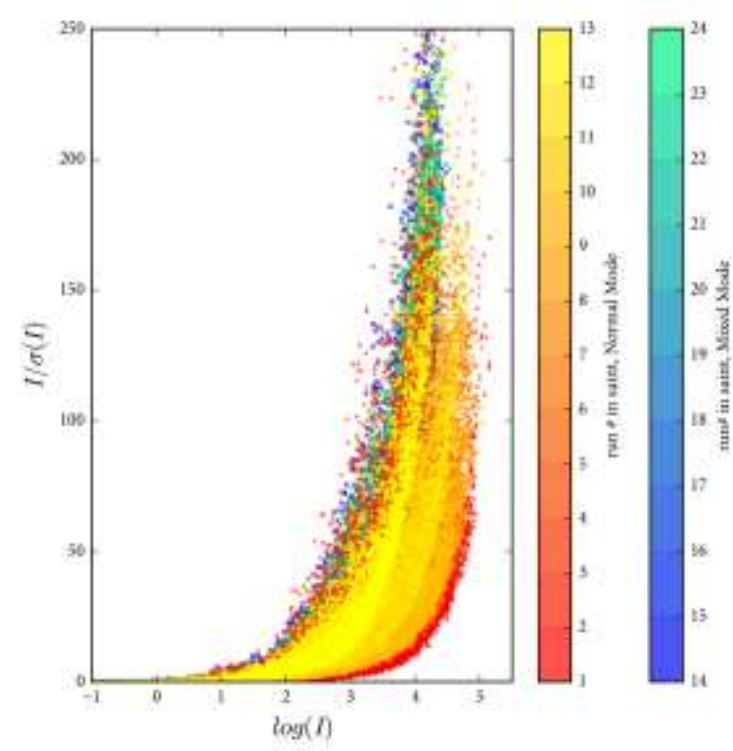

Figure 4.10: Diederichsplot (Diederichs, 2010) of selected data for dataset $\mathbf{G}$. Run 1 to 13 were collected in the in the Normal Mode and 14 to 24 in Mixed Mode.

Especially low intensity reflections should hence be collected with higher accuracy. In order to collect a full dataset at best configuration, the dataset has been collected once in normal and once in the mixed mode. All data were integrated, using the best plane algorithm. W eak reflections are more accurate in the mixed mode, while strong reflections are missing. In order to construct a full dataset with good overlap between the runs, various approaches weretested, considering $\mathrm{I}, \mathrm{l} / \sigma$, the resolution and exposure time. In the end, simply merging all data showed to be the best approach.

\subsection{Charge Density Refinement}

To obtain an adequate image of the charge density, the aspherical density was refined in the multipole approach ( $\mathrm{H}$ ansen $\&$ Coppens, 1978). Suitable models were then interpreted according to the Quantum Theory of Atoms in M olecules (QTAIM) (Bader, 1990).

The first step in the charge density refinement is always the preparation of a suitable starting model. The starting models were refined in the IAM in SH ELXL (Sheldrick, 2015) using the SHELXLE GUI (Hübschle et al., 2011). The objective in preparing a good starting model is to find correct atom positions and vibrational parameters, ideally unaffected by the aspherical density. Therefore, the heavy atom positions and vibrational parameters were refined only with the high-resolution data $(<0.6 \AA)$. The hydrogen positions were located in the residual density map of the low-resolution data $(>0.6 \AA)$ and the vibrational parameters were constrained to the heavy atoms' vibration.

Starting models were prepared for all datasets and subsequently used for the charge density refinement. In order to retain comparability, all datasets had to be refined with the same refinement strategy and with the same number of parameters. Therefore, all datasets had to be considered in the 
development of the refinement strategies. Refinement strategies were developed under consideration of thefree R-value (Krause et al., 2017), the residual density (M eindl \& Henn, 2008), the probability density function (PDF) (Herbst-Irmer \& Stalke, 2017), Kuhs's Rule (Kuhs, 1992) and DRK plot (Zavodnik et al., 1999; A dam Stash, 2007; Zhurov et al., 2008).

Two program packages were used to refine and interpret the aspherical density in the multipole approach and the subsequent interpretation in the QTAIM framework. The XD2006 program package (V olkov et al., 2007), which has been well established for years, and the M oPro program package (Jelsch et al., 2001; Guillot et al., 2001; Zarychta et al., 2007; Jelsch et al., 2018), which has been steadily developed in recent years and features new options in aspherical density refinement. An overview of all refinement results is shown in Table 4.5 .

\subsubsection{Charge Density in XD}

The XD program package was first released in 2003 as a compilation of prior existing programs and has been steadily improved ever since. Therefore, the software is very well established in the charge density community and is the standard tool for charge density refinements in the Stalke group. Based on this package, numerous further applications and scripts were developed, like XD Rfree (Krause et al., 2017) or a TDS correction (N iepötter et al., 2015). It has therefore become more user-friendly to work with the package.

In order to refine a meaningful density model, parameters are included stepwise in the refinement. After each of these refinement steps, the refinement needs to be checked for significance, convergence, and correctness. Furthermore, the same refinement strategy needed to be applied to all datasets in order to retain comparability. Therefore, the development of the refinement strategy is of particular importance for this work.

The first step in strategy development is the choice of local coordinate systems and subsequently the choice of local symmetry. By the application of local symmetry, no multipoles that contradict the symmetry are refined. Parameters are thereby saved effectively. Furthermore, chemical constraints are applied, constraining all multipole parameters of two or more chemically equivalent atoms.

Table 4.3: Gram-Charlier-level, local symmetry and chemical constraints used in the charge density refinements in XD and MoPro.

\begin{tabular}{llll}
\hline ATOM & GC & Symm & CON \\
$\mathrm{Se}(1)$ & 4 & $\mathrm{~m} \rightarrow \mathrm{no}$ & \\
$\mathrm{C}(1)$ & 2 & $\mathrm{~m}$ & \\
$\mathrm{C}(2)$ & 2 & $\mathrm{~mm} 2$ & \\
$\mathrm{C}(3)$ & 2 & $\mathrm{~mm} 2$ & \\
$\mathrm{C}(4)$ & 2 & $\mathrm{~mm} 2$ & $\mathrm{C}(3)$ \\
$\mathrm{C}(5)$ & 2 & $\mathrm{~mm} 2$ & $\mathrm{C}(3)$ \\
$\mathrm{C}(6)$ & 2 & $\mathrm{~mm} 2$ & $\mathrm{C}(3)$ \\
$\mathrm{C}(7)$ & 2 & $\mathrm{~mm} 2$ & $\mathrm{C}(3)$ \\
$\mathrm{H}(1 \mathrm{~A})$ & 1 & cyc & \\
$\mathrm{H}(1 \mathrm{~B})$ & 1 & cyc & $\mathrm{H}(1 \mathrm{~A})$ \\
$\mathrm{H}(3)$ & 1 & cyc & \\
$\mathrm{H}(4)$ & 1 & cyc & $\mathrm{H}(3)$ \\
$\mathrm{H}(5)$ & 1 & cyc & $\mathrm{H}(3)$ \\
$\mathrm{H}(6)$ & 1 & cyc & $\mathrm{H}(3)$ \\
$\mathrm{H}(7)$ & 1 & cyc & $\mathrm{H}(3)$ \\
\hline
\end{tabular}

Thelocal coordinates were selected according to TableS4.8 (A ppendix) and theapplied symmetry, given in Table 4.3, was selected to be maximal. In addition, the degree of chemical constraints for the benzyl moiety was the highest possible. During the development of the refinement strategy, loosening of local symmetry was tested and showed no significant improvement to the fit. The only exception was 
the local symmetry at the selenium atom position, as disbanding its local symmetry lead to significant improvements in the fit.

Gradually, parameters were introduced until the full refinement of all multipoles at selenium. H exadecapoles were refined for carbon and one bond-directed dipole for hydrogen. Furthermore, the refinement of anharmonic motion coefficients (Gram-Charlier level 4) was introduced for selenium, while all carbon atoms were refined anisotropically (GC level 2 ) and hydrogen atoms isotropically (GC level 1).

Table 4.4: Refinement strategy for the charge density refinement in XD. The ratio of data to parameter and the R-values are exemplary given for dataset $\mathbf{C}$. A bbreviations: Para: parameter; M P: M ultipole; M : monopoles; D: dipoles; Q: quadrupoles; O: octupoles; $\mathrm{H}$ : hexadecapoles, U 2, U 3, U 4: Gram Charlier $2^{\text {nd }}, 3^{\text {rd }}$ and $4^{\text {th }}$ order, $\mathrm{H}-\mathrm{XYZ}$ : hydrogen position against data up to $0.5 \sin (\theta) / \lambda$.

\begin{tabular}{llllll}
\hline Step & New Para & MP-Para & Para & Data/Para & WR(F⿻) \\
1 & Scalefactor & 0 & 1 & 6774 & 0.0430 \\
2 & DQOH & 46 & 47 & 144.1 & 0.0279 \\
3 & U2 & 46 & 95 & 71.3 & 0.0182 \\
4 & $\kappa$ & 46 & 98 & 69.1 & 0.0173 \\
5 & $\mathrm{XYZ}$ & 46 & 122 & 55.5 & 0.0163 \\
6 & $\mathrm{M}$ & 52 & 127 & 53.3 & 0.0154 \\
7 & $\mathrm{H}-\mathrm{XYZ}$ & 0 & 22 & 29.3 & 0.0140 \\
8 & all prior & 52 & 127 & 53.3 & 0.0147 \\
9 & $\mathrm{U} 3(\mathrm{Se})$ & 0 & 11 & 615.8 & 0.0146 \\
10 & all prior +U3(Se) & 52 & 137 & 49.5 & 0.0145 \\
11 & $\mathrm{U} 4(\mathrm{Se})$ & 0 & 16 & 423.4 & 0.0143 \\
12 & all prior +U4(Se) & 52 & 152 & 44.6 & 0.0137 \\
13 & Se NoSymm & 62 & 162 & 41.8 & 0.0132 \\
14 & $\kappa$ & 0 & 4 & 1693.5 & 0.0131 \\
15 & all prior & 62 & 162 & 41.8 & 0.0130 \\
\hline
\end{tabular}

The refinement strategy, given in Table 4.4 was applied to all datasets $\mathbf{A}$ to $\mathbf{G}$. Refinement procedures for all datasets are given in Tables S4.9 to S4.15 in the appendix. .The significance of all refined parameters in all datasets is demonstrated by the progression of $\mathrm{R}_{\text {cross }}$ throughout the refinement procedure in Figure 54.1 to $\$ 4.7$.

The refinement of the anharmonic motion of selenium remains debatable, as Kuhs's Rule (Kuhs, 1992) is not strictly fulfilled for all datasets (see Table S4.18). N evertheless, the refined parameters are significant (Table S4.17) and the probability density function is physically meaningful (Table S4.16). Also, the refinement of Gram-Charlier parameters of $4^{\text {th }}$ order leads to a significantly improved fit to the data. Therefore, it was adopted to the refinement strategy.

The refinement results are given in Table 4.2. The multipole refinement in XD clearly improves the model compared to the IAM refinement. The R-values and residual density peaks clearly drop for all datasets. N evertheless, the overall refinement results are unsatisfactory. All datasets and in particular datasets $\mathbf{A}$ and $\mathbf{D}$, which were collected with rotating anodes, show high values for the GOF as well as residual density peaks that are too high for a suitable refinement. The best results are achieved with 
Table 4.5: Charge density refinement result overview.

\begin{tabular}{|c|c|c|c|c|c|c|c|}
\hline Dataset & A & B & C & D & $\mathbf{E}$ & $\mathbf{F}$ & G \\
\hline Detector & Apex2 & Apex2 & Pila. 3 & Pila. 3 & Phot.2 & Phot. 2 & Phot. 3 \\
\hline X-ray source & TXS & $\mathrm{I} \mu \mathrm{S}$ & $\mathrm{I} \mu \mathrm{S}$ & 007 & $\mathrm{I} \mu \mathrm{S} 3$ & $M$. Jet & $\mathrm{I} \mu \mathrm{S} 3$ \\
\hline Power [W] & 1200 & 30 & 30 & 1200 & 70 & 140 & 70 \\
\hline R1(I) (all data, IAM ) [\%] & 2.72 & 2.67 & 2.54 & 2.44 & 2.25 & 2.83 & 2.57 \\
\hline wR2(I) (all data, IAM ) [\%] & 7.29 & 6.47 & 6.71 & 7.56 & 5.45 & 6.69 & 0.56 \\
\hline $\operatorname{GOF}\left(F^{2}, I A M\right)$ & 1.09 & 1.06 & 1.111 & 1.110 & 1.102 & 1.098 & 1.077 \\
\hline Diff peak & 1.909 & 1.585 & 1.444 & 1.690 & 1.144 & 1.103 & 1.047 \\
\hline /hole $\left(F^{2}, I A M\right)\left[e \AA^{-3}\right]$ & -1.160 & -0.838 & -0.833 & -0.448 & -0.604 & -0.690 & -0.809 \\
\hline Data in XD and M oPro & 7032 & 6719 & 6774 & 6952 & 6777 & 6660 & 6866 \\
\hline R1(I) (all data, merged, XD) [\%] & 2.11 & 1.18 & 1.09 & 1.43 & 1.21 & 1.42 & 1.27 \\
\hline wR(I) (all data, merged, XD) [\%] & 2.30 & 1.27 & 1.30 & 1.89 & 1.28 & 1.42 & 1.51 \\
\hline $\operatorname{GOF}(F, X D)$ & 4.901 & 2.027 & 1.143 & 5.636 & 2.123 & 1.676 & 1.992 \\
\hline Diff peak & 1.603 & 0.508 & 0.309 & 0.992 & 0.371 & 0.574 & 0.776 \\
\hline /hole $\left(F^{2}, X D\right)\left[e^{-3}\right]$ & -0.626 & -0.441 & -0.209 & -0.531 & -0.401 & -0.310 & -0.501 \\
\hline R1(I) (all data, merged, M oPro) [\%] & 2.01 & 1.20 & 1.09 & 1.57 & 1.25 & 1.10 & 1.23 \\
\hline wR2(I) (all data, merged, M oPro) [\%] & 2.24 & 1.29 & 1.28 & 2.77 & 1.18 & 1.17 & 1.44 \\
\hline GOF (F, M oPro, 287 Para) & 4.799 & 2.059 & 1.504 & 5.517 & 2.072 & 1.646 & 1.900 \\
\hline Diff peak & 1.719 & 0.582 & 0.438 & 1.206 & 0.447 & 0.712 & 0.983 \\
\hline /hole (F², MoPro) $\left[\mathrm{e}^{-3}\right]$ & -0.778 & -0.461 & -0.344 & -0.572 & -0.509 & -0.360 & -0.638 \\
\hline
\end{tabular}

dataset $\mathbf{C}$, but still the residual density peaks are quite high with a level of approximately $0.3 \mathrm{e}^{-3}$. By the analysis of the DRK-Plots, systematic and resolution-dependent errors were excluded (Figure S4.8 to S4.14).

Nevertheless, the results of the charge density refinement have been interpreted according to the QTAIM, but the results remained inconclusive. Especially the properties of selenium differed greatly between the datasets and none of them even remotely resembled the theoretically expected density around selenium.

The reason for this was that the residual density was not featureless for any dataset. Shown in Figure $\mathrm{S} 4.15$ to $\mathrm{S} 4.21$, the highest residual density peaks reside al ways at two positions in the elongation of the C-Se bond and near the Se-Se axis. The peak positions very closely resemble those shown in Table 4.7 or Figure 4.11. These features were of great importance for the further strategy, as they appear independent from the underlying dataset. Various possible reasons for these features have been taken into consideration - each particular one was excluded.

Crystal defects, like twinning, were excluded, as data were collected from different crystals and different batches of (BzSe) 2 . N one of the used crystals showed optical defects. Simple bad data quality is unlikely for the wide variety of all seven different datasets. The effects of $X$-ray fluorescence have been cancelled out for the datasets, collected with Pilatus3 detectors (C and D). As the peaks reside around 
selenium, absorption could bea cause for such features. But absorption features occur more isotropically around the heavy atoms (see also Krause et al., 2015a) and are furthermore dependent on the wavelengths. As dataset $\mathbf{A}$ has been collected with molybdenum radiation, dataset $\mathbf{F}$ with indium and other datasets with silver radiation but all feature residual density peaks in the same general positions, absorption was also excluded to be the origin of those peaks. Disorder was also excluded as an error source, as the peak positions did not resemble a chemically sound structure. One option left, however, was that the density truly was part of the selenium valence density that was not perfectly described by the applied model. This seemed plausible, as selenium is rather heavy for a charge density structure and therefore the charge density could be delicately textured.

\subsubsection{Charge Density in MoPro}

As the limits of theXD 2006 program package were reached but still residual density was observable around selenium for all datasets, an expansion of the model was only feasible by a change of the software to the M oPro suite.

M oPro was introduced in 2001 and has been improved ever since. In contrast to XD, M oPro comes with a graphical user interface and provides many automated refinement options, were XD is based on command line input and relies on a rather strict user defined code for refinement. In contrast to XD, which has been more or less finalized in 2016 and is currently not updated on a regular basis, development in MoPro is vivid and new versions are published multiple times a year.

One of the latest add-ons to M oPro was the possibility to refine multipoles up to 64-poles in 2017. In order to apply this new feature to the $(\mathrm{BzSe})_{2}$ datasets, a new refinement strategy had to be compiled. The strategy should be analogous to the refinement procedure in XD in order to retain comparability between the refinements in different program packages.

Table 4.6: Refinement strategy for the charge density refinement in M oPro. The ratio of Data to parameter and the R-values are exemplary given for dataset $\mathbf{C}$. A bbreviations: Para: parameter; M P: M ultipole; M : monopoles; $\mathrm{D}$ : dipoles; $\mathrm{Q}$ : quadrupoles; $\mathrm{O}$ : octupoles; $\mathrm{H}$ : hexadecapoles; 32P: 32-poles; 64P: 64-poles; U2, U 4: Gram Charlier $2^{\text {nd }}$ and $4^{\text {th }}$ order, $\mathrm{H}$-XYZ: hydrogen position against data up to $0.5 \sin (\theta) / \lambda$.

\begin{tabular}{llllll}
\hline Step & New Para & MP-Para & Para & Data/Para & wR(F2) \\
1 & SCALE & 0 & 1 & 6770.0 & 4.751 \\
2 & DQOH & 46 & 47 & 144.0 & 3.517 \\
3 & U2 & 46 & 95 & 71.3 & 2.679 \\
4 & K & 46 & 98 & 69.1 & 1.914 \\
5 & XYZ & 46 & 122 & 55.5 & 1.816 \\
6 & M & 46 & 127 & 53.3 & 1.759 \\
7 & H-XYZ only & 0 & 22 & 29.3 & 1.673 \\
8 & all prior & 46 & 127 & 53.3 & 1.713 \\
9 & U4 (Se) & 46 & 152 & 44.6 & 1.573 \\
10 & Se NoSymm & 56 & 162 & 41.8 & 1.534 \\
11 & K' & 0 & 4 & 1690.0 & 1.514 \\
12 & all prior & 56 & 162 & 41.8 & 1.495 \\
13 & 32P (Se) & 67 & 173 & 39.2 & 1.484 \\
14 & 64P (Se) & 80 & 186 & 36.4 & 1.461 \\
\hline
\end{tabular}


Due to program restrictions, the refinement strategy used in XD was not entirely devolved to M oPro. N evertheless, the same parameters were introduced to the refinement in the same sequence, as shown in Table 4.6. In addition to the prior in XD refined parameters, 32-poles, and 64-poles were introduced at selenium in the final steps of the refinement procedure.

The refinement converged and the model changes were significant. The refinements for all datasets are given in Tables $\$ 4.19$ to $\$ 4.24$. The significance of the additional refined parameters was checked by the progression of the $\left\langle R_{\text {free }}\right\rangle$ (Figure S4.22 to S4.28).

Unfortunately, the refinement of the additional parameters did not bring the expected improvements. A QTAIM-interpretation of the models showed a more textured charge density with an overall increased number of VSCCS around selenium. But again, the model properties did not correspond between the datasets, and furthermore none corresponded to the theoretical expectations. Ultimately, the overall fit of the data did improve only little, as shown by the R-values and residual density in Table 4.2. A gain, high residual density peaks were observed for all datasets at the same general positions, corresponding well to those found in the XD-refinement and shown in Table 4.7 and Figure 4.11. As the extended multipole model with 64-poles at selenium could not fit the residual density, it was deduced that the residual density was not part of the selenium valence density.

Comparing the two program packages XD2006 and MoPro, slightly improved results were observed for XD, although the same starting models were used and the same parameters were refined in the same order. It is therefore assumed that the differences originate from the refinement algorithms applied in the programs.

\subsection{Residual Density Peak Analysis}

As the origin of the residual density peaks could not be assigned to experimental errors, twinning or disorder and also was not part of the selenium valence density, the next step was to quantify the residual density. In order to do so, a standard model of the electron density was needed, generated independently from all possible effects that lead to the peaks. Therefore, an invariom-like approach was applied.

In the invariom approach (Dittrich et al., 2004), aspherical density in the form of multipole populations are computed for DFT calculated molecules and then applied atom-wise to experimental structures, based on the atoms chemical environment. For this, aspherical scattering factors are transferred from a database to any structure, leading to an improved description and allowing for the QTAIM -interpretation of those structures (see also Schürmann et al., 2012; Dittrich et al., 2016).

Unfortunately, no suitable entries were present in the invariom-database, therefore new calculations werenecessary. Thorsten $\mathrm{L}$. T euteberg from the M ata group of the Institut für Physikalische Chemie at the Göttingen University took on the task. He started a series of extended DFT calculations of (BzSe) ${ }_{2}$ further discussed in Chapter 4.5. Among others, he optimized $(\mathrm{BzSe})_{2}$ in the ground sate, 
starting from the atom positions determined from dataset $\mathbf{C}$. This optimization result is further on called $1\left(S_{0}\right)$.

On the one hand, the optimised wave function $\mathbf{1}\left(\mathbf{S}_{\mathbf{0}}\right)$ was interpreted according to the QTAIM approach, using the AIM ALL package (Keith, 2017) to compare the properties of the theoretical density with the experimental ones. Theoretical scattering factors werecalculated from that wavefunction $\mathbf{1}\left(\mathbf{S}_{0}\right)$, using the program DenProp (V olkov et al., 2016a). For those scattering factors, a multipole model was developed by the refinement of only the multipoles and no positional or vibrational parameters. The resulting models deformation- and residual density are shown in Figure S4.32 and Figure S4.33. The latter shows only spherical features of density around the selenium positions. This is a common feature for this kind of refinements and originates from the atoms core-polarization.

Table 4.7: Residual density peak analysis result overview. $E_{\text {gross }}$ is determined according to ( $M$ eindl $\& \mathrm{H}$ enn, 2008), Peak 1 resides in the elongation of the C-Se axis and Peak 2 lies near the Se-Se axis. In the figures, red spheres with the spheresize proportional to the peak height represent theresidual density peaks. N ote that also the symmetry equivalents of the residual peaks are depicted.

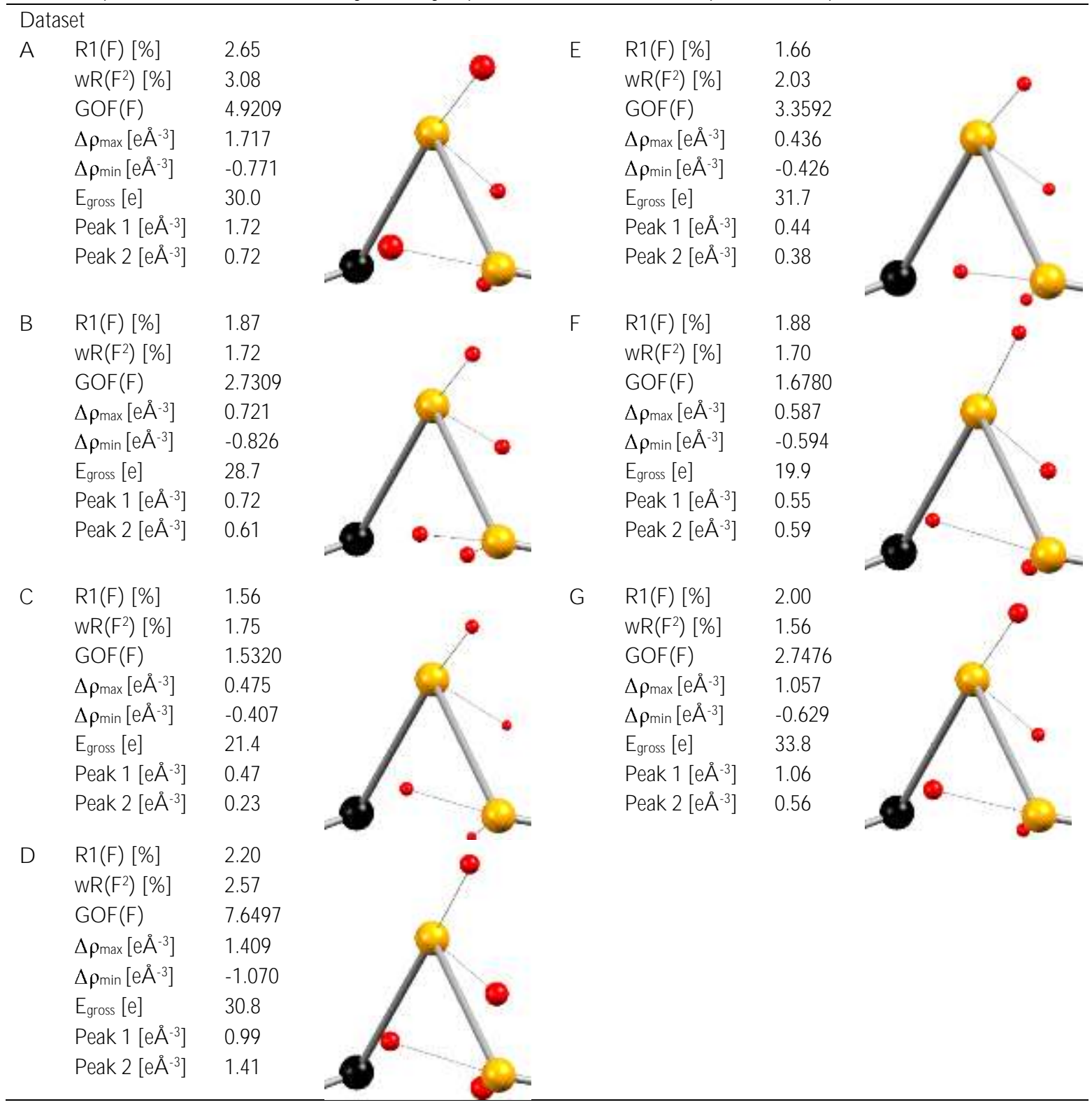


The multipole populations determined on the basis of $\mathbf{1}\left(\mathbf{S}_{0}\right)$ were then applied to the datasets $\mathbf{A}$ to G and only the positional and vibrational parameters were refined. Thereby, the molecules valence density was described at best, while highlighting the data features in the residual density.

The results of the refinement with the invariom-like approach are given in Table 4.7 and images of all residual density maps are shown in Figure S4.34 to S4.40. Like in the prior approaches by charge density refinements in XD and MoPro, the two largest residual density peaks are located in very similar positions for all datasets. Peak 1 resides in the elongation of the C-Se axis and Peak 2 lies near the Se-Se axis.

By the application of a standard model for the aspheric density, the peak height is now comparable between the datasets. While the datasets $\mathbf{C}$ and $\mathbf{E}$ show the lowest peaks, $\mathbf{A}$ and $\mathbf{D}$ tower out with residual density peaks higher than $1.4 \mathrm{e}^{-3}$. The only thing the two datasets have in common is the fact that they were collected using rotating anodes as radiation sources. The power and subsequently the beam intensity of the rotating anodes is approximately one order of magnitude higher than any other used Xray source. It was therefore deduced that the effect responsible for the residual peaks roughly scaled with the beam intensity. As a result, the idea emerged that the peaks originated from an X-ray activated form of $(\mathrm{BzSe})_{2}$.

The mean positions of the two residual density peaks are shown in Figure 4.11 with distances to the atoms. The distance from the selenium positions to the residual density peaks of 0.86 and $1.02 \AA$ is a sensible shift in bond lengths. The distance from carbon to peak 2 resembles approximately a $\mathrm{C}$-Se bond of $2.14 \AA$ and the distance from Peak 2 to the symmetry equivalent of Peak 1 of $2.31 \AA$ resembles approximately a Se-Se bond.

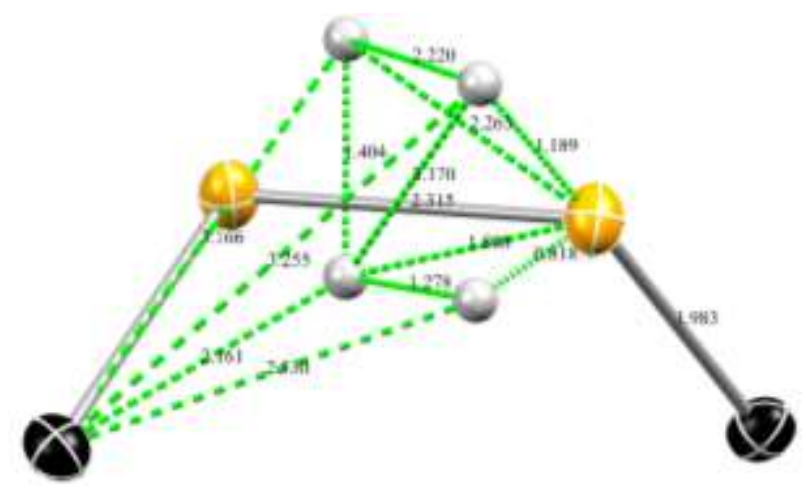

Figure 4.11: Mean residual density peak positions with distances in $\AA$. Hence the implied structure of the activated (BzSe) ${ }_{2}$ resembled a C-Se bond cleavage to give BzSeSe* (2) in Figure 4.13.

\subsection{DFT Optimizations}

All calculations were carried out by Thorsten $\mathrm{L}$. Teuteberg with the additive crystal quantum mechanics / molecule mechanics model (AC-QM/M M) (Teuteberg et al., 2018) at the B3LYP/def2TZVP level of theory. Within the AC-QM/M M approach, the molecule is optimized on the QM level, while it interacts on the $M M$ level with the surrounding crystal environment. In order to model the crystal environment, a sphere of $40 \AA$ around the molecule is populated with the optimized molecule, according to the crystal symmetry. This process is permutated until conversion. 
This procedure was carried out for the ground state structure of $(\mathrm{BzSe})_{2} \mathbf{1}\left(\mathbf{S}_{0}\right)$. The structure served as baseline for the experimentally determined properties of $\mathbf{A}$ to $\mathbf{G}$ and was therefore analyzed in the QTAIM framework. The analysis found two nonbonding Laplacian critical points around selenium with a Se-CP distance of $1.71 \AA$ and a CP-Se-CP angle of $150.0^{\circ}$. The Se-Se BCP features a rather low electron density of $0.725 \mathrm{e}^{-3}$ and a Laplacian of $-0.930 \mathrm{e} \AA^{-5}$, which is near zero. The bond can therefore be

Figure 4.12: BCP (red), bond paths (black) and LapCP (yellow) for $\mathbf{1}\left(\mathbf{S}_{0}\right)$. characterized as a weak closed-shell interaction. Further results of theQTAIM analysis are shown in Figures $\$ 4.29$ to S4.31 and Tables S4.26 and S4.27.

For the optimization of activated molecules, the $\mathbf{1}\left(\mathbf{S}_{\mathbf{0}}\right)$-environment was adopted and kept fixed for the optimization of possible electronically excited states. Furthermore, the starting geometry of the molecule was changed, in order to mimic the molecular geometry of the electronically excited states. For the emulation of the C-Se bond-breakage, the selenium atoms were placed at the residual density peaks (see Figure 4.14). For the emulation of the Se-Se bond breakage BzSe• (3), the Se-Se distance was elongated to $2.894 \AA$, the mean value of intra- and intermolecular Se-Se distance.

For the three starting geometries, resembling the ground structure $\mathbf{1}$, the $\mathrm{C}$-Se bond breakage 2 and the Se-Se cleavage $\mathbf{3}$, different electronic states were taken into account: thesinglet ground state $\left(\mathbf{S}_{0}\right)$ and first excited state $\left(\mathbf{S}_{\mathbf{1}}\right)$, the lowest triplet state $\left(\mathbf{T}_{\mathbf{0}}\right)$, and a broken symmetry state (BS). Furthermore, the singly charged cation and anion in their doublet ground states $\left(\mathbf{D}_{0}\right)$ were considered. Selected distances of all

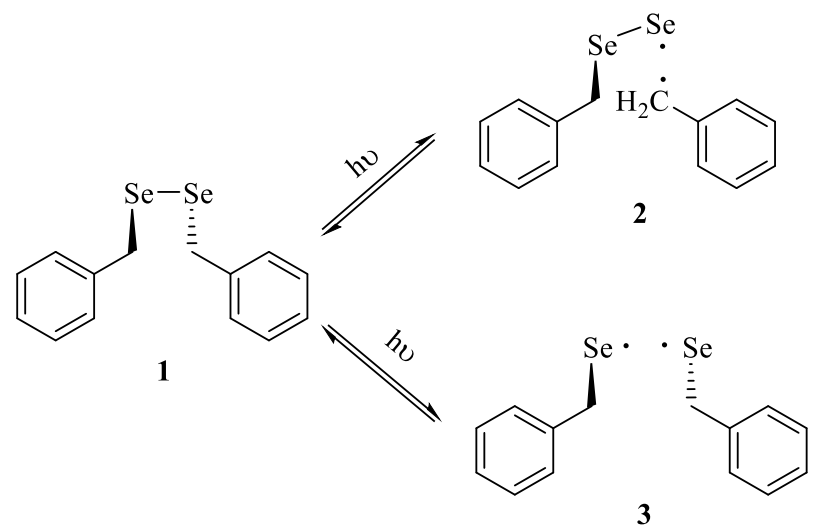

Figure 4.13: Activation of (BzSe) 2 . optimized geometries and electronic states are shown in Table 4.8.

For $\mathbf{1}\left(\mathbf{S}_{0}\right)$ and $\mathbf{1}(\mathbf{B S})$, the starting geometry was retained. For $\mathbf{1}\left(\mathbf{S}_{\mathbf{1}}\right)$ and $\mathbf{1}\left(\mathbf{T}_{0}\right)$, an elongation of the Se-Se bond was observed, resembling $\mathbf{3}$. The cation $\mathbf{1}\left(\mathbf{D}_{0^{+}}\right)$leads to a significant torsion of the $\mathrm{C}-\mathrm{Se}-\mathrm{Se}-$ $\mathrm{C}$ dihedral angle, slightly smaller Se-Se and slightly larger $\mathrm{C}$-Se distances, that do not resemble the experimental expectation. For the anion $\mathbf{1}\left(\mathbf{D}_{0^{-}}\right)$, an elongated Se-Se bond is observed, but quite similar residual geometry parameters. 
Table 4.8: Selected geometry parameters of optimized structures for different starting structures (Init) optimized in different electronic states (State) and compared to the experimental parameters (Exp), as well as the residual density peak positions as Se-atoms (ExpQ).

\begin{tabular}{|c|c|c|c|c|c|c|c|}
\hline \multirow[t]{3}{*}{ Init } & State & $\mathrm{r}(\mathrm{Se}-\mathrm{Se})[\AA]$ & \multicolumn{2}{|c|}{$r(\mathrm{C}-\mathrm{Se})[\AA]$} & \multicolumn{2}{|c|}{$\mathrm{a}(\mathrm{C}-\mathrm{Se}-\mathrm{Se})\left[^{\circ}\right]$} & $d(\mathrm{C}-\mathrm{Se}-\mathrm{Se}-\mathrm{C})\left[^{\circ}\right]$ \\
\hline & Exp & 2.315 & 1.984 & 1.984 & 101.1 & 101.1 & 93.6 \\
\hline & ExpQ & 2.170 & 2.167 & 3.155 & 97.3 & 52.7 & 126.0 \\
\hline \multirow[t]{6}{*}{1} & $\mathrm{~S}_{0}$ & 2.328 & 1.996 & 1.996 & 101.3 & 101.3 & 95.6 \\
\hline & $\mathrm{S}_{1}$ & 2.785 & 1.986 & 1.985 & 92.9 & 93.0 & 112.2 \\
\hline & $\mathrm{T}_{0}$ & 2.766 & 1.991 & 1.991 & 91.8 & 91.8 & 114.2 \\
\hline & BS & 2.328 & 1.998 & 1.998 & 101.6 & 101.6 & 95.1 \\
\hline & $\mathrm{D}_{0}^{+}($Cation $)$ & 2.268 & 2.056 & 2.056 & 101.1 & 101.0 & 132.1 \\
\hline & $\mathrm{D}_{0}^{-}$(A nion) & 2.862 & 1.983 & 1.984 & 95.9 & 95.3 & 85.3 \\
\hline \multirow[t]{6}{*}{2} & $\mathrm{~S}_{0}$ & 2.328 & 1.997 & 1.998 & 101.3 & 101.6 & 94.8 \\
\hline & $\mathrm{S}_{1}$ & - & - & - & - & - & - \\
\hline & $T_{0}$ & 2.243 & 2.004 & 3.132 & 100.8 & 79.7 & 109.2 \\
\hline & BS & 2.328 & 1.998 & 1.998 & 101.5 & 101.6 & 94.7 \\
\hline & $\mathrm{D}_{0}^{+}($Cation $)$ & 2.284 & 2.040 & 2.040 & 106.5 & 106.4 & 79.4 \\
\hline & $\mathrm{D}_{0}^{-}$(Anion) & 2.325 & 1.994 & 2.734 & 99.9 & 95.1 & 98.4 \\
\hline \multirow[t]{6}{*}{3} & $\mathrm{~S}_{0}$ & 2.329 & 1.998 & 1.998 & 101.6 & 101.6 & 95.6 \\
\hline & $S_{1}$ & 2.819 & 1.979 & 1.977 & 100.2 & 102.2 & 73.4 \\
\hline & $\mathrm{T}_{0}$ & 2.766 & 1.991 & 1.991 & 92.4 & 92.3 & 114.8 \\
\hline & BS & 2.329 & 1.998 & 1.999 & 101.6 & 101.6 & 95.6 \\
\hline & $\mathrm{D}_{0}^{+}$(Cation) & 2.284 & 2.041 & 2.041 & 106.9 & 106.9 & 80.0 \\
\hline & $\mathrm{D}_{0}^{-}$(Anion) & 2.860 & 1.983 & 1.983 & 94.5 & 94.5 & 85.8 \\
\hline
\end{tabular}

Starting from structure $\mathbf{3}$ with elongated Se-Se bond, the optimizations $\mathbf{3}\left(\mathbf{S}_{0}\right)$ to $\mathbf{3}\left(\mathbf{D}_{0}{ }^{-}\right)$yielded very similar, virtually unchanged structures for all electronic states with only negligible differences in geometry.

The optimization starting from the experimental residual density peak positions 2 provided results that were more interesting. A s expected, $\mathbf{2}\left(\mathbf{S}_{\mathbf{0}}\right)$ and $\mathbf{2}(\mathbf{B S})$ relaxed to the ground state structure. For $\mathbf{2}\left(\mathbf{S}_{\mathbf{1}}\right)$ no energy minimum could be determined and $\mathbf{2}\left(\mathbf{T}_{0}\right)$ did not relax to a $\mathbf{3}$ akin structure, as it had been the case for both other starting geometries. Although the starting structure was not retained, the general agreement of key geometry parameters between the experimental residual density peak positions and $\mathbf{2}\left(\mathbf{T}_{0}\right)$ is quite convincing, as shown in Figure 4.14.

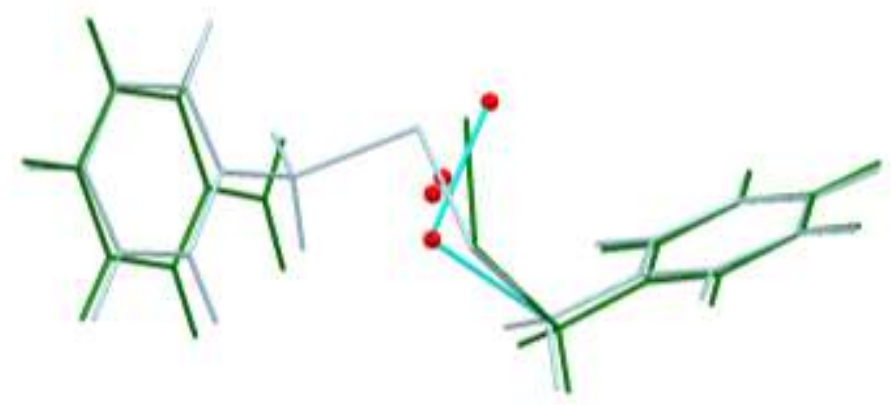

Figure 4.14: Optimized structures of the ground state $\mathbf{1}\left(\mathbf{S}_{0}\right)$ (blue), the starting positions at the mean residual density peaks (red and turquise) and the optimized structure $\mathbf{2}\left(\mathbf{T}_{0}\right)$ (green). 
With $2.243 \AA$, the Se-Se distance is significantly longer than the residual density peak distance $(2.170 \AA)$, while the $C$-Se bond is shorter $(2.004 \AA)$ than in the experimental estimation $(2.167 \AA)$. The broken C-Se bond is very similar $(3.132 \AA)$ to the experimental estimation ( $3.155 \AA$ ) and the C-Se-Se angles are very close $\left(100.8^{\circ}\right.$ to $\left.93.3^{\circ}\right)$. For the $\mathrm{C}$...Se-Se angle $\left(79.7^{\circ}\right)$ the agreement to the experimental estimation $\left(52.7^{\circ}\right)$ is notably worse, as well for the C-Se-Se-C dihedral $\left(109.2^{\circ}\right.$ to $\left.126^{\circ}\right)$.

Nonetheless, the results obtained from $\mathbf{2}\left(\mathbf{T}_{0}\right)$ are not only in much better agreement than all other results, but also the deviations from $\mathbf{1}\left(\mathbf{S}_{0}\right)$ are in the direction of the residual density peaks. Hence, it was considered not clear evidence of the experimental structure representing a triplet state, but it strongly supported the assumption.

\subsection{EPR Investigation}

As the theoretical investigation hinted towards an activated triplet state structure that was observed as a minute contribution in the diffraction experiment, an investigation using Electron Paramagnetic Resonance (EPR) was started to find and characterize radicals in irradiated (BzSe) ${ }_{2}$. In EPR, a strong magnetic field is applied to the sample. The spins of unpaired electrons align themselves to the magnetic field. By the irradiation of the sample with microwaves, the resonance of these aligned spins can be detected. Hence, the occurrence and to some extent also the nature of radicals can be determined.

When solid (BzSe) ${ }_{2}$ is UV-irradiated for one to several hours at $77 \mathrm{~K}$, it is known to give EPRactive species (W indle et al., 1964). In order to investigatethe presence of radicals and clarify their nature in X-ray and UV -irradiated samples, (BzSe) ${ }_{2}$ was freshly recrystallized, ground up and exposed to a highpower quartz UV lamp and the radiation of several X-ray sources in a cooled EPR tube. Samples were irradiated first in the monochromatic and collimated beams of single crystal X-ray diffractometers. As this lead to weak signals only, a sample was irradiated in the polychromatic and un-collimated "pink" beam of a $1.2 \mathrm{~kW}$ Rigaku M icroM ax $007 \mathrm{Cu}$ rotating anode, provided by J. Frohn from the group of Prof. Salditt. In collaboration with Dr. A. C. Stückl, EPR spectra were collected immediately after irradiation.

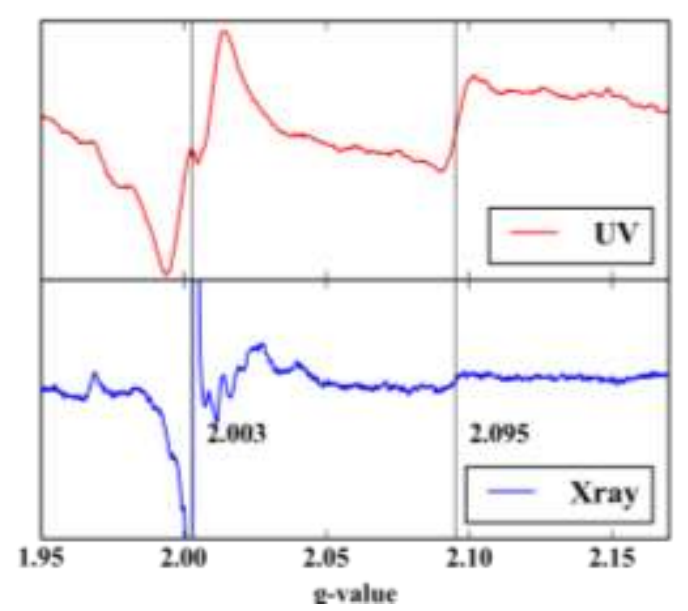

Figure 4.15: EPR signals of UV- and X-ray irradiated probes.
The irradiated, cooled samples were directly exposed to microwave excitation in the magnetic field at $142 \mathrm{~K}$. The resulting EPR signals in Figure 4.15 clearly show the emergence of unpaired spins upon X-ray and UV-irradiation. In both spectra a signal at $\mathrm{g}=2.003$ can be observed. This is indicative for an aromatic radical like Bz* (Ohnishi et al., 1962), but also selenium-centered radicals (Sampath, 1966; Windle et al., 1964). The UV-irradiated samples feature broad, unresolved peak pattern, assigned to typical Bz• with a 30 - 40 G spread (Bridge, 1960) and 
with a small feature at $g=2.003$, while the $X$-ray irradiated samples feature a sharp peak with satellite peaks at ca. $60 \mathrm{G}$, underlain with a broad signal. The ca. $60 \mathrm{G}$ coupling is indicative for a seleniumcentered species. Therefore both spectra are interpreted as an overlay of a Bz and a selenium-centered radical species, like BzSeSe in varying proportion. While X-rays are mainly absorbed at selenium and hence lead to Se-centered radical species, UV -light is absorbed at the aromatic moieties and leads to Bzcentered radicals. Furthermore, in both spectra a signal at $\mathrm{g}=2.095$ can be observed, that was assigned to BzSe• by Windle et al..

The progression of the EPR signals upon aging of the samples in Figure 4.16 yield further information about the underlying effects. The signal, induced upon UV-irradiation, is depleted after two days, leaving only a very broad signal around $g=2.1$, that could be assigned to amorphous selenium. For the X-ray irradiated sample, the selenium signal with a coupling of ca. $60 \mathrm{G}$ gets stronger and stays strong for a month, while the underlying broad signal vanishes. It is therefore confirmed that radical species are induced by $\mathrm{X}$-ray irradiation. While Bz probably reacts with oxygen and evaporates as benzaldehyde, the selenium species are stable, which is not uncommon according to Sampath (1966). The full EPR analysis is given in Figures $\$ 4.41$ to $\$ 4.48$.

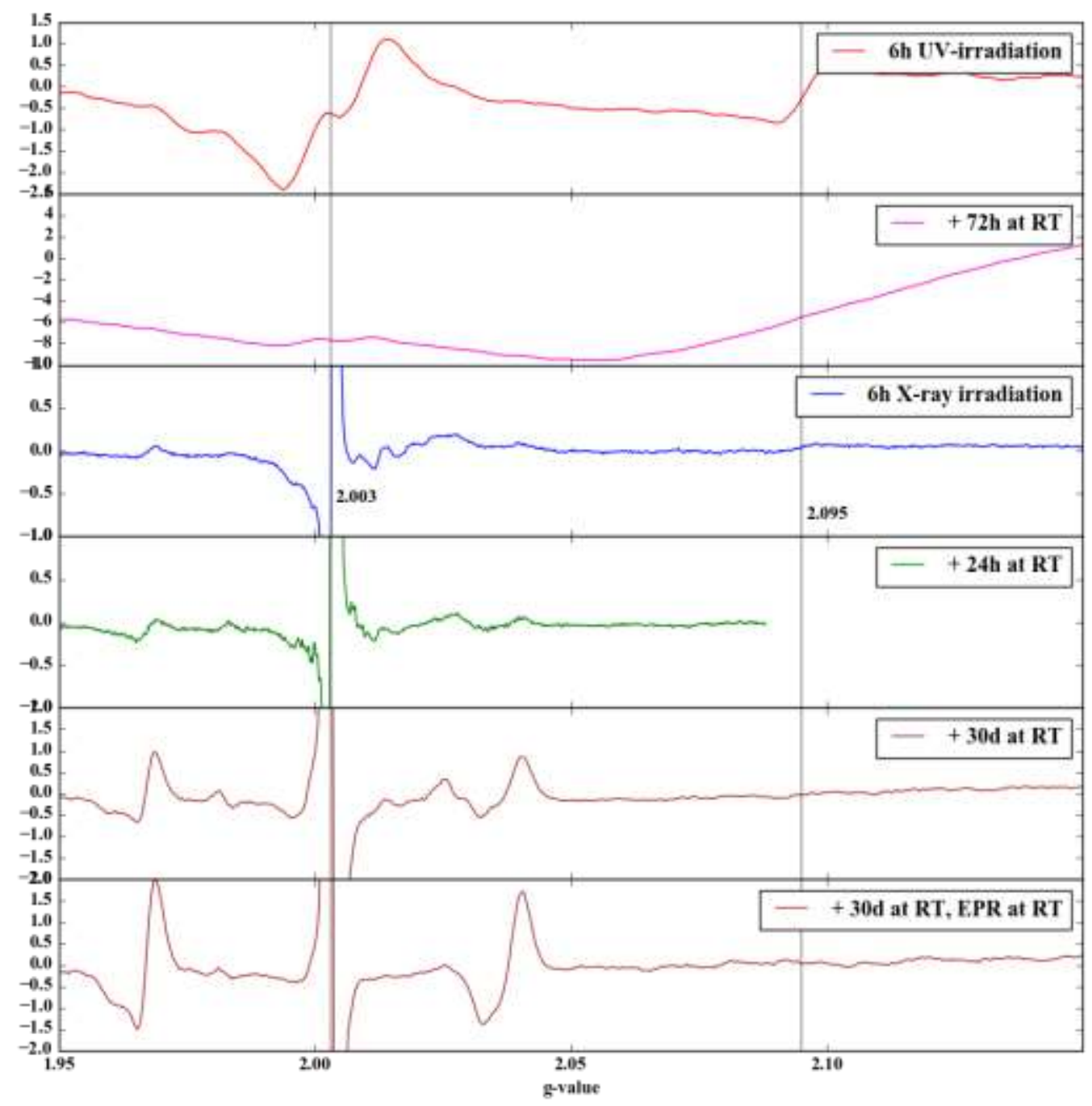

Figure 4.16: EPR signals of UV - and X-ray irradiated samples upon aging. 


\subsection{Conclusion}

The multiple high-resolution charge density determination of $(\mathrm{BzSe})_{2}(\mathbf{1})$ with various X-ray intensities and wavelengths unearthed the trapping of a persistent radiation-induced radical species BzSeSe $/ \cdot \mathrm{Bz}(2)$ in the single crystalline state. The related site occupation factors correlate directly with the power of the used X-ray source. The other structural changes, apart from the hemolytic Se-C bond cleavage, are so minute that a phase transition is not observed and the structural periphery is not affected. Hybrid QM/M M calculations confirmed $\mathbf{2}$ to be a minimum on the energy hyper phase and identified the residual peaks from the diffraction experiments to be the selenium positions of $\mathbf{2}$. UV- and X-ray induced radiation damage on the crystalline sample of $\mathbf{1}$ followed by EPR spectroscopy confirmed both radical species to be present. This effect is particularly important in structural biology, because especially in selenium MAD phasing the present organic radical causes a vivid unforeseen reaction cascade to the protein, i. e. not only resulting in the simple protonation of the related cysteine residue, but also might result in hydroxygenation, oxidation or $\mathrm{C}-\mathrm{C}$ bond formation. In protein structure determination, it is important to consider those radical-induced processes.

Revisiting some charge density investigations of organo-selenium compounds proved that this radiation induced Se-C bond cleavage not only occurs $\operatorname{in}(\mathrm{BzSe})_{2}(\mathbf{1})$ but is of more general concern. The works of Brezgunova et al. (2013) and Buhl et al. (2016) show the charge density investigation of Secontaining structures to be challenging and their refinements show so-far unassigned residual density peaks around selenium, possibly due to radiation damage and formation of radical species. 


\section{Chapter 5 Data Quality Assessment of New X-RAY Area DETECTORS}

\subsection{Introduction}

Recently Bruker AXS and Dectris introduced a new generation of X-ray area detectors for in-house use - the Bruker PHOTON II (Jarzembska et al., 2015), the Dectris PILATUS 3 R CdTe 300K (Brönnimann et al., 2006; Kraft et al., 2009) and the Bruker PHOTON III. Although relying on essentially different $X$-ray detection technologies, all three detectors shine with a large active area, a high dynamic range and very low noise (Stalke, 2014). The objective of this chapter is to compare this new generation of detectors among each other and with the well-established Bruker A PEX II by the means of actual structure data.

\subsubsection{Precision and Accuracy}

The Detectors are compared in three categories: precision, accuracy and to a minor extent also detection speed and price. Precision means the ability to determine the same intensity at repetitive measurements with small deviation and accuracy means to collect correct data without systematic errors that therefore truly reflects the charge density distribution in the crystal as illustrated in Figure 5.1.

The multiplicity-independent merging R-value $\mathrm{R}_{\text {rim }}$ (W eiss, 2001) (Eq. 3.3) serves as a tool for the evaluation of the data precision. The $R_{\text {rim }}$ gives a multiplicity-corrected measure for the

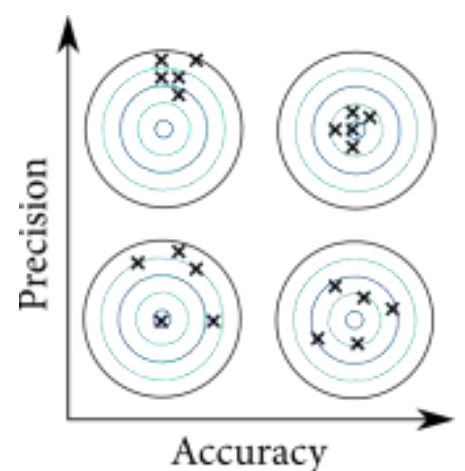

Figure 5.1: Schematic illustration of accuracy and precision. deviation of a single intensity from its mean value. By the resolution-dependent application of the $\mathrm{R}_{\text {rim, }}$ the progression of the precision can be compared for the different detectors.

The assessment of the accuracy on the other hand is less straightforward. The true value of a reflection intensity is in no other way accessible, than by the experiment itself. All theoretical approximations fail to take into account experimental circumstances, like temperature, mosaicity, and vibration. Therefore, the datasets have to be compared with each other by the means of their 
corresponding model: how well the model describes the data and whether all models show the same features.

\subsubsection{The Latest Generation of X-ray A rea D etectors}

O ver the course of the last years, the leading companies in the field of X-ray area detectors for inhouse single-crystal diffraction launched a series of new detectors. In 2014, Dectris introduced the PILATUS 3 R-series. In 2015, Bruker followed with the introduction of the PHOTON II and the PH OTON III, which is currently achieving market maturity, was introduced at the IU CR-M eeting 2017. All three detectors rely on new technologies, which distinct them from each other and earlier generations of detectors like the very popular and well established A PEX II.

\subsubsection{Bruker APEX II}

The Bruker APEX II (short A pex2) (Bruker AXS Inc., 2011) is a classical scintillating charge coupled device (CCD) detector. As high-energy photons are notoriously difficult to detect directly, impinging $X$-rays are converted to visible light by a layer of scintillating phosphorous material and the

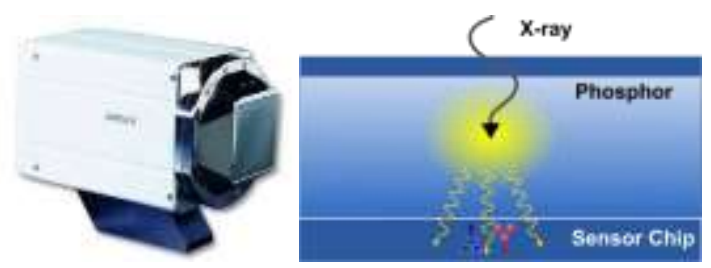

Figure 5.2: Bruker APEX II CCD detector and a schematic representation of its working principle. visible light is then detected by a CCD camera chip.

Thus, the first important building unit of the detector is the scintillation layer. Depending on the used material and its thickness, the conversion rate is optimized. H owever, whilea thicker layer increases theX-ray absorption and conversion rate, the resulting light also has to travel through the layer to reach theCCD. On the way, it is partly extinct and dissipated (Gruner et al., 2002), therefore thelayer thickness is always a compromise between these two factors (Schulz et al., 2009). The used APEX II detector was adapted to Ag-radiation with a thicker phosphor layer. Thereby, the smaller gain caused by the shorter wavelength was compensated, resulting in a photon efficiency of $91 \%$.

In the A pex2, an array of four Fairchild CCD6161 sensors, attached to the scintillation layer by a fiber optic faceplate is used to convert the visible light into electric charge with a characteristic quantum yield of 204 e per $\mathrm{X}$-ray photon $\left(\mathrm{Ag} \mathrm{K}_{\mathrm{a}}\right.$.) The photon-generated charge is read out, amplified pixelwise, and converted into a current. Ther efore, the detector requires a relatively long read-out time in the range of $500 \mathrm{~ms}$. The detector chip relies in a 16 bit well depth, allowing a dynamic range of 65535 counts per pixel and image. For the collection of high-quality $X$-ray diffraction data, this very limited dynamic range proves very problematic (W olf, 2014). Severe overexposure for the low-resolution range is often unavoidable, as the exposure time cannot be reduced as necessary. The active area of the A pex 2 is with $62.62 \mathrm{~mm}^{2}$ very small, compared to thenewer detectors, whilethe $15.15 \mu \mathrm{m}^{2}$ pixels size is comparable.

Frames are usually detected in two correlated scans, taking half the total exposure time. Thereby the dynamic range is doubled and noise is reduced. On the other hand, this procedure also lengthens the data collection immensely. 


\subsubsection{Dectris PILATUS3 R CdTe 300K}

The Dectris PILATUS 3 R CdTe 300K (short Pilatus3) is a specially adapted version of the PILATUS 3 series for the detection of hard radiation from Ag- (22.1 keV) or In-radiation (24.1 $\mathrm{keV}$ ) sources. All detectors in the PILATUS 3 series are Hybrid Pixel Array Detectors (HPAD) or so-

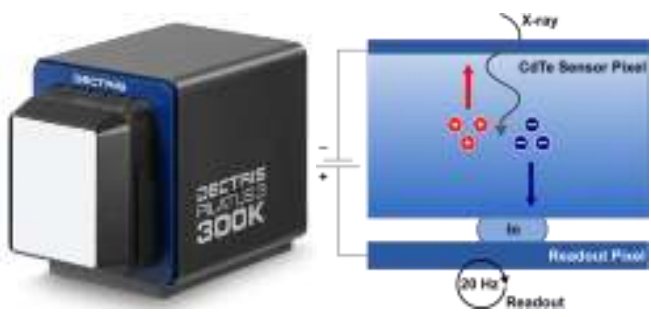

Figure 5.3: Dectris PILATUS3 R CdTe 300K pixel detector and a schematic representation of its working principle. called "Pixel detectors" that are capable of a direct pixelwise detection of X-ray photons. Each sensor pixel consists of a semiconductor layer, on which a high voltage is applied. While this layer usually consists of silicon, in the PILATUS3 R CdTe 300K a $1 \mathrm{~mm}$ cadmium telluride layer is used, resulting in a photon efficiency near $100 \%$. Impinging photons induce a charge-separation in the semiconductor layer that is directly detected by a readout pixel or application specific integrated circuit (ASIC). Semiconductor and readout pixel are attached pixel-wise by an interlayer (DECTRIS Ltd., 2015).

The direct conversion of X-ray photons into charge brings with it some mayor advantages to scintillating detectors. Primarily, the charge directly corresponds to the photons' energy. Therefore, an energy-discrimination for the detection of photon events is applicable. U sually, this energy-threshold is set to half of the photon energy. In the case of a photon impinging between two pixels, the induced charge is shared between them and the photon would only be counted once in the pixel where more than $50 \%$ of the photons energy is induced. All events that induce less than half of the photons' energy are ignored, resulting in virtually noise-free images. The energy cutoff can be adjusted to a desired value between 8 and $40 \mathrm{keV}$, so that for example in the case of X-ray fluorescent materials, the fluorescence can be effectively omitted (see also Figure 4.6).

The count-rate of $20 \mathrm{~Hz}$ and the 20 bit memory well depth are sufficient for the detection of intense radiation. H owever, at very high intensities in the $10^{6} \mathrm{cps}$ range, the detector approaches its count-ratelimit, as the $20 \mathrm{~Hz}$ read-out can no longer resolve each singular photon event in time. The Pilatus3 is capable of shutter-less readout, allowing for fast and continuous data collection. No additional errors are introduced through speed-shutters and principally, time-resolved crystallography is feasible.

The direct detection of X-ray photons also brings some drawbacks. The ASIC architecture requires a quite large pixel size of $172.172 \mu \mathrm{m}^{2}$, drastically decreasing the detector's resolution. The total active area is with $83.8 \cdot 106.5 \mathrm{~mm}^{2}$ about 2.3 times larger than the A pex2. However, the high voltage applied to the sensor chips requires one vertical and two horizontal spacers. As all reflections that touch the inactive area cannot be integrated correctly, the effectively usable detector area is further reduced.

The specific sensitivity of each pixel is scaled by a so-called flood-field correction. This correction has been determined experimentally for a series of typical energy cutoffs and is interpolated in between the experimentally determined values. If the energy cutoff is set to a non-tabled value, the interpolated flood-field correction can lead to minor features on the detector in the range of one count, as shown by Paul Niklas Ruth (2017). 


\subsubsection{Bruker PHOTON II}

The Bruker PHOTON II (short Photon2) is a charge-integrating pixel array detector (CPAD). Like the Apex2, the Photon2 detects $X$-rays via a scintillating phosphor that converts $X$-rays into visible light that is subsequently detected by a sensor chip. For the Photon2, the technique has been further developed in many aspects.

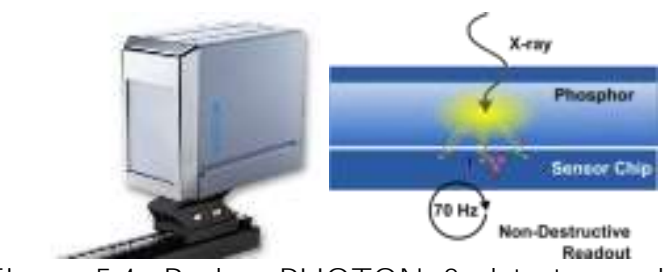

Figure 5.4: Bruker PHOTON 2 detector and a schematic representation of its working principle.

The detector shines with a very large active area of $110.140 \mathrm{~mm}^{2}, 1.6$ times larger than the Pilatus3, and a better resolution with a pixel size of $135.135 \mu \mathrm{m}^{2}$. This is achieved by the use of a large CMOS sensor chip. For effective noise cancellation, the Photon2 relies on real-time processing of the intensities during collection. Each pixels is measured at $70 \mathrm{~Hz}$, much faster than necessary for the diffraction experiment. This manifold measurement allows for oversampling. In oversampling, the mean value is calculated for a series of data points and thereby noise is effectively cancelled out. In the Photon2, this is also an adaptive oversampling ( $\mathrm{AO}$ ), so the oversampling rate is adapted to the exposure time. Therefore, especially at long exposure times, the Poton 2 is virtually noise-free and gives rise to the term 'single photon sensitivity'. In order to perform adaptive oversampling, the Photon 2 is equipped with a powerful digital signal-processing unit that processes the signal in real time.

While the Photon2 can hardly be physically oversaturated, as there is no count-rate limitation, it suffers from something called full-well saturation. As the signal is read non-destructively, the 10.14 bit (163810 counts) memory well runs full for high intensities. This count rate limit is in the same range as the Apex 2 limit for correlated measurements ( 2.16 bit, 131072 counts). Therefore, also in the Photon2, oversaturation of the strong, low-resolution reflections can be observed. This problem can be reduced by collecting data at a higher frame rate in an extra fast scan mode. The Photon 2 is capable of shutterless readout, allowing for fast and continuous data collection.

The scintillator has been vastly improved by the application of "structured scintillators" and micro-machined substrates to confine the scintillation light to a single pixel. Thereby, lateral spreading of the light is prevented and a superior point spread function is achieved. However, this improvement in point spread is accompanied by a decreased quantum gain.

The detector is advertised with high absorption and photon efficiency over $90 \%$, also for hard radiation like Ag-(22.1 keV) or In-radiation $(24.1 \mathrm{keV})$. N evertheless, as standard values for the collected frames, the phosphor absorbance is given as 70\% for $\mathrm{Ag}$ - and $53 \%$ for In-radiation. These are very low values that contradict the advertisement (Bruker AXS GmbH, 2015). 


\subsubsection{Bruker PHOTON III 14}

The Bruker PHOTON III (short Photon3) relies on the same basic principles and largely the same technology as the Photon2. The Photon 3 comes in two active area sizes: the same size as the Photon2 $\left(100 \times 140 \mathrm{~mm}^{2}\right)$ and twice its size

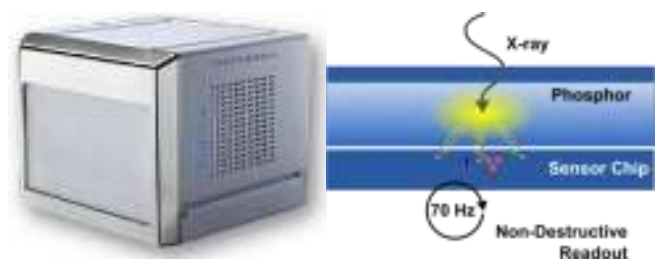

Figure 5.5: Bruker PHOTON III 28 detector and a schematic representation of its working principle. $\left(200 \times 140 \mathrm{~mm}^{2}\right)$, called PHOTON III 14 and PH OTON III 28. For this comparison, the small version with $14 \mathrm{~cm}^{2}$ was used, as it proved sufficient to collect the whole resolution range in one frame. The other huge improvement to the Photon 2 lies in its vastly improved processing power. The on-board real-time signal processing allows for data collection in the so-called "Mixed Mode". In "Normal Mode", the detector functions exactly like a Photon2, whereas in the M ixed Mode, the Detector has two unique features: On the one hand, it is capable to emulate the single photon counting capabilities of a pixel detector and on the other hand, the dynamic range truly scales with the exposure time.

Single photon detection is achieved by pixel wise real-timeevaluation of photon events. The charge, induced in each CM OS pixel plus a $3 \times 3$ array around it, is evaluated. Strong reflections are collected in integrative mode, like in the Photon2. W eak intensities are scaled to the energy of an integer number of photon events ( $329.4 \mathrm{e}$. for the case of the $\mathrm{Ag} \mathrm{K}_{\alpha}$ radiation used in this data collection). This includes the application of an energy threshold like in conventional photon-counting detectors. Thereby, the background noise is further reduced and especially weak reflections benefit from this procedure.

In Mixed Mode, the dynamic range scales with the exposure time by the factor $14 \mathrm{bit} \cdot \mathrm{s}^{-1}$. This means that at short exposure times, e.g. $1 \mathrm{~s}$, the dynamic range is one order of magnitude smaller, than for the A pex 2 or the Photon2 and ten orders of magnitude smaller than for the Pilatus3. This changes with exposure time and goes toward infinity at high exposure times. Peak intensity and dynamic range rise constantly with exposure time, therefore reflections that do not saturate the detector at a short exposure time will also not do so at any other given longer one. At least in theory, this allows for new data collection strategies, were all data are collected in one setup and with a long exposure time, as the detector is large enough to cover the whole resolution range and thanks to the $M$ ixed $M$ ode gives best intensities for all resolution ranges at highest exposure time. If however, a reflection is overexposed in the Mixed Mode, there is no chance to collect it in this mode at all. This causes problems for well diffracting crystals, as they are used in experimental charge density determination. It is shown that numerous strong reflections are missing in the M ixed M ode datasets, as they were overexposed. Strong reflections are better determined in the N ormal Mode and in order to achieve complete datasets, data, collected in Normal and M ixed M ode, have to be combined. The question of how this combination of the data and modes is achieved best is the subject of ongoing discussions between Bruker developers and the relatively few beta testers like ourselves. For the present work, best results were achieved by the merging of two full datasets - one collected in N ormal and the other in M ixed M ode. 


\subsection{Benchmark Structures}

For the comparison of the detectors performance, two compounds have been selected that diffract well and make high-resolution data accessible. On the other hand, they represent the scope of molecular crystallography. For the comparison, data sets of both compounds were collected with all four detectors.

[2,2]-Paracyclophane (Figure 5.6) is representative for organic structures that only contain light elements and are therefore weakly diffracting. In its high-temperature phase over 45.2K it adopts the highly symmetric space-group $\mathrm{P} 4{ }_{2} / \mathrm{mnm}$ (W olf et al., 2015a; Wolf et al., 2015b). The compound has been heavily investigated and served as benchmarking system for the development of theoretical methods, the comparison of $X$-ray diffraction facilities (Wolf, 2014) and X-ray detectors (Krause, 2017). The

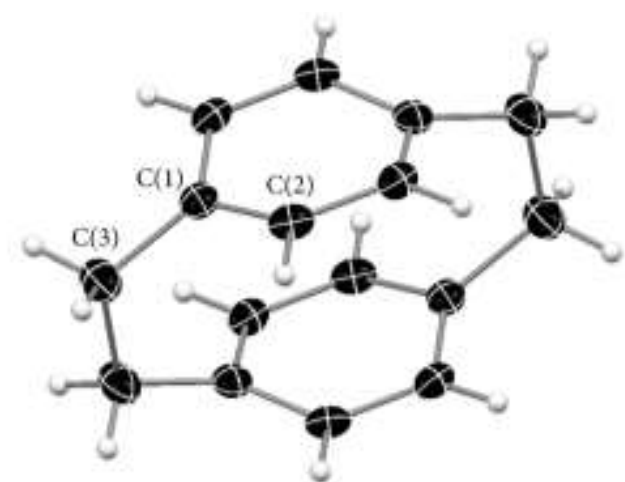

Figure 5.6: Crystal structure of [2,2]Paracyclophane. The asymmetric unit contains $1 / 8$ molecule. diffraction pattern of paracyclophane crystals features very strong low-resolution reflections and very weak high-resolution reflections that rarely exceed resolutions of $0.5 \AA$. The challenge for the detectors is to determine both weak and strong data correctly.

Dibenzyldiselenide (BzSe) ${ }_{2}$ is al ready discussed in depth in Chapter 4. It is representative for heavy atom compounds that diffract well up to a resolution of $0.45 \AA$ and furthermore shows $x$-ray fluorescence (see also Figure 4.6), resulting in an elevated background. As discussed in Chapter 4, the structure of $(B z S e)_{2}$ is superimposed with a lower percentage of the $X$-ray activated bi-radical formation of $\mathrm{Bz}^{\bullet}{ }^{\bullet} \mathrm{SeSeBz}$ and therefore it is currently impossible to model the charge density correctly. As the true density distribution is impossible to describe, no true peak intensities are accessible for this compound. Subsequently, the detectors' accuracy cannot be assessed with this dataset. Nevertheless, raw peak intensities are perfectly comparable and ther efor it can be used for the assessment of the detectors.

\subsection{Experimental D etails}

The Pilatus3 datasets have been collected in the summer of 2015, when the Stalke group was offered the opportunity to gather experience with the new detector. It was fitted into a Bruker D8 housing on a A pex II SM ART 3-circle goniometer, combined with a $30 \mathrm{~W}$ Ag I $\mathrm{S}$ S by Felix Engelhard (Engelhardt, 2017). Paul Niklas Ruth converted the frames into the Bruker frame format (Ruth, 2017). The A pex 2 datasets have been collected at the same setup. The paracyclophane dataset was collected by HilkeW olf in 2013 (W olf, 2014) and the (BzSe) ${ }_{2}$ dataset was collected in 2015. All Photon2 and Photon3 datasets were collected by Holger Ott at the Bruker AXS facilities in Karlsruhe between 2016 and 2018, using a Bruker Venture housing, a Kappa goniometer and a $70 \mathrm{~W}$ A g I $\mathrm{SS3}$. 
Therefore, all datasets have been collected from similar crystals on largely similar setups, using silver radiation from Incoatec M icrofocus Sources (I $\mu S$ ) of different generations (A rndt, 1990; Storm et al., 2004). All resulting datasets showed good multiplicity, full completeness up to the full selected resolution and were therefore suitable for charge density refinements.

All datasets were processed individually. They were integrated in SAINT (Bruker AXS Inc., 2016), scaled in SADABS (Krause et al., 2015a) while retaining the experimental weights and refined in SHELXL (Sheldrick, 2015) and XD2006 (V olkov et al., 2007), using always the same refinement strategy. The processing of the (BzSe) ${ }_{2}$ datasets has been discussed in Chapter 4 , the Apex2 was used in dataset $\mathbf{B}$, Pilatus3 in dataset $\mathbf{C}$, Photon2 in $\mathbf{E}$ and Photon 3 in $\mathbf{G}$. For paracyclophane, the Apex2 and Pilatus3 datasets were integrated, using the SAINT defaults. Photon2 \& 3 data were integrated, using the PLANEBG algorithm. All datasets are named further on according to their compound and the used detector, e.g. Para_A pex2.
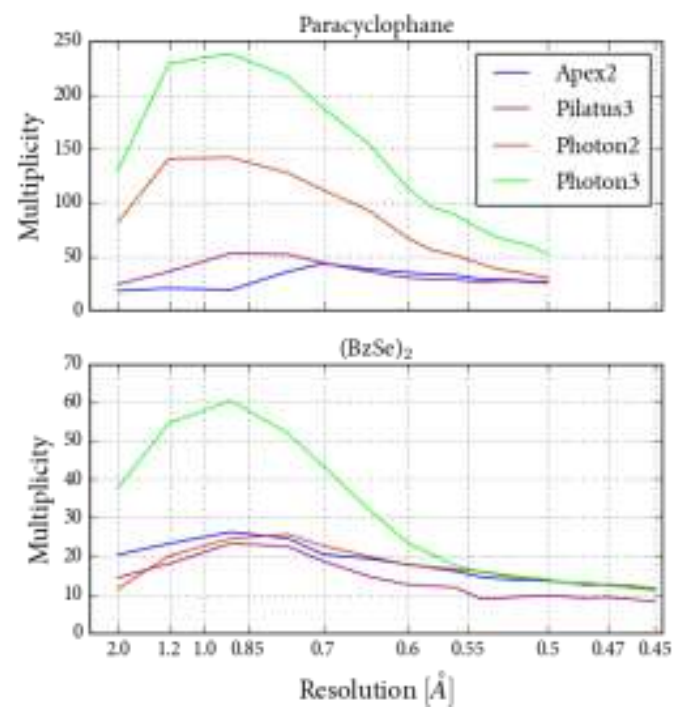

Figure 5.7: Evaluation of the Multiplicity for all compared datasets.

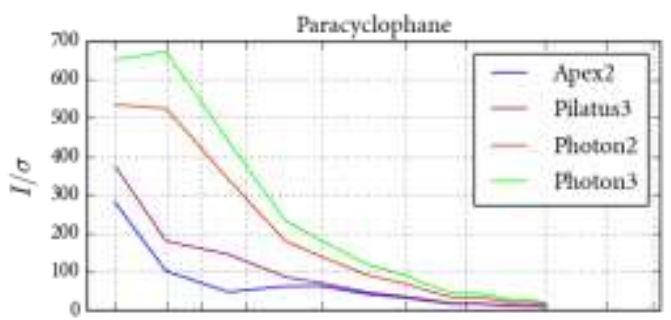

$(\mathrm{BzSe})_{2}$

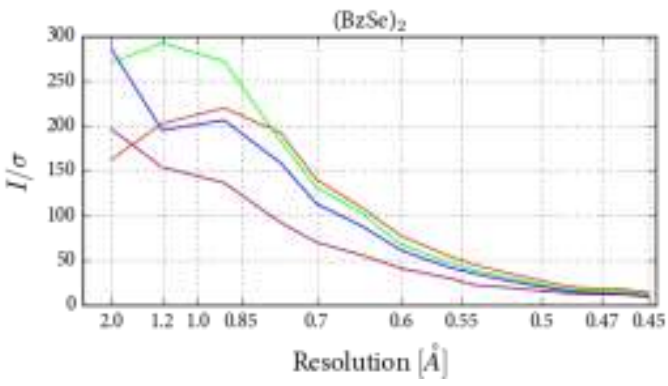

Figure 5.8: Evaluation of the $I / \sigma$ for all compared datasets.

XPREP (Sheldrick, 2014a) statistics were computed for all datasets (seeT ables S4.2, S4.3, S4.5, S4.7, and S5.2 to S5.5). By putting the statistics into context, the comparability of the datasets was evaluated.

First objective was to select collected runs for each dataset, in order to achieve a similar multiplicity for all datasets. As can be observed in Figure 5.7, this goal was achieved somewhat limited. Due to the large active area of the Photon $2 \& 3$, regardless of the detectors position almost always the full resolution range was seen in one frame, leading to a higher multiplicity especially in the paracyclophane datasets. The multiplicity of the Photon 3 datasets is in all cases twice as large, as it is comprised of two full datasets from Mixed and Normal Mode.

Thel/ $\sigma$ in Figure 5.8 shows highest values in most of the resolution range for Photon $2 \& 3$, due to the more powerful X-ray sources. However, for the innermost resolution shell the I/ $\sigma$ drops, due to the 
limited dynamic range of the detectors. The Apex2 and Pilatus3 datasets show very similar features. Therefore, the datasets are well comparable, but one has to keep in mind, that the Photon $2 \& 3$ datasets were collected with approximately twice the radiation intensity.

The refinement procedure of all (BzSe) ${ }_{2}$ datasets is given in Chapter 4, therefore here only an introduction to the refinement of paracyclophane is given. A smallest possible number of parameters was achieved by the application of maximal local symmetry (Table 5.1). The refinement strategy in Table 5.2 was tested for all datasets by the means of $R_{\text {free }}$ (Figures S5.1 to S5.4), significance (Tables S5.6 to S5.9), systematic errors (Figures S5.5 to S5.8) and evaluation of the Probability Density Function (PDF) of the anharmonically refined atom C(3) (Tables S5.10 to S5.12). Multipoles were applied to carbon up to the octupole and to the dipole for hydrogen. As shown by Köhler (2017), and Krause (2017), the anisotropic refinement of hydrogen is generally for high resolution $X$-ray data and in particular for paracyclophane feasible. In addition, the course of the $R_{\text {free }}$ gave no indication of a problematic refinement. Therefore, the shown strategy in Table 5.2 was found to be optimal for the refinement in this comparison.

Table 5.2: Refinement strategy for the charge density refinements of paracyclophane datasets. The ratio of data to parameter and the R-values are exemplary given for dataset Para_Pilatus3. A bbreviations: Para: parameter; M P: M ultipole; M : monopoles; D: dipoles; Q: quadrupoles; O: octupoles; U2, U 3: Gram Charlier $2^{\text {nd }}$ and $3^{\text {rd }}$ order, $\mathrm{H}$-XYZ: hydrogen position against data up to $0.5 \sin (\theta) / \lambda$.

\begin{tabular}{lllll}
\hline Step & New Parameter & Para & Data/Para & $w R\left(F^{2}\right)$ \\
1 & SCALE & 1 & 1227 & 0.1508 \\
2 & DQO & 28 & 43.8 & 0.0968 \\
3 & U2 & 42 & 29.2 & 0.0684 \\
4 & H-XYZ & 7 & 25.4 & 0.0553 \\
5 & XYZ & 49 & 25 & 0.0606 \\
6 & H-XYZ & 7 & 25.4 & 0.0449 \\
7 & M & 53 & 23.1 & 0.056 \\
8 & U3 & 59 & 20.8 & 0.0532 \\
9 & U2(H) & 71 & 17.3 & 0.0459 \\
10 & K & 74 & 16.6 & 0.045 \\
\hline
\end{tabular}

\subsection{Assessment of Precision}

The assessment of the detectors' precision is based on the evaluation of the multiplicityindependent $\mathrm{R}_{\text {rim }}$ of the compared datasets in Figure 5.9. For the paracyclophane-datasets, the Pilatus3 
shows the lowest $R$-values, up to a resolution of $0.6 \AA$, followed by the $A$ pex 2 dataset that shows a similar progression. At high resolution $(<0.6 \AA)$, the Photon $2 \& 3$ datasets show the best R-values. The course of the Photon $2 \& 3$ datasets is almost the same. For the (BzSe) ${ }_{2}$ datasets, the best results over the full resolution range are achieved by the Photon2 and Apex2 datasets. Here, the Pilatus3 dataset is inferior and shows almost twice as high $\mathrm{R}$-values over the full resolution range and astonishingly the Photon2 here performs better than the Photon3. It has to be stated that the "old" A pex2 detector and the subsequent generation of detectors perform very similar in this assessment of precision. In fact, in the combined analysis of
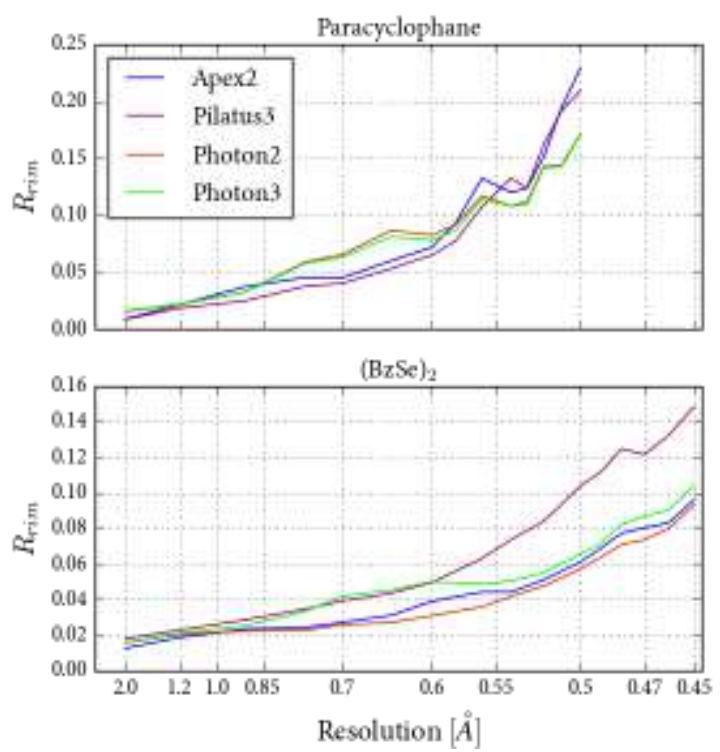

Figure 5.9: Evaluation of the $R_{\text {rim }}$ for all compared all datasets, the Apex 2 results show the best. The intensities of all datasets seem to be determined with good precision that corresponds to the experimental errors.

\subsection{Assessment of Accuracy}

The Assessment of accuracy is based on the evaluation of refined charge density models of Paracyclophane. The (BzSe) ${ }_{2}$ datasets are not addressed, because of the density features, discussed in Chapter 4.

The overall R-values give a measure for the accordance of data and parameter. As the model should be able to refine all physically meaningful density, differences of data and model should only occur due to experimental errors. Therefore the residual density is analyzed according to $M$ eindl \& $\mathrm{Henn}$ as a measure of data quality. No weighting is applied to the data during scaling or refinement, so the experimentally determined weights were retained throughout the procedure. The goodness of fit (GOF) indicates therefore whether the experimental errors fit the deviations between data and model. So if the GOF deviates from 1, the experimental and theoretical errors are not in accordance (see also part 1.6.).

Table 5.3: Selected model quality indicators and selected properties of the derived model. The observed BCP is the $C(1)-C(3) B C P$

\begin{tabular}{lllll}
\hline Detector & A pex2a & Pilatus3 & Photon2 & Photon3 \\
$\mathrm{R}\left(\mathrm{F}^{2}\right)[\%]$ & 0.0143 & 0.0118 & 0.0128 & 0.0126 \\
$\mathrm{wR}\left(\mathrm{F}^{2}\right)[\%]$ & 0.0188 & 0.0169 & 0.0142 & 0.0141 \\
$\mathrm{GOF}$ & 1.8921 & 2.5359 & 4.2661 & 5.4566 \\
$\rho_{\min }\left[\mathrm{e} \AA^{-3}\right]$ & -0.116 & -0.087 & -0.107 & -0.108 \\
$\rho_{\max }\left[\mathrm{e} \AA^{-3}\right]$ & 0.103 & 0.147 & 0.094 & 0.097 \\
$e_{\text {gross }}$ & 5.3134 & 4.3258 & 2.9607 & 2.9328 \\
$\rho(\mathrm{BCP})\left[\mathrm{e} \AA^{-3}\right]$ & $1.672(12)$ & $1.703(5)$ & $1.671(9)$ & $1.673(12)$ \\
$\nabla^{2} \rho(\mathrm{BCP})\left[\mathrm{e}^{-5}\right]$ & $12.7(3)$ & $-13.6(1)$ & $-12.3(2)$ & $-12.3(3)$ \\
\hline
\end{tabular}


The models of all datasets refine to very good fits with low R-values and low residuals. The quality of all datasets is sufficient for the experimental charge density investigation. N evertheless, differences can be observed between the datasets.

Looking at the R-values in Table 5.3, the A pex2 dataset shows the worst accordance between model and data. Photon 2 and Photon3 perform very similar. The Pilatus3 shows the best $R\left(F^{2}\right)$, while the Photon detectors show the best $w R\left(F^{2}\right)$. This hints to differences in the weighting and is reflected by the GOF, that is lowest for the A pex2, slightly raised for the Pilatus3 and definitely too high for the Photon detectors. The very high GOF values are a salient feature for all Photon $2 \& 3$ data, discussed in this work (see also Table 4.5) and plead for an incorrect determination of the experimental errors.

The derived properties in Table 5.3 and Table S5.13 are mostly within standard deviation for all datasets. For the Pilatus2 and Pilatus3 datasets, they are almost the same, while they generally differ slightly but significantly from the values of the Pilatus3 and Apex2 dataset. This advocates for only minor, systematic difference between the datasets.

The residual density in Table 5.3 and Table 5.4 shows highest features for the A pex 2 dataset. The residual density map is noisy and the fractal dimension plot is the broadest. The Pilatus3 dataset performs little better, with less divergent residual maxima and minima, a less noisy residual map and a narrower fractal dimension plot. The residual density of the Photon2 and Photon3 datasets is very similar in height. The residual map and fractal dimension plot are nearly indistinguishable. The residual density on the Photon datasets is furthermore the lowest. The residual density map shows nearly no features and the fractal dimension plots are very narrow, reflecting a flat and featureless residual density.

Table 5.4: Residual density analysis for all compared datasets.

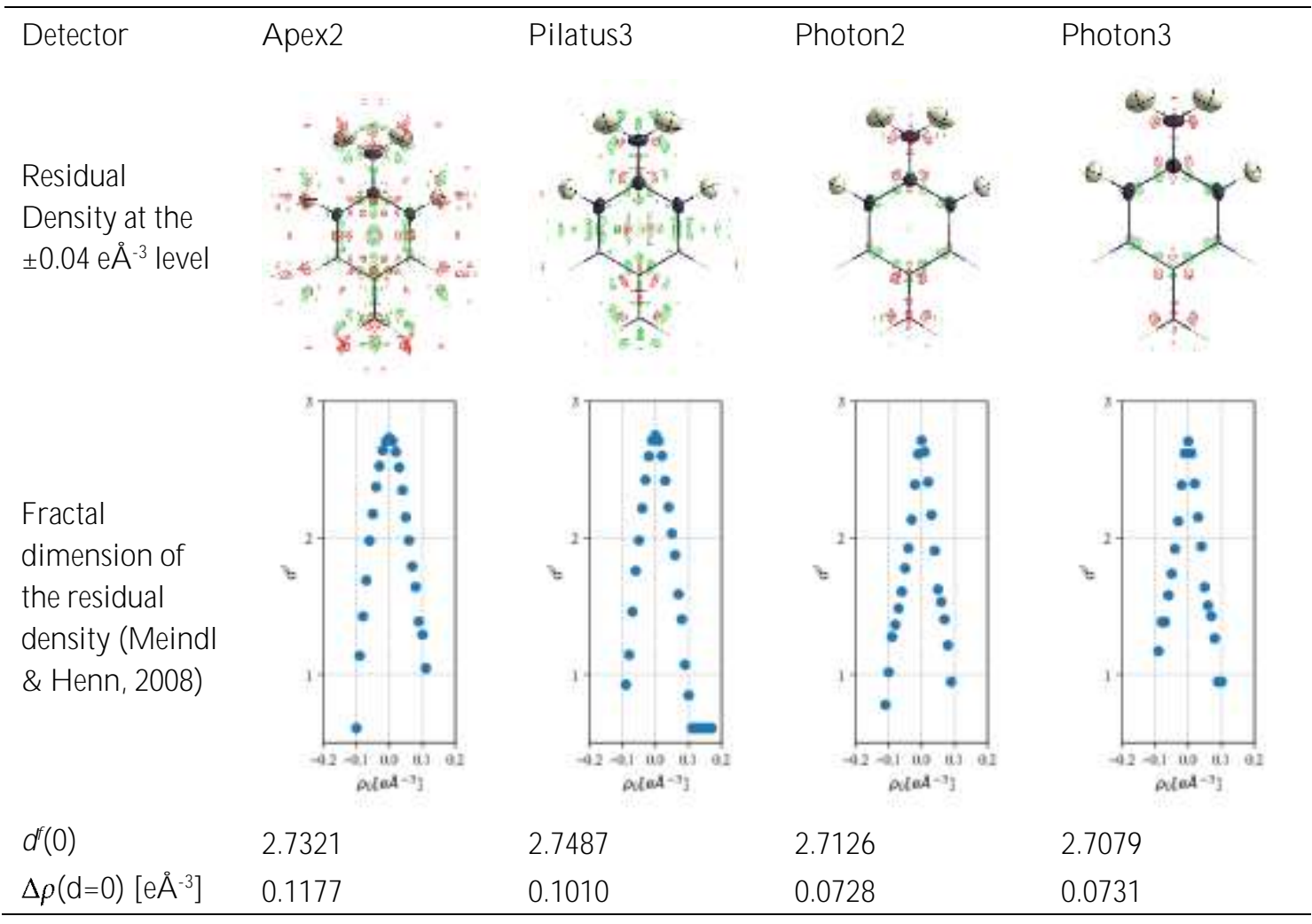




\subsection{Conclusion}

The new generation of detectors performs very well in the experiment, but the differences to the old A pex 2 lie foremost in the better practicability. Due to shutter-less data collection and larger active areas, the data collection is sped up significantly. Experimental problems, like icing and crystal decomposition, are thereby reduced. The increased dynamic range of the Pilatus3 allows for higher intensities and the M ixed M ode of the Photon3 allows for new data collection strategies.

The precision of the collected data is very similar for all detectors. It is therefore concluded, that all detectors determine the intensities exact and do not add random errors to the signal. The accuracy was assessed via the refined models. The refined models showed very similar properties with only slight divergence. The Pilatus3 showed the best overall fit of data and model $\left(R\left(F^{2}\right)\right)$, but the Photon datasets showed the best weighted fit $\left(w R\left(F^{2}\right)\right)$ and the flattest residual density. The weights of the Photon $2 \& 3$ datasets however, prove to be incorrect, as shown by the high GOF. This is a grave problem for the Photon2 and Photon3 detectors. The Photon detectors probably collected the most accurate datasets, but simultaneously the most inaccurate experimental errors are determined. W ith respect to the used $X$-ray source, with higher intensity for the Photon datasets, it must be concluded that all detectors determine intensities with very similar accuracy.

Between the Photon2 and Photon3 datasets, no significant difference could be found that vindicates experimental effort to collect a combined Mixed Mode/Normal Mode dataset and the substantial difference in pricing. Considering also the very substantial price difference between Photon2 and Pilatus3, this comparison clearly advocates for the moderately prized Photon2 detector - if the problem of the erroneous experimental errors can be fixed. 



\section{Chapter 6 ChARGE Density INVESTIGATIONS ON Frustrated LeWIS PAIRS}

\subsection{Introduction}

In 2011/12, Daniel Kratzert collected data on a pair of structures, emerging from a cooperation with the Erker group of M ünster U niversity. The structures comprised one Frustrated Lewis Pair (FLP) (3) and the reaction product of an FLP (2). Although the crystal- and data quality were fine, the datasets proved to be problematic and while one was refined by Dr. R. H erbst-Irmer, the other one was handed down to a latter generation of PhD students and ended with me. The objective was to receive the experimental charge density of this compound and put it into context with the other compound and further FLPS.
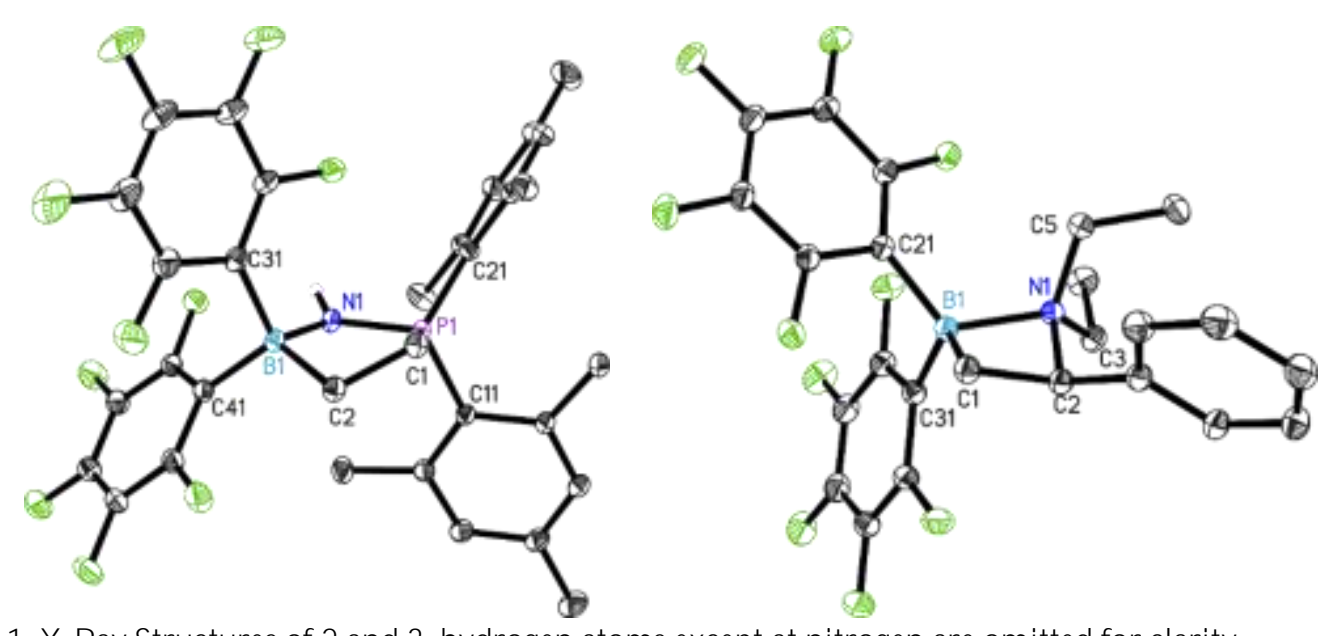

Figure 6.1: X-Ray Structures of $\mathbf{2}$ and $\mathbf{3}$, hydrogen atoms except at nitrogen are omitted for clarity

FLPs are sterically encumbered Lewis acid and Lewis base combinations and are applied in the activation of small molecules (Frenking $\&$ Shaik, 2014; Stephan $\&$ Erker, 2010). Here, they offer new reaction pathways (Kehr \& Erker, 2017; Caputo \& Stephan, 2017) which include the activation of elemental hydrogen and the catalysis of hydrogenation reactions (Özgün et al., 2016). Examples for such catalytically active compounds are intramolecular B-N and B-P FLPs with the so-called Piers' borane (Piers \& Chivers, 1997) $\mathrm{HB}\left(\mathrm{C}_{6} \mathrm{~F}_{5}\right)_{2}$ as acid, connected by carbon bridges to the pnictogen (nitrogengroup element) with variable residues. One of the most catalytically active and well-investigated compounds of this class is $\mathbf{1}$. Here, Piers' borane is connected to PMes 2 by an ethyl bridge (Spies et al., 
2007). U nfortunately, 1 has not been structurally characterized so far, due to the lack of suitable single crystals (Spies et al., 2009) and therefore only has been structurally investigated by DFT methods.

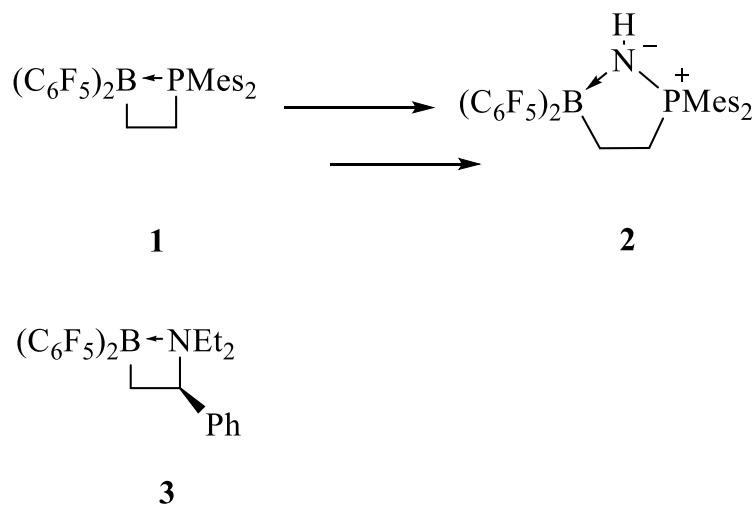

Figure 6.2: Donor-acceptor diagrams of the investigated Figure 6.3: suggested Lewis diagram of $\mathbf{2}$ as FLP structures published by (Schwendemann et al., 2011).

However, various reaction products of $\mathbf{1}$ were characterized by X-ray diffraction, like the hydrogenation product, reaction products with carbonyl groups and olefins ( $M$ ömming et al., 2009a) or $\mathrm{CO}_{2}$. (M ömming et al., 2009b) and, most noticeably for this work, the reaction product of an anomalous Staudinger reaction $\mathbf{2}$ (Stute et al., 2012). The substitution of phosphorous by nitrogen leads to another wide range of FLPs. Schwendemann et al. synthesized and characterized a series of $\mathrm{C}_{2}$-bound intramolecular B-N FLPs (3 to 6) with varying catalytic abilities (M ömming et al., 2009b). O ne particular example is $\mathbf{3}$ that resulted in crystals suitable for an experimental charge density investigation and is isostructural to 1.

FLPs often feature the activation of $\mathrm{H}_{2}$ at mild conditions. This catalytic activity is anticipated to correlate to the B-N coordinating strength and subsequently to the bond lengths in the solid state. Furthermore, weakly coordinated boron is prone to a trigonal-planar coordination, so the angular sum of C-B-C angles is anticipated to be near $360^{\circ}$. Correlating the reactivity with the conformation, $\mathbf{3}$ and $\mathbf{5}$ show the shortest bond distances, comparably low C-B-C angular sum and do not activate $\mathrm{H}_{2} .6$ and 7 show slightly longer bond distances, higher sum of C-B-C angles and readily activate $\mathrm{H}_{2}$, while in $\mathbf{8}$ and 9 the distance is even larger than the sum of the van der Waals radii, almost trigonal-planar boron coordination and the activity is outstanding. In contrast to $\mathbf{3}$, the structurally closely related $\mathbf{4}$ is able to activate $\mathrm{H}_{2}$. Nevertheless, both molecules show the same $\delta^{11} \mathrm{~B}-\mathrm{N} M \mathrm{R}$ chemical shift of $2.1 \mathrm{ppm}$, which suggests a very similar bonding situation.

The bonding situation of intramolecular FLPs seems unclear in some cases and manifests itself in inconsistent Lewis diagrams. The acidic boron is presented as a formally negative charged boride, while the pnictogen is oxidized, what seems surprising with regard to the electronegativities. In other cases, the atoms are connected by a dotted line or an arrow, indicating a donating bond (Himmel et al., 2014a, 2014b; Frenking, 2014). The charge density analysis of $\mathbf{1}$ and $\mathbf{3}$ can give rise to a more physically meaningful charge density distribution of intramolecular FLPs. Furthermore, the similarities and 
differences by the introduction of different pnictogen atoms can be analyzed. The charge density analysis of $\mathbf{2}$ can provide information about the general reactivity of FLPs in terms of charge transfers and oxidation states. The bonding situation raises questions, as it was introduced as an phosphinimine with a formal double bond between $\mathrm{N}$ and $\mathrm{P}$ and a negative formal charge at B (Stute et al., 2012). This seems surprising with regard to the electronegativities and the previous experimental charge density investigation of a phosphinimine. This indicated that the formal $\mathrm{P}=\mathrm{N}$ double bond is better described as a nonhypervalent electrostatically reinforced $\mathrm{P}^{\delta+}$ $\mathrm{N}^{\delta-}$ polar bond (Kocher et al., 2004). In order to clarify here, we investigate the experimental electron density distribution in $\mathbf{2}$.

In order to attain the charge density distribution of $\mathbf{1}, \mathrm{T}$. Teuteberg performed an extended DFT optimization at the B3LYPD3/def2-TZVP level of theory. In order to test the agreement of the DFT results of $\mathbf{1}$ with the charge density distribution of compound $\mathbf{3} \mathrm{He}$ also optimized this at the same level of theory as $\mathbf{3}^{\prime}$. By the comparison of $\mathbf{3}$ and $\mathbf{3}$ ' the comparability of experimental and theoretical charge densities in the QTAIM approach should be confirmed.
Table 6.1: Overview of structurally related B-N FLPs, their solid-state $\mathrm{N}-\mathrm{B}$ distance and their reactivity towards hydrogen. Compounds 3 to 6 were published by Schwendemann et al., 2011, 7 and 8 by Chernichenko et al., 2012 and 9 by $W$ ang et al., 2016.

3




\subsection{Experimental}

Compound 3 was processed and refined by Dr. R. H erbstIrmer and was published in Krause et al. (2017). Only the charge density analysis is part of this thesis.

Compound 2 crystallizes in space group $P 2_{1} / n$, with one molecule in the asymmetric unit. The dataset exhibits high completeness, multiplicity, and resolution, which made a multipole refinement feasible. The refinement in the multipole formalism (Hansen \& Coppens, 1978) and QTAIM analysis (Bader, 1991) were carried out in the XD2006 program package (Volkov et al., 2006).

One highly important feature for this refinement was that 2 contains three disordered methyl groups. In order to refine a valid model of the charge density, special care and caution needed to be taken with the refinement of these disordered groups, as disorder is normally fatal for a charge density refinement.

The theoretical wave functions of $\mathbf{1}$ and $\mathbf{3}$ ', optimized by Thorsten Teuteberg, were analyzed in the QTAIM framework, using the AIM ALL software suite (Keith, 2017).
Table6.2: Crystallographic details for 2.

\begin{tabular}{|c|c|}
\hline & 2 \\
\hline Formula & $\mathrm{C}_{32} \mathrm{H}_{27} \mathrm{BF}_{10} \mathrm{NP}$ \\
\hline CCDC no. & 1819123 \\
\hline Space group & $\mathrm{P} 21 / \mathrm{n}$ \\
\hline $\mathrm{a}[\AA]$ & $11.045(1)$ \\
\hline $\mathrm{b}[\AA]$ & $14.357(2)$ \\
\hline$c[\AA]$ & $18.753(2)$ \\
\hline$\beta\left[^{\circ}\right]$ & $100.390(9)$ \\
\hline$V\left[\AA^{-3}\right]$ & $2925.0(6)$ \\
\hline crystal size & $0.174 \times 0.233 \times 0.280$ \\
\hline$\theta$ range $\left[^{\circ}\right]$ & 1.797 to 52.190 \\
\hline Collected ref. & 252458 \\
\hline Independent ref. & 33664 \\
\hline Rint & 0.0395 \\
\hline R1 (all data, IAM ) & 0.0433 \\
\hline wR2 (all data, IAM) & 0.0995 \\
\hline $\operatorname{GOF}\left(F^{2}, I A M\right)$ & 0.957 \\
\hline Data XD used & 31140 \\
\hline Parameter & 742 \\
\hline$R\left(F^{2}, X D\right)$ & 0.023 \\
\hline$w R\left(F^{2}, X D\right)$ & 0.022 \\
\hline $\mathrm{GOF}\left(\mathrm{F}^{2}, \mathrm{XD}\right)$ & 1.215 \\
\hline \multicolumn{2}{|l|}{ Diff peak/hole } \\
\hline$(\mathrm{XD})\left[\mathrm{e}^{-3}\right]$ & $0.198 /-0.185$ \\
\hline
\end{tabular}

\subsubsection{Charge Density Refinement}

For the preparation of a starting model, the heavy element positions and vibrational parameters were refined against high-resolution data $(<0.8 \AA)$, while hydrogen atoms were placed on the difference Fourier maxima of low-resolution data $(>0.8 \AA)$ and set to neutron data distances. Only disordered hydrogen atoms were placed on idealized positions with neutron diffraction data derived bond lengths and tetrahedral angles. For the introduction of multipole parameters, the highest possible local symmetry was applied. M ultipole populations of chemically equivalent atoms were constrained to bethe same (Table S6.1). All atoms except hydrogen were refined to the hexadecapole level, while hydrogen atoms were refined to the dipolelevel. The final refinement strategy is given in Table S6.2. Selected atoms were refined with anharmonic motion (Gram-Charlier $3^{\text {rd }}$ order). Special care was taken to refine meaningful anharmonic motion, as ten additional parameters need to be refined for every anharmonic atom position, thereby raising the number of parameter significantly. Therefore, Kuhs's Rule (Kuhs, 1992) was applied (Table S6.3), the significance of refined Gram-Charlier parameters was tested (Table S6.4) and the atomic probability density function was tested to be physically meaningful (Table S6.5) (Herbst-Irmer \& Stalke, 2017). The refinement procedures were tested under consideration of the resolution-dependent error and statistic error distribution (Figure S6.2), the residual density distribution (FigureS6.3 and FigureS6.4), and cross-validation results (FigureS6.1) (Krauseet al., 2017). 


\subsubsection{Refinement of Disordered $H$ ydrogen}

Disorder is usually fatal for an electron density refinement. Beside the problems regarding data quality and resolution, the interpretation of disordered models in the QTAIM framework is not feasible. One moiety of the disordered model must be selected in order to obtain meaningful results. Nevertheless, the refined model should describe the disorder at best in order to correctly assign the electron density to the disordered moieties and subsequently obtain a correct representation of the valence electron density for each moiety.

Three of the six methyl groups in the structure are disordered in a rotation of the hydrogen atoms. The disorder was described by two staggered conformations of the methyl group: The two carbon atoms were constrained to have the same position and vibrational parameters, but had differing multipole orientations, regarding to their hydrogen atoms (Figure 6.4).

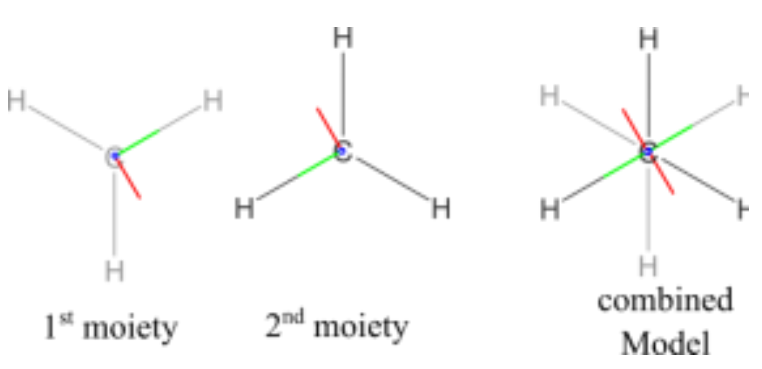

Figure 6.4: Principal orientation of the local coordinate systems for a disordered methyl group. Red: $x$, green: $y$, blue: $z$.

The multipole parameters of all methyl groups in the molecule (disordered and not disordered) were constrained to be equal, so that only single sets of multipole parameters for carbon and hydrogen were refined. For the application of these global parameters to the disordered moieties, the multipole populations were multiplied by the population coefficient of the corresponding moiety. However, the refined parameters were slightly biased by the disorder. Therefore, as a final step, the multipole parameters of the non-disordered groups were refined without constraint to the disordered and the parameters were adopted to the disordered atoms. The population of the disordered moieties were determined by the ratio of the freely refined hydrogen monopoles.

\subsubsection{Identification of Bad Data}

By the application of cross-validation (Krause et al., 2017), three reflections of the dataset were identified to be outliers, unfitting to the residual dataset. During the refinement of 20 cross-validation sets, two sets stood out to exhibit significantly higher $R_{\text {free }}$ values (see Figure 6.5). Furthermore, the check for parameter outliers identified outliers belonging to only three test sets (Figure 6.6). Careful inspection of these two validation sets showed that both sets contain one very

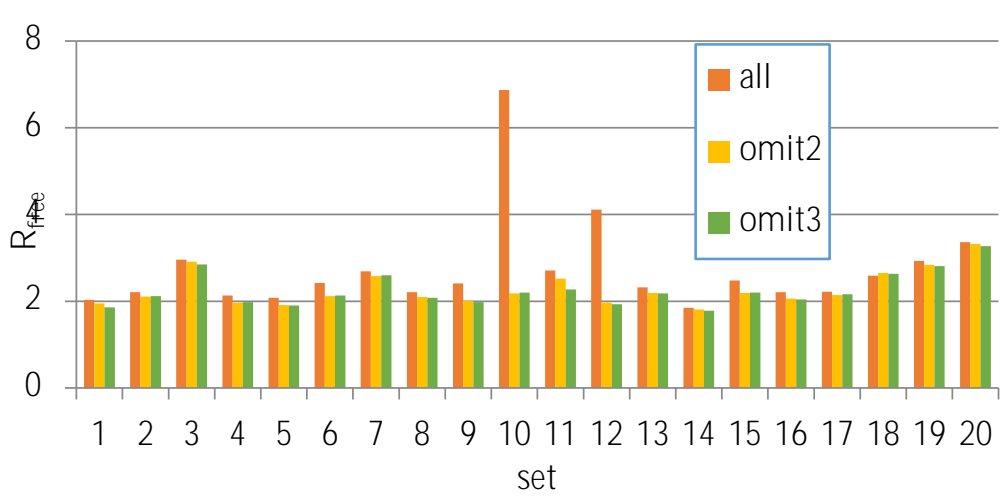

Figure 6.5: Rfree values for all 20 validations sets without and with omitted reflections. 
strong low order reflection. These two reflections were overexposed. Strong low order reflections strongly influence the multipole populations, therefore bad determination of such reflections is very problematic. Omitting these two reflections from the whole data set improved the model indicated by a featureless parameter distribution.

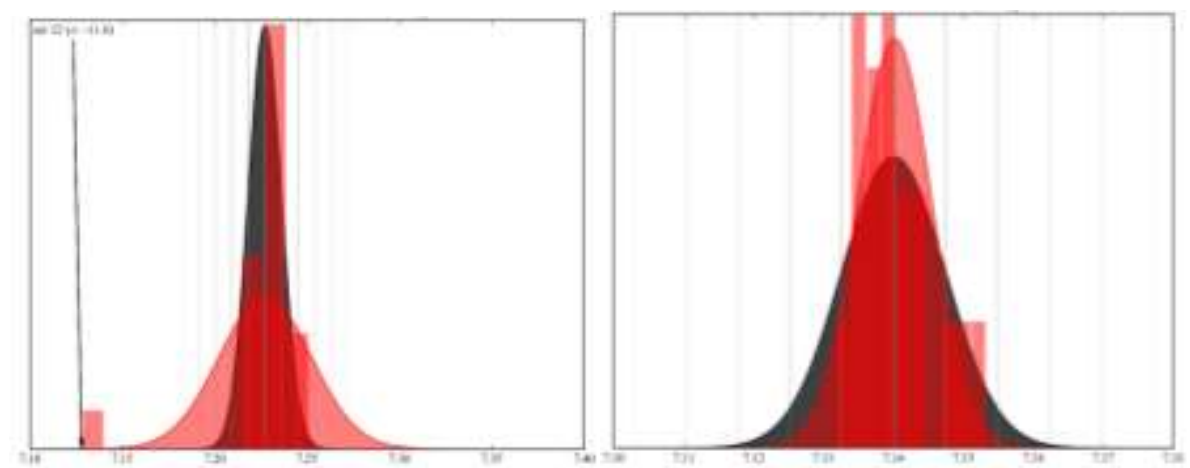

Figure 6.6: Distribution of the monopole population of atom $F(34)$ using all data and after omitting two outlier reflections.

\subsection{Evaluation}

The comparison of $\mathbf{3}$ and $\mathbf{3}$ ' shows that almost all bond lengths and properties as well as the integrated charges are very similar (see Table6.3). Theonly larger difference is that the B-N bond lengths of $\mathbf{3}^{\prime}$ is $4 \mathrm{pm}$ longer than in 3. As in so many other cases, the difference between theoretical and experimental structure could be explained by packing effects that in this case manifest themselves in the molecule's weakest bond. Nevertheless, the properties at the BCP and subsequently the bond classification coincide. Therefore, the comparison of the theoretical results of $\mathbf{1}$ and the experimental results of $\mathbf{2}$ is principally allowed.

The B-P distance in $\mathbf{1}$ is extremely long and similar to the longest experimentally confirmed B-P adduct in a four-membered heterocycle of 2.206(5) $\AA$ (A xenov et al., 2010). O nly for this bond, the bond path is substantially longer than the interatomic distances, resembling a non-linear orbital overlap. This correlates with a more planar coordination of the boron (sum of C-B-C angles: $350^{\circ}$ ). The B-P bond exhibits the properties of a very weak and non-covalent interaction, as $\rho\left(\mathbf{r}_{\mathrm{bcp}}\right)$ is very low, while $\nabla^{2} \rho\left(\mathbf{r}_{\mathrm{bcp}}\right)$ is slightly positive (see Table 6.3). The analysis of $\nabla^{2} \rho(\mathbf{r})$ along the BP (Figure 6.7) exhibits a mild polarization towards phosphorous, as implied by the almost equal electronegativity. This should also be mirrored by the charges (Table 6.4). The B and $P$ charges are positive and almost equal, but they also contain bond polarization effects. In order to obtain an estimation of the charge transfer, the molecule
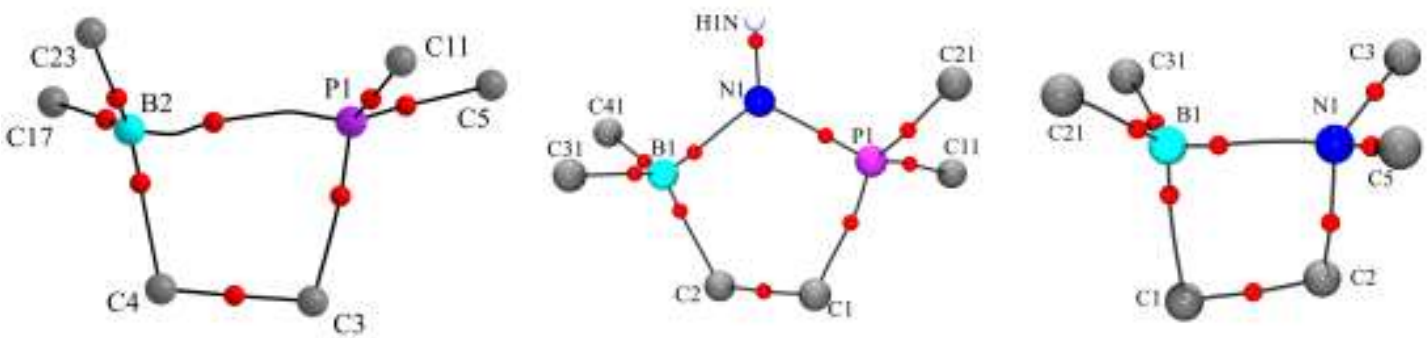

Figure 6.7: Bond Paths (black) and BCPs (red) of compounds $\mathbf{1}, \mathbf{2}$ and $\mathbf{3}$. 
Table 6.3: Topological parameters of selected bonds in $\mathbf{1}$ (theoretical data), $\mathbf{2}$ (experimental data), $\mathbf{3}$ (experimental data) and $\mathbf{3}^{\prime}$ (theoretical data). *marked ESD s are determined by 20 cross-validation sets. In any case, the larger ESD was chosen.

\begin{tabular}{|c|c|c|c|c|c|c|c|c|}
\hline & Bond & $\begin{array}{l}\text { Bond } \\
\text { Length } \\
{[\AA]}\end{array}$ & $\begin{array}{l}\text { Bond Path } \\
\text { Length } \\
{[\AA]}\end{array}$ & $\mathbf{r}_{\mathrm{Al-BCP}}[\AA]$ & $\mathbf{r}_{\mathrm{AZ}-\mathrm{BCP}}[\AA]$ & $\begin{array}{l}\rho\left(\mathbf{r}_{\text {cc }}\right) \\
{\left[\mathrm{e} \AA^{-3}\right]}\end{array}$ & $\begin{array}{l}\nabla^{2} \rho\left(\mathbf{r}_{\mathrm{bc}}\right) \\
{\left[\mathrm{e} \AA^{-5}\right]}\end{array}$ & $\varepsilon\left(\mathbf{r}_{\mathrm{bcp}}\right)$ \\
\hline \multirow[t]{6}{*}{1} & $P 1-B 4$ & 2.2016 & 2.2436 & 1.3691 & 0.8354 & 0.513 & -1.38 & 0.16 \\
\hline & B4 - C3 & 1.6469 & 1.6471 & 0.5554 & 1.0916 & 1.134 & -8.62 & 0.03 \\
\hline & $\mathrm{C} 2-\mathrm{C} 3$ & 1.5467 & 1.5468 & 0.7971 & 0.7496 & 1.578 & -12.98 & 0.01 \\
\hline & $\mathrm{P} 1-\mathrm{C} 2$ & 1.8325 & 1.8366 & 0.7440 & 1.0921 & 1.140 & -7.67 & 0.05 \\
\hline & B4 - C17 & 1.6128 & 1.6134 & 0.5225 & 1.0908 & 1.099 & -3.18 & 0.08 \\
\hline & $\mathrm{B} 4-\mathrm{C} 23$ & 1.6133 & 1.6136 & 0.5231 & 1.0905 & 1.101 & -3.30 & 0.05 \\
\hline \multirow[t]{7}{*}{2} & $\mathrm{P1} 1-\mathrm{N} 1$ & $1.6201(2)$ & $1.6203(1) *$ & $0.6636(6) *$ & $0.9567(6) *$ & $1.39(2)$ & $2.2(3)^{*}$ & $0.04(1) *$ \\
\hline & $\mathrm{B} 1-\mathrm{N} 1$ & $1.5657(2)$ & $1.5662(3) *$ & $0.4980(9) *$ & $1.0683(9) *$ & $1.01(2)$ & $1.3(4)^{*}$ & $0.01(2)^{*}$ \\
\hline & $\mathrm{B} 1-\mathrm{C} 2$ & $1.6386(2)$ & $1.6390(2) *$ & $0.524(3)^{*}$ & $1.114(3)^{*}$ & $1.16(2)$ & $-8.9(4) *$ & $0.09(3)^{*}$ \\
\hline & $\mathrm{C} 1-\mathrm{C} 2$ & $1.5505(2)$ & $1.5509(1) *$ & $0.807(3)^{*}$ & $0.743(3)^{*}$ & $1.54(2)$ & $-12.3(3)^{*}$ & $0.01(1) *$ \\
\hline & $\mathrm{P} 1-\mathrm{C} 1$ & $1.8139(2)$ & $1.8182(4) *$ & $0.820(5) *$ & $0.997(5) *$ & $1.172(9)$ & $-8.52(3)^{*}$ & $0.03(1) *$ \\
\hline & $\mathrm{C} 31-\mathrm{B} 1$ & $1.6638(2)$ & $1.6642(3) *$ & $1.1239(3) *$ & $0.5402(3) *$ & $1.017(5)$ & $-7.1(4)^{*}$ & $0.19(3)^{*}$ \\
\hline & $\mathrm{C} 41-\mathrm{B} 1$ & $1.6504(2)$ & $1.6508(3)^{*}$ & $1.1177(3)^{*}$ & $0.5331(3) *$ & $1.028(5)$ & $-6.4(4) *$ & $0.13(3)^{*}$ \\
\hline \multirow[t]{6}{*}{3} & $\mathrm{~B} 1-\mathrm{N} 1$ & $1.7102(3)$ & $1.7109(2) *$ & $0.528(2)^{*}$ & $1.182(2) *$ & $0.75(1)$ & $1.1(4)^{*}$ & $0.56(9)^{*}$ \\
\hline & $\mathrm{B} 1-\mathrm{C} 1$ & $1.6296(3)$ & $1.6316(4) *$ & $0.511(2)^{*}$ & $1.121(2)^{*}$ & $1.16(2)$ & $-6.8(5)^{*}$ & $0.04(3)^{*}$ \\
\hline & $\mathrm{C} 1-\mathrm{C} 2$ & $1.5201(3)$ & $1.5207(2) *$ & $0.752(4)^{*}$ & $0.768(4)^{*}$ & $1.69(1)$ & $-14.9(2) *$ & $0.07(1) *$ \\
\hline & $\mathrm{N} 1-\mathrm{C} 2$ & $1.5624(3)$ & $1.5633(3) *$ & $0.884(2) *$ & $0.680(2)^{*}$ & $1.45(1)$ & $-8.8(2)^{*}$ & $0.02(1) *$ \\
\hline & $C 21-B 1$ & $1.6393(2)$ & $1.6398(2) *$ & $1.1216(2) *$ & $0.5182(2) *$ & $1.05(1)$ & $-4.9(4) *$ & $0.11(2) *$ \\
\hline & C31 - B1 & $1.6455(3)$ & $1.6461(2) *$ & $1.1233(3) *$ & $0.5228(3) *$ & $1.04(1)$ & $-5.2(4)^{*}$ & $0.19(2) *$ \\
\hline \multirow[t]{6}{*}{$3^{\prime}$} & $\mathrm{B} 1-\mathrm{N} 1$ & 1.7502 & 1.7543 & 0.5593 & 1.1950 & 0.686 & 1.73 & 0.58 \\
\hline & $\mathrm{B} 1-\mathrm{C} 1$ & 1.6286 & 1.6306 & 0.5432 & 1.0874 & 1.162 & -8.15 & 0.07 \\
\hline & $\mathrm{C} 1-\mathrm{C} 2$ & 1.5167 & 1.5178 & 0.7205 & 0.7973 & 1.708 & -15.19 & 0.03 \\
\hline & $\mathrm{N} 1-\mathrm{C} 2$ & 1.5579 & 1.5590 & 0.9108 & 0.6481 & 1.468 & -9.56 & 0.03 \\
\hline & $\mathrm{C} 21-\mathrm{B} 1$ & 1.6277 & 1.6289 & 1.1024 & 0.5264 & 1.075 & -3.52 & 0.17 \\
\hline & C31-B1 & 1.6444 & 1.6450 & 1.1137 & 0.5313 & 1.033 & -3.25 & 0.13 \\
\hline
\end{tabular}

is divided at the merely polarized bridging $\mathrm{C}-\mathrm{C}$ bond and group charges for the $\left(\mathrm{F}_{5} \mathrm{C}_{6}\right)_{2} \mathrm{BCH}_{2}$ and $\mathrm{CH}_{2} \mathrm{PM} \mathrm{es}_{2}$ fragments are calculated. This results in a minute charge transfer of 0.29 .

In comparison to $\mathbf{1}$, the boron atom in $\mathbf{3}$ is closer to a tetrahedral arrangement with a sum of $\mathrm{C}$-B-C angles of $339^{\circ}$. The $B-N$ bond lengths of $1.7106(3) \AA$ is $0.22 \AA$ longer than the typical single bond (Rademacher, 1987) but not exceptionally long for such a four membered heterocyclic system. Again, the bond is characterized as a weak, non-covalent interaction, because the charge density $\rho\left(\mathbf{r}_{\text {bcp }}\right)$ is low while the Laplacian $\nabla^{2} \rho\left(\mathbf{r}_{\text {bcp }}\right)$ is slightly positive (see Table 6.3). Nevertheless, $\rho\left(\mathbf{r}_{\text {bcp }}\right)$ and subsequently the strength of the interaction is much higher, compared to $\mathbf{1}$. The position of the B-N BCP in $\mathbf{3}$ is strongly shifted towards the boron atom and in the analysis of $\nabla^{2} \rho(\mathbf{r})$ along the Bond Path (BP) (Figure 6.7) shows a strong bond polarization towards nitrogen. This is al so reflected by the charges (Table 6.4). The charge transfer between boron and the pnicogen again is very low, as reflected by the $\left(\mathrm{F}_{5} \mathrm{C}_{6}\right)_{2} \mathrm{BCH}_{2}$ and $\mathrm{Et}_{2} \mathrm{NCH}$ Ph group charges. In both molecules, $\mathbf{1}$ and $\mathbf{3}$, the ellipticity at the B-pnictogen $\mathrm{BCP}$ is distinctly non zero. This is a result of the very low charge density at the $\mathrm{BCP} \rho\left(\mathbf{r}_{\mathrm{bcp}}\right)$ which manifests itself in very low H essian Eigenvalues (Table S6.7), leading to a high uncertainty of the ellipticity. The $\rho\left(\mathbf{r}_{\mathrm{bcp}}\right)$ is biased by the density fluctuations of the surrounding molecule - here the gradient between the central heterocyclic ring and the outer parts of the molecule. In $\mathbf{1}$ and $\mathbf{3}$, we find weak non-coval ent interactions 
Table 6.4: Integrated Charges or Bader Charges for specific atoms as well as the sum of charges for the most relevant moieties.

\begin{tabular}{|c|c|c|c|c|c|c|c|}
\hline \multirow{2}{*}{$\begin{array}{l}1 \\
\text { Fragment } \\
\text { /A tom }\end{array}$} & \multicolumn{3}{|c|}{2} & \multicolumn{2}{|l|}{3} & \multicolumn{2}{|l|}{$3^{\prime}$} \\
\hline & $\begin{array}{l}\text { Charge } \\
{[e]}\end{array}$ & $\begin{array}{l}\text { Fragment } \\
\text { /Atom }\end{array}$ & $\begin{array}{l}\text { Charge } \\
\text { [e] }\end{array}$ & $\begin{array}{l}\text { Fragment } \\
\text { /Atom }\end{array}$ & $\begin{array}{l}\text { Charge } \\
{[\mathrm{e}]}\end{array}$ & $\begin{array}{l}\text { Fragment } \\
\text { /A tom }\end{array}$ & $\begin{array}{l}\text { Charge } \\
\text { [e] }\end{array}$ \\
\hline$B$ & 1.71 & $B$ & 2.04 & $B$ & 2.07 & $\mathrm{~B}$ & 1.89 \\
\hline C2 & -0.53 & C2 & -0.47 & $\mathrm{Cl}$ & -0.71 & $\mathrm{C} 1$ & -0.48 \\
\hline C3 & -0.50 & $\mathrm{C} 1$ & -0.37 & C2 & 0.32 & C2 & 0.23 \\
\hline \multirow[t]{2}{*}{$\mathrm{P}$} & 1.60 & $P$ & 2.10 & & & & \\
\hline & & $\mathrm{N}$ & -1.84 & $\mathrm{~N}$ & -1.20 & $\mathrm{~N}$ & -1.05 \\
\hline $\mathrm{B}\left(\mathrm{C}_{6} \mathrm{~F}_{5}\right)_{2}$ & 0.21 & $\mathrm{~B}\left(\mathrm{C}_{6} \mathrm{~F}_{5}\right)_{2}$ & 0.59 & $\mathrm{~B}\left(\mathrm{C}_{6} \mathrm{~F}_{5}\right)_{2}$ & 0.64 & $\mathrm{~B}\left(\mathrm{C}_{6} \mathrm{~F}_{5}\right)_{2}$ & 0.44 \\
\hline $\mathrm{C}_{2} \mathrm{H}_{4}$ & -0.94 & $\mathrm{C}_{2} \mathrm{H}_{4}$ & -0.77 & $\mathrm{CH}_{2} \mathrm{CHPh}$ & -0.18 & $\mathrm{CH}_{2} \mathrm{CHPh}$ & -0.20 \\
\hline \multirow[t]{2}{*}{$\mathrm{PMes}_{2}$} & 0.73 & $\mathrm{PMeS}_{2}$ & 1.46 & & & & \\
\hline & & $\mathrm{NH}$ & -1.35 & $\mathrm{NEt}_{2}$ & -0.35 & $\mathrm{NEt}_{2}$ & -0.24 \\
\hline$\left(\mathrm{F}_{5} \mathrm{C}_{6}\right)_{2} \mathrm{BCH}_{2}$ & -0.28 & $\left(\mathrm{~F}_{5} \mathrm{C}_{6}\right)_{2} \mathrm{BCH}_{2}$ & 0.16 & $\left(\mathrm{~F}_{5} \mathrm{C}_{6}\right)_{2} \mathrm{BCH}_{2}$ & 0.08 & $\left(\mathrm{~F}_{5} \mathrm{C}_{6}\right)_{2} \mathrm{BCH}_{2}$ & -0.04 \\
\hline $\mathrm{CH}_{2} \mathrm{PMeS}_{2}$ & 0.28 & $\mathrm{CH}_{2} \mathrm{PM}_{\mathrm{eS}}$ & 1.12 & $\mathrm{Et}_{2} \mathrm{NCHPh}$ & 0.03 & $\mathrm{Et}_{2} \mathrm{NCHPh}$ & 0.04 \\
\hline
\end{tabular}

between $B$ and the pnictogen. Therefore, these bonds should be depicted as dative bonds (Figure 6.2). The main differences in the otherwise isoelectronic molecules $\mathbf{1}$ and $\mathbf{3}$ are the strengths of the B-pnictogen bonds and the bond polarization to the pnictogen. The stronger B-pnictogen interaction of $\mathbf{3}$ resembles a more stable coordination that has to be overcome for any reaction at the FLP. More strained B-N moieties, like in $\mathbf{6}$ to $\mathbf{9}$, result in a weaker coordination and open a pathway to a similar reactivity as $\mathbf{1}$. The B-pnictogen bond polarization in $\mathbf{1}$ and $\mathbf{3}$ resembles the different electronegativities and also accounts for the higher basicity of phosphorous.

In 2, the $B-N$ bond-lengths of $1.5657(2) \AA$ is $0.08 \AA$ longer than the typical single bond lengths, (Rademacher, 1987) whilethe B-P distance of 1.6201(2) $\AA$ is $0.08 \AA$ shorter. The nitrogen atom is almost trigonal planar arranged with a sum of angles of $358^{\circ}$, while boron and phosphorous are tetrahedrally coordinated. This bonding pattern is very unusual. The positions of the B-N and N-P BCP is strongly shifted away from the nitrogen position. $\rho\left(\mathbf{r}_{\text {bcp }}\right)$ for the $B-N$ bond is with $1.01(2) e \AA^{-3}$ relatively low and $\nabla^{2} \rho\left(\mathbf{r}_{\text {bcp }}\right)$ with $1.3(4) e \AA^{-5}$ distinctly positive. So as in $\mathbf{1}$ and $\mathbf{3}$, the B-pnictogen bond is unambiguously a

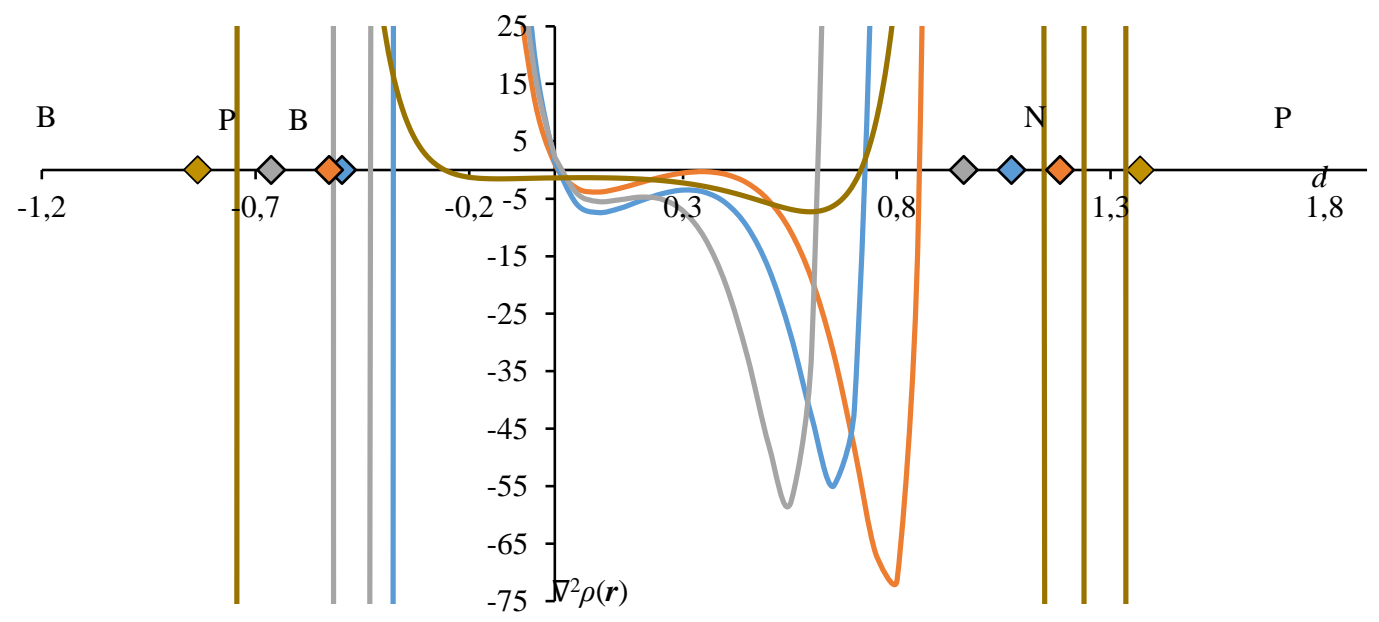

Figure 6.8: $\nabla^{2} \rho(\mathbf{r})$ along the B-P bond of $\mathbf{1}$ (brass), the B-N bonds of $\mathbf{2}$ (orange) and $\mathbf{3}$ (blue) and the P-N bond of $\mathbf{2}$ (grey) with $d$ being the distance from theBCP. Thezero value of the $d$ axis indicates the position of the BCP. The basins of the acidic atoms $N$ and $P$ spans for positive values, while the basic $B / P$ basins span for negative values. A tom positions are marked as squares. 

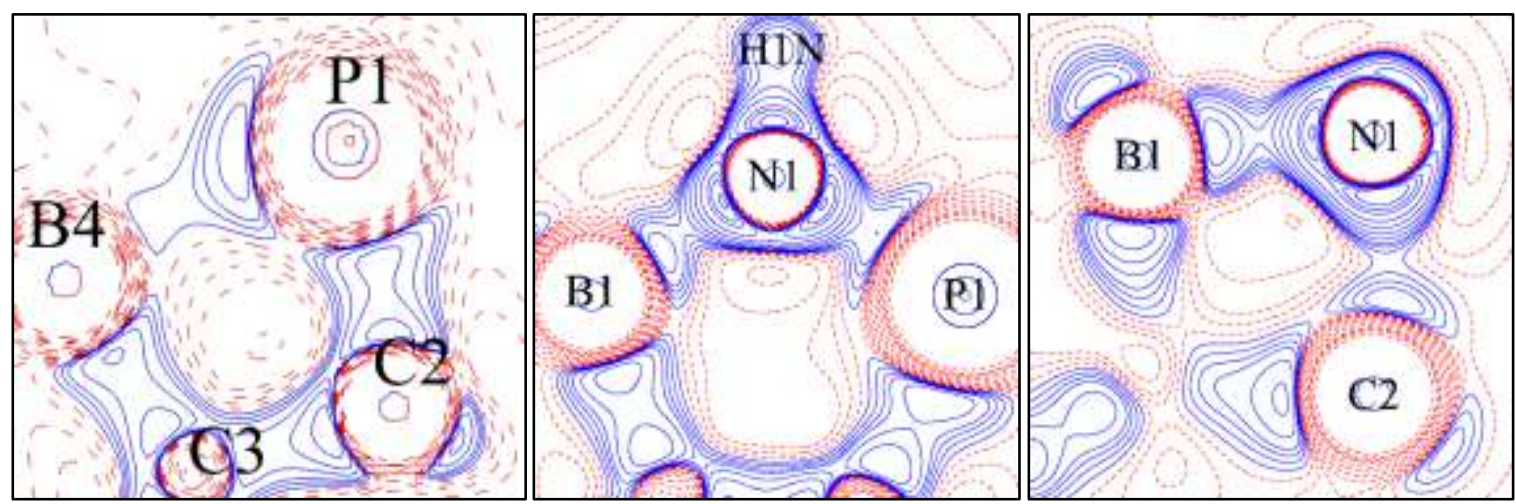

Figure 6.9: Laplacian distributions in the B4-P1-C2 plane of 1, the $B(1)-N(1)-P(1)$ plane of 2 , and the $B(1)-$

$\mathrm{N}(1)-C(2)$ plane of 3. Isolevels +(red)/ -(blue) $0,1,2,3,5,8,13,21,34,43,55$ and $89 \mathrm{e}^{-5}$.

non-covalent interaction but here this nature is much stronger pronounced. The bond properties correlate well with the experimental characterisation of the $\mathrm{B}-\mathrm{N}$ bond of bis(pentafluorophenyl)(Npyrrolidinyl)borane with $\mathrm{R}_{\mathrm{ij}}=1.4094 \AA, \rho\left(\mathbf{r}_{\mathrm{bcp}}\right)=1.472 \mathrm{e}^{-3}$ and $\nabla^{2} \rho\left(\mathbf{r}_{\mathrm{bcp}}\right)=0.504 \mathrm{e}^{-5}$ (Flierler et al., 2009). For the N-P bond $\rho\left(\mathbf{r}_{\text {bcp }}\right)$ is with $1.39(2)$ e $\AA^{-3}$ quite high and $\nabla^{2} \rho\left(\mathbf{r}_{\text {bcp }}\right)$ with $2.2(3)$ e $\AA^{-5}$ clearly positive. The ellipticity on the other hand is with $0.04(1)$ very low. This qualifies the bond as an intense, but distinctly non-covalent interaction without major $\pi$-contribution. The analysis of $\nabla^{2} \rho(\mathbf{r})$ along the bond path (Figure 6.7) shows analogous features for the B-N bonds of $\mathbf{2}$ and $\mathbf{3}$, as well as the P-N bond of 2: The charge concentrations are exclusively located in the nitrogen basins, while they are depleted in the boron and phosphorous basins. Two minima in the interatomic region are observed in all bonds. These minima originate from valence shell charge concentrations (VSCCs) at both bond partners, indicating shared, but severely polarized interactions. Again, the bridging $C_{-} C$ bond is assumed to be mostly covalent and non-polarized. Therefore, the summation of the charges of all moieties connected to $B, N$, or $P$ should give an estimate of the $B / N$ and $P / N$ charge transfer. The $\left(F_{5} C_{6}\right)_{2} B_{C} H_{2}$ fragment gives a reasonably small charge of 0.17 e. Therefore, like in 3, no $B-N$ charge transfer can be observed and the bonding ED mostly originates from the nitrogen atom. The $\mathrm{CH}_{2} \mathrm{PM}_{2} \mathrm{~S}_{2}$ group charge of $1.12 \mathrm{e}$ and the $\mathrm{NH}$ group charge of -1.35 e resemble a charge transfer from the phosphorous to nitrogen atom, resulting in a located negative charge at nitrogen and a positive charge at the phosphorous atom. So, while the B-N bond can be classified as a dative bond like in $\mathbf{1}$ and $\mathbf{3}$, the P-N bond can be classified as a covalent bond with a major electrostatic contribution, implying localized charges at $\mathrm{N}$ and $\mathrm{P}$. The properties agree very well with experimental characterization of a formal $\mathrm{P}-\mathrm{N}$ phosphinimine that was shown to be a non-hypervalent $\mathrm{P}^{\delta+}-\mathrm{N}^{\delta-}$ moiety with $\mathrm{R}_{\mathrm{ij}}=1.5903 \AA, \rho\left(\mathbf{r}_{\mathrm{bcp}}\right)=1.508 \mathrm{e} \AA^{-3}, \quad \nabla$ ${ }^{2} \rho\left(\mathbf{r}_{\text {bcp }}\right)=5.874 \AA^{-5}$ and $\varepsilon\left(\mathbf{r}_{\text {bcp }}\right)=0.05$ (Kocher et al., 2004).

The trigonal-planar coordinated nitrogen atom in $\mathbf{2}$ implies $\mathrm{sp}^{2}$ hybridization, which should be resembled by the Laplacian distribution. The nitrogen atom features three Valence Shell Charge Concentrations (VSCCS) in the B-N-P-plane, facing the three bonding partners (see Figure 6.9 and Figure 6.10). Perpendicular to that plane, above and below the nitrogen atom, no VSCCs can be found. However, the Laplacian in these regions is higher than between the VSCCS, indicating charge accumulation. For B and P, four VSCC s are found facing their bond partners and the same is true for $\mathbf{1}$, 
as well as B and N in $\mathbf{3}$ and $\mathbf{3}$ '. The non-linear overlap of the VSCC s in the four-membered heterocycle is well observable in Figure 6.9.

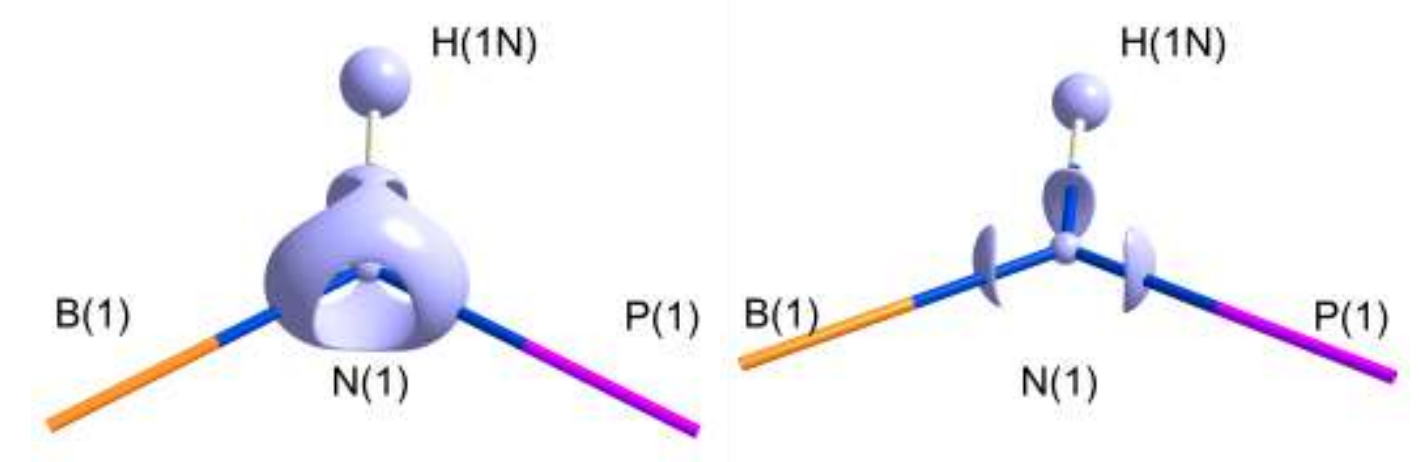

Figure 6.10: Laplacian isosurface representation of the VSCCs around $N$ of 2 at $-37 e^{-5}$ and $-50 e^{-5}$.

\subsection{Conclusion}

As determined by the topological analysis, the B-pnictogen bonds of $\mathbf{1}, \mathbf{2}$ and $\mathbf{3}$ show low charge density at the BCP and a slightly positive Laplacian. They are therefore characterized as weak, noncovalent interactions and are consequently best described as dative bonds. A charge transfer between $B$ and pnictogen is not observed.

Comparing $\mathbf{1}$ and $\mathbf{3}$, the B-pnictogen interaction of $\mathbf{3}$ is much stronger and therefore inhibits the catalytic activity. An exchange to sterically more demanding substituents like in 6 to 9 opens a pathway to catalytically active compounds.

The nitrogen atom of $\mathbf{2}$ features a negative charge, due to a charge transfer from the phosphorus atom. Consequently, the bonding situation is best described by a non-hypervalent $\mathrm{P}^{\delta+}-\mathrm{N}^{\delta-}$ moiety. The nitrogen atom features three VSCC in the B-N-P plane, as well as charge accumulations perpendicular to that plane. Together these features indicate a negatively charged, $\mathrm{sp}^{2}$ hybridized nitrogen atom. During the reaction from $\mathbf{1}$ to $\mathbf{2}$, the oxidation took placeat the base of the FLP, while the oxidation state of the acid remained unchanged. This finding can lead to an improved understanding of FLP reactivity. Under consideration of the shown findings, the Lewis diagrams in Figure 6.2 are found to be suitable. 


\section{Chapter 7 ChARGe Density InVESTIGATIONS ON A SULFUR TETRAYNE}

\subsection{Introduction}

As group 16 elements, such as oxygen, sulfur, selenium, and tellurium are notoriously electron rich, they are not expected to form particularly favorable contacts. However, chalcogen-chalcogen contacts are very common in X-ray crystal structures, and have therefore become known as chalcogenbonding interactions. They have been investigated thoroughly by theoretical methods (M urray et al., 2007; M urray et al., 2008; Beno et al., 2015) and are mostly described as $\sigma$-holeinteractions. The $\sigma$-hole interactions are established by the donation of non-bonding orbitals into the $\sigma^{*}$-orbitals of another chalcogen. This $n \rightarrow \sigma^{*}$ interaction has recently been differentiated from other non-covalent interactions, like electrostatic and V an der W aals interactions to be the origin of chalcogen bonding (Pascoe et al., 2017).

In order to contribute to the current discussion by the means of experimental charge density distributions of chalcogen-chalcogen interactions, a cooperation with the Werz-group (TU Braunschweig) was strived for in 2014/15. Prof. Werz had published a series of chalcogenheterocycles (cyclical tetra- and hexaynes) and tellurium-capped rods which formed tubular structures and macromolecular grids presumably by chalcogen-chalcogen bonds (Gleiter et al., 2003; Schulte et al., 2003; Werz et al., 2002a; 2002b; 2003; 2004) and were al ready subjected to a theoretical investigation by Bleiholder et al.. In order to contribute here, the published crystal structures of Werz's heterocycles were searched for suitable compounds for the experimental charge density determination. After

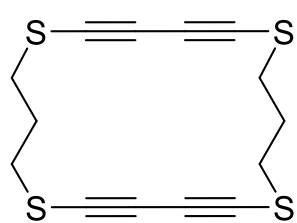

a

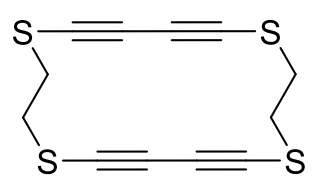

$\mathbf{b}$

Figure 7.1: Selected tetraynes. thorough investigation of the published crystal structures, a (CCDC\# 223392) and $\mathbf{b}$ (223393) were selected as suitablecandidates. $\mathbf{a}$ and $\mathbf{b}$ feature intermolecular S-S distances of 3.558 and $3.600 \AA$, in the range of the sum of $V$ an der $W$ aals radii of $3.6 \AA$. Furthermore, the molecules are reasonably small. The published crystal structures showed small ADPs and the published crystal sizes were reasonably large. All these points made them promising candidates for an experimental charge density determination. 
Therefore, both compounds were resynthesized in the Werz group and shipped to Göttingen. Unfortunately, the samples of $\mathbf{b}$ did not survive the shipment and disintegrated into a black, carbon like substance. Presumably, the high ring tension of $\mathbf{b}$, combined with

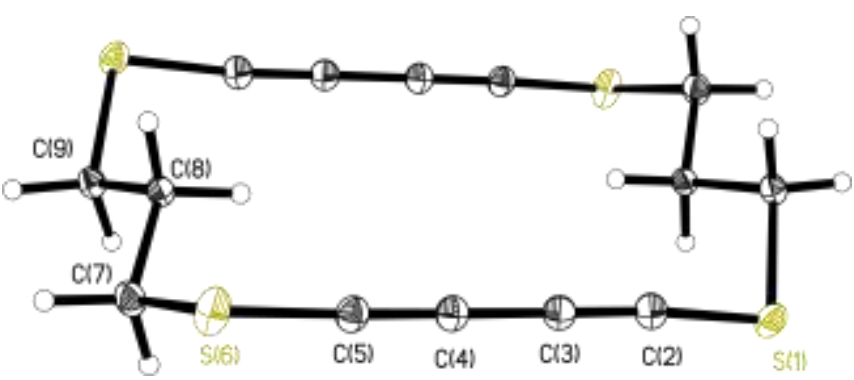

Figure 7.2: Crystal structure of a. the unsuitable shipment-conditions, lead to the disintegration. As the synthesis was very demanding, further investigations on $\mathbf{b}$ were dismissed. H owever, the samples of a only partly disintegrated and were re-crystallized under Schlenkconditions. High-resolution diffraction data were collected from one of these crystals, suitable for an experimental charge density determination.

\subsection{Theoretical Investigation}

In order to quantify the intermolecular interactions in $\mathbf{b}$, to visualize the intermolecular coordination of Selenium atoms and to compare the strengths of these interactions, intermolecular interactions were determined with CrystalExplorer (Turner et al., 2018) on the B3LYP/6-31G(d,p) level of theory.
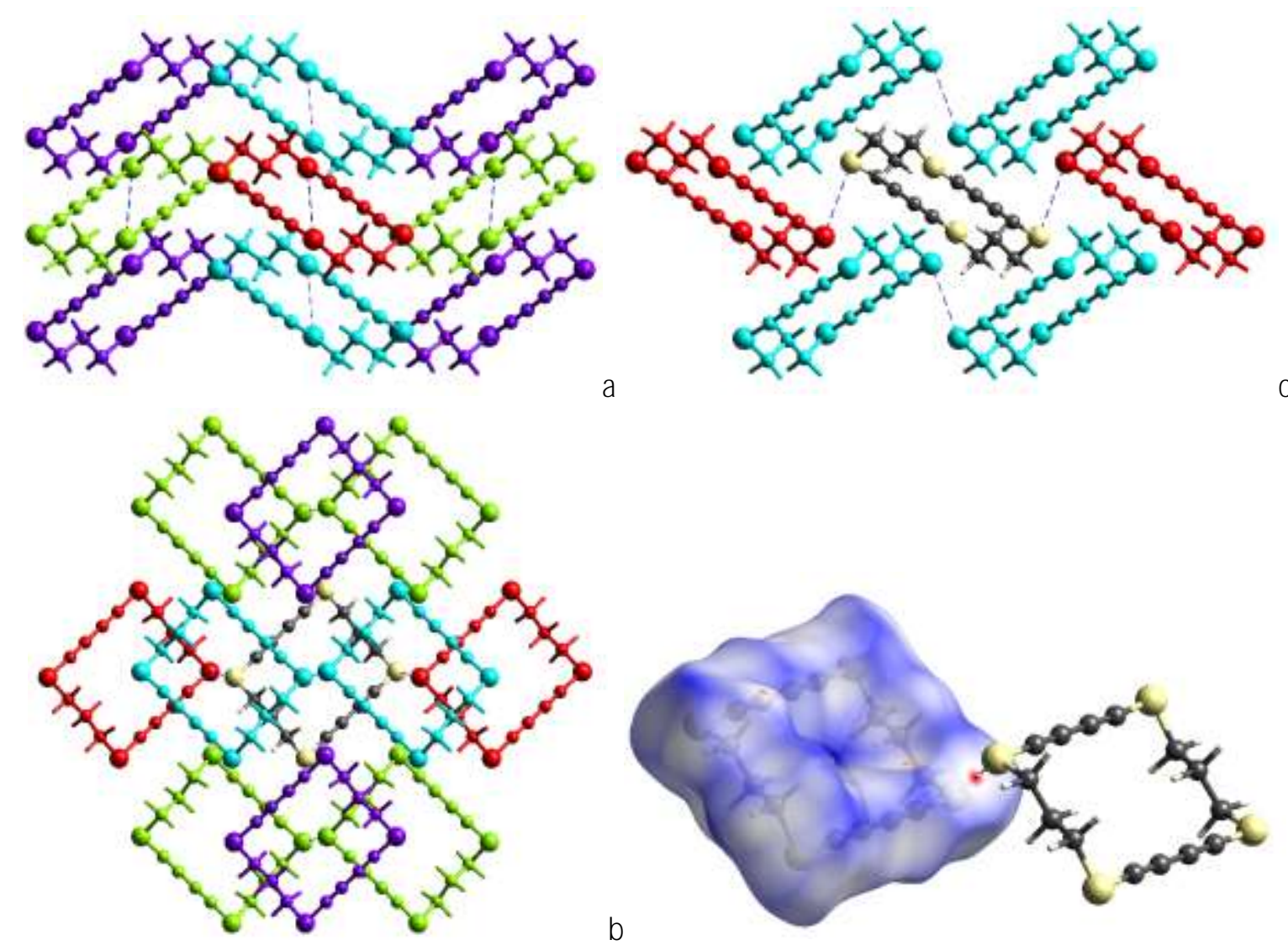

Figure 7.3: CrystalExploter investigatipon of a. Shortest S-S distance of $3.548 \AA$ are marked as blue dashed lines. Depictions of the crystal lattice along viewing axes a, b, c and a Hirshfeld-surface highlighting the chalcogen-chalcogen interaction. The color-code regards to Table 7.1. 
Table 7.1: Crystal Explorer (Turner et al., 2017) interaction Energies $(\mathrm{kJ} / \mathrm{mol})$. $\mathrm{R}$ is the distance between molecular centroids (mean atomic position) in $\AA$. The color code regards to Figure 7.3

\begin{tabular}{|l|l|l|l|l|l|l|l|l|}
\hline & $\mathrm{N}$ & Symop & $\mathrm{R}$ & E_ele & E_pol & E_dis & E_rep & E_tot \\
\hline & 2 & $\mathrm{x}, \mathrm{y}, \mathrm{z}$ & 9.54 & -5.7 & -0.6 & -11.5 & 17.9 & -5.6 \\
\hline & 4 & $-\mathrm{x}+1 / 2,-\mathrm{y}, \mathrm{z}+1 / 2$ & 9.68 & -5.9 & -1.5 & -19.7 & 22 & -10.9 \\
\hline & 4 & $\mathrm{x}+1 / 2,-\mathrm{y}+1 / 2,-\mathrm{z}$ & 6.55 & -13.2 & -3.3 & -44.3 & 35.1 & -33 \\
\hline & 4 & $-\mathrm{x}, \mathrm{y}+1 / 2,-\mathrm{z}+1 / 2$ & 9.55 & -5.4 & -1.2 & -14.9 & 11.6 & -12.4 \\
\hline
\end{tabular}

The H irshfeld surface in Figure 7.3 clearly indicates close intermolecular S...S contacts. Thereby strands of molecule are connected along the a-viewing axis, well observable along the $c$ viewing axis in Figure 7.3. However, comparted to the total intermolecular interaction, the interactions of the pairs, forming the chalcogen-chalcogen bond are only minor. All intermolecular interactions are dominated by the dispersive term. The most intense interactions are observableto the $[x+1 / 2,-y+1 / 2,-z]$ equivalent (blue) which has a good overlap with the central molecule (Figure 7.3 and Table 7.1). The chalcogeninteraction performing $[x, y, z]$ equivalent (red) exhibits only a small surface area and small values of intermolecular interaction. Chalcogen interactions are therefore not the predominant force that builds up the crystal. Theobjective and challenge for an experimental charge density determination is therefore to find these weak interactions and to characterize them.

\subsection{Experimental}

The solid-statestructure exhibits the space group Pbca with $1 \frac{1}{2}$ molecule per asymmetric unit. Data were collected at a Bruker SM ART A PEX2 D8 3-circle diffractometer, equipped with a SRA TXS rotating anode (Mo Ka) with Montel mirror optics and an APEX2 detector. Diffraction data were collected and integrated to the high resolution of $0.45 \AA$. The data were corrected for thermal diffuse scattering (TDS) (Niepötter et al., 2015). Crystallographic details and an overview on the refinement results are given in Table 7.2 and the data quality statistics are given in Table S7.1 in the appendix.

Table 7.2: Crystallographic details for $\mathbf{a}$.

\begin{tabular}{|c|c|c|c|}
\hline Space group & Pbca & & \\
\hline $\mathrm{a}[\AA]$ & $9.5357(3)$ & Data XD used & 6571 \\
\hline $\mathrm{b}[\AA]$ & $8.9814(3)$ & Parameter & 154 \\
\hline$c[\AA]$ & $16.8508(5) \AA$ & $R(F 2, X D)$ & $1.82 \%$ \\
\hline Collected ref. & 125240 & $w R(F 2, X D)$ & $2.00 \%$ \\
\hline Independent ref. & 8296 & GOF (F2, XD) & 1.7052 \\
\hline Rint & $3.06 \%$ & Diff peak/hole & -0.159 \\
\hline R1 (all data, IAM) & $3.54 \%$ & (XD) [e̊̊-3] & 0.253 \\
\hline wR2 (all data, IAM ) & $8.79 \%$ & & \\
\hline GOF (F2, IAM) & 1.063 & & \\
\hline
\end{tabular}

A starting model was prepared in the IAM using SHELXL (Sheldrick, 2015) and SHELXLE (Hübschle et al., 2011) by the refinement of the heavy element positions and vibrational parameters 
against high resolution data $(<0.6 \AA)$, while hydrogen atoms were placed on the difference Fourier maxima of low resolution data $(>1.0 \AA)$ and set to neutron data distances. The anisotropic density was refined in the Multipole M odel (H ansen \& Coppens, 1978), using theXD program package (Volkov et al., 2006). For the introduction of multipole parameters, the
Table 7.3: Applied chemical constraints, GramCharlier-Level and Symmetry restraints for the charge density refinement.

\begin{tabular}{lll}
\hline Constrained Atoms & CG-Level & Symmetry \\
$\mathrm{S}(1), \mathrm{S}(6)$ & 3 & $\mathrm{~m}$ \\
$\mathrm{C}(2), \mathrm{C}(5)$ & 2 & cylindrical \\
$\mathrm{C}(3), \mathrm{C}(4)$ & 2 & cylindrical \\
$\mathrm{C}(7) ; \mathrm{C}(8) ; \mathrm{C}(9)$ & 2 & mm2 \\
all H & 1 & cylindrical \\
\hline
\end{tabular}

highest possible local symmetry was applied. M ultipole populations of chemically equivalent atoms were constrained to be the same (Table 7.3). All atoms except hydrogen were refined to the hexadecapole level, while hydrogen atoms were refined to the dipole level. The final refinement strategy is given in Table 7.3. The refinement procedure was tested under consideration of the resolution-dependent error and statistic error distribution (Figure S7.2), the residual density distribution (Figure S7.4) and crossvalidation results (Krause et al., 2017) (Figure S7.1). The refinement of anharmonic motion was tested under consideration of Kuhs's Rule (Table S7.4), the significance of refined parameters (Table 7.5) and the analysis of the probability density function (PDF) (Table S7.6). All tests evidenced the refinement to be sensible and it resulted in a flat and featureless residual density (Figure S7.3).

\subsection{Evaluation}

The refined model was analyzed in the QTAIM (Bader, 1991) frame work using the XD program package (Volkov et al., 2006). The objective of the experimental charge density analysis were to characterize the bonding pattern within the molecule, to analyze the intermolecular chalcogen interaction and attempt to characterize the orbital overlap within the capabilities of the QTAIM approach and possibly find $\mathrm{a} n \rightarrow \sigma^{*}$ interaction.

Table 7.4: BCP Analysis. * marked value errors are determined by cross-validation. In any case the larger error valuewas chosen. S1_symm is the symmetry equivalent of S1 that makes up the other half of the molecule.

\begin{tabular}{|c|c|c|c|c|c|c|c|}
\hline A tom 1 & Atom2 & $\begin{array}{l}\rho\left(\mathbf{r}_{b c p}\right) \\
{\left[\mathrm{e}^{-3}\right]}\end{array}$ & $\begin{array}{l}\nabla^{2} \rho\left(\mathbf{r}_{\mathrm{bcp}}\right) \\
{\left[\mathrm{e} \AA^{-5}\right]}\end{array}$ & $\begin{array}{l}\text { Bond Path } \\
\text { Length }[\AA]\end{array}$ & $\mathbf{r}_{\mathrm{Al-BCP}}[\AA]$ & $\mathbf{r}_{\mathrm{A} 2-\mathrm{BCP}}[\AA]$ & $\varepsilon\left(\mathbf{r}_{\mathrm{bcp}}\right)$ \\
\hline S1 & $\mathrm{C} 2$ & $1.438(8)$ & $-8.11(16)^{*}$ & $1.67070(11)^{*}$ & $0.781(5)^{*}$ & $0.890(5)^{*}$ & $0.240(15)^{*}$ \\
\hline$C 2$ & $\mathrm{C} 3$ & $2.692(15)$ & $-25.0(4)^{*}$ & $1.22100(10)^{*}$ & $0.597(7)^{*}$ & $0.624(7)^{*}$ & $0.0(0)^{*}$ \\
\hline C3 & $\mathrm{C} 4$ & $2.169(9)^{*}$ & $-18.6(4)^{*}$ & $1.35520(12)^{*}$ & $0.67760(7)^{*}$ & $0.67760(5)^{*}$ & $0.0(0)^{*}$ \\
\hline C4 & $\mathrm{C} 5$ & $2.695(7)^{*}$ & $-25.1(3)^{*}$ & $1.22030(16)^{*}$ & $0.624(7)^{*}$ & $0.596(7)^{*}$ & $0.0(0)^{*}$ \\
\hline $\mathrm{C} 5$ & S6 & $1.444(4)^{*}$ & $-8.27(17)^{*}$ & $1.66800(15)^{*}$ & $0.891(5)^{*}$ & $0.777(5)^{*}$ & $0.250(17)^{*}$ \\
\hline S6 & $\mathrm{C} 7$ & $1.187(8)$ & $-6.17(13)^{*}$ & $1.82370(13)^{*}$ & $0.957(3)^{*}$ & $0.867(3) *$ & $0.080(10)^{*}$ \\
\hline $\mathrm{C7}$ & $\mathrm{C} 8$ & $1.724(8)$ & $-19.25(18) *$ & $1.51560(15)^{*}$ & $0.768(7) *$ & $0.748(7)^{*}$ & $0.010(4)^{*}$ \\
\hline $\mathrm{C} 8$ & $\mathrm{C} 9$ & $1.717(12)$ & $-19.06(17)^{*}$ & $1.51900(17)^{*}$ & $0.750(7)^{*}$ & $0.769(7)^{*}$ & $0.010(4)^{*}$ \\
\hline C9 & S1_symm. & $1.191(4)$ & $-6.22(13)^{*}$ & $1.82160(15)^{*}$ & $0.866(3)^{*}$ & $0.956(3)^{*}$ & $0.080(11)^{*}$ \\
\hline S1 & S1_intermol. & $0.0620(4)^{*}$ & $0.589(3)^{*}$ & $3.54850(13)^{*}$ & $1.77430(8) *$ & $1.77430(8)^{*}$ & $0.440(13)^{*}$ \\
\hline
\end{tabular}




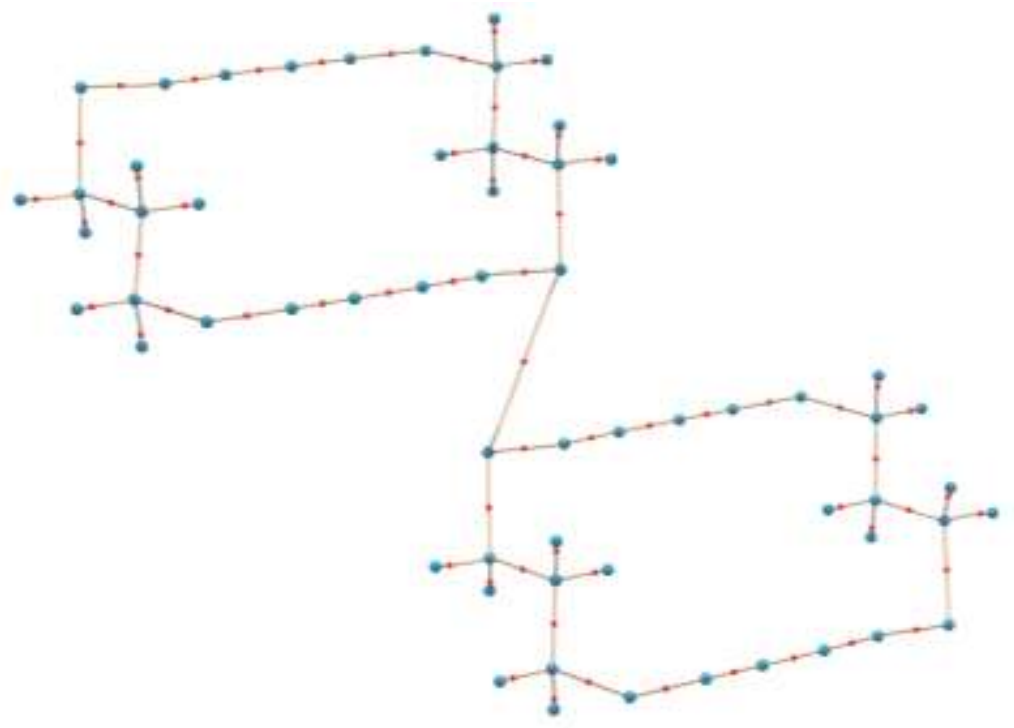

Figure 7.4: Molecular graph with atom positions (blue) bond-paths (brown) and BCPS (red) of two moieties of a, connected by a chalcogen-chalcogen interaction.

The BCP analysis in Table 7.4 and Figure 7.4 yields the properties of the molecular and intermolecular bonds. The $\mathrm{S}-\mathrm{C}(\mathrm{sp}) \mathrm{BCPs}$ are slightly shifted towards the sulfur position, while the S$\mathrm{C}\left(\mathrm{sp}^{3}\right) \mathrm{BCPs}$ are shifted towards carbon. For both $\mathrm{S}-\mathrm{C}(\mathrm{sp})$ and $\mathrm{S}-\mathrm{C}\left(\mathrm{sp}^{3}\right) \mathrm{BCPS}, \rho\left(\mathbf{r}_{\mathrm{bcc}}\right)$ is with ca. 1.44 and $1.19 \mathrm{e}^{-3}$ moderately high and $\nabla^{2} \rho\left(\mathbf{r}_{\text {bcp }}\right)$ is with ca. -8 and $-6 e^{-5}$ moderately negative, making these bonds moderately strong, slightly polarized covalent interactions. The $\mathrm{S}-\mathrm{C}(\mathrm{sp})$ is distinctly stronger, than the $\mathrm{S}-\mathrm{C}\left(\mathrm{sp}^{3}\right)$ interaction. This correllates with a shorter bond and bond-path length. The $\mathrm{S}-\mathrm{C}(\mathrm{sp})$ BCPs feature furthermore a distinctly non-zero $\varepsilon\left(\mathbf{r}_{\mathrm{bcp}}\right)$, implying a $\pi$-contribution to the bond. The alternating triple-and single-bonded carbon atoms are well observable by the differing bond lengths of 1.22 (triple) and $1.35 \AA$ (single bond) and in the properties at the BCP. The BCPs feature an ellipticity of zero, omitting any $\pi$-contribution to the single bond. $\rho\left(\mathbf{r}_{\text {bc }}\right)$ and $\nabla^{2} \rho\left(\mathbf{r}_{\text {bcp }}\right)$ are with ca. $2.69 \mathrm{e} \AA^{-3}$ and $-25 \mathrm{e}^{-5}$ distinctly higher than the single bond with $2.17 \mathrm{e}^{-3}$ and $-19 \mathrm{e}^{-5}$. The $\mathrm{C}\left(\mathrm{sp}^{3}\right) \mathrm{BCP}$ properties arefull within the expected range. A BCP is also found for the intermolecular $\mathrm{S} \cdots . \mathrm{S}$ interaction. It features a very low $\rho\left(\mathbf{r}_{\text {bcp }}\right)$ of $0.0620(4)$ e $\AA^{-3}$ and a positive, near zero $\nabla^{2} \rho\left(\mathbf{r}_{\text {bcp }}\right)$ of $0.589(3) e \AA^{-5}$ and is therefore classified as a weak, non-covalent interaction.

Table 7.5: Integrated Charges or Bader Charges.

\begin{tabular}{ll|ll|ll|ll|ll}
\hline $\mathrm{S}(1)$ & 0.3567 & $\mathrm{C}(4)$ & -0.0851 & $\mathrm{C}(7)$ & -0.4191 & $\mathrm{C}(8)$ & -0.3068 & $\mathrm{C}(9)$ & -0.4423 \\
$\mathrm{C}(2)$ & -0.269 & $\mathrm{C}(5)$ & -0.273 & $\mathrm{H}(7 \mathrm{~A})$ & 0.2027 & $\mathrm{H}(8 \mathrm{~A})$ & 0.1907 & $\mathrm{H}(9 \mathrm{~A})$ & 0.2017 \\
$\mathrm{C}(3)$ & -0.0848 & $\mathrm{~S}(6)$ & 0.352 & $\mathrm{H}(7 \mathrm{~B})$ & 0.1996 & $\mathrm{H}(8 \mathrm{~B})$ & 0.1899 & $\mathrm{H}(9 \mathrm{~B})$ & 0.2032 \\
\hline
\end{tabular}

The integrated charges or Bader charges in Table 7.5 yield a charge-shift from sulfur to the $C(\mathrm{sp})$ ), mainly by bond polarization, leaving sulfur with a low positive polarization. The $\mathrm{C}(\mathrm{sp})$ and sulfur charges add up to near zero, as do the $\mathrm{C}\left(\mathrm{sp}^{3}\right)$ and hydrogen charges, resulting in a neglectible chargeshift between $\mathrm{C}\left(\mathrm{sp}^{3}\right)$ and sulfur.

The analysis of the Laplacian around sulfur yields the positions of four VSCCs: two bonding and two non-bonding (Figure 7.5). The non-bonding VSCCS are with $\rho\left(\mathbf{r}_{v s c C}\right)=1.196 e \AA^{-3}$ and 
$\nabla^{2} \rho\left(\mathbf{r}_{\mathrm{vsc}}\right)=-11.98 \mathrm{e}^{-5}$ very pronounced and feature an $\mathrm{n}-\mathrm{S}-\mathrm{n}$ angle of $144.8^{\circ}$. They correspond to the positions of the nonbonding orbitals that are the source for the $n \rightarrow \sigma^{*}$ interaction, establishing chalcogen-chalcogen interactions. The $\mathrm{S}(1)$ atom position, the positions of the VSCCs, and the intermolecular VSCC are within a plane. In Figure 7.6, the VSCCS are well observable as minima in the Laplacian map. The VSCCs are not pointing directly into the direction of the bonding partner, as is commonly observed for covalent interactions, but are arranged parallel to each other. The bond path does not pass through the

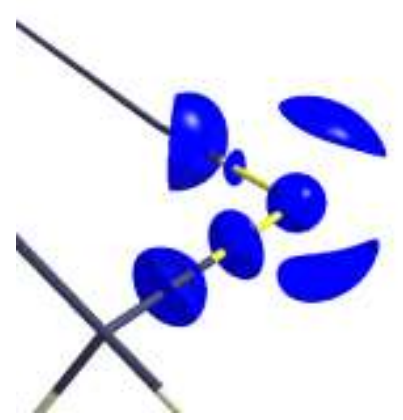

Figure 7.5: $\nabla^{2} \rho\left(\mathbf{r}_{\mathrm{bcp}}\right)$ iso-surface arouns $S(1)$ at the $-9 e^{-5}$ level. VSCC, as is commonly observed for covalent interactions. It only brushes the VSCC and proceeds straight in the direction of the other atom. This can al so be observed in the course of the Laplacian along the bond path in Figure 7.7. The minima of the Laplacian along the bond path reach values of $-7 e^{-5}$, that are distinctly higher than the Laplacian at the VSCC $\left(-11.98 \mathrm{e}^{-5}\right)$, when it brushes the VSCC and rises to low, positive values around the $\mathrm{BCP}$, corresponding of the electronic depletion in the intermolecular region.

The Laplacian map (Figure 7.6) also shows areas of electronic depletion around the sulfur positions. They reside perpendicular to the VSCC positions and the VSCC seem to point in the direction of that electronic depletion. One could envisage that this is the donation of the non-bonding orbital to the non-populated and hence depleted $\sigma^{\star}$ orbital.

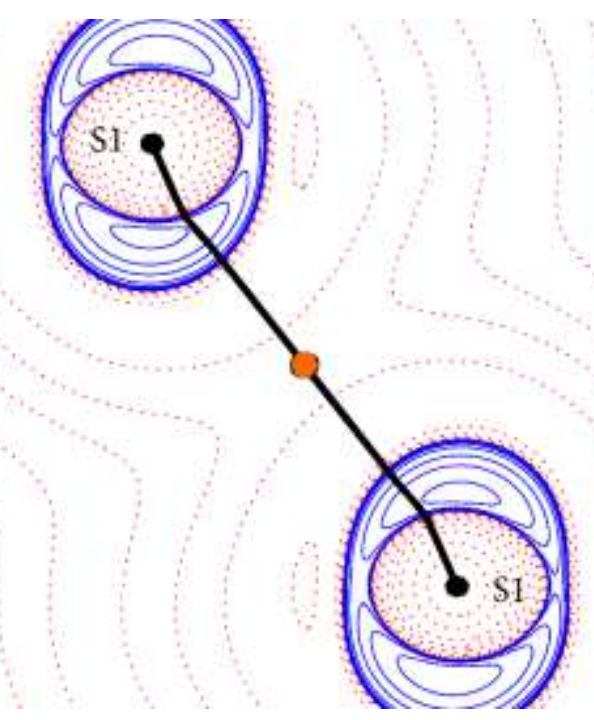

Figure 7.6: $\nabla^{2} \rho\left(\mathbf{r}_{\mathrm{bcp}}\right)$ map of the intermolecular bonding area in the plane of $\mathrm{S} 1$ and its non-bonding VSCCs. Isolevels +(red)/ -(blue) 1, 2, 3, 4, 5, 6, 7, 8, 9, 10, 11, 12, 13,14 and $15 e^{-5}$. BCP in orange; atom positions and bond path in black.

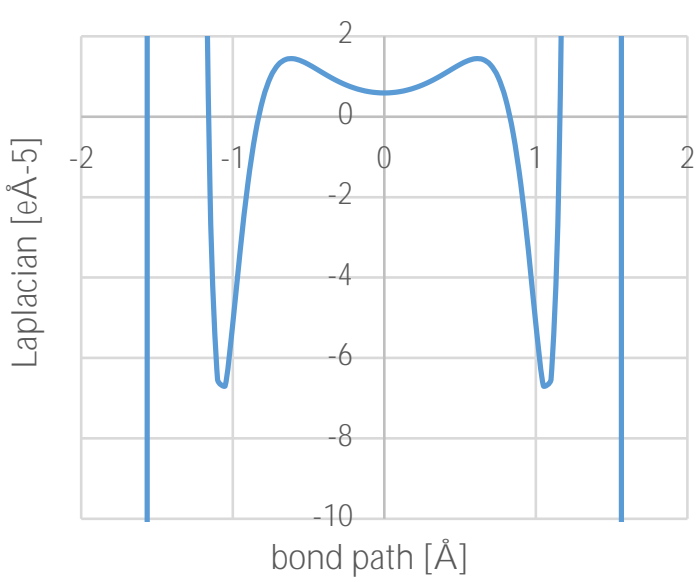

Figure 7.7: $\nabla^{2} \rho\left(\mathbf{r}_{\text {bcp }}\right)$ along the intermolecular S...S bond path. 


\subsection{Conclusion}

The experimental charge density determination yielded a complete characterization of the bonds within the molecule. The alternating single and triple bonds of the $\mathrm{sp}^{\mathrm{n}}$-hybridized carbon atoms is well observable in the analysis of the BCP. Furthermore, a slight charge-shift from the sulfur atoms through bond polarization to the $\mathrm{C}(\mathrm{sp})$ is detected in the integrated charges. The intermolecular interaction is witnessed by a BCP in the intermolecular region. The bond path connecting the two sulfur atoms does not pass through the VSCCS, but merely brushes them. The VSCCS and atom positions of both intermolecular interaction partners reside within a plane. Also visible in that plane is a region of electronic depletion near sulfur, which the VSCC of the bonding partner seems to point at. This arrangement can be interpreted as the observation of the $n \rightarrow \sigma^{*}$ interaction, postulated by Pascoe et al..

On the other hand, these results have to be received with caution. The experimental charge density has only one observable, which is the (existing) charge density. The charge density within the intermolecular region is notoriously low and therefore exhibits relatively large estimated standard deviation. The position of non-populated orbitals can be deduced indirectly from the charge density and the subtle effects of the donation into such orbitals are prone to be influenced by systematic or statistic errors. Although considerable care has been taken, the experimental charge density reaches its limits when it comes to the characterization of such weak interactions. N evertheless, the position of the VSCCS hints to the conclusion that there is in fact an $n \rightarrow \sigma^{*}$ overlap. However, it needs to be verified with other structures of similar kind, with further experiments or experimental methods, whether this is a salient feature or just circumstantial. 



\section{Chapter 8 CRYstal Structure Determinations}

Crystals were screened and selected on a custom movable table, equipped with a Schlenk line and a X-Temp2 crystal cooling device (Kottke \& Stalke, 1993; Stalke, 1998) under a polarization microscope. $M$ oisture, air, and temperature sensitive crystals were directly transferred from the Schlenk flask into perfluorinated polyether oil and cooled with the X-Temp2 device. The crystals were manipulated with fine needles and knives and mounted on either a glass fiber or a M iT eGen M icromount. Diffraction data were collected on Bruker A pex II UItra or Q uazar three circle diffractometers. They were equipped with a Bruker ( $\mathrm{Mo-K}$ ) Turbo X-ray Source (TXS) rotating anode with M olden multilayer mirror optics, an Incoatec M icrosource $(\mathrm{I} \mu \mathrm{S})$ with silver $\left(\mathrm{Ag}-\mathrm{K}_{\mathrm{a}}\right)$ or molybdenum $\left(\mathrm{M} \mathrm{o-K}_{\mathrm{a}}\right)$ as anode material and Incoatec multilayer mirror optics, or a rotating anode $\left(\mathrm{Cu} \mathrm{K}_{\alpha}\right.$ radiation) with multilayer optics and a SMART 6000 CCD detector. The diffractometers were controlled via the APEX2 (Bruker AXS Inc., 2012) suite. The data were integrated using SAINT (Bruker AXS Inc., 2016). Scaling, absorption correction and in some cases a $3 \lambda$ correction (K rause et al., 2015b) were applied using SADABS (K rause et al., 2015a). The structure solution was performed in SHELXT (Sheldrick, 2014b) and the subsequent structure refinement was carried out in SHELXL (Sheldrick, 2016), using the SHELXLE graphical user interface (Hübschle et al., 2011). 


\subsection{Crystal Structures in Collaboration with Rajendra Ghadwal}

In the course of the years 2014 to 2016, over 25 successful crystal structure determinations have been performed for Rajendra Ghadwal resulting in currently 6 publications and 10 unpublished structures.

\subsubsection{Unprecedented Borylene Insertion into a C-N Bond}

The following crystal structures were published in Ghadwal, R. S., Schürmann, C. J., Engelhardt, F. \& Steinmetzger, C. 2014. Eur. J. Inorg. Chem. 2014, 4921-4926.

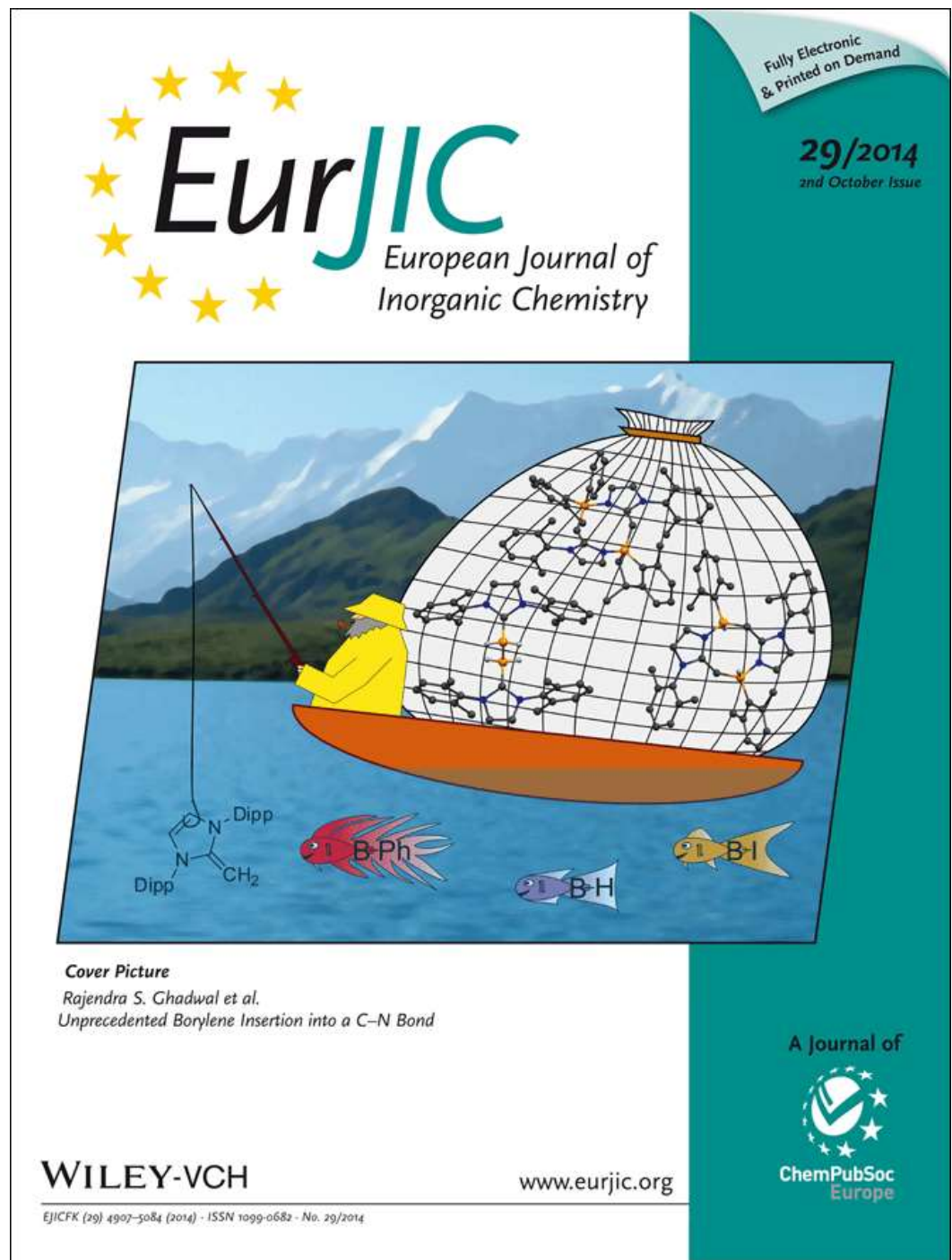

Figure 8.1: Frontispiece associated to "U nprecedented Borylene Insertion into a C-N Bond" (Ghadwal et al., 2014). 


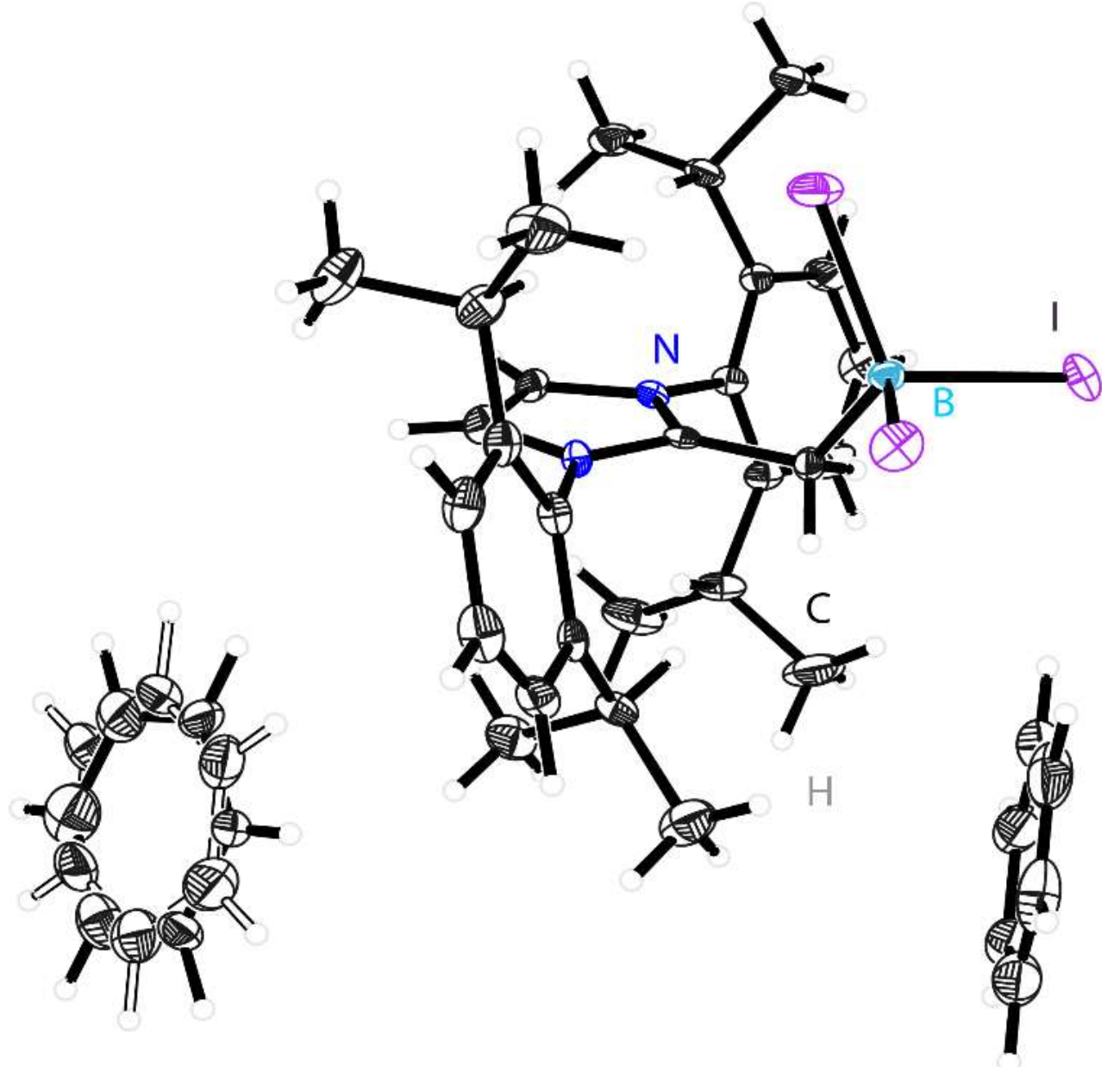

\begin{tabular}{|c|c|c|c|}
\hline Structure code & CS_RAJ_282_a_Ag & CCDC Number & 1010606 \\
\hline Empirical Formula & $\mathrm{C}_{40} \mathrm{H}_{50} \mathrm{BI}_{3} \mathrm{~N}_{2}$ & $\mu\left[\mathrm{mm}^{-1}\right]$ & 1.25 \\
\hline Formula weight $\left[\mathrm{g} \mathrm{mol}^{-1}\right]$ & 950.33 & $F(000)$ & 936 \\
\hline Sample temperature [K] & $100(2)$ & $\theta$ range $\left[{ }^{\circ}\right]$ & 1.808 to 20.018 \\
\hline Wavelength $[\AA]$ & 0.56086 & Reflections collected & 81014 \\
\hline Crystal System & Monoclinic & Unique reflections & 7688 \\
\hline \multirow[t]{2}{*}{ Space group } & $P 2_{1}($ Flack $=-0.018(12))$ & $\mathrm{R}_{\text {int }}$ & 0.0659 \\
\hline & $a=9.717(2)$ & Completeness to $\theta_{\max }[\%]$ & 99.9 \\
\hline U nit cell dimensions $\left[\AA,{ }^{\circ}\right]$ & $\begin{array}{l}b=20.578(2) \quad \beta=113.81(2) \\
c=11.048(3)\end{array}$ & $\begin{array}{l}\text { restraints/parameter } \\
\text { GooF }\end{array}$ & $\begin{array}{l}637 / 478 \\
1.076\end{array}$ \\
\hline Volume $\left[\AA^{3}\right]$ & $2021.1(8)$ & R1 (all data) & 0.0262 \\
\hline Z & 2 & wR2 (all data) & 0.0544 \\
\hline Crystal dimensions [mm] & $0.104 \times 0.085 \times 0.079$ & max. diff. peak / hole $\left[\mathrm{e}^{-3}\right]$ & 0.774 and -0.597 \\
\hline
\end{tabular}




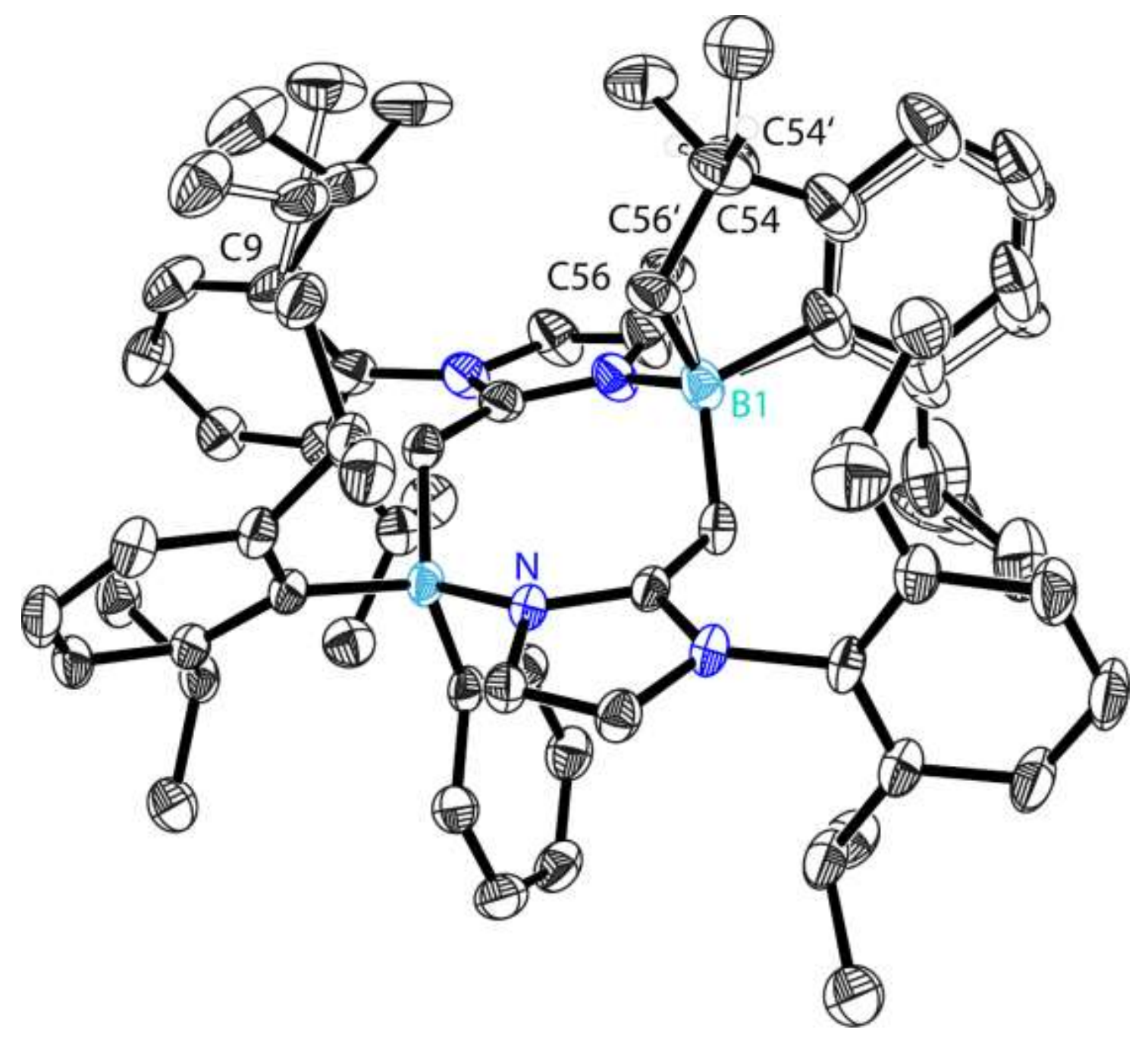

\begin{tabular}{|c|c|c|c|}
\hline Structure code & CS_RAJ_285 & CCDC N umber & 1010610 \\
\hline Empirical Formula & $\mathrm{C}_{62} \mathrm{H}_{80} \mathrm{~B}_{2} \mathrm{~N}_{4}$ & $\mu\left[\mathrm{mm}^{-1}\right]$ & 0.471 \\
\hline Formula weight $\left[\mathrm{g} \mathrm{mol}^{-1}\right]$ & 902.92 & $F(000)$ & 1960 \\
\hline Sample temperature [K] & $100(2)$ & $\theta$ range $\left[{ }^{\circ}\right]$ & 2.185 to 68.244 \\
\hline Wavelength $[\AA]$ & 1.54178 & Reflections collected & 92165 \\
\hline Crystal System & Monoclinic & Unique reflections & 9955 \\
\hline Space group & $\mathrm{P} 2 \mathrm{l} / \mathrm{C}$ & $\mathrm{R}_{\text {int }}$ & 0.0411 \\
\hline & $a=20.269(3) 2)$ & Completeness to $\theta_{\max }[\%]$ & 99.9 \\
\hline U nit cell dimensions $\left[\AA,{ }^{\circ}\right]$ & $\begin{array}{l}\mathrm{b}=10.673(2) \quad \beta=94.00(2) \\
\mathrm{c}=25.213(2)\end{array}$ & $\begin{array}{l}\text { restraints/parameter } \\
\text { GooF }\end{array}$ & $\begin{array}{l}810 / 754 \\
1.015\end{array}$ \\
\hline V olume $\left[\AA^{3}\right]$ & $5441.1(14)$ & R1 (all data) & 0.0480 \\
\hline $\mathrm{Z}$ & 4 & wR2 (all data) & 0.1033 \\
\hline Crystal dimensions [mm] & $0.300 \times 0.180 \times 0.070$ & max. diff. peak / hole $\left[\mathrm{e}^{-3}\right]$ & 0.308 and -0.191 \\
\hline
\end{tabular}




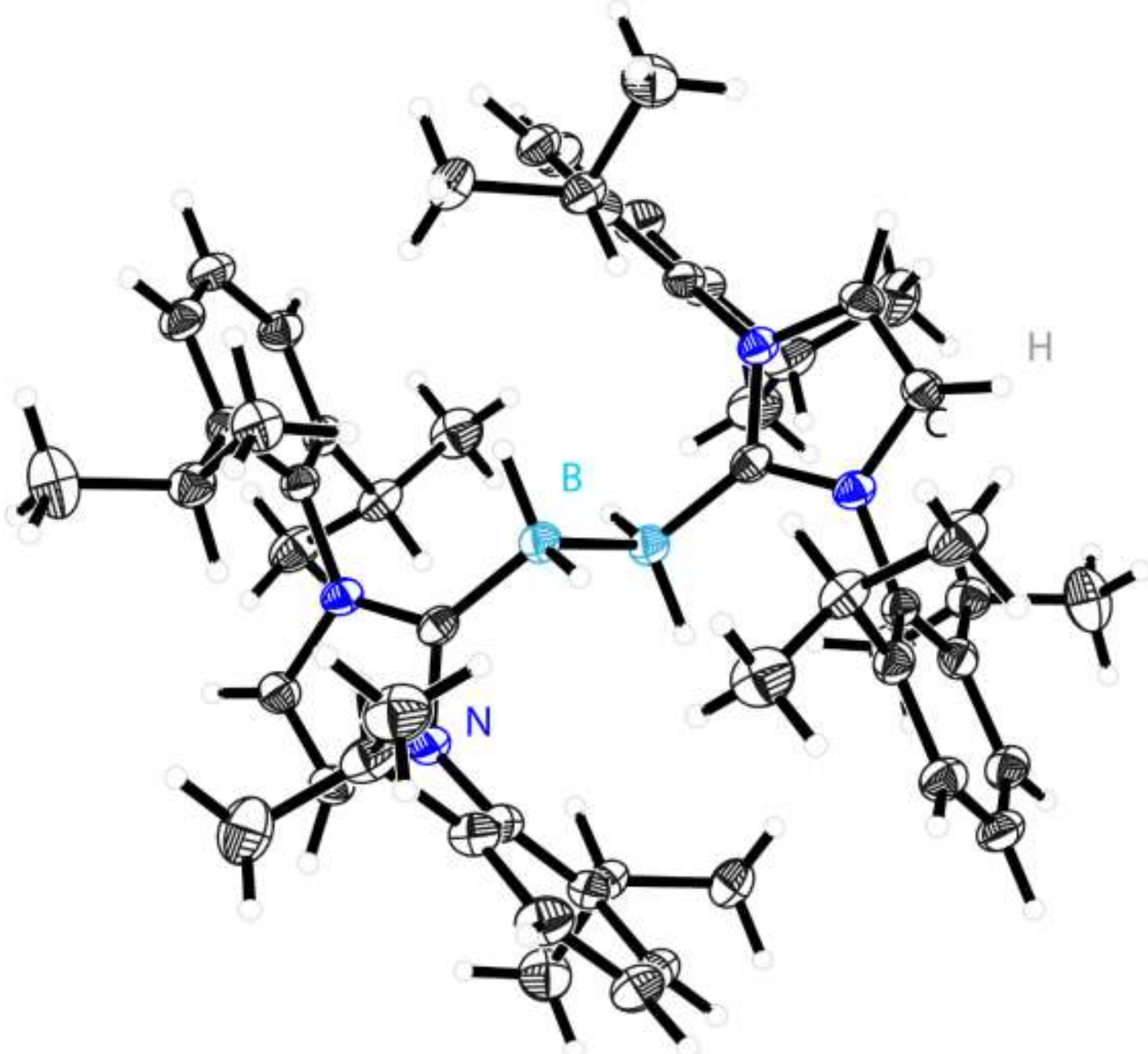

\begin{tabular}{|c|c|c|c|}
\hline Structure code & CS_RAJ_286 & CCDC Number & 1010607 \\
\hline Empirical Formula & $\mathrm{C}_{46} \mathrm{H}_{55} \mathrm{BCl}_{2} \mathrm{~N}_{2}$ & $\mu\left[\mathrm{mm}^{-1}\right]$ & 0.469 \\
\hline Formula weight $\left[\mathrm{g} \mathrm{mol}^{-1}\right]$ & 717.63 & $F(000)$ & 876 \\
\hline Sample temperature [K] & $100(2)$ & $\theta$ range $\left[{ }^{\circ}\right]$ & 3.723 to 70.133 \\
\hline Wavelength $[\AA]$ & 1.54178 & Reflections collected & 60305 \\
\hline Crystal System & Monoclinic & Unique reflections & 7889 \\
\hline \multirow[t]{2}{*}{ Space group } & $\mathrm{P} 2_{1} / \mathrm{n}$ & $\mathrm{R}_{\text {int }}$ & 0.0223 \\
\hline & $a=14.765(2)$ & Completeness to $\theta_{\max }[\%]$ & 99.9 \\
\hline \multirow[t]{2}{*}{ U nit cell dimensions $\left[\AA,{ }^{\circ}\right]$} & $b=16.594(2)$ & restraints/parameter & $0 / 468$ \\
\hline & $c=17.071(2)$ & GooF & 1.035 \\
\hline Volume $\left[\AA^{3}\right]$ & $4164.1(9)$ & R1 (all data) & 0.0356 \\
\hline Z & 4 & wR2 (all data) & 0.0938 \\
\hline Crystal dimensions [mm] & $0.3 \times 0.2 \times 0.2$ & max. diff. peak / hole $\left[e^{-3}\right]$ & 0.337 and -0.226 \\
\hline
\end{tabular}




\subsubsection{Mono- and Di-Cationic Hydrido Boron Compounds}

The following Crystal Structures have been published together with one re-integrated and -refined structure by Dr. Julia M atusseck (M atussek, 2014, CCDC\# 1401734, str. code: raj_243) in Ghadwal, R. S., Schürmann, C. J., Andrada, D. M . \& Frenking, G. 2015. Dalton Trans. 44, 14359-14367.

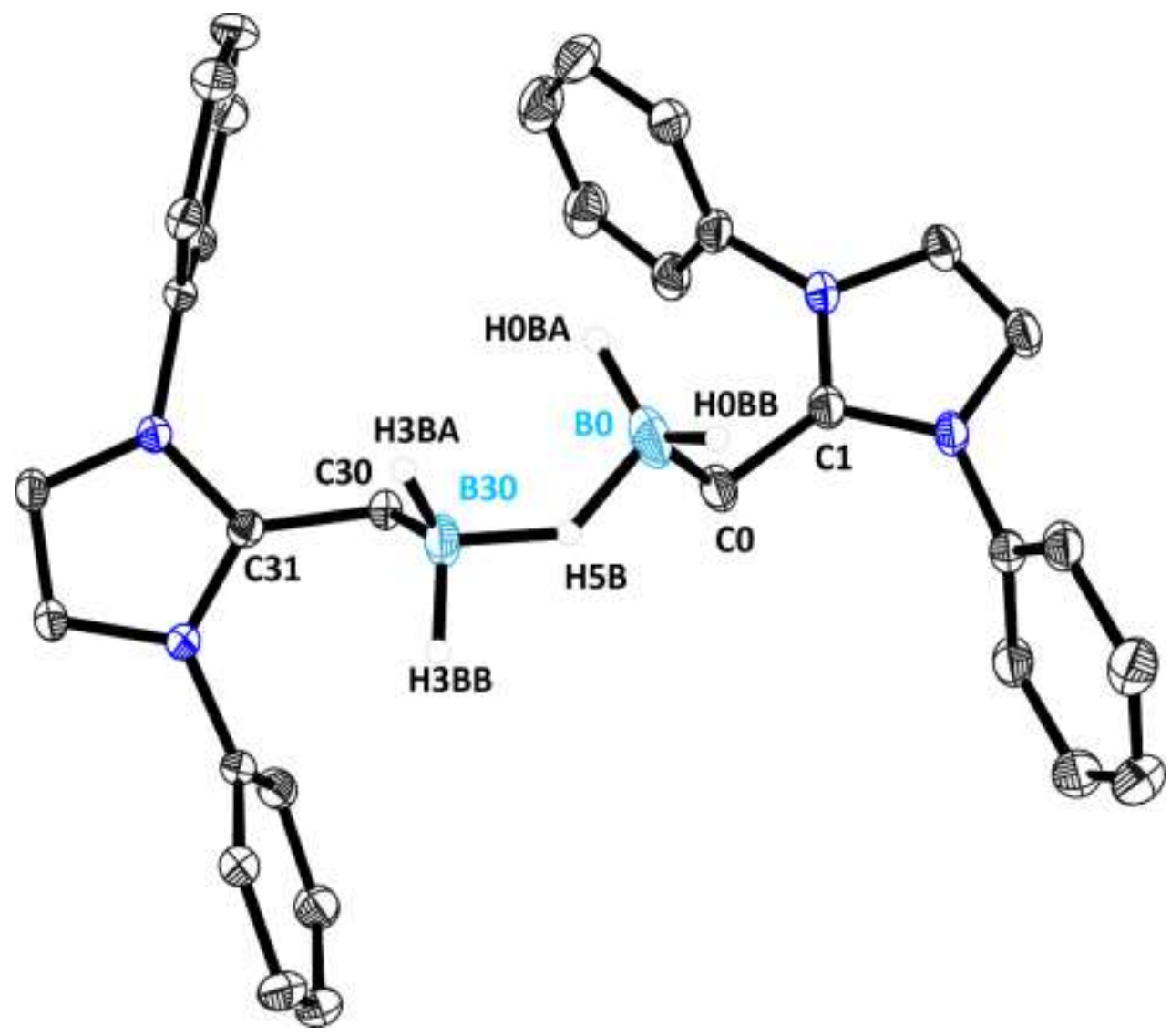

One $\left(\mathrm{CF}_{3} \mathrm{SO}_{2}\right)_{2} \mathrm{~N}$ counter ion is omitted for clarity.

\begin{tabular}{|c|c|c|c|c|}
\hline Structure code & \multicolumn{2}{|c|}{ CS_RAJ_325 } & CCDC Number & 1060035 \\
\hline Empirical Formula & \multicolumn{2}{|c|}{$\mathrm{C}_{58} \overline{\mathrm{H}}_{81} \mathrm{~B}_{2} \mathrm{~F}_{6} \mathrm{~N}_{5} \mathrm{O}_{4} \mathrm{~S}_{2}$} & $\mu\left[\mathrm{mm}^{-1}\right]$ & 0.085 \\
\hline Formula weight $\left[\mathrm{g} \mathrm{mol}^{-1}\right]$ & \multicolumn{2}{|c|}{1112.01} & $F(000)$ & 1184 \\
\hline Sample temperature [K ] & \multicolumn{2}{|l|}{$100(2)$} & $\theta$ range $\left[{ }^{\circ}\right]$ & 1.289 to 22.073 \\
\hline W avelength $[\AA]$ & \multicolumn{2}{|l|}{0.56086} & Reflections collected & 238549 \\
\hline Crystal System & \multicolumn{2}{|l|}{ Triclinic } & Unique reflections & 15500 \\
\hline \multirow[t]{2}{*}{ Space group } & $\mathrm{P} \overline{1}$ & & $\mathrm{R}_{\text {int }}$ & 0.0605 \\
\hline & $a=10.754(2)$ & $\alpha=83.78(2)$ & Completeness to $\theta_{\max }[\%]$ & 100.0 \\
\hline \multirow[t]{2}{*}{ U nit cell dimensions $\left[\AA{ }^{\circ}{ }^{\circ}\right]$} & $b=16.407(2)$ & $\beta=73.47(2)$ & restraints/parameter & $513 / 768$ \\
\hline & $c=18.510(2)$ & $y=83.41(2)$ & GooF & 1.024 \\
\hline Volume $\left[\AA^{3}\right]$ & $3100.4(4)$ & & R1 (all data) & 0.0550 \\
\hline Z & 2 & & wR2 (all data) & 0.0999 \\
\hline Crystal dimensions [mm] & $0.195 \times 0.194$ & 0.104 & max. diff. peak / hole $\left[\AA^{-3}\right]$ & 0.334 and -0.440 \\
\hline
\end{tabular}




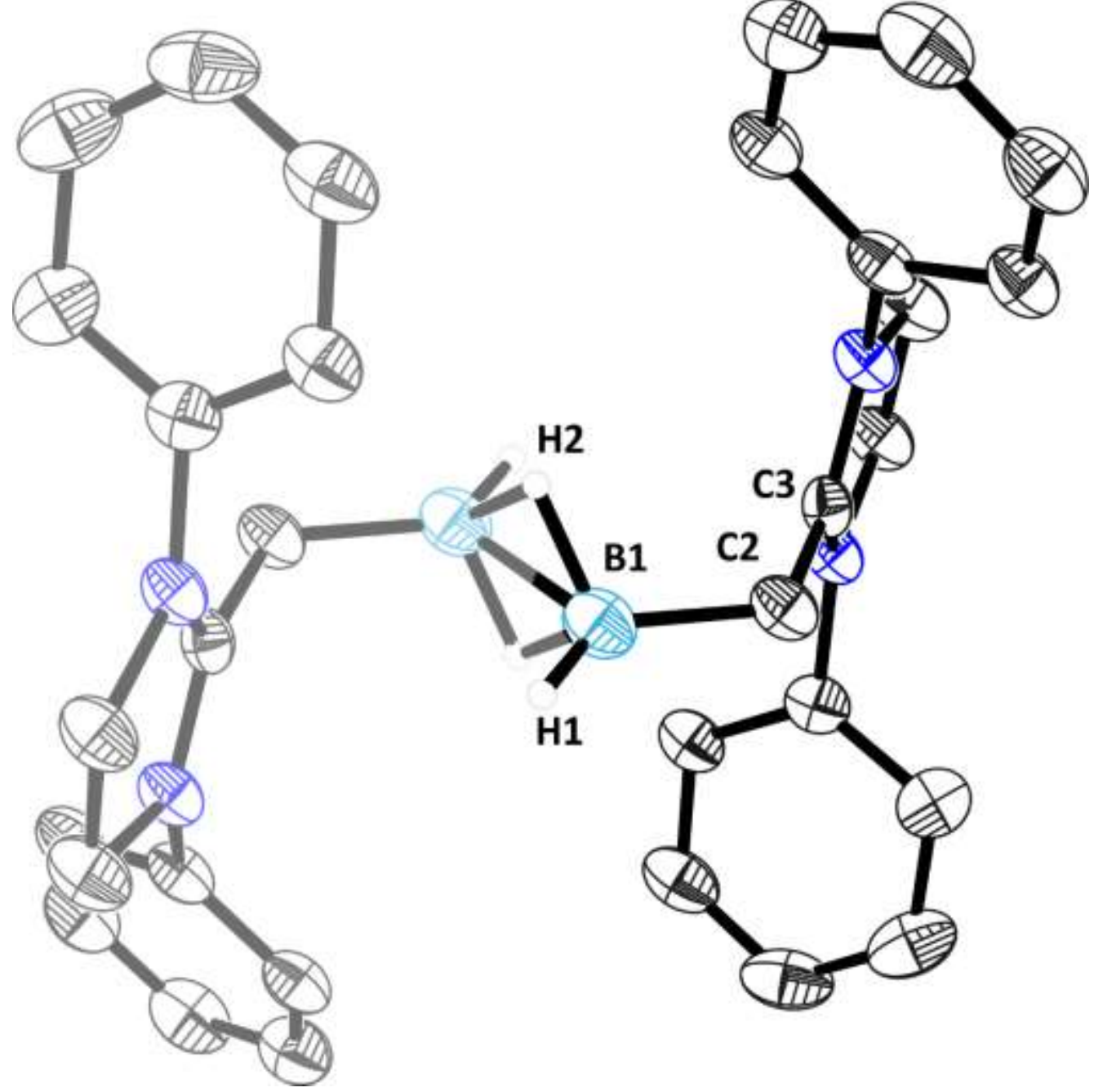

Two disordered $\left(\mathrm{CF}_{3} \mathrm{SO}_{2}\right)_{2} \mathrm{~N}$ and one disordered $\mathrm{CH}_{2} \mathrm{Cl}_{2}$ are omitted for clarity

\begin{tabular}{|c|c|c|c|c|}
\hline Structure code & \multicolumn{2}{|c|}{ CS_RAJ_365_Ag } & CCDC N umber & 1060034 \\
\hline Empirical Formula & \multicolumn{2}{|c|}{$\mathrm{C}_{60.66} \mathrm{H}_{85.32} \mathrm{~B}_{2} \overline{\mathrm{C}} \mathrm{l}_{1.32} \mathrm{~F}_{12} \mathrm{~N}_{6} \mathrm{O}_{8} \mathrm{~S}_{4}$} & $\mu\left[\mathrm{mm}^{-1}\right]$ & 0.146 \\
\hline Formula weight $\left[\mathrm{g} \mathrm{mol}^{-1}\right]$ & \multicolumn{2}{|c|}{1451.24} & $F(000)$ & 760 \\
\hline Sample temperature [K ] & \multicolumn{2}{|l|}{$100(2)$} & $\theta$ range $\left[{ }^{\circ}\right]$ & 2.508 to 20.125 \\
\hline W avelength $[\AA]$ & \multicolumn{2}{|l|}{0.56086} & Reflections collected & 52767 \\
\hline Crystal System & \multicolumn{2}{|l|}{ Triclinic } & Unique reflections & 6769 \\
\hline \multirow[t]{2}{*}{ Space group } & \multicolumn{2}{|l|}{$P \overline{1}$} & $\mathrm{R}_{\text {int }}$ & 0.0554 \\
\hline & $a=12.306(2)$ & $\alpha=95.14(2)$ & Completeness to $\theta_{\max }[\%]$ & 99.8 \\
\hline \multirow[t]{2}{*}{ U nit cell dimensions $[\AA]$} & $b=12.691(2)$ & $\beta=101.53(2)$ & restraints/parameter & $1636 / 863$ \\
\hline & $c=13.389(2)$ & $y=118.07(2)$ & GooF & 1.044 \\
\hline Volume $\left[\AA^{3}\right]$ & \multicolumn{2}{|l|}{$1766.6(6)$} & R1 (all data) & 0.0956 \\
\hline Z & \multicolumn{2}{|l|}{1} & wR2 (all data) & 0.2259 \\
\hline Crystal dimensions [mm] & \multicolumn{2}{|c|}{$0.317 \times 0.301 \times 0.110$} & max. diff. peak / hole $\left[\AA^{-3}\right]$ & 0.785 and -0.449 \\
\hline
\end{tabular}




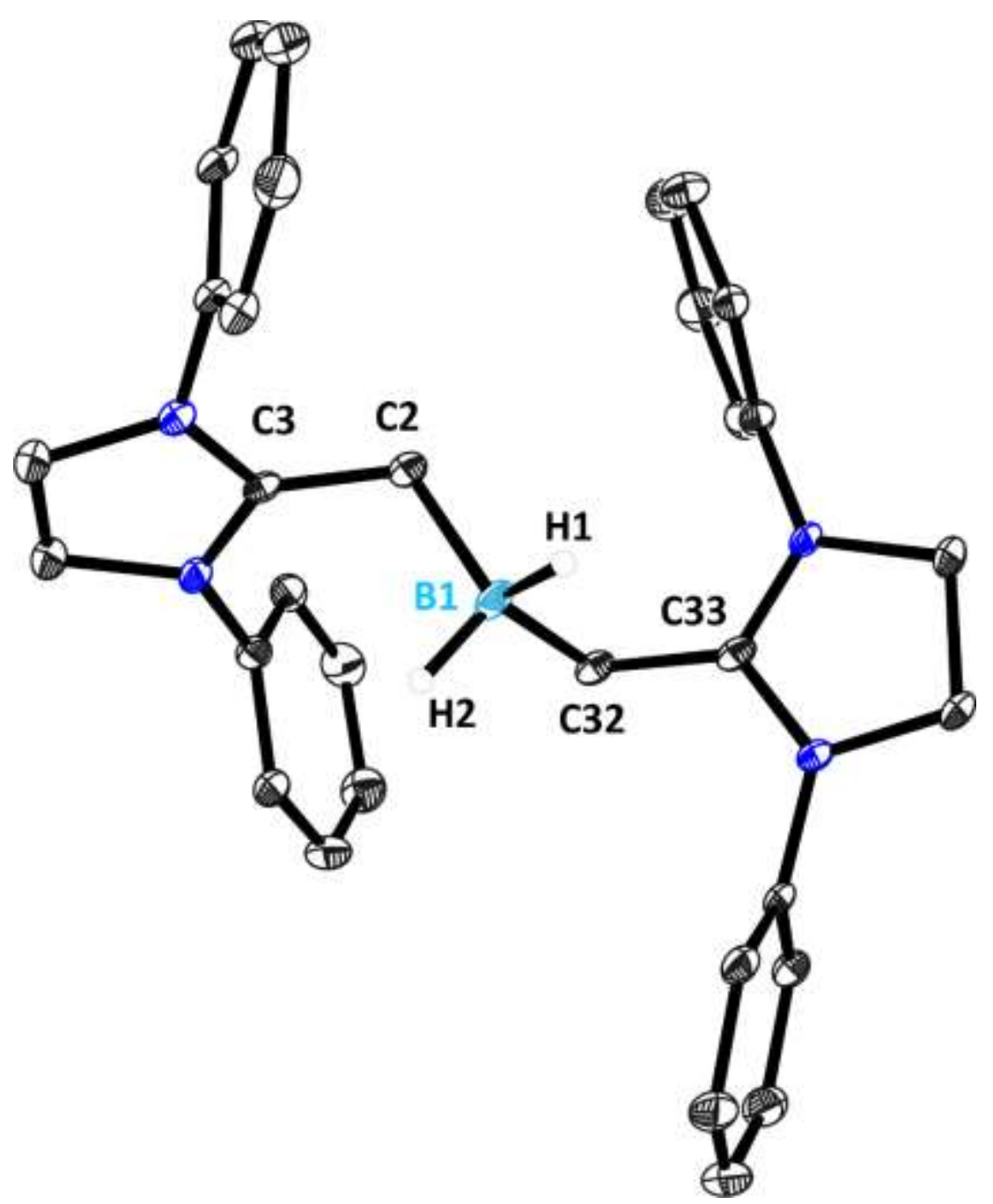

One $\left(\mathrm{CF}_{3} \mathrm{SO}_{2}\right)_{2} \mathrm{~N}$ counter ion and two $\mathrm{C}_{6} \mathrm{H}_{5} \mathrm{~F}$ solvent molecules have been omitted for clarity.

\begin{tabular}{|c|c|c|c|c|}
\hline Structure code & \multicolumn{2}{|l|}{ CS_RAJ_364 } & CCDC Number & 1060033 \\
\hline Empirical Formula & \multicolumn{2}{|c|}{$\mathrm{C}_{67} \mathrm{H}_{89.50} \mathrm{BF}_{7.50} \mathrm{~N}_{5} \mathrm{O}_{4} \mathrm{~S}_{2}$} & $\mu\left[\mathrm{mm}^{-1}\right]$ & 0.088 \\
\hline Formula weight $\left[\mathrm{g} \mathrm{mol}^{-1}\right]$ & \multicolumn{2}{|c|}{1246.36} & $\mathrm{~F}(000)$ & 1326 \\
\hline Sample temperature [K ] & \multicolumn{2}{|l|}{$100(2)$} & $\theta$ range $\left[{ }^{\circ}\right]$ & 1.739 to 19.601 \\
\hline W avelength $[\AA]$ & \multicolumn{2}{|l|}{0.56086} & Reflections collected & 161683 \\
\hline Crystal System & \multicolumn{2}{|l|}{ Triclinic } & Unique reflections & 11676 \\
\hline \multirow[t]{2}{*}{ Space group } & \multicolumn{2}{|l|}{$\mathrm{P} \overline{1}$} & $\mathrm{R}_{\text {int }}$ & 0.0810 \\
\hline & $a=13.061(3)$ & $\alpha=96.88(2)$ & Completeness to $\theta_{\max }[\%]$ & 99.6 \\
\hline \multirow[t]{2}{*}{ Unit cell dimensions $[\AA]$ ] } & $b=15.613(4)$ & $\beta=101.45(2)$ & restraints/parameter & $111 / 808$ \\
\hline & $c=16.727(4)$ & $y=98.29(2)$ & GooF & 1.044 \\
\hline Volume $\left[\AA^{3}\right]$ & \multicolumn{2}{|l|}{$3269.2(14)$} & R1 (all data) & 0.0737 \\
\hline Z & \multicolumn{2}{|c|}{2} & wR2 (all data) & 0.1589 \\
\hline Crystal dimensions [mm] & \multicolumn{2}{|c|}{$0.180 \times 0.153 \times 0.079$} & max. diff. peak / hole $\left[\mathrm{e}^{-3}\right]$ & 0.746 and -0.565 \\
\hline
\end{tabular}




\subsubsection{Abnormal-NHC Palladium(II) Complexes: Rational Synthesis, Structural Elucidation, and Catalytic Activity}

The following structures have been published in Rottschäfer, D., Schürmann, C. J., Lamm, J.-H., Paesch, A. N., Neumann, B. \& Ghadwal, R. S. 2016. Organometallics. 35, 3421-3429.

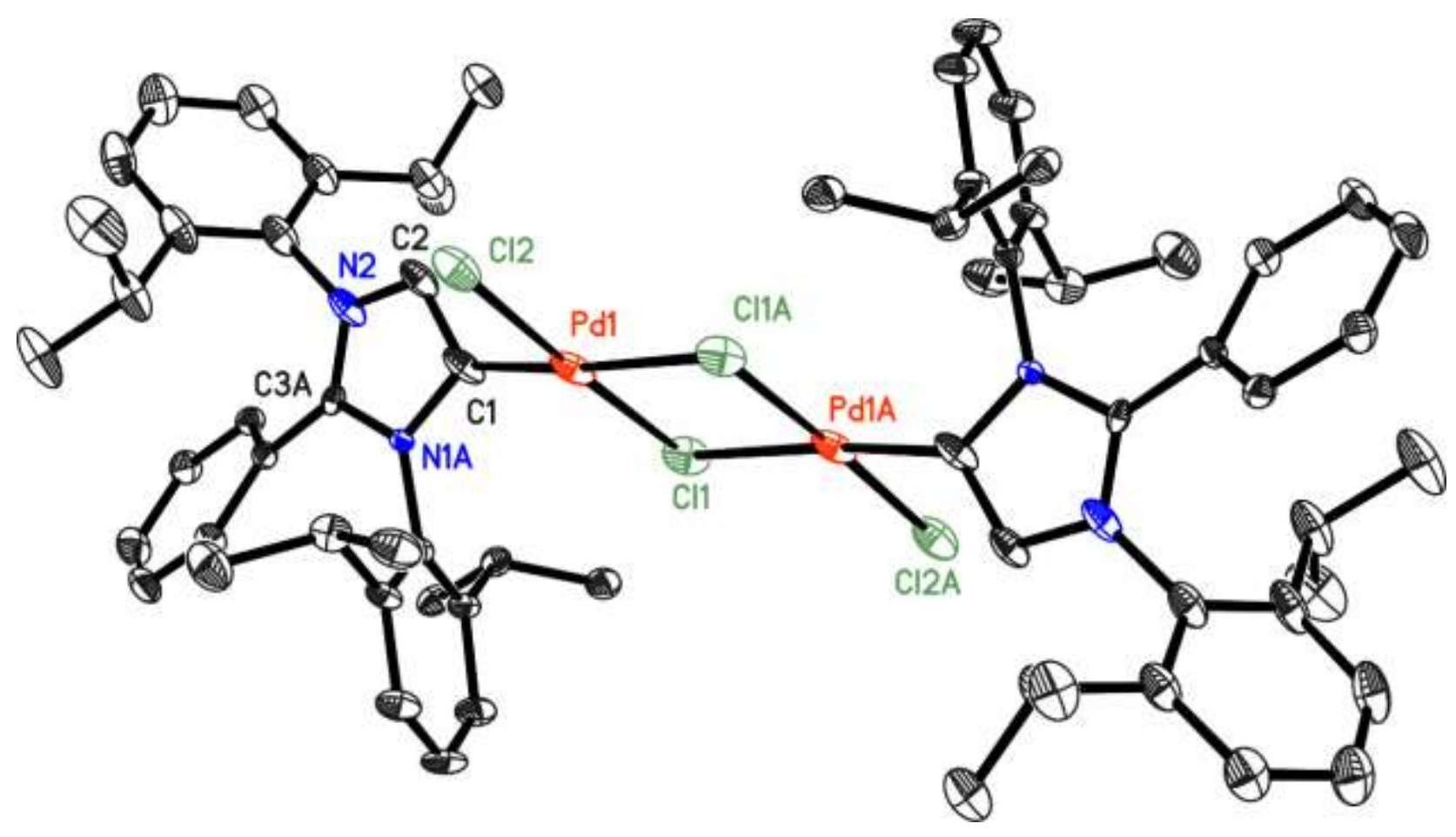

\begin{tabular}{|c|c|c|c|}
\hline Structure code & CS_RAJ_343_new_c2 & CCDC Number & 1487623 \\
\hline Empirical Formula & $\mathrm{C}_{68}-\mathrm{H}_{84} \mathrm{Cl}_{8} \mathrm{~N}_{4} \overline{P d}_{2}$ & $\mu\left[\mathrm{mm}^{-1}\right]$ & 0.865 \\
\hline Formula weight $\left[\mathrm{g} \mathrm{mol}^{-1}\right]$ & 1453.79 & $F(000)$ & 748 \\
\hline Sample temperature [K] & $100(2)$ & $\theta$ range $\left[{ }^{\circ}\right]$ & 1.380 to 28.255 \\
\hline W avelength $[\AA]$ & 0.71073 & Reflections collected & 60420 \\
\hline Crystal System & Monoclinic & Unique reflections & 8524 \\
\hline \multirow[t]{2}{*}{ Space group } & $\mathrm{P} \overline{1}$ & $\mathrm{R}_{\text {int }}$ & 0.0288 \\
\hline & $a=10.282(2)$ & Completeness to $\theta_{\max }[\%]$ & 100 \\
\hline \multirow[t]{2}{*}{ Unit cell dimensions $[\AA]$} & $b=11.538(2)$ & restraints/parameter & 1084 / 582 \\
\hline & $c=15.223(3)$ & GooF & 1.126 \\
\hline Volume $\left[\AA^{3}\right]$ & $1740.6(6)$ & R1 (all data) & 0.0346 \\
\hline Z & 1 & wR2 (all data) & 0.0624 \\
\hline Crystal dimensions [mm] & $0.274 \times 0.096 \times 0.078$ & max. diff. peak / hole $\left[\mathrm{e} \AA^{-3}\right]$ & 0.676 and -1.232 \\
\hline
\end{tabular}




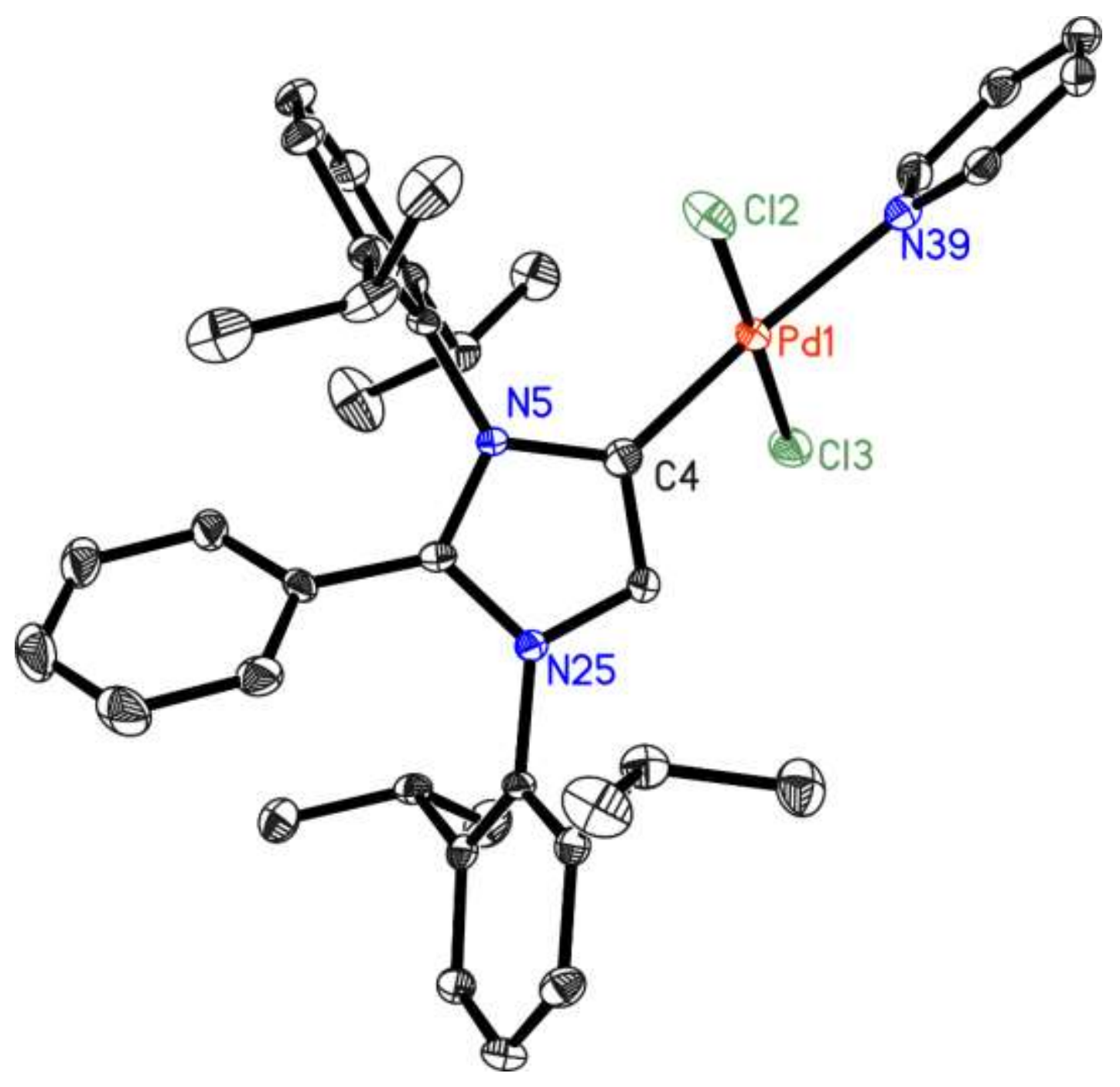

\begin{tabular}{llll}
\hline Structure code & $\mathrm{CS} \mathrm{RA}_{-} 368$ Hex & $\mathrm{CCDC}$ Number & 1445486 \\
Empirical Formula & $\mathrm{C}_{38} \mathrm{H}_{45} \mathrm{Cl}_{2} \mathrm{~N}_{3} \mathrm{Pd}$ & $\mu\left[\mathrm{mm}^{-1}\right]$ & 0.379 \\
Formula weight $\left[\mathrm{g} \mathrm{mol}^{-1}\right]$ & 721.07 & $\mathrm{~F}(000)$ & 1496 \\
Sampletemperature $[\mathrm{K}]$ & $100(2)$ & $\theta$ range $\left.{ }^{\circ}\right]$ & 1.256 to 20.556 \\
Wavelength $[\AA]$ & 0.56086 & Reflections collected & 75942 \\
Crystal System & $\mathrm{M}$ onoclinic & Unique reflections & 7228 \\
Space group & $\mathrm{P} 2{ }_{1} / \mathrm{n}$ & $\mathrm{R}_{\text {int }}$ & 0.0834 \\
& $\mathrm{a}=9.437(2)$ & Completeness to $\theta_{\max }[\%]$ & 100 \\
Unit cell dimensions $[\AA]$ & $\mathrm{b}=25.014(3) \quad \beta=100.00(2)$ & restraints/parameter & $0 / 406$ \\
& $\mathrm{C}=15.126(2)$ & GooF & 1.022 \\
Volume $\left[\AA^{3}\right]$ & $3516.4(10)$ & $\mathrm{R} 1$ (all data) & 0.0596 \\
$Z$ & 4 & wR2 (all data) & 0.0913 \\
Crystal dimensions $[\mathrm{mm}]$ & $0.193 \times 0.126 \times 0.057$ & max. diff. peak / hole $\left[\mathrm{e} \AA^{-3}\right]$ & 1.783 and -0.793 \\
\hline
\end{tabular}




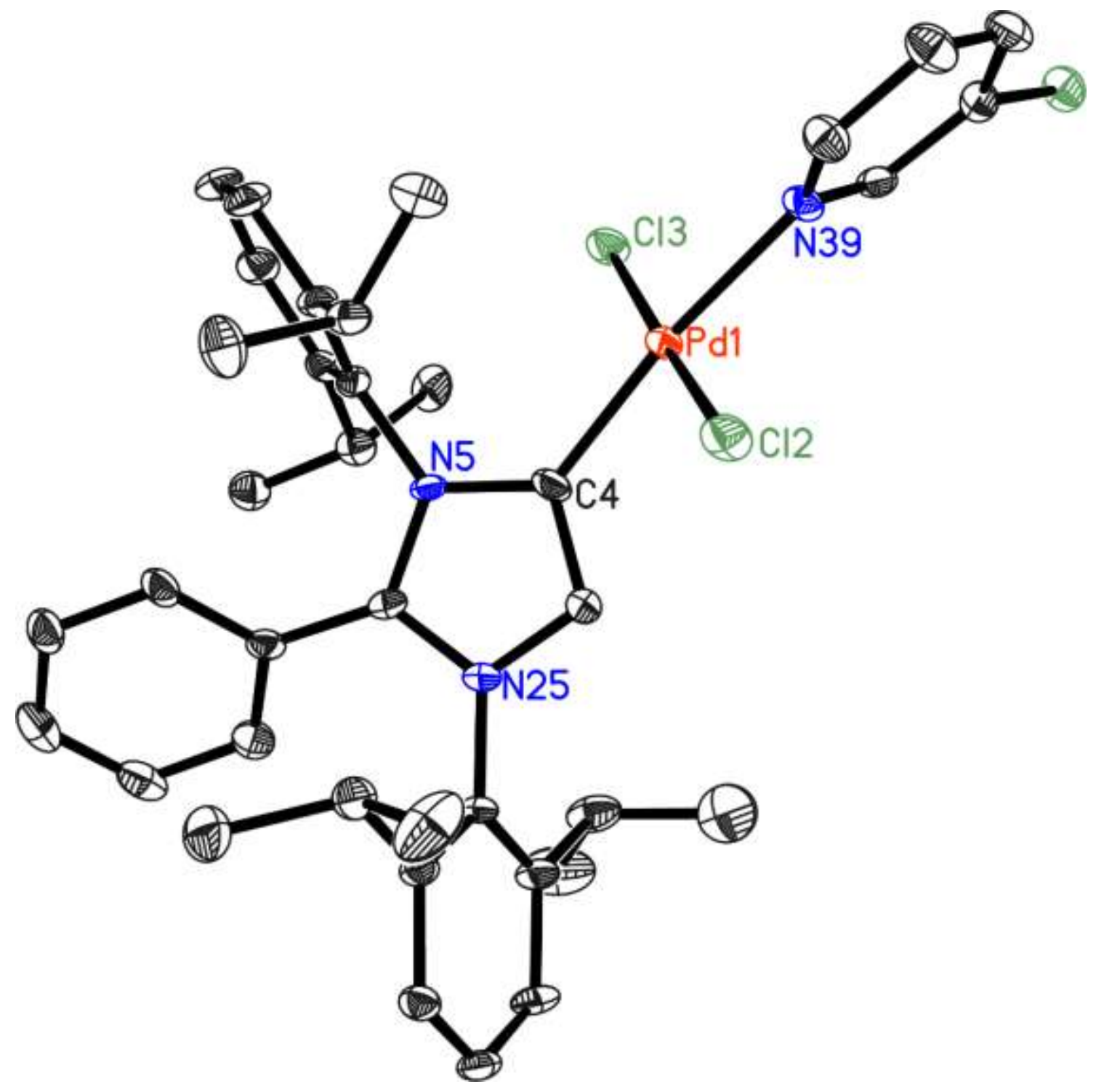

\begin{tabular}{|c|c|c|c|c|}
\hline Structure code & \multicolumn{2}{|c|}{ CS_RAJ_374_Mo } & CCDC Number & 1445487 \\
\hline Empirical Formula & \multicolumn{2}{|c|}{$\mathrm{C}_{38} \mathrm{H}_{44} \mathrm{Cl}_{3} \mathrm{~N}_{3} \mathrm{Pd}$} & $\mu\left[\mathrm{mm}^{-1}\right]$ & 0.088 \\
\hline Formula weight $\left[\mathrm{g} \mathrm{mol}^{-1}\right]$ & \multicolumn{2}{|l|}{755.51} & $\mathrm{~F}(000)$ & 1326 \\
\hline Sample temperature [K] & \multicolumn{2}{|l|}{$100(2)$} & $\theta$ range $\left[{ }^{\circ}\right]$ & 1.347 to 28.307 \\
\hline W avelength $[\AA]$ & \multicolumn{2}{|l|}{0.71073} & Reflections collected & - (twin) \\
\hline Crystal System & \multicolumn{2}{|l|}{ Triclinic } & Unique reflections & 17928 \\
\hline \multirow[t]{2}{*}{ Space group } & \multicolumn{2}{|l|}{$\mathrm{P} \overline{1}$} & $\mathrm{R}_{\text {int }}$ & - merged \\
\hline & $a=9.537(2)$ & $\alpha=91.36(2))$ & Completeness to $\theta_{\max }[\%]$ & 100 \\
\hline U nit cell dimensions $[\AA]$ & $\begin{array}{l}b=15.348(2) \\
c=24.812(3)\end{array}$ & $\begin{array}{l}\beta=91.14(2) \\
\gamma=99.93(3)\end{array}$ & $\begin{array}{l}\text { restraints/parameter } \\
\text { GooF }\end{array}$ & $\begin{array}{l}0 / 828 \\
1.195\end{array}$ \\
\hline Volume $\left[\AA^{3}\right]$ & $3610.2(10)$ & & R1 (all data) & 0.0543 \\
\hline Z & 4 & & wR2 (all data) & 0.1335 \\
\hline Crystal dimensions [mm] & $0.217 \times 0.213$ & 0.117 & max. diff. peak / hole $\left[\mathrm{e}^{-3}\right]$ & 2.051 and -1.110 \\
\hline
\end{tabular}




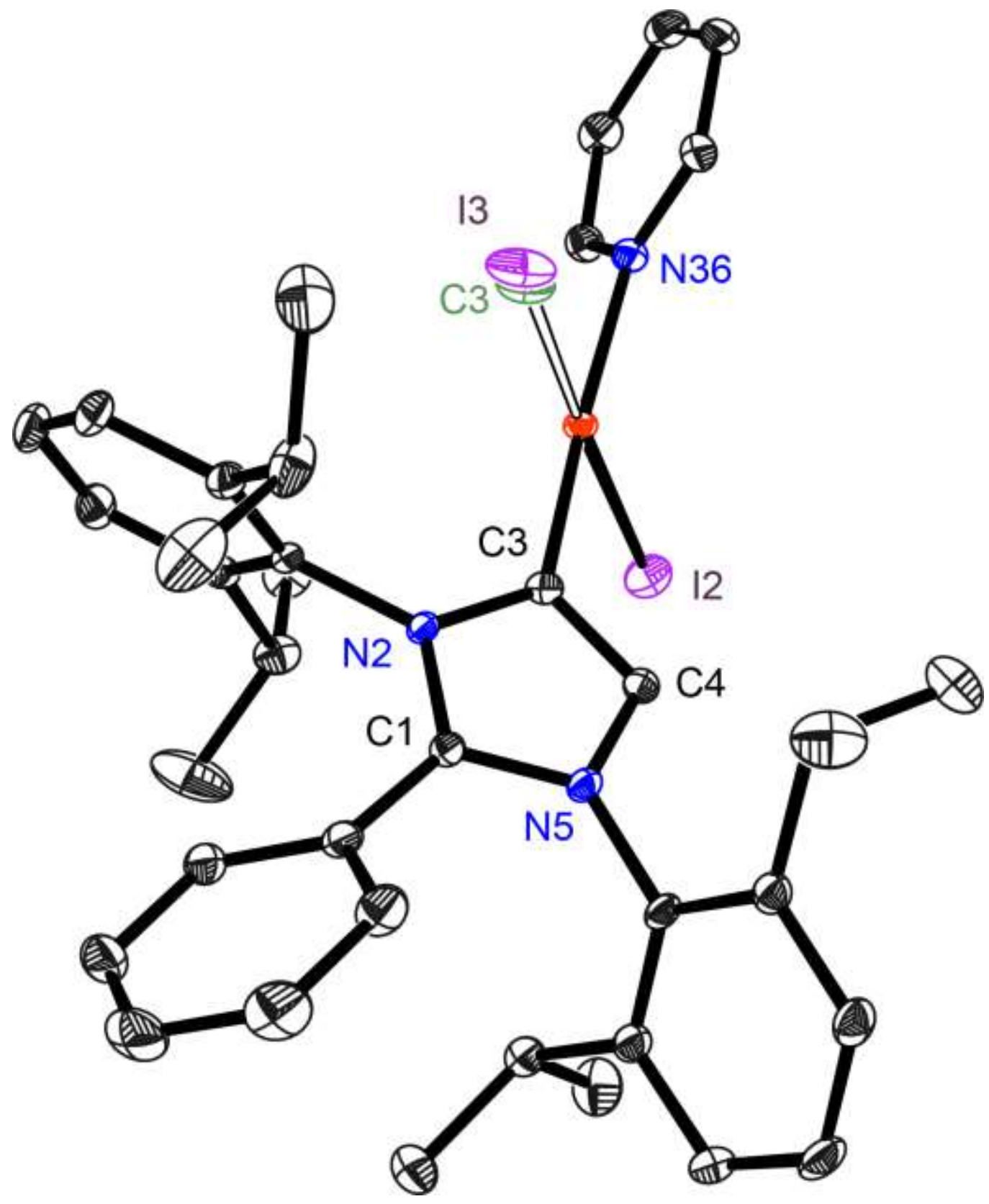

\begin{tabular}{|c|c|c|c|}
\hline Structure code & CS_RAJ_352 & CCDC Number & 1445487 \\
\hline Empirical Formula & $\mathrm{C}_{38} \mathrm{H}_{45} \mathrm{Cl}_{1.06} \mathrm{I}_{0.94} \mathrm{~N}_{3} \mathrm{Pd}$ & $\mu\left[\mathrm{mm}^{-1}\right]$ & 1.438 \\
\hline Formula weight $\left[\mathrm{g} \mathrm{mol}^{-1}\right]$ & 807.46 & $\mathrm{~F}(000)$ & 1632 \\
\hline Sample temperature [K] & $100(2)$ & $\theta$ range $\left[{ }^{\circ}\right]$ & 1.574 to 29.044 \\
\hline Wavelength $[\AA]$ & 0.71073 & Reflections collected & 187613 \\
\hline Crystal System & Monoclinic & Unique reflections & 9067 \\
\hline \multirow[t]{2}{*}{ Space group } & $\mathrm{P} 2 / / \mathrm{n}$ & $\mathrm{R}_{\text {int }}$ & 0.0323 \\
\hline & $a=9.151(3)$ & Completeness to $\theta_{\max }[\%]$ & 100 \\
\hline \multirow[t]{2}{*}{ Unit cell dimensions $[\AA]$} & $b=24.824(3)$ & restraints/parameter & $7 / 413$ \\
\hline & $c=15.418(2)$ & GooF & 1.199 \\
\hline Volume $\left[\AA^{3}\right]$ & $3589.4(10)$ & R1 (all data) & 0.0247 \\
\hline Z & 4 & wR2 (all data) & 0.0514 \\
\hline Crystal dimensions [mm] & $0.280 \times 0.079 \times 0.068$ & max. diff. peak / hole $\left[\mathrm{e}^{-3}\right]$ & 0.568 and -0.506 \\
\hline
\end{tabular}




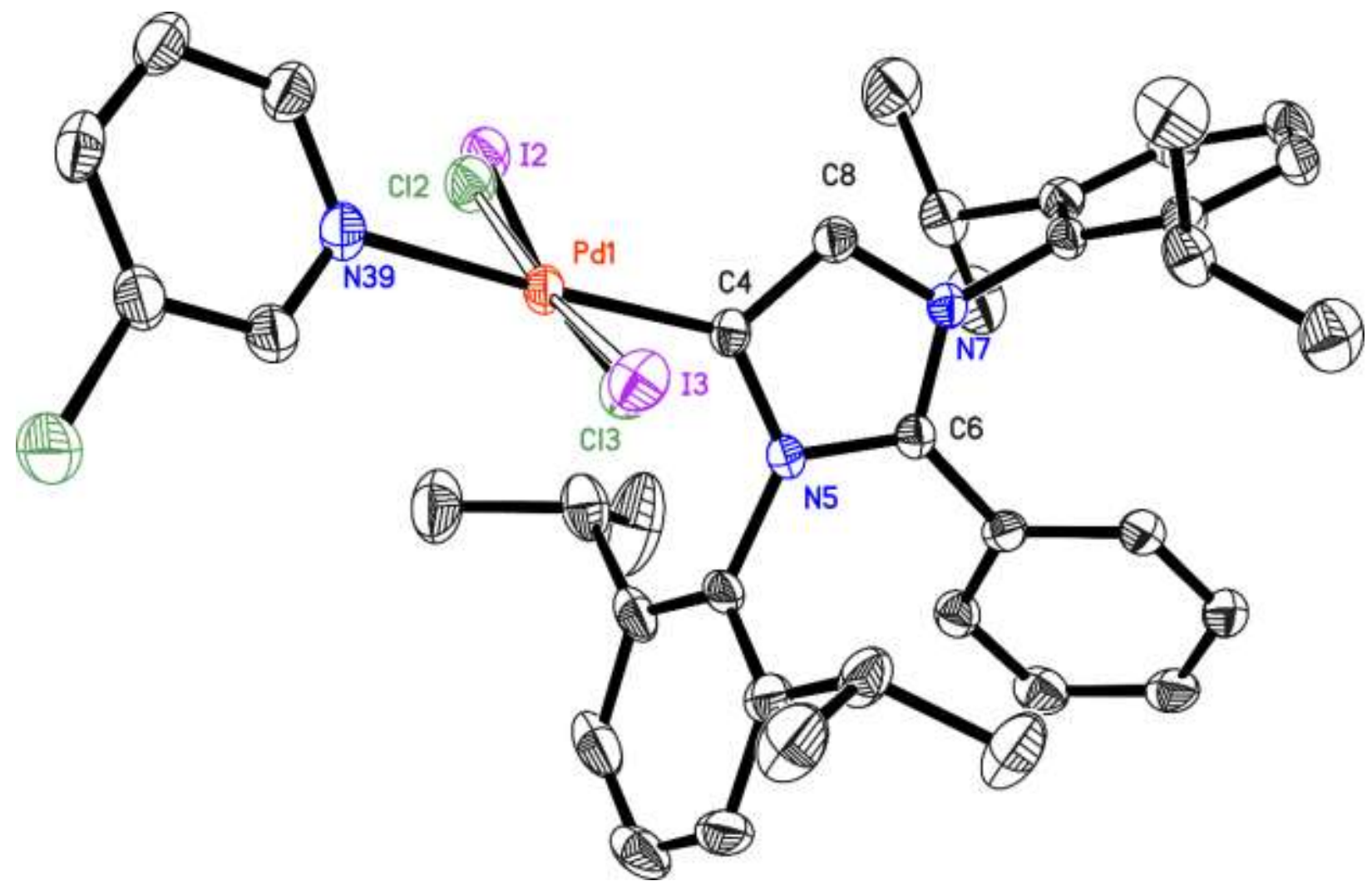

\begin{tabular}{|c|c|c|c|}
\hline Structure code & CS_RAJ_355 & CCDC Number & 1445485 \\
\hline Empirical Formula & $\mathrm{C}_{38} \mathrm{H}_{44} \mathrm{Cl}_{1.74} \mathrm{I}_{1.26} \mathrm{~N}_{3} \mathrm{Pd}$ & $\mu\left[\mathrm{mm}^{-1}\right]$ & 1.705 \\
\hline Formula weight $\left[\mathrm{g} \mathrm{mol}^{-1}\right]$ & 871.06 & $F(000)$ & 871 \\
\hline Sample temperature [K] & $100(2)$ & $\theta$ range $\left[{ }^{\circ}\right]$ & 1.154 to 26.664 \\
\hline W avelength $[\AA]$ & 0.71073 & Reflections collected & 54131 \\
\hline Crystal System & Triclinic & Unique reflections & 7826 \\
\hline \multirow[t]{2}{*}{ Space group } & $\mathrm{P} \overline{1}$ & $\mathrm{R}_{\text {int }}$ & 0.0401 \\
\hline & $a=9.2685(3)$ & Completeness to $\theta_{\max }[\%]$ & 100 \\
\hline \multirow[t]{2}{*}{ Unit cell dimensions $[\AA]$} & $b=11.811(3)$ & restraints/parameter & $6 / 422$ \\
\hline & $c=18.420(6)$ & GooF & 1.102 \\
\hline Volume $\left[\AA^{3}\right]$ & $1857.0(10)$ & R1 (all data) & 0.0343 \\
\hline Z & 2 & wR2 (all data) & 0.0641 \\
\hline Crystal dimensions [mm] & $0.278 \times 0.082 \times 0.077$ & max. diff. peak / hole $\left[\mathrm{e}^{-3}\right]$ & 0.765 and -0.549 \\
\hline
\end{tabular}




\subsubsection{Normal-to-Abnormal Rearrangement of an N-heterocyclic Carbene with a Silylene Transition Metal Complex}

The following crystal structures have been published together with reintegrated and -refined structures by Dr. Julia Matussek (Matussek, 2014 CCDC\#. 1514799, str. code: raj71) Dr. Markus Granitzka (Granitzka, 2013, CCDC\#. 1514798 and 1514801, str. code: mg_raj_962 and M G_Raj_943) in Ghadwal, R. S., Rottschäfer, D., Andrada, D. M., Frenking, G., Schürmann, C. J. \& Stammler, H.-G. 2017. Dalton Trans. 46, 7791-7799

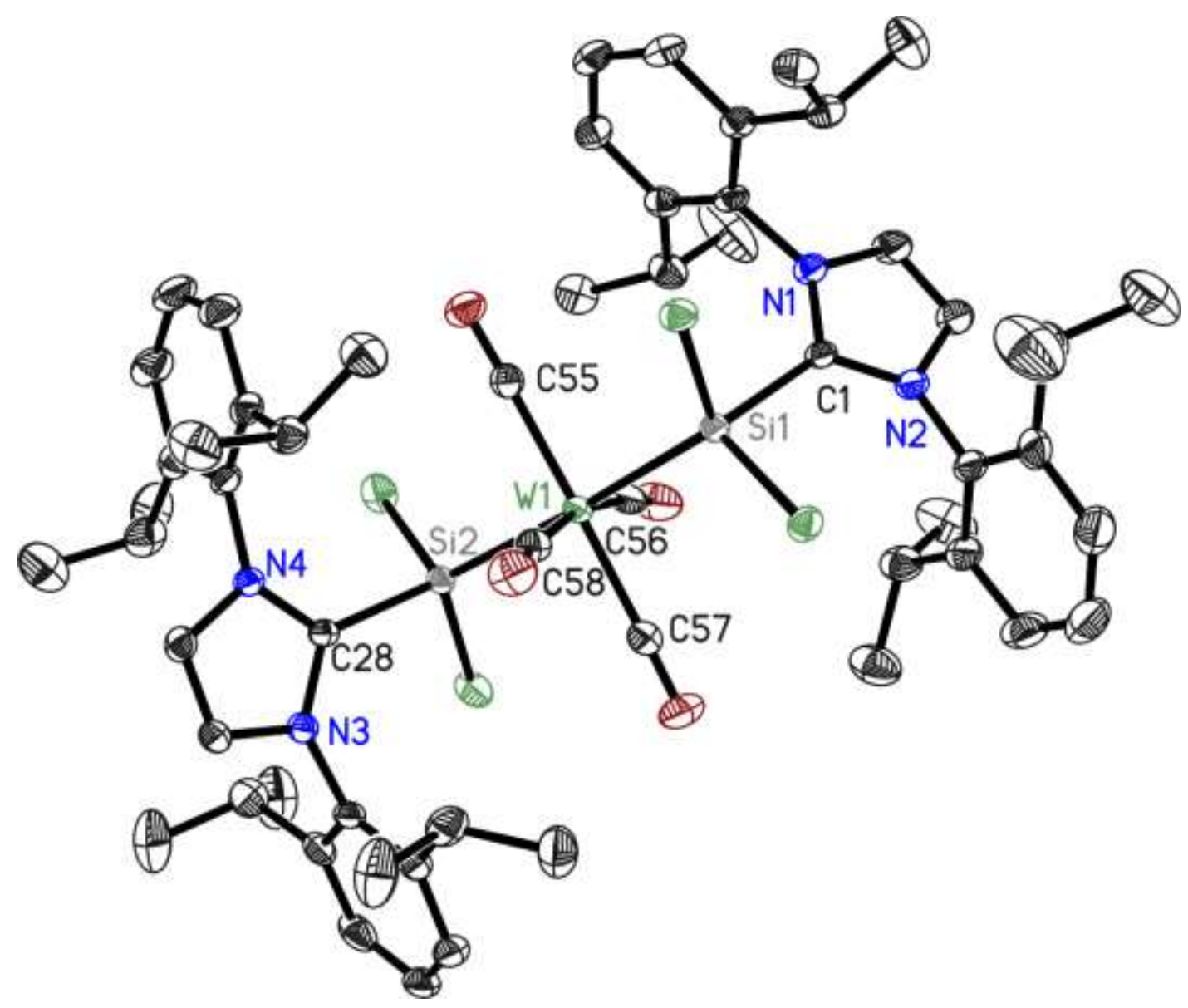

\begin{tabular}{llll}
\hline Structure code & CS_RA]_SiWCO5 & CCDC Number & 1514798 \\
Empirical Formula & $\mathrm{C}_{64} \mathrm{H}_{79} \mathrm{Cl}_{6} \mathrm{~N}_{5} \mathrm{O}_{4} \mathrm{Si}_{2} \mathrm{~W}$ & $\mu\left[\mathrm{mm}^{-1}\right]$ & 2.035 \\
Formula weight $\left[\mathrm{g} \mathrm{mol}^{-1}\right]$ & 1435.05 & $\mathrm{~F}(000)$ & 1468 \\
Sampletemperature $[\mathrm{K}]$ & $100(2)$ & $\theta$ range $\left.{ }^{\circ}\right]$ & 1.231 to 27.487 \\
Wavelength $[\AA]$ & 0.71073 & Reflections collected & 57844 \\
Crystal System & M onoclinic & Unique reflections & 15424 \\
Space group & $\mathrm{Pn}(\mathrm{Flack}=0.493(4)$, Twin) & $\mathrm{R}_{\text {int }}$ & 0.0219 \\
& $\mathrm{a}=12.696(2)$ & Completeness to $\theta_{\max }[\%]$ & 100 \\
Unit cell dimensions $[\AA]$ & $\mathrm{b}=16.539(2) \quad \beta=103.35(2)$ & restraints/parameter & $377 / 829$ \\
& $\mathrm{C}=16.499(3)$ & GooF & 1.043 \\
Volume $\left[\AA^{3}\right]$ & $3370.8(11)$ & $\mathrm{R} 1$ (all data) & 0.0196 \\
$Z$ & 2 & wR2 (all data) & 0.0447 \\
Crystal dimensions $[\mathrm{mm}]$ & $0.371 \times 0.159 \times 0.074$ & max. diff. peak / hole $\left[\mathrm{e} \AA^{-3}\right]$ & 0.765 and -0.288 \\
\hline
\end{tabular}




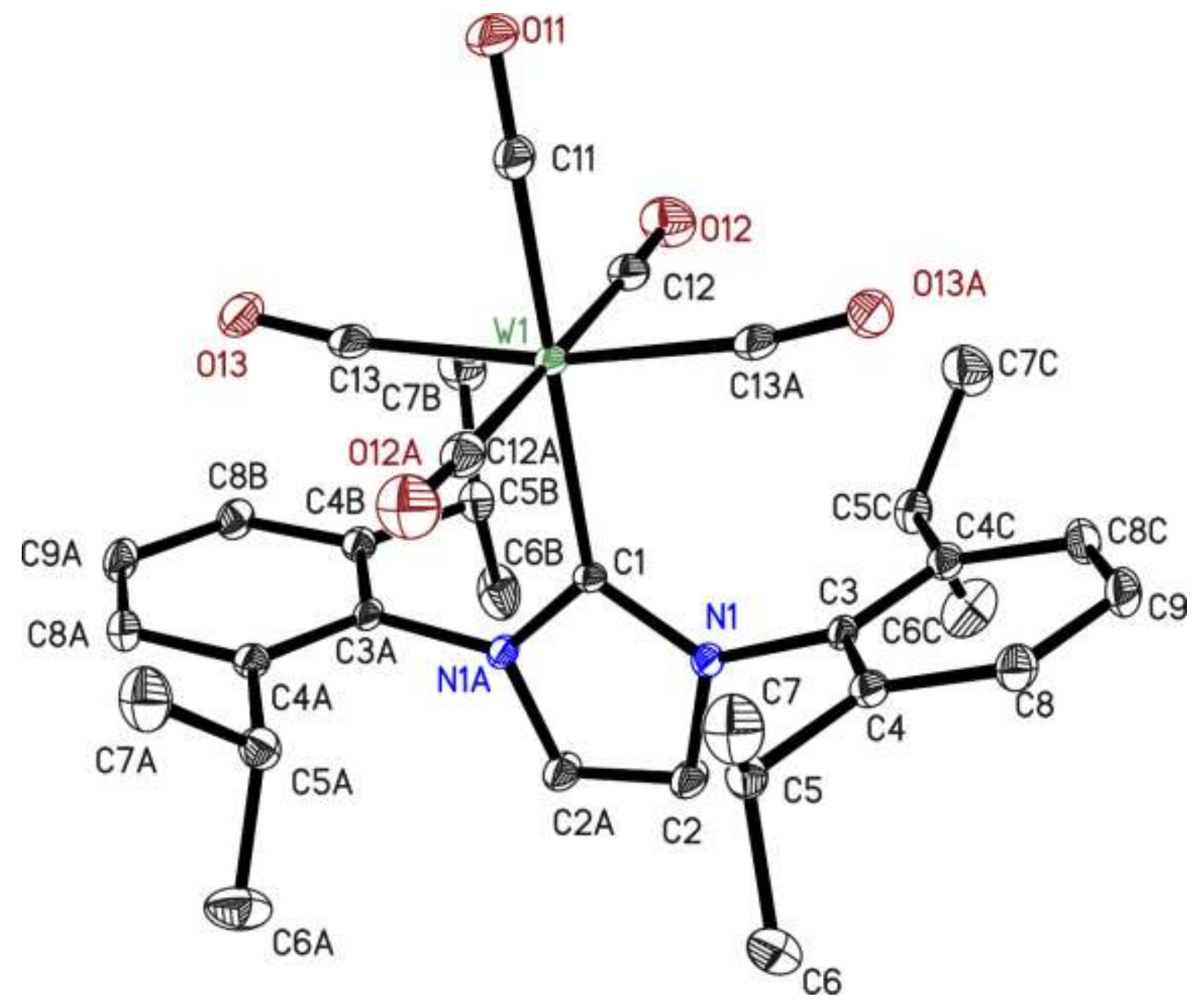

\begin{tabular}{llll}
\hline Structure code & CS_RA]_WCO5 & CCDC Number & 1514800 \\
Empirical Formula & $\mathrm{C}_{32} \mathrm{H}_{36} \mathrm{~N}_{2} \mathrm{O}_{5} \mathrm{~W}$ & $\mu\left[\mathrm{mm}^{-1}\right]$ & 2.035 \\
Formula weight $\left[\mathrm{g} \mathrm{mol}^{-1}\right]$ & 862.61 & $\mathrm{~F}(000)$ & 1424 \\
Sample temperature $[\mathrm{K}]$ & $100(2)$ & $\theta$ range $\left.{ }^{\circ}\right]$ & 1.627 to 23.659 \\
Wavelength $[\AA]$ & 0.56086 & Reflections collected & 23603 \\
Crystal System & Orthorhombic & Unique reflections & 2539 \\
Space group & $\mathrm{Cmcm}$ & $\mathrm{R}_{\text {int }}$ & 0.0290 \\
& $\mathrm{a}=11.250(2)$ & Completeness to $\theta_{\text {max }}[\%]$ & 100 \\
Unit cell dimensions $[\AA]$ & $\mathrm{b}=13.869(2)$ & restraints/parameter & $0 / 112$ \\
& $\mathrm{C}=19.759(3)$ & GooF & 1.112 \\
Volume $\left[\AA^{3}\right]$ & $3082.9(8)$ & R1 (all data) & 0.0137 \\
$Z$ & 4 & wR2 (all data) & 0.0308 \\
Crystal dimensions $[\mathrm{mm}]$ & $0.230 \times 0.224 \times 0.200$ & max. diff. peak / hole $\left[\mathrm{e} \AA^{-3}\right]$ & 0.499 and -1.841 \\
\hline
\end{tabular}




\subsubsection{Abnormal-NHC-Cobalt(II) Complexes}

The following crystal structures have been published in Ghadwal, R. S., Lamm, J.-H., Rottschäfer, D., Schürmann, C. J. \& Demeshko, S. 2017. Dalton Trans. 46, 7664-7667.

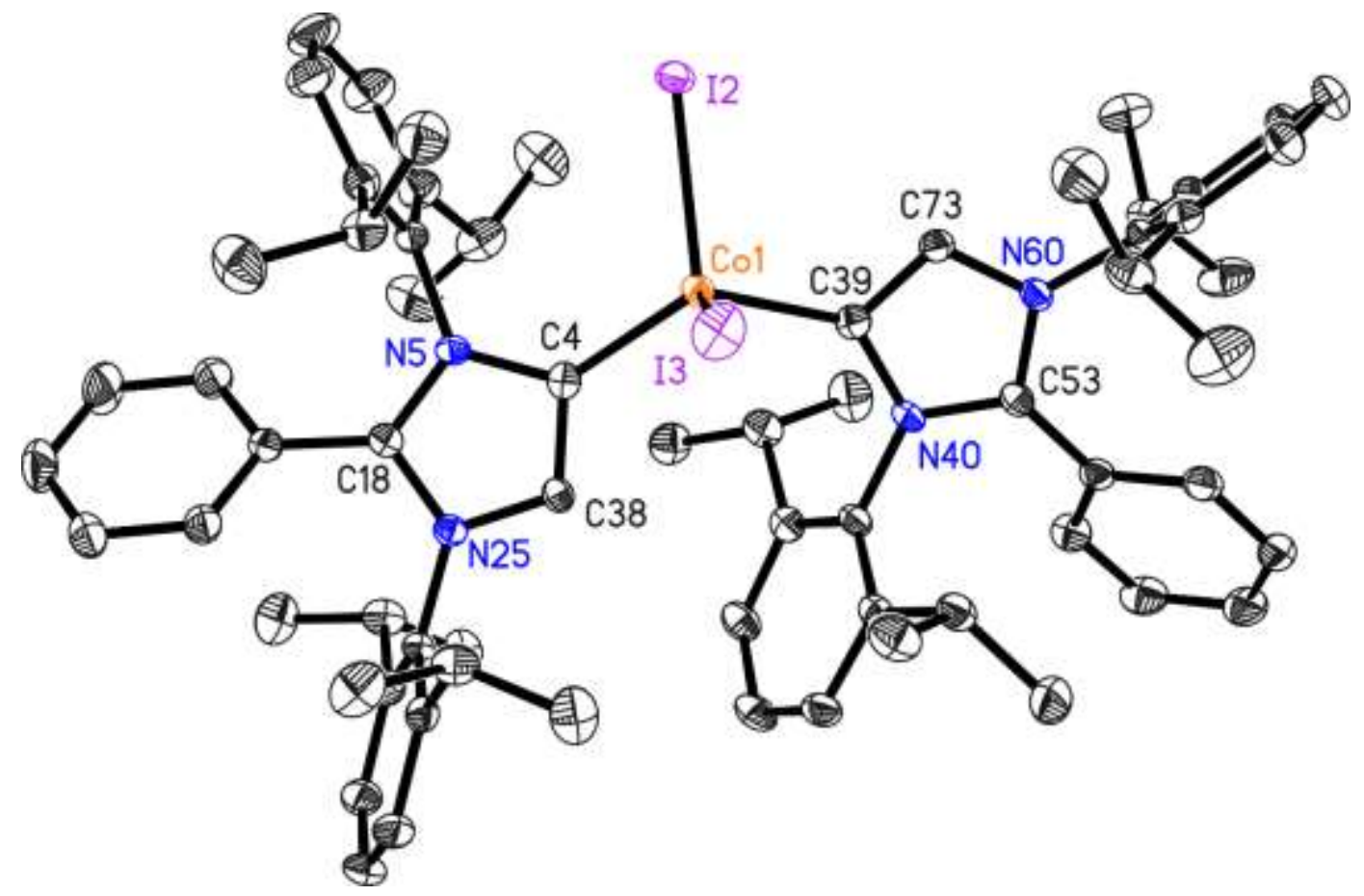

\begin{tabular}{|c|c|c|c|c|}
\hline Structure code & \multicolumn{2}{|l|}{ CS_RAJ_336 } & CCDC Number & 1503206 \\
\hline Empirical Formula & \multicolumn{2}{|c|}{$\mathrm{C}_{70.50} \mathrm{H}_{87.50} \mathrm{Col}_{2} \mathrm{~N}_{4} \mathrm{O}_{0.75}$} & $\mu\left[\mathrm{mm}^{-1}\right]$ & 0.661 \\
\hline Formula weight $\left[\mathrm{g} \mathrm{mol}^{-1}\right]$ & \multicolumn{2}{|c|}{1315.67} & $F(000)$ & 2710 \\
\hline Sample temperature [K] & \multicolumn{2}{|l|}{$100(2)$} & $\theta$ range $\left[{ }^{\circ}\right]$ & 1.540 to 21.490 \\
\hline W avelength $[\AA]$ & \multicolumn{2}{|l|}{0.56086} & Reflections collected & 292417 \\
\hline Crystal System & \multicolumn{2}{|l|}{ Triclinic } & Unique reflections & 30586 \\
\hline \multirow[t]{2}{*}{ Space group } & \multicolumn{2}{|l|}{$P \overline{1}$} & $\mathrm{R}_{\text {int }}$ & 0.0768 \\
\hline & $a=10.437(2)$ & $\alpha=90.142(2)$ & Completeness to $\theta_{\max }[\%]$ & 100 \\
\hline \multirow[t]{2}{*}{ U nit cell dimensions [ $\AA$ ] } & $b=21.065(3)$ & $\beta=91.419(2)$ & restraints/parameter & 1079 / 1644 \\
\hline & $c=30.004(3)$ & $y=90.082(2)$ & GooF & 1.033 \\
\hline Volume $\left[\AA^{3}\right]$ & $6594.5(17)$ & & R1 (all data) & 0.0535 \\
\hline Z & 4 & & wR2 (all data) & 0.0743 \\
\hline Crystal dimensions [mm] & $0.259 \times 0.088$ & 0.088 & max. diff. peak / hole $\left[\mathrm{e}^{-3}\right]$ & 0.672 and -0.682 \\
\hline
\end{tabular}




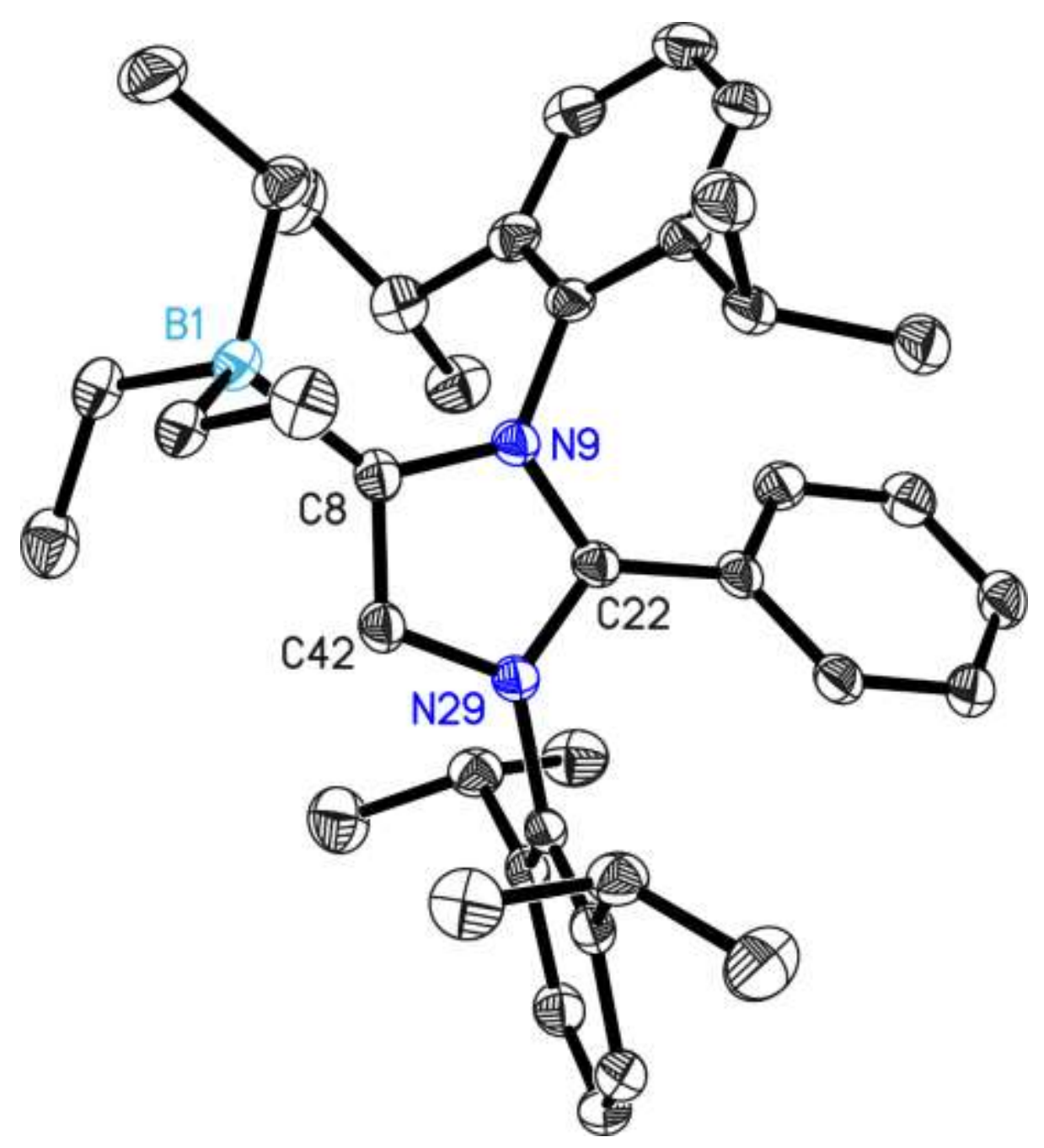

\begin{tabular}{|c|c|c|c|}
\hline Structure code & CS RS iPrB & CCDC Number & 1503204 \\
\hline Empirical Formula & $\mathrm{C}_{39.50}^{-} \mathrm{H}_{55.92}^{-} \mathrm{BCIN} 2$ & $\mu\left[\mathrm{mm}^{-1}\right]$ & 0.130 \\
\hline Formula weight $\left[\mathrm{g} \mathrm{mol}^{-1}\right]$ & 605.04 & $\mathrm{~F}(000)$ & 2631 \\
\hline Sample temperature [K] & $100(2)$ & $\theta$ range $\left[{ }^{\circ}\right]$ & 1.014 to 25.729 \\
\hline W avelength $[\AA]$ & 0.71073 & Reflections collected & 132794 \\
\hline Crystal System & Monoclinic & Unique reflections & 14184 \\
\hline \multirow[t]{2}{*}{ Space group } & $P 2_{1} / \mathrm{c}$ & $\mathrm{R}_{\text {int }}$ & 0.0390 \\
\hline & $a=21.075(2)$ & Completeness to $\theta_{\max }[\%]$ & 100 \\
\hline \multirow[t]{2}{*}{ Unit cell dimensions $[\AA]$} & $b=17.000(2)$ & restraints/parameter & $673 / 979$ \\
\hline & $c=21.788(3)$ & GooF & 1.015 \\
\hline Volume $\left[\AA^{3}\right]$ & $7436.2(17)$ & R1 (all data) & 0.0598 \\
\hline Z & 8 & wR2 (all data) & 0.1068 \\
\hline Crystal dimensions [mm] & $0.177 \times 0.156 \times 0.070$ & max. diff. peak / hole $\left[\mathrm{e}^{\AA}{ }^{-3}\right]$ & 0.352 and -0.222 \\
\hline
\end{tabular}




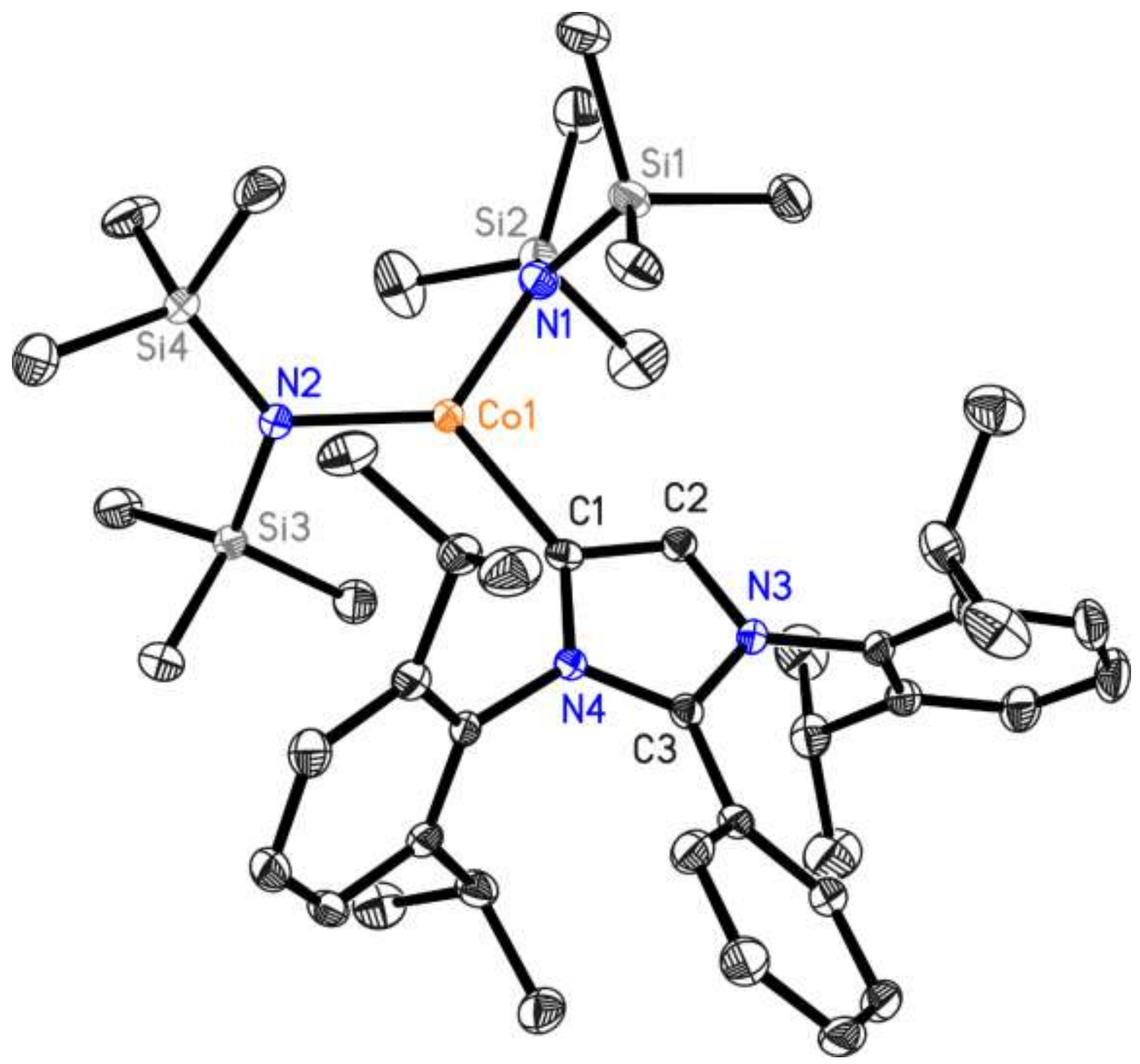

\begin{tabular}{|c|c|c|c|c|}
\hline Structure code & \multicolumn{2}{|l|}{ CS_RAJ_413 } & CCDC Number & 1503205 \\
\hline Empirical Formula & \multicolumn{2}{|l|}{$\mathrm{C}_{45} \mathrm{H}_{76} \mathrm{CoN}_{4} \mathrm{Si}_{4}$} & $\mu\left[\mathrm{mm}^{-1}\right]$ & 0.247 \\
\hline Formula weight $\left[\mathrm{g} \mathrm{mol}^{-1}\right]$ & \multicolumn{2}{|l|}{844.38} & $F(000)$ & 1828 \\
\hline Sample temperature [K ] & \multicolumn{2}{|l|}{$100(2)$} & $\theta$ range $\left[{ }^{\circ}\right]$ & 1.205 to 19.751 \\
\hline Wavelength $[\AA]$ & \multicolumn{2}{|l|}{0.56086} & Reflections collected & 156211 \\
\hline Crystal System & \multicolumn{2}{|l|}{ Triclinic } & Unique reflections & 18549 \\
\hline \multirow[t]{2}{*}{ Space group } & \multicolumn{2}{|l|}{$P \overline{1}$} & $\mathrm{R}_{\text {int }}$ & 0.0398 \\
\hline & $a=13.047(2)$ & $\alpha=85.11(2)$ & Completeness to $\theta_{\max }[\%]$ & 100 \\
\hline \multirow[t]{2}{*}{ Unit cell dimensions $[\AA]$} & $b=19.641(2)$ & $\beta=79.66(2)$ & restraints/parameter & $1098 / 1140$ \\
\hline & $c=20.141(3)$ & $y=89.90(2)$ & GooF & 1.031 \\
\hline Volume $\left[\AA^{3}\right]$ & $5058.5(12)$ & & R1 (all data) & 0.0406 \\
\hline Z & 4 & & wR2 (all data) & 0.0769 \\
\hline Crystal dimensions [mm] & $0.364 \times 0.325$ & 0.162 & max. diff. peak / hole $\left[\mathrm{e}^{-3}\right]$ & 0.316 and -0.300 \\
\hline
\end{tabular}




\subsubsection{Normal- and A bnormal- N-H eterocyclic Carbene (NHC) Magnesium Compounds}

The following crystal structure has been published, together with two re-integrated and re refined crystal structures by Sebastian M erkel (M erkel, 2009, CCDC\#1500885/6, structure code: Inder1/2).

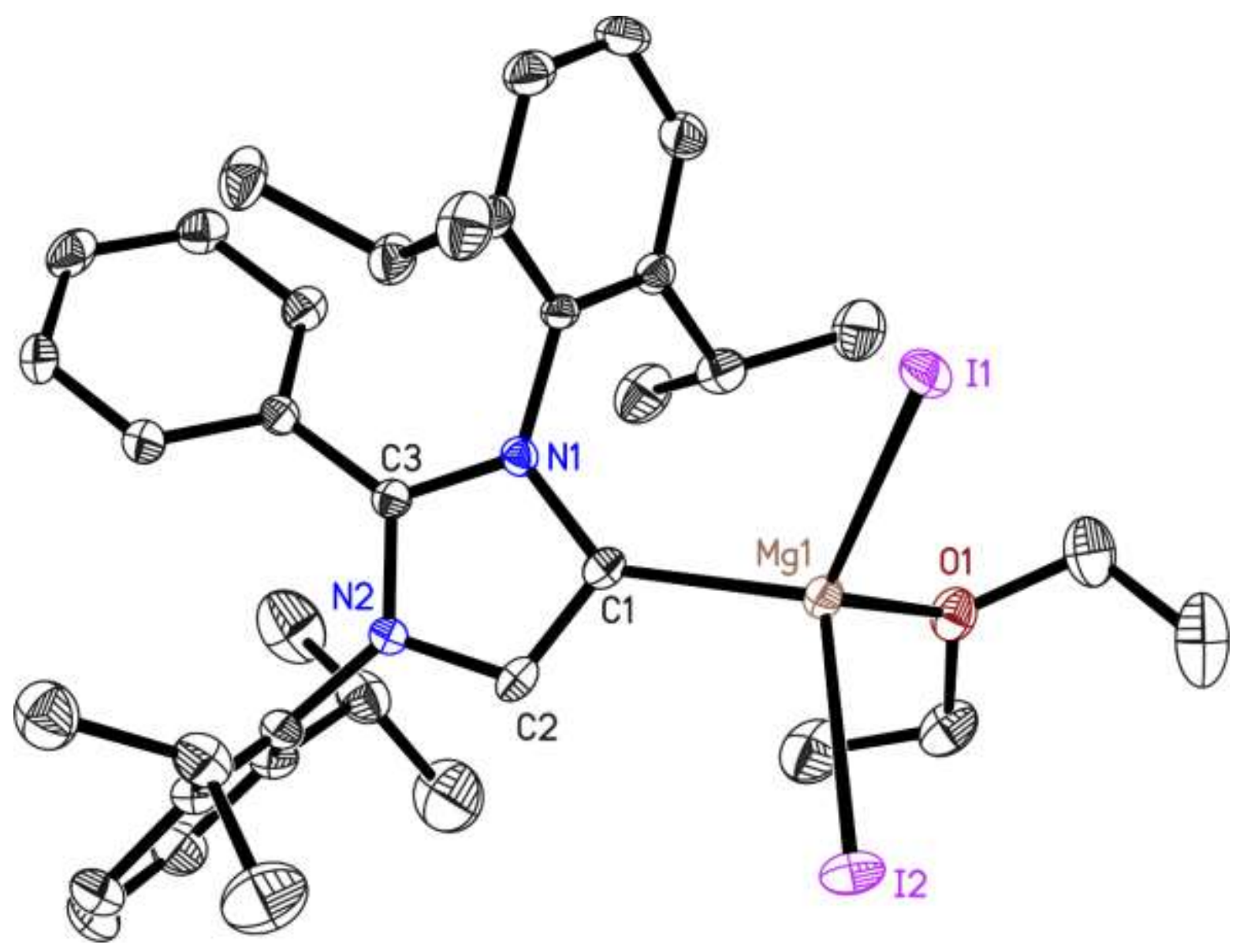

\begin{tabular}{llll}
\hline Structure code & CS_RA]_413 & CCDC Number & 1500887 \\
Empirical Formula & $\mathrm{C}_{43.75} \mathrm{H}_{57.42} l_{2.06} \mathrm{M} \mathrm{gN}_{2} \mathrm{O}$ & $\mu\left[\mathrm{mm}^{-1}\right]$ & 0.805 \\
Formula weight $\left[\mathrm{g} \mathrm{mol}^{-1}\right]$ & 913.54 & $\mathrm{~F}(000)$ & 3706 \\
Sampletemperature $[\mathrm{K}]$ & $100(2)$ & $\theta$ range $\left.{ }^{\circ}\right]$ & 1.486 to 19.784 \\
Wavelength $[\AA]$ & 0.56086 & Reflections collected & 160636 \\
Crystal System & Orthorhombic & Unique reflections & 8159 \\
Space group & $\mathrm{Pbca}$ & $\mathrm{R}_{\text {int }}$ & 0.0695 \\
& $\mathrm{a}=18.390(2)$ & Completeness to $\theta_{\max }[\%]$ & 100 \\
Unit cell dimensions $[\AA]$ & $\mathrm{b}=14.613(2)$ & restraints/parameter & $123 / 508$ \\
& $\mathrm{C}=33.027(3)$ & GooF & 1.031 \\
Volume $\left[\AA^{3}\right]$ & $8875.4(17)$ & $\mathrm{R} 1$ (all data) & 0.0461 \\
$Z$ & 8 & wR2 (all data) & 0.0650 \\
Crystal dimensions $[\mathrm{mm}]$ & $0.214 \times 0.203 \times 0.200$ & max. diff. peak / hole $\left[\mathrm{e} \AA^{-3}\right]$ & 0.404 and -0.493 \\
\hline
\end{tabular}




\subsubsection{Unpublished Crystal Structures}

The following crystal structures have been collected, integrated, refined, and prepared for publication, but are currently still unpublished.

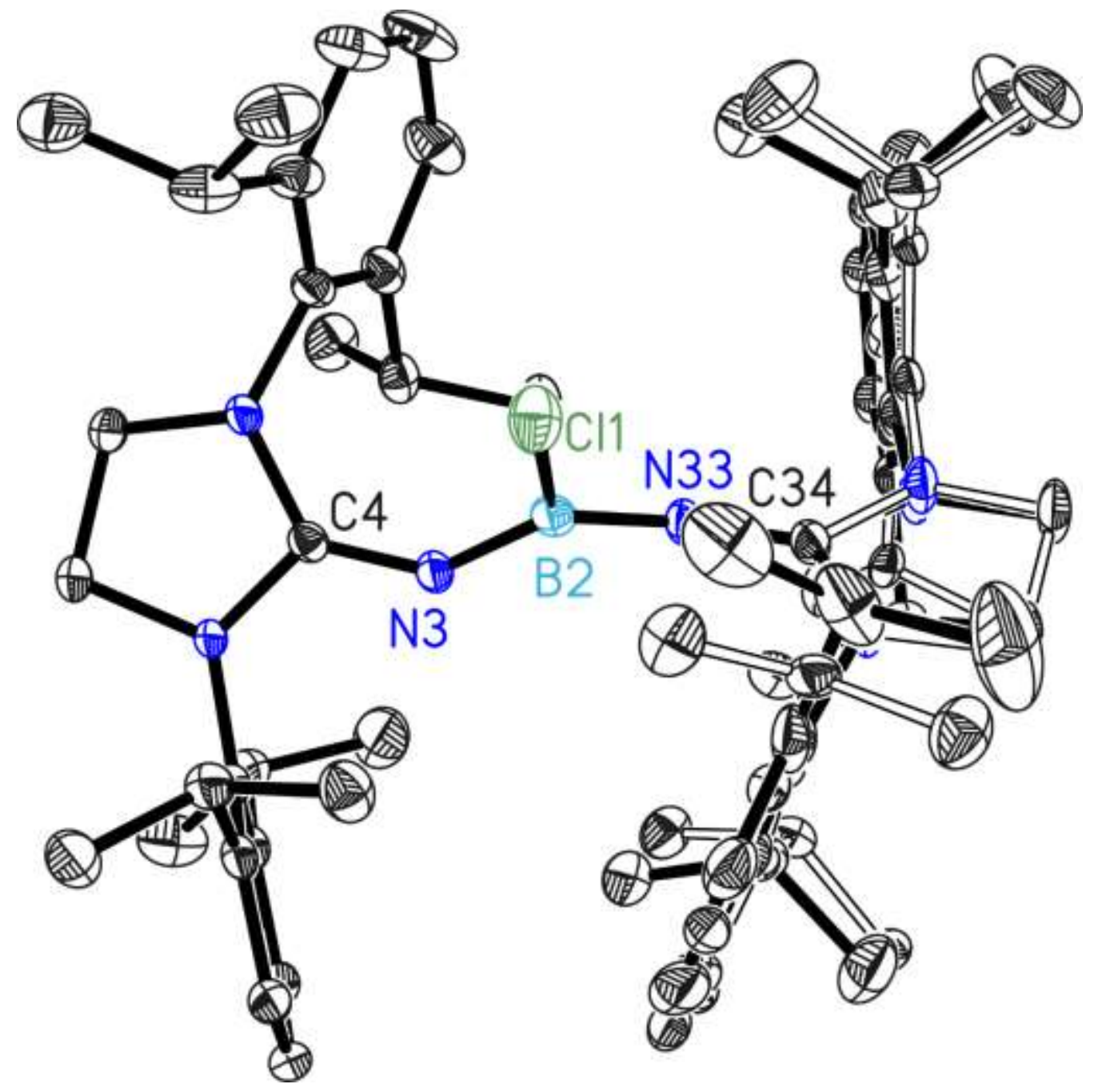

\begin{tabular}{|c|c|c|c|}
\hline Structure code & CS_RAJ_264_Pnsol & $\mu\left[\mathrm{mm}^{-1}\right]$ & 0.117 \\
\hline Empirical Formula & $\mathrm{C}_{55} \mathrm{H}_{78} \mathrm{BCl}_{3} \mathrm{~N}_{6}$ & $F(000)$ & 2024 \\
\hline Formula weight $\left[\mathrm{g} \mathrm{mol}^{-1}\right]$ & 940.39 & $\theta$ range $\left[{ }^{\circ}\right]$ & 1.515 to 19.607 \\
\hline Sample temperature [K] & $100(2)$ & Reflections collected & 260884 \\
\hline W avelength $[\AA]$ & 0.56086 & Unique reflections & 9545 \\
\hline Crystal System & Monoclinic & $\mathrm{R}_{\text {int }}$ & 0.0853 \\
\hline Space group & $\begin{array}{l}P 2_{1} / c \\
a=20.745(2)\end{array}$ & $\begin{array}{l}\text { Completeness to } \theta_{\max }[\%] \\
\text { restraints/parameter }\end{array}$ & $\begin{array}{l}99.6 \\
2801 / 841\end{array}$ \\
\hline Unit cell dimensions $[\AA]$ & $\begin{array}{l}\mathrm{b}=12.315(2) \quad \beta=96.05(2) \\
\mathrm{c}=21.012(3)\end{array}$ & $\begin{array}{l}\text { GooF } \\
\text { R1 (all data) }\end{array}$ & $\begin{array}{l}1.066 \\
0.0649\end{array}$ \\
\hline Volume $\left[\AA^{3}\right]$ & $5338.1(13)$ & wR2 (all data) & 0.1005 \\
\hline Z & 4 & max. diff. peak / hole $\left[\mathrm{e}^{-3}\right]$ & 0.292 and -0.485 \\
\hline
\end{tabular}




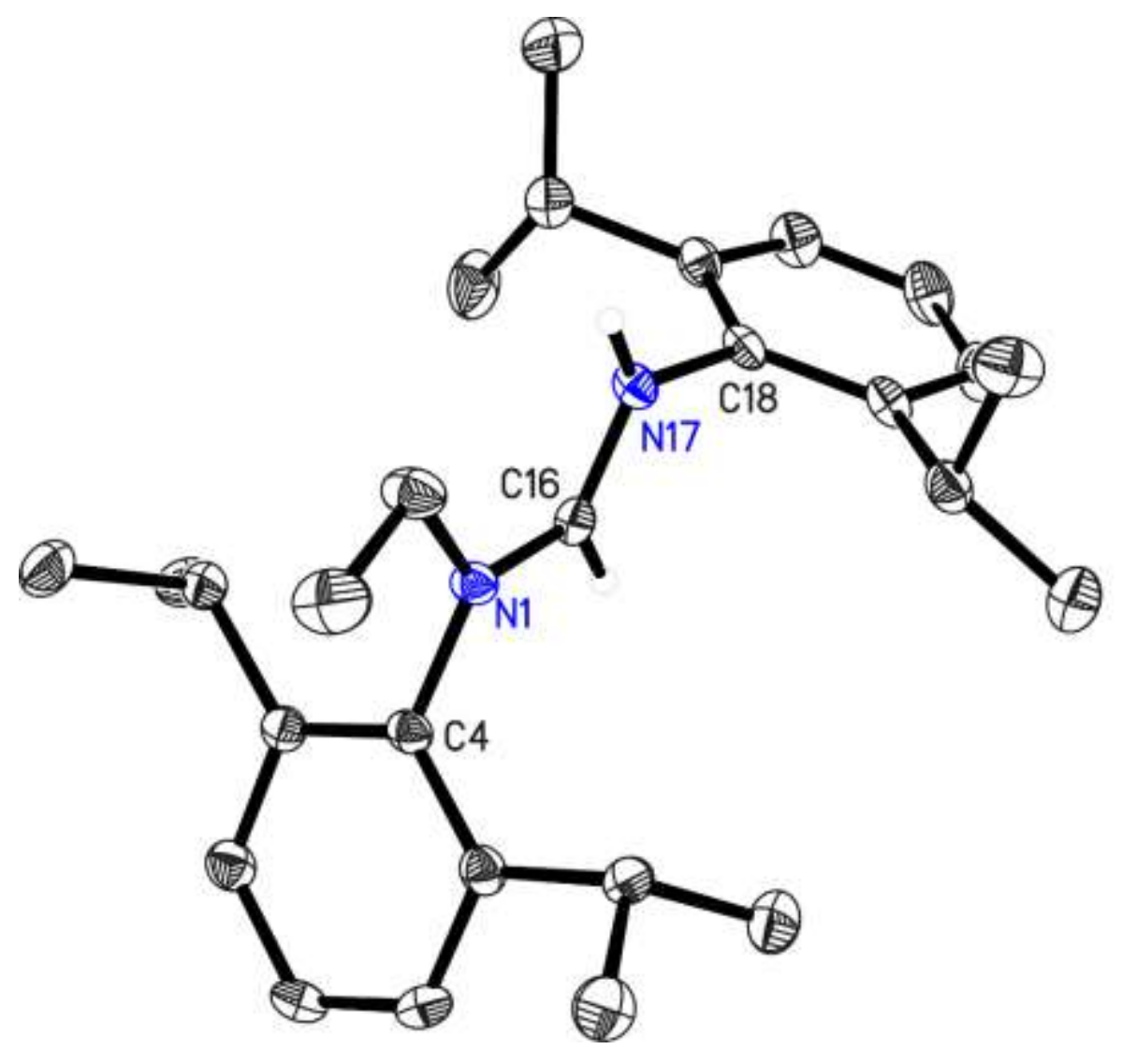

\begin{tabular}{|c|c|c|c|}
\hline Structure code & CS_RAJ_319 & $\mu\left[\mathrm{mm}^{-1}\right]$ & 0.555 \\
\hline Empirical Formula & $\mathrm{C}_{34} \mathrm{H}_{49} \mathrm{IN}_{2}$ & $F(000)$ & 1280 \\
\hline Formula weight $\left[\mathrm{g} \mathrm{mol}^{-1}\right]$ & 612.65 & $\theta$ range $\left[{ }^{\circ}\right]$ & 1.740 to 20.550 \\
\hline Sample temperature [K] & $100(2)$ & Reflections collected & 61205 \\
\hline W avelength $[\AA]$ & 0.56086 & Unique reflections & 6497 \\
\hline Crystal System & Monoclinic & $\mathrm{R}_{\text {int }}$ & 0.0755 \\
\hline Space group & $\begin{array}{l}P 2_{1} / \mathrm{c} \\
a=11.857(2)\end{array}$ & $\begin{array}{l}\text { Completeness to } \theta_{\max }[\%] \\
\text { restraints/parameter }\end{array}$ & $\begin{array}{l}100 \\
763 / 472\end{array}$ \\
\hline Unit cell dimensions $[\AA]$ & $\begin{array}{l}b=15.867(2) \quad \beta=106.62(2) \\
c=17.583(3)\end{array}$ & $\begin{array}{l}\text { GooF } \\
\text { R1 (all data) }\end{array}$ & $\begin{array}{l}1.036 \\
0.0506\end{array}$ \\
\hline Volume $\left[\AA^{3}\right]$ & $3169.8(9)$ & wR2 (all data) & 0.0753 \\
\hline Z & 4 & max. diff. peak / hole $\left[e^{-3}{ }^{-3}\right]$ & 0.711 and -0.549 \\
\hline
\end{tabular}




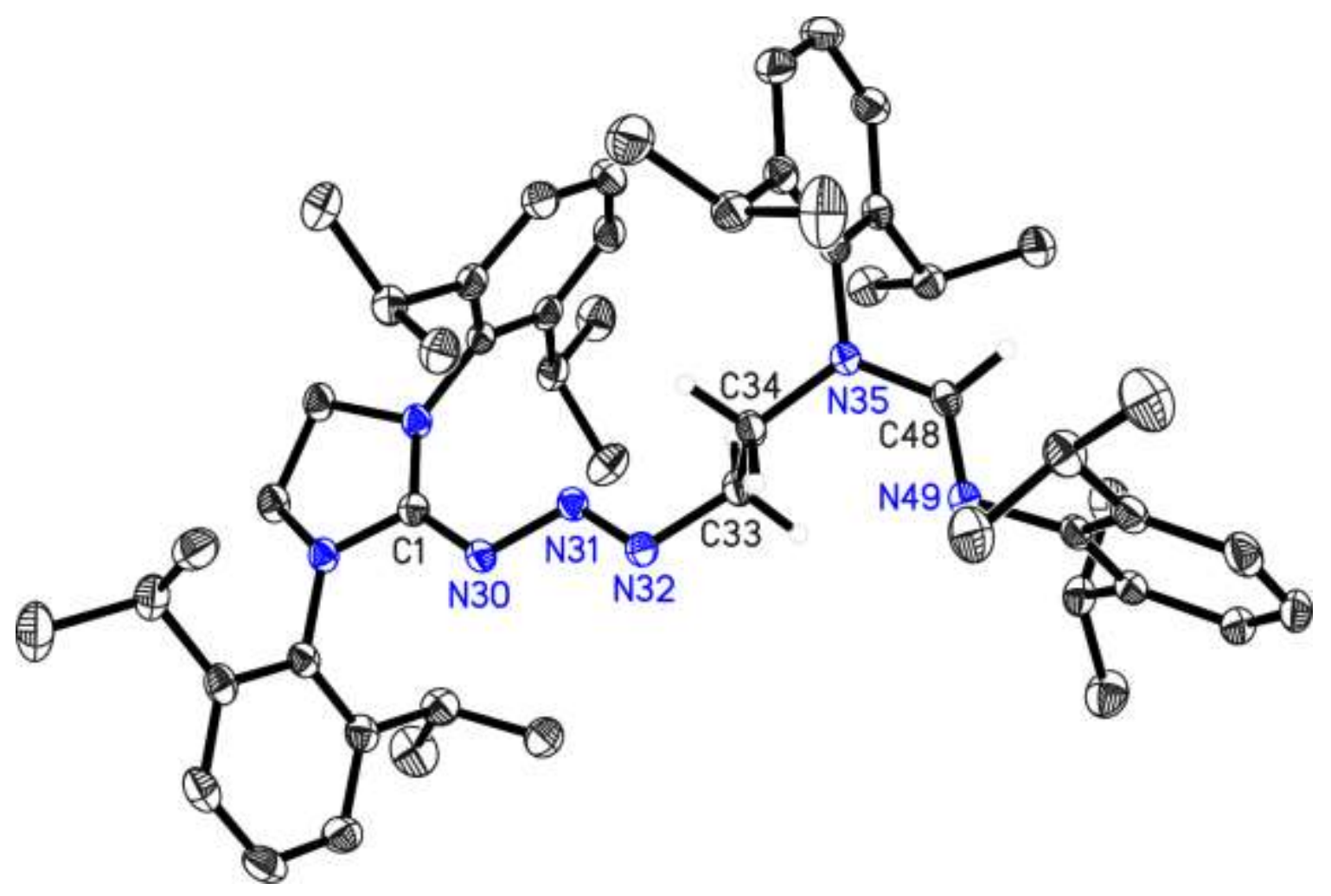

\begin{tabular}{|c|c|c|c|}
\hline Structure code & CS_RAJ_361b & & \\
\hline Empirical Formula & $\mathrm{C}_{54} \overline{\mathrm{H}}_{77} \mathrm{~N}_{7}$ & $\mu\left[\mathrm{mm}^{-1}\right]$ & 0.065 \\
\hline Formula weight $\left[\mathrm{g} \mathrm{mol}^{-1}\right]$ & 824.22 & $\mathrm{~F}(000)$ & 1800 \\
\hline Sample temperature [K] & $100(2)$ & $\theta$ range $\left[{ }^{\circ}\right]$ & 1.361 to 26.362 \\
\hline W avelength $[\AA]$ & 0.71073 & Reflections collected & 78477 \\
\hline Crystal System & Monoclinic & Unique reflections & 10084 \\
\hline \multirow[t]{2}{*}{ Space group } & $\mathrm{P} 2_{1} / \mathrm{C}$ & $\mathrm{R}_{\text {int }}$ & 0.0296 \\
\hline & $a=13.247(2)$ & Completeness to $\theta_{\max }[\%]$ & 99.9 \\
\hline \multirow[t]{2}{*}{ Unit cell dimensions $[\AA]$} & $b=29.933(3)$ & restraints/parameter & $0 / 567$ \\
\hline & $c=12.658(2)$ & GooF & 1.025 \\
\hline Volume $\left[\AA^{3}\right]$ & $4944.0(12)$ & R1 (all data) & 0.0500 \\
\hline Z & 4 & wR2 (all data) & 0.0986 \\
\hline Crystal dimensions [mm] & $0.317 \times 0.201 \times 0.148$ & max. diff. peak / hole $\left[\mathrm{e}^{-3}\right]$ & 0.236 and -0.185 \\
\hline
\end{tabular}




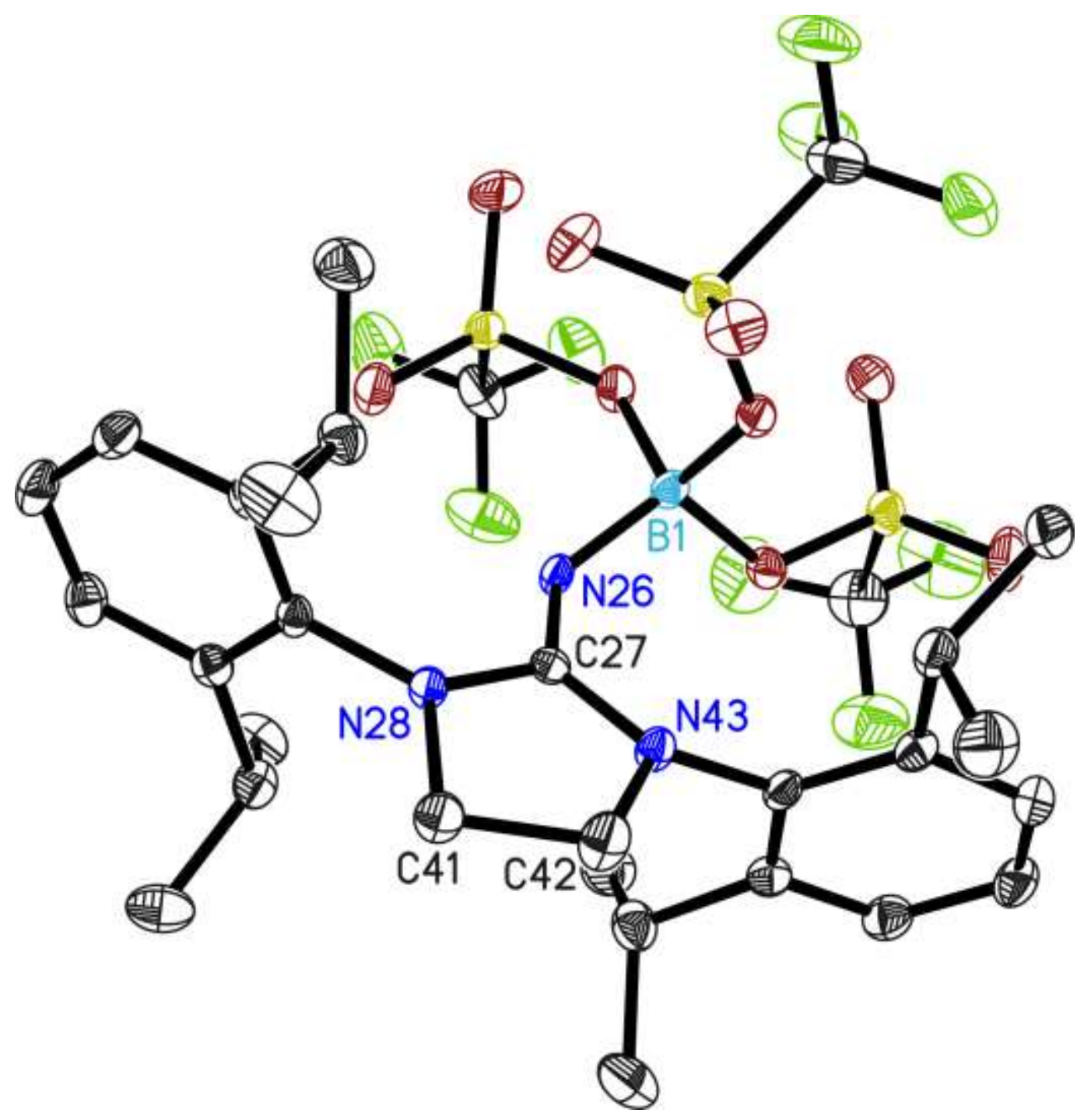

\begin{tabular}{|c|c|c|c|c|}
\hline Structure code & \multicolumn{2}{|c|}{ CS_RAJ_367b_Mo } & $\mu\left[\mathrm{mm}^{-1}\right]$ & 0.279 \\
\hline Empirical Formula & \multicolumn{2}{|c|}{$\mathrm{C}_{33} \overline{\mathrm{H}}_{41} \mathrm{BF}_{9.50} \mathrm{~N}_{3} \overline{\mathrm{O}}_{9} \mathrm{~S}_{3}$} & $F(000)$ & 941 \\
\hline Formula weight [ $\left.\mathrm{g} \mathrm{mol}^{-1}\right]$ & \multicolumn{2}{|c|}{911.18} & $\theta$ range $\left[{ }^{\circ}\right]$ & 1.036 to 26.391 \\
\hline Sample temperature [K] & \multicolumn{2}{|l|}{$100(2)$} & Reflections collected & 59460 \\
\hline W avelength $[\AA]$ & \multicolumn{2}{|l|}{0.71073} & Unique reflections & 8332 \\
\hline Crystal System & \multicolumn{2}{|l|}{ Triclinic } & $\mathrm{R}_{\text {int }}$ & 0.0379 \\
\hline \multirow[t]{2}{*}{ Space group } & \multicolumn{2}{|l|}{$\mathrm{P} \overline{1}$} & Completeness to $\theta_{\max }[\%]$ & 100 \\
\hline & $a=10.495(2)$ & $\alpha=81.83(2)$ & restraints/parameter & $0 / 544$ \\
\hline \multirow[t]{2}{*}{ Unit cell dimensions $[\AA]$} & $b=10.518(2)$ & $\beta=89.61(3)$ & GooF & 1.022 \\
\hline & $c=19.872(3)$ & $y=70.00(2)$ & R1 (all data) & 0.0465 \\
\hline V olume $\left[\AA^{3}\right]$ & \multicolumn{2}{|l|}{$2038.3(7)$} & wR2 (all data) & 0.0852 \\
\hline Z & \multicolumn{2}{|l|}{2} & max. diff. peak / hole $\left[\mathrm{e}^{-3}\right]$ & 0.382 and -0.392 \\
\hline
\end{tabular}




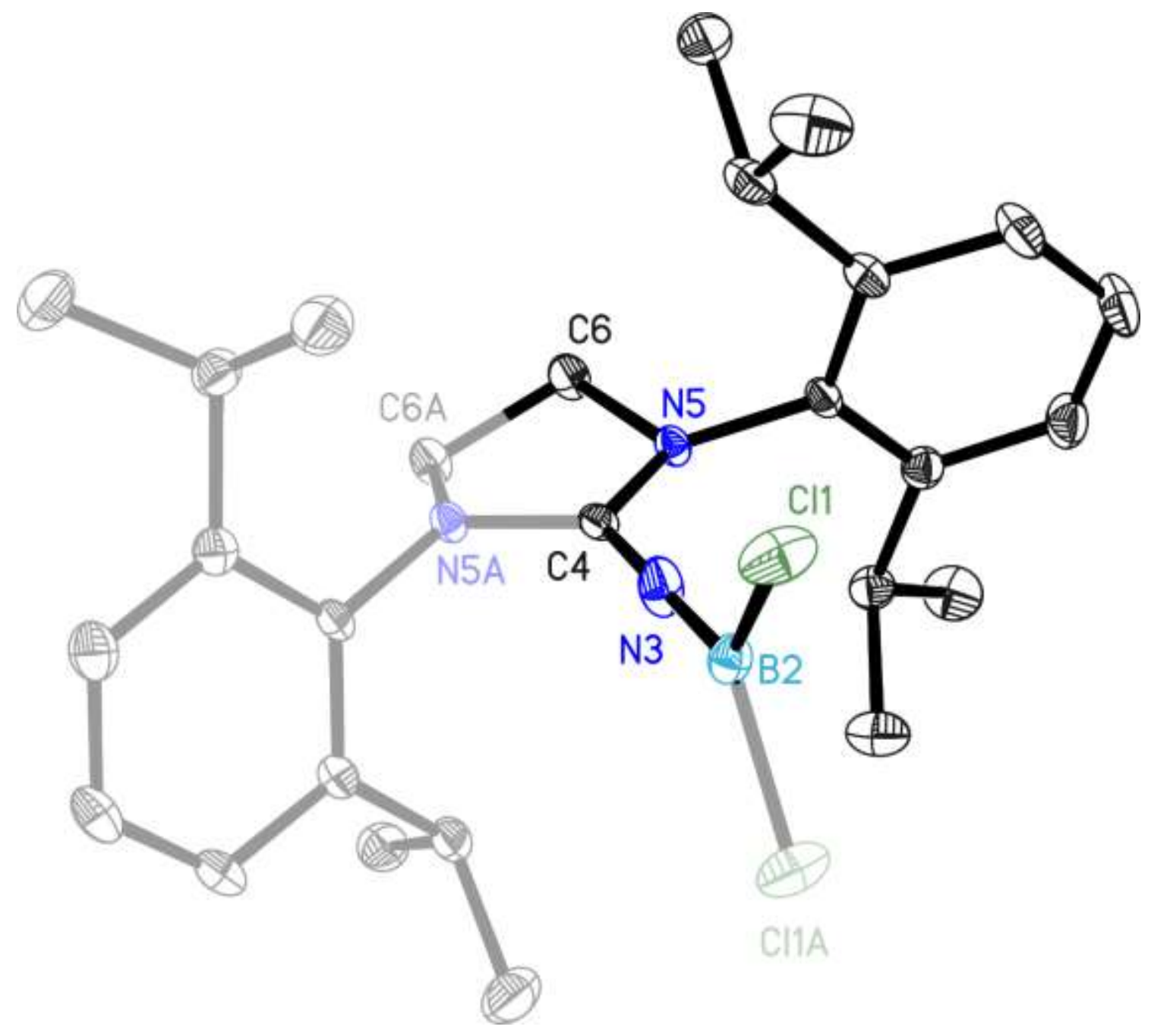

\begin{tabular}{|c|c|c|c|}
\hline Structure code & CS_RAJ_370 & & \\
\hline Empirical Formula & $\mathrm{C}_{27} \mathrm{H}_{38} \mathrm{BCl}_{2} \mathrm{~N}_{3}$ & $\mu\left[\mathrm{mm}^{-1}\right]$ & 0.139 \\
\hline Formula weight $\left[\mathrm{g} \mathrm{mol}^{-1}\right]$ & 486.31 & $F(000)$ & 1040 \\
\hline Sample temperature [K] & $100(2)$ & $\theta$ range $\left[{ }^{\circ}\right]$ & 2.573 to 23.304 \\
\hline W avelength $[\AA]$ & 0.56086 & Reflections collected & 38882 \\
\hline Crystal System & Monoclinic & Unique reflections & 3989 \\
\hline \multirow[t]{2}{*}{ Space group } & $\mathrm{C} 2 / \mathrm{c}$ & $\mathrm{R}_{\text {int }}$ & 0.0470 \\
\hline & $a=17.175(2)$ & Completeness to $\theta_{\max }[\%]$ & 99.8 \\
\hline \multirow[t]{2}{*}{ Unit cell dimensions $[\AA]$} & $b=8.969(2)$ & restraints/parameter & $0 / 155$ \\
\hline & $c=17.630(3)$ & GooF & 1.037 \\
\hline Volume $\left[\AA^{3}\right]$ & $2714.5(8)$ & R1 (all data) & 0.0457 \\
\hline Z & 4 & wR2 (all data) & 0.0958 \\
\hline Crystal dimensions [mm] & $0.232 \times 0.171 \times 0.146$ & max. diff. peak / hole $\left[\mathrm{e}^{-3}\right]$ & 0.430 and -0.468 \\
\hline
\end{tabular}




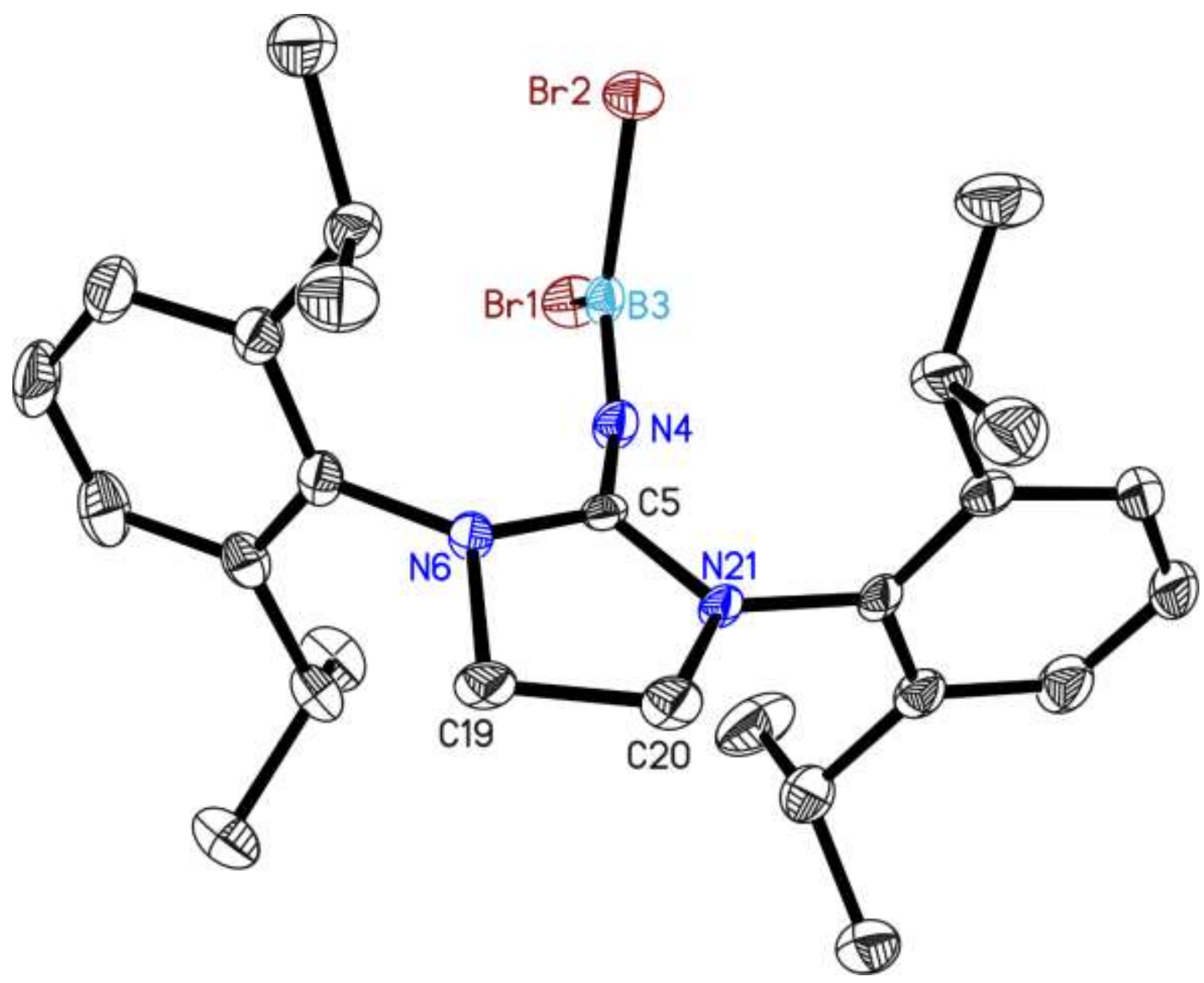

\begin{tabular}{|c|c|c|c|}
\hline Structure code & CS_RAJ_371 & & \\
\hline Empirical Formula & $\mathrm{C}_{27.33} \mathrm{H}_{38.67} \mathrm{BBr}_{2} \mathrm{Cl}_{0.67} \mathrm{~N}_{3}$ & $\mu\left[\mathrm{mm}^{-1}\right]$ & 1.552 \\
\hline Formula weight $\left[\mathrm{g} \mathrm{mol}^{-1}\right]$ & 603.54 & $F(000)$ & 3720 \\
\hline Sample temperature [K] & $100(2)$ & $\theta$ range $\left[{ }^{\circ}\right]$ & 2.214 to 19.646 \\
\hline W avelength $[\AA]$ & 0.56086 & Reflections collected & 166981 \\
\hline Crystal System & Monoclinic & Unique reflections & 15522 \\
\hline \multirow[t]{2}{*}{ Space group } & $\mathrm{P} 2 \mathrm{I} / \mathrm{C}$ & $\mathrm{R}_{\text {int }}$ & 0.0837 \\
\hline & $a=27.407(3)$ & Completeness to $\theta_{\max }[\%]$ & 99.4 \\
\hline \multirow[t]{2}{*}{ Unit cell dimensions $[\AA]$} & $b=17.209(2)$ & restraints/parameter & $0 / 943$ \\
\hline & $c=18.591(2)$ & GooF & 1.037 \\
\hline Volume $\left[\AA^{3}\right]$ & $8657.3(17)$ & R1 (all data) & 0.0639 \\
\hline Z & 12 & wR2 (all data) & 0.0943 \\
\hline Crystal dimensions [mm] & $0.251 \times 0.160 \times 0.046$ & max. diff. peak / hole $\left[\mathrm{e}^{-3}\right]$ & 0.746 and -0.675 \\
\hline
\end{tabular}




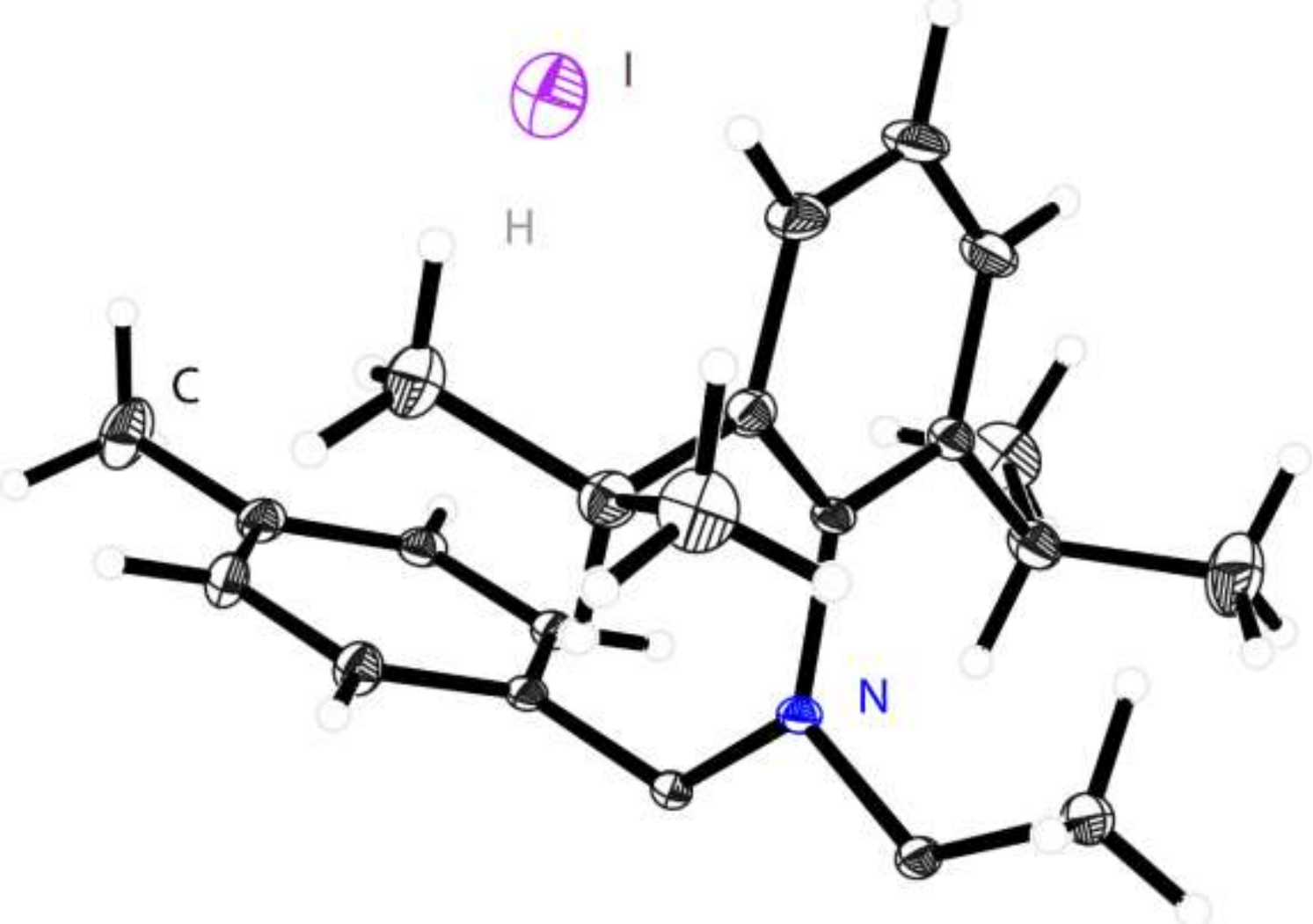

\begin{tabular}{|c|c|c|c|}
\hline Structure code & CS_RAJ_309 & & \\
\hline Empirical Formula & $\mathrm{C}_{36} \mathrm{H}_{47} \mathrm{~N}_{2}$ & $\mu\left[\mathrm{mm}^{-1}\right]$ & 0.550 \\
\hline Formula weight $\left[\mathrm{g} \mathrm{mol}^{-1}\right]$ & 634.65 & $F(000)$ & 660 \\
\hline Sample temperature [K] & $100(2)$ & $\theta$ range $\left[{ }^{\circ}\right]$ & 1.713 to 23.679 \\
\hline Wavelength $[\AA]$ & 0.56086 & Reflections collected & 47287 \\
\hline Crystal System & Monoclinic & Unique reflections & 5081 \\
\hline \multirow[t]{2}{*}{ Space group } & $\mathrm{P} 2 \mathrm{l} / \mathrm{C}$ & $\mathrm{R}_{\text {int }}$ & 0.0522 \\
\hline & $a=9.897(2)$ & Completeness to $\theta_{\max }[\%]$ & 100 \\
\hline \multirow[t]{2}{*}{ Unit cell dimensions $[\AA]$} & $b=17.276(3)$ & restraints/parameter & $0 / 196$ \\
\hline & $c=10.122(2)$ & GooF & 1.049 \\
\hline Volume $\left[\AA^{3}\right]$ & $1604.2(6)$ & R1 (all data) & 0.0357 \\
\hline Z & 2 & wR2 (all data) & 0.0640 \\
\hline Crystal dimensions [mm] & $0.112 \times 0.092 \times 0.087$ & max. diff. peak / hole $\left[\mathrm{e}^{-3}\right]$ & 0.866 and -0.471 \\
\hline
\end{tabular}




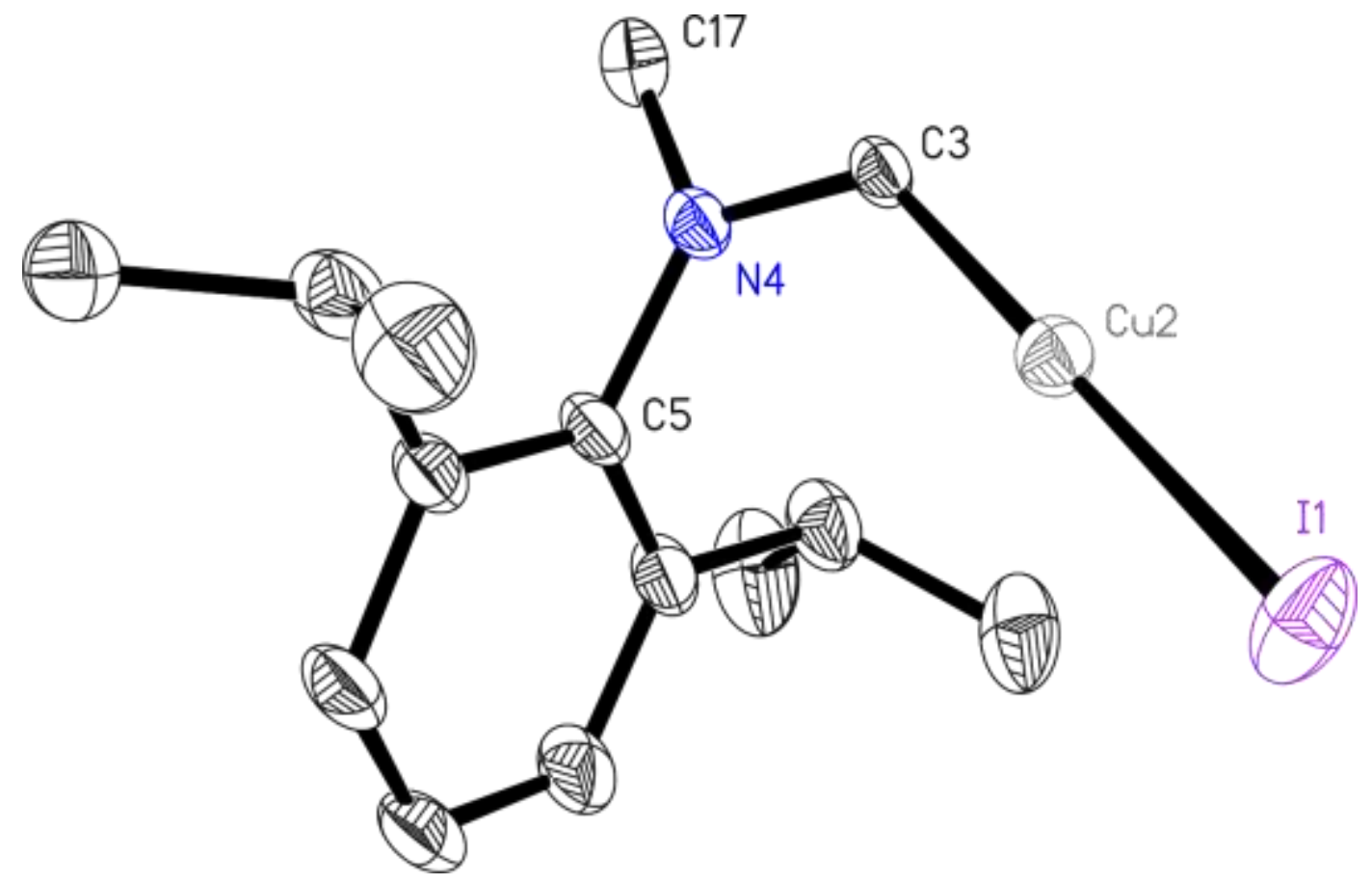

\begin{tabular}{llll}
\hline Structure code & CS_RA]_329 & & \\
Empirical Formula & $\mathrm{C}_{27} \mathrm{H}_{36} \mathrm{CuIIN}_{2}$ & $\mu\left[\mathrm{mm}^{-1}\right]$ & 1.912 \\
Formula weight $\left[\mathrm{g} \mathrm{mol}^{-1}\right]$ & 579.02 & $\mathrm{~F}(000)$ & 1176 \\
Sampletemperature $[\mathrm{K}]$ & $100(2)$ & $\theta$ range $\left.{ }^{\circ}\right]$ & 2.021 to 26.420 \\
Wavelength $[\AA]$ & 0.71073 & Reflections collected & 26740 \\
Crystal System & Orthorhombic & Unique reflections & 2866 \\
Space group & $\mathrm{Pccn}$ & $\mathrm{R}_{\text {int }}$ & 0.0583 \\
& $\mathrm{a}=10.889(2)$ & Completeness to $\theta_{\max }[\%]$ & 100 \\
Unit cell dimensions $[\AA]$ & $\mathrm{b}=12.663(2)$ & restraints/parameter & $0 / 146$ \\
& $\mathrm{C}=20.156(3)$ & GooF & 1.019 \\
Volume $\left[\AA^{3}\right]$ & $2779.3(6)$ & $\mathrm{R} 1$ (all data) & 0.0611 \\
$Z$ & 4 & wR2 (all data) & 0.0833 \\
Crystal dimensions $[\mathrm{mm}]$ & $0.129 \times 0.069 \times 0.024$ & max. diff. peak / hole $\left[\mathrm{e} \AA^{-3}\right]$ & 0.904 and -0.931 \\
\hline
\end{tabular}




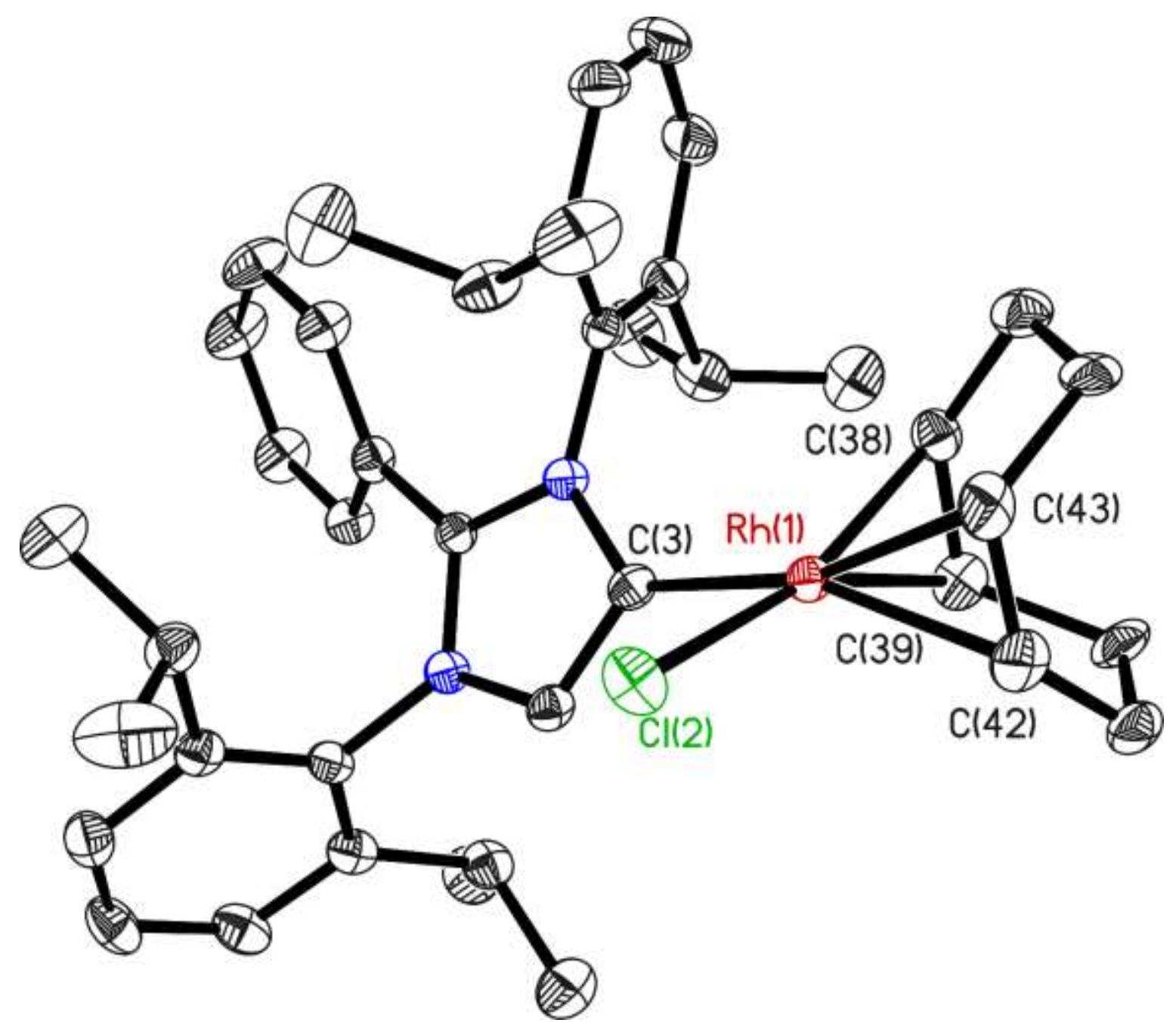

\begin{tabular}{|c|c|c|c|}
\hline Structure code & CS_RAJ_381 & & \\
\hline Empirical Formula & $\mathrm{C}_{56} \mathrm{H}_{67} \mathrm{CIN}{ }_{2} \mathrm{Rh}$ & $\mu\left[\mathrm{mm}^{-1}\right]$ & 0.242 \\
\hline Formula weight $\left[\mathrm{g} \mathrm{mol}^{-1}\right]$ & 906.47 & $\mathrm{~F}(000)$ & 1916 \\
\hline Sample temperature [K] & $100(2)$ & $\theta$ range $\left[{ }^{\circ}\right]$ & 0.861 to 20.612 \\
\hline W avelength $[\AA]$ & 0.56086 & Reflections collected & 90047 \\
\hline Crystal System & Monoclinic & Unique reflections & 9966 \\
\hline \multirow[t]{2}{*}{ Space group } & $P 2 \sqrt{1} \mathrm{C}$ & $\mathrm{R}_{\text {int }}$ & 0.0598 \\
\hline & $a=11.887(2)$ & Completeness to $\theta_{\max }[\%]$ & 100 \\
\hline \multirow[t]{2}{*}{ Unit cell dimensions $[\AA]$} & $b=37.338(4)$ & restraints/parameter & 444 / 616 \\
\hline & $c=11.347(2)$ & GooF & 1.043 \\
\hline Volume $\left[\AA^{3}\right]$ & $4829.8(14)$ & R1 (all data) & 0.0439 \\
\hline Z & 4 & wR2 (all data) & 0.0712 \\
\hline Crystal dimensions [mm] & $0.576 \times 0.402 \times 0.245$ & max. diff. peak / hole $\left[\mathrm{e}^{-3}\right]$ & 0.305 and -0.544 \\
\hline
\end{tabular}




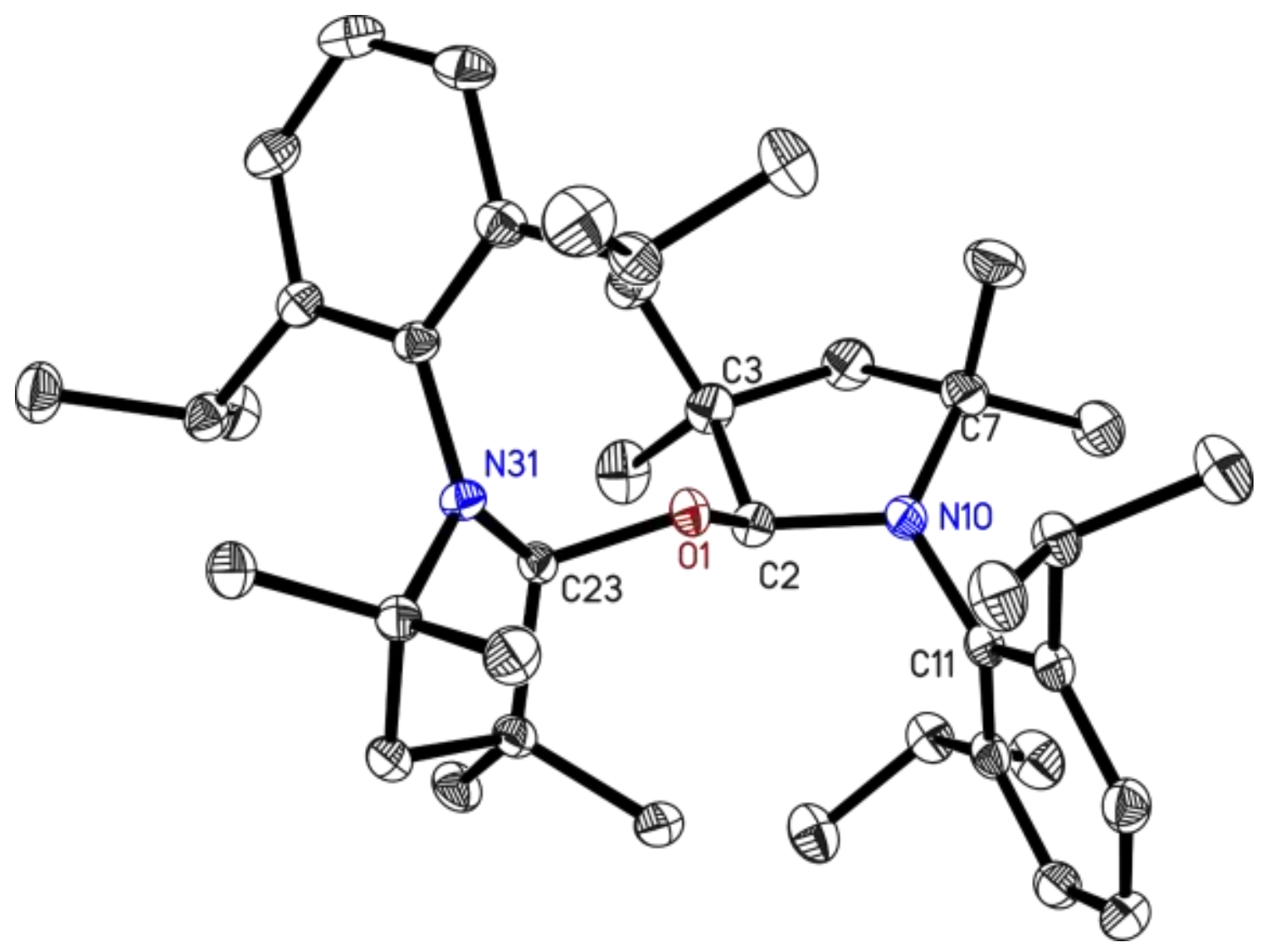

\begin{tabular}{|c|c|c|c|c|}
\hline Structure code & CS_RAJ_391 & & $\mu\left[\mathrm{mm}^{-1}\right]$ & 0.042 \\
\hline Empirical Formula & $\mathrm{C}_{40} \mathrm{H}_{64} \mathrm{~N}_{2} \mathrm{O}$ & & $F(000)$ & 652 \\
\hline Formula weight $\left[\mathrm{g} \mathrm{mol}^{-1}\right]$ & 588.93 & & $\theta$ range $\left[{ }^{\circ}\right]$ & 1.110 to 22.047 \\
\hline Sample temperature [K] & $100(2)$ & & Reflections collected & 131677 \\
\hline W avelength $[\AA]$ & 0.56086 & & Unique reflections & 9063 \\
\hline Crystal System & Triclinic & & $\mathrm{R}_{\text {int }}$ & 0.0441 \\
\hline \multirow[t]{2}{*}{ Space group } & $P \overline{1}$ & & Completeness to $\theta_{\max }[\%]$ & 100 \\
\hline & $a=10.771(5)$ & $\alpha=79.77(2)$ & restraints/parameter & $0 / 404$ \\
\hline \multirow[t]{2}{*}{ Unit cell dimensions $[\AA]$} & $b=11.885(6)$ & $\beta=86.71(2)$ & GooF & 1.024 \\
\hline & $c=14.713(7)$ & $v=78.07(2)$ & R1 (all data) & 0.0562 \\
\hline Volume $\left[\AA^{3}\right]$ & $1813.1(15)$ & & wR2 (all data) & 0.1157 \\
\hline Z & 2 & & max. diff. peak / hole $\left[\mathrm{e}^{-3}\right]$ & 0.366 and -0.225 \\
\hline
\end{tabular}




\subsection{Crystal Structures in Collaboration with Sudipta Roy}

In the course of the years 2016/17, five very interesting crystal structures have been determined for Sudipta Roy, resulting in two publications.

\subsubsection{Activation of Elemental Sulfur at a T wo-C oordinate Platinum(0) Center}

The following structures have been published in Roy, S., Schürmann, C. J., M ondal, T., Koley, D., Herbst-Irmer, R., Stalke, D. \& Roesky, H. W. 2016. Chem. Eur. J. 22, 12629-12633.

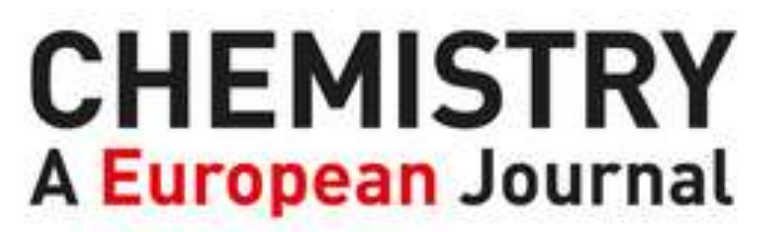

www.chemeurj.org

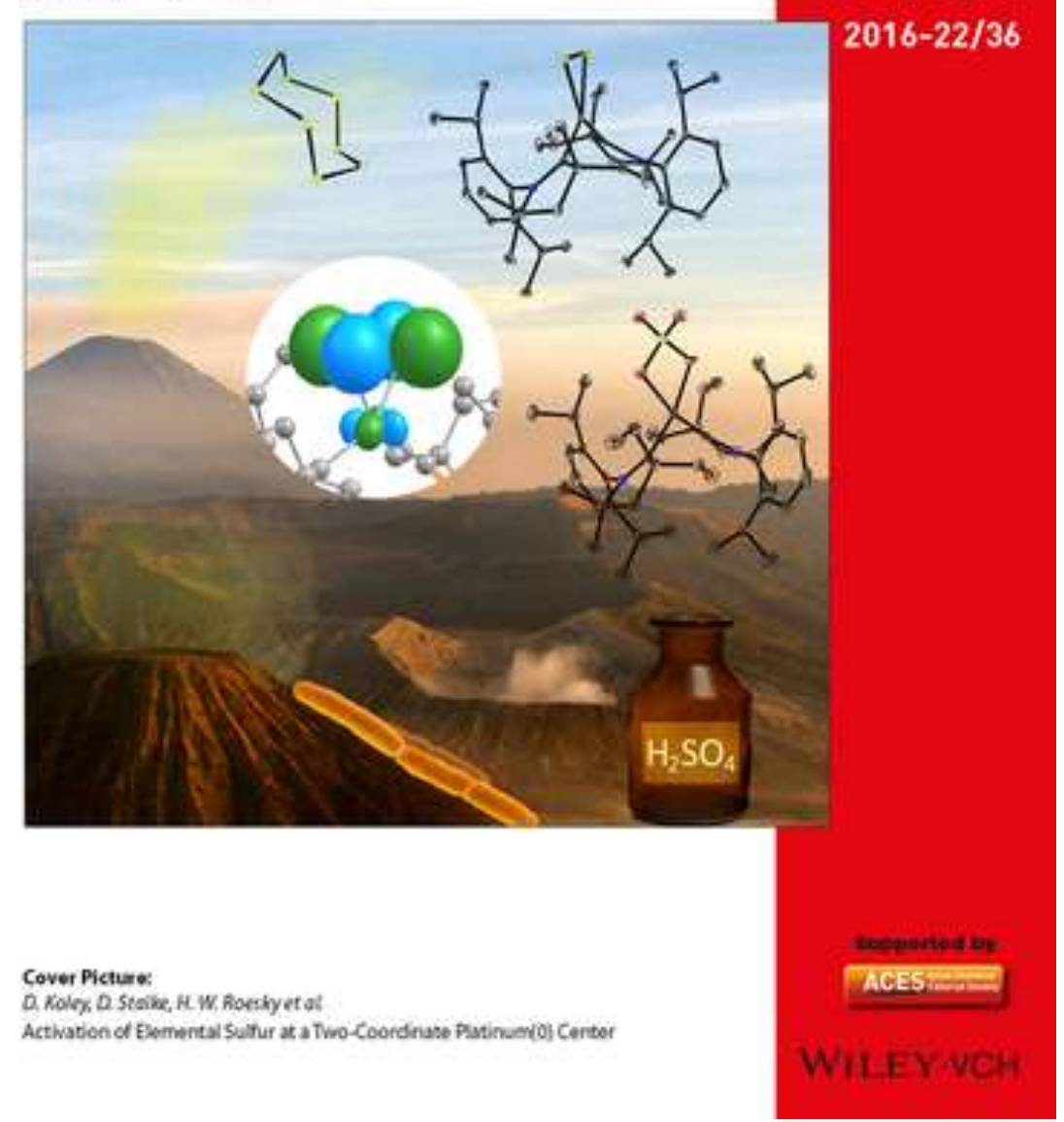

Figure 8.2: Back cover associated to "A ctivation of elemental Sulfur at a T wo-Coordinate Platinum(0) Center" (Roy et al., 2016). 


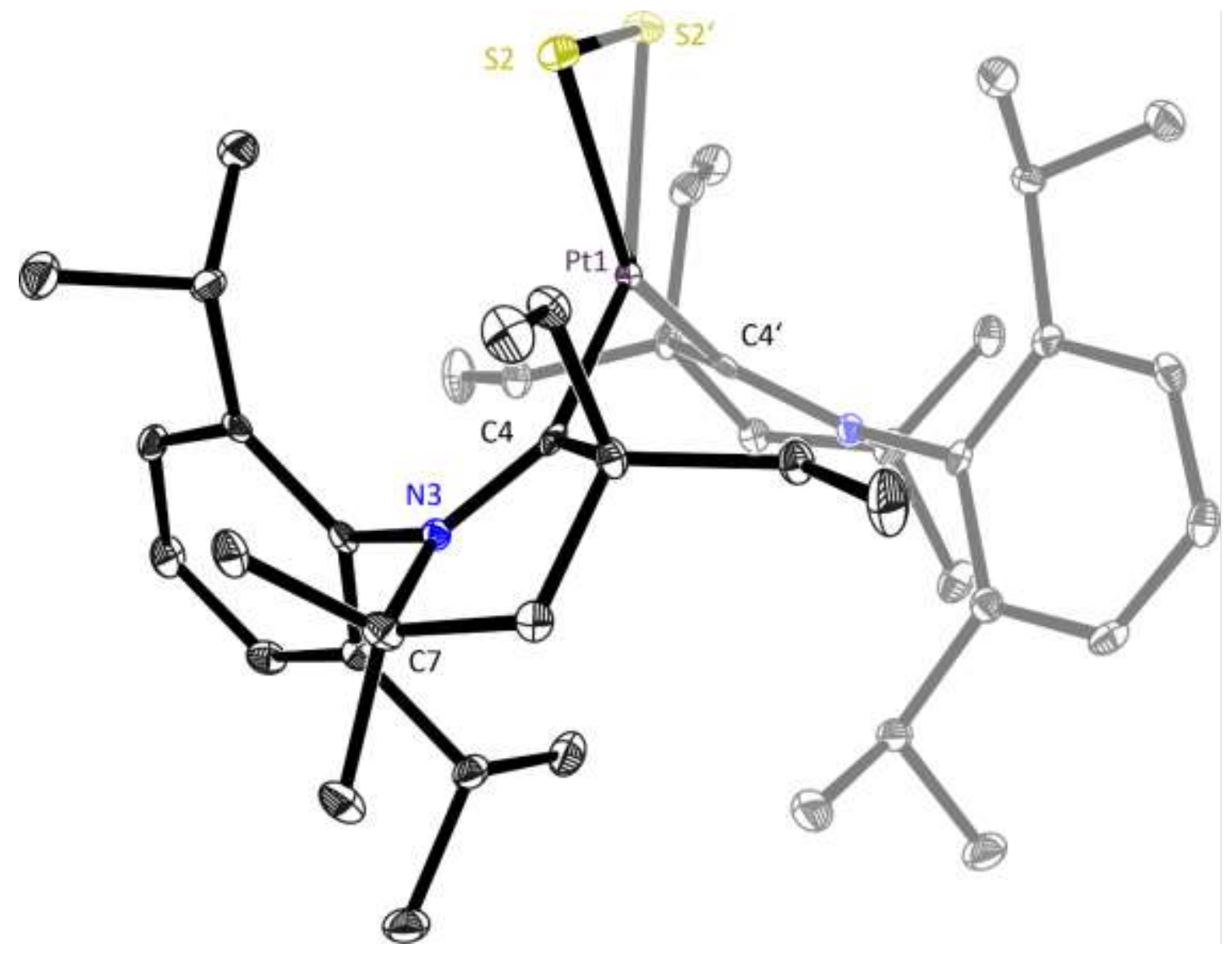

\begin{tabular}{llll}
\hline Structure code & $\mathrm{CS} S \mathrm{SR} 22 \mathrm{~S}$ & $\mathrm{CCDC}$ Number & 1444046 \\
Empirical Formula & $\mathrm{C}_{52} \mathrm{H}_{80} \mathrm{~N}_{2} \mathrm{O}_{2} \mathrm{PtS}_{2}$ & $\mu\left[\mathrm{mm}^{-1}\right]$ & 1.929 \\
Formula weight $\left[\mathrm{g} \mathrm{mol}^{-1}\right]$ & 1030.43 & $\mathrm{~F}(000)$ & 2152 \\
Sampletemperature $[\mathrm{K}]$ & $100(2)$ & $\theta$ range $\left[{ }^{\circ}\right]$ & 1.324 to 22.016 \\
Wavelength $[\AA]$ & 0.56086 & Reflections collected & 51764 \\
Crystal System & M onoclinic & Unique reflections & 5942 \\
Space group & $\mathrm{C} 2 / \mathrm{c}$ & R & 0.0591 \\
& $\mathrm{a}=29.646(2)$ & Completeness to $\theta_{\text {max }}[\%]$ & 100 \\
Unit cell dimensions $[\AA]$ & $\mathrm{b}=10.870(2) \quad \beta=125.02(2)$ & restraints/parameter & $0 / 275$ \\
& $\mathrm{C}=18.065(3)$ & GooF & 1.049 \\
Volume $\left[\AA^{3}\right]$ & $4767.5(16)$ & R1 (all data) & 0.0271 \\
$Z$ & 4 & wR2 (all data) & 0.0436 \\
Crystal dimensions $[\mathrm{mm}]$ & $0.178 \times 0.114 \times 0.065$ & max. diff. peak / hole $\left[e \AA^{-3}\right]$ & 0.578 and -0.751 \\
\hline
\end{tabular}




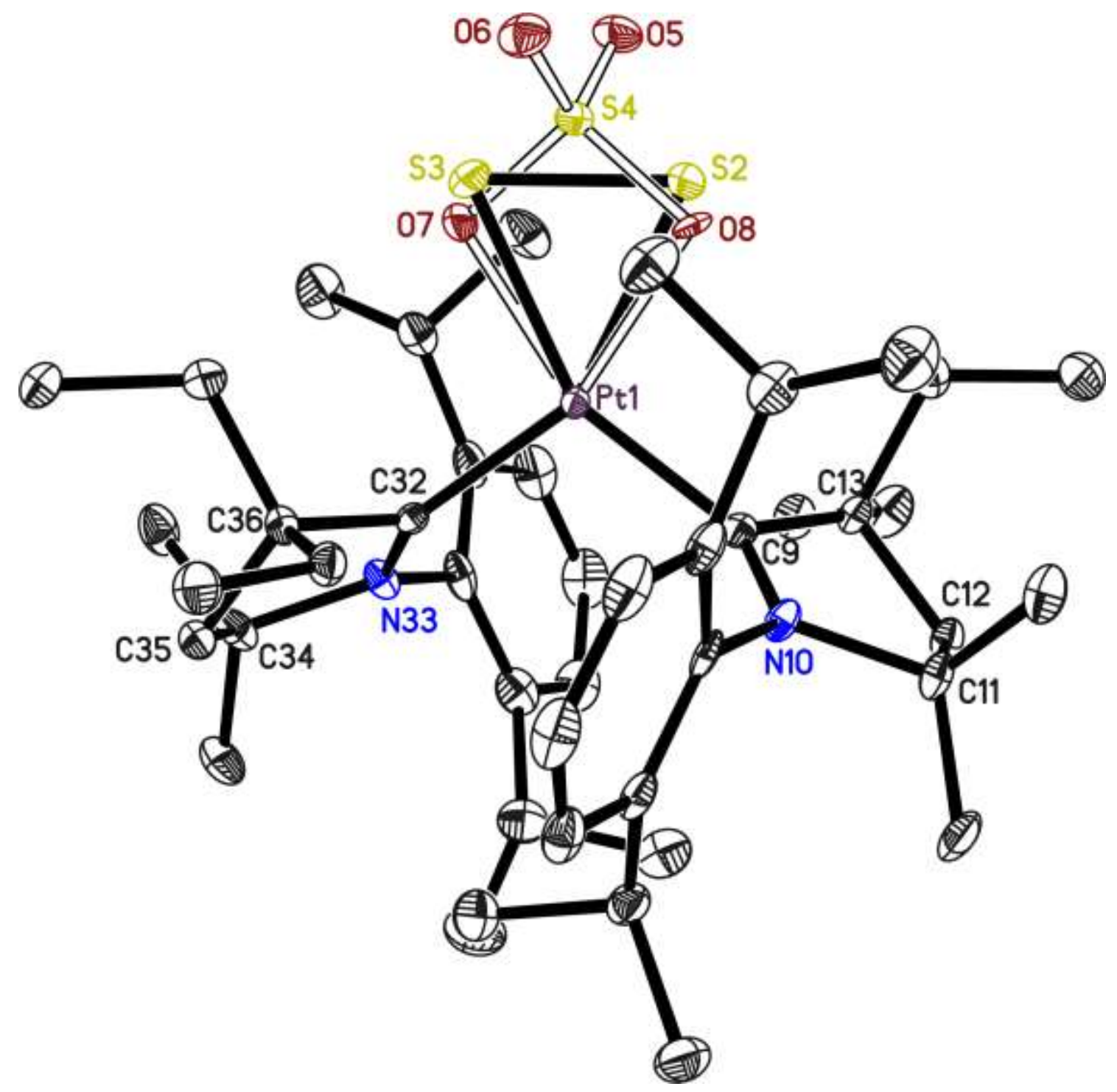

The structure features an interesting 1:1 disorder of the prior investigated structure CS_SR_22S and the oxidation product.

\begin{tabular}{llll}
\hline Structure code & CS_SR_23s & CCDC Number & 1444047 \\
Empirical Formula & $\mathrm{C}_{44}-\mathrm{H}_{70} \mathrm{~N}_{2} \mathrm{O}_{1.98} \mathrm{Pt} \mathrm{S}_{1.50}$ & $\mu\left[\mathrm{mm}^{-1}\right]$ & 1.929 \\
Formula weight $\left[\mathrm{g} \mathrm{mol}^{-1}\right]$ & 902.08 & $\mathrm{~F}(000)$ & 1864 \\
Sampletemperature $[\mathrm{K}]$ & $100(2)$ & $\theta$ range $\left.{ }^{\circ}\right]$ & 1.214 to 22.013 \\
Wavelength $[\AA]$ & 0.56086 & Reflections collected & 121963 \\
Crystal System & $\mathrm{M}$ onoclinic & Unique reflections & 10139 \\
Space group & $\mathrm{P} 2 / \mathrm{C}$ & $\mathrm{R}_{\text {int }}$ & 0.0728 \\
& $\mathrm{a}=11.473(2)$ & Completeness to $\theta_{\max }[\%]$ & 100 \\
Unit cell dimensions $[\AA]$ & $\mathrm{b}=20.772(3) \quad \beta=95.42(2)$ & restraints/parameter & $95 / 504$ \\
& $\mathrm{C}=17.246(2)$ & GooF & 1.162 \\
Volume $\left[\AA^{3}\right]$ & $4091.6(10)$ & $\mathrm{R} 1$ (all data) & 0.0522 \\
$Z$ & 4 & wR2 (all data) & 0.0548 \\
Crystal dimensions $[\mathrm{mm}]$ & $0.158 \times 0.123 \times 0.088$ & max. diff. peak / hole $\left[\mathrm{e} \AA^{-3}\right]$ & 0.899 and -1.489 \\
\hline
\end{tabular}




\subsubsection{Two Structurally Characterized Conformational I somers with D ifferent C-P Bonds}

The following structures have been published in Roy, S., Mondal, K. C., Kundu, S., Li, B., Schürmann, C. J., Dutta, S., Koley, D., H erbst-Irmer, R., Stalke, D. \& Roesky, H. W. 2017. Chem. Eur. J. 23, 12153-12157. All three structures show a disorder that resembles the two reaction products in Figure 8.3 .

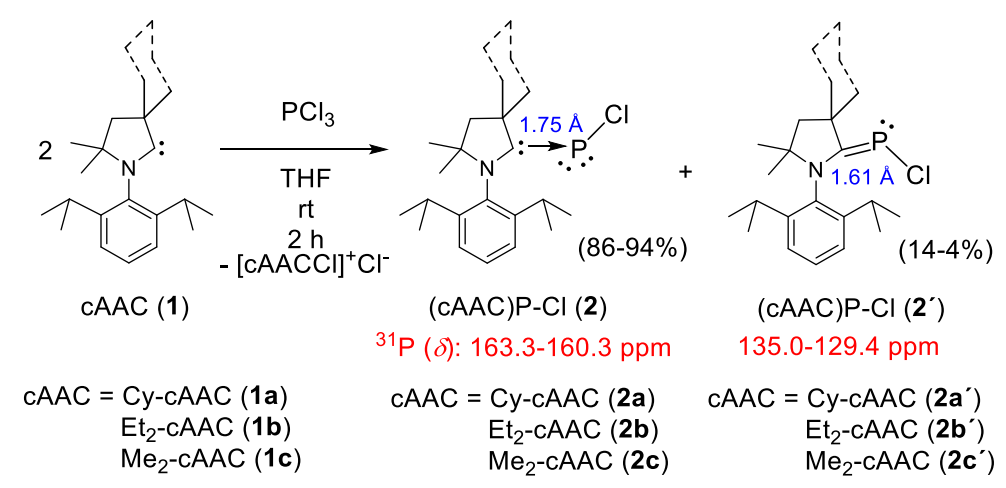

Figure 8.3: Synthesis of compounds 2a-c/a'-c'.

After refinement of the major component the residual density in all three structures reveals unexplained residual density (see Figure 8.4). The two highest residual density peaks had a distance of ca. 2.1 $\AA$. In accordance with the ${ }^{31} \mathrm{P}-\mathrm{N} M \mathrm{R}$ results these peaks were interpreted as a second position of $\mathrm{P}$ and $\mathrm{Cl}$. Because no disorder of the CAAC could be resolved this means a co-crystallization of two conformational isomers. In all three structures the NCP angle of the minor component refines to $\mathrm{ca}$. $150^{\circ}$ and the $\mathrm{CPCl}$ angle to ca. $97^{\circ}$ in contrast to the major component with 117 and $104^{\circ}$, respectively. The P- $\mathrm{C}_{\text {CAAC }}$ distance of the minor component is in all three structures much smaller than in the major component. In $\mathbf{2} \mathbf{b}^{\prime}$ 'the refined value of $1.435 \AA$ seems to be much too small (and could not be forced to a reasonable value by using a distance restraint), while the values for $2 \mathbf{a}^{\prime}$ and $\mathbf{2} \mathbf{c}^{\prime}$ fit to the theoretical values. This makes clear that in disordered groups with such low occupancy any detailed discussion on bond lengths is excluded.

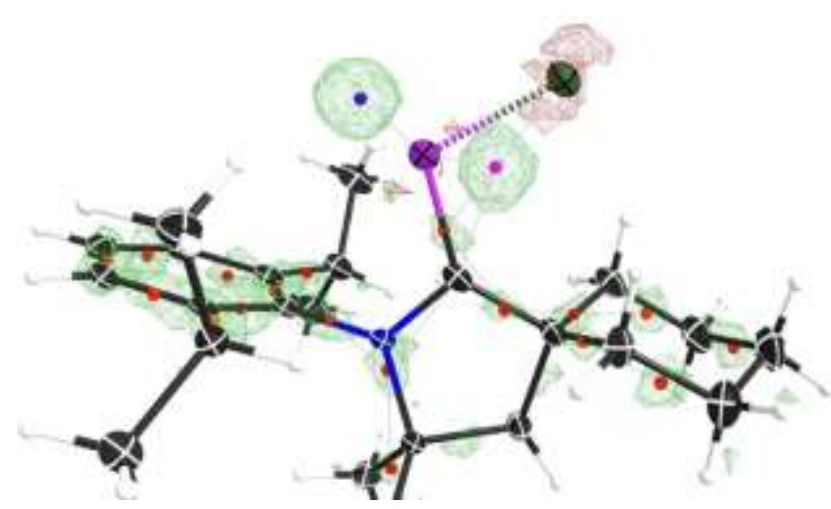

Figure 8.4: Difference electron density after refinement with one major PCl-group of 2 at the $0.2 \mathrm{e}^{-3}$ level. 
Table 8.1: Selected bond lengths and angles.

\begin{tabular}{|c|c|c|c|c|c|c|}
\hline Compound & $2 a$ & $2 a^{\prime}$ & $2 b$ & $2 b^{\prime}$ & $2 c$ & $2 c^{\prime}$ \\
\hline Occupancy[\%] & $86.27(13)$ & 13.73(13) & $96.3(1)$ & $3.7(1)$ & $94.5(1)$ & $5.4(1)$ \\
\hline$C-P[\AA ̊]$ & $1.7513(15)$ & $1.615(4)$ & $1.7404(12)$ & $1.435(12)$ & $1.7355(11)$ & $1.686(7)$ \\
\hline P-CI $[\AA]]$ & $2.0982(7)$ & $2.048(5)$ & $2.1004(4)$ & $2.101(16)$ & $2.1049(5)$ & $2.066(9)$ \\
\hline $\mathrm{N}-\mathrm{C}-\mathrm{P}\left[{ }^{\circ}\right]$ & $116.17(11)$ & $149.27(18)$ & $117.10(7)$ & $149.9(5)$ & $117.47(7)$ & $150.8(2)$ \\
\hline C-P-CI [ $\left.{ }^{\circ}\right]$ & $104.51(5)$ & $98.0(2)$ & $104.06(4)$ & $98.3(7)$ & $103.64(4)$ & $96.3(3)$ \\
\hline
\end{tabular}

Although this interpretation is in good accordance with the NM R and the theoretical results there are still some deficiencies in this model. In all three structures the anisotropic displacement parameters for the minor component are much larger than in the major component In spite of the used restraints for the anisotropic displacement parameters. Enforcing them to be equal of course influences the occupancies and leads to higher R-values as well as residual density. The effect on the bond lengths is negligible. Even with this disorder model there is small unexplained residual density (see Figure 8.5).

Figure 8.5: Difference electron density after refinement with disordered PCl-groups at the $0.082 \mathrm{e}^{-3}$ level. 


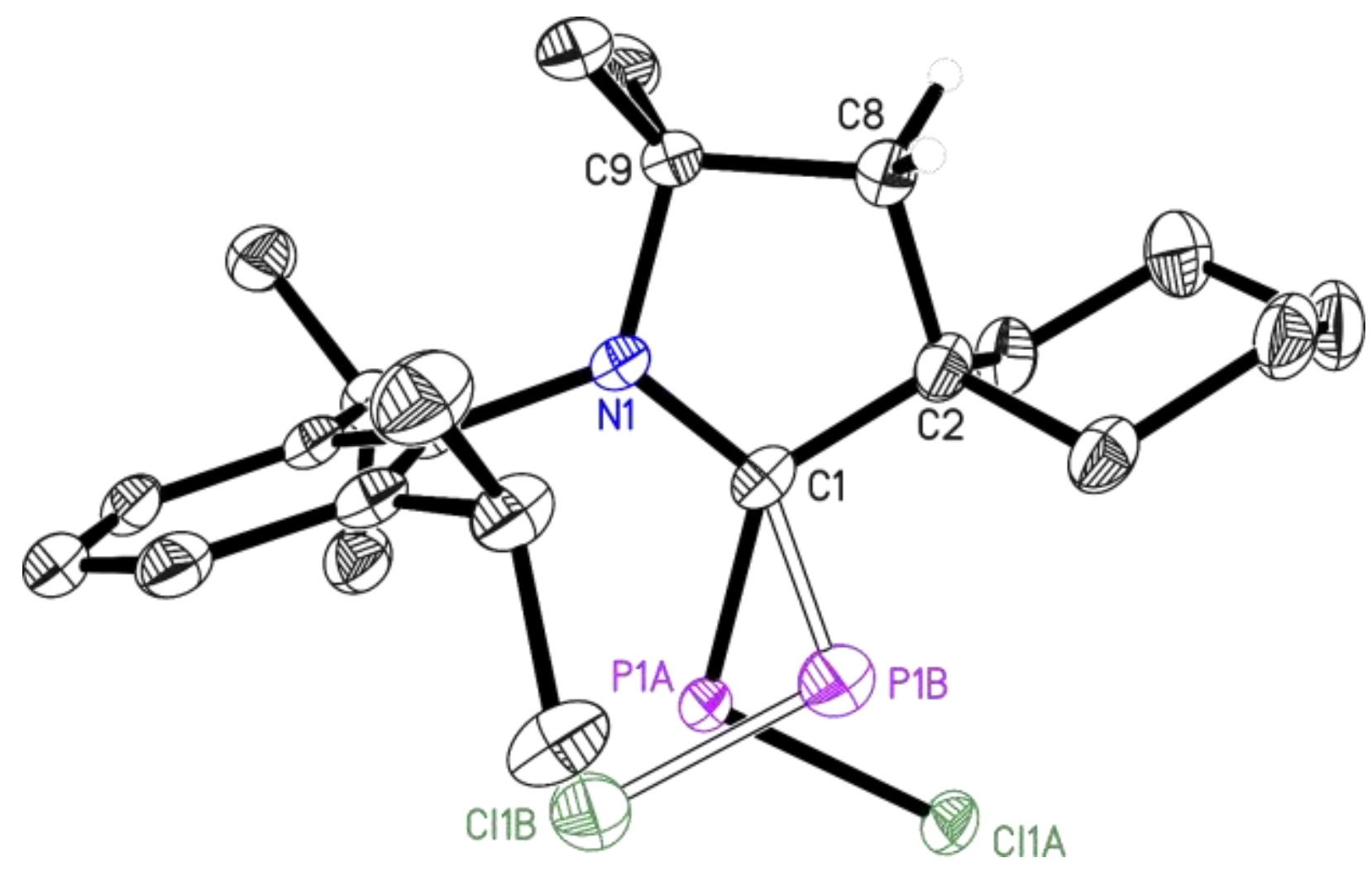

\begin{tabular}{|c|c|c|c|c|}
\hline Structure code & \multicolumn{2}{|c|}{ CS_SR_SKM_367 } & CCDC Number & 1498552 \\
\hline Empirical Formula & \multicolumn{2}{|c|}{$\mathrm{C}_{23} \mathrm{H}_{35} \mathrm{CINP}$} & $\mu\left[\mathrm{mm}^{-1}\right]$ & 0.254 \\
\hline Formula weight $\left[\mathrm{g} \mathrm{mol}^{-1}\right]$ & \multicolumn{2}{|l|}{391.94} & $F(000)$ & 424 \\
\hline Sample temperature [K] & \multicolumn{2}{|l|}{$100(2)$} & $\theta$ range $\left[{ }^{\circ}\right]$ & 1.679 to 28.272 \\
\hline W avelength $[\AA]$ & \multicolumn{2}{|l|}{0.71073} & Reflections collected & 26769 \\
\hline Crystal System & \multicolumn{2}{|l|}{ Triclinic } & Unique reflections & 5430 \\
\hline \multirow[t]{2}{*}{ Space group } & \multicolumn{2}{|l|}{$\mathrm{P} \overline{1}$} & $\mathrm{R}_{\text {int }}$ & 0.0310 \\
\hline & $a=8.868(2)$ & $\alpha=86.77(2)$ & Completeness to $\theta_{\max }[\%]$ & 99.9 \\
\hline \multirow[t]{2}{*}{ Unit cell dimensions $[\AA]$} & $b=10.197(2)$ & $\beta=82.10(2)$ & restraints/parameter & $60 / 260$ \\
\hline & $c=12.260(2)$ & $y=88.59(2)$ & GooF & 1.028 \\
\hline Volume $\left[\AA^{3}\right]$ & \multicolumn{2}{|l|}{$1096.2(4)$} & R1 (all data) & 0.0530 \\
\hline Z & \multicolumn{2}{|l|}{2} & wR2 (all data) & 0.1097 \\
\hline Crystal dimensions [mm] & \multicolumn{2}{|c|}{$0.170 \times 0.130 \times 0.110$} & max. diff. peak / hole $\left[\mathrm{e}^{-3}{ }^{-3}\right]$ & 0.447 and -0.256 \\
\hline
\end{tabular}




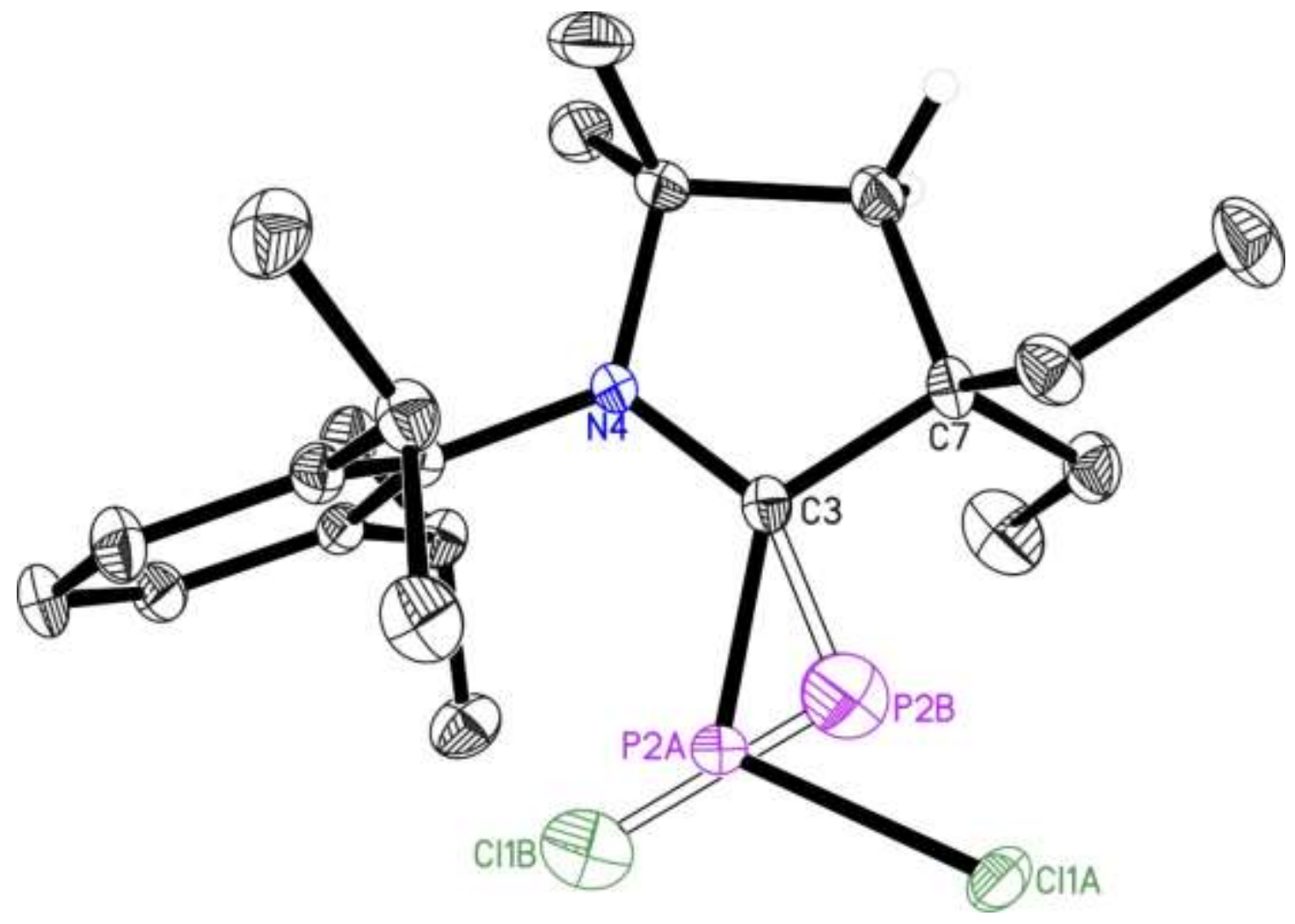

\begin{tabular}{|c|c|c|c|}
\hline Structure code & CS_SR_SKM_368 & CCDC Number & 1498554 \\
\hline Empirical Formula & $\mathrm{C}_{22} \mathrm{H}_{35} \mathrm{CINP}$ & $\mu\left[\mathrm{mm}^{-1}\right]$ & 0.254 \\
\hline Formula weight $\left[\mathrm{g} \mathrm{mol}^{-1}\right]$ & 379.93 & $F(000)$ & 824 \\
\hline Sample temperature [K] & $100(2)$ & $\theta$ range $\left[{ }^{\circ}\right]$ & 1.752 to 30.019 \\
\hline W avelength $[\AA]$ & 0.71073 & Reflections collected & 53338 \\
\hline Crystal System & Monoclinic & Unique reflections & 6347 \\
\hline \multirow[t]{3}{*}{ Space group } & $\mathrm{P} 2 \mathrm{~J} / \mathrm{C}$ & $\mathrm{R}_{\text {int }}$ & 0.0337 \\
\hline & $a=7.807(2)$ & Completeness to $\theta_{\max }[\%]$ & 99.9 \\
\hline & $b=14.711(2)$ & restraints/parameter & $60 / 253$ \\
\hline Unit cell dimensions [ $\AA \AA]$ & $c=19.173(3)$ & GooF & 1.040 \\
\hline Volume $\left[\AA^{3}\right]$ & $4091.6(10)$ & R1 (all data) & 0.0411 \\
\hline Z & 4 & wR2 (all data) & 0.0897 \\
\hline Crystal dimensions [mm] & $0.359 \times 0.101 \times 0.071$ & max. diff. peak / hole $\left[\mathrm{e}^{-3}\right]$ & 0.433 and -0.241 \\
\hline
\end{tabular}




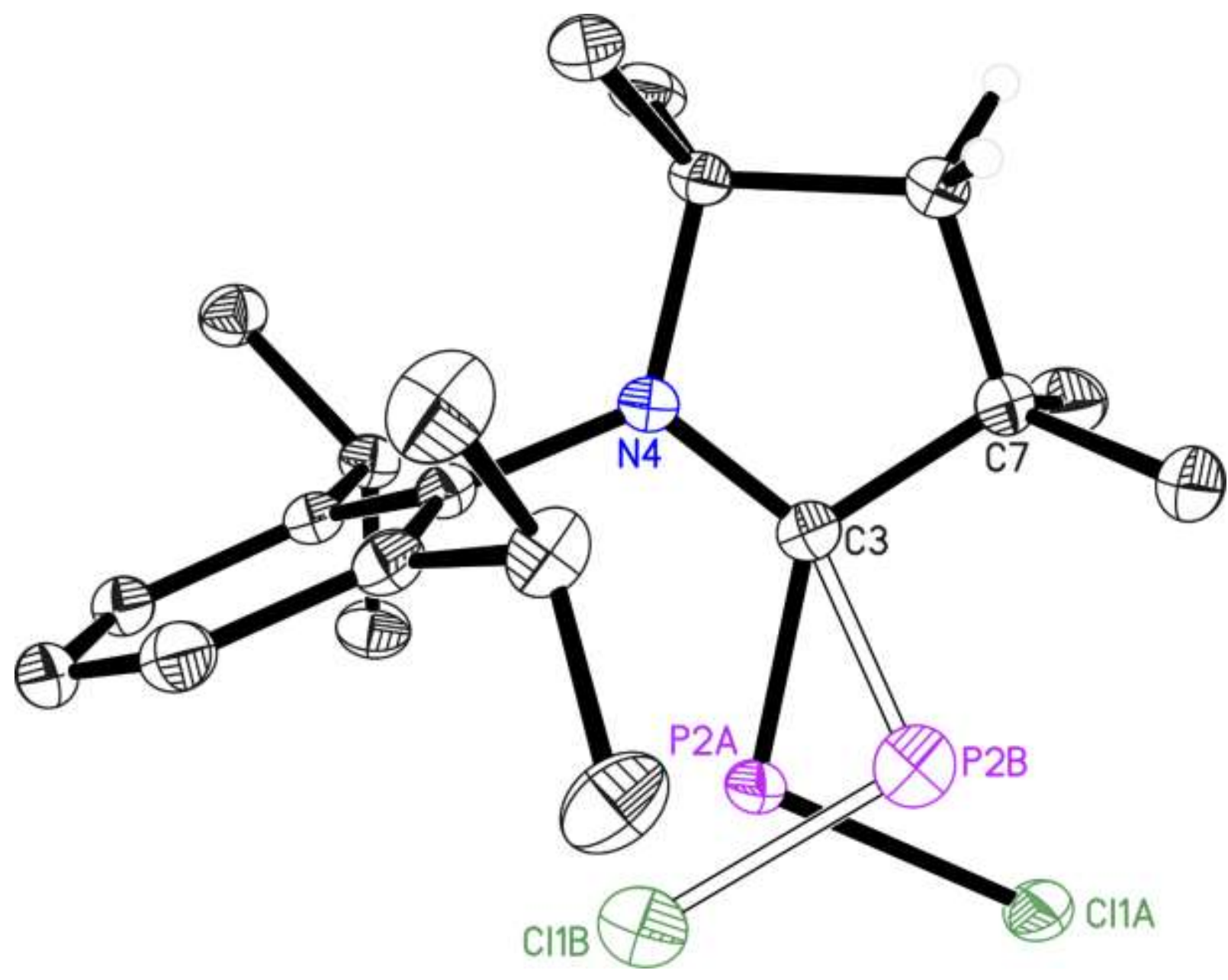

\begin{tabular}{|c|c|c|c|}
\hline Structure code & CS_SR_SKM_369 & CCDC Number & 1498553 \\
\hline Empirical Formula & $\mathrm{C}_{20} \mathrm{H}_{31} \mathrm{CINP}$ & $\mu\left[\mathrm{mm}^{-1}\right]$ & 0.272 \\
\hline Formula weight $\left[\mathrm{g} \mathrm{mol}^{-1}\right]$ & 351.88 & $F(000)$ & 760 \\
\hline Sample temperature [K] & $100(2)$ & $\theta$ range $\left[{ }^{\circ}\right]$ & 1.949 to 29.168 \\
\hline W avelength $[\AA]$ & 0.71073 & Reflections collected & 40708 \\
\hline Crystal System & Monoclinic & Unique reflections & 5384 \\
\hline \multirow[t]{2}{*}{ Space group } & $P 2_{1} / \mathrm{C}$ & $\mathrm{R}_{\text {int }}$ & 0.0366 \\
\hline & $a=10.597(2)$ & Completeness to $\theta_{\max }[\%]$ & 100 \\
\hline \multirow[t]{2}{*}{ U nit cell dimensions [ $\AA$ ] } & $b=9.000(2)$ & restraints/parameter & $60 / 235$ \\
\hline & $c=21.437(3)$ & GooF & 1.042 \\
\hline Volume $\left[\AA^{3}\right]$ & $1992.8(7)$ & R1 (all data) & 0.0371 \\
\hline Z & 4 & wR2 (all data) & 0.0841 \\
\hline Crystal dimensions [mm] & $0.267 \times 0.164 \times 0.080$ & max. diff. peak / hole $\left[\mathrm{e}^{-3}\right]$ & 0.391 and -0.195 \\
\hline
\end{tabular}




\subsection{Crystal Structures in Collaboration with Chandrajeet Mohapatra}

In 2016, two crystal structures have been determined for Chandrajeet M ohapatra and published in M ohapatra, C., Samuel, P. P., Li, B., Niepötter, B., Schürmann, C. J., Herbst-Irmer, R., Stalke, D., M aity, B., Koley, D. \& Roesky, H. W . 2016. Inorg. Chem. 55, 1953-1955.

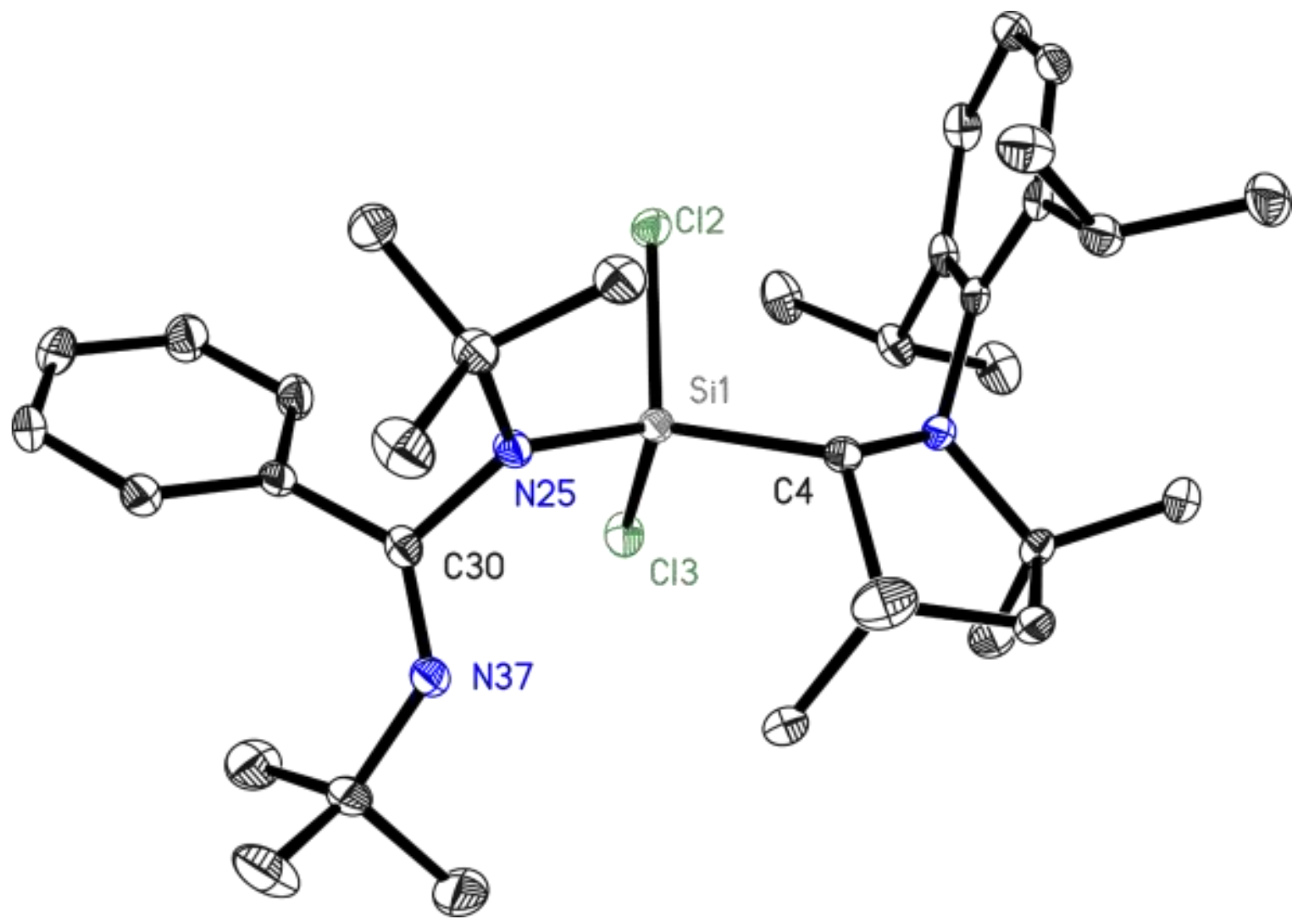

\begin{tabular}{|c|c|c|c|}
\hline Structure code & CS_CM_101 & CCDC Number & 1443739 \\
\hline Empirical Formula & $\mathrm{C}_{35} \mathrm{H}_{55} \mathrm{Cl}_{2} \mathrm{~N}_{3} \mathrm{Si}$ & $\mu\left[\mathrm{mm}^{-1}\right]$ & 0.132 \\
\hline Formula weight $\left[\mathrm{g} \mathrm{mol}^{-1}\right]$ & 616.81 & $F(000)$ & 1336 \\
\hline Sample temperature [K ] & $100(2)$ & $\theta$ range $\left[{ }^{\circ}\right]$ & 1.305 to 19.510 \\
\hline W avelength $[\AA]$ & 0.56086 & Reflections collected & 104005 \\
\hline Crystal System & Monoclinic & Unique reflections & 6257 \\
\hline \multirow[t]{2}{*}{ Space group } & $P 2_{1} / \mathrm{C}$ & $\mathrm{R}_{\text {int }}$ & 0.0842 \\
\hline & $a=13.314(2)$ & Completeness to $\theta_{\max }$ [\%] & 100 \\
\hline \multirow[t]{2}{*}{ Unit cell dimensions $[\AA]$} & $b=16.570(2)$ & restraints/parameter & $0 / 387$ \\
\hline & $c=17.340(3)$ & GooF & 1.030 \\
\hline Volume $\left[\AA^{3}\right]$ & $3537.3(10)$ & R1 (all data) & 0.0504 \\
\hline Z & 4 & wR2 (all data) & 0.0841 \\
\hline Crystal dimensions [mm] & $0.196 \times 0.170 \times 0.062$ & max. diff. peak / hole $\left[\mathrm{e}^{-3}\right]$ & 0.381 and -0.319 \\
\hline
\end{tabular}




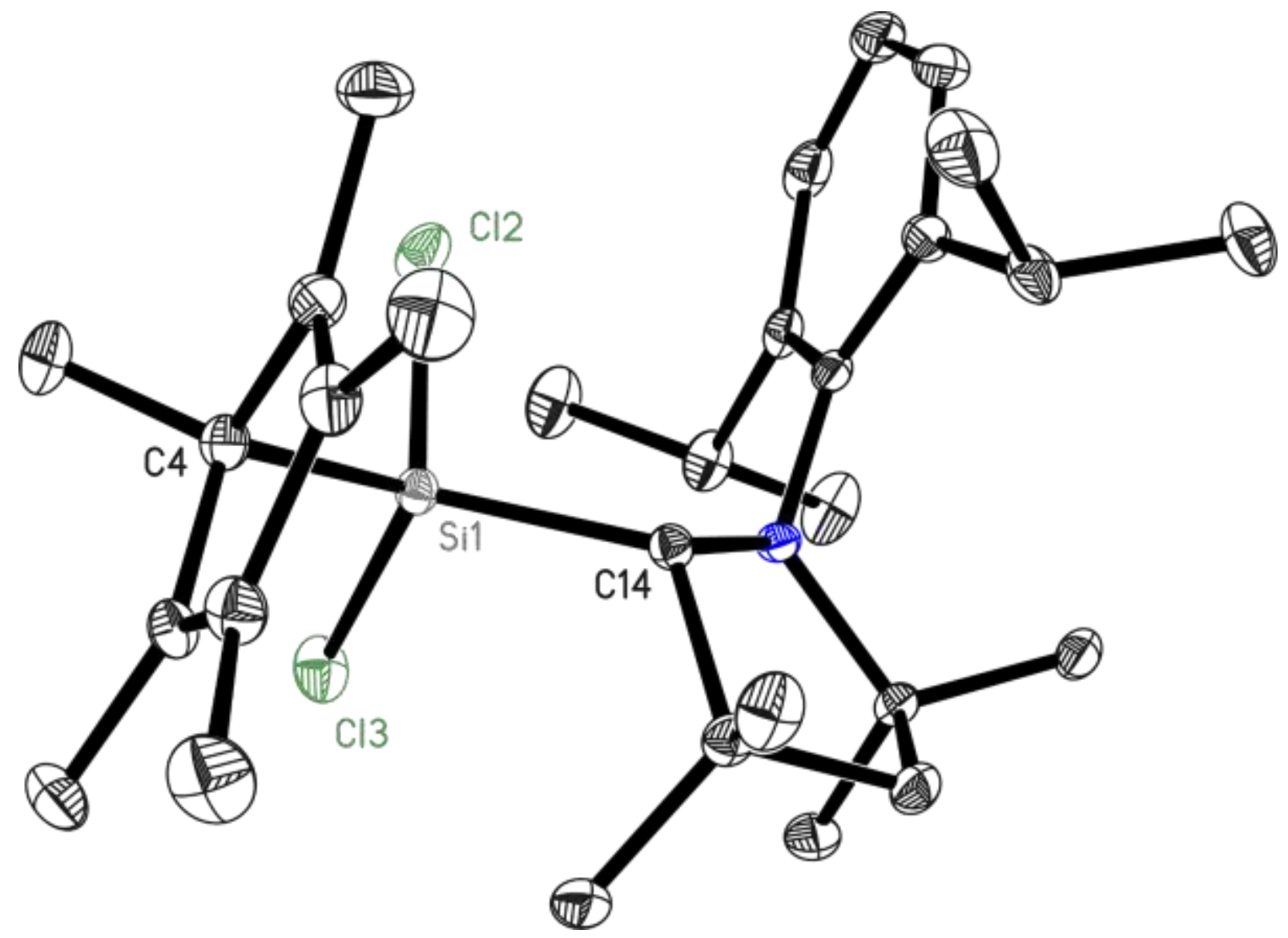

\begin{tabular}{llll}
\hline Structure code & $\mathrm{CS}{ }_{-} \mathrm{CM}-103$ & $\mathrm{CCDC}$ Number & 1443738 \\
Empirical Formula & $\mathrm{C}_{30} \mathrm{H}_{47} \mathrm{Cl}_{2} \mathrm{NSi}$ & $\mu\left[\mathrm{mm}^{-1}\right]$ & 0.151 \\
Formula weight $\left[\mathrm{g} \mathrm{mol}^{-1}\right]$ & 520.67 & $\mathrm{~F}(000)$ & 564 \\
Sampletemperature $[\mathrm{K}]$ & $100(2)$ & $\theta$ range $\left.{ }^{\circ}\right]$ & 1.599 to 23.694 \\
Wavelength $[\AA]$ & 0.56086 & Reflections collected & 60081 \\
Crystal System & Monoclinic & Unique reflections & 8956 \\
Space group & $\mathrm{P} 2_{1}(\mathrm{Flack}=-0.027(18))$ & $\mathrm{R}_{\text {int }}$ & 0.0385 \\
& $\mathrm{a}=8.815(2)$ & Completeness to $\theta_{\max }[\%]$ & 100 \\
Unit cell dimensions $[\AA]$ & $\mathrm{b}=16.427(3) \quad \beta=113.64(2)$ & restraints/parameter & $1 / 323$ \\
& $\mathrm{C}=10.972(2)$ & GooF & 1.025 \\
Volume $\left[\AA^{3}\right]$ & $1455.5(5)$ & $\mathrm{R} 1$ (all data) & 0.0293 \\
$Z$ & 2 & wR2 (all data) & 0.0633 \\
Crystal dimensions $[\mathrm{mm}]$ & $0.404 \times 0.385 \times 0.245$ & max. diff. peak / hole $\left[\mathrm{e} \AA^{-3}\right]$ & 0.244 and -0.169 \\
\hline
\end{tabular}





\section{Chapter 9 APPENDIX}

\section{Supplementary information to Chapter $\mathbf{4}$}

Table S4.1: D ata quality statistics from XPREP of dataset $\mathbf{A}$.

\begin{tabular}{|c|c|c|c|c|c|c|c|c|c|c|c|c|}
\hline \multicolumn{3}{|c|}{ Resolution } & \multirow{2}{*}{$\begin{array}{l}\text { Number } \\
\text { of Data } \\
95\end{array}$} & \multirow{2}{*}{$\begin{array}{l}\text { Theory } \\
95\end{array}$} & \multirow{2}{*}{$\begin{array}{l}\text { Complete- } \\
\text { ness [\%] } \\
100\end{array}$} & \multirow{2}{*}{$\begin{array}{l}\text { M ultiplicity } \\
37.21\end{array}$} & \multirow{2}{*}{$\begin{array}{c}\langle I\rangle \\
115.28\end{array}$} & \multirow{2}{*}{$\begin{array}{r}\langle I / \sigma\rangle \\
405.04\end{array}$} & \multirow{2}{*}{$\begin{array}{l}R_{\text {merge }} \\
0.0537\end{array}$} & \multirow{2}{*}{$\begin{array}{l}R_{\text {sigma }} \\
0.0014\end{array}$} & \multirow{2}{*}{$\begin{array}{l}R_{\text {rim }} \\
0.0546\end{array}$} & \multirow{2}{*}{$\begin{array}{l}R_{\text {pim }} \\
0.0098\end{array}$} \\
\hline Inf & - & 2 & & & & & & & & & & \\
\hline 2 & - & 1.2 & 333 & 333 & 100 & 34.91 & 62.18 & 262.71 & 0.0411 & 0.002 & 0.0417 & 0.007 \\
\hline 1.2 & - & 0.9 & 542 & 542 & 100 & 23.58 & 33.24 & 155.65 & 0.0438 & 0.0038 & 0.0448 & 0.0091 \\
\hline 0.9 & - & 0.76 & 624 & 624 & 100 & 18.5 & 16.52 & 196.09 & 0.0584 & 0.0031 & 0.0601 & 0.0138 \\
\hline 0.76 & - & 0.7 & 436 & 436 & 100 & 18.06 & 11.04 & 213.9 & 0.0401 & 0.0032 & 0.0412 & 0.0096 \\
\hline 0.7 & - & 0.64 & 608 & 608 & 100 & 17.99 & 8.39 & 173.2 & 0.0317 & 0.0041 & 0.0326 & 0.0077 \\
\hline 0.64 & - & 0.6 & 551 & 551 & 100 & 17.22 & 5.39 & 111.63 & 0.0347 & 0.0067 & 0.0358 & 0.0085 \\
\hline 0.6 & - & 0.56 & 716 & 716 & 100 & 16.54 & 3.87 & 75.4 & 0.0392 & 0.0099 & 0.0404 & 0.0099 \\
\hline 0.56 & - & 0.54 & 433 & 433 & 100 & 16.09 & 3.14 & 58.37 & 0.0443 & 0.013 & 0.0458 & 0.0114 \\
\hline 0.54 & - & 0.52 & 513 & 513 & 100 & 15.37 & 2.53 & 46.85 & 0.0522 & 0.0167 & 0.054 & 0.0138 \\
\hline 0.52 & - & 0.5 & 583 & 583 & 100 & 14.89 & 1.85 & 33.78 & 0.061 & 0.0232 & 0.0632 & 0.0163 \\
\hline 0.5 & - & 0.49 & 357 & 357 & 100 & 13.62 & 1.59 & 28.85 & 0.0637 & 0.0282 & 0.0661 & 0.0176 \\
\hline 0.49 & - & 0.48 & 356 & 356 & 100 & 12.68 & 1.27 & 23.42 & 0.0637 & 0.0345 & 0.0664 & 0.0183 \\
\hline 0.48 & - & 0.47 & 390 & 390 & 100 & 12.03 & 1.21 & 20.63 & 0.0666 & 0.0384 & 0.0696 & 0.0199 \\
\hline 0.47 & - & 0.46 & 441 & 441 & 100 & 11.84 & 1.19 & 20.76 & 0.0695 & 0.0384 & 0.0727 & 0.0211 \\
\hline 0.46 & - & 0.45 & 473 & 473 & 100 & 10.74 & 0.97 & 17.21 & 0.0798 & 0.0493 & 0.0839 & 0.0253 \\
\hline 0.45 & - & 0.45 & 16 & 25 & 64 & 1.84 & 0.56 & 5.5 & 0.1144 & 0.1815 & 0.1339 & 0.0679 \\
\hline 0.55 & - & 0.45 & 3352 & 3361 & 99.7 & 13.24 & 1.63 & 29.49 & 0.0598 & 0.0275 & 0.0622 & 0.0167 \\
\hline Inf & - & 0.45 & 7467 & 7476 & 99.9 & 17.07 & 10.96 & 101.76 & 0.0454 & 0.0049 & 0.0464 & 0.0092 \\
\hline
\end{tabular}


Table S4.2: Data quality statistics from XPREP of dataset B.

\begin{tabular}{|c|c|c|c|c|c|c|c|c|c|c|c|c|}
\hline \multicolumn{3}{|c|}{ Resolution } & \multirow{2}{*}{$\begin{array}{l}\text { Number } \\
\text { of Data } \\
95\end{array}$} & \multirow{2}{*}{$\begin{array}{l}\text { Theory } \\
95\end{array}$} & \multirow{2}{*}{$\begin{array}{l}\text { Complete- } \\
\text { ness [\%] } \\
100\end{array}$} & \multirow{2}{*}{$\begin{array}{l}\text { Multipli } \\
\text { city } \\
20.37\end{array}$} & \multirow{2}{*}{$\begin{array}{c}\langle I\rangle \\
137.66\end{array}$} & \multirow{2}{*}{$\begin{array}{l}\langle I / \sigma\rangle \\
285.6\end{array}$} & \multirow{2}{*}{$\begin{array}{l}R_{\text {merge }} \\
0.0123\end{array}$} & \multirow{2}{*}{$\begin{array}{l}R_{\text {sigma }} \\
0.0018\end{array}$} & \multirow{2}{*}{$\begin{array}{l}\text { Rrim } \\
0.0126\end{array}$} & \multirow{2}{*}{$\begin{array}{l}R_{\text {pim }} \\
0.003\end{array}$} \\
\hline Inf & - & 2 & & & & & & & & & & \\
\hline 2 & - & 1.2 & 334 & 334 & 100 & 23.23 & 74.76 & 194.7 & 0.0182 & 0.0027 & 0.0186 & 0.004 \\
\hline 1.2 & - & 0.9 & 544 & 544 & 100 & 26.27 & 39.1 & 206.12 & 0.0229 & 0.0027 & 0.0234 & 0.0047 \\
\hline 0.9 & - & 0.76 & 627 & 627 & 100 & 24.62 & 19.77 & 157.92 & 0.0237 & 0.0035 & 0.0242 & 0.005 \\
\hline 0.76 & - & 0.7 & 434 & 434 & 100 & 20.49 & 13.13 & 112.81 & 0.0265 & 0.0054 & 0.0272 & 0.0061 \\
\hline 0.7 & - & 0.64 & 616 & 616 & 100 & 19.31 & 10 & 87.67 & 0.0302 & 0.0076 & 0.0311 & 0.0071 \\
\hline 0.64 & - & 0.6 & 555 & 555 & 100 & 17.88 & 6.68 & 60.97 & 0.0378 & 0.0117 & 0.039 & 0.0093 \\
\hline 0.6 & - & 0.56 & 717 & 717 & 100 & 16.1 & 4.78 & 42.21 & 0.0427 & 0.0172 & 0.0442 & 0.011 \\
\hline 0.56 & - & 0.54 & 438 & 438 & 100 & 14.5 & 3.88 & 34.51 & 0.0426 & 0.0228 & 0.0442 & 0.0114 \\
\hline 0.54 & - & 0.52 & 512 & 512 & 100 & 13.88 & 3.11 & 27.24 & 0.0492 & 0.0298 & 0.0511 & 0.0135 \\
\hline 0.52 & - & 0.5 & 585 & 585 & 100 & 13.59 & 2.33 & 20.21 & 0.0587 & 0.0416 & 0.0611 & 0.0164 \\
\hline 0.5 & - & 0.49 & 358 & 358 & 100 & 13.11 & 2.01 & 16.82 & 0.0658 & 0.0505 & 0.0686 & 0.0187 \\
\hline 0.49 & - & 0.48 & 352 & 352 & 100 & 12.5 & 1.67 & 13.86 & 0.0739 & 0.063 & 0.0772 & 0.0216 \\
\hline 0.48 & - & 0.47 & 392 & 392 & 100 & 12.66 & 1.61 & 13.4 & 0.077 & 0.0671 & 0.0803 & 0.0221 \\
\hline 0.47 & - & 0.46 & 444 & 444 & 100 & 12.06 & 1.52 & 12.35 & 0.0794 & 0.0748 & 0.083 & 0.0233 \\
\hline 0.46 & - & 0.45 & 472 & 472 & 100 & 11.55 & 1.21 & 9.55 & 0.0917 & 0.0964 & 0.0961 & 0.0278 \\
\hline 0.45 & - & 0.45 & 27 & 29 & 93.1 & 4.1 & 2.05 & 8.76 & 0.0614 & 0.1172 & 0.0694 & 0.0305 \\
\hline 0.55 & - & 0.45 & 3373 & 3375 & 99.9 & 12.87 & 2.06 & 17.5 & 0.0626 & 0.0506 & 0.0652 & 0.0178 \\
\hline Inf & - & 0.45 & 7502 & 7504 & 100 & 17.07 & 13.14 & 71.66 & 0.0244 & 0.0075 & 0.0251 & 0.0056 \\
\hline
\end{tabular}

Table S4.3: Data quality statistics from XPREP of dataset C.

\begin{tabular}{|c|c|c|c|c|c|c|c|c|c|c|c|c|}
\hline \multicolumn{3}{|c|}{ Resolution } & \multirow{2}{*}{$\begin{array}{l}\text { Number } \\
\text { of Data } \\
95\end{array}$} & \multirow{2}{*}{$\begin{array}{l}\text { Theory } \\
95\end{array}$} & \multirow{2}{*}{$\begin{array}{l}\text { Complete- } \\
\text { ness [\%] } \\
100\end{array}$} & \multirow{2}{*}{$\begin{array}{l}\text { Multiplicity } \\
14.42\end{array}$} & \multirow{2}{*}{$\begin{array}{c}\langle I\rangle \\
136.87\end{array}$} & \multirow{2}{*}{$\begin{array}{r}\langle I / \sigma\rangle \\
196.31\end{array}$} & \multirow{2}{*}{$\begin{array}{l}\text { Rmerge } \\
0.0172\end{array}$} & \multirow{2}{*}{$\begin{array}{l}\text { Rsigma } \\
0.003\end{array}$} & \multirow{2}{*}{$\begin{array}{l}\text { Rrim } \\
0.0179\end{array}$} & \multirow{2}{*}{$\begin{array}{l}R_{\text {pim }} \\
0.0049\end{array}$} \\
\hline Inf & - & 2 & & & & & & & & & & \\
\hline 2 & - & 1.2 & 334 & 334 & 100 & 18 & 73.59 & 153.5 & 0.0221 & 0.0036 & 0.0228 & 0.0055 \\
\hline 1.2 & - & 0.9 & 542 & 542 & 100 & 23.29 & 38.38 & 136.28 & 0.0278 & 0.0038 & 0.0285 & 0.0059 \\
\hline 0.9 & - & 0.76 & 626 & 626 & 100 & 22.51 & 19.17 & 91.7 & 0.0338 & 0.0056 & 0.0346 & 0.0072 \\
\hline 0.76 & - & 0.7 & 435 & 435 & 100 & 18.69 & 12.83 & 69.78 & 0.0381 & 0.0078 & 0.0392 & 0.0089 \\
\hline 0.7 & - & 0.64 & 607 & 611 & 99.3 & 14.69 & 9.91 & 55.35 & 0.042 & 0.0105 & 0.0435 & 0.0111 \\
\hline 0.64 & - & 0.6 & 551 & 555 & 99.3 & 12.59 & 6.48 & 40.89 & 0.0476 & 0.0147 & 0.0496 & 0.0138 \\
\hline 0.6 & - & 0.56 & 707 & 717 & 98.6 & 11.9 & 4.72 & 30.18 & 0.0603 & 0.0192 & 0.063 & 0.018 \\
\hline 0.56 & - & 0.54 & 434 & 437 & 99.3 & 8.8 & 3.79 & 21.99 & 0.0696 & 0.0284 & 0.0737 & 0.0236 \\
\hline 0.54 & - & 0.52 & 502 & 512 & 98 & 9.29 & 3.18 & 19.43 & 0.0794 & 0.032 & 0.0839 & 0.0265 \\
\hline 0.52 & - & 0.5 & 575 & 583 & 98.6 & 9.69 & 2.34 & 15.66 & 0.0983 & 0.0392 & 0.1037 & 0.0326 \\
\hline 0.5 & - & 0.49 & 347 & 352 & 98.6 & 9.39 & 2 & 13.8 & 0.1057 & 0.0448 & 0.1115 & 0.0352 \\
\hline 0.49 & - & 0.48 & 347 & 354 & 98 & 9.12 & 1.66 & 12.22 & 0.1176 & 0.0524 & 0.1247 & 0.0408 \\
\hline 0.48 & - & 0.47 & 395 & 399 & 99 & 9.37 & 1.6 & 12.06 & 0.1149 & 0.053 & 0.1215 & 0.0391 \\
\hline 0.47 & - & 0.46 & 433 & 442 & 98 & 8.79 & 1.53 & 11.65 & 0.1248 & 0.0567 & 0.1322 & 0.0431 \\
\hline 0.46 & - & 0.45 & 461 & 469 & 98.3 & 8.17 & 1.22 & 8.89 & 0.1393 & 0.0725 & 0.1484 & 0.0502 \\
\hline 0.45 & - & 0.45 & 29 & 45 & 64.4 & 1.02 & 1.61 & 5.21 & 0.0897 & 0.1595 & 0.1231 & 0.0839 \\
\hline 0.55 & - & 0.45 & 3315 & 3385 & 97.9 & 8.91 & 2.06 & 13.82 & 0.1009 & 0.0453 & 0.1069 & 0.0346 \\
\hline Inf & - & 0.45 & 7420 & 7508 & 98.8 & 13.19 & 13.03 & 48.57 & 0.0323 & 0.0086 & 0.0333 & 0.0081 \\
\hline
\end{tabular}


Table S4.4: Data quality statistics from XPREP of dataset $\mathbf{D}$.

\begin{tabular}{|c|c|c|c|c|c|c|c|c|c|c|c|c|}
\hline \multicolumn{3}{|c|}{ Resolution } & \multirow{2}{*}{$\begin{array}{l}\text { Number } \\
\text { of Data } \\
94\end{array}$} & \multirow{2}{*}{$\begin{array}{l}\text { Theory } \\
95\end{array}$} & \multirow{2}{*}{$\begin{array}{l}\text { Complete- } \\
\text { ness [\%] } \\
98.9\end{array}$} & \multirow{2}{*}{$\begin{array}{l}\text { M ultipli } \\
\text { city } \\
21.67\end{array}$} & \multirow{2}{*}{$\begin{array}{c}\langle I\rangle \\
157.86\end{array}$} & \multirow{2}{*}{$\begin{array}{r}\langle I / \sigma\rangle \\
808.65\end{array}$} & \multirow{2}{*}{$\begin{array}{l}R_{\text {merge }} \\
0.0049\end{array}$} & \multirow{2}{*}{$\begin{array}{l}R_{\text {sigma }} \\
0.0007\end{array}$} & \multirow{2}{*}{$\begin{array}{l}R_{\text {rim }} \\
0.005\end{array}$} & \multirow{2}{*}{$\begin{array}{l}R_{\text {pim }} \\
0.0013\end{array}$} \\
\hline Inf & - & 2 & & & & & & & & & & \\
\hline 2 & - & 1.2 & 334 & 334 & 100 & 28.51 & 88.66 & 568.52 & 0.0079 & 0.0009 & 0.0081 & 0.0017 \\
\hline 1.2 & - & 0.9 & 545 & 545 & 100 & 31.3 & 45.31 & 355.89 & 0.0126 & 0.0015 & 0.0128 & 0.0025 \\
\hline 0.9 & - & 0.76 & 629 & 629 & 100 & 32.86 & 22.23 & 221.1 & 0.0184 & 0.0024 & 0.0188 & 0.0035 \\
\hline 0.76 & - & 0.7 & 431 & 431 & 100 & 27.49 & 14.47 & 154.47 & 0.0257 & 0.0036 & 0.0262 & 0.0053 \\
\hline 0.7 & - & 0.64 & 618 & 618 & 100 & 31.5 & 10.94 & 134.05 & 0.0299 & 0.0044 & 0.0305 & 0.0056 \\
\hline 0.64 & - & 0.6 & 557 & 557 & 100 & 31.39 & 7.2 & 98.62 & 0.0383 & 0.0064 & 0.039 & 0.0071 \\
\hline 0.6 & - & 0.56 & 713 & 713 & 100 & 31.12 & 5.07 & 70.6 & 0.0456 & 0.0088 & 0.0464 & 0.0084 \\
\hline 0.56 & - & 0.54 & 440 & 440 & 100 & 30.79 & 4.14 & 58.96 & 0.0512 & 0.011 & 0.0521 & 0.0094 \\
\hline 0.54 & - & 0.52 & 515 & 515 & 100 & 30.03 & 3.34 & 48.65 & 0.0578 & 0.0138 & 0.0588 & 0.0107 \\
\hline 0.52 & - & 0.5 & 585 & 585 & 100 & 28.86 & 2.45 & 36.21 & 0.0658 & 0.0191 & 0.067 & 0.0124 \\
\hline 0.5 & - & 0.49 & 363 & 363 & 100 & 27.02 & 2.11 & 30.74 & 0.073 & 0.0232 & 0.0744 & 0.0141 \\
\hline 0.49 & - & 0.48 & 350 & 350 & 100 & 26.88 & 1.76 & 25.66 & 0.0831 & 0.0281 & 0.0847 & 0.0163 \\
\hline 0.48 & - & 0.47 & 391 & 391 & 100 & 25.49 & 1.68 & 23.18 & 0.0869 & 0.0316 & 0.0887 & 0.0175 \\
\hline 0.47 & - & 0.46 & 445 & 445 & 100 & 24.39 & 1.58 & 21.36 & 0.092 & 0.035 & 0.094 & 0.0190 \\
\hline 0.46 & - & 0.45 & 471 & 471 & 100 & 23.42 & 1.34 & 17.48 & 0.105 & 0.0428 & 0.1074 & 0.0221 \\
\hline 0.45 & - & 0.45 & 22 & 25 & 88 & 4.36 & 2.18 & 11.62 & 0.0939 & 0.0865 & 0.1024 & 0.0383 \\
\hline 0.55 & - & 0.45 & 3375 & 3378 & 99.9 & 26.83 & 2.18 & 31.19 & 0.0713 & 0.0235 & 0.0727 & 0.0139 \\
\hline Inf & - & 0.45 & 7503 & 7507 & 99.9 & 28.96 & 14.95 & 129.68 & 0.0219 & 0.0036 & 0.0223 & 0.0043 \\
\hline
\end{tabular}

Table S4.5: D ata quality statistics from XPREP of dataset $\mathbf{E}$.

\begin{tabular}{|c|c|c|c|c|c|c|c|c|c|c|c|c|}
\hline \multicolumn{3}{|c|}{ Resolution } & \multirow{2}{*}{$\begin{array}{l}\text { N umber } \\
\text { of Data } \\
92\end{array}$} & \multirow{2}{*}{$\begin{array}{l}\text { Theory } \\
95\end{array}$} & \multirow{2}{*}{$\begin{array}{l}\text { Complete- } \\
\text { ness [\%] } \\
96.8\end{array}$} & \multirow{2}{*}{$\begin{array}{l}\text { Multiplicity } \\
11.49\end{array}$} & \multirow{2}{*}{$\begin{array}{c}\langle I\rangle \\
132.82\end{array}$} & \multirow{2}{*}{$\begin{array}{r}\langle I / \sigma\rangle \\
161.72\end{array}$} & \multirow{2}{*}{$\begin{array}{l}R_{\text {merge }} \\
0.0143\end{array}$} & \multirow{2}{*}{$\begin{array}{l}R_{\text {sigma }} \\
0.0052\end{array}$} & \multirow{2}{*}{$\begin{array}{l}\text { R } \operatorname{rim} \\
0.0157\end{array}$} & \multirow{2}{*}{$\begin{array}{l}R_{\text {pim }} \\
0.0063\end{array}$} \\
\hline Inf & - & 2 & & & & & & & & & & \\
\hline 2 & - & 1.2 & 334 & 334 & 100 & 20.04 & 74.76 & 202.35 & 0.0193 & 0.0039 & 0.0204 & 0.0063 \\
\hline 1.2 & - & 0.9 & 544 & 544 & 100 & 24.57 & 39.96 & 219.61 & 0.0216 & 0.0032 & 0.0223 & 0.0053 \\
\hline 0.9 & - & 0.76 & 625 & 625 & 100 & 25.69 & 20.31 & 191.71 & 0.0222 & 0.0028 & 0.0227 & 0.0045 \\
\hline 0.76 & - & 0.7 & 434 & 434 & 100 & 22.63 & 13.69 & 139.98 & 0.0252 & 0.0041 & 0.0258 & 0.0054 \\
\hline 0.7 & - & 0.64 & 615 & 615 & 100 & 19.92 & 10.49 & 108.14 & 0.0264 & 0.0055 & 0.0271 & 0.006 \\
\hline 0.64 & - & 0.6 & 552 & 552 & 100 & 17.73 & 7.03 & 76.86 & 0.03 & 0.0083 & 0.0309 & 0.0073 \\
\hline 0.6 & - & 0.56 & 717 & 717 & 100 & 16.58 & 5.14 & 54.99 & 0.0348 & 0.0115 & 0.0359 & 0.0088 \\
\hline 0.56 & - & 0.54 & 433 & 433 & 100 & 15.96 & 4.16 & 44.21 & 0.0404 & 0.0147 & 0.0418 & 0.0104 \\
\hline 0.54 & - & 0.52 & 513 & 513 & 100 & 14.78 & 3.42 & 35.96 & 0.0459 & 0.0186 & 0.0476 & 0.0123 \\
\hline 0.52 & - & 0.5 & 591 & 591 & 100 & 13.85 & 2.59 & 27.06 & 0.055 & 0.0253 & 0.0571 & 0.0153 \\
\hline 0.5 & - & 0.49 & 359 & 359 & 100 & 13.01 & 2.16 & 22.17 & 0.0611 & 0.0312 & 0.0636 & 0.0175 \\
\hline 0.49 & - & 0.48 & 346 & 346 & 100 & 12.75 & 1.82 & 19.1 & 0.0681 & 0.0375 & 0.0709 & 0.0197 \\
\hline 0.48 & - & 0.47 & 398 & 398 & 100 & 12.42 & 1.77 & 17.97 & 0.0705 & 0.0401 & 0.0736 & 0.0209 \\
\hline 0.47 & - & 0.46 & 443 & 443 & 100 & 12.31 & 1.69 & 17.27 & 0.0764 & 0.0429 & 0.0797 & 0.0225 \\
\hline 0.46 & - & 0.45 & 468 & 470 & 99.6 & 11.47 & 1.39 & 13.66 & 0.0891 & 0.0541 & 0.0932 & 0.0271 \\
\hline 0.45 & - & 0.45 & 8 & 23 & 34.8 & 1.13 & 1.8 & 7.93 & 0.1389 & 0.108 & 0.163 & 0.0848 \\
\hline 0.55 & - & 0.45 & 3353 & 3370 & 99.5 & 13.13 & 2.27 & 23.61 & 0.0586 & 0.0302 & 0.061 & 0.0166 \\
\hline Inf & - & 0.45 & 7472 & 7492 & 99.7 & 17.15 & 13.41 & 83.01 & 0.0256 & 0.0064 & 0.0265 & 0.0065 \\
\hline
\end{tabular}


Table S4.6: D ata quality statistics from XPREP of dataset $\mathbf{F}$.

\begin{tabular}{|c|c|c|c|c|c|c|c|c|c|c|c|c|}
\hline \multicolumn{3}{|c|}{ Resolution } & \multirow{2}{*}{$\begin{array}{l}\text { Number } \\
\text { of Data } \\
90\end{array}$} & \multirow{2}{*}{$\begin{array}{l}\text { Theory } \\
95\end{array}$} & \multirow{2}{*}{$\begin{array}{l}\text { Complete- } \\
\text { ness [\%] } \\
94.7\end{array}$} & \multirow{2}{*}{$\begin{array}{l}\text { M ultipli } \\
\text { city } \\
14.45\end{array}$} & \multirow{2}{*}{$\begin{array}{c}\langle I\rangle \\
124.61\end{array}$} & \multirow{2}{*}{$\begin{array}{r}\langle I / \sigma\rangle \\
160.41\end{array}$} & \multirow{2}{*}{$\begin{array}{l}R_{\text {merge }} \\
0.0244\end{array}$} & \multirow{2}{*}{$\begin{array}{l}R_{\text {sigma }} \\
0.0049\end{array}$} & \multirow{2}{*}{$\begin{array}{l}R_{\text {rim }} \\
0.0261\end{array}$} & \multirow{2}{*}{$\begin{array}{l}R_{\text {pim }} \\
0.0086\end{array}$} \\
\hline Inf & - & 2 & & & & & & & & & & \\
\hline 2 & - & 1.2 & 334 & 334 & 100 & 21.63 & 64.77 & 201.11 & 0.0233 & 0.0033 & 0.0241 & 0.006 \\
\hline 1.2 & - & 0.9 & 545 & 545 & 100 & 23.74 & 33.79 & 166.02 & 0.0241 & 0.0032 & 0.0246 & 0.0051 \\
\hline 0.9 & - & 0.76 & 627 & 627 & 100 & 22.88 & 16.74 & 98.86 & 0.0302 & 0.0053 & 0.0309 & 0.0064 \\
\hline 0.76 & - & 0.7 & 434 & 434 & 100 & 21.6 & 11.31 & 74.04 & 0.0353 & 0.0077 & 0.0362 & 0.0077 \\
\hline 0.7 & - & 0.64 & 615 & 615 & 100 & 20.03 & 8.37 & 56.18 & 0.0416 & 0.0105 & 0.0427 & 0.0095 \\
\hline 0.64 & - & 0.6 & 557 & 557 & 100 & 18.48 & 5.55 & 38.85 & 0.0553 & 0.016 & 0.0568 & 0.0131 \\
\hline 0.6 & - & 0.56 & 714 & 714 & 100 & 16.66 & 3.96 & 26.8 & 0.0669 & 0.0227 & 0.0691 & 0.0169 \\
\hline 0.56 & - & 0.54 & 439 & 439 & 100 & 14.97 & 3.23 & 21.94 & 0.0744 & 0.029 & 0.077 & 0.0197 \\
\hline 0.54 & - & 0.52 & 517 & 517 & 100 & 13.91 & 2.57 & 17.33 & 0.0838 & 0.0372 & 0.087 & 0.0232 \\
\hline 0.52 & - & 0.5 & 582 & 582 & 100 & 13.09 & 1.9 & 12.83 & 0.1062 & 0.0509 & 0.1106 & 0.0305 \\
\hline 0.5 & - & 0.49 & 362 & 362 & 100 & 12.47 & 1.64 & 11.16 & 0.1173 & 0.06 & 0.1224 & 0.0345 \\
\hline 0.49 & - & 0.48 & 353 & 353 & 100 & 12.14 & 1.35 & 9.42 & 0.1386 & 0.0723 & 0.1447 & 0.0414 \\
\hline 0.48 & - & 0.47 & 390 & 390 & 100 & 11.83 & 1.28 & 8.7 & 0.1504 & 0.0801 & 0.1573 & 0.0458 \\
\hline 0.47 & - & 0.46 & 445 & 445 & 100 & 11.68 & 1.2 & 8.31 & 0.1592 & 0.0857 & 0.1666 & 0.0488 \\
\hline 0.46 & - & 0.45 & 471 & 472 & 99.8 & 10.65 & 0.99 & 6.61 & 0.1806 & 0.1111 & 0.1897 & 0.0577 \\
\hline 0.45 & - & 0.45 & 2 & 3 & 66.7 & 1.33 & 3.25 & 7.21 & 0.0724 & 0.0978 & 0.1024 & 0.0724 \\
\hline 0.55 & - & 0.45 & 3354 & 3356 & 99.9 & 12.47 & 1.68 & 11.44 & 0.1145 & 0.0595 & 0.1193 & 0.0335 \\
\hline Inf & - & 0.45 & 7477 & 7484 & 99.9 & 16.67 & 11.26 & 51.51 & 0.0346 & 0.0095 & 0.0356 & 0.0085 \\
\hline
\end{tabular}

Table S4.7: Data quality statistics from XPREP of dataset G.

\begin{tabular}{|c|c|c|c|c|c|c|c|c|c|c|c|c|}
\hline \multicolumn{3}{|c|}{ Resolution } & \multirow{2}{*}{$\begin{array}{l}\text { Number } \\
\text { of Data } \\
92\end{array}$} & \multirow{2}{*}{$\begin{array}{l}\text { Theory } \\
94\end{array}$} & \multirow{2}{*}{$\begin{array}{l}\text { Complete- } \\
\text { ness [\%] } \\
97.9\end{array}$} & \multirow{2}{*}{$\begin{array}{l}\text { Multiplicity } \\
17.01\end{array}$} & \multirow{2}{*}{$\begin{array}{c}\langle I\rangle \\
140.02\end{array}$} & \multirow{2}{*}{$\begin{array}{r}\langle I / \sigma\rangle \\
233.74\end{array}$} & \multirow{2}{*}{$\begin{array}{l}R_{\text {merge }} \\
0.0115\end{array}$} & \multirow{2}{*}{$\begin{array}{l}R_{\text {sigma }} \\
0.0028\end{array}$} & \multirow{2}{*}{$\begin{array}{l}\text { R }_{\text {rim }} \\
\text { Inf }\end{array}$} & \multirow{2}{*}{$\begin{array}{l}R_{\text {pim }} \\
-\end{array}$} \\
\hline Inf & - & 2 & & & & & & & & & & \\
\hline 2 & - & 1.2 & 334 & 334 & 100 & 23.69 & 80.43 & 221.38 & 0.0167 & 0.0027 & 2 & - \\
\hline 1.2 & - & 0.9 & 543 & 543 & 100 & 26.12 & 43.2 & 183.24 & 0.0227 & 0.0031 & 1.2 & - \\
\hline 0.9 & - & 0.76 & 626 & 626 & 100 & 22.6 & 22.11 & 122.76 & 0.0332 & 0.0043 & 0.9 & - \\
\hline 0.76 & - & 0.7 & 434 & 434 & 100 & 19.43 & 14.91 & 93.88 & 0.0402 & 0.0061 & 0.76 & - \\
\hline 0.7 & - & 0.64 & 609 & 609 & 100 & 15.06 & 11.7 & 75.33 & 0.0345 & 0.0081 & 0.7 & - \\
\hline 0.64 & - & 0.6 & 554 & 554 & 100 & 11.84 & 7.54 & 51.02 & 0.0341 & 0.0127 & 0.64 & - \\
\hline 0.6 & - & 0.56 & 716 & 716 & 100 & 9.92 & 5.55 & 35.88 & 0.0369 & 0.0182 & 0.6 & - \\
\hline 0.56 & - & 0.54 & 435 & 435 & 100 & 9.38 & 4.51 & 28.77 & 0.0395 & 0.0236 & 0.56 & - \\
\hline 0.54 & - & 0.52 & 514 & 514 & 100 & 8.79 & 3.71 & 23.21 & 0.0471 & 0.0304 & 0.54 & - \\
\hline 0.52 & - & 0.5 & 582 & 582 & 100 & 8.49 & 2.79 & 17.1 & 0.0591 & 0.0417 & 0.52 & - \\
\hline 0.5 & - & 0.49 & 358 & 358 & 100 & 7.99 & 2.38 & 14.16 & 0.0649 & 0.0517 & 0.5 & - \\
\hline 0.49 & - & 0.48 & 355 & 355 & 100 & 8.11 & 1.98 & 12.29 & 0.0768 & 0.0606 & 0.49 & - \\
\hline 0.48 & - & 0.47 & 393 & 393 & 100 & 7.62 & 1.93 & 11.54 & 0.0804 & 0.0655 & 0.48 & - \\
\hline 0.47 & - & 0.46 & 443 & 443 & 100 & 7.32 & 1.9 & 11.14 & 0.0854 & 0.0709 & 0.47 & - \\
\hline 0.46 & - & 0.45 & 461 & 464 & 99.4 & 7.27 & 1.53 & 9.1 & 0.0991 & 0.0871 & 0.46 & - \\
\hline 0.55 & - & 0.45 & 3330 & 3336 & 99.8 & 8.07 & 2.47 & 15.15 & 0.0638 & 0.0493 & 0.55 & - \\
\hline Inf & - & 0.45 & 7450 & 7458 & 99.9 & 13.14 & 14.52 & 63.07 & 0.0258 & 0.0082 & Inf & - \\
\hline Inf & - & 2 & 92 & 94 & 97.9 & 17.01 & 140.02 & 233.74 & 0.0115 & 0.0028 & Inf & - \\
\hline
\end{tabular}


Table S4.8: Local coordinate system for the charge density refinement in XD and M oPro.

\begin{tabular}{|c|c|c|c|c|c|c|c|c|c|}
\hline ATOM & ATOM 0 & $\mathrm{AX1}$ & ATOM 1 & ATOM 2 & $A \times 2$ & GC-level & K-set & SITESYM & CHEMCON \\
\hline $\mathrm{Se}(1)$ & DUM 0 & Z & $\mathrm{Se}(1)$ & $C(1)$ & Y & 4 & 1 & $\mathrm{mZ} \rightarrow \mathrm{NO}$ & \\
\hline$C(1)$ & $\mathrm{Se}(1)$ & Z & $C(1)$ & $C(2)$ & Y & 2 & 2 & mZ & \\
\hline$C(2)$ & $C(1)$ & Z & $C(2)$ & $C(3)$ & Y & 2 & 3 & _mXmY $2 Z$ & \\
\hline$C(3)$ & $C(6)$ & Z & $C(3)$ & $C(2)$ & Y & 2 & 3 & $m X m Y 2 Z$ & \\
\hline$C(4)$ & $C(7)$ & Z & $C(4)$ & $C(3)$ & Y & 2 & 3 & mXmY $2 Z$ & $C(3)$ \\
\hline$C(5)$ & $C(2)$ & Z & $C(5)$ & $C(4)$ & Y & 2 & 3 & _mXmY $2 Z$ & $C(3)$ \\
\hline$C(6)$ & $C(3)$ & Z & $C(6)$ & $C(7)$ & Y & 2 & 3 & _mXmY $2 Z$ & $C(3)$ \\
\hline$C(7)$ & $C(4)$ & Z & $C(7)$ & $C(2)$ & Y & 2 & 3 & $m X m Y 2 Z$ & $C(3)$ \\
\hline$H(1 A)$ & $C(1)$ & Z & $H(1 A)$ & $H(1 B)$ & Y & 1 & 4 & _cy & \\
\hline$H(1 B)$ & $C(1)$ & Z & $H(1 B)$ & $H(1 A)$ & Y & 1 & 4 & _cy & $H(1 A)$ \\
\hline$H(3)$ & $C(3)$ & Z & $H(3)$ & $C(2)$ & Y & 1 & 4 & _cy & \\
\hline$H(4)$ & $C(4)$ & Z & $H(4)$ & $C(5)$ & Y & 1 & 4 & _cy & $H(3)$ \\
\hline$H(5)$ & $C(5)$ & Z & $H(5)$ & $C(4)$ & Y & 1 & 4 & _cy & $H(3)$ \\
\hline$H(6)$ & $C(6)$ & Z & $H(6)$ & $C(7)$ & Y & 1 & 4 & _cy & $H(3)$ \\
\hline$H(7)$ & $C(7)$ & Z & $H(7)$ & $C(6)$ & Y & 1 & 4 & _cy & $H(3)$ \\
\hline
\end{tabular}

Table S4.9: XD refinement strategy for dataset A. A bbreviations: M P: Multipole, M: monopoles; D: dipoles; Q: quadrupoles; O: octupoles; H: hexadecapoles, K: $\kappa$, U2, U3, U4: Gram Charlier $2^{\text {nd }}, 3^{\text {rd }}$ and $4^{\text {th }}$ order, HXYZ: hydrogen position against data up to $0.5 \sin (\theta) / \lambda$.

\begin{tabular}{|c|c|c|c|c|c|c|c|c|c|}
\hline Step & New Parameter & $D<0.5 \sin ($ th $) / l$ & \#M P-Param & Data & Para & Data/Para & $\mathrm{R}\left(\mathrm{F}^{2}\right)$ & $\mathrm{WR}\left(\mathrm{F}^{2}\right)$ & GOF \\
\hline 1 & SCALE & 644 & 0 & 7032 & 1 & 7032 & 0.033 & 0.041 & 8.619 \\
\hline 2 & $\mathrm{DQOH}$ & 644 & 46 & 7032 & 47 & 149.6 & 0.026 & 0.030 & 6.247 \\
\hline 3 & U2 & 644 & 46 & 7032 & 95 & 74 & 0.025 & 0.028 & 5.937 \\
\hline 4 & к & 644 & 46 & 7032 & 98 & 71.8 & 0.025 & 0.028 & 5.874 \\
\hline 5 & $X Y Z$ & 644 & 46 & 7032 & 122 & 57.6 & 0.025 & 0.027 & 5.723 \\
\hline 6 & M & 644 & 52 & 7032 & 127 & 55.4 & 0.024 & 0.027 & 5.691 \\
\hline 7 & $H-X Y Z$ & 644 & 0 & 643 & 22 & 29.2 & 0.024 & 0.026 & 10.137 \\
\hline 8 & all prior & 644 & 52 & 7032 & 127 & 55.4 & 0.024 & 0.027 & 5.662 \\
\hline 9 & U3(Se) & 644 & 0 & 7032 & 11 & 639.3 & 0.025 & 0.027 & 5.625 \\
\hline 10 & all prior + U3(Se) & 644 & 52 & 7032 & 137 & 51.3 & 0.025 & 0.027 & 5.660 \\
\hline 11 & $\mathrm{U} 4(\mathrm{Se})$ & 644 & 0 & 7032 & 16 & 439.5 & 0.024 & 0.025 & 5.322 \\
\hline 12 & all prior $+U 4(\mathrm{Se})$ & 644 & 52 & 7032 & 152 & 46.3 & 0.023 & 0.024 & 5.138 \\
\hline 13 & Se NoSymm & 644 & 62 & 7032 & 162 & 43.4 & 0.021 & 0.023 & 4.938 \\
\hline 14 & $\kappa^{\prime}$ & 644 & 0 & 7032 & 4 & 1758 & 0.021 & 0.023 & 4.867 \\
\hline 15 & all prior & 644 & 62 & 7032 & 162 & 43.4 & 0.021 & 0.023 & 4.901 \\
\hline
\end{tabular}


Table S4.10: XD refinement strategy for dataset B. Abbreviations: M P: M ultipole, M: monopoles; D: dipoles; Q: quadrupoles; O: octupoles; H: hexadecapoles, K: $\kappa$, U2, U3, U4: Gram Charlier $2^{\text {nd }}$, $3^{\text {rd }}$ and $4^{\text {th }}$ order, HXYZ: hydrogen position against data up to $0.5 \sin (\theta) / \lambda$.

\begin{tabular}{|c|c|c|c|c|c|c|c|c|c|}
\hline Step & New Parameter & $D<0.5 \sin ($ th) $/ /$ & \#M P-Param & Data & Para & Data/Para & $\mathrm{R}\left(\mathrm{F}^{2}\right)$ & $w R\left(F^{2}\right)$ & GOF \\
\hline 1 & SCALE & 644 & 0 & 6719 & 1 & 6719 & 0.0351 & 0.0449 & 7.085 \\
\hline 2 & $\mathrm{DQOH}$ & 644 & 46 & 6719 & 47 & 143 & 0.0301 & 0.0329 & 5.216 \\
\hline 3 & U2 & 644 & 46 & 6719 & 95 & 70.7 & 0.0214 & 0.0242 & 3.841 \\
\hline 4 & к & 644 & 46 & 6719 & 98 & 68.6 & 0.0168 & 0.0197 & 3.127 \\
\hline 5 & $X Y Z$ & 644 & 46 & 6719 & 122 & 55.1 & 0.0159 & 0.0176 & 2.805 \\
\hline 6 & M & 644 & 52 & 6719 & 127 & 52.9 & 0.0157 & 0.0172 & 2.744 \\
\hline 7 & $H-X Y Z$ & 644 & 0 & 644 & 22 & 29.3 & 0.0154 & 0.0178 & 5.696 \\
\hline 8 & all prior & 644 & 52 & 6719 & 127 & 52.9 & 0.0154 & 0.0167 & 2.666 \\
\hline 9 & U3(Se) & 644 & 0 & 6719 & 11 & 610.8 & 0.0151 & 0.0165 & 2.611 \\
\hline 10 & all prior $+\mathrm{U} 3(\mathrm{Se})$ & 644 & 52 & 6719 & 137 & 49 & 0.0148 & 0.0161 & 2.57 \\
\hline 11 & U $4(\mathrm{Se})$ & 644 & 0 & 6719 & 16 & 419.9 & 0.0141 & 0.0153 & 2.41 \\
\hline 12 & all prior $+\mathrm{U} 4(\mathrm{Se})$ & 644 & 52 & 6719 & 152 & 44.2 & 0.0124 & 0.0133 & 2.13 \\
\hline 13 & Se NoSymm & 644 & 62 & 6719 & 162 & 41.5 & 0.0119 & 0.0129 & 2.057 \\
\hline 14 & $\kappa^{\prime}$ & 644 & 0 & 6719 & 4 & 1679.8 & 0.0119 & 0.0128 & 2.021 \\
\hline 15 & all prior & 644 & 62 & 6719 & 162 & 41.5 & 0.0118 & 0.0127 & 2.027 \\
\hline
\end{tabular}

Table S4.11: XD refinement strategy for dataset C. Abbreviations: M P: M ultipole, M: monopoles; D: dipoles; Q: quadrupoles; O: octupoles; H: hexadecapoles, K: $\kappa$, U2, U3, U4: Gram Charlier $2^{\text {nd }}, 3^{\text {rd }}$ and $4^{\text {th }}$ order, HXYZ: hydrogen position against data up to $0.5 \sin (\theta) / \lambda$.

\begin{tabular}{llllllllll}
\hline Step & New Parameter & D $<0.5 \sin ($ th)/l & \#M P-Param & Data & Para & Data/Para & $R\left(F^{2}\right)$ & wR $\left(F^{2}\right)$ & $G O F$ \\
1 & SCALE & 644 & 0 & 6774 & 1 & 6774 & 0.0329 & 0.0430 & 3.750 \\
2 & DQOH & 644 & 46 & 6774 & 47 & 144.1 & 0.0254 & 0.0279 & 2.442 \\
3 & U2 & 644 & 46 & 6774 & 95 & 71.3 & 0.0148 & 0.0182 & 1.595 \\
4 & $\kappa$ & 644 & 46 & 6774 & 98 & 69.1 & 0.0143 & 0.0173 & 1.521 \\
5 & XYZ & 644 & 46 & 6774 & 122 & 55.5 & 0.0137 & 0.0163 & 1.436 \\
6 & M & 644 & 52 & 6774 & 127 & 53.3 & 0.0128 & 0.0154 & 1.355 \\
7 & $\mathrm{H}-\mathrm{XYZ}$ & 644 & 0 & 644 & 22 & 29.3 & 0.0125 & 0.0140 & 3.156 \\
8 & all prior & 644 & 52 & 6774 & 127 & 53.3 & 0.0125 & 0.0147 & 1.296 \\
9 & U3(Se) & 644 & 0 & 6774 & 11 & 615.8 & 0.0124 & 0.0146 & 1.274 \\
10 & all prior +U3(Se) & 644 & 52 & 6774 & 137 & 49.5 & 0.0123 & 0.0145 & 1.275 \\
11 & U4(Se) & 644 & 0 & 6774 & 16 & 423.4 & 0.0119 & 0.0143 & 1.244 \\
12 & all prior +U4(Se) & 644 & 52 & 6774 & 152 & 44.6 & 0.0115 & 0.0137 & 1.204 \\
13 & Se NoSymm & 644 & 62 & 6774 & 162 & 41.8 & 0.0111 & 0.0132 & 1.162 \\
14 & $\kappa$ & 644 & 0 & 6774 & 4 & 1693.5 & 0.0111 & 0.0131 & 1.139 \\
15 & all prior & 644 & 62 & 6774 & 162 & 41.8 & 0.0109 & 0.0130 & 1.144 \\
\hline
\end{tabular}


Table S4.12: XD refinement strategy for dataset $\mathbf{D}$. A bbreviations: M P: M ultipole, M : monopoles; D: dipoles; Q: quadrupoles; O: octupoles; H: hexadecapoles, K: $\kappa$, U2, U3, U4: Gram Charlier $2^{\text {nd }}$, $3^{\text {rd }}$ and $4^{\text {th }}$ order, HXYZ: hydrogen position against data up to $0.5 \sin (\theta) / \lambda$.

\begin{tabular}{|c|c|c|c|c|c|c|c|c|c|}
\hline Step & New Parameter & $D<0.5 \sin ($ th) $/ /$ & \#M P-Param & Data & Para & Data/Para & $\mathrm{R}\left(\mathrm{F}^{2}\right)$ & $w R\left(F^{2}\right)$ & GOF \\
\hline 1 & SCALE & 644 & 0 & 6952 & 1 & 6952 & 0.061 & 0.0747 & 22.062 \\
\hline 2 & $\mathrm{DQOH}$ & 644 & 46 & 6952 & 47 & 147.9 & 0.0544 & 0.0599 & 17.754 \\
\hline 3 & U2 & 644 & 46 & 6952 & 95 & 73.2 & 0.0289 & 0.0336 & 9.988 \\
\hline 4 & к & 644 & 46 & 6952 & 98 & 70.9 & 0.0187 & 0.0258 & 7.675 \\
\hline 5 & $X Y Z$ & 644 & 46 & 6952 & 122 & 57 & 0.0181 & 0.0243 & 7.227 \\
\hline 6 & M & 644 & 52 & 6952 & 127 & 54.7 & 0.0178 & 0.0239 & 7.134 \\
\hline 7 & $H-X Y Z$ & 643 & 0 & 643 & 22 & 29.2 & 0.0177 & 0.0245 & 19.845 \\
\hline 8 & all prior & 644 & 52 & 6952 & 127 & 54.7 & 0.0178 & 0.0235 & 6.997 \\
\hline 9 & U3(Se) & 644 & 0 & 6952 & 11 & 632 & 0.0176 & 0.0233 & 6.902 \\
\hline 10 & all prior $+\mathrm{U} 3(\mathrm{Se})$ & 644 & 52 & 6952 & 137 & 50.7 & 0.0175 & 0.0231 & 6.885 \\
\hline 11 & U $4(\mathrm{Se})$ & 644 & 0 & 6952 & 16 & 434.5 & 0.0166 & 0.0227 & 6.723 \\
\hline 12 & all prior $+\mathrm{U} 4(\mathrm{Se})$ & 644 & 52 & 6952 & 152 & 45.7 & 0.0159 & 0.0206 & 6.151 \\
\hline 13 & Se NoSymm & 644 & 62 & 6952 & 162 & 42.9 & 0.015 & 0.0196 & 5.871 \\
\hline 14 & $\mathrm{~K}^{\prime}$ & 644 & 0 & 6952 & 4 & 1738 & 0.015 & 0.0193 & 5.697 \\
\hline 15 & all prior & 644 & 62 & 6952 & 162 & 42.9 & 0.0143 & 0.0189 & 5.636 \\
\hline
\end{tabular}

Table S4.13: XD refinement strategy for dataset E. A bbreviations: M P: M ultipole, M : monopoles; D: dipoles; Q: quadrupoles; O: octupoles; H: hexadecapoles, K: $\kappa$, U2, U3, U4: Gram Charlier $2^{\text {nd }}, 3^{\text {rd }}$ and $4^{\text {th }}$ order, HXYZ: hydrogen position against data up to $0.5 \sin (\theta) / \lambda$.

\begin{tabular}{|c|c|c|c|c|c|c|c|c|c|}
\hline Step & N ew Parameter & $D<0.5 \sin ($ th $) / l$ & \#M P-Param & Data & Para & Data/Para & $\mathrm{R}\left(\mathrm{F}^{2}\right)$ & $w R\left(F^{2}\right)$ & GOF \\
\hline 1 & SCALE & 643 & 0 & 6777 & 1 & 6777.0 & 0.0305 & 0.0445 & 7.303 \\
\hline 2 & $\mathrm{DQOH}$ & 643 & 46 & 6777 & 47 & 144.2 & 0.0255 & 0.0255 & 4.208 \\
\hline 3 & U2 & 643 & 46 & 6777 & 95 & 71.3 & 0.0200 & 0.0218 & 3.606 \\
\hline 4 & $\kappa$ & 643 & 46 & 6777 & 98 & 69.2 & 0.0152 & 0.0189 & 3.123 \\
\hline 5 & $X Y Z$ & 643 & 46 & 6777 & 122 & 55.5 & 0.0147 & 0.0165 & 2.730 \\
\hline 6 & M & 643 & 52 & 6777 & 127 & 53.4 & 0.0142 & 0.0154 & 2.560 \\
\hline 7 & $H-X Y Z$ & 643 & 0 & 643 & 22 & 29.2 & 0.0141 & 0.0186 & 5.142 \\
\hline 8 & all prior & 643 & 52 & 6777 & 127 & 53.4 & 0.0141 & 0.0151 & 2.495 \\
\hline 9 & U3(Se) & 643 & 0 & 6777 & 11 & 616.1 & 0.0138 & 0.0148 & 2.438 \\
\hline 10 & all prior $+\mathrm{U} 3(\mathrm{Se})$ & 643 & 52 & 6777 & 137 & 49.5 & 0.0136 & 0.0144 & 2.387 \\
\hline 11 & U $4(\mathrm{Se})$ & 643 & 0 & 6777 & 16 & 423.6 & 0.0130 & 0.0139 & 2.284 \\
\hline 12 & all prior $+\cup 4(\mathrm{Se})$ & 643 & 52 & 6777 & 152 & 44.6 & 0.0124 & 0.0132 & 2.187 \\
\hline 13 & Se NoSymm & 643 & 62 & 6777 & 162 & 41.8 & 0.0121 & 0.0128 & 2.132 \\
\hline 14 & $\kappa^{\prime}$ & 643 & 0 & 6777 & 4 & 1694.2 & 0.0122 & 0.0128 & 2.104 \\
\hline 15 & all prior & 643 & 62 & 6777 & 162 & 41.8 & 0.0121 & 0.0128 & 2.123 \\
\hline
\end{tabular}


Table S4.14: XD refinement strategy for dataset F. Abbreviations: M P: M ultipole, M: monopoles; D: dipoles; Q: quadrupoles; O: octupoles; H: hexadecapoles, K: $\kappa$, U2, U3, U4: Gram Charlier $2^{\text {nd }}$, $3^{\text {rd }}$ and $4^{\text {th }}$ order, HXYZ: hydrogen position against data up to $0.5 \sin (\theta) / \lambda$.

\begin{tabular}{|c|c|c|c|c|c|c|c|c|c|}
\hline Step & New Parameter & $D<0.5 \sin ($ th $) / l$ & \#M P-Param & Data & Para & Data/Para & $\mathrm{R}\left(\mathrm{F}^{2}\right)$ & $w R\left(F^{2}\right)$ & GOF \\
\hline 1 & SCALE & 640 & 0 & 6660 & 1 & 6660.0 & 0.0333 & 0.0448 & 5.213 \\
\hline 2 & $\mathrm{DQOH}$ & 640 & 46 & 6660 & 47 & 141.7 & 0.0277 & 0.0283 & 3.308 \\
\hline 3 & U2 & 640 & 46 & 6660 & 95 & 70.1 & 0.0243 & 0.0245 & 2.878 \\
\hline 4 & к & 640 & 46 & 6660 & 98 & 68.0 & 0.0171 & 0.0199 & 2.337 \\
\hline 5 & $X Y Z$ & 640 & 46 & 6660 & 122 & 54.6 & 0.0166 & 0.0181 & 2.131 \\
\hline 6 & M & 640 & 52 & 6660 & 127 & 52.4 & 0.0163 & 0.0163 & 1.915 \\
\hline 7 & $H-X Y Z$ & 640 & 0 & 640 & 22 & 29.1 & 0.0163 & 0.0158 & 4.417 \\
\hline 8 & all prior & 640 & 52 & 6660 & 127 & 52.4 & 0.0163 & 0.0160 & 1.877 \\
\hline 9 & U3(Se) & 640 & 0 & 6660 & 11 & 605.5 & 0.0161 & 0.0158 & 1.843 \\
\hline 10 & all prior $+\mathrm{U} 3(\mathrm{Se})$ & 640 & 52 & 6660 & 137 & 48.6 & 0.0159 & 0.0155 & 1.826 \\
\hline 11 & U $4(\mathrm{Se})$ & 640 & 0 & 6660 & 16 & 416.2 & 0.0156 & 0.0153 & 1.787 \\
\hline 12 & all prior $+\cup 4(\mathrm{Se})$ & 640 & 52 & 6660 & 152 & 43.8 & 0.0151 & 0.0150 & 1.766 \\
\hline 13 & Se NoSymm & 640 & 62 & 6660 & 162 & 41.1 & 0.0145 & 0.0144 & 1.694 \\
\hline 14 & $\kappa^{\prime}$ & 640 & 0 & 6660 & 4 & 1665.0 & 0.0145 & 0.0143 & 1.661 \\
\hline 15 & all prior & 640 & 62 & 6660 & 162 & 41.1 & 0.0142 & 0.0142 & 1.669 \\
\hline
\end{tabular}

Table S4.15: XD refinement strategy for dataset G. Abbreviations: M P: M ultipole, M: monopoles; D: dipoles; Q: quadrupoles; O: octupoles; H: hexadecapoles, K: $\kappa$, U2, U3, U4: Gram Charlier $2^{\text {nd }}, 3^{\text {rd }}$ and $4^{\text {th }}$ order, HXYZ: hydrogen position against data up to $0.5 \sin (\theta) / \lambda$.

\begin{tabular}{|c|c|c|c|c|c|c|c|c|c|}
\hline Step & N ew Parameter & $D<0.5 \sin ($ th $) / l$ & \#M P-Param & Data & Para & Data/Para & $\mathrm{R}\left(\mathrm{F}^{2}\right)$ & $w R\left(F^{2}\right)$ & GOF \\
\hline 1 & SCALE & 644 & 0 & 6866 & 1 & 6866 & 0.0309 & 0.0464 & 6.043 \\
\hline 2 & $\mathrm{DQOH}$ & 644 & 46 & 6866 & 47 & 146.1 & 0.0241 & 0.0289 & 3.772 \\
\hline 3 & U2 & 644 & 46 & 6866 & 95 & 72.3 & 0.016 & 0.0213 & 2.789 \\
\hline 4 & $\kappa$ & 644 & 46 & 6866 & 98 & 70.1 & 0.0157 & 0.0204 & 2.67 \\
\hline 5 & $X Y Z$ & 644 & 46 & 6866 & 122 & 56.3 & 0.0155 & 0.0196 & 2.576 \\
\hline 6 & M & 644 & 52 & 6866 & 127 & 54.1 & 0.0148 & 0.0175 & 2.297 \\
\hline 7 & $H-X Y Z$ & 644 & 0 & 644 & 22 & 29.3 & 0.0146 & 0.0167 & 4.941 \\
\hline 8 & all prior & 644 & 52 & 6866 & 127 & 54.1 & 0.0145 & 0.0168 & 2.213 \\
\hline 9 & U3(Se) & 644 & 0 & 6866 & 11 & 624.2 & 0.0144 & 0.0167 & 2.178 \\
\hline 10 & all prior $+\mathrm{U} 3(\mathrm{Se})$ & 644 & 52 & 6866 & 137 & 50.1 & 0.0143 & 0.0166 & 2.183 \\
\hline 11 & U $4(\mathrm{Se})$ & 644 & 0 & 6866 & 16 & 429.1 & 0.0135 & 0.016 & 2.091 \\
\hline 12 & all prior $+\cup 4(\mathrm{Se})$ & 644 & 52 & 6866 & 152 & 45.2 & 0.0133 & 0.0158 & 2.075 \\
\hline 13 & Se NoSymm & 644 & 62 & 6866 & 162 & 42.4 & 0.0129 & 0.0153 & 2.017 \\
\hline 14 & $\kappa^{\prime}$ & 644 & 0 & 6866 & 4 & 1716.5 & 0.0128 & 0.0152 & 1.98 \\
\hline 15 & all prior & 644 & 62 & 6866 & 162 & 42.4 & 0.0127 & 0.0151 & 1.992 \\
\hline
\end{tabular}




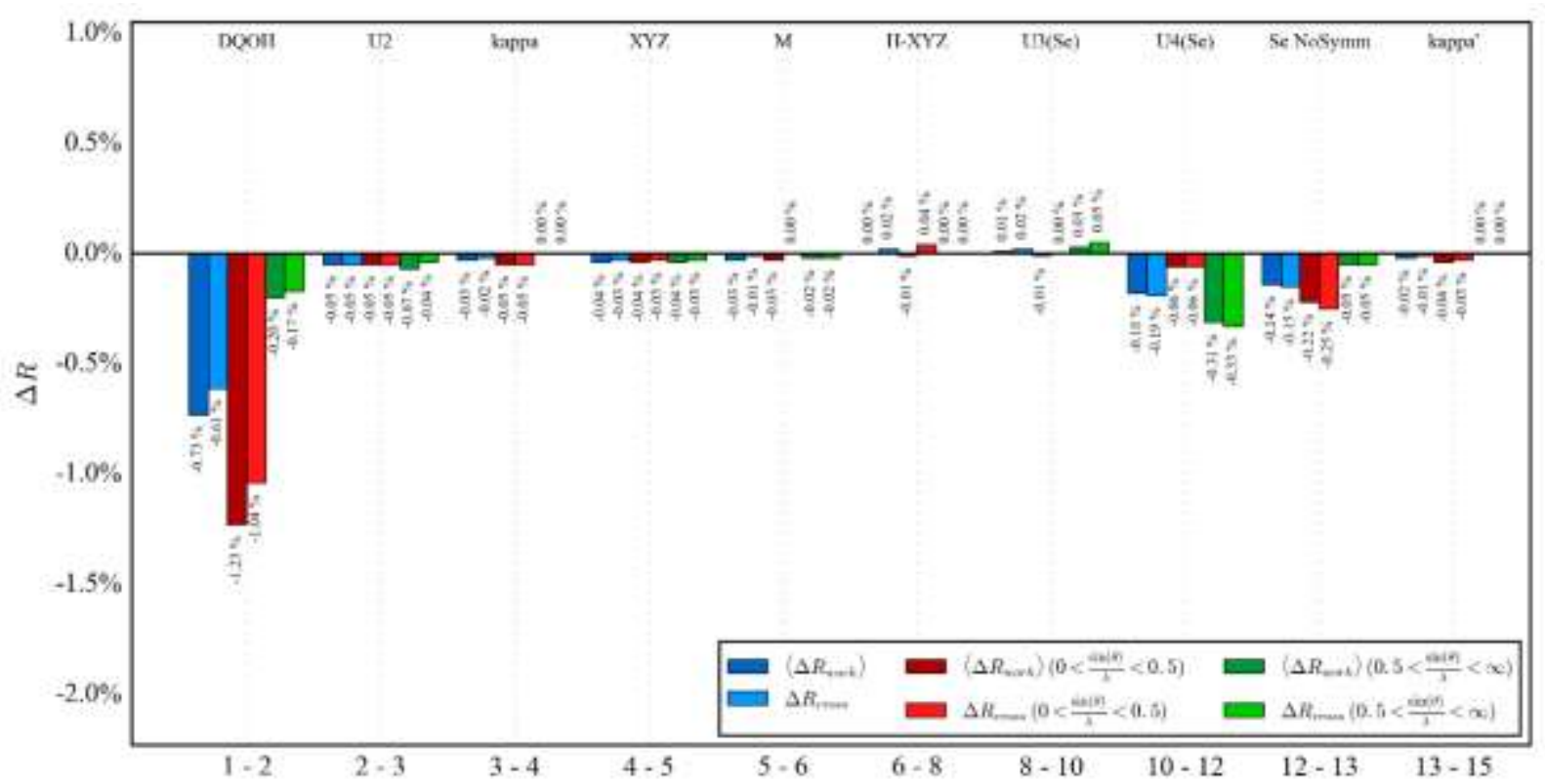

Figure S4.1: $\Delta \mathrm{R}_{\text {cross }}$ values for the XD refinement of dataset $\mathbf{A}$. A bbreviations: $\mathrm{M}$ : monopoles; D: dipoles; Q: quadrupoles; O: octupoles; $\mathrm{H}$ : hexadecapoles, U2, U 3, U 4: Gram Charlier $2^{\text {nd }}, 3^{\text {rd }}$ and $4^{\text {th }}$ order, HXYZ: hydrogen position against data up to $0.5 \sin (\theta) / \lambda$.

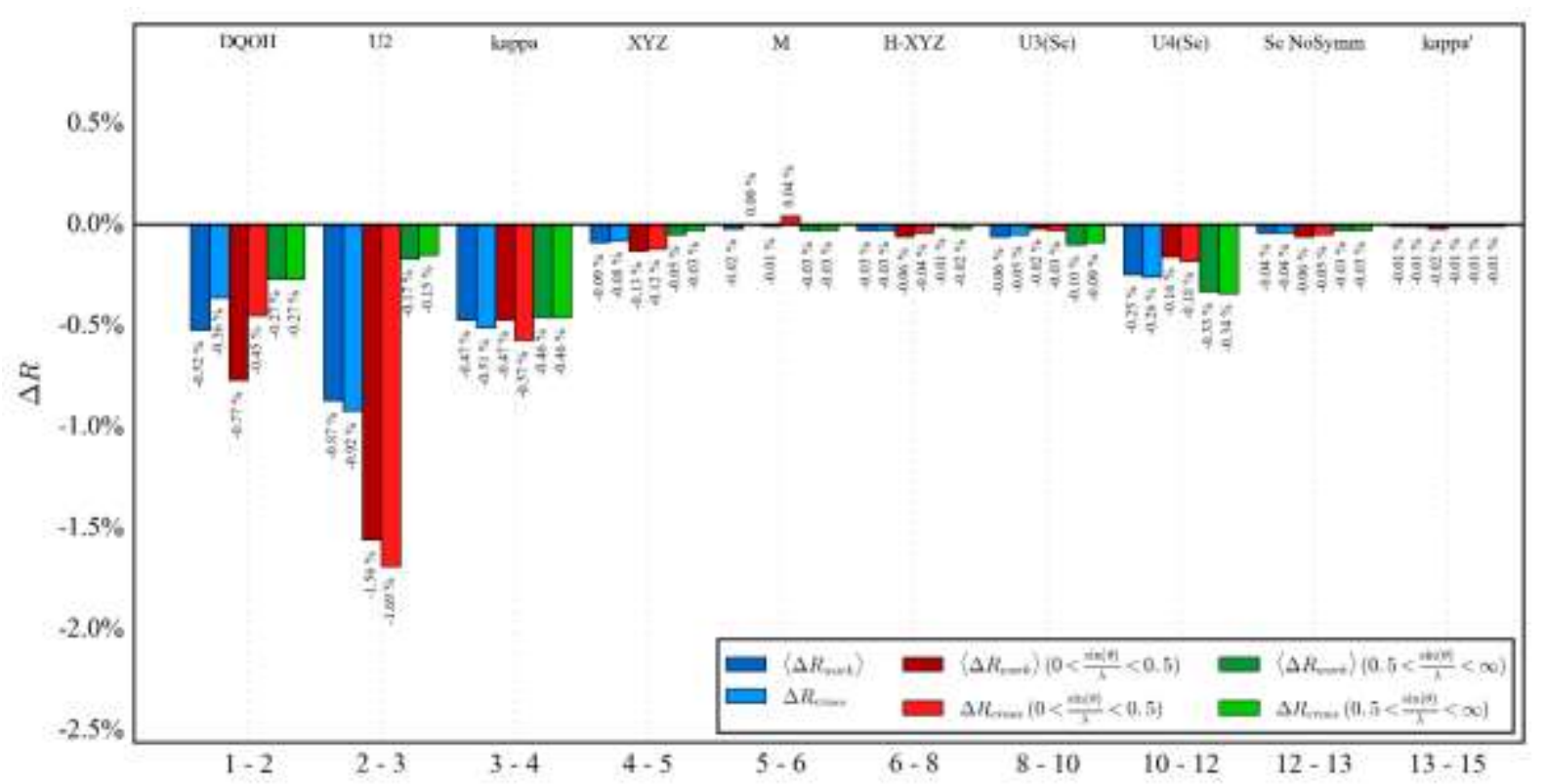

Figure S4.2: $\Delta \mathrm{R}_{\text {cross }}$ values for the $X D$ refinement of dataset $\mathbf{B}$. Abbreviations: $M$ : monopoles; $D$ : dipoles; $Q$ : quadrupoles; O: octupoles; $\mathrm{H}$ : hexadecapoles, U2, U 3, U 4: Gram Charlier $2^{\text {nd }}, 3^{\text {rd }}$ and $4^{\text {th }}$ order, $\mathrm{HXYZ \text {: }}$ hydrogen position against data up to $0.5 \sin (\theta) / \lambda$. 


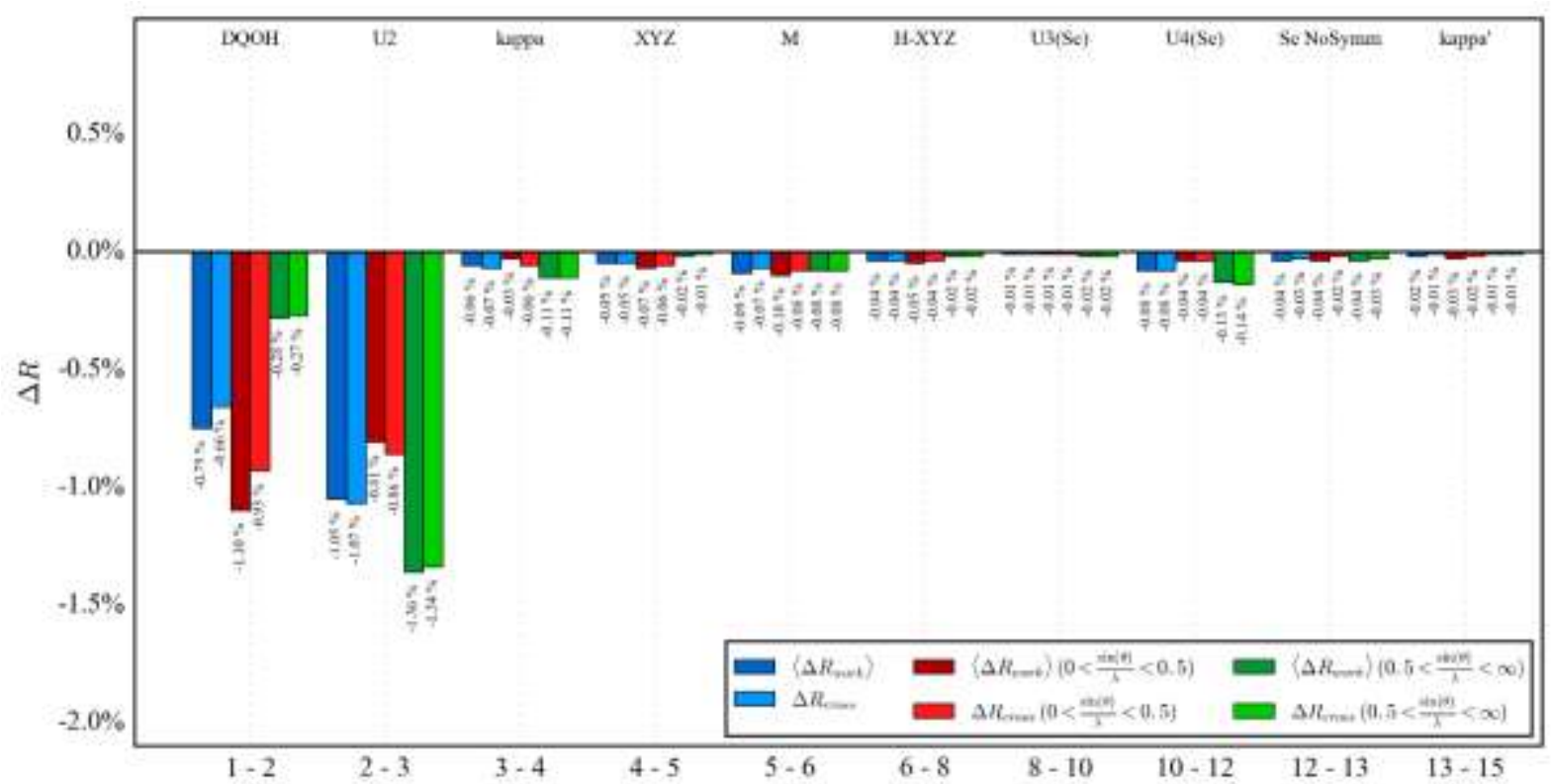

Figure S4.3: $\Delta \mathrm{R}_{\text {cross }}$ values for the $\mathrm{XD}$ refinement of dataset $\mathbf{C}$. Abbreviations: $\mathrm{M}$ : monopoles; $\mathrm{D}$ : dipoles; $\mathrm{Q}$ : quadrupoles; O: octupoles; $\mathrm{H}$ : hexadecapoles, U2, U 3, U4: Gram Charlier $2^{\text {nd }}, 3^{\text {rd }}$ and $4^{\text {th }}$ order, $\mathrm{HXYZ \text {: }}$ hydrogen position against data up to $0.5 \sin (\theta) / \lambda$.

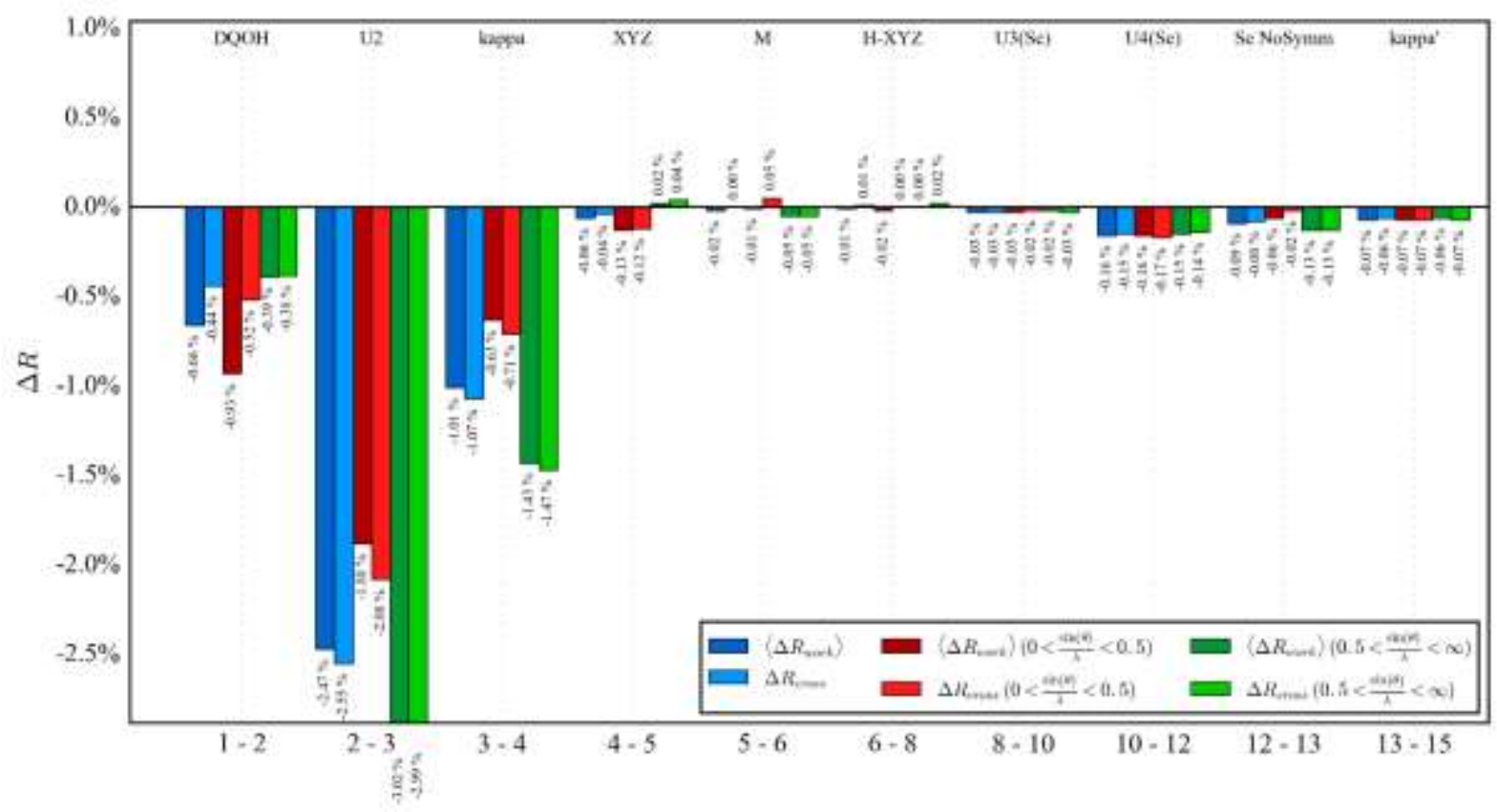

Figure S4.4: $\Delta R_{\text {cross }}$ values for the $X D$ refinement of dataset $\mathbf{D}$. Abbreviations: $M$ : monopoles; $D$ : dipoles; $Q$ : quadrupoles; O: octupoles; $\mathrm{H}$ : hexadecapoles, U 2, U 3, U 4: Gram Charlier $2^{\text {nd }}, 3^{\text {rd }}$ and $4^{\text {th }}$ order, HXYZ: hydrogen position against data up to $0.5 \sin (\theta) / \lambda$. 


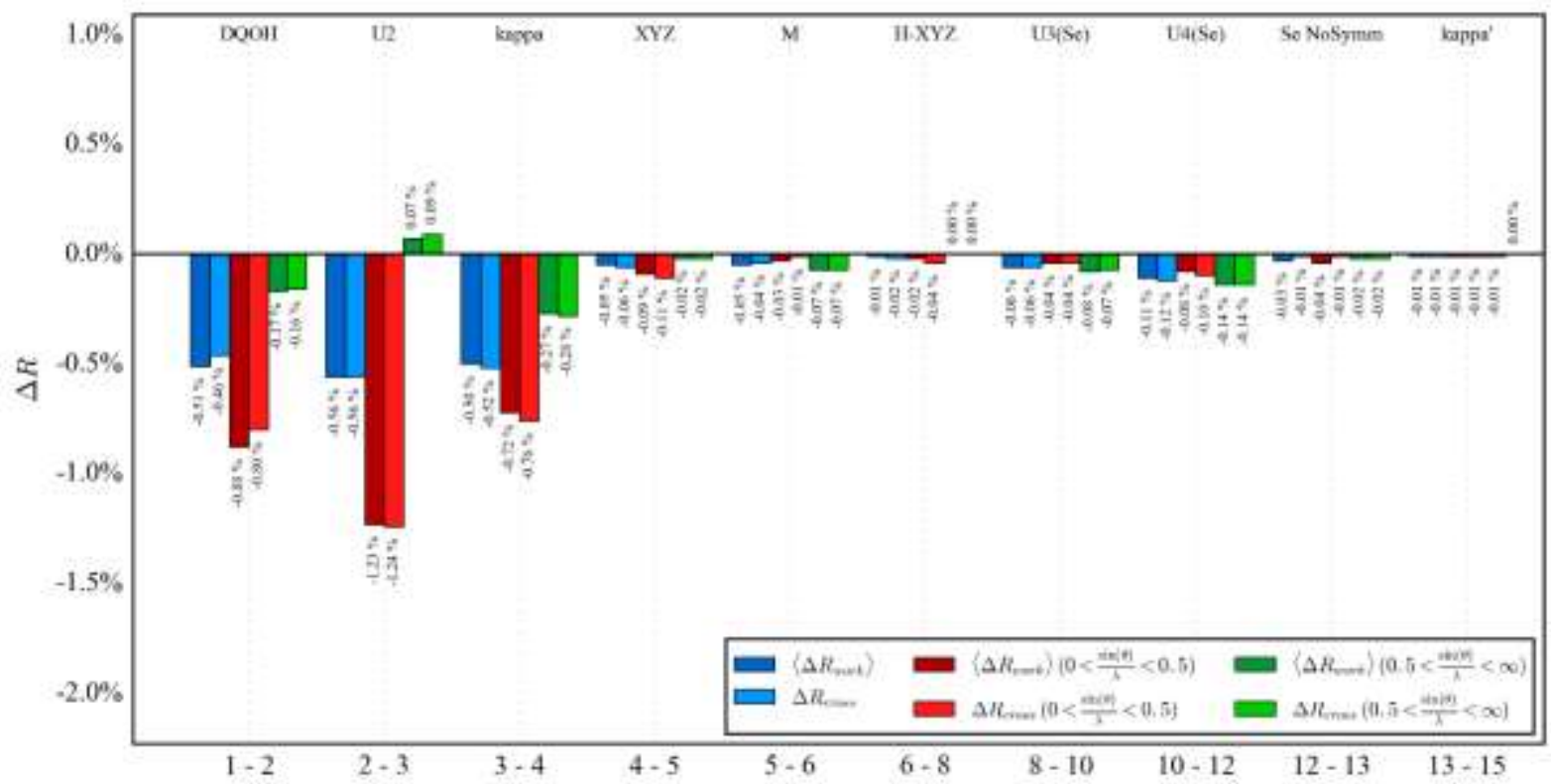

Figure S4.5: $\Delta R_{\text {cross }}$ values for the $X D$ refinement of dataset $\mathbf{E}$. Abbreviations: $M$ : monopoles; $D$ : dipoles; $Q$ : quadrupoles; O: octupoles; $\mathrm{H}$ : hexadecapoles, U2, U3, U4: Gram Charlier $2^{\text {nd }}, 3^{\text {rd }}$ and $4^{\text {th }}$ order, HXYZ: hydrogen position against data up to $0.5 \sin (\theta) / \lambda$.

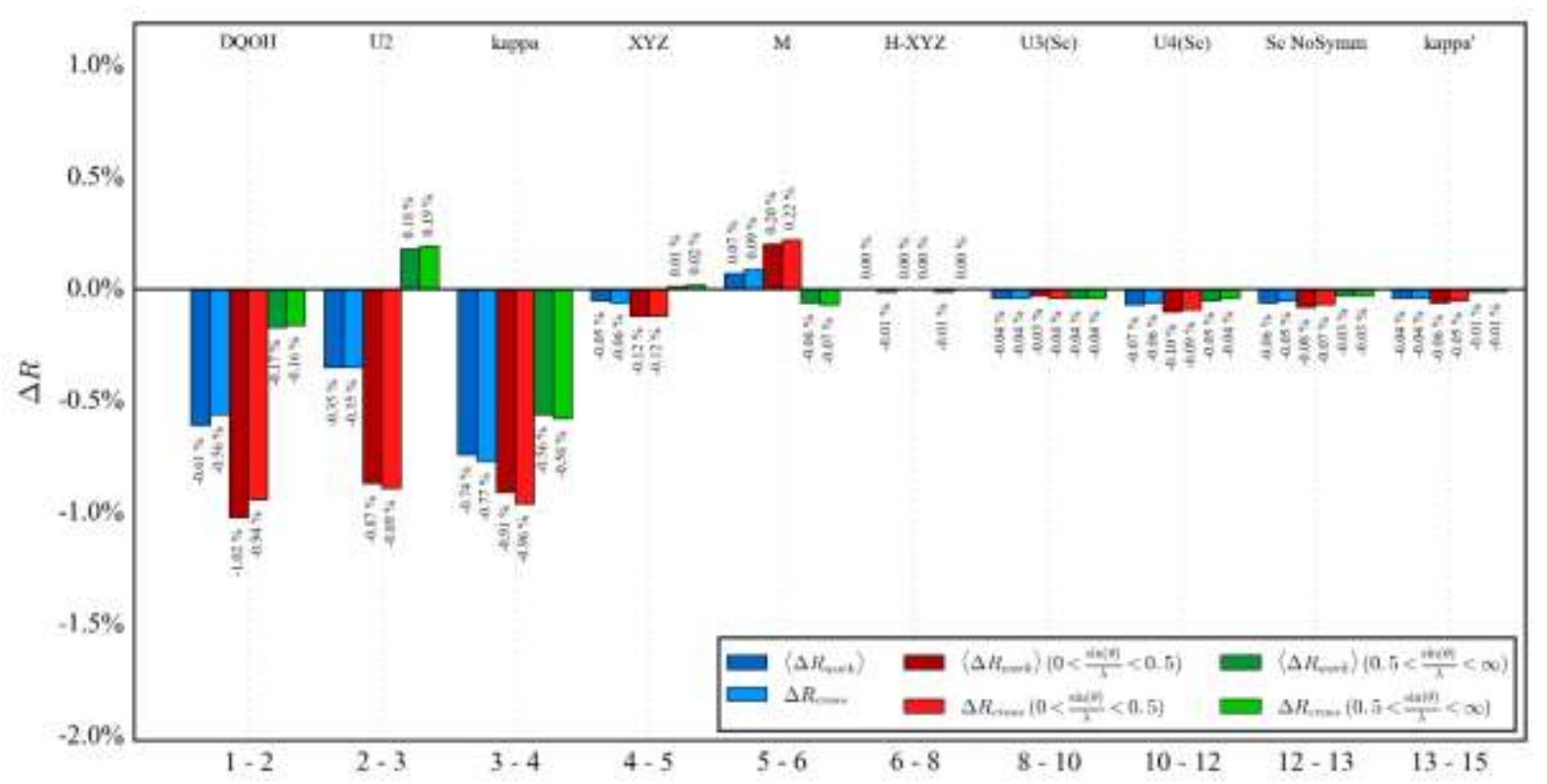

Figure S4.6: $\Delta \mathrm{R}_{\text {cross }}$ values for the $X D$ refinement of dataset $\mathbf{F}$. Abbreviations: $M$ : monopoles; $D$ : dipoles; $Q$ : quadrupoles; O: octupoles; $\mathrm{H}$ : hexadecapoles, U2, U3, U4: Gram Charlier $2^{\text {nd }}, 3^{\text {rd }}$ and $4^{\text {th }}$ order, HXYZ: hydrogen position against data up to $0.5 \sin (\theta) / \lambda$. 


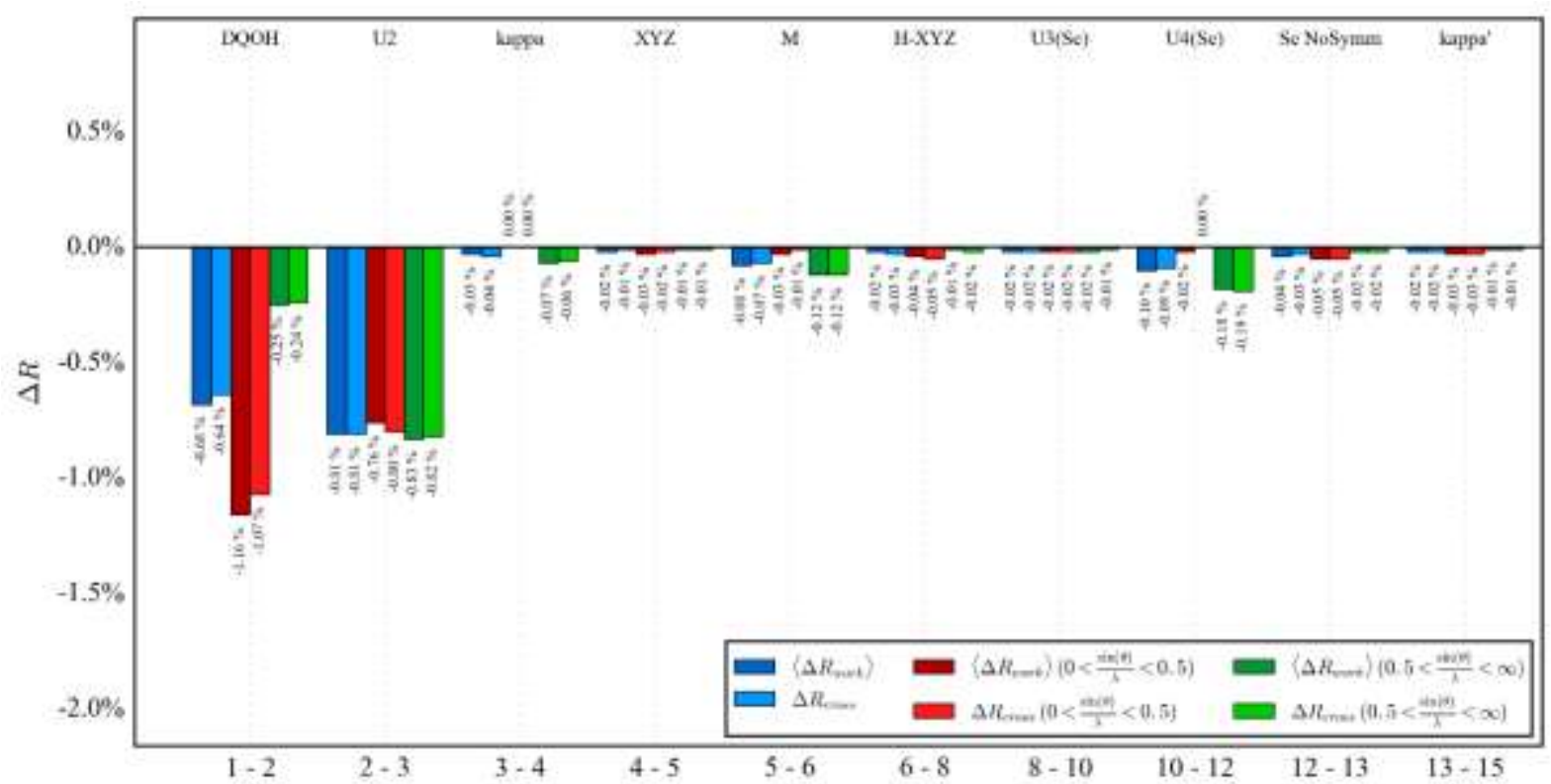

Figure S4.7: $\Delta \mathrm{R}_{\text {cross }}$ values for the $X D$ refinement of dataset $\mathbf{G}$. Abbreviations: $M$ : monopoles; $D$ : dipoles; $Q$ : quadrupoles; O: octupoles; $\mathrm{H}$ : hexadecapoles, U2, U3, U4: Gram Charlier $2^{\text {nd }}, 3^{\text {rd }}$ and $4^{\text {th }}$ order, HXYZ: hydrogen position against data up to $0.5 \sin (\theta) / \lambda$.

Table S4.16: Consistency check for the refinement of Gram Charlier parameters of $3^{\text {rd }}$ and $4^{\text {th }}$ order at Se(1) in XD. Analysis of the Probability Density Function (PDF). Extreme displacements in the map from the equilibrium position. $\Delta \mathrm{X} / \mathrm{Y} / \mathrm{Z}=-0.80$ to $0.80 \AA$. For Datasets $\mathbf{B}$ to $\mathbf{F}$, the minimum PSD value and integrated volume for negative probability are reasonably low, while for dataset $\mathbf{A}$, the values are slightly elevated. In order to retain comparability, anharmonic motion was refined anyway.

\begin{tabular}{|c|c|c|c|c|c|c|}
\hline Set & $\begin{array}{l}\text { M inimum } \\
\text { PDF value }\end{array}$ & $\begin{array}{l}\text { Maximum } \\
\text { PDF Value }\end{array}$ & $\begin{array}{l}\text { Total integrated } \\
\text { negative } \\
\text { probability [\%] }\end{array}$ & $\begin{array}{l}\text { Integrated volume } \\
\text { for negative } \\
\text { probability }\left[\AA^{-3}\right]\end{array}$ & $\begin{array}{l}\text { Total integrated } \\
\text { positive } \\
\text { probability [\%] }\end{array}$ & $\begin{array}{l}\text { Integrated volume } \\
\text { for positive } \\
\left.\text { probability [ } \AA^{-3}\right]\end{array}$ \\
\hline A & -159.77 & 53291.07 & -0.23 & 1.79 & 100.23 & 2.50 \\
\hline B & -33.65 & 60841.29 & -0.02 & 0.58 & 100.02 & 3.72 \\
\hline C & -0.04 & 33440.04 & 0.00 & 0.73 & 100.00 & 3.56 \\
\hline D & 0.00 & 56247.16 & 0.00 & 0.00 & 100.00 & 4.29 \\
\hline $\mathbf{E}$ & -6.81 & 63790.20 & 0.00 & 0.57 & 100.00 & 3.72 \\
\hline $\mathbf{F}$ & -15.72 & 55020.99 & -0.01 & 0.68 & 100.01 & 3.62 \\
\hline G & -48.6 & 64549.91 & -0.022 & 0.45 & 100.02 & 3.84 \\
\hline
\end{tabular}


Table S4.17: Significance check for the refinement of Gram Charlier parameters of $3^{\text {rd }}$ and $4^{\text {th }}$ order at Se(1) in XD. Gram-Charlier parameter divided by their error - in order to besignificant, values need to be larger than $3 \sigma$.

\begin{tabular}{lllllllllll}
\hline \multirow{2}{*}{ Dataset } & $\mathrm{C} 111 / \sigma$ & $\mathrm{C} 222 / \sigma$ & $\mathrm{C} 333 / \sigma$ & $\mathrm{C} 112 / \sigma$ & $\mathrm{C} 122 / \sigma$ & $\mathrm{C} 113 / \sigma$ & $\mathrm{C} 133 / \sigma$ & $\mathrm{C} 223 / \sigma$ & $\mathrm{C} 233 / \sigma$ & $\mathrm{C} 123 / \sigma$ \\
& $\mathrm{D} 1111 / \sigma$ & $\mathrm{D} 2222 / \sigma$ & $\mathrm{D} 3333 / \sigma$ & $\mathrm{D} 1112 / \sigma$ & $\mathrm{D} 1222 / \sigma$ & $\mathrm{D} 1113 / \sigma$ & $\mathrm{D} 1333 / \sigma$ & $\mathrm{D} 2223 / \sigma$ & $\mathrm{D} 2333 / \sigma$ & $\mathrm{D} 1122 / \sigma$ \\
& $\mathrm{D} 1133 / \sigma$ & $\mathrm{D} 2233 / \sigma$ & $\mathrm{D} 1123 / \sigma$ & $\mathrm{D} 1223 / \sigma$ & $\mathrm{D} 1233 / \sigma$ & & & & & \\
& 3.8 & 7.1 & 0.5 & 0.1 & 4.9 & 1.5 & 2.7 & 0.4 & 2.4 & 2.0 \\
& 3.0 & 11.1 & 14.3 & 4.0 & 4.4 & 3.0 & 3.0 & 2.7 & 3.8 & 5.5 \\
& 6.0 & 5.3 & 2.5 & 29.0 & 2.5 & & & & & \\
& 13.0 & 2.2 & 13.5 & 0.8 & 17.4 & 21.0 & 15.5 & 8.2 & 1.0 & 2.0 \\
& 2.0 & 10.0 & 44.5 & 7.5 & 3.4 & $\mathrm{inf}$ & 4.0 & 4.3 & 8.0 & 21.0 \\
& 2.0 & 18.5 & 7.0 & 9.0 & 5.5 & & & & & \\
& 4.7 & 4.8 & 0.0 & 1.5 & 11.0 & 4.5 & 8.0 & 2.5 & 4.0 & 2.0 \\
& 3.0 & 16.8 & 6.3 & 1.5 & 3.4 & 8.0 & 12.0 & 0.3 & 0.0 & 0.5 \\
& 8.0 & 9.0 & 5.0 & 7.0 & 6.0 & & & & & \\
& 9.5 & 1.43 & 3.43 & 0.67 & 6.75 & 5.67 & 7 & 2.89 & 2.44 & 0.5 \\
& 12.5 & 21.61 & 25.25 & 4.5 & 5.11 & 4 & 14 & 4.82 & 7 & 11 \\
& 17 & 21.25 & 4.5 & 4.5 & 9 & & & & & \\
E & 11.0 & 4.0 & 6.3 & 1.0 & 15.8 & 12.0 & 17.0 & 3.4 & 1.0 & 2.0 \\
& 1.0 & 22.7 & 12.0 & 5.0 & 5.0 & inf & 0.0 & 2.2 & 5.0 & 6.0 \\
& 3.0 & 4.0 & 3.0 & 5.0 & 8.0 & & & & & \\
F & 9.0 & 4.2 & 0.8 & 0.2 & 11.0 & 5.0 & 7.5 & 1.5 & 1.7 & 1.5 \\
& 9.0 & 2.6 & 11.7 & 2.5 & 5.2 & 1.0 & 6.0 & 2.0 & 3.7 & 1.5 \\
& 4.0 & 3.0 & 2.0 & 1.0 & 4.5 & & & & & \\
G & 3.5 & 7.6 & 4.5 & 1.3 & 4.2 & 0.5 & 0.5 & 1.7 & 3.0 & 1.0 \\
& 19.0 & 12.3 & 7.5 & 1.5 & 4.0 & 1.0 & 2.0 & 5.7 & 0.7 & 0.0 \\
& 3.0 & 2.0 & 3.0 & 5.0 & 5.0 & & & & & \\
\hline & & & & & & & & & &
\end{tabular}

Table S4.18: For the anharmonic refinement of Se(1), Kuhs's rule (Kuhs, 1992) should be fulfilled. The used resolution is $\sin (\theta) / \lambda=1.12 \AA^{-1}$, therefore Kuhs's rule is only fulfilled for dataset $\mathbf{C}$. Anharmonic motion of $\mathrm{Se}(1)$ was refined anyway for all datasets in order to retain comparability.

\begin{tabular}{llllll}
\hline Dataset & \multicolumn{2}{c}{ Principal M.D.A's $(\mathrm{A})$} & & \multicolumn{2}{c}{ M in. resolution $\left[\AA^{-1}\right]$} \\
& & & & $\mathbf{n}=\mathbf{3}$ & $\mathbf{n = 4}$ \\
A & 0.129 & 0.118 & 0.096 & 1.14 & 1.32 \\
B & 0.136 & 0.118 & 0.095 & 1.12 & 1.3 \\
C & 0.153 & 0.139 & 0.115 & 0.96 & 1.11 \\
D & 0.145 & 0.124 & 0.104 & 1.05 & 1.22 \\
E & 0.132 & 0.111 & 0.092 & 1.17 & 1.35 \\
F & 0.134 & 0.118 & 0.097 & 1.12 & 1.3 \\
G & 0.131 & 0.110 & 0.094 & 1.17 & 1.35 \\
\hline
\end{tabular}



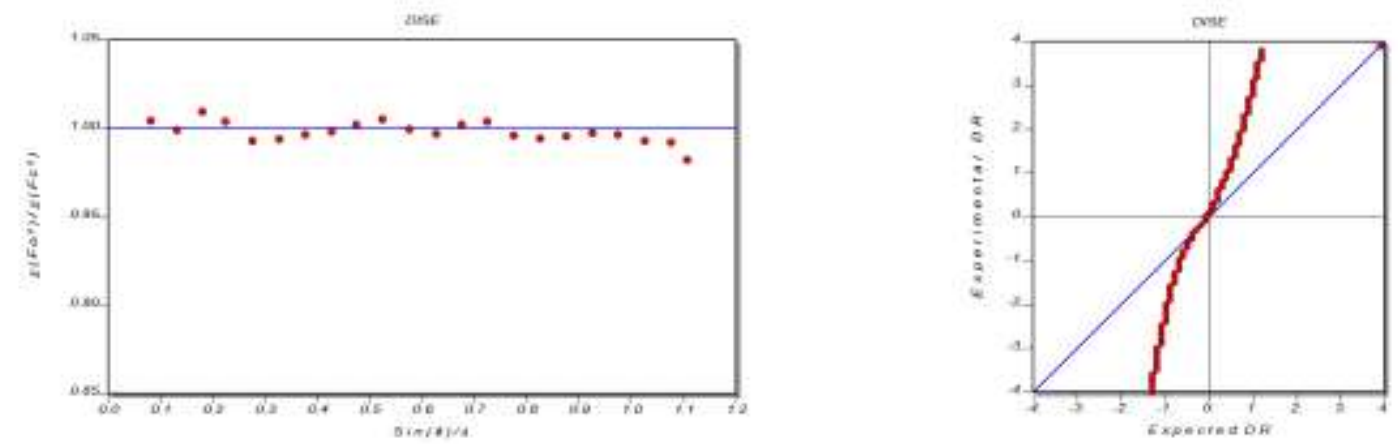

Figure S4.8: Resolution dependent error and Quantile/Quantile DRK-Plots (Zhurov et al., 2008; Zavodnik et al., 1999; A dam Stash, 2007) for the XD refinement of dataset $\mathbf{A}$.
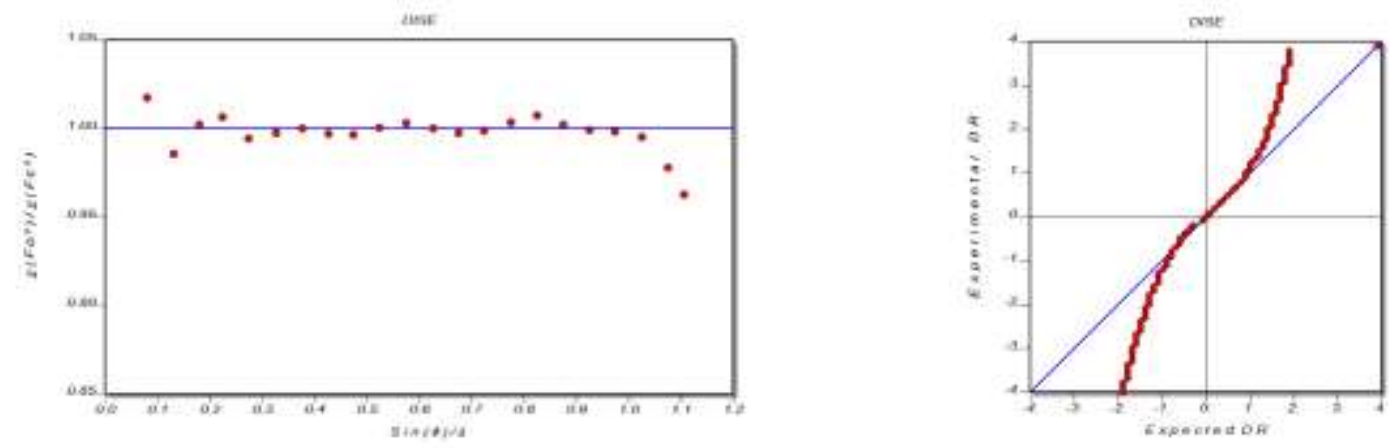

Figure S4.9: Resolution dependent error and Quantile/Quantile DRK-Plots (Zhurov et al., 2008; Zavodnik et al., 1999; A dam Stash, 2007) for the XD refinement of dataset B.
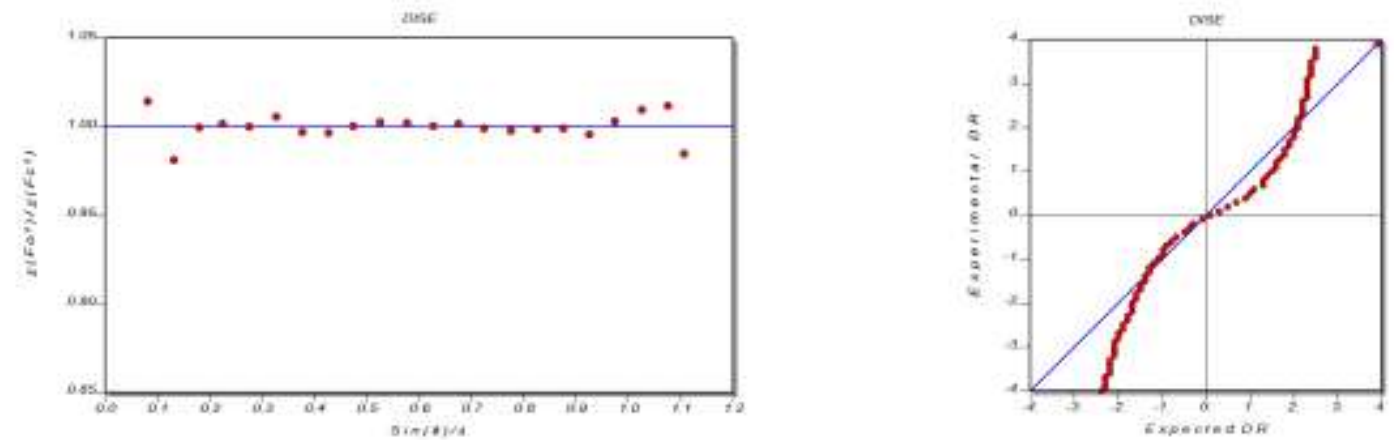

Figure S4.10: Resolution dependent error and Quantile/ Quantile DRK-Plots (Zhurov et al., 2008; Zavodnik et al., 1999; A dam Stash, 2007) for the XD refinement of dataset C.
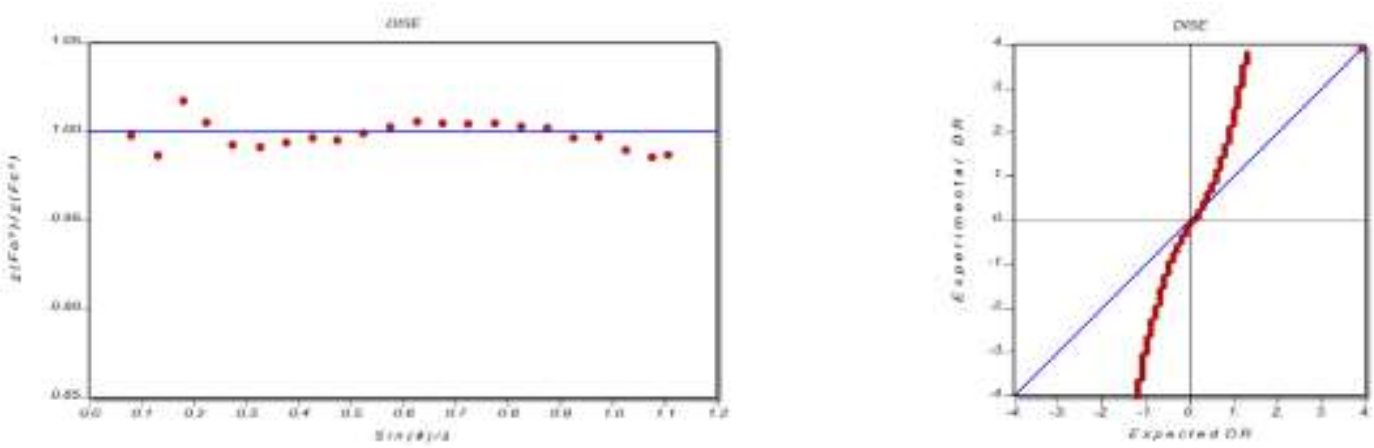

Figure S4.11: Resolution dependent error and Quantile/Quantile D RK -Plots (Zhurov et al., 2008; Zavodnik et al., 1999; A dam Stash, 2007) for theXD refinement of dataset $\mathbf{D}$. 

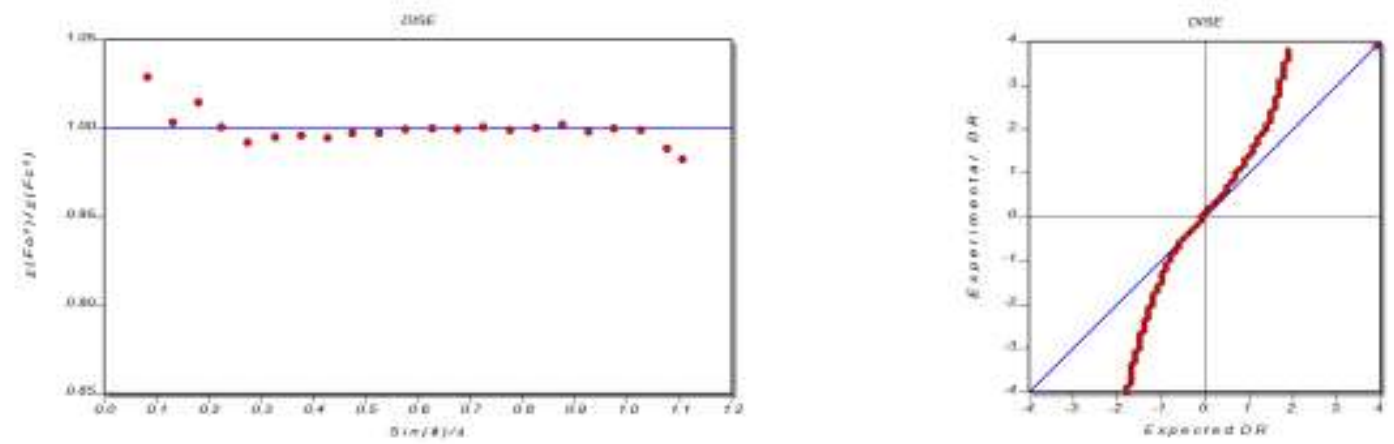

Figure S4.12: Resolution dependent error and Quantile/Quantile DRK -Plots (Zhurov et al., 2008; Zavodnik et al., 1999; A dam Stash, 2007) for theXD refinement of dataset $\mathbf{E}$.
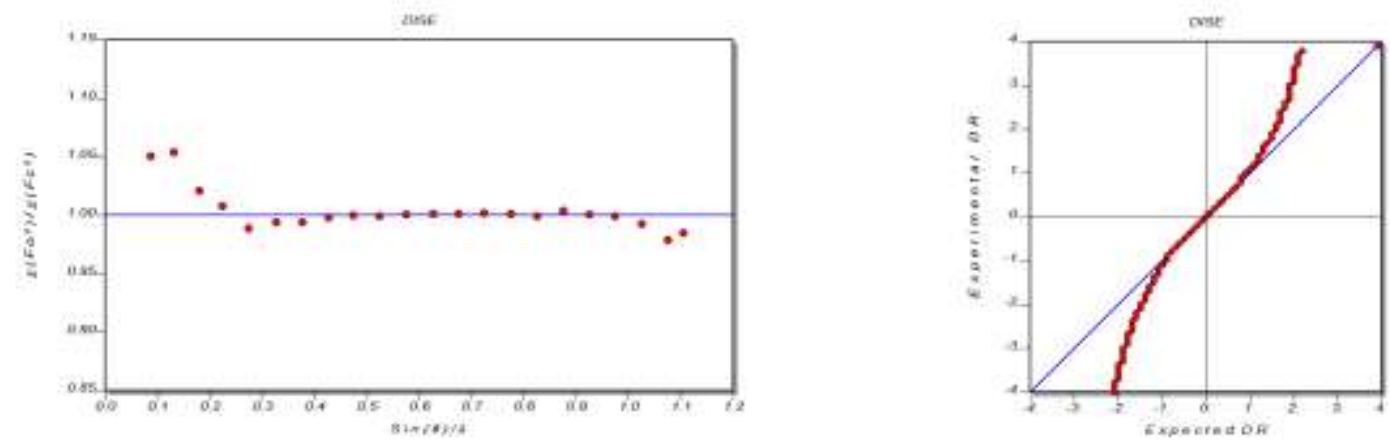

Figure S4.13: Resolution dependent error and Quantile/Quantile DRK -Plots (Zhurov et al., 2008; Zavodnik et al., 1999; A dam Stash, 2007) for theXD refinement of dataset $\mathbf{F}$.
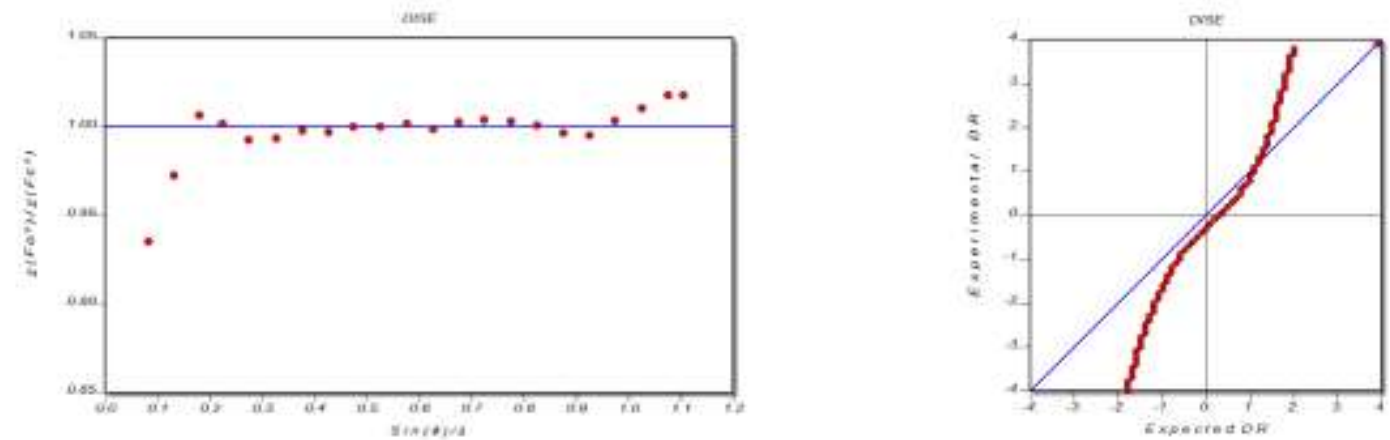

Figure S4.14: Resolution dependent error and Quantile/ Quantile DRK-Plots (Zhurov et al., 2008; Zavodnik et al., 1999; A dam Stash, 2007) for the XD refinement of dataset G. 

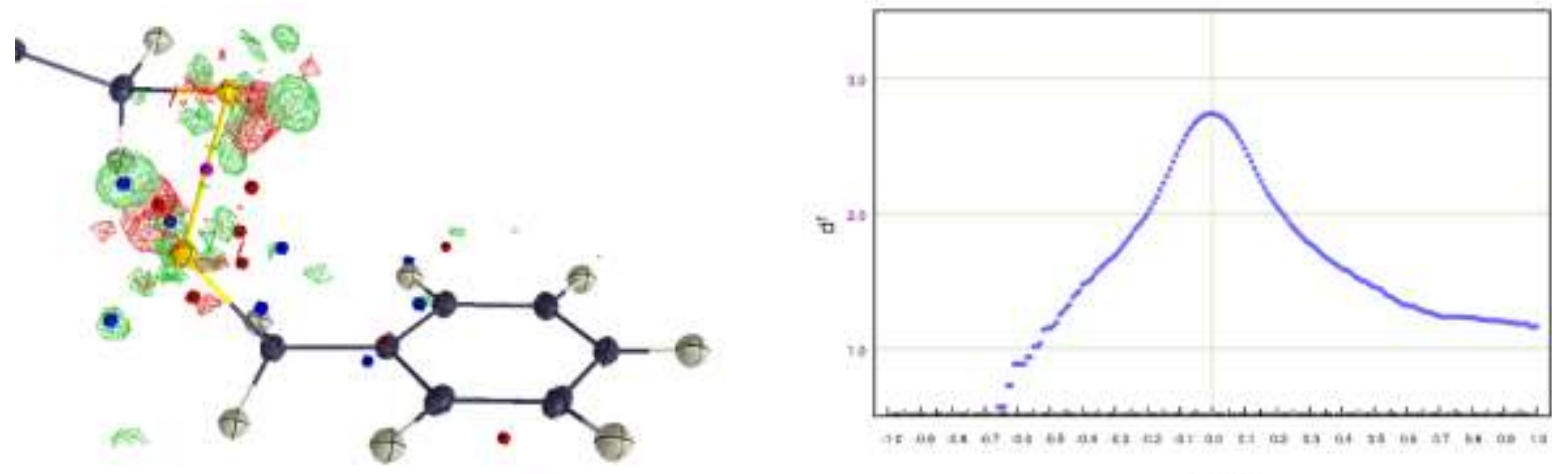

$\rho_{0}\left[e A^{2}\right]$

Figure S4.15: Residual density plot at the $\pm 0.30 \mathrm{e}^{-1}$ level and fractal dimension plot ( $M$ eindl \& Henn, 2008) for the XD refinement of dataset $\mathbf{A}$. Residual density isolevels: positive green, negative red; maxima blue, minima red.
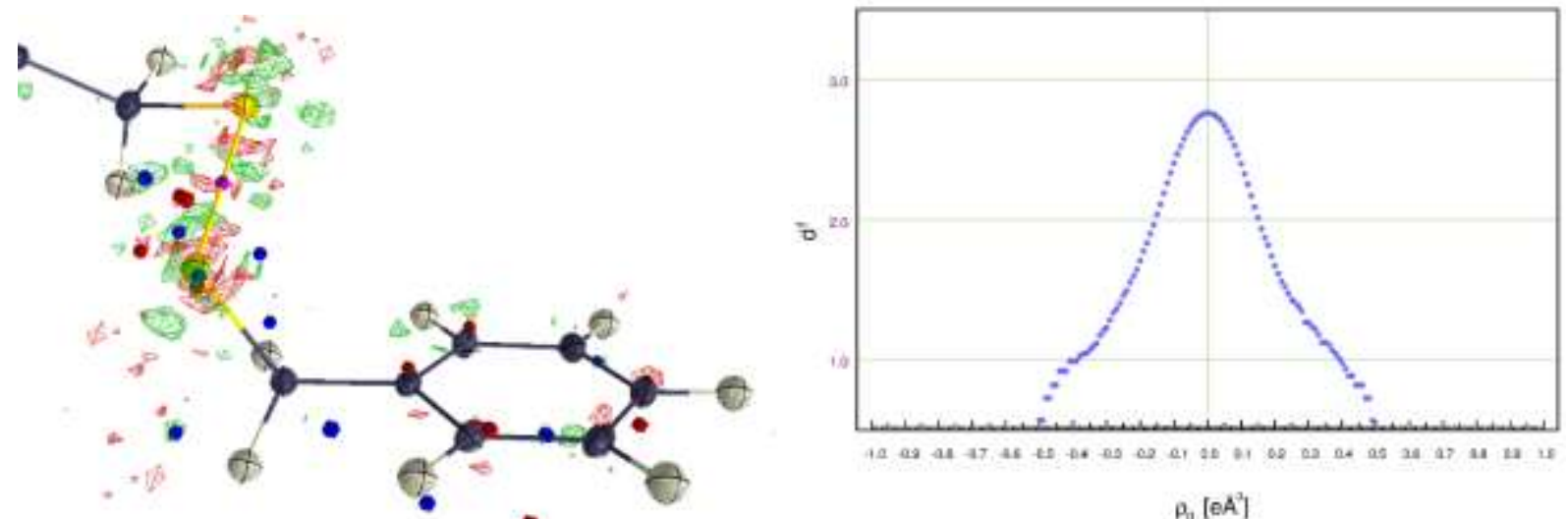

$p_{0}\left[\in A^{3}\right]$

Figure S4.16: Residual density plot at the $\pm 0.16 \mathrm{e}^{-1}$ level and fractal dimension plot (M eindl \& Henn, 2008) for the XD refinement of dataset $\mathbf{B}$. Residual density isolevels: positive green, negative red; maxima blue, minima red.
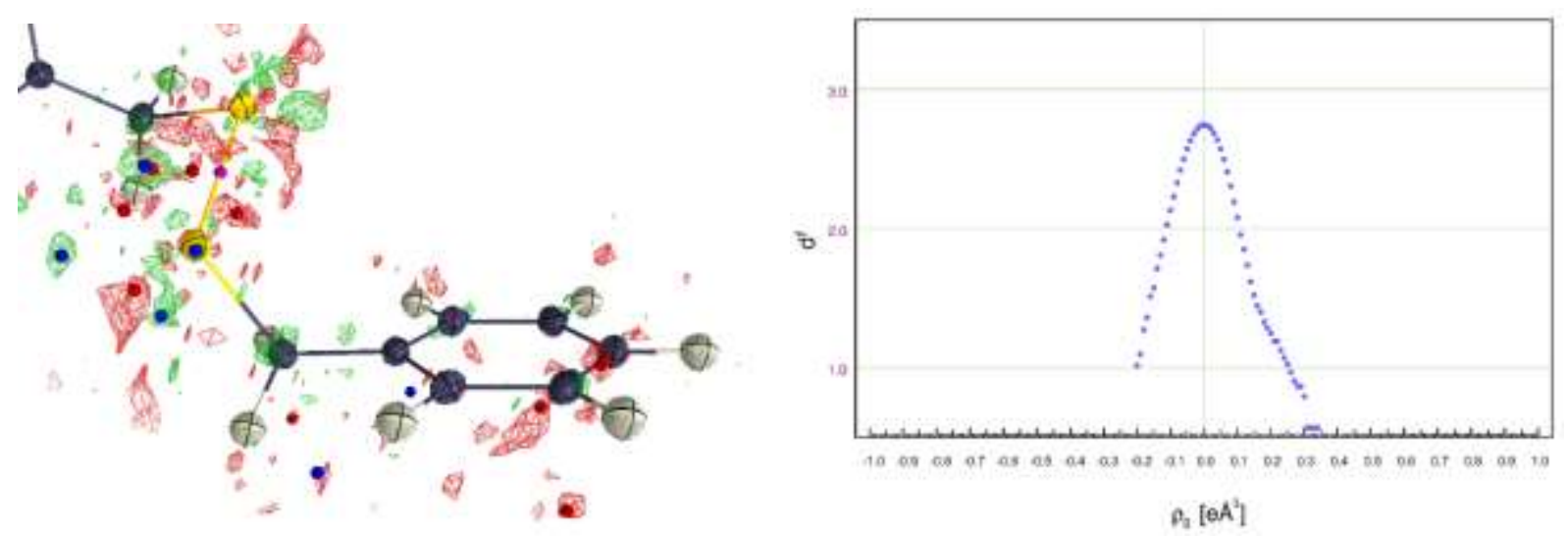

Figure S4.17: Residual density plot at the $\pm 0.10 \mathrm{e} \AA^{-1}$ level and fractal dimension plot (M eindl $\&$ Henn, 2008) for the XD refinement of dataset $\mathbf{C}$. Residual density isolevels: positive green, negative red; maxima blue, minima red. 

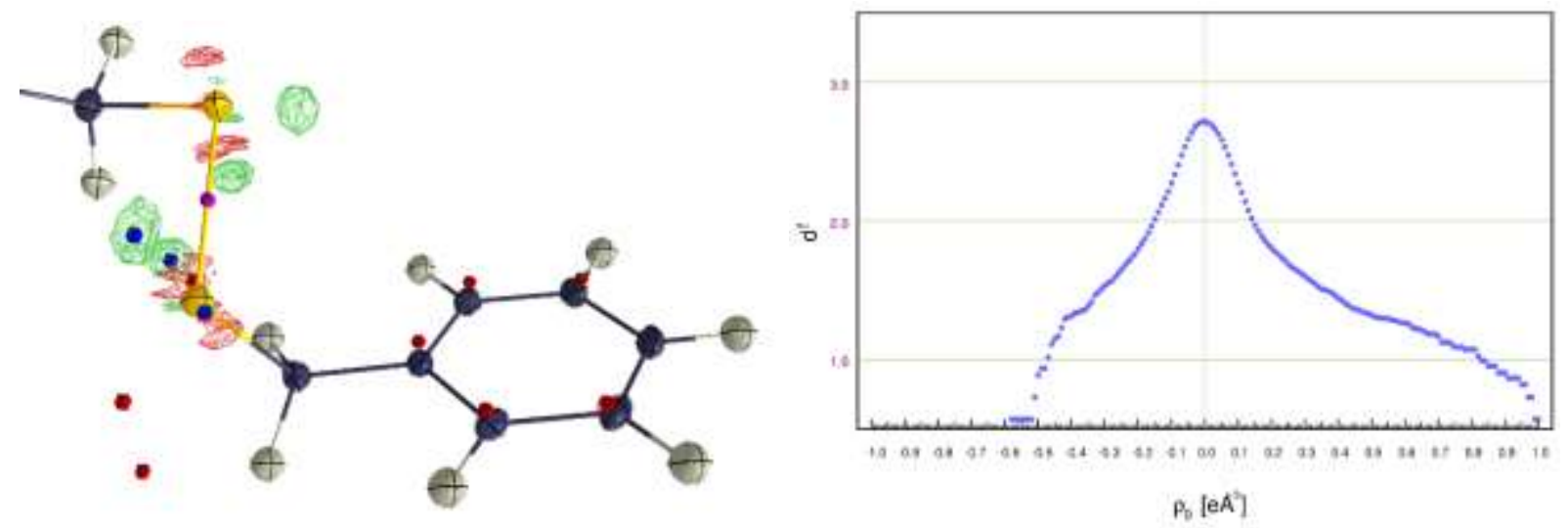

Figure S4.18: Residual density plot at the $\pm 0.30 \mathrm{e}^{-1}$ level and fractal dimension plot ( $M$ eindl \& Henn, 2008) for the $X D$ refinement of dataset $\mathbf{D}$. Residual density isolevels: positive green, negative red; maxima blue, minima red.
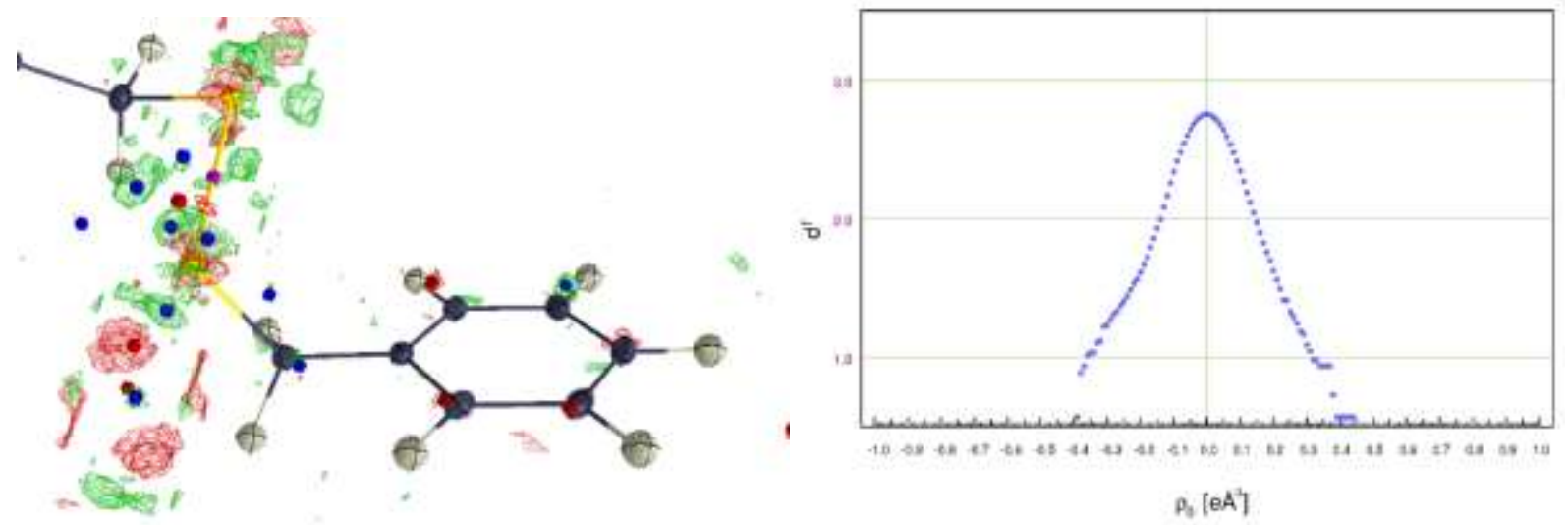

Figure S4.19: Residual density plot at the $\pm 0.15 \mathrm{e}^{-1}$ level and fractal dimension plot (M eindl \& Henn, 2008) for the $X D$ refinement of dataset $\mathbf{E}$. Residual density isolevels: positive green, negative red; maxima blue, minima red.
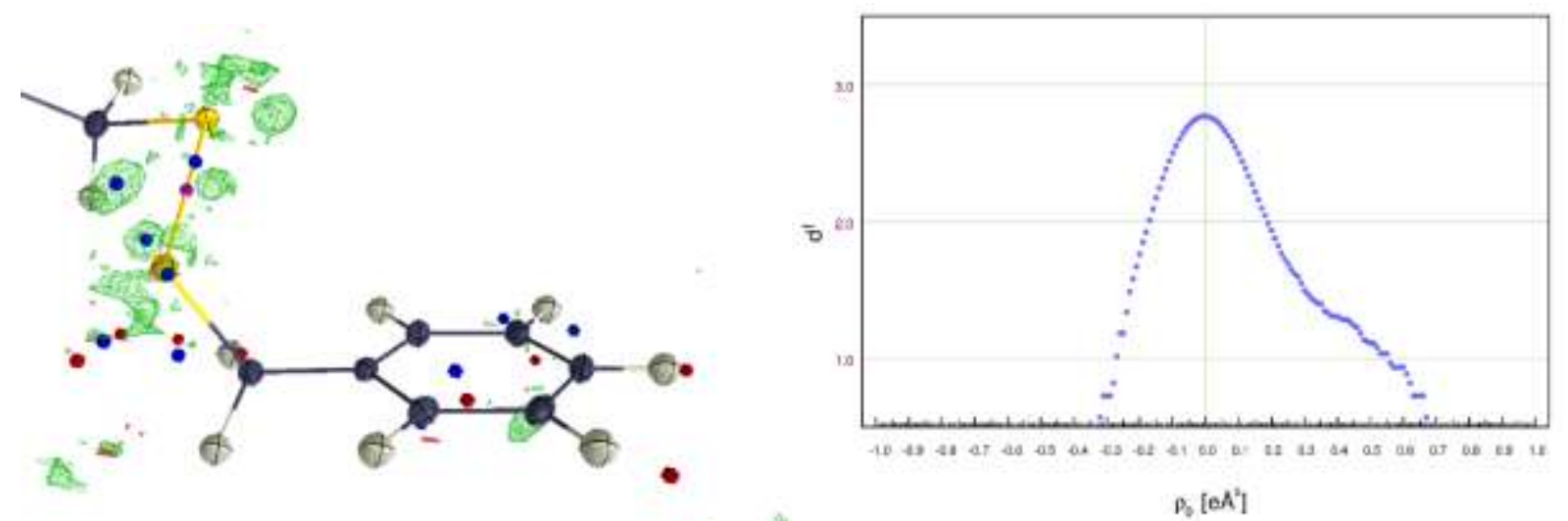

Figure S4.20: Residual density plot at the $\pm 0.20 \mathrm{e}^{-1}$ level and fractal dimension plot ( $M$ eindl \& Henn, 2008) for the XD refinement of dataset $\mathbf{F}$. Residual density isolevels: positive green, negative red; maxima blue, minima red. 

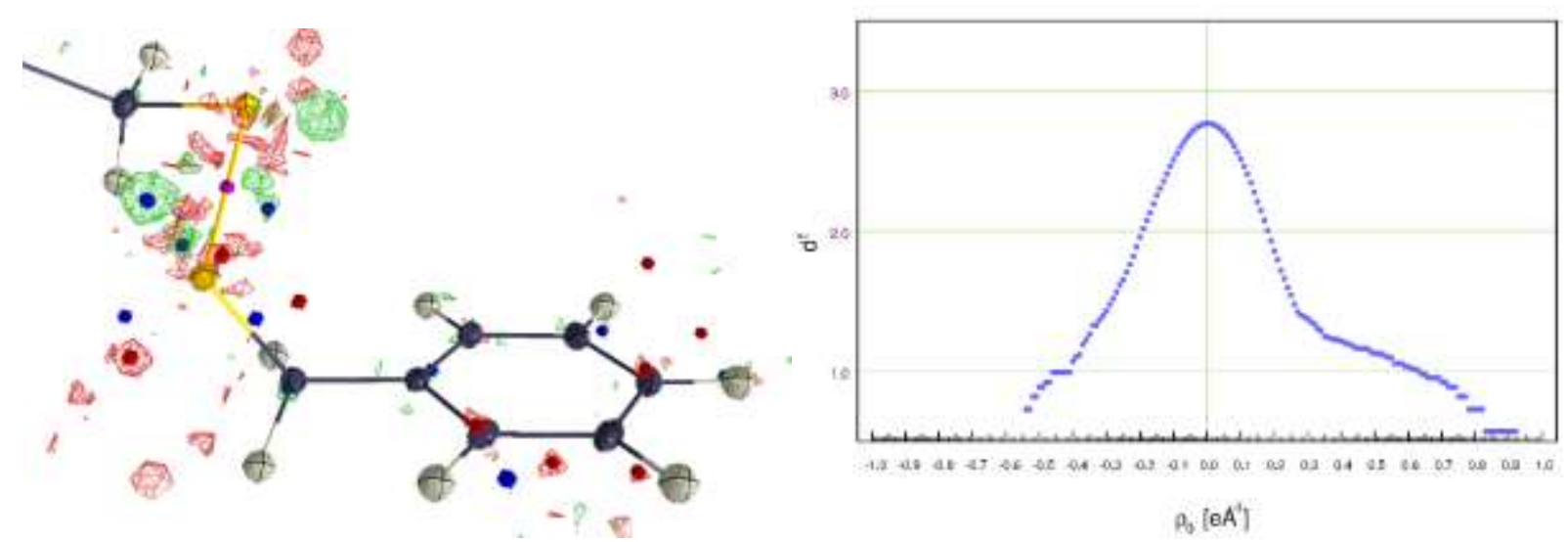

Figure S4.21: Residual density plot at the $\pm 0.20 \mathrm{e}^{-1}$ level and fractal dimension plot ( $M$ eindl \& Henn, 2008) for the XD refinement of dataset $\mathbf{G}$. Residual density isolevels: positive green, negative red; maxima blue, minima red.

Table S4.19: M oPro refinement strategy, for dataset A. A bbreviations: M : monopoles; D: dipoles; Q: quadrupoles; O: octupoles; $\mathrm{H}$ : hexadecapoles, K: $\kappa, \mathrm{U} 2, \mathrm{U} 3$, U4: Gram Charlier $2^{\text {nd }}, 3^{\text {rd }}$ and $4^{\text {th }}$ order, HXYZ: hydrogen position against data up to $0.5 \sin (\theta) / \lambda$.

\begin{tabular}{|c|c|c|c|c|c|c|c|c|c|}
\hline step & New Para & $\begin{array}{l}\mathrm{D}<0.5 \\
\sin (\text { th) } / \mathrm{I}\end{array}$ & \#M P-Param & Data & Para & Data/Para & $\mathrm{R}\left(\mathrm{F}^{2}\right)$ & $w R\left(F^{2}\right)$ & GOF \\
\hline 1 & SCALE & 643 & 0 & 7032 & 1 & 7030.0 & 4.714 & 5.906 & 12.565 \\
\hline 2 & DQOH & 643 & 46 & 7032 & 47 & 150.0 & 4.127 & 5.118 & 10.815 \\
\hline 3 & U2 & 643 & 46 & 7032 & 95 & 74.0 & 2.694 & 3.002 & 6.397 \\
\hline 4 & $\kappa$ & 643 & 46 & 7032 & 98 & 71.8 & 2.672 & 2.97 & 6.327 \\
\hline 5 & $X Y Z$ & 643 & 46 & 7032 & 122 & 57.6 & 2.589 & 2.82 & 6.016 \\
\hline 6 & M & 643 & 46 & 7032 & 127 & 55.4 & 2.571 & 2.8 & 5.976 \\
\hline 7 & $H-X Y Z$ & 643 & 0 & 643 & 22 & 29.2 & 2.33 & 2.792 & 10.797 \\
\hline 8 & all prior & 643 & 46 & 7032 & 127 & 55.4 & 2.561 & 2.781 & 5.934 \\
\hline 9 & U4 & 643 & 46 & 7032 & 152 & 46.3 & 2.367 & 2.495 & 5.337 \\
\hline 10 & Se NoSymm & 643 & 56 & 7032 & 162 & 43.4 & 2.122 & 2.351 & 5.036 \\
\hline 11 & $\kappa^{\prime}$ & 643 & 0 & 7032 & 4 & 1760.0 & 2.115 & 2.337 & 4.949 \\
\hline 12 & all prior & 643 & 56 & 7032 & 162 & 43.4 & 2.096 & 2.32 & 4.97 \\
\hline 13 & $32 \mathrm{P}(\mathrm{Se})$ & 643 & 67 & 7032 & 173 & 40.6 & 2.039 & 2.283 & 4.895 \\
\hline 14 & $64 \mathrm{P}(\mathrm{Se})$ & 643 & 80 & 7032 & 186 & 37.8 & 2.026 & 2.244 & 4.82 \\
\hline
\end{tabular}


Table S4.20: M oPro refinement strategy, for dataset B. A bbreviations: M : monopoles; D: dipoles; Q: quadrupoles;

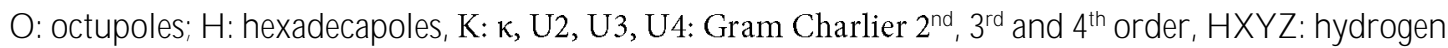
position against data up to $0.5 \sin (\theta) / \lambda$.

\begin{tabular}{|c|c|c|c|c|c|c|c|c|c|}
\hline step & N ew Para & $\begin{array}{l}D<0.5 \\
\sin (\text { th) } / 1\end{array}$ & \#M P-Param & Data & Para & Data/Para & $R\left(F^{2}\right)$ & $w R\left(F^{2}\right)$ & GOF \\
\hline 1 & SCALE & 644 & 0 & 6719 & 1 & 6720.0 & 3.518 & 4.503 & 7.267 \\
\hline 2 & $\mathrm{DQOH}$ & 644 & 46 & 6719 & 47 & 143.0 & 2.971 & 3.303 & 5.280 \\
\hline 3 & U2 & 644 & 46 & 6719 & 95 & 70.7 & 2.168 & 2.428 & 3.899 \\
\hline 4 & $\kappa$ & 644 & 46 & 6719 & 98 & 68.6 & 1.701 & 1.950 & 3.124 \\
\hline 5 & $X Y Z$ & 644 & 46 & 6719 & 122 & 55.1 & 1.643 & 1.808 & 2.901 \\
\hline 6 & M & 644 & 46 & 6719 & 127 & 52.9 & 1.615 & 1.775 & 2.849 \\
\hline 7 & $H-X Y Z$ & 644 & 0 & 644 & 22 & 29.3 & 1.432 & 1.839 & 5.917 \\
\hline 8 & all prior & 644 & 46 & 6719 & 127 & 52.9 & 1.589 & 1.731 & 2.778 \\
\hline 9 & U4 & 644 & 46 & 6719 & 152 & 44.2 & 1.282 & 1.380 & 2.221 \\
\hline 10 & Se NoSymm & 644 & 56 & 6719 & 162 & 41.5 & 1.233 & 1.333 & 2.146 \\
\hline 11 & $\kappa^{\prime}$ & 644 & 0 & 6719 & 4 & 1680.0 & 1.231 & 1.324 & 2.107 \\
\hline 12 & all prior & 644 & 56 & 6719 & 162 & 41.5 & 1.221 & 1.316 & 2.120 \\
\hline 13 & $32 \mathrm{P}(\mathrm{Se})$ & 644 & 67 & 6719 & 173 & 38.8 & 1.212 & 1.300 & 2.096 \\
\hline 14 & $64 \mathrm{P}(\mathrm{Se})$ & 644 & 80 & 6719 & 186 & 36.1 & 1.205 & 1.288 & 2.078 \\
\hline
\end{tabular}

Table S4.21: M oPro refinement strategy, for dataset C. A bbreviations: M : monopoles; D: dipoles; Q: quadrupoles; O: octupoles; $\mathrm{H}$ : hexadecapoles, K: $\kappa, \mathrm{U} 2, \mathrm{U} 3$, U4: Gram Charlier $2^{\text {nd }}, 3^{\text {rd }}$ and $4^{\text {th }}$ order, HXYZ: hydrogen position against data up to $0.5 \sin (\theta) / \lambda$.

\begin{tabular}{|c|c|c|c|c|c|c|c|c|c|}
\hline step & New Para & $\begin{array}{l}D<0.5 \\
\sin (\text { th) } / /\end{array}$ & \#M P-Param & Data & Para & Data/Para & $\mathrm{R}\left(\mathrm{F}^{2}\right)$ & $w R\left(F^{2}\right)$ & GOF \\
\hline 1 & SCALE & 644 & 0 & 6774 & 1 & 6770.0 & 3.736 & 4.751 & 4.936 \\
\hline 2 & DQOH & 644 & 46 & 6774 & 47 & 144.0 & 3.156 & 3.517 & 3.631 \\
\hline 3 & U2 & 644 & 46 & 6774 & 95 & 71.3 & 2.330 & 2.679 & 2.783 \\
\hline 4 & $\kappa$ & 644 & 46 & 6774 & 98 & 69.1 & 1.516 & 1.914 & 1.990 \\
\hline 5 & $X Y Z$ & 644 & 46 & 6774 & 122 & 55.5 & 1.463 & 1.816 & 1.889 \\
\hline 6 & M & 644 & 46 & 6774 & 127 & 53.3 & 1.409 & 1.759 & 1.831 \\
\hline 7 & $H-X Y Z$ & 644 & 0 & 644 & 22 & 29.3 & 1.223 & 1.673 & 4.050 \\
\hline 8 & all prior & 644 & 46 & 6774 & 127 & 53.3 & 1.388 & 1.713 & 1.783 \\
\hline 9 & U4 & 644 & 46 & 6774 & 152 & 44.6 & 1.287 & 1.573 & 1.639 \\
\hline 10 & Se NoSymm & 644 & 56 & 6774 & 162 & 41.8 & 1.252 & 1.534 & 1.600 \\
\hline 11 & $\kappa^{\prime}$ & 644 & 0 & 6774 & 4 & 1690.0 & 1.247 & 1.514 & 1.560 \\
\hline 12 & all prior & 644 & 56 & 6774 & 162 & 41.8 & 1.222 & 1.495 & 1.559 \\
\hline 13 & $32 \mathrm{P}(\mathrm{Se})$ & 644 & 67 & 6774 & 173 & 39.2 & 1.215 & 1.484 & 1.549 \\
\hline 14 & $64 \mathrm{P}(\mathrm{Se})$ & 644 & 80 & 6774 & 186 & 36.4 & 1.197 & 1.461 & 1.527 \\
\hline
\end{tabular}


Table S4.22: M oPro refinement strategy, for dataset $\mathbf{D}$. A bbreviations: M : monopoles; D: dipoles; Q: quadrupoles; $\mathrm{O}$ : octupoles; $\mathrm{H}$ : hexadecapoles, K: $\kappa, \mathrm{U} 2, \mathrm{U} 3, \mathrm{U} 4$ : Gram Charlier $2^{\text {nd }}$, $3^{\text {rd }}$ and $4^{\text {th }}$ order, $\mathrm{HXYZ}$ : hydrogen position against data up to $0.5 \sin (\theta) / \lambda$.

\begin{tabular}{|c|c|c|c|c|c|c|c|c|c|}
\hline step & New Para & $\begin{array}{l}D<0.5 \\
\sin (\text { th) } / /\end{array}$ & \#M P-Param & Data & Para & Data/Para & $\mathrm{R}\left(\mathrm{F}^{2}\right)$ & $W R\left(F^{2}\right)$ & GOF \\
\hline 1 & SCALE & 641 & 0 & 6955 & 1 & 6960.0 & 6.063 & 7.606 & 21.019 \\
\hline 2 & DQOH & 641 & 46 & 6955 & 47 & 148.0 & 5.470 & 6.177 & 16.855 \\
\hline 3 & U2 & 641 & 46 & 6955 & 95 & 73.2 & 3.012 & 3.838 & 10.420 \\
\hline 4 & $\kappa$ & 641 & 46 & 6955 & 98 & 71.0 & 2.066 & 3.258 & 8.747 \\
\hline 5 & $X Y Z$ & 641 & 46 & 6955 & 122 & 57.0 & 2.023 & 3.158 & 8.485 \\
\hline 6 & M & 641 & 46 & 6955 & 127 & 54.8 & 1.987 & 3.102 & 8.378 \\
\hline 7 & $\mathrm{H}-\mathrm{XYZ}$ & 641 & 0 & 641 & 22 & 29.1 & 2.095 & 3.500 & 24.880 \\
\hline 8 & all prior & 641 & 46 & 6955 & 127 & 54.8 & 1.985 & 3.076 & 8.306 \\
\hline 9 & U4 & 641 & 46 & 6955 & 152 & 45.8 & 1.778 & 2.893 & 7.764 \\
\hline 10 & Se NoSymm & 641 & 56 & 6955 & 162 & 42.9 & 1.685 & 2.836 & 7.605 \\
\hline 11 & $\kappa^{\prime}$ & 641 & 0 & 6955 & 4 & 1740.0 & 1.688 & 2.807 & 7.437 \\
\hline 12 & all prior & 641 & 56 & 6955 & 162 & 42.9 & 1.639 & 2.778 & 7.449 \\
\hline 13 & $32 \mathrm{P}(\mathrm{Se})$ & 641 & 67 & 6955 & 173 & 40.2 & 1.606 & 2.731 & 7.322 \\
\hline 14 & $64 \mathrm{P}(\mathrm{Se})$ & 641 & 80 & 6955 & 186 & 37.4 & 1.599 & 2.715 & 7.283 \\
\hline
\end{tabular}

Table S4.23: M oPro refinement strategy, for dataset E. A bbreviations: M : monopoles; D: dipoles; Q: quadrupoles; $\mathrm{O}$ : octupoles; $\mathrm{H}$ : hexadecapoles, $\mathrm{K}: \kappa, \mathrm{U} 2, \mathrm{U} 3$, U4: Gram Charlier $2^{\text {nd }}, 3^{\text {rd }}$ and $4^{\text {th }}$ order, HXYZ: hydrogen position against data up to $0.5 \sin (\theta) / \lambda$.

\begin{tabular}{|c|c|c|c|c|c|c|c|c|c|}
\hline step & New Para & $\begin{array}{l}<<0.5 \\
\sin (\text { th) } / /\end{array}$ & \#M P-Param & Data & Para & Data/Para & $R\left(F^{2}\right)$ & $W R\left(F^{2}\right)$ & GOF \\
\hline 1 & SCALE & 643 & 0 & 6777 & 1 & 6777.0 & 3.056 & 4.463 & 7.57 \\
\hline 2 & DQOH & 643 & 46 & 6777 & 47 & 144.0 & 2.568 & 2.57 & 4.301 \\
\hline 3 & U2 & 643 & 46 & 6777 & 95 & 71.3 & 2.007 & 2.186 & 3.665 \\
\hline 4 & $\kappa$ & 643 & 46 & 6777 & 98 & 69.2 & 1.526 & 1.852 & 3.103 \\
\hline 5 & $X Y Z$ & 643 & 46 & 6777 & 122 & 55.5 & 1.493 & 1.636 & 2.746 \\
\hline 6 & M & 643 & 46 & 6777 & 127 & 53.4 & 1.43 & 1.562 & 2.624 \\
\hline 7 & $H-X Y Z$ & 643 & 0 & 643 & 22 & 29.2 & 1.455 & 1.849 & 5.22 \\
\hline 8 & all prior & 643 & 46 & 6777 & 127 & 53.4 & 1.418 & 1.521 & 2.557 \\
\hline 9 & U4 & 643 & 46 & 6777 & 152 & 44.6 & 1.249 & 1.331 & 2.248 \\
\hline 10 & Se NoSymm & 643 & 56 & 6777 & 162 & 41.8 & 1.224 & 1.298 & 2.194 \\
\hline 11 & $\kappa^{\prime}$ & 643 & 0 & 6777 & 4 & 6777.0 & 1.225 & 1.295 & 2.164 \\
\hline 12 & all prior & 643 & 56 & 6777 & 162 & 41.8 & 1.222 & 1.293 & 2.185 \\
\hline 13 & $32 \mathrm{P}(\mathrm{Se})$ & 643 & 67 & 6777 & 173 & 39.2 & 1.212 & 1.268 & 2.145 \\
\hline 14 & $64 \mathrm{P}(\mathrm{Se})$ & 643 & 80 & 6777 & 186 & 36.4 & 1.201 & 1.235 & 2.094 \\
\hline
\end{tabular}


Table S4.24: M oPro refinement strategy, for dataset F. A bbreviations: M : monopoles; D: dipoles; Q: quadrupoles; $\mathrm{O}$ : octupoles; $\mathrm{H}$ : hexadecapoles, K: $\mathrm{K}, \mathrm{U} 2, \mathrm{U} 3$, U4: Gram Charlier $2^{\text {nd }}, 3^{\text {rd }}$ and $4^{\text {th }}$ order, HXYZ: hydrogen position against data up to $0.5 \sin (\theta) / \lambda$.

\begin{tabular}{|c|c|c|c|c|c|c|c|c|c|}
\hline step & New Para & $\begin{array}{l}D<0.5 \\
\sin (\text { th }) / /\end{array}$ & \#M P-Param & Data & Para & Data/Para & $R\left(F^{2}\right)$ & $w R\left(F^{2}\right)$ & GOF \\
\hline 1 & SCALE & 640 & 0 & 6660 & 1 & 6660.0 & 14.605 & 13.394 & 16.59 \\
\hline 2 & $\mathrm{DQOH}$ & 640 & 46 & 6660 & 47 & 142.0 & 14.746 & 12.896 & 16.114 \\
\hline 3 & U2 & 640 & 46 & 6660 & 95 & 70.1 & 2.355 & 2.328 & 2.761 \\
\hline 4 & $\kappa$ & 640 & 46 & 6660 & 98 & 68.0 & 1.609 & 1.836 & 2.171 \\
\hline 5 & $X Y Z$ & 640 & 46 & 6660 & 122 & 54.6 & 1.568 & 1.67 & 1.973 \\
\hline 6 & M & 640 & 46 & 6660 & 127 & 52.4 & 1.603 & 1.631 & 1.926 \\
\hline 7 & $H-X Y Z$ & 640 & 0 & 640 & 22 & 29.1 & 1.582 & 1.612 & 4.538 \\
\hline 8 & all prior & 640 & 46 & 6660 & 127 & 52.4 & 1.607 & 1.597 & 1.884 \\
\hline 9 & U4 & 640 & 46 & 6660 & 152 & 43.8 & 1.51 & 1.506 & 1.781 \\
\hline 10 & Se NoSymm & 640 & 56 & 6660 & 162 & 41.1 & 1.472 & 1.456 & 1.722 \\
\hline 11 & $\kappa^{\prime}$ & 640 & 0 & 6660 & 4 & 1660.0 & 1.468 & 1.444 & 1.687 \\
\hline 12 & all prior & 640 & 56 & 6660 & 162 & 41.1 & 1.439 & 1.433 & 1.695 \\
\hline 13 & $32 \mathrm{P}(\mathrm{Se})$ & 640 & 67 & 6660 & 173 & 38.5 & 1.423 & 1.403 & 1.661 \\
\hline 14 & $64 \mathrm{P}(\mathrm{Se})$ & 640 & 80 & 6660 & 186 & 35.8 & 1.415 & 1.394 & 1.651 \\
\hline
\end{tabular}

Table S4.25: M oPro refinement strategy, for dataset G. Abbreviations: M : monopoles; D: dipoles; Q: quadrupoles; $\mathrm{O}$ : octupoles; $\mathrm{H}$ : hexadecapoles, K: $\mathrm{K}, \mathrm{U} 2, \mathrm{U} 3$, U4: Gram Charlier $2^{\text {nd }}, 3^{\text {rd }}$ and $4^{\text {th }}$ order, HXYZ: hydrogen position against data up to $0.5 \sin (\theta) / \lambda$.

\begin{tabular}{|c|c|c|c|c|c|c|c|c|c|}
\hline step & New Para & $\begin{array}{l}\mathrm{D}<0.5 \\
\sin (\text { th) } / \mathrm{l}\end{array}$ & \#M P-Param & Data & Para & Data/Para & $\mathrm{R}\left(\mathrm{F}^{2}\right)$ & $w R\left(F^{2}\right)$ & GOF \\
\hline 1 & SCALE & 644 & 0 & 6866 & 1 & 6870.0 & 3.08 & 4.646 & 6.231 \\
\hline 2 & DQOH & 644 & 46 & 6866 & 47 & 146.0 & 2.388 & 2.807 & 3.733 \\
\hline 3 & U2 & 644 & 46 & 6866 & 95 & 72.3 & 1.55 & 1.983 & 2.686 \\
\hline 4 & $\kappa$ & 644 & 46 & 6866 & 98 & 70.1 & 1.525 & 1.893 & 2.564 \\
\hline 5 & $X Y Z$ & 644 & 46 & 6866 & 122 & 56.3 & 1.497 & 1.821 & 2.471 \\
\hline 6 & M & 644 & 46 & 6866 & 127 & 54.1 & 1.475 & 1.779 & 2.415 \\
\hline 7 & $H-X Y Z$ & 644 & 0 & 644 & 22 & 29.3 & 1.3 & 1.756 & 5.381 \\
\hline 8 & all prior & 644 & 46 & 6866 & 127 & 54.1 & 1.456 & 1.717 & 2.326 \\
\hline 9 & U4 & 644 & 46 & 6866 & 152 & 45.2 & 1.341 & 1.601 & 2.178 \\
\hline 10 & Se NoSymm & 644 & 56 & 6866 & 162 & 42.4 & 1.301 & 1.561 & 2.127 \\
\hline 11 & $\kappa^{\prime}$ & 644 & 0 & 6866 & 4 & 1720.0 & 1.297 & 1.557 & 2.096 \\
\hline 12 & all prior & 644 & 56 & 6866 & 162 & 42.4 & 1.294 & 1.553 & 2.115 \\
\hline 13 & $32 \mathrm{P}(\mathrm{Se})$ & 644 & 67 & 6866 & 173 & 39.7 & 1.251 & 1.481 & 2.022 \\
\hline 14 & $64 \mathrm{P}(\mathrm{Se})$ & 644 & 80 & 6866 & 186 & 36.9 & 1.229 & 1.439 & 1.97 \\
\hline
\end{tabular}




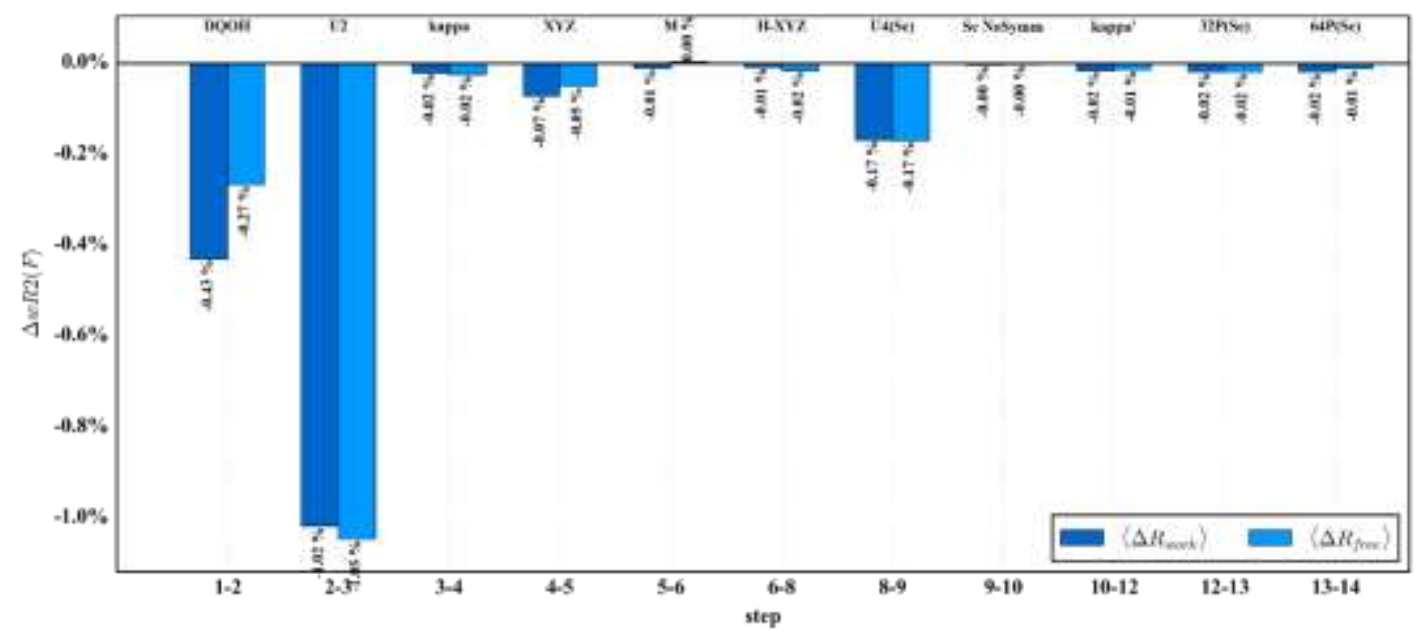

Figure S4.22: $\left\langle R_{\text {free }}\right\rangle$ values for the M oPro refinement of dataset A. A bbreviations: M : monopoles; D: dipoles; Q: quadrupoles; O: octupoles; $\mathrm{H}$ : hexadecapoles; 32P; 32-poles; 64P: 64-poles; U2, U3, U 4: Gram Charlier $2^{\text {nd }}, 3^{\text {rd }}$ and $4^{\text {th }}$ order, HXYZ: hydrogen position against data up to $0.5 \sin (\theta) / \lambda$.

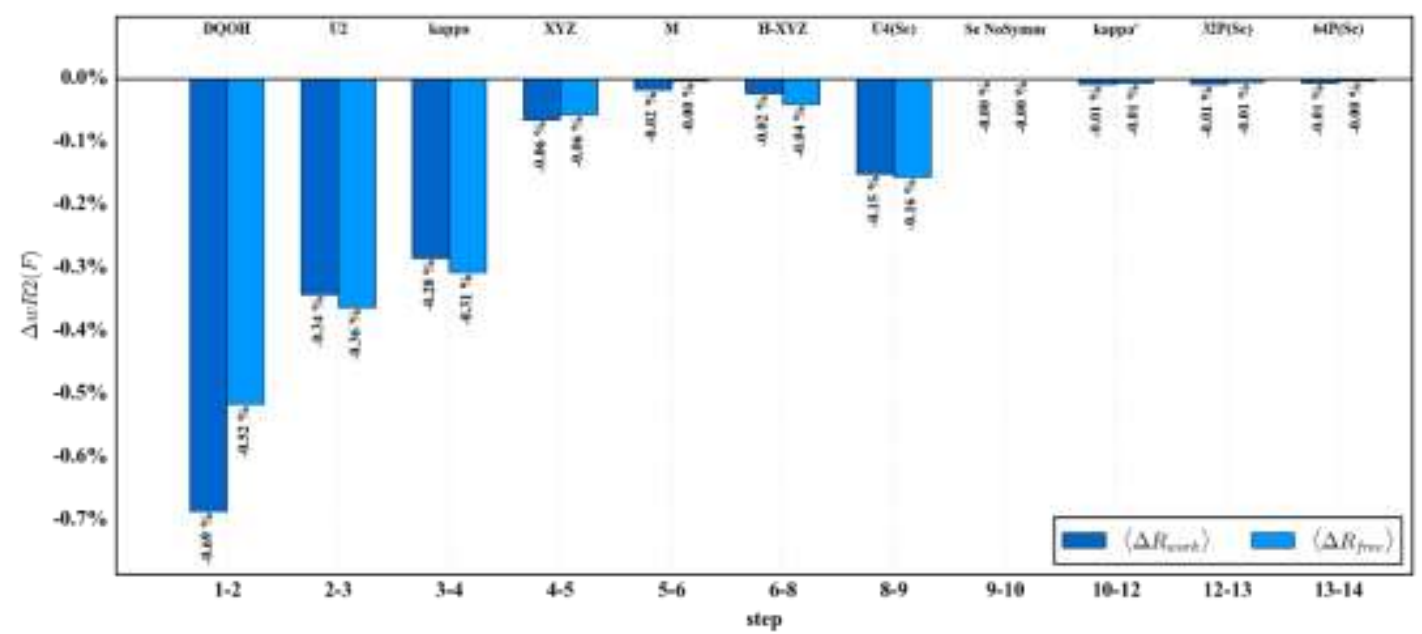

Figure S4.23: $\left\langle R_{\text {free }}\right\rangle$ values for the M oPro refinement of dataset B. A bbreviations: M: monopoles; D: dipoles; Q: quadrupoles; O: octupoles; H: hexadecapoles; 32P; 32-poles; 64P: 64-poles; U2, U3, U 4: Gram Charlier $2^{\text {nd }}, 3^{\text {rd }}$ and $4^{\text {th }}$ order, HXYZ: hydrogen position against data up to $0.5 \sin (\theta) / \lambda$.

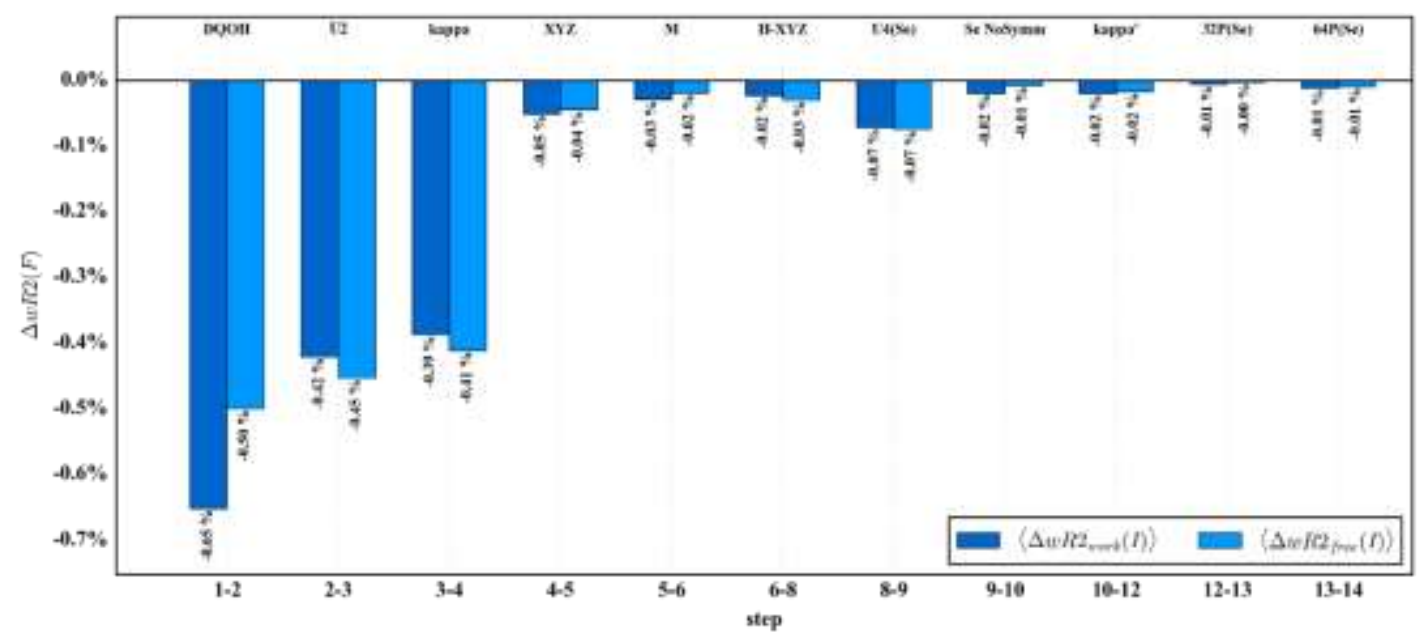

Figure S4.24: $\left\langle R_{\text {free }}\right\rangle$ values for the M oPro refinement of dataset C. A bbreviations: M : monopoles; D: dipoles; Q: quadrupoles; O: octupoles; $\mathrm{H}$ : hexadecapoles; 32P; 32-poles; 64P: 64-poles; U2, U3, U 4: Gram Charlier $2^{\text {nd }}, 3^{\text {rd }}$ and $4^{\text {th }}$ order, HXYZ: hydrogen position against data up to $0.5 \sin (\theta) / \lambda$. 


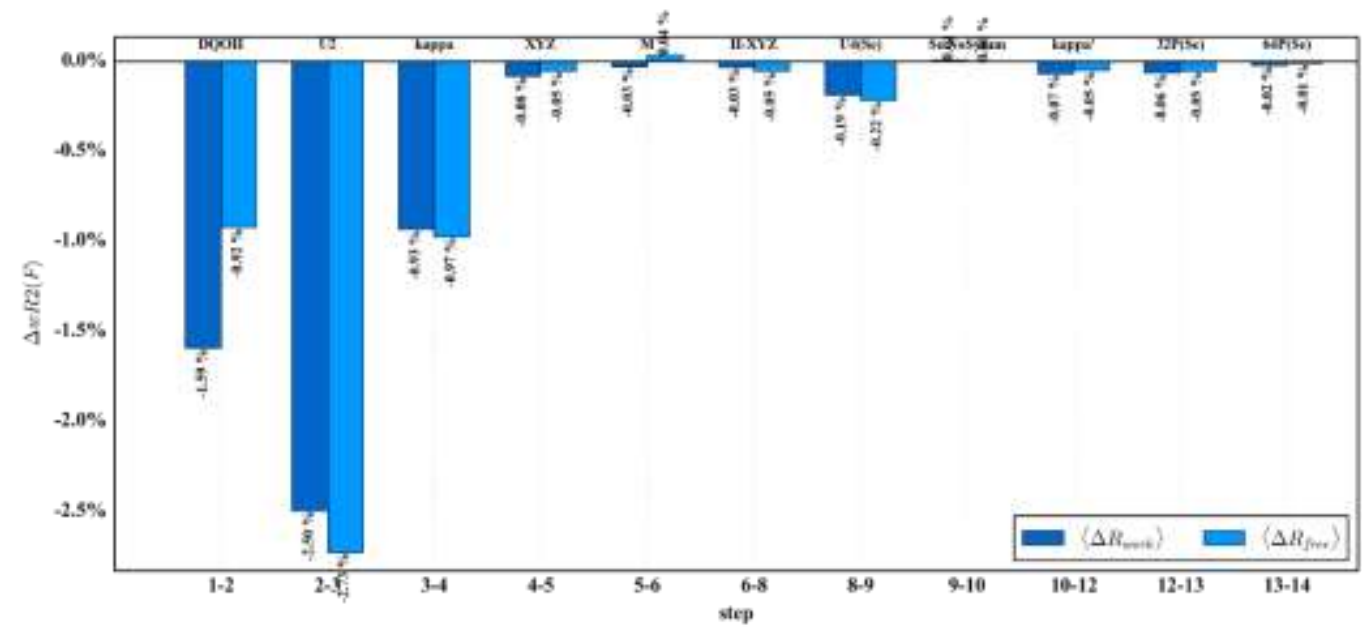

Figure S4.25: $\left\langle R_{\text {free }}\right\rangle$ values for the M oPro refinement of dataset $\mathbf{D}$. A bbreviations: M : monopoles; D: dipoles; Q: quadrupoles; O: octupoles; $\mathrm{H}$ : hexadecapoles; 32P; 32-poles; 64P: 64-poles; U2, U3, U 4: Gram Charlier $2^{\text {nd }}, 3^{\text {rd }}$ and $4^{\text {th }}$ order, $H X Y Z$ : hydrogen position against data up to $0.5 \sin (\theta) / \lambda$.

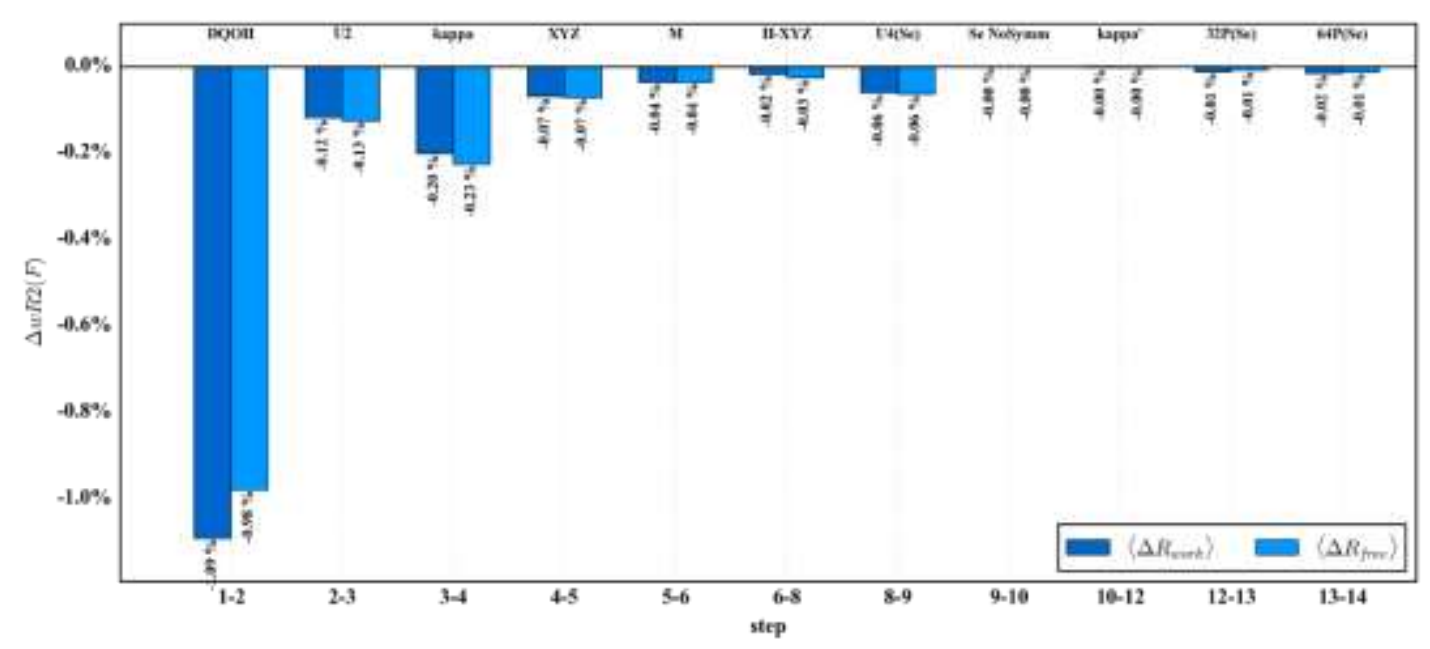

Figure S4.26: $\left\langle R_{\text {free }}\right\rangle$ values for the M oPro refinement of dataset E. A bbreviations: M : monopoles; D: dipoles; Q: quadrupoles; O: octupoles; H: hexadecapoles; 32P; 32-poles; 64P: 64-poles; U2, U3, U 4: Gram Charlier $2^{\text {nd }}, 3^{\text {rd }}$ and $4^{\text {th }}$ order, HXYZ: hydrogen position against data up to $0.5 \sin (\theta) / \lambda$.

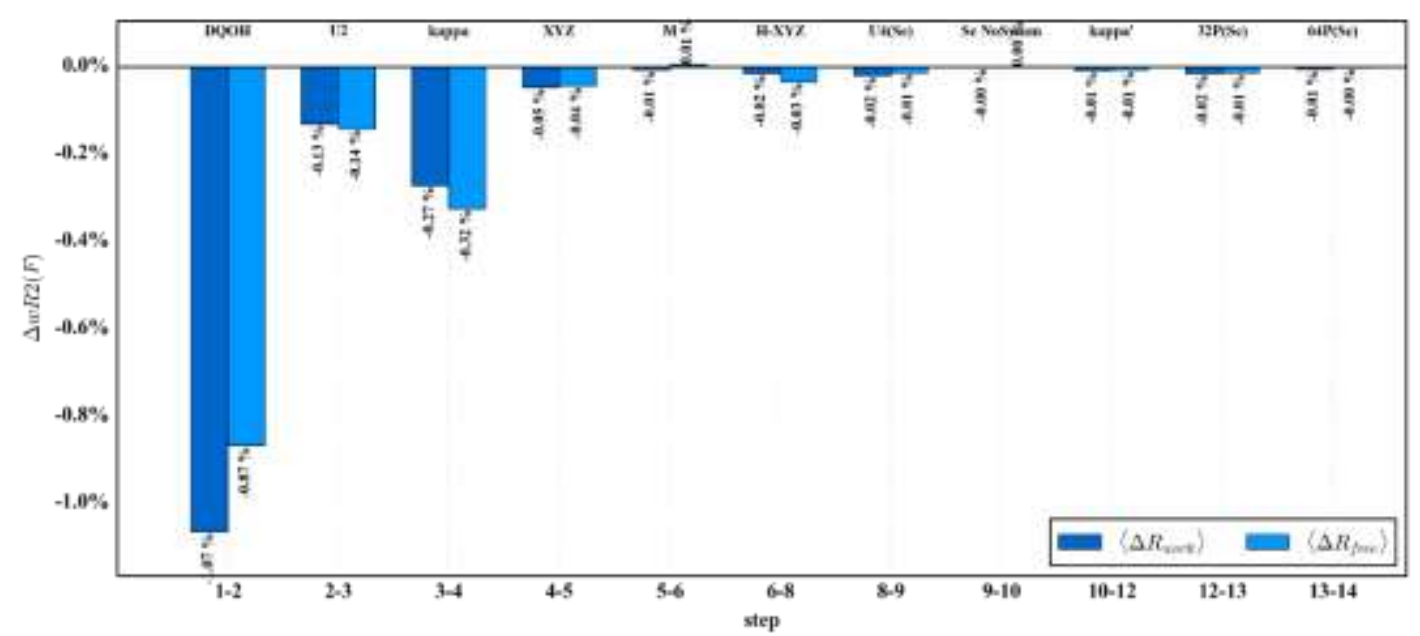

Figure S4.27: $\left\langle R_{\text {free }}\right\rangle$ values for the M oPro refinement of dataset F. A bbreviations: M : monopoles; $D$ : dipoles; Q : quadrupoles; 0 : octupoles; H : hexadecapoles; 32P; 32-poles; 64P: 64-poles; U 2, U 3, U 4:

Gram Charlier $2^{\text {nd }}, 3^{\text {rd }}$ and $4^{\text {th }}$ order, HXYZ: hydrogen position against data up to $0.5 \sin (\theta) / \lambda$. 


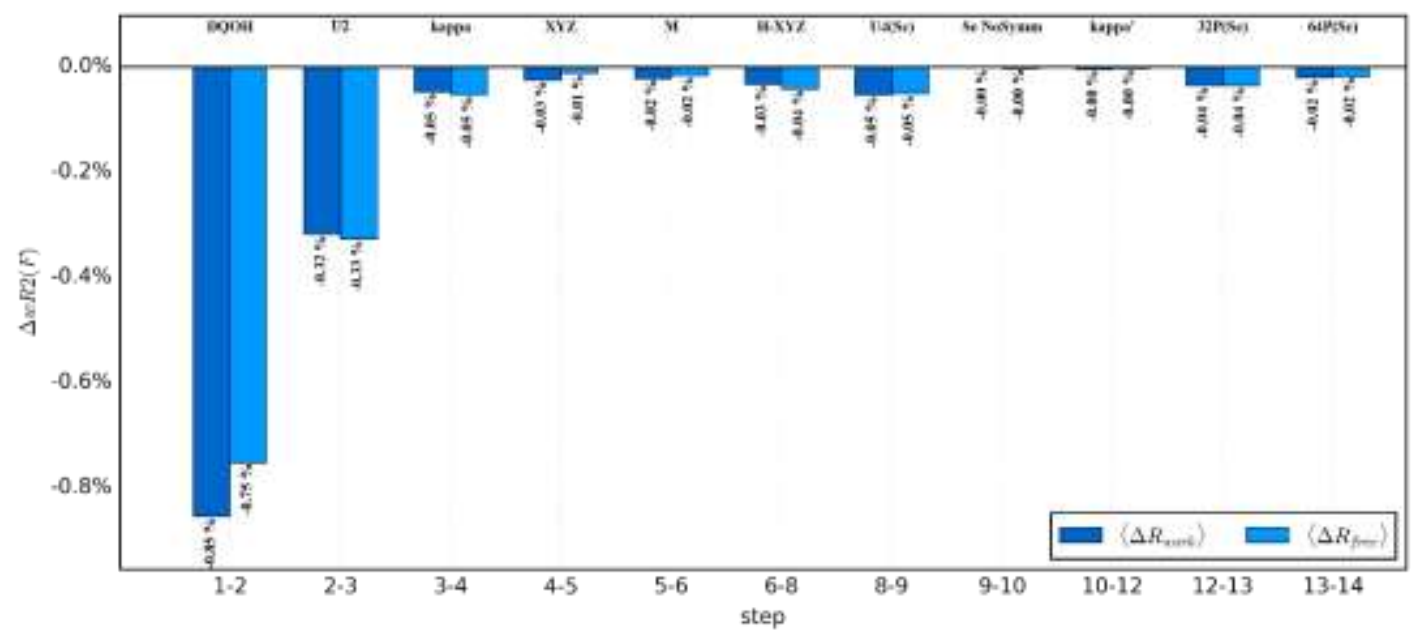

Figure S4.28: $\left\langle R_{\text {free }}\right\rangle$ values for the M oPro refinement of dataset G. A bbreviations: M : monopoles; D: dipoles; Q: quadrupoles; O: octupoles; H: hexadecapoles; 32P; 32-poles; 64P: 64-poles; U2, U3, U4: Gram Charlier $2^{\text {nd }}, 3^{\text {rd }}$ and $4^{\text {th }}$ order, $H X Y Z$ : hydrogen position against data up to $0.5 \sin (\theta) / \lambda$

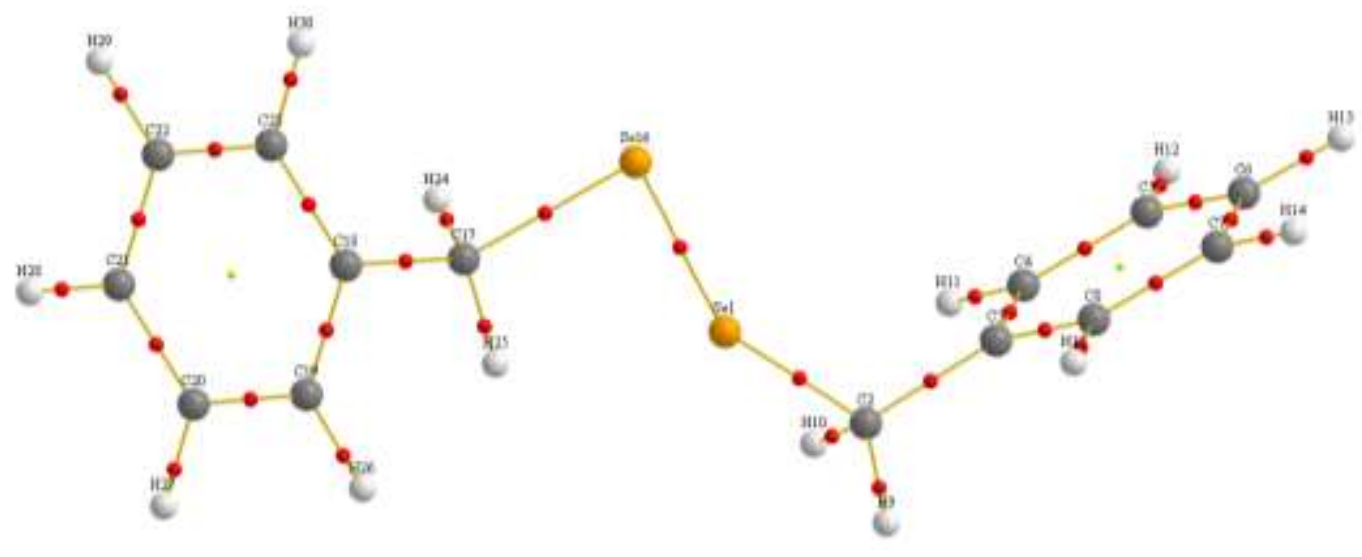

Figure S4.29: Bond Paths, BCP (red) and RCP (yellow) of $\mathbf{1}\left(\mathbf{S}_{0}\right)$. 
Table S4.26: BCP analysis of $\mathbf{1}\left(\mathbf{S}_{0}\right)$.

\begin{tabular}{|c|c|c|c|c|c|c|c|c|}
\hline A toms & $\begin{array}{l}\text { Atom dis- } \\
\text { tance }[\AA]]\end{array}$ & $\begin{array}{l}\text { Bond Path } \\
\text { Length }[\AA]\end{array}$ & $\begin{array}{l}\rho\left(\mathbf{r}_{\mathrm{bcp}}\right) \\
{\left[\AA^{-3}\right]}\end{array}$ & $\begin{array}{l}\nabla^{2} \rho\left(\mathbf{r}_{\text {bcp }}\right) \\
{\left[\AA^{-5}\right]}\end{array}$ & $\varepsilon\left(\mathbf{r}_{\mathrm{bcp}}\right)$ & \multicolumn{3}{|c|}{ Hessian Eigenvalues [a.u.] } \\
\hline Sel - Sel6 & 2.32164 & 2.32195 & 0.725 & -0.978 & 0.01 & -0.0557 & -0.0546 & 0.0889 \\
\hline Sel - C2 & 1.99480 & 1.99506 & 0.930 & -3.078 & 0.04 & -0.0956 & -0.0895 & 0.1175 \\
\hline$C 2-C 3$ & 1.48865 & 1.48901 & 1.811 & -17.453 & 0.02 & -0.2881 & -0.2775 & 0.1823 \\
\hline C3-C4 & 1.39411 & 1.39415 & 2.156 & -22.789 & 0.10 & -0.3710 & -0.3102 & 0.1808 \\
\hline$C 4-C 5$ & 1.38980 & 1.38981 & 2.168 & -23.151 & 0.10 & -0.3740 & -0.3148 & 0.1804 \\
\hline C5 - C6 & 1.38820 & 1.38822 & 2.172 & -23.128 & 0.11 & -0.3753 & -0.3124 & 0.1798 \\
\hline C3-C8 & 1.39853 & 1.39860 & 2.139 & -22.521 & 0.10 & -0.3669 & -0.3086 & 0.1809 \\
\hline C6 - C7 & 1.39427 & 1.39428 & 2.147 & -22.718 & 0.10 & -0.3691 & -0.3101 & 0.1803 \\
\hline $\mathrm{C} 8-\mathrm{H} 15$ & 1.06979 & 1.06980 & 1.937 & -24.716 & 0.01 & -0.4172 & -0.4113 & 0.2858 \\
\hline $\mathrm{C} 7-\mathrm{C} 8$ & 1.38696 & 1.38696 & 2.176 & -23.163 & 0.11 & -0.3759 & -0.3123 & 0.1795 \\
\hline $\mathrm{C} 2-\mathrm{H} 9$ & 1.07636 & 1.07663 & 1.905 & -23.732 & 0.00 & -0.4063 & -0.4028 & 0.2880 \\
\hline $\mathrm{C} 2-\mathrm{H} 10$ & 1.07109 & 1.07129 & 1.932 & -24.207 & 0.01 & -0.4117 & -0.4078 & 0.2880 \\
\hline $\mathrm{C} 4-\mathrm{H} 11$ & 1.06678 & 1.06679 & 1.959 & -25.397 & 0.01 & -0.4290 & -0.4226 & 0.2939 \\
\hline $\mathrm{C} 5-\mathrm{H} 12$ & 1.06885 & 1.06885 & 1.934 & -24.610 & 0.01 & -0.4147 & -0.4079 & 0.2821 \\
\hline $\mathrm{C} 6-\mathrm{H} 13$ & 1.06508 & 1.06508 & 1.952 & -25.018 & 0.01 & -0.4212 & -0.4137 & 0.2855 \\
\hline $\mathrm{C} 7-\mathrm{H} 14$ & 1.06969 & 1.06969 & 1.931 & -24.554 & 0.01 & -0.4150 & -0.4074 & 0.2832 \\
\hline Se16 - C17 & 1.99479 & 1.99506 & 0.930 & -3.078 & 0.04 & -0.0956 & -0.0895 & 0.1175 \\
\hline C17 - C18 & 1.48871 & 1.48908 & 1.811 & -17.448 & 0.02 & -0.2880 & -0.2774 & 0.1823 \\
\hline C18 - C19 & 1.39408 & 1.39411 & 2.156 & -22.793 & 0.10 & -0.3711 & -0.3102 & 0.1808 \\
\hline C19- C20 & 1.38991 & 1.38992 & 2.168 & -23.141 & 0.10 & -0.3739 & -0.3147 & 0.1804 \\
\hline C20 - C21 & 1.38815 & 1.38816 & 2.172 & -23.133 & 0.11 & -0.3753 & -0.3124 & 0.1798 \\
\hline $\mathrm{C} 18-\mathrm{C} 23$ & 1.39853 & 1.39860 & 2.139 & -22.520 & 0.10 & -0.3669 & -0.3086 & 0.1809 \\
\hline C21 - C22 & 1.39416 & 1.39417 & 2.147 & -22.727 & 0.10 & -0.3692 & -0.3102 & 0.1803 \\
\hline $\mathrm{C} 23-\mathrm{H} 30$ & 1.06974 & 1.06974 & 1.937 & -24.722 & 0.01 & -0.4173 & -0.4114 & 0.2859 \\
\hline C22 - C23 & 1.38708 & 1.38709 & 2.175 & -23.152 & 0.11 & -0.3758 & -0.3122 & 0.1795 \\
\hline $\mathrm{C} 17-\mathrm{H} 24$ & 1.07635 & 1.07662 & 1.905 & -23.732 & 0.00 & -0.4063 & -0.4028 & 0.2880 \\
\hline $\mathrm{C} 17-\mathrm{H} 25$ & 1.07108 & 1.07128 & 1.932 & -24.208 & 0.01 & -0.4117 & -0.4078 & 0.2880 \\
\hline $\mathrm{C} 19-\mathrm{H} 26$ & 1.06676 & 1.06677 & 1.959 & -25.399 & 0.01 & -0.4290 & -0.4226 & 0.2939 \\
\hline $\mathrm{C} 20-\mathrm{H} 27$ & 1.06881 & 1.06881 & 1.934 & -24.614 & 0.01 & -0.4147 & -0.4079 & 0.2821 \\
\hline $\mathrm{C} 21-\mathrm{H} 28$ & 1.06517 & 1.06518 & 1.951 & -25.010 & 0.01 & -0.4211 & -0.4135 & 0.2855 \\
\hline $\mathrm{C} 22-\mathrm{H} 29$ & 1.06968 & 1.06968 & 1.931 & -24.555 & 0.01 & -0.4150 & -0.4074 & 0.2832 \\
\hline
\end{tabular}

Table S4.27: Integrated Charges of $\mathbf{1}\left(\mathbf{S}_{\mathbf{0}}\right)$.

\begin{tabular}{|c|c|c|c|c|c|c|c|}
\hline Name & $\mathrm{q}(\mathrm{A})$ & Name & $q(A)$ & Name & $q(A)$ & Name & $\mathrm{q}(\mathrm{A})$ \\
\hline Se1 & 0.03 & & & Se16 & 0.03 & & \\
\hline $\mathrm{C} 2$ & -0.14 & H9 & 0.08 & $\mathrm{C} 17$ & -0.14 & $\mathrm{H} 24$ & 0.08 \\
\hline C3 & 0.01 & H10 & 0.04 & $\mathrm{C} 18$ & 0.01 & $\mathrm{H} 25$ & 0.04 \\
\hline $\mathrm{C} 4$ & -0.04 & H11 & 0.08 & C19 & -0.04 & $\mathrm{H} 26$ & 0.08 \\
\hline C5 & -0.05 & H12 & 0.02 & $\mathrm{C} 20$ & -0.05 & $\mathrm{H} 27$ & 0.02 \\
\hline C6 & -0.05 & H13 & 0.03 & $\mathrm{C} 21$ & -0.05 & $\mathrm{H} 28$ & 0.03 \\
\hline $\mathrm{C} 7$ & -0.05 & H14 & 0.03 & $\mathrm{C} 22$ & -0.05 & $\mathrm{H} 29$ & 0.03 \\
\hline $\mathrm{C} 8$ & -0.03 & H15 & 0.04 & $\mathrm{C} 23$ & -0.03 & H30 & 0.04 \\
\hline
\end{tabular}




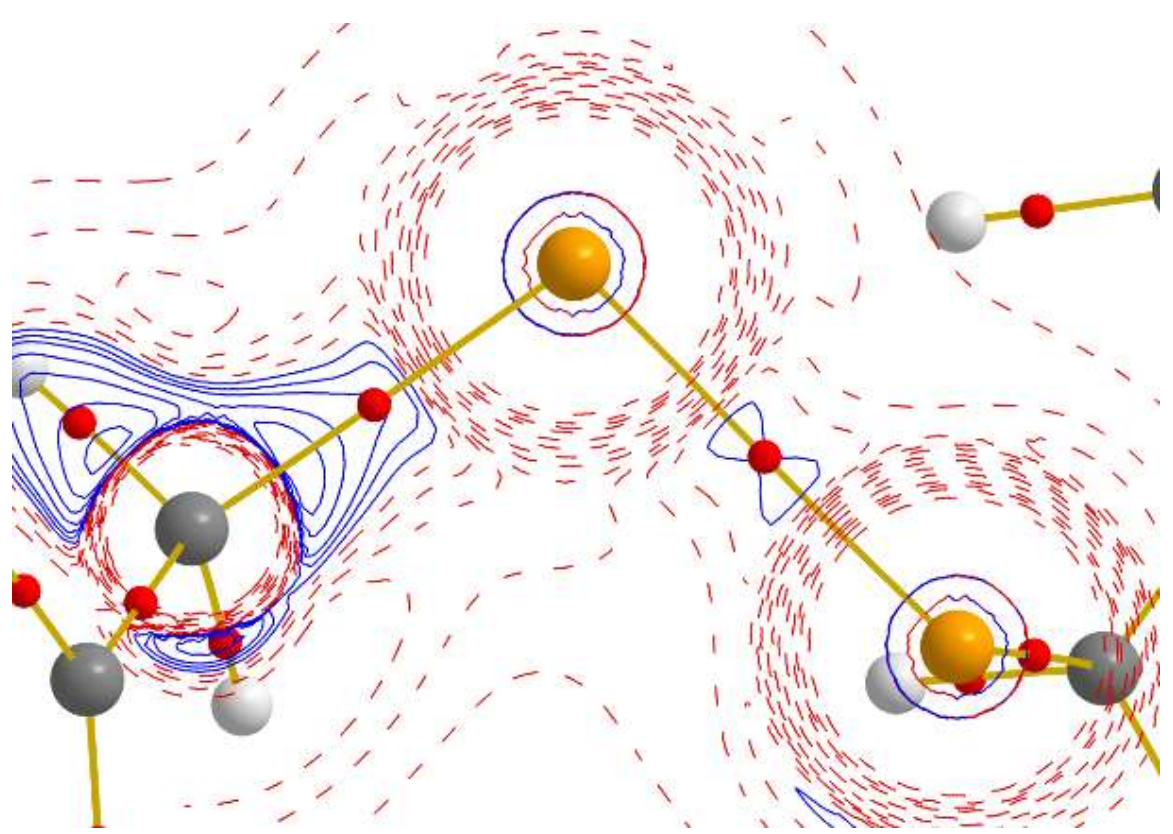

Figure S4.30: Laplacian map and BCPs (red) of $\mathbf{1}\left(\mathbf{S}_{0}\right)$. Isolevels +(red)/ -(blue) $0,1,2,3,5,8,13,21,34,43,55$ and $89 \mathrm{e}^{-5}$.

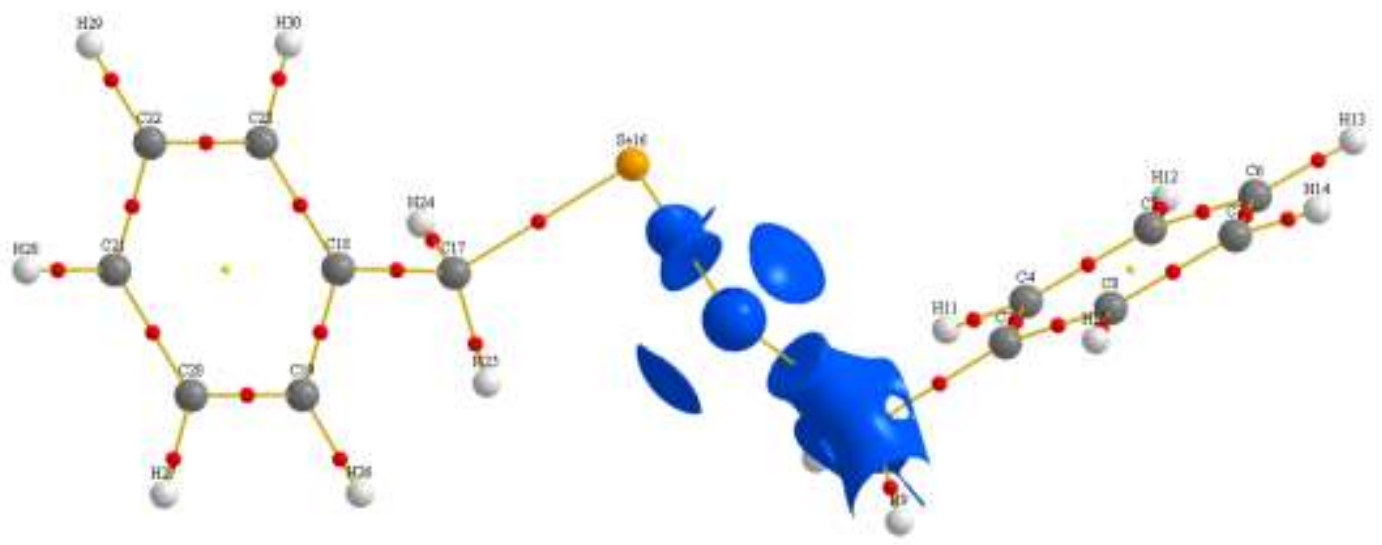

Figure S4.31: Se-VSCCs on the-0.01 a.u. Laplacian isolevel of $\mathbf{1}\left(\mathbf{S}_{\mathbf{0}}\right)$. 


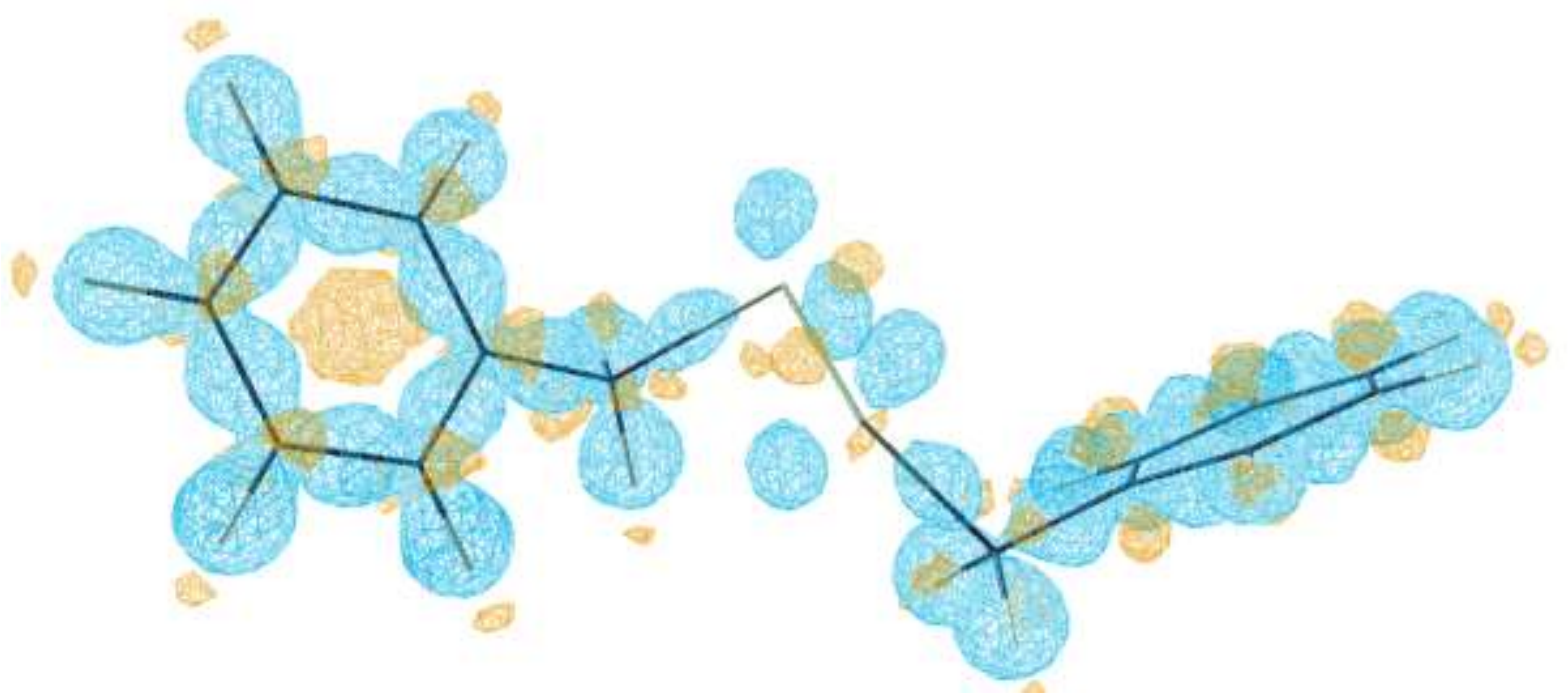

Figure S4.32: Deformation density map of the refined multipole model against calculated scattering factors of the optimized structure $\mathbf{1}\left(\mathbf{S}_{0}\right)$. Levels: $+0.1 \mathrm{e}^{-1}$ (blue) and $-0.1 \mathrm{e}^{-1}$ (orange).

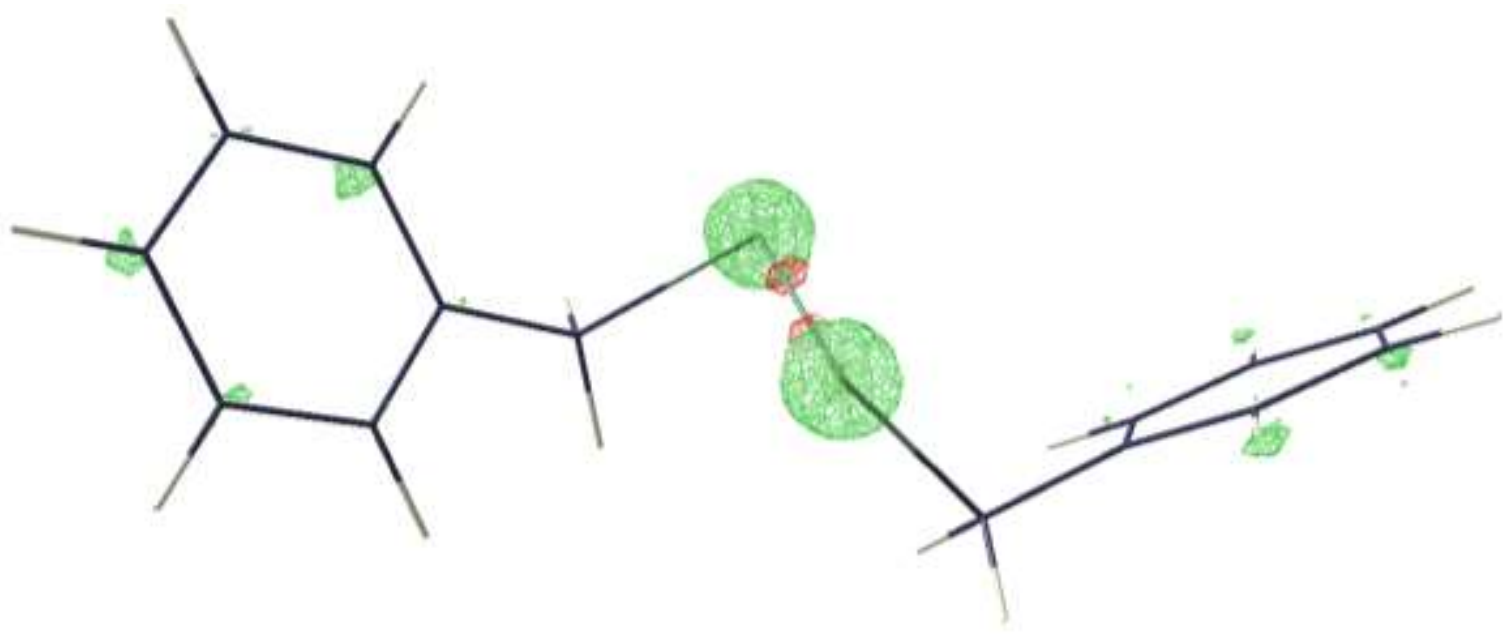

Figure S4.33: Residual density map of the refined multipole model against calculated scattering factors of the optimized structure $\mathbf{1}\left(\mathbf{S}_{\mathbf{0}}\right)$. Levels: $+0.1 \AA^{-1}$ (green) and $-0.1 \AA^{-1}$ (red).

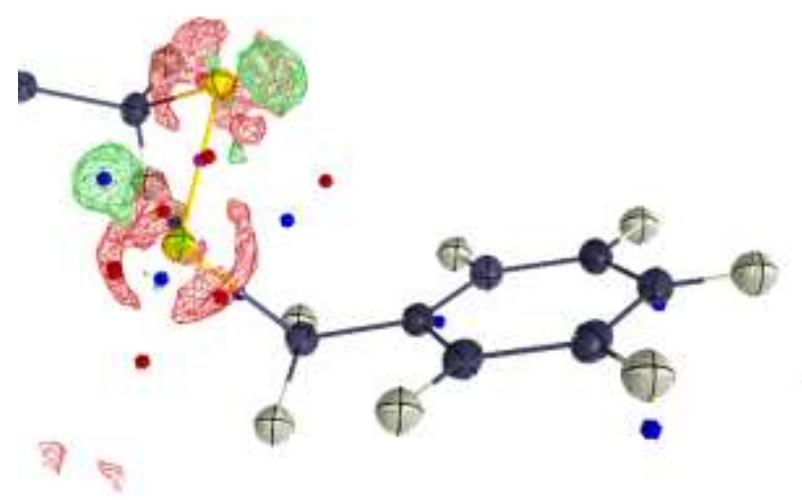

Figure S4.34: Residual density map of dataset $\mathbf{A}$ with theoretically determined anisotropic scattering factors based on $\mathbf{1}\left(\mathbf{S}_{\mathbf{0}}\right)$. Levels: $+0.4 \mathrm{e} \AA^{-1}$ (green) and $-0.4 \mathrm{e}^{-1}$ (red). 


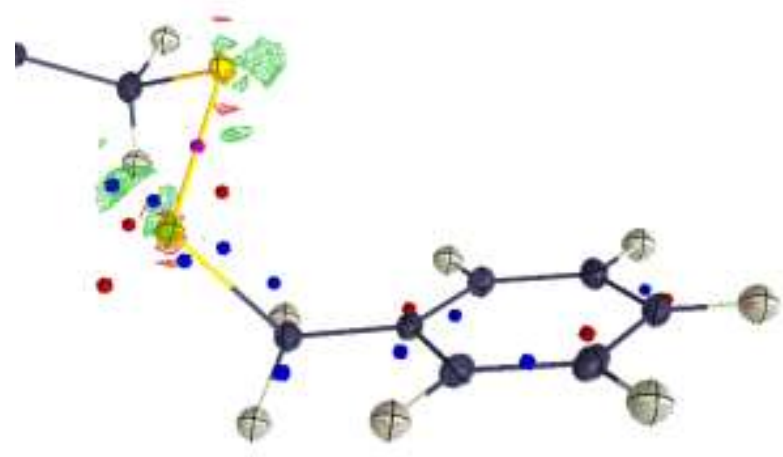

Figure S4.35: Residual density map of dataset $\mathbf{B}$ with theoretically determined aspherical scattering factors based on $\mathbf{1}\left(\mathbf{S}_{\mathbf{0}}\right)$. Levels: $+0.4 e^{-1}$ (green) and $-0.4 e^{-1}$ (red).

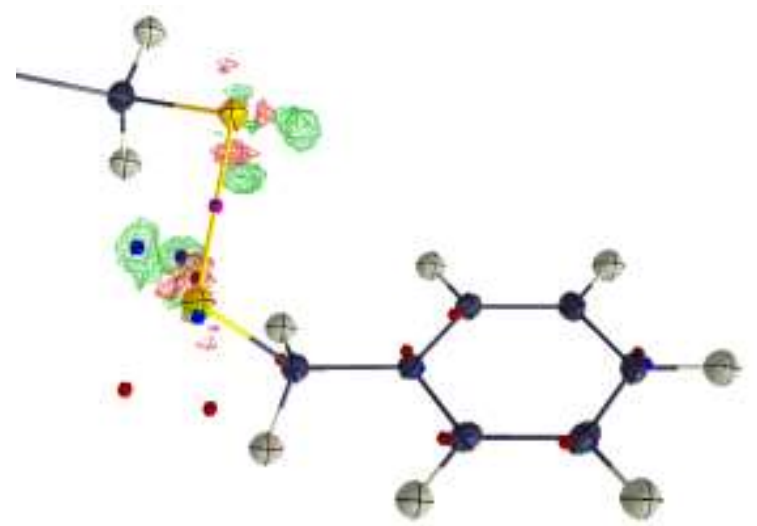

Figure S4.37: Residual density map of dataset $\mathbf{D}$ with theoretically determined aspherical scattering factors based on $\mathbf{1}\left(\mathbf{S}_{0}\right)$. Levels: $+0.5 e^{-1}$ (green) and $-0.5 e^{-1}$ (red).

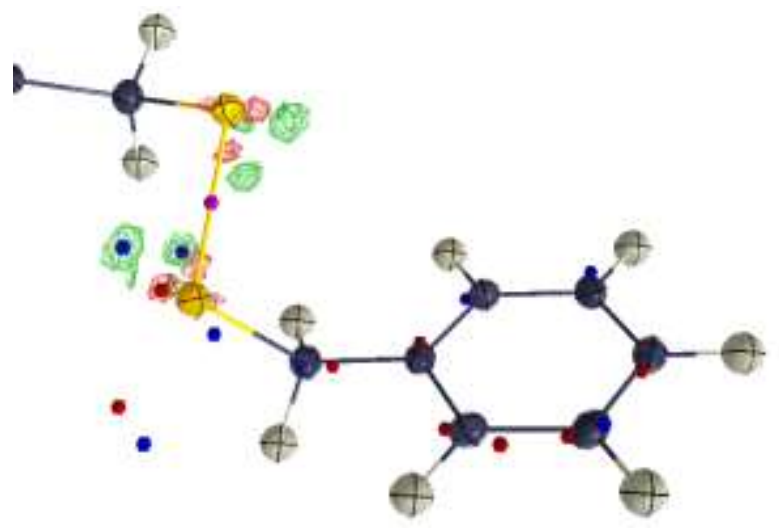

Figure S4.39: Residual density map of dataset $\mathbf{F}$ with theoretically determined aspherical scattering factors based on $\mathbf{1}\left(\mathbf{S}_{0}\right)$. Levels: $+0.3 \AA^{-1}$ (green) and $-0.3 e \AA^{-1}$ (red).

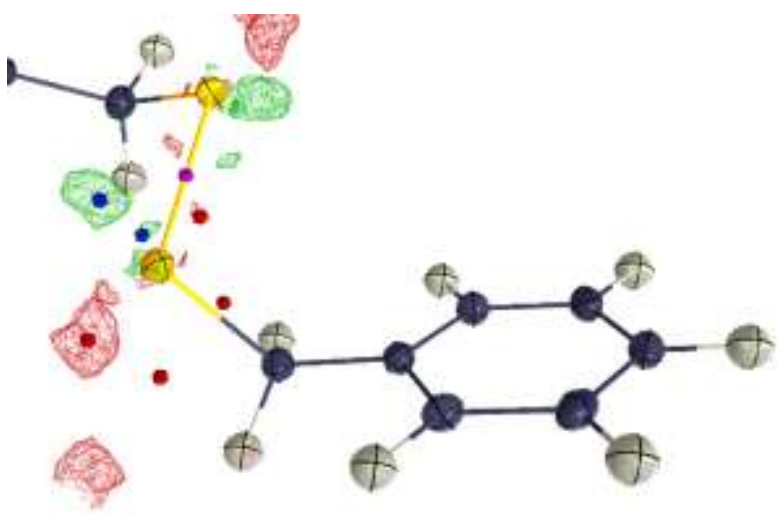

Figure S4.36: Residual density map of dataset $\mathbf{C}$ with theoretically determined aspherical scattering factors based on $\mathbf{1}\left(\mathbf{S}_{\mathbf{0}}\right)$. Levels: $+0.2 \mathrm{e}^{-1}$ (green) and $-0.2 \mathrm{e} \AA^{-1}$ (red).

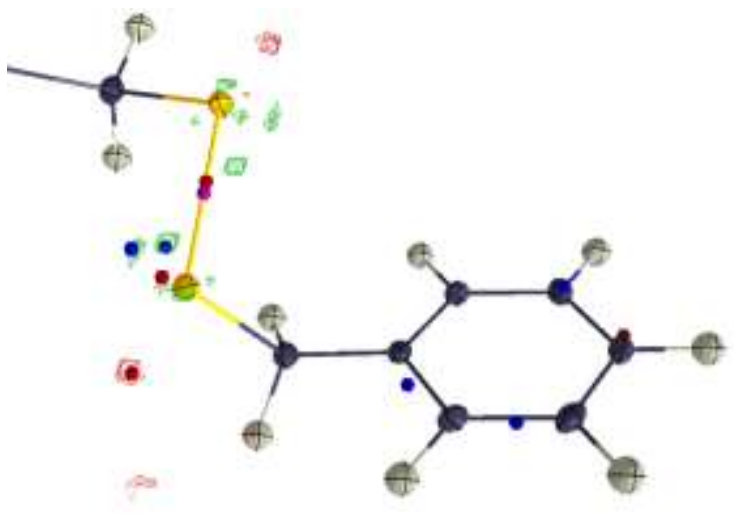

Figure S4.38: Residual density map of dataset $\mathbf{E}$ with theoretically determined aspherical scattering factors based on $\mathbf{1}\left(\mathbf{S}_{0}\right)$. Levels: $+0.4 \mathrm{e}^{-1}$ (green) and $-0.4 \mathrm{e}^{-1}$ (red).

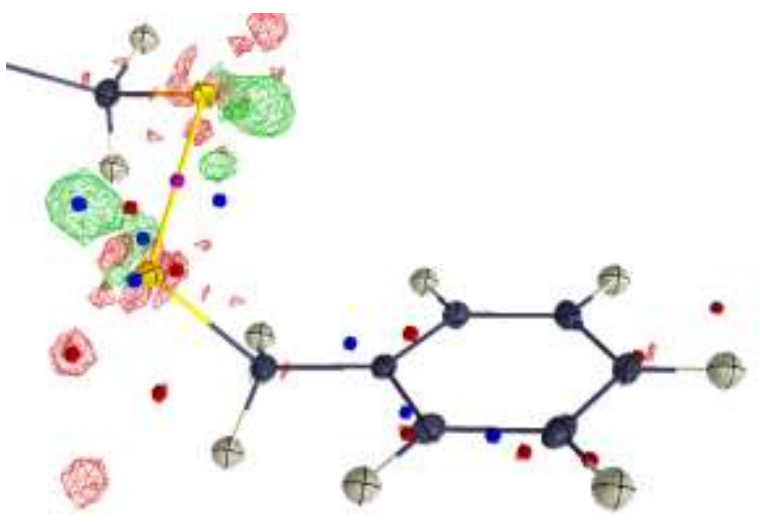

Figure S4.40: Residual density map of dataset $\mathbf{G}$ with theoretically determined aspherical scattering factors based on $\mathbf{1}\left(\mathbf{S}_{\mathbf{0}}\right)$. Levels: $+0.25 e \AA^{-1}$ (green) and $-0.25 e \AA^{-1}$ (red). 


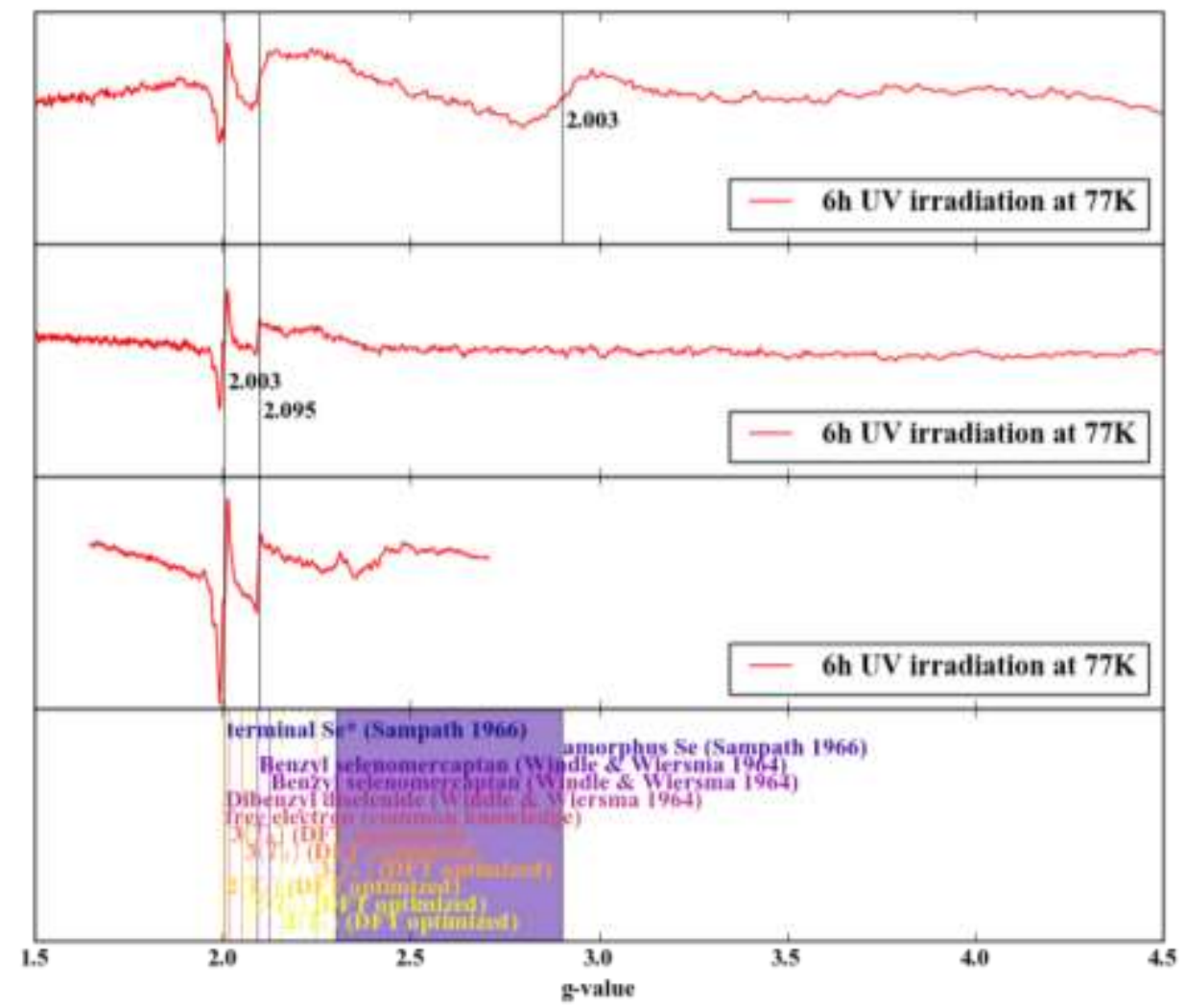

Figure S4.41: EPR signals after UV irradiation, low resolution with values from literature.

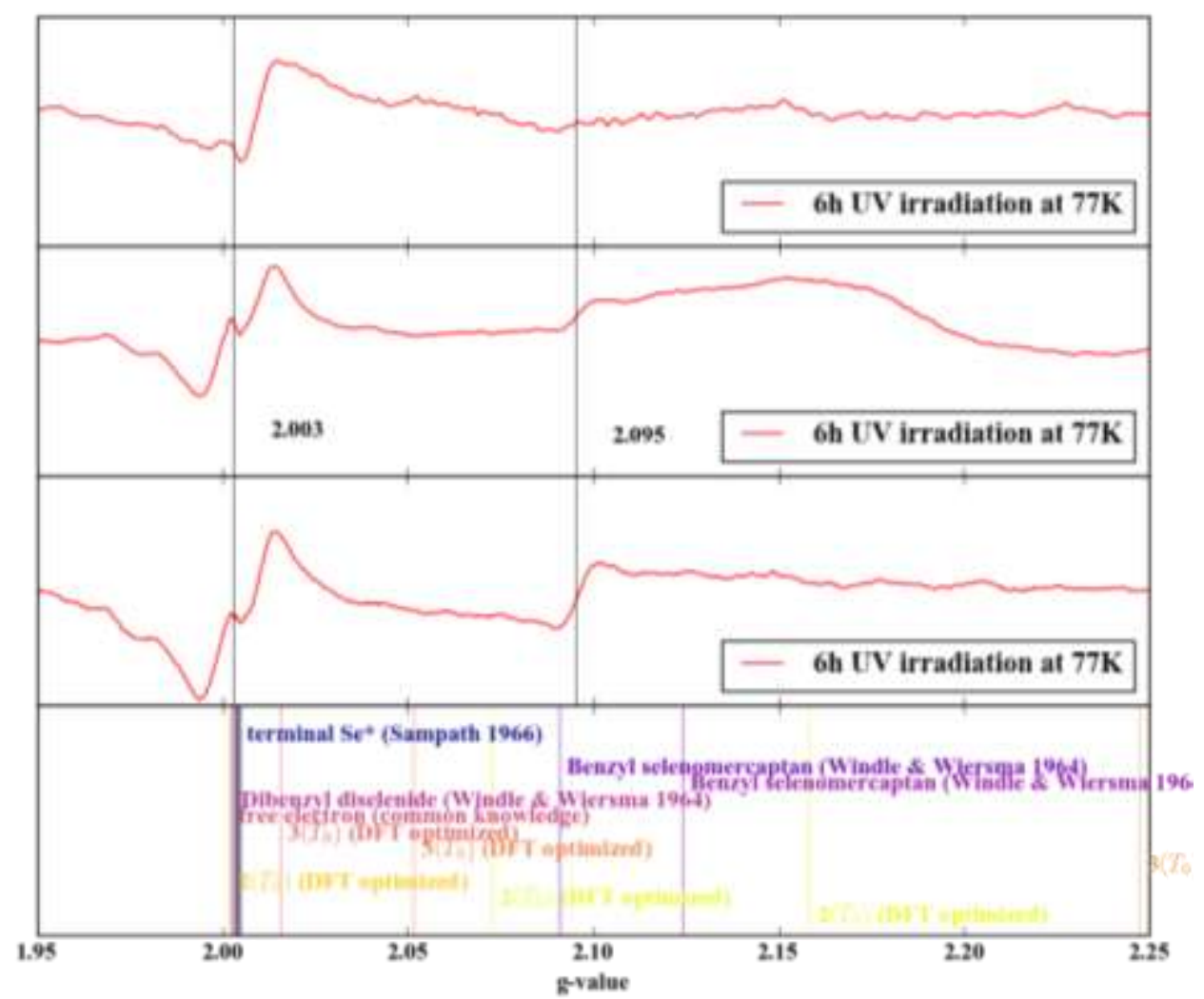

Figure S4.42: EPR signals after UV irradiation, medium resolution with values from literature. 


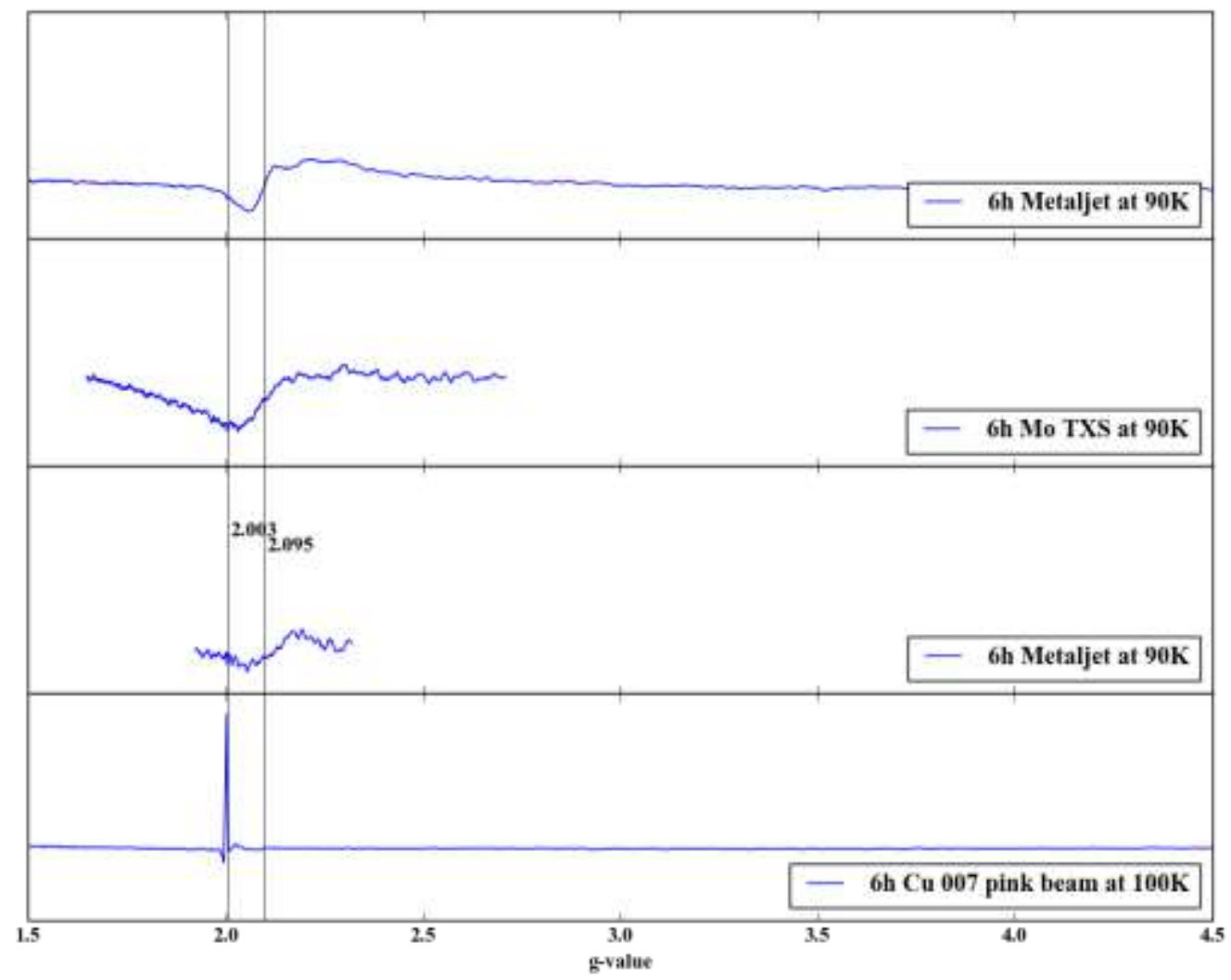

Figure S4.43: EPR signals after $X$-ray irradiation, low resolution.

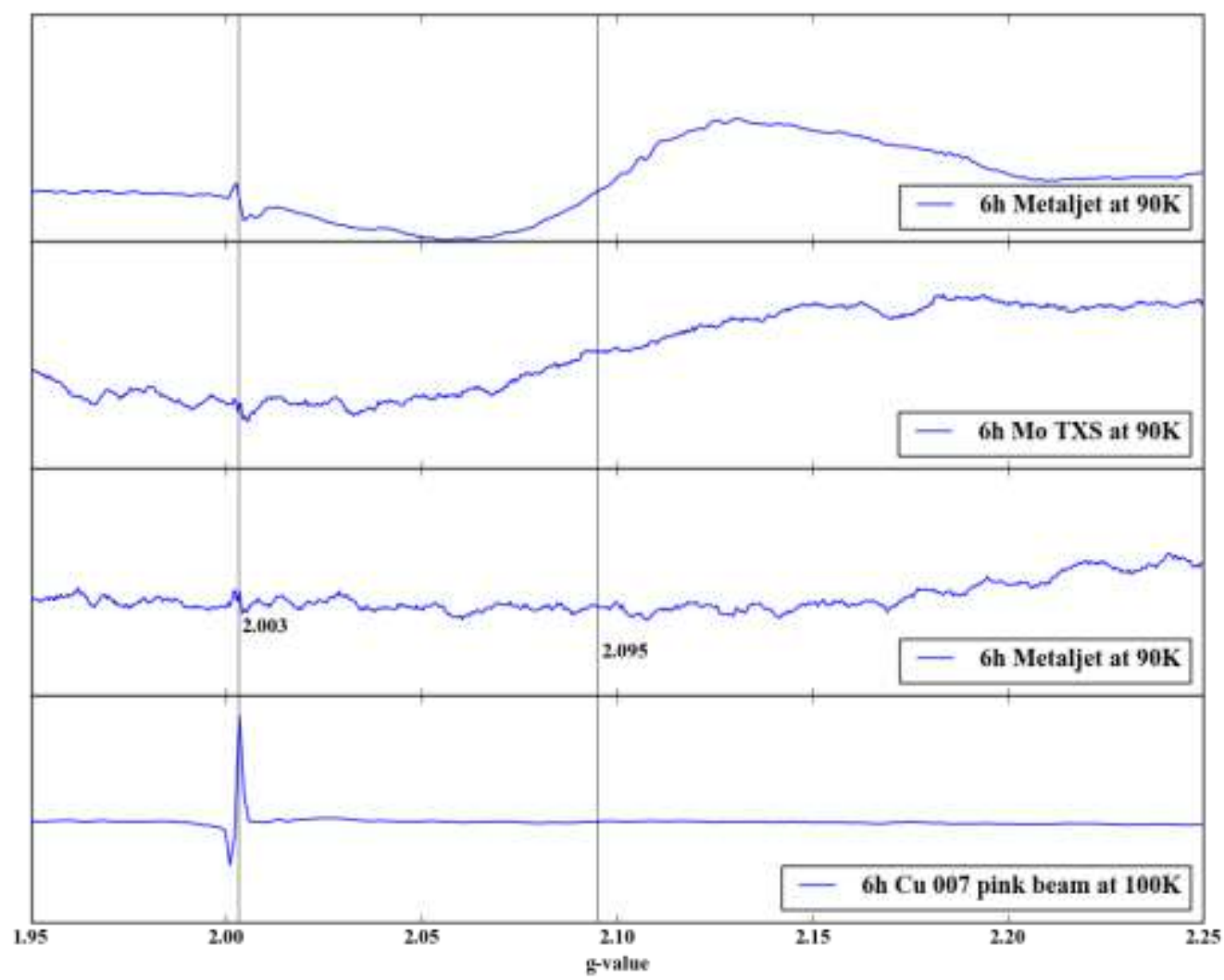

Figure S4.44: EPR signals after $\mathrm{X}$-ray irradiation, medium resolution. 


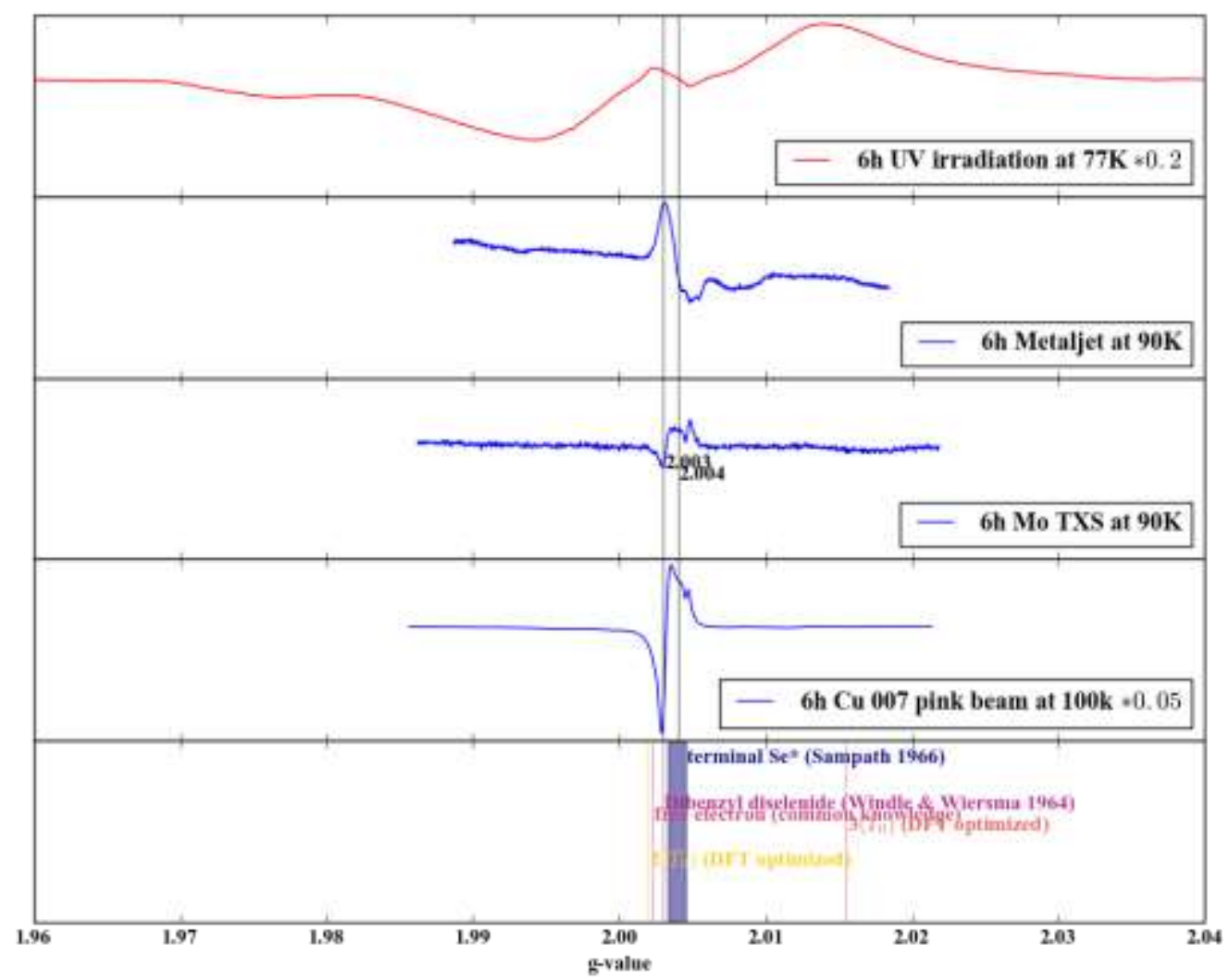

Figure S4.45: EPR signals after UV or X-ray irradiation, high resolution with values from literature.

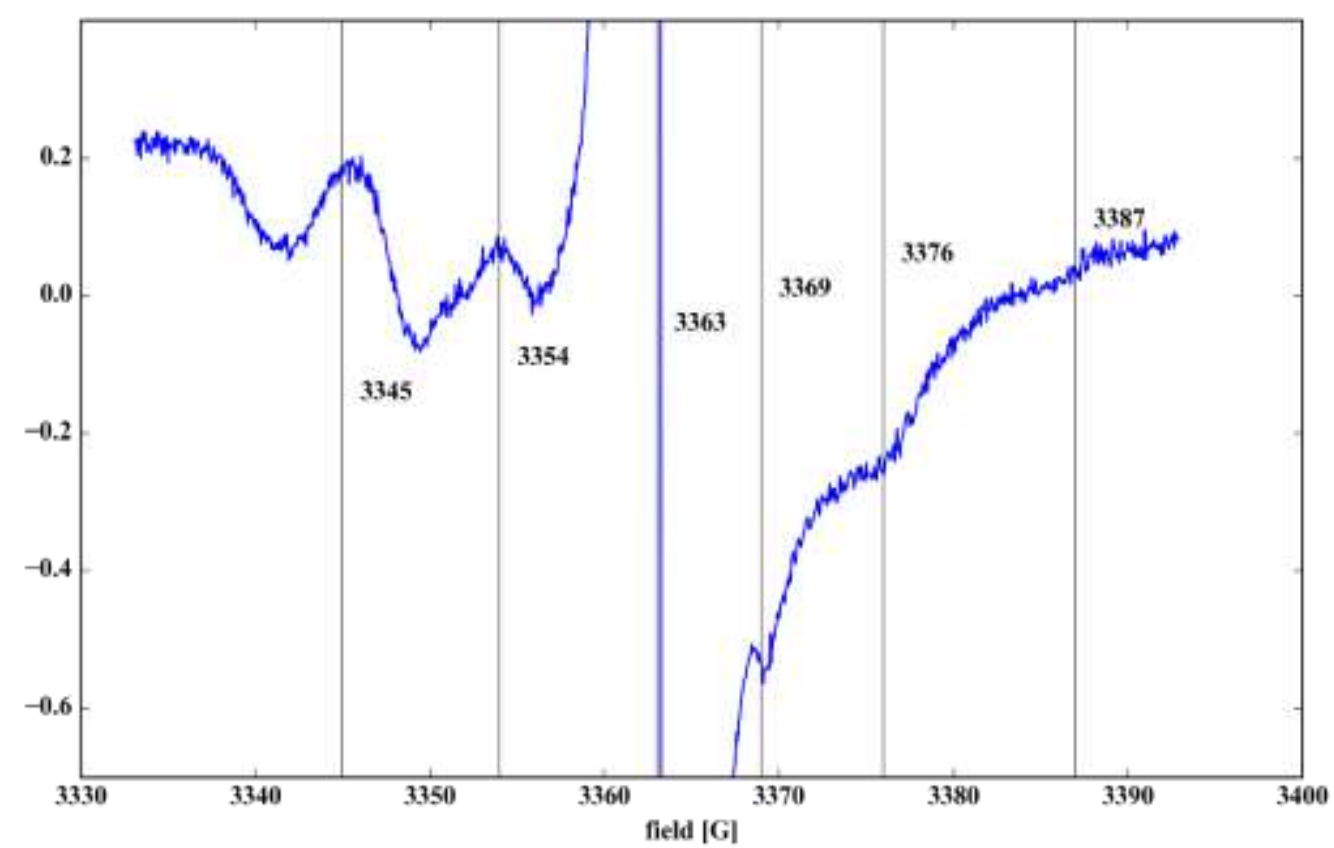

Figure S4.46: EPR signals after X-ray irradiation, detailed spectra of main peak. 


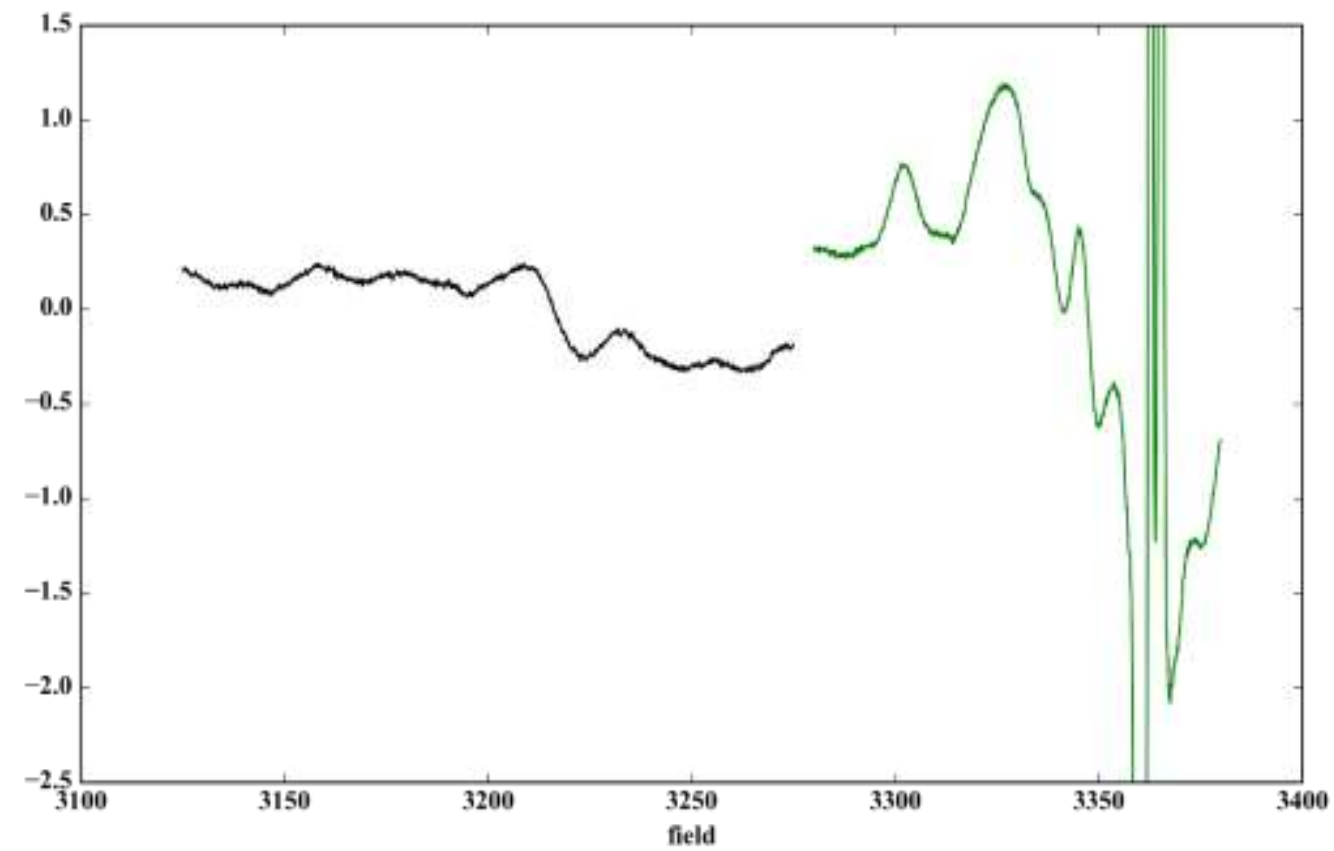

Figure S4.47: EPR signals after X-ray irradiation, detailed spectra of main and second peak. 


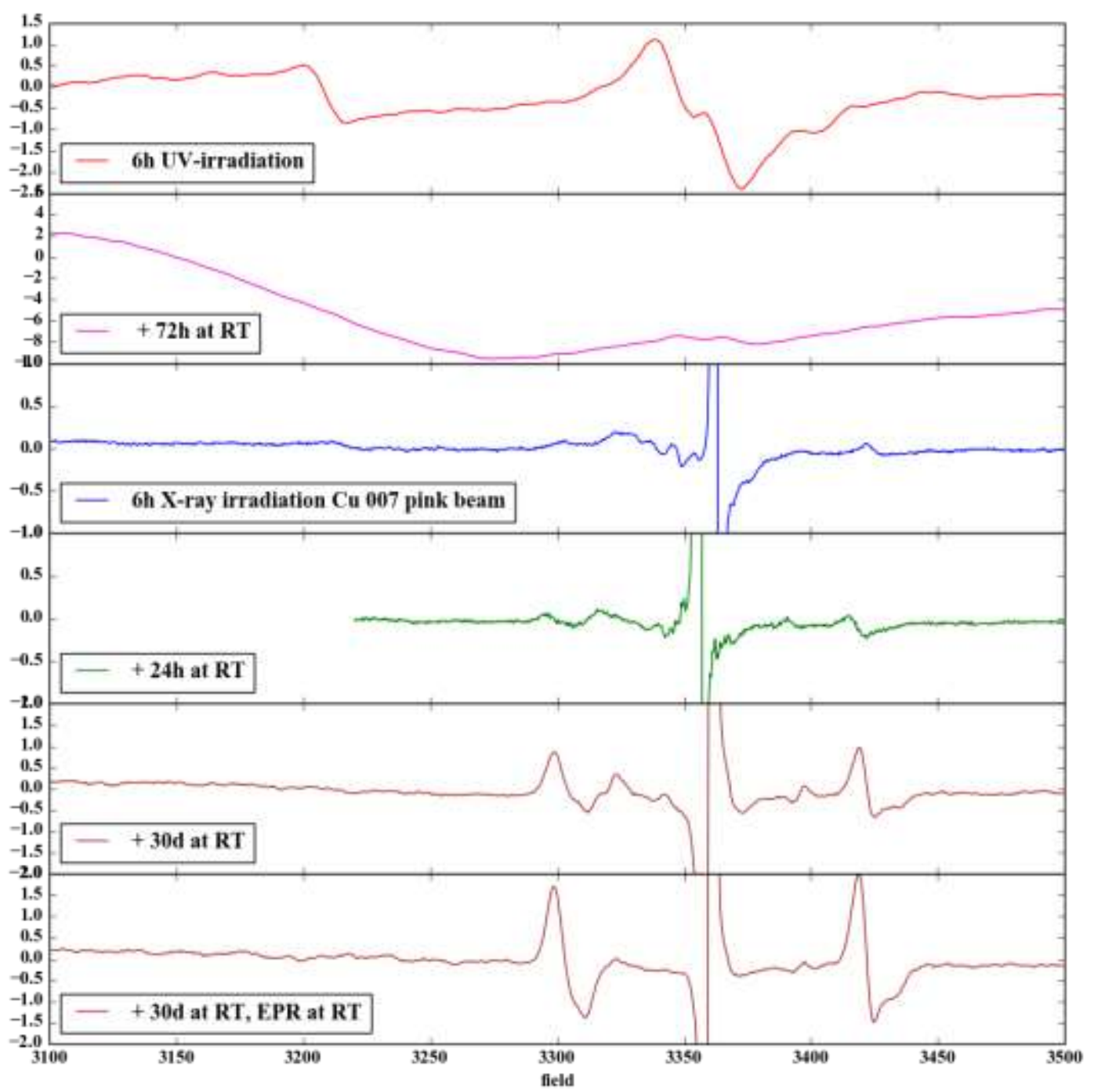

Figure S4.48: EPR signals of UV and X-ray irradiated sample after aging. 


\section{Supplementary Information to Chapter 5}

Table S5.1: Overview of Paracyclophane-datasets in comparison.

\begin{tabular}{|c|c|c|c|c|}
\hline Detector / Dataset & Apex2 & Pilatus3 & Photon2 & Photon3 \\
\hline X-ray source & $\mathrm{I} \mu \mathrm{S} 2$ & $\mathrm{I} \mu \mathrm{S} 2$ & $\mathrm{I} \mu \mathrm{S} 3$ & $\mathrm{I} \mu \mathrm{S} 3$ \\
\hline Source power [W] & 30 & 30 & 70 & 70 \\
\hline$\lambda[\AA]$ & 0.56086 & 0.56086 & 0.56086 & 0.56086 \\
\hline $\mathrm{a}[\AA]$ & $7.6987(3)$ & $7.7025(5)$ & $7.6942(2)$ & $7.6941(2)$ \\
\hline$c[\AA]$ & $9.2163(4)$ & $9.2204(6)$ & $9.2161(2)$ & $9.2161(2)$ \\
\hline$V\left[\AA^{-3}\right]$ & $546.25(5)$ & $547.03(8)$ & $545.61(4)$ & $545.59(3)$ \\
\hline Crystal size $\left[\mathrm{mm}^{3}\right]$ & $0.2 \times 0.3 \times 0.4$ & $0.2 \times 0.3 \times 0.4$ & $0.206 \times 0.315 \times 0.434$ & $0.206 \times 0.315 \times 0.434$ \\
\hline Absorption coefficient $\left[\mathrm{mm}^{-1}\right]$ & 0.047 & 0.047 & 0.047 & 0.047 \\
\hline$F(000)$ & 224 & 224 & 224 & 224 \\
\hline$\theta$ range $\left[{ }^{\circ}\right] \mathrm{min}$. & 2.72 & 2.719 & 2.72 & 3.432 \\
\hline $\max$. & 34.122 & 34.173 & 34.122 & 34.117 \\
\hline Resolution Range $[\AA]$ min. & 5.909 & 5.912 & 5.909 & 4.684 \\
\hline $\max$ & 0.500 & 0.499 & 0.500 & 0.500 \\
\hline Collected ref. & 40583 & 47140 & 40583 & 185261 \\
\hline Independent ref. & 1275 & 1278 & 1275 & 1272 \\
\hline $\mathrm{R}_{\text {int }}[\%]$ & 3.56 & 2.47 & 3.56 & 3.71 \\
\hline Non-default SAINT input & Queuehalf 11 & & PLANEBG & PLANEBG \\
\hline R1(I) (all data, IAM ) [\%] & 4.35 & 4.25 & 4.35 & 3.77 \\
\hline wR2(I) (all data, IAM ) [\%] & 13.98 & 14.11 & 13.98 & 13.17 \\
\hline GOF $\left(F^{2}, I A M\right)$ & 1.171 & 1.096 & 1.171 & 1.127 \\
\hline \multirow{2}{*}{ Diff peak/hole $\left(F^{2}, I A M\right)\left[\AA^{-3}\right]$} & 0.535 & 0.496 & 0.535 & 0.536 \\
\hline & -0.166 & -0.196 & -0.166 & -0.186 \\
\hline Data in XD & 1210 & 1227 & 1239 & 1233 \\
\hline Parameter in XD & 74 & 74 & 74 & 74 \\
\hline $\mathrm{R} 1(\mathrm{I})$ (all data, merged, XD) [\%] & 0.0143 & 0.0118 & 0.0128 & 0.0126 \\
\hline wR(I) (all data, merged, XD) [\%] & 0.0188 & 0.0169 & 0.0142 & 0.0141 \\
\hline $\operatorname{GOF}(I, X D)$ & 1.8921 & 2.5359 & 4.2661 & 5.4566 \\
\hline \multirow[t]{2}{*}{ Diff peak/hole $\left(F^{2}, X D\right)\left[e \AA^{-3}\right]$} & -0.116 & -0.087 & -0.107 & -0.108 \\
\hline & 0.103 & 0.147 & 0.094 & 0.097 \\
\hline egross & 5.3134 & 4.3258 & 2.9607 & 2.9328 \\
\hline
\end{tabular}


Table S5.2: Data quality statistics from XPREP of dataset Para_A pex2.

\begin{tabular}{|c|c|c|c|c|c|c|c|c|c|c|c|c|}
\hline \multicolumn{3}{|c|}{ Resolution } & \multirow{2}{*}{$\begin{array}{l}\text { Number } \\
\text { of Data } \\
33\end{array}$} & \multirow{2}{*}{$\begin{array}{l}\text { Theory } \\
34\end{array}$} & \multirow{2}{*}{$\begin{array}{l}\text { Complete- } \\
\text { ness [\%] } \\
97.1\end{array}$} & \multirow{2}{*}{$\begin{array}{l}\text { Multipli } \\
\text { city } \\
17.62\end{array}$} & \multirow{2}{*}{$\begin{array}{c}\langle I\rangle \\
189.06\end{array}$} & \multirow{2}{*}{$\begin{array}{r}\langle I / \sigma\rangle \\
279.85\end{array}$} & \multirow{2}{*}{$\begin{array}{l}R_{\text {merge }} \\
0.0085\end{array}$} & \multirow{2}{*}{$\begin{array}{l}R_{\text {sigma }} \\
0.002\end{array}$} & \multirow{2}{*}{$\begin{array}{l}\text { Rrim } \\
0.0088\end{array}$} & \multirow{2}{*}{$\begin{array}{l}R_{\text {pim }} \\
0.0021\end{array}$} \\
\hline Inf & - & 2 & & & & & & & & & & \\
\hline 2 & - & 1.2 & 95 & 95 & 100 & 20.34 & 41.69 & 101.56 & 0.0207 & 0.0058 & 0.0212 & 0.0047 \\
\hline 1.2 & - & 0.9 & 146 & 146 & 100 & 18.69 & 17.57 & 47 & 0.036 & 0.0141 & 0.0371 & 0.0085 \\
\hline 0.9 & - & 0.76 & 163 & 163 & 100 & 35.77 & 7.03 & 61.97 & 0.0445 & 0.0123 & 0.0452 & 0.0075 \\
\hline 0.76 & - & 0.7 & 107 & 107 & 100 & 43.11 & 5.94 & 62.83 & 0.0445 & 0.0108 & 0.0451 & 0.0068 \\
\hline 0.7 & - & 0.64 & 152 & 152 & 100 & 38.18 & 4.03 & 41.67 & 0.0591 & 0.0161 & 0.0599 & 0.0096 \\
\hline 0.64 & - & 0.6 & 138 & 138 & 100 & 34.73 & 3.1 & 31.87 & 0.0707 & 0.0216 & 0.0718 & 0.0122 \\
\hline 0.6 & - & 0.58 & 87 & 87 & 100 & 33.38 & 2.34 & 24.29 & 0.0916 & 0.0288 & 0.093 & 0.0158 \\
\hline 0.58 & - & 0.56 & 94 & 94 & 100 & 32.62 & 1.4 & 16.02 & 0.1302 & 0.0452 & 0.1322 & 0.0228 \\
\hline 0.56 & - & 0.54 & 109 & 109 & 100 & 28.58 & 1.47 & 16.3 & 0.1178 & 0.0486 & 0.1199 & 0.0221 \\
\hline 0.54 & - & 0.53 & 62 & 62 & 100 & 28.32 & 1.44 & 13.36 & 0.1213 & 0.0487 & 0.1236 & 0.0234 \\
\hline 0.53 & - & 0.52 & 65 & 65 & 100 & 27.95 & 1.08 & 11.32 & 0.1482 & 0.0646 & 0.151 & 0.0286 \\
\hline 0.52 & - & 0.51 & 66 & 66 & 100 & 26.61 & 1.02 & 10.17 & 0.1903 & 0.074 & 0.1941 & 0.0376 \\
\hline 0.51 & - & 0.5 & 78 & 78 & 100 & 26.1 & 0.71 & 8.04 & 0.2229 & 0.1025 & 0.2274 & 0.0443 \\
\hline 0.6 & - & 0.5 & 561 & 561 & 100 & 29.32 & 1.38 & 14.72 & 0.1268 & 0.0503 & 0.1291 & 0.0235 \\
\hline Inf & - & 0.5 & 1395 & 1396 & 99.9 & 30.62 & 11.73 & 44.13 & 0.035 & 0.0092 & 0.0357 & 0.0067 \\
\hline
\end{tabular}

Table S5.3: Data quality statistics from XPREP of dataset Para_Pilatus3

\begin{tabular}{|c|c|c|c|c|c|c|c|c|c|c|c|c|}
\hline \multicolumn{3}{|c|}{ Resolution } & $\begin{array}{l}\text { Number } \\
\text { of Data } \\
34\end{array}$ & $\begin{array}{l}\text { Theory } \\
34\end{array}$ & $\begin{array}{l}\text { Complete- } \\
\text { ness [\%] } \\
100\end{array}$ & $\begin{array}{l}\text { Multipli } \\
\text { city } \\
2409\end{array}$ & $\begin{array}{c}\langle I\rangle \\
18273\end{array}$ & $\begin{array}{r}\langle I / \sigma\rangle \\
37469\end{array}$ & $\begin{array}{l}R_{\text {merge }} \\
0.0083\end{array}$ & $\begin{array}{l}\text { Rsigma } \\
0.0014\end{array}$ & $\begin{array}{l}\text { Rrim } \\
0.0085\end{array}$ & $\begin{array}{l}R_{\text {pim }} \\
0.0018\end{array}$ \\
\hline 2 & - & 1.2 & 95 & 95 & 100 & 35.6 & 39.81 & 179.48 & 0.0179 & 0.0033 & 0.0182 & 0.0031 \\
\hline 1.2 & - & 0.9 & 146 & 146 & 100 & 52.75 & 15.86 & 145.44 & 0.0244 & 0.0043 & 0.0247 & 0.0034 \\
\hline 0.9 & - & 0.76 & 163 & 164 & 99.4 & 51.8 & 6.01 & 86.21 & 0.0371 & 0.0078 & 0.0375 & 0.0052 \\
\hline 0.76 & - & 0.7 & 107 & 107 & 100 & 43.92 & 4.9 & 70.69 & 0.0397 & 0.0092 & 0.0402 & 0.0061 \\
\hline 0.7 & - & 0.64 & 152 & 152 & 100 & 35.8 & 3.22 & 46.71 & 0.0522 & 0.0139 & 0.053 & 0.0091 \\
\hline 0.64 & - & 0.6 & 139 & 139 & 100 & 30.08 & 2.31 & 34.97 & 0.0637 & 0.0187 & 0.0649 & 0.0119 \\
\hline 0.6 & - & 0.58 & 87 & 87 & 100 & 28.75 & 1.74 & 27.14 & 0.076 & 0.0243 & 0.0774 & 0.0146 \\
\hline 0.58 & - & 0.56 & 95 & 95 & 100 & 28.39 & 0.99 & 17.7 & 0.1051 & 0.039 & 0.107 & 0.0202 \\
\hline 0.56 & - & 0.54 & 105 & 107 & 98.1 & 25.8 & 0.91 & 16.85 & 0.1297 & 0.0463 & 0.1324 & 0.0259 \\
\hline 0.54 & - & 0.53 & 63 & 63 & 100 & 27.06 & 0.96 & 14.38 & 0.1211 & 0.0427 & 0.1236 & 0.0245 \\
\hline 0.53 & - & 0.52 & 63 & 63 & 100 & 27.54 & 0.73 & 12.7 & 0.1588 & 0.0528 & 0.1619 & 0.0315 \\
\hline 0.52 & - & 0.51 & 66 & 66 & 100 & 25.98 & 0.63 & 10.65 & 0.1874 & 0.0682 & 0.1913 & 0.0377 \\
\hline 0.51 & - & 0.5 & 85 & 85 & 100 & 23.46 & 0.42 & 8.12 & 0.2051 & 0.1045 & 0.2097 & 0.0421 \\
\hline 0.6 & - & 0.5 & 564 & 566 & 99.6 & 26.69 & 0.93 & 15.8 & 0.1184 & 0.0445 & 0.1208 & 0.0234 \\
\hline Inf & - & 0.5 & 1400 & 1403 & 99.8 & 35.52 & 10.81 & 66.79 & 0.0246 & 0.0053 & 0.025 & 0.0041 \\
\hline
\end{tabular}


Table S5.4: Data quality statistics from XPREP of dataset Para_Photon2.

\begin{tabular}{|c|c|c|c|c|c|c|c|c|c|c|c|c|}
\hline \multicolumn{3}{|c|}{ Resolution } & \multirow{2}{*}{$\begin{array}{l}\text { Number } \\
\text { of Data } \\
33\end{array}$} & \multirow{2}{*}{$\begin{array}{l}\text { Theory } \\
34\end{array}$} & \multirow{2}{*}{$\begin{array}{l}\text { Complete- } \\
\text { ness [\%] } \\
97.1\end{array}$} & \multirow{2}{*}{$\begin{array}{l}\text { Multipli } \\
\text { city } \\
81.76\end{array}$} & \multirow{2}{*}{$\begin{array}{c}\langle I\rangle \\
187.25\end{array}$} & \multirow{2}{*}{$\begin{array}{r}\langle I / \sigma\rangle \\
534.38\end{array}$} & \multirow{2}{*}{$\begin{array}{l}R_{\text {merge }} \\
0.0152\end{array}$} & \multirow{2}{*}{$\begin{array}{l}\text { Rsigma } \\
0.0024\end{array}$} & \multirow{2}{*}{$\begin{array}{l}\text { Rrim } \\
0.0155\end{array}$} & \multirow{2}{*}{$\begin{array}{l}R_{\text {pim }} \\
0.0022\end{array}$} \\
\hline Inf & - & 2 & & & & & & & & & & \\
\hline 2 & - & 1.2 & 95 & 95 & 100 & 140.62 & 40.92 & 524.47 & 0.0214 & 0.0012 & 0.0215 & 0.0019 \\
\hline 1.2 & - & 0.9 & 146 & 146 & 100 & 141.78 & 16.9 & 341.59 & 0.0323 & 0.0018 & 0.0324 & 0.0027 \\
\hline 0.9 & - & 0.76 & 163 & 163 & 100 & 127.4 & 6.62 & 180.11 & 0.0584 & 0.0038 & 0.0587 & 0.0051 \\
\hline 0.76 & - & 0.7 & 107 & 107 & 100 & 111.59 & 5.55 & 139.88 & 0.0652 & 0.0047 & 0.0655 & 0.0061 \\
\hline 0.7 & - & 0.64 & 149 & 149 & 100 & 92.37 & 3.71 & 91.68 & 0.086 & 0.0071 & 0.0865 & 0.0089 \\
\hline 0.64 & - & 0.6 & 141 & 141 & 100 & 67.04 & 2.87 & 68.47 & 0.0818 & 0.0096 & 0.0824 & 0.0099 \\
\hline 0.6 & - & 0.58 & 87 & 87 & 100 & 56.3 & 2.12 & 50.15 & 0.0909 & 0.0133 & 0.0917 & 0.0121 \\
\hline 0.58 & - & 0.56 & 94 & 94 & 100 & 51.11 & 1.25 & 33.18 & 0.1157 & 0.0205 & 0.1168 & 0.0161 \\
\hline 0.56 & - & 0.54 & 106 & 106 & 100 & 42.5 & 1.29 & 32.93 & 0.1068 & 0.0229 & 0.1081 & 0.0162 \\
\hline 0.54 & - & 0.53 & 64 & 64 & 100 & 38.09 & 1.26 & 26.49 & 0.1102 & 0.0241 & 0.1118 & 0.0182 \\
\hline 0.53 & - & 0.52 & 62 & 62 & 100 & 36 & 0.9 & 20.79 & 0.1404 & 0.0328 & 0.1425 & 0.0239 \\
\hline 0.52 & - & 0.51 & 68 & 68 & 100 & 33.25 & 0.94 & 21.31 & 0.1418 & 0.036 & 0.144 & 0.0247 \\
\hline 0.51 & - & 0.5 & 76 & 76 & 100 & 30.03 & 0.6 & 14.83 & 0.1682 & 0.0528 & 0.1711 & 0.0309 \\
\hline 0.6 & - & 0.5 & 557 & 557 & 100 & 42.05 & 1.23 & 29.68 & 0.1107 & 0.024 & 0.112 & 0.0167 \\
\hline Inf & - & 0.5 & 1391 & 1392 & 99.9 & 83.46 & 11.4 & 144.86 & 0.0358 & 0.0035 & 0.036 & 0.0035 \\
\hline
\end{tabular}

Table S5.5: Data quality statistics from XPREP of dataset Para_Photon3.

\begin{tabular}{|c|c|c|c|c|c|c|c|c|c|c|c|c|}
\hline \multicolumn{3}{|c|}{ Resolution } & $\begin{array}{l}\text { Number } \\
\text { of Data } \\
33\end{array}$ & $\begin{array}{l}\text { Theory } \\
34\end{array}$ & $\begin{array}{l}\text { Complete- } \\
\text { ness [\%] } \\
97.1\end{array}$ & $\begin{array}{l}\text { Multipli } \\
\text { city } \\
12968\end{array}$ & $\begin{array}{c}\langle I\rangle \\
18745\end{array}$ & $\begin{array}{r}\langle I / \sigma\rangle \\
65138\end{array}$ & $\begin{array}{l}R_{\text {merge }} \\
0.016\end{array}$ & $\begin{array}{l}\text { Rsigma } \\
0.0024\end{array}$ & $\begin{array}{l}\text { Rrim } \\
0.0162\end{array}$ & $\begin{array}{l}R_{\text {pim }} \\
0.0019\end{array}$ \\
\hline 2 & - & 1.2 & 95 & 95 & 100 & 229.09 & 40.96 & 672.58 & 0.022 & 0.001 & 0.022 & 0.0015 \\
\hline 1.2 & - & 0.9 & 146 & 146 & 100 & 238.17 & 16.92 & 441.4 & 0.0321 & 0.0014 & 0.0322 & 0.0021 \\
\hline 0.9 & - & 0.76 & 163 & 163 & 100 & 216.97 & 6.63 & 232.98 & 0.0575 & 0.0029 & 0.0576 & 0.0038 \\
\hline 0.76 & - & 0.7 & 107 & 107 & 100 & 187.67 & 5.57 & 181.48 & 0.0629 & 0.0036 & 0.0631 & 0.0046 \\
\hline 0.7 & - & 0.64 & 149 & 149 & 100 & 154.3 & 3.71 & 119.47 & 0.0807 & 0.0055 & 0.081 & 0.0065 \\
\hline 0.64 & - & 0.6 & 141 & 141 & 100 & 113.48 & 2.88 & 89.73 & 0.0777 & 0.0073 & 0.0781 & 0.0073 \\
\hline 0.6 & - & 0.58 & 87 & 87 & 100 & 96.48 & 2.13 & 66.21 & 0.0859 & 0.0099 & 0.0864 & 0.0088 \\
\hline 0.58 & - & 0.56 & 94 & 94 & 100 & 88.48 & 1.26 & 43.5 & 0.1132 & 0.0155 & 0.1138 & 0.0119 \\
\hline 0.56 & - & 0.54 & 106 & 106 & 100 & 74.52 & 1.29 & 43.23 & 0.1075 & 0.0173 & 0.1082 & 0.0123 \\
\hline 0.54 & - & 0.53 & 64 & 64 & 100 & 67.53 & 1.27 & 34.98 & 0.1083 & 0.0182 & 0.1091 & 0.0134 \\
\hline 0.53 & - & 0.52 & 62 & 62 & 100 & 63.81 & 0.9 & 27.1 & 0.14 & 0.0249 & 0.1411 & 0.0178 \\
\hline 0.52 & - & 0.51 & 68 & 68 & 100 & 59.81 & 0.94 & 28.17 & 0.1414 & 0.0268 & 0.1427 & 0.0183 \\
\hline 0.51 & - & 0.5 & 77 & 78 & 98.7 & 52.1 & 0.59 & 19.36 & 0.1689 & 0.0421 & 0.1705 & 0.023 \\
\hline 0.6 & - & 0.5 & 558 & 559 & 99.8 & 73.38 & 1.23 & 38.99 & 0.1087 & 0.0183 & 0.1094 & 0.0124 \\
\hline Inf & - & 0.5 & 1392 & 1394 & 99.9 & 140.89 & 11.4 & 186.38 & 0.0368 & 0.0029 & 0.037 & 0.0028 \\
\hline
\end{tabular}


Table S5.6: XD refinement strategy for dataset Para_Apex2. Abbreviations: M: monopoles; D: dipoles; Q: quadrupoles; O: octupoles; K: $\kappa$, U2, U3: Gram Charlier $2^{\text {nd }}$ and $3^{\text {rd }}$ order, H-XYZ: only hydrogen position against data up to $0.5 \sin (\theta) / \lambda$.

\begin{tabular}{llllllllll}
\hline Step & New Parameter & $\mathrm{D}<0.5 \sin ($ th)/I & \#M P-Param & Data & Para & Data/Para & $\mathrm{R}\left(\mathrm{F}^{2}\right)$ & $\mathrm{wR}\left(\mathrm{F}^{2}\right)$ & $\mathrm{GOF}$ \\
1 & SCALE & 176 & 0 & 1210 & 1 & 1210 & 0.0727 & 0.0789 & 7.683 \\
2 & DQO & 176 & 27 & 1210 & 28 & 43.2 & 0.0228 & 0.0289 & 2.85 \\
3 & $\mathrm{U} 2$ & 176 & 27 & 1210 & 42 & 28.8 & 0.0221 & 0.0284 & 2.812 \\
4 & $\mathrm{H}-\mathrm{XYZ}$ & 175 & 0 & 175 & 7 & 25 & 0.0202 & 0.0196 & 4.31 \\
5 & $\mathrm{XYZ}$ & 176 & 27 & 1210 & 49 & 24.7 & 0.0194 & 0.0248 & 2.465 \\
6 & $\mathrm{H}-\mathrm{XYZ}$ & 175 & 0 & 175 & 7 & 25 & 0.0191 & 0.0174 & 3.821 \\
7 & $\mathrm{M}$ & 176 & 32 & 1210 & 53 & 22.8 & 0.0181 & 0.0231 & 2.298 \\
8 & $\mathrm{U} 3$ & 176 & 32 & 1210 & 59 & 20.5 & 0.0176 & 0.022 & 2.201 \\
9 & $\mathrm{U} 2(\mathrm{H})$ & 176 & 32 & 1210 & 71 & 17 & 0.0147 & 0.019 & 1.906 \\
10 & $\kappa$ & 176 & 32 & 1210 & 74 & 16.4 & 0.0143 & 0.0188 & 1.892 \\
\hline
\end{tabular}

Table S5.7: XD refinement strategy for dataset Para_Pilatus3. Abbreviations: M: monopoles; D: dipoles; Q: quadrupoles; O: octupoles; K: K, U2, U3: Gram Charlier $2^{\text {nd }}$ and $3^{\text {rd }}$ order, H-XYZ: only hydrogen position against data up to $0.5 \sin (\theta) / \lambda$.

\begin{tabular}{llllllllll}
\hline Step & New Parameter & D $<0.5 \sin ($ th $) / l$ & \#M P-Param & Data & Para & Data/Para & R $\left(F^{2}\right)$ & WR $\left(F^{2}\right)$ & $G O F$ \\
1 & SCALE & 178 & 0 & 1227 & 1 & 1227 & 0.0676 & 0.0969 & 14.129 \\
2 & DQO & 178 & 27 & 1227 & 28 & 43.8 & 0.045 & 0.0559 & 8.248 \\
3 & U2 & 178 & 27 & 1227 & 42 & 29.2 & 0.0215 & 0.0324 & 4.812 \\
4 & H-XYZ & 178 & 0 & 178 & 7 & 25.4 & 0.0205 & 0.0232 & 7.902 \\
5 & XYZ & 178 & 27 & 1227 & 49 & 25 & 0.0177 & 0.0265 & 3.946 \\
6 & H-XYZ & 178 & 0 & 178 & 7 & 25.4 & 0.0186 & 0.0193 & 6.585 \\
7 & M & 178 & 32 & 1227 & 53 & 23.1 & 0.0172 & 0.0234 & 3.488 \\
8 & U3 & 178 & 32 & 1227 & 59 & 20.8 & 0.0166 & 0.0226 & 3.374 \\
9 & U2(H) & 178 & 32 & 1227 & 71 & 17.3 & 0.0128 & 0.0175 & 2.632 \\
10 & K & 178 & 32 & 1227 & 74 & 16.6 & 0.0118 & 0.0169 & 2.536 \\
\hline
\end{tabular}


Table S5.8: XD refinement strategy for dataset Para_Photon2. Abbreviations: M: monopoles; D: dipoles; Q: quadrupoles; O: octupoles; K: $k$, U2, U3: Gram Charlier $2^{\text {nd }}$ and $3^{\text {rd }}$ order, $\mathrm{H}-\mathrm{XYZ}$ : only hydrogen position against data up to $0.5 \sin (\theta) / \lambda$.

\begin{tabular}{|c|c|c|c|c|c|c|c|c|c|}
\hline Step & New Parameter & $D<0.5 \sin ($ th $) / l$ & \#M P-Param & Data & Para & Data/Para & $R\left(F^{2}\right)$ & $w R\left(F^{2}\right)$ & GOF \\
\hline 1 & SCALE & 177 & 0 & 1239 & 1 & 1239 & 0.0746 & 0.1237 & 36.164 \\
\hline 2 & DQO & 177 & 27 & 1239 & 28 & 44.2 & 0.0249 & 0.0375 & 11.067 \\
\hline 3 & U2 & 177 & 27 & 1239 & 42 & 29.5 & 0.025 & 0.0358 & 10.647 \\
\hline 4 & $H-X Y Z$ & 177 & 0 & 177 & 7 & 25.3 & 0.0239 & 0.031 & 21.041 \\
\hline 5 & $X Y Z$ & 177 & 27 & 1239 & 49 & 25.3 & 0.0219 & 0.0292 & 8.691 \\
\hline 6 & $H-X Y Z$ & 177 & 0 & 177 & 7 & 25.3 & 0.0219 & 0.0258 & 17.486 \\
\hline 7 & M & 177 & 32 & 1239 & 53 & 23.4 & 0.0194 & 0.0234 & 6.979 \\
\hline 8 & U3 & 177 & 32 & 1239 & 59 & 21 & 0.0189 & 0.0226 & 6.763 \\
\hline 9 & $\mathrm{U} 2(\mathrm{H})$ & 177 & 32 & 1239 & 71 & 17.4 & 0.0129 & 0.0144 & 4.338 \\
\hline 10 & к & 177 & 32 & 1239 & 74 & 16.7 & 0.0128 & 0.0142 & 4.266 \\
\hline
\end{tabular}

Table S5.9: XD refinement strategy for dataset Para_Photon3. Abbreviations: M: monopoles; D: dipoles; Q: quadrupoles; O: octupoles; K: $\mathrm{k}$, U2, U3: Gram Charlier $2^{\text {nd }}$ and $3^{\text {rd }}$ order, $\mathrm{H}-\mathrm{XYZ}$ : only hydrogen position against data up to $0.5 \sin (\theta) / \lambda$.

\begin{tabular}{llllllllll}
\hline Step & New Parameter & $\mathrm{D}<0.5 \sin ($ th)/I & \#M P-Param & Data & Para & Data/Para & $\mathrm{R}\left(\mathrm{F}^{2}\right)$ & WR $\left(\mathrm{F}^{2}\right)$ & $\mathrm{GOF}$ \\
1 & SCALE & 177 & 0 & 1245 & 1 & 1245 & 0.0728 & 0.124 & 46.523 \\
2 & DQO & 177 & 27 & 1245 & 28 & 44.5 & 0.0248 & 0.0375 & 14.242 \\
3 & $\mathrm{U} 2$ & 177 & 27 & 1245 & 42 & 29.6 & 0.0249 & 0.036 & 13.732 \\
4 & $\mathrm{H}-\mathrm{XYZ}$ & 177 & 0 & 177 & 7 & 25.3 & 0.0236 & 0.0313 & 27.28 \\
5 & $\mathrm{XYZ}$ & 177 & 27 & 1245 & 49 & 25.4 & 0.0219 & 0.0294 & 11.257 \\
6 & $\mathrm{H}-\mathrm{XYZ}$ & 177 & 0 & 177 & 7 & 25.3 & 0.0216 & 0.0261 & 22.727 \\
7 & $\mathrm{M}$ & 177 & 32 & 1245 & 53 & 23.5 & 0.019 & 0.0237 & 9.087 \\
8 & $\mathrm{U} 3$ & 177 & 32 & 1245 & 59 & 21.1 & 0.0183 & 0.0229 & 8.784 \\
9 & $\mathrm{U} 2(\mathrm{H})$ & 177 & 32 & 1245 & 71 & 17.5 & 0.0126 & 0.0143 & 5.532 \\
10 & K & 177 & 32 & 1245 & 74 & 16.8 & 0.0126 & 0.0141 & 5.457 \\
\hline
\end{tabular}




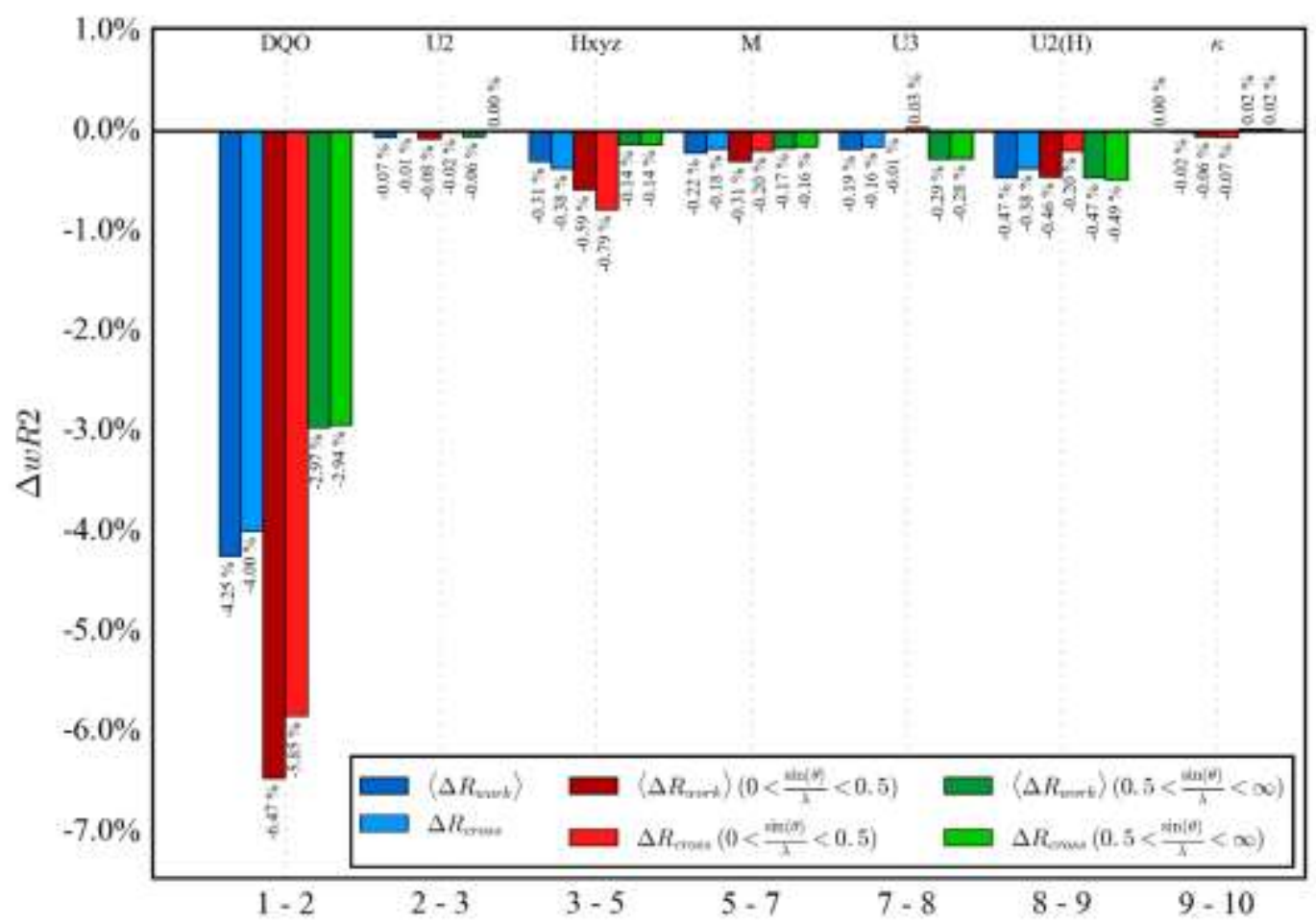

Figure S5.1: $\Delta R_{\text {cross }}$ values for the XD refinement of dataset Para_A pex2. A bbreviations: $M$ : monopoles; $D$ : dipoles; Q: quadrupoles; O: octupoles; K: $\kappa, \mathrm{U} 2$, U3: Gram Charlier $2^{\text {nd }}$ and $3^{\text {rd }}$ order, H-XYZ: only hydrogen position against data up to $0.5 \sin (\theta) / \lambda$.

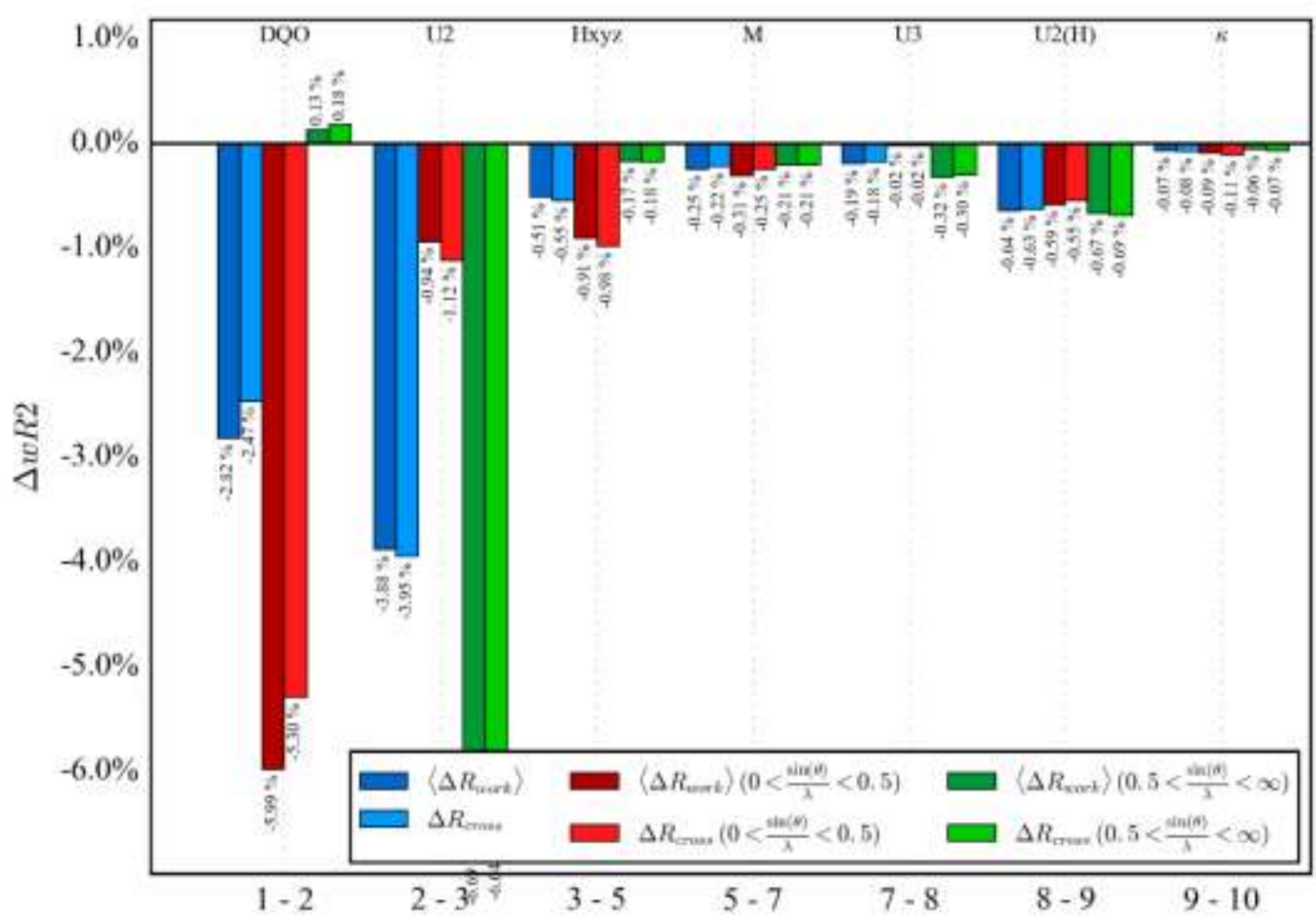

Figure S5.2: $\Delta R_{\text {cross }}$ values for the XD refinement of dataset Para_Pilatus3. A bbreviations: $M$ : monopoles; $D$ : dipoles; Q: quadrupoles; O: octupoles; K: $\kappa$, U2, U3: Gram Charlier $2^{\text {nd }}$ and $3^{\text {rd }}$ order, $\mathrm{H}-\mathrm{XYZ}$ : only hydrogen position against data up to $0.5 \sin (\theta) / \lambda$. 


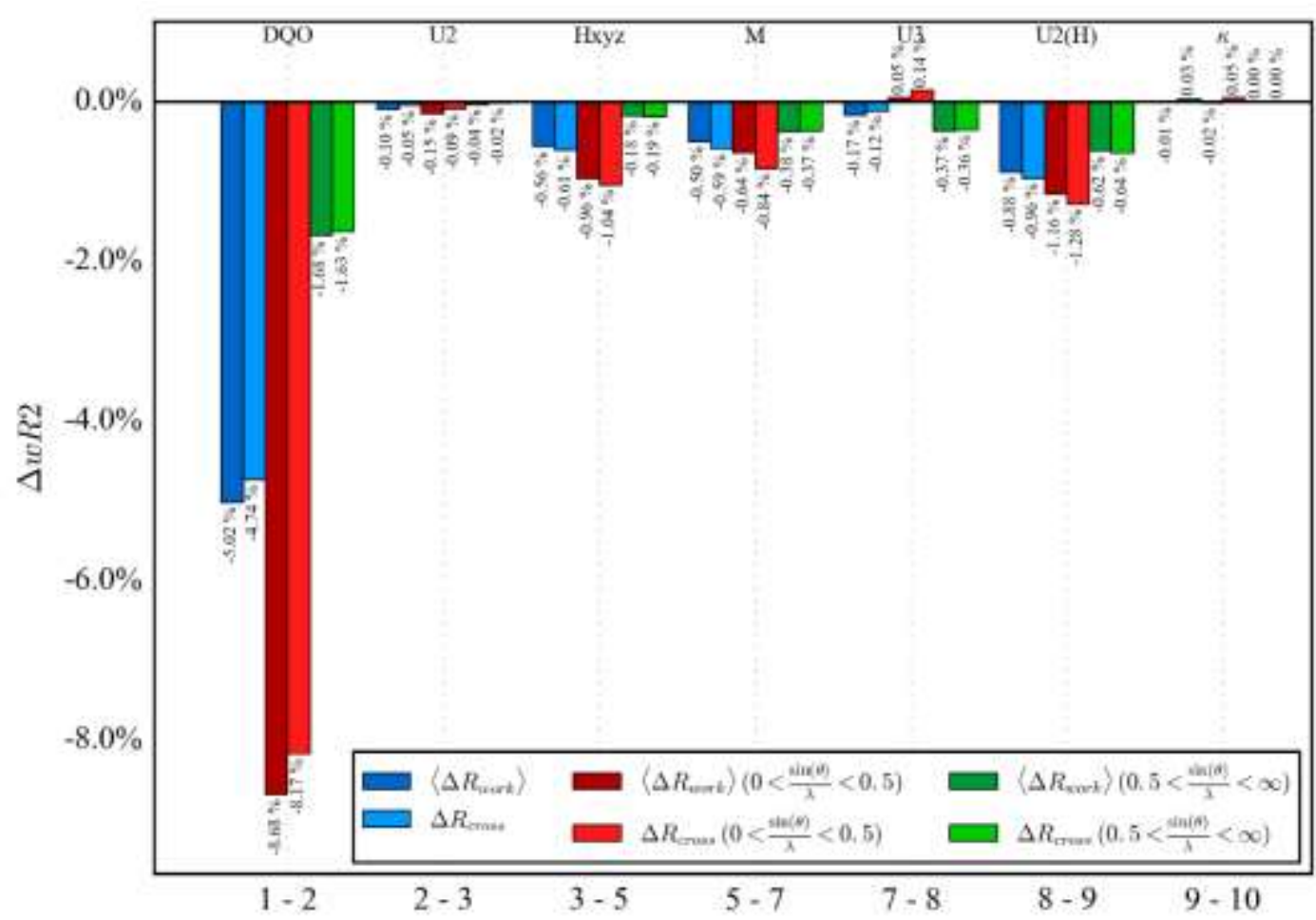

Figure S5.3: $\Delta R_{\text {cross }}$ values for the XD refinement of dataset Para Photon2. A bbreviations: $M$ : monopoles; $D$ : dipoles; Q: quadrupoles; O: octupoles; K: $\kappa$, U2, U3: Gram Charlier $2^{\text {nd }}$ and $3^{\text {rd }}$ order, H-XYZ: only hydrogen position against data up to $0.5 \sin (\theta) / \lambda$.

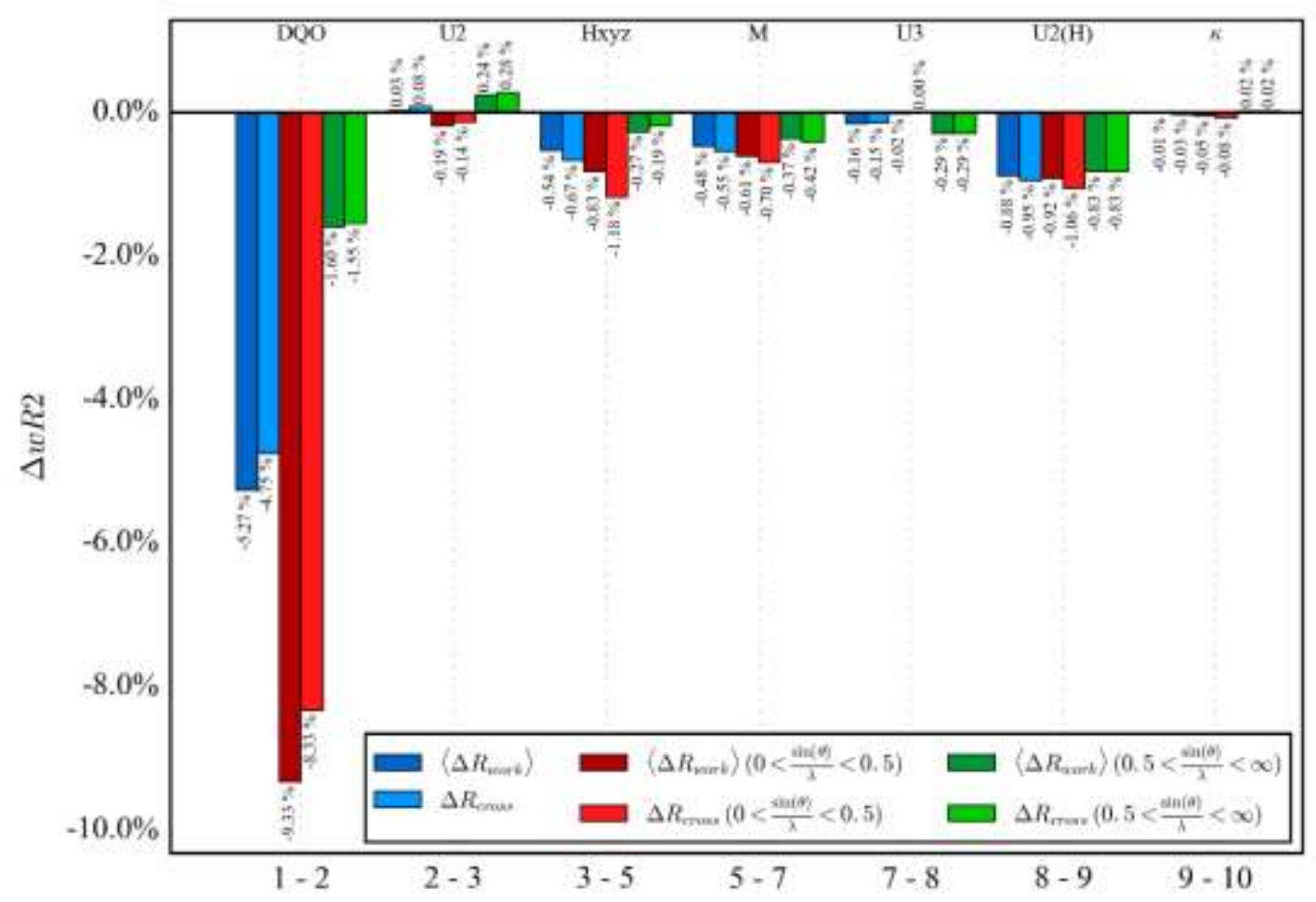

Figure S5.4: $\Delta R_{\text {cross }}$ values for the XD refinement of dataset Para_Photon3. A bbreviations: $M$ : monopoles; $D$ : dipoles; Q: quadrupoles; O: octupoles; K: $\kappa$, U2, U3: Gram Charlier $2^{\text {nd }}$ and $3^{\text {rd }}$ order, H-XYZ: only hydrogen position against data up to $0.5 \sin (\theta) / \lambda$. 

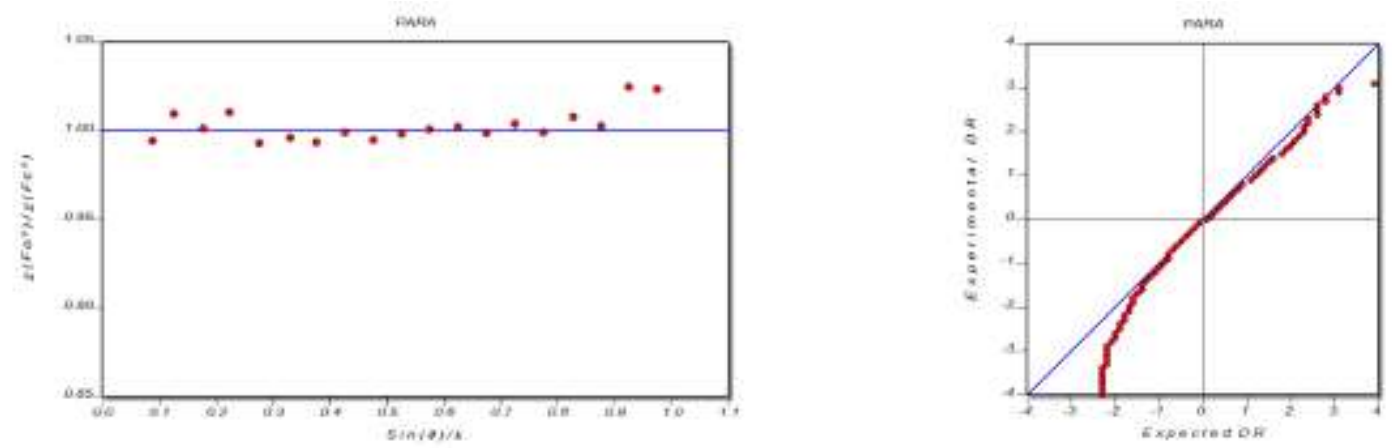

Figure S5.5: Resolution dependent error and Quantile/Quantile DRK-Plots (Zhurov et al., 2008; Zavodnik et al., 1999; A dam Stash, 2007) for theXD refinement of dataset Para_A pex2.
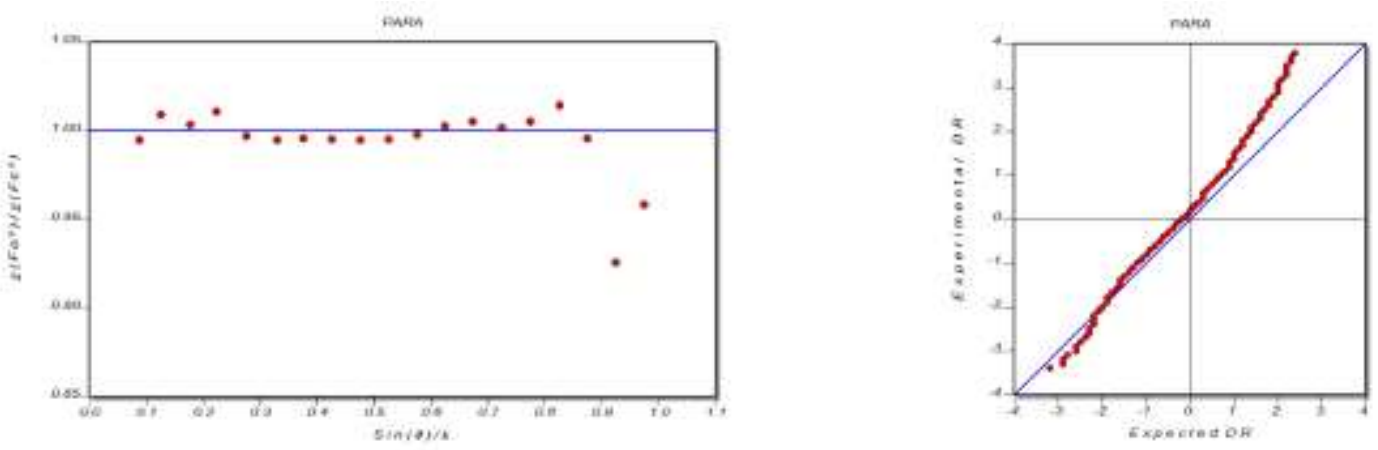

Figure S5.6: Resolution dependent error and Quantile/Quantile DRK-Plots (Zhurov et al., 2008; Zavodnik et al., 1999; A dam Stash, 2007) for theXD refinement of dataset Para_Pilatus3.
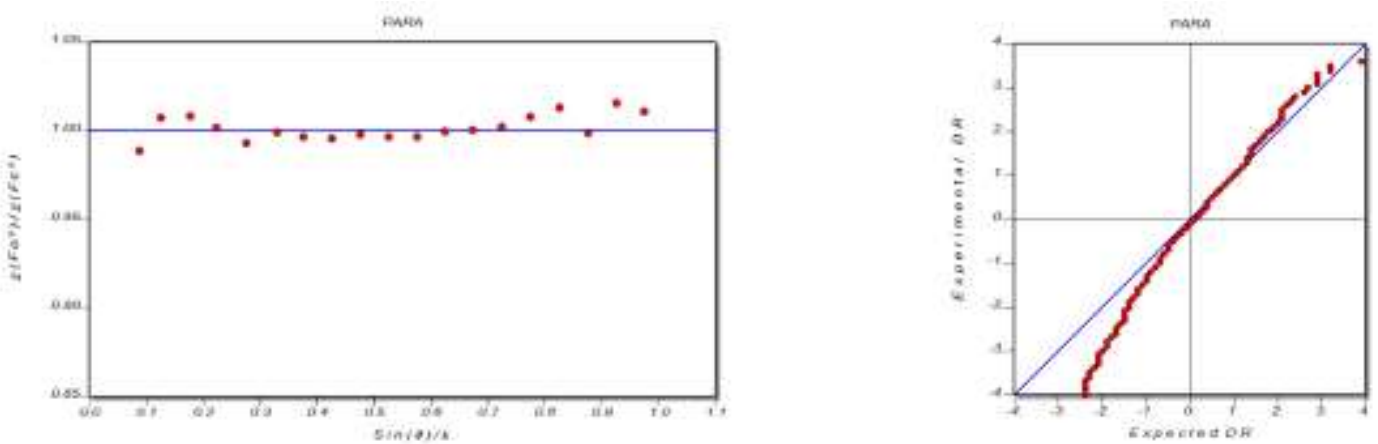

Figure S5.7: Resolution dependent error and Quantile/Quantile DRK-Plots (Zhurov et al., 2008; Zavodnik et al., 1999; A dam Stash, 2007) for theXD refinement of dataset Para_Photon2.
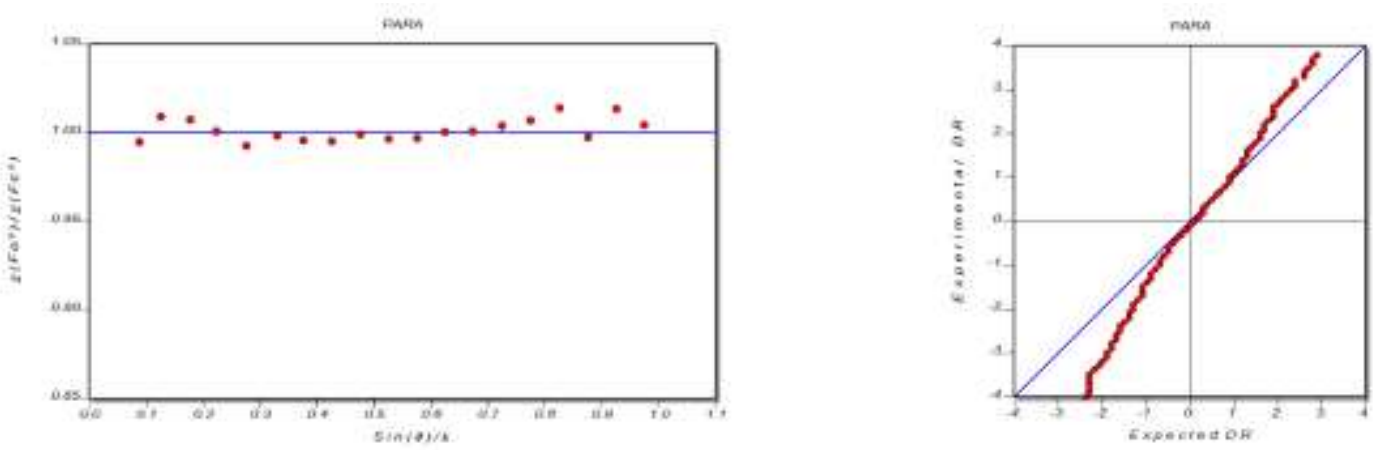

Figure S5.8: Resolution dependent error and Quantile/Quantile DRK-Plots (Zhurov et al., 2008; Zavodnik et al., 1999; A dam Stash, 2007) for theXD refinement of dataset Para_Photon3. 
Table S5.10: For the anharmonic refinement of $C(3)$, Kuhs's rule (Kuhs, 1992) should be fulfilled. The used resolution is $\sin (\theta) / \lambda=1.0 \AA^{-1}$, therefore Kuhs's rule is fulfilled for all datasets.

\begin{tabular}{llllll}
\hline Dataset & \multicolumn{2}{c}{ Principal M.D.A's (A) } & & \multicolumn{2}{c}{ M in. resolution $\left[\AA^{-1}\right]$} \\
& & & & $\mathbf{n}=\mathbf{3}$ & $\mathbf{n = 4}$ \\
Para_Apex2 & $\mathrm{C}(3)$ & 0.212 & 0.14 & 0.125 & 0.84 \\
Para_Pilatus3 & $\mathrm{C}(3)$ & 0.22 & 0.15 & 0.138 & 0.78 \\
Para_Photon2 & $\mathrm{C}(3)$ & 0.214 & 0.142 & 0.129 & 0.82 \\
Para_Photon3 & $\mathrm{C}(3)$ & 0.214 & 0.142 & 0.129 & 0.82 \\
\hline
\end{tabular}

Table S5.11: Consistency check for the refinement of Gram Charlier parameters of $3^{\text {rd }}$ order at $C(3)$. A nalysis of the Probability Density Function (PDF). Extreme displacements in the map from the equilibrium position. $\triangle \mathrm{X} / \mathrm{Y} / \mathrm{Z}=-0.80$ to $0.80 \AA$. For all datasets, the minimum PSD value and integrated volume for negative probability are reasonably low.

\begin{tabular}{|c|c|c|c|c|c|c|}
\hline Dataset & $\begin{array}{l}\text { Minimum } \\
\text { PDF value }\end{array}$ & $\begin{array}{l}\text { Maximum } \\
\text { PDF } \\
\text { Value }\end{array}$ & $\begin{array}{l}\text { Total } \\
\text { integrated } \\
\text { negative } \\
\text { probability } \\
{[\%]}\end{array}$ & $\begin{array}{l}\text { Integrated } \\
\text { volume for } \\
\text { negative } \\
\text { probability } \\
{\left[\AA^{-3}\right]}\end{array}$ & $\begin{array}{l}\text { Total } \\
\text { integrated } \\
\text { positive } \\
\text { probability } \\
{[\%]}\end{array}$ & $\begin{array}{l}\text { Integrated } \\
\text { volume for } \\
\text { positive } \\
\text { probability } \\
{\left[\AA^{-3}\right]}\end{array}$ \\
\hline Para_A pex2 & -0.84 & 9335.2 & -0.003 & 0.824 & 100.003 & 3.508 \\
\hline Para_Pilatus3 & -0.66 & 7637.31 & -0.003 & 0.538 & 100.001 & 3.8 \\
\hline Para_Photon2 & -1.11 & 8861.48 & -0.005 & 0.93 & 100.004 & 3.433 \\
\hline Para_Photon3 & -1.3 & 8847.77 & -0.007 & 0.956 & 100.006 & 3.407 \\
\hline
\end{tabular}

Table S5.12: Significance check for the refinement of Gram Charlier parameters of $3^{\text {rd }}$ order at C (3) in XD. GramCharlier parameter divided by their error - in order to be significant, values need to be larger than $3 \sigma$. For all datasets, the refined PDF are significant.

\begin{tabular}{lllllllllll}
\hline Dataset & $\mathrm{C} 111 / \sigma$ & $\mathrm{C} 222 / \sigma$ & $\mathrm{C} 333 / \sigma$ & $\mathrm{C} 112 / \sigma$ & $\mathrm{C} 122 / \sigma$ & $\mathrm{C} 113 / \sigma$ & $\mathrm{C} 133 / \sigma$ & $\mathrm{C} 223 / \sigma$ & $\mathrm{C} 233 / \sigma$ & $\mathrm{C} 123 / \sigma$ \\
Para_A pex2 & 3.02 & 3.02 & 0.44 & 1.28 & 1.28 & 7.36 & 1.48 & 7.36 & 1.48 & 11.31 \\
Para_Pilatus3 & 3.46 & 3.46 & 0.7 & 2.27 & 2.27 & 9.28 & 0.95 & 9.28 & 0.95 & 12.76 \\
Para_Photon2 & 7.78 & 7.78 & 2.06 & 7.24 & 7.24 & 15.4 & 2.68 & 15.4 & 2.68 & 19.76 \\
Para_Photon3 & 7.94 & 7.94 & 2.85 & 7.11 & 7.11 & 16.09 & 3.08 & 16.09 & 3.08 & 20.3 \\
\hline
\end{tabular}


Table S5.13: BCP analysis of Para_A pex2. *marked value errors are determined by cross-validation. In any case, the larger error value was chosen.

\begin{tabular}{|c|c|c|c|c|c|c|c|c|c|c|}
\hline Al & A2 & $\begin{array}{l}\rho\left(\mathbf{r}_{\mathrm{rcc}}\right) \\
{\left[\mathrm{e}^{-3}\right]}\end{array}$ & $\begin{array}{l}\nabla^{2} \rho\left(\mathbf{r}_{\mathrm{bcc}}\right) \\
{\left[\mathrm{e} \AA^{-5}\right]}\end{array}$ & $\begin{array}{l}\text { Bond Path } \\
\text { Length }[\AA]]\end{array}$ & $\mathbf{r}_{A 1-B C P}[\AA]$ & $\mathbf{r}_{\mathrm{A} 2-\mathrm{BCP} P}[\AA]$ & $\varepsilon\left(\mathbf{r}_{\mathrm{bcp}}\right)$ & \multicolumn{3}{|c|}{ Hessian Eigenvalues [e $\AA^{-5}$ ] } \\
\hline $\mathrm{Cl}$ & C2 & 2.113(5)* & $-19.82(16) *$ & $1.39900(6) *$ & $0.6897(15) *$ & $0.7092(15) *$ & $0.210(7)^{*}$ & $-15.97(9)^{*}$ & $-13.21(7)^{*}$ & $9.37(13)^{*}$ \\
\hline $\mathrm{C} 1$ & C3 & $1.672(12)^{*}$ & $-12.7(3)^{*}$ & $1.5054(6)^{*}$ & 0.783(3)* & $0.723(3)^{*}$ & $0.010(8)^{*}$ & $-11.34(17)^{*}$ & $-11.18(14) *$ & 9.79(14)* \\
\hline $\mathrm{C} 2$ & $\mathrm{H} 2$ & $1.77(2)$ & $-17.2(2)^{*}$ & $1.083(0) *$ & $0.691(10)^{*}$ & $0.392(10) *$ & $0.050(4)^{*}$ & $-15.64(12)^{*}$ & $-14.91(15) *$ & $13.4(4)^{*}$ \\
\hline C3 & $\mathrm{H} 3$ & $1.60(4)$ & $-13.1(4) *$ & $1.105(2) *$ & $0.680(10)^{*}$ & $0.425(12) *$ & $0.090(13)^{*}$ & $-13.61(17)^{*}$ & $-12.47(18)^{*}$ & $13.0(2)^{*}$ \\
\hline
\end{tabular}

Table S5.14: BCP analysis of Para_Pilatus3. *marked value errors are determined by cross-validation. In any case, the larger error value was chosen.

\begin{tabular}{|c|c|c|c|c|c|c|c|c|c|c|}
\hline A1 & A2 & $\begin{array}{l}\rho\left(\mathbf{r}_{\text {bcp }}\right) \\
{\left[\mathrm{e}^{-3}\right]}\end{array}$ & $\begin{array}{l}\nabla^{2} \rho\left(\mathbf{r b c}_{\mathrm{bcp}}\right) \\
{\left[\mathrm{e}^{-5}{ }^{-5}\right]}\end{array}$ & $\begin{array}{l}\text { Bond Path } \\
\text { Length }[\AA]]\end{array}$ & $\mathbf{r}_{A 1-B C P}[\AA]$ & $\mathbf{r}_{A 2-B C P}[\AA]$ & $\varepsilon\left(r_{b c p}\right)$ & \multicolumn{3}{|c|}{ Hessian Eigenvalues [ $\mathrm{e} \AA^{-5}$ ] } \\
\hline $\mathrm{C} 1$ & C2 & $2.112(4)$ & $-20.31(9)^{*}$ & 1.39930(4)* & $0.6910(14) *$ & $0.7083(13)^{*}$ & $0.210(5)^{*}$ & $-15.95(4)^{*}$ & $-13.13(5) *$ & $8.76(11)^{*}$ \\
\hline $\mathrm{C} 1$ & C3 & $1.703(5)^{*}$ & $-13.6(1)^{*}$ & $1.5052(6)^{*}$ & $0.763(3)^{*}$ & $0.742(3)^{*}$ & $0.040(8)^{*}$ & $-11.83(11)^{*}$ & $-11.32(8) *$ & $9.54(12)^{*}$ \\
\hline $\mathrm{C} 2$ & $\mathrm{H} 2$ & $1.76(2)$ & $-17.43(17)^{*}$ & $1.083(0) *$ & $0.698(7) *$ & $0.385(7)^{*}$ & $0.060(3)^{*}$ & $-15.77(8)^{*}$ & $-14.86(9) *$ & $13.2(3)^{*}$ \\
\hline $\mathrm{C} 3$ & H3 & $1.61(4)$ & $-13.59(14)^{*}$ & 1.1056(7)* & $0.671(6)^{*}$ & $0.434(6)^{*}$ & $0.080(11)^{*}$ & $-13.45(8)^{*}$ & $-12.5(10) *$ & $12.36(13)^{*}$ \\
\hline
\end{tabular}

Table S5.15: BCP analysis of Para_Photon2. *marked value errors are determined by cross-validation. In any case, the larger error value was chosen.

\begin{tabular}{|c|c|c|c|c|c|c|c|c|c|c|}
\hline A1 & A2 & $\begin{array}{l}\rho\left(\mathbf{r}_{\text {rcco }}\right) \\
{\left[\mathrm{e}^{-3}{ }^{-3}\right]}\end{array}$ & $\begin{array}{l}\nabla^{2} \rho\left(\mathbf{r}_{\mathrm{bcp}}\right) \\
{\left[\mathrm{e}^{-5}{ }^{-5}\right]}\end{array}$ & $\begin{array}{l}\text { Bond Path } \\
\text { Length }[\AA \AA]\end{array}$ & $\mathbf{r}_{A 1-B C P}[\AA]$ & $\mathbf{r}_{A 2-B C P}[\AA]$ & $\varepsilon\left(\mathbf{r}_{\mathrm{bcp}}\right)$ & \multicolumn{3}{|c|}{ Hessian Eigenvalues [ $\mathrm{e}^{\AA-5}$ ] } \\
\hline $\mathrm{C} 1$ & C2 & $2.107(3)$ & $-19.59(15)^{*}$ & $1.39810(6) *$ & $0.6903(8)^{*}$ & $0.7079(8)^{*}$ & $0.210(6)^{*}$ & $-16.05(4)^{*}$ & $-13.21(5)^{*}$ & $9.7(2)^{*}$ \\
\hline $\mathrm{C} 1$ & C3 & $1.671(9)^{*}$ & $-12.3(2) *$ & $1.5055(4)^{*}$ & $0.767(6)^{*}$ & $0.738(6) *$ & $0.020(8)^{*}$ & $-11.31(15)^{*}$ & $-11.08(10)^{*}$ & $10.11(14)^{*}$ \\
\hline $\mathrm{C} 2$ & $\mathrm{H} 2$ & $1.761(17)$ & $-17.36(14)^{*}$ & $1.083(0)^{*}$ & $0.710(5)^{*}$ & $0.373(5)^{*}$ & $0.050(4)^{*}$ & $-16.08(5)^{*}$ & $-15.25(5)^{*}$ & $14.0(1)^{*}$ \\
\hline C3 & H3 & $1.62(3)$ & $-13.2(3)^{*}$ & $1.1041(12)^{*}$ & $0.682(7)^{*}$ & $0.422(7)^{*}$ & $0.090(11)^{*}$ & $-13.67(13)^{*}$ & $-12.56(9)^{*}$ & $13.0(2) *$ \\
\hline
\end{tabular}

Table S5.16: BCP analysis of Para_Photon3. *marked value errors are determined by cross-validation. In any case, the larger error value was chosen.

\begin{tabular}{|c|c|c|c|c|c|c|c|c|c|c|}
\hline Al & A2 & $\begin{array}{l}\rho\left(\mathbf{r b c}_{\mathrm{bc}}\right) \\
{\left[\mathrm{e}^{-3}\right]}\end{array}$ & $\begin{array}{l}\nabla^{2} \rho\left(\mathbf{r}_{\mathrm{bc}}\right) \\
{\left[\mathrm{e} \AA^{-5}\right]}\end{array}$ & $\begin{array}{l}\text { Bond Path } \\
\text { Length }[\AA]]\end{array}$ & $\mathbf{r}_{\mathrm{A} 1-\mathrm{BCP}}[\AA]$ & $\mathbf{r}_{\mathrm{A} 2 \mathrm{BCP}[\AA]}[\AA]$ & $\varepsilon\left(\mathbf{r}_{\mathrm{ccp}}\right)$ & \multicolumn{3}{|c|}{ Hessian Eigenvalues [e $\AA^{-5}$ ] } \\
\hline $\mathrm{C} 1$ & $\mathrm{C} 2$ & $2.111(3)^{*}$ & $-19.55(14)^{*}$ & $1.39810(8)^{*}$ & $0.6912(12)^{*}$ & $0.7069(12) *$ & $0.210(4)^{*}$ & $-16.13(5)^{*}$ & $-13.29(4)^{*}$ & $9.87(13)^{*}$ \\
\hline $\mathrm{C} 1$ & C3 & $1.673(12)^{*}$ & $-12.3(3)^{*}$ & $1.5056(11)^{*}$ & $0.766(9) *$ & $0.740(8)^{*}$ & $0.020(7)^{*}$ & $-11.4(2)^{*}$ & $-11.12(15) *$ & $10.24(10) *$ \\
\hline C2 & $\mathrm{H} 2$ & $1.762(15)$ & $-17.29(11)^{*}$ & $1.083(0)^{*}$ & $0.712(4)^{*}$ & $0.371(4)^{*}$ & $0.060(5)^{*}$ & $-16.15(6)^{*}$ & $-15.3(6)^{*}$ & 14.17(14)* \\
\hline C3 & $\mathrm{H} 3$ & $1.63(3)$ & $-13.3(7)^{*}$ & $1.1041(9)^{*}$ & $0.680(14)^{*}$ & $0.424(14)^{*}$ & $0.090(10)^{*}$ & $-13.7(2)^{*}$ & $-12.7(2)^{*}$ & $13.1(3)^{*}$ \\
\hline
\end{tabular}




\section{Supplementary Information to Chapter 6}

Table S6.1: Local coordinate systems, $\kappa$-set assignment and chemical constraints for the refinements. The indicated Gram-Charlier-level corresponds to the final refinement. The three disordered methyl groups [C(141), H (14A), H(14B), H (14C)/ C(142), H(14D), H (14E), H(14F)], [C(221), H (22A), $\mathrm{H}(22 \mathrm{~B}), \mathrm{H}(22 \mathrm{C}) / \mathrm{C}(222), \mathrm{H}(22 \mathrm{D}), \mathrm{H}(22 \mathrm{E}), \mathrm{H}(22 \mathrm{~F})]$ and [C(241), H (24A), H (24B), H (24C)/ C(242), $H(24 D), H(24 E), H(24 F)]$ are constrained to the non-disordered groups with their corresponding population of $0.61 / 0.39,0.83 / 0.17$ and $0.54 / 0.46$.

\begin{tabular}{|c|c|c|c|c|c|c|c|c|c|}
\hline$\overline{\text { ATOM }}$ & ATOM 0 & $A X 1$ & ATOM 1 & ATOM 2 & $A \times 2$ & GC-level & к-set & SITESYM & CHEMCON \\
\hline $\mathrm{P}(1)$ & $N(1)$ & $x$ & $P(1)$ & $C(1)$ & Y & 2 & 1 & _mZ & \\
\hline$F(32)$ & $C(32)$ & Z & $F(32)$ & $C(31)$ & $Y$ & 3 & 2 & _cy & \\
\hline$F(33)$ & $C(33)$ & Z & $F(33)$ & $C(32)$ & $Y$ & 3 & 2 & _cy & $F(32)$ \\
\hline$F(34)$ & $C(34)$ & Z & $F(34)$ & $C(33)$ & $Y$ & 3 & 2 & cy & $F(32)$ \\
\hline$F(35)$ & $C(35)$ & Z & $F(35)$ & $C(36)$ & $Y$ & 3 & 2 & cy & $F(32)$ \\
\hline$F(36)$ & $C(36)$ & Z & $F(36)$ & $C(31)$ & $Y$ & 3 & 2 & cy & $F(32)$ \\
\hline$F(42)$ & $C(42)$ & Z & $F(42)$ & $C(41)$ & $Y$ & 3 & 2 & cy & $F(32)$ \\
\hline$F(43)$ & $C(43)$ & Z & $F(43)$ & $C(42)$ & $Y$ & 3 & 2 & cy & $F(32)$ \\
\hline$F(44)$ & $C(44)$ & Z & $F(44)$ & $C(43)$ & $Y$ & 3 & 2 & _cy & $F(32)$ \\
\hline$F(45)$ & $C(45)$ & Z & $F(45)$ & $C(46)$ & $Y$ & 3 & 2 & _cy & $F(32)$ \\
\hline$F(46)$ & $C(46)$ & Z & $F(46)$ & $C(41)$ & $Y$ & 3 & 2 & _cy & $F(32)$ \\
\hline$N(1)$ & $P(1)$ & $x$ & $N(1)$ & $B(1)$ & $Y$ & 2 & 3 & $\mathrm{mz}$ & \\
\hline$C(1)$ & $P(1)$ & $x$ & $C(1)$ & $C(2)$ & $Y$ & 2 & 5 & $\mathrm{mZ}$ & \\
\hline$C(2)$ & $B(1)$ & $x$ & $C(2)$ & $C(1)$ & $Y$ & 2 & 5 & _mZ & \\
\hline$C(11)$ & $P(1)$ & Z & $C(11)$ & $C(16)$ & $Y$ & 2 & 4 & cy & \\
\hline$C(12)$ & $C(15)$ & Z & $C(12)$ & $C(11)$ & $Y$ & 2 & 4 & cy & \\
\hline$C(13)$ & $C(16)$ & Z & $C(13)$ & $C(12)$ & $Y$ & 2 & 4 & cy & \\
\hline$C(14)$ & $C(11)$ & Z & $C(14)$ & $C(13)$ & $Y$ & 2 & 4 & cy & $C(12)$ \\
\hline$C(15)$ & $C(12)$ & Z & $C(15)$ & $C(16)$ & $Y$ & 2 & 4 & _cy & $C(13)$ \\
\hline$C(16)$ & $C(13)$ & Z & $C(16)$ & $C(11)$ & $Y$ & 2 & 4 & cy & $C(12)$ \\
\hline$C(21)$ & $P(1)$ & Z & $C(21)$ & $C(26)$ & $Y$ & 2 & 4 & _cy & $C(11)$ \\
\hline$C(22)$ & $C(25)$ & Z & $C(22)$ & $C(21)$ & $Y$ & 2 & 4 & cy & $C(12)$ \\
\hline$C(23)$ & $C(26)$ & z & $C(23)$ & $C(22)$ & $Y$ & 2 & 4 & cy & $C(13)$ \\
\hline$C(24)$ & $C(21)$ & Z & $C(24)$ & $C(23)$ & $Y$ & 2 & 4 & cy & $C(12)$ \\
\hline$C(25)$ & $C(22)$ & Z & $C(25)$ & $C(26)$ & $Y$ & 2 & 4 & _cy & $C(13)$ \\
\hline$C(26)$ & $C(23)$ & Z & $C(26)$ & $C(21)$ & $Y$ & 2 & 4 & _cy & $C(12)$ \\
\hline$C(31)$ & $B(1)$ & Z & $C(31)$ & $C(36)$ & $Y$ & 2 & 4 & _cy & \\
\hline$C(32)$ & $C(35)$ & z & $C(32)$ & $C(31)$ & $Y$ & 3 & 4 & _cy & \\
\hline$C(33)$ & $C(36)$ & Z & $C(33)$ & $C(32)$ & $Y$ & 3 & 4 & _cy & $C(32)$ \\
\hline$C(34)$ & $C(31)$ & Z & $C(34)$ & $C(33)$ & $Y$ & 3 & 4 & _cy & $C(32)$ \\
\hline$C(35)$ & $C(32)$ & Z & $C(35)$ & $C(36)$ & $Y$ & 3 & 4 & cy & $C(32)$ \\
\hline$C(36)$ & $C(33)$ & Z & $C(36)$ & $C(31)$ & $Y$ & 3 & 4 & cy & $C(32)$ \\
\hline$C(41)$ & $B(1)$ & Z & $C(41)$ & $C(46)$ & $Y$ & 2 & 4 & _cy & $C(31)$ \\
\hline$C(42)$ & $C(45)$ & Z & $C(42)$ & $C(41)$ & $Y$ & 2 & 4 & _cy & $C(32)$ \\
\hline$C(43)$ & $C(46)$ & Z & $C(43)$ & $C(42)$ & $Y$ & 2 & 4 & _cy & $C(32)$ \\
\hline$C(44)$ & $C(41)$ & Z & $C(44)$ & $C(43)$ & $Y$ & 2 & 4 & cy & $C(32)$ \\
\hline$C(45)$ & $C(42)$ & Z & $C(45)$ & $C(46)$ & $Y$ & 2 & 4 & cy & $C(32)$ \\
\hline$C(46)$ & $C(43)$ & z & $C(46)$ & $C(41)$ & $Y$ & 2 & 4 & cy & $C(32)$ \\
\hline$C(121)$ & $C(12)$ & Z & $C(121)$ & $H(12 A)$ & $Y$ & 3 & 6 & $3 m$ & \\
\hline$C(141)$ & $C(14)$ & Z & $C(141)$ & $H(14 A)$ & $Y$ & 3 & 6 & $3 m$ & $C(121)^{*}$ pop \\
\hline$C(142)$ & $C(14)$ & Z & $C(142)$ & $H(14 D)$ & $Y$ & 3 & 6 & $3 m$ & $C(121) *$ pop \\
\hline$C(161)$ & $C(16)$ & Z & $C(161)$ & $H(16 A)$ & $Y$ & 2 & 6 & $3 \mathrm{~m}$ & $C(121)$ \\
\hline$C(221)$ & $C(22)$ & Z & $C(221)$ & $H(22 A)$ & $Y$ & 3 & 6 & $3 m$ & $C(121)^{*}$ pop \\
\hline$C(222)$ & $C(22)$ & Z & $C(222)$ & $H(22 D)$ & $Y$ & 3 & 6 & $3 m$ & $C(121)^{*}$ pop \\
\hline$C(241)$ & $C(24)$ & Z & $C(241)$ & $H(24 A)$ & $Y$ & 3 & 6 & $3 \mathrm{~m}$ & $C(121) *$ pop \\
\hline
\end{tabular}




\begin{tabular}{|c|c|c|c|c|c|c|c|c|c|}
\hline$C(242)$ & $C(24)$ & Z & $C(242)$ & $H(24 D)$ & Y & 3 & 6 & $-3 m$ & $C(121) *$ pop \\
\hline$C(261)$ & $C(26)$ & Z & $C(261)$ & $H(26 A)$ & Y & 3 & 6 & $-3 m$ & $C(121)$ \\
\hline $\mathrm{B}(1)$ & $\mathrm{N}(1)$ & Z & $B(1)$ & $C(2)$ & Y & 2 & 7 & _mZ & \\
\hline$H(1 N)$ & $N(1)$ & Z & $H(1 N)$ & $B(1)$ & Y & 1 & 8 & _cy & \\
\hline$H(1 A)$ & $C(1)$ & Z & $H(1 A)$ & $\mathrm{H}(1 \mathrm{~B})$ & Y & 1 & 8 & _cy & \\
\hline$H(1 B)$ & $C(1)$ & Z & $H(1 B)$ & $H(1 A)$ & Y & 1 & 8 & _cy & $H(1 A)$ \\
\hline$H(2 A)$ & $C(2)$ & Z & $H(2 A)$ & $H(2 B)$ & Y & 1 & 8 & _cy & $H(1 A)$ \\
\hline$H(2 B)$ & $C(2)$ & Z & $H(2 B)$ & $H(2 A)$ & Y & 1 & 8 & _cy & $H(1 A)$ \\
\hline$H(13)$ & $C(13)$ & Z & $H(13)$ & $C(14)$ & Y & 1 & 8 & _cy & \\
\hline$H(15)$ & $C(15)$ & Z & $\mathrm{H}(15)$ & $C(16)$ & Y & 1 & 8 & _cy & $H(13)$ \\
\hline$H(23)$ & $C(23)$ & Z & $H(23)$ & $C(22)$ & Y & 1 & 8 & _cy & $H(13)$ \\
\hline$H(25)$ & $C(25)$ & Z & $H(25)$ & $C(26)$ & Y & 1 & 8 & _cy & $H(13)$ \\
\hline$H(12 A)$ & $C(121)$ & Z & $H(12 A)$ & $\mathrm{H}(12 \mathrm{C})$ & Y & 1 & 8 & _cy & \\
\hline$H(12 B)$ & $C(121)$ & Z & $H(12 B)$ & $H(12 A)$ & Y & 1 & 8 & _cy & $H(12 A)$ \\
\hline $\mathrm{H}(12 \mathrm{C})$ & $C(121)$ & Z & $H(12 C)$ & $H(12 A)$ & Y & 1 & 8 & _cy & $H(12 A)$ \\
\hline$H(14 A)$ & $C(141)$ & Z & $H(14 A)$ & $H(14 B)$ & Y & 1 & 8 & _cy & $H(12 A) *$ pop \\
\hline$H(14 B)$ & $C(141)$ & Z & $H(14 B)$ & $H(14 C)$ & Y & 1 & 8 & _cy & $H(14 A)$ \\
\hline $\mathrm{H}(14 \mathrm{C})$ & $C(141)$ & Z & $H(14 C)$ & $H(14 A)$ & Y & 1 & 8 & _cy & $H(14 A)$ \\
\hline$H(14 D)$ & $C(142)$ & Z & $H(14 D)$ & $H(14 E)$ & Y & 1 & 8 & _cy & $H(12 A) *$ pop \\
\hline$H(14 E)$ & $C(142)$ & Z & $\mathrm{H}(14 \mathrm{E})$ & $H(14 F)$ & Y & 1 & 8 & _cy & $H(14 D)$ \\
\hline$H(14 F)$ & $C(142)$ & Z & $H(14 F)$ & $H(14 D)$ & Y & 1 & 8 & _cy & $H(14 D)$ \\
\hline$H(16 A)$ & $C(161)$ & Z & $H(16 A)$ & $H(16 B)$ & Y & 1 & 8 & _cy & $H(12 A)$ \\
\hline$H(16 B)$ & $C(161)$ & Z & $H(16 B)$ & $H(16 C)$ & Y & 1 & 8 & _cy & $H(12 A)$ \\
\hline$H(16 C)$ & $C(161)$ & Z & $H(16 C)$ & $H(16 A)$ & Y & 1 & 8 & _cy & $H(12 A)$ \\
\hline$H(22 A)$ & $C(221)$ & Z & $H(22 A)$ & $\mathrm{H}(22 \mathrm{~B})$ & Y & 1 & 8 & _cy & $H(12 A) *$ pop \\
\hline $\mathrm{H}(22 \mathrm{~B})$ & $C(221)$ & Z & $H(22 B)$ & $\mathrm{H}(22 \mathrm{C})$ & Y & 1 & 8 & _cy & $H(22 A)$ \\
\hline$H(22 C)$ & $C(221)$ & Z & $H(22 C)$ & $H(22 A)$ & Y & 1 & 8 & _cy & $H(22 A)$ \\
\hline$H(22 D)$ & $C(222)$ & Z & $H(22 D)$ & $\mathrm{H}(22 \mathrm{E})$ & Y & 1 & 8 & _cy & $H(12 A) *$ pop \\
\hline$H(22 E)$ & $C(222)$ & Z & $H(22 E)$ & $H(22 F)$ & Y & 1 & 8 & _cy & $H(22 D)$ \\
\hline $\mathrm{H}(22 \mathrm{~F})$ & $C(222)$ & Z & $H(22 F)$ & $H(22 D)$ & Y & 1 & 8 & _cy & $\mathrm{H}(22 \mathrm{D})$ \\
\hline$H(24 A)$ & $C(241)$ & Z & $H(24 A)$ & $H(24 B)$ & Y & 1 & 8 & _cy & $H(12 A) *$ pop \\
\hline$H(24 B)$ & $C(241)$ & Z & $H(24 B)$ & $H(24 C)$ & Y & 1 & 8 & _cy & $H(24 A)$ \\
\hline$H(24 C)$ & $C(241)$ & Z & $H(24 C)$ & $H(24 A)$ & Y & 1 & 8 & _cy & $H(24 A)$ \\
\hline$H(24 D)$ & $C(242)$ & Z & $H(24 D)$ & $H(24 E)$ & Y & 1 & 8 & _cy & $H(12 A) *$ pop \\
\hline$H(24 E)$ & $C(242)$ & Z & $H(24 E)$ & $H(24 F)$ & Y & 1 & 8 & _cy & $H(24 D)$ \\
\hline$H(24 F)$ & $C(242)$ & Z & $H(24 F)$ & $H(24 D)$ & Y & 1 & 8 & _cy & $H(24 D)$ \\
\hline$H(26 A)$ & $C(261)$ & Z & $H(26 A)$ & $H(26 B)$ & Y & 1 & 8 & _cy & $H(12 A)$ \\
\hline$H(26 B)$ & $C(261)$ & Z & $H(26 B)$ & $H(26 C)$ & Y & 1 & 8 & _cy & $H(12 A)$ \\
\hline $\mathrm{H}(26 \mathrm{C})$ & $C(261)$ & Z & $H(26 C)$ & $H(26 A)$ & Y & 1 & 8 & _cy & $H(12 A)$ \\
\hline
\end{tabular}


Table S6.2: XD refinement strategy for dataset A. A bbreviations: M P: M ultipole, M : monopoles; D: dipoles; Q: quadrupoles; O: octupoles; H: hexadecapoles, K: $\kappa$, U2, U3, U4: Gram Charlier $2^{\text {nd }}, 3^{\text {rd }}$ and $4^{\text {th }}$ order, HXYZ: hydrogen position against data up to $0.5 \sin (\theta) / \lambda$. Local

\begin{tabular}{llllllllll}
\hline Step & New Para. & $\begin{array}{l}\text { Data }< \\
0.5\end{array}$ & M P-Para & Data & Para & Data/Para & R(F2) & wR(F2) & GOF \\
& & $\begin{array}{l}\text { sin }(\theta) / \lambda \\
\text { 1 }\end{array}$ & & & & & & & \\
2 & scalefactor & 3028 & 0 & 31140 & 1 & 31140 & 0.0494 & 0.0539 & 2.9047 \\
3 & DQOH & 3028 & 89 & 31140 & 125 & 249.12 & 0.0286 & 0.0335 & 1.8049 \\
4 & M & 3028 & 89 & 31140 & 132 & 235.91 & 0.0274 & 0.0328 & 1.7695 \\
5 & U2 & 3028 & 117 & 31140 & 147 & 211.84 & 0.0271 & 0.0322 & 1.7404 \\
6 & XYZ & 3028 & 117 & 31140 & 417 & 74.68 & 0.0244 & 0.0291 & 1.5771 \\
7 & H-XYZ & 3027 & 117 & 31140 & 552 & 56.41 & 0.0240 & 0.0284 & 1.5426 \\
& All & & 3027 & 55 & 55.04 & 0.0239 & 0.0255 & 2.7619 \\
8 & previous & 3028 & 117 & 31140 & 552 & 56.41 & 0.0239 & 0.0283 & 1.5381 \\
9 & U3 & 3028 & 117 & 31140 & 742 & 41.97 & 0.0206 & 0.0230 & 1.2523 \\
10 & א' & 3028 & 0 & 31140 & 8 & 3892.5 & 0.0205 & 0.0228 & 1.2275 \\
11 & & 3028 & 117 & 31140 & 742 & 41.97 & 0.0202 & 0.0226 & 1.2328 \\
& SHADE- & & & & & & & & \\
12 & ADP & 3028 & 117 & 31140 & 742 & 41.97 & 0.0200 & 0.0223 & 1.2162 \\
13 & H-XYZ & 3027 & 0 & 3027 & 55 & 55.04 & 0.0200 & 0.0225 & 2.4363 \\
14 & & 3028 & 117 & 31140 & 742 & 41.97 & 0.0200 & 0.0223 & 1.2158 \\
& Me & & & & & & & & \\
15 & transfer* & 3028 & 81 & 31140 & 742 & 41.97 & 0.0200 & 0.0223 & 1.2148 \\
\hline
\end{tabular}

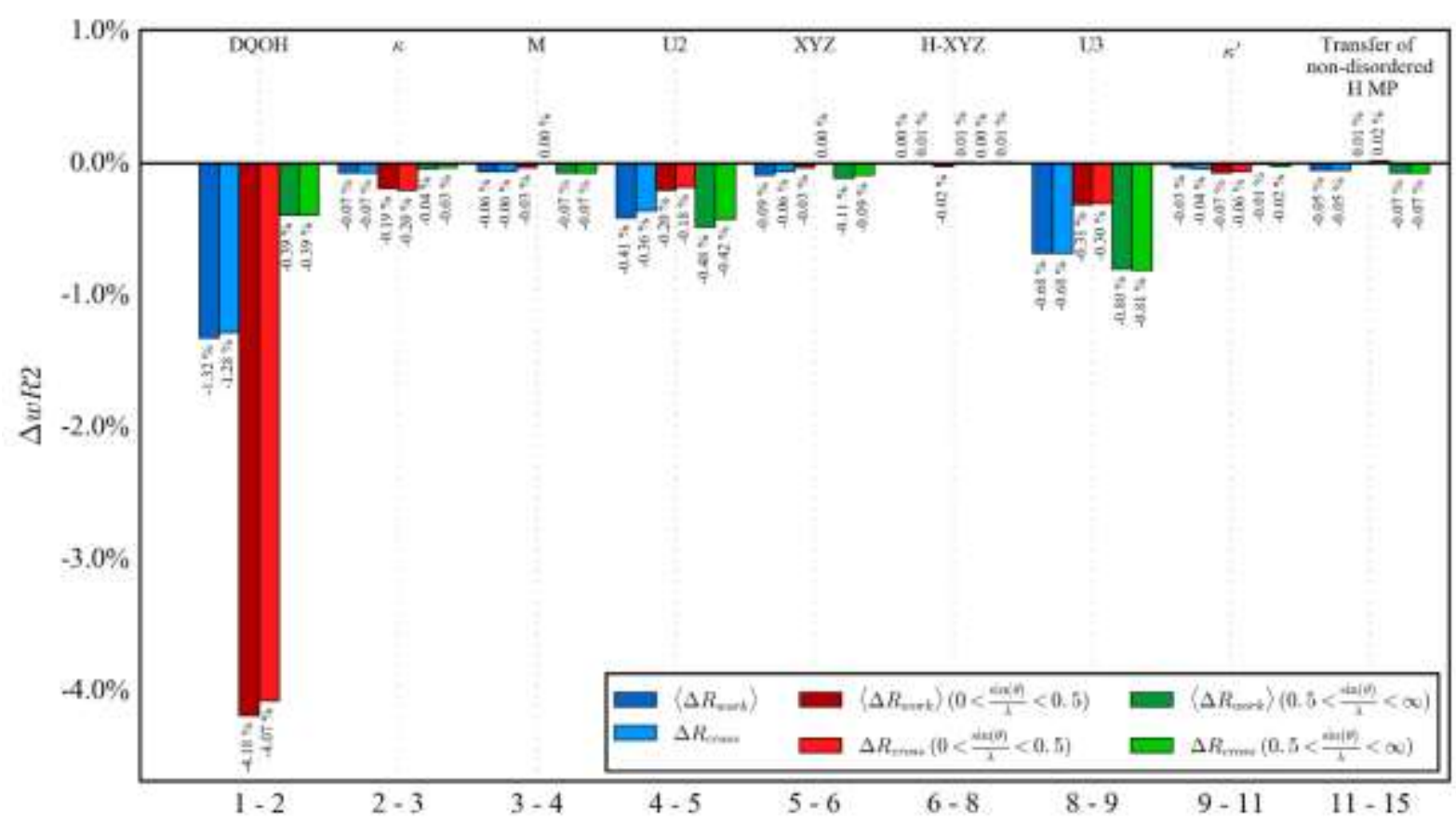

Figure S6.1: $\Delta \mathrm{R}_{\text {cross }}$ values for the XD refinement. A bbreviations: $\mathrm{M}$ : monopoles; $\mathrm{D}$ : dipoles; $\mathrm{Q}$ : quadrupoles; $\mathrm{O}$ : octupoles; K: $\kappa, U 2$, U3: Gram Charlier $2^{\text {nd }}$ and $3^{\text {rd }}$ order, $\mathrm{H}-\mathrm{XYZ}$ : only hydrogen position against data up to $0.5 \sin (\theta) / \lambda$. 

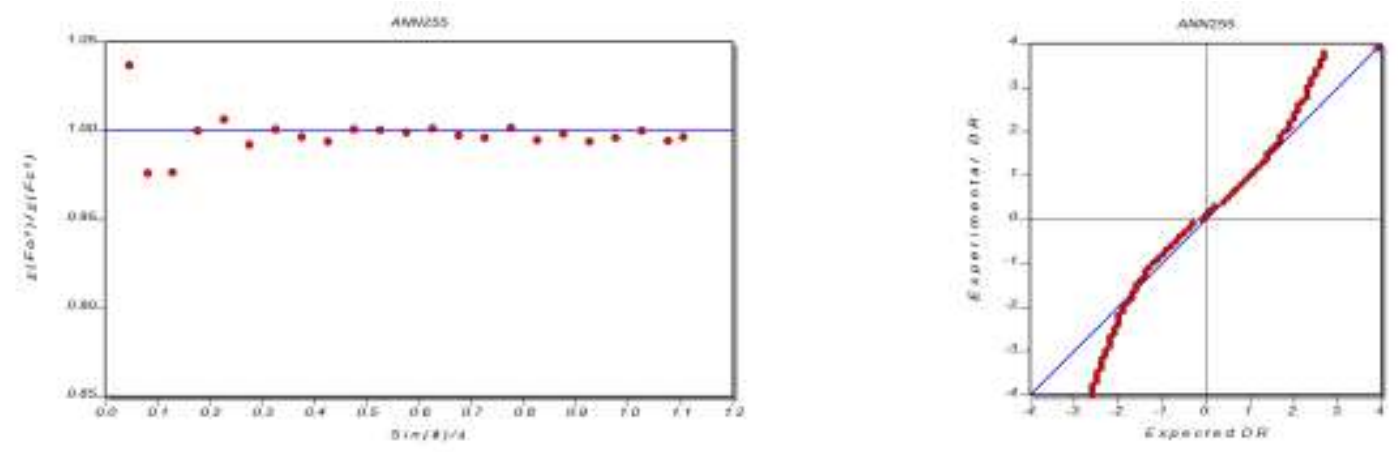

Figure S6.2: DRK-Plots(Adam Stash, 2007; Zhurov et al., 2008; Zavodnik et al., 1999) on F².

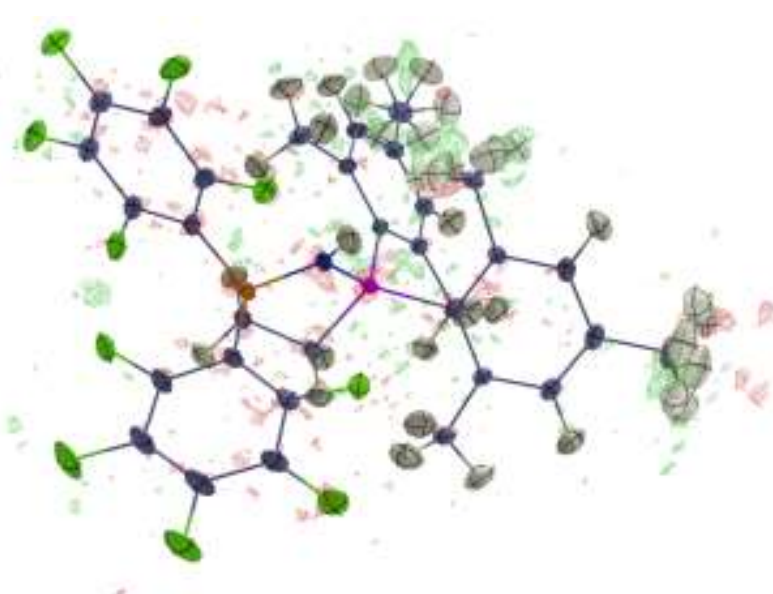

Figure S6.3: Difference electron density of $\mathbf{2}$ after the final refinement at the $+/-0.084 \mathrm{e}^{-3}$-level.

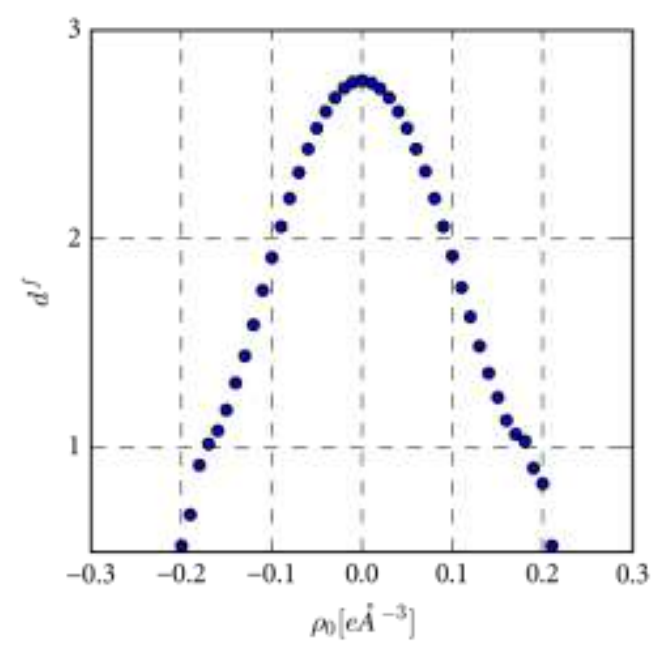

Figure A 0.1: Fractal dimension analysis (M eindl \& Henn, 2008) 

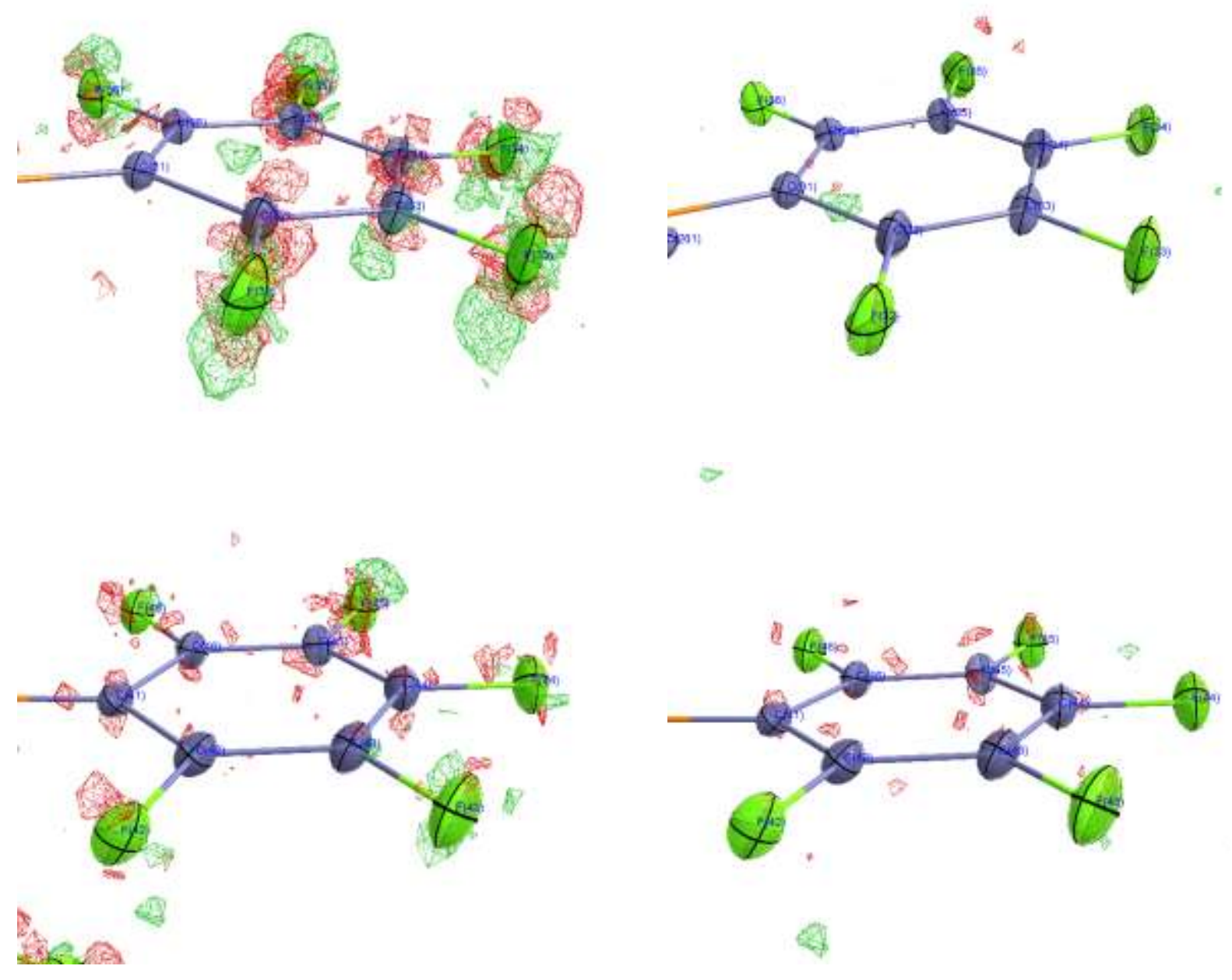

Figure S6.4: Residual density isosurfaces before (left) and after (right) introduction on Gram-Charlier Coefficients. The green density is positive and red negative, isolevel \pm 0.1 e $\AA^{-3}$.

Table S6.3: Kuhs's Rule

\begin{tabular}{llllllllll}
\hline A tom & \multicolumn{2}{l|l}{ Principal M.D.A's $[\AA]$} & M in. resolution $\left[\AA^{-1}\right]$ & \multicolumn{2}{l}{ Atom } & \multicolumn{3}{l}{ Principal M.D.A's $[\AA]$} & M in. resolution $\left[\AA^{-1}\right]$ \\
$F(32)$ & 0.25 & 0.176 & 0.107 & 0.77 & $C(32)$ & 0.186 & 0.152 & 0.104 & 0.9 \\
$F(33)$ & 0.301 & 0.208 & 0.103 & 0.7 & $C(33)$ & 0.219 & 0.164 & 0.105 & 0.83 \\
$F(34)$ & 0.276 & 0.174 & 0.114 & 0.73 & $C(34)$ & 0.214 & 0.144 & 0.114 & 0.85 \\
$F(35)$ & 0.235 & 0.171 & 0.127 & 0.75 & $C(35)$ & 0.183 & 0.134 & 0.121 & 0.9 \\
$F(36)$ & 0.204 & 0.134 & 0.122 & 0.87 & $C(36)$ & 0.153 & 0.122 & 0.117 & 1 \\
$F(42)$ & 0.22 & 0.144 & 0.108 & 0.86 & $C(121)$ & 0.185 & 0.138 & 0.118 & 0.9 \\
$F(43)$ & 0.236 & 0.173 & 0.111 & 0.78 & $C(141)$ & 0.193 & 0.171 & 0.115 & 0.83 \\
$F(44)$ & 0.202 & 0.178 & 0.124 & 0.79 & $C(161)$ & 0.164 & 0.133 & 0.112 & 0.96 \\
$F(45)$ & 0.216 & 0.155 & 0.123 & 0.81 & $C(221)$ & 0.194 & 0.14 & 0.114 & 0.89 \\
$F(46)$ & 0.19 & 0.133 & 0.11 & 0.92 & $C(241)$ & 0.237 & 0.189 & 0.11 & 0.76 \\
$C(31)$ & 0.147 & 0.121 & 0.106 & 1.05 & $C(261)$ & 0.152 & 0.144 & 0.122 & 0.93 \\
\hline
\end{tabular}


Table S6.4: Significance test for Gram-Charlier parameters colored by significance - not significant (value $<3 \sigma)$ : red, significant (value $>3 \sigma$ ): white.

\begin{tabular}{|c|c|c|c|c|c|c|c|c|c|c|}
\hline Atom & $\mathrm{C} 111 / \sigma$ & $\mathrm{C} 222 / \sigma$ & $\mathrm{C} 333 / \sigma$ & $\mathrm{C} 112 / \sigma$ & $\mathrm{C} 122 / \sigma$ & $\mathrm{Cl13} / \sigma$ & $\mathrm{C} 133 / \sigma$ & $\mathrm{C} 223 / \sigma$ & $\mathrm{C} 233 / \sigma$ & $\mathrm{Cl} 23 / \sigma$ \\
\hline$F(32)$ & 3.79 & 16.75 & 2.35 & 7.69 & 19.47 & 7.65 & 9 & 18.46 & 14 & 27.86 \\
\hline$F(33)$ & 4.55 & 29.71 & 13.24 & 25.24 & 45 & 14.42 & 3.64 & 38.08 & 6.91 & 30.36 \\
\hline$F(34)$ & 2.06 & 7.33 & 17.76 & 3.74 & 19.43 & 3.18 & 1.73 & 1.09 & 20.8 & 1.9 \\
\hline$F(35)$ & 4.27 & 16.11 & 0.12 & 24.93 & 15.77 & 11.86 & 4.75 & 15.62 & 26.5 & 20.86 \\
\hline$F(36)$ & 0.52 & 5.94 & 1 & 6.2 & 15.55 & 10.8 & 9.83 & 14.2 & 5.6 & 16.67 \\
\hline$F(42)$ & 0.77 & 1.85 & 0.83 & 10.38 & 3.92 & 4.92 & 1.56 & 7.4 & 1 & 1.6 \\
\hline$F(43)$ & 2.29 & 8.56 & 1.31 & 7 & 0.5 & 3.35 & 6.89 & 5.2 & 6 & 6.57 \\
\hline$F(44)$ & 3.68 & 0.77 & 4.54 & 1.44 & 1.58 & 0.63 & 0.12 & 0.22 & 5.4 & 12.5 \\
\hline$F(45)$ & 3.12 & 5.7 & 9.67 & 3.16 & 12.86 & 6.36 & 3.78 & 20.33 & 11.17 & 11.17 \\
\hline$F(46)$ & 0.97 & 0.2 & 2.2 & 3.4 & 5.33 & 3 & 5 & 2.57 & 6.67 & 9.75 \\
\hline$C(31)$ & 0 & 2.36 & 2.57 & 0.4 & 1.86 & 0.11 & 4.8 & 4.2 & 1.25 & 1 \\
\hline$C(32)$ & 0.5 & 9.07 & 4.11 & 6.15 & 13.9 & 4.09 & 6.14 & 12 & 4.33 & 10.83 \\
\hline$C(33)$ & 2.29 & 15.95 & 9.5 & 11.88 & 21.08 & 4.93 & 4.6 & 14.22 & 1.25 & 13.38 \\
\hline$C(34)$ & 0.68 & 5.53 & 13 & 1.93 & 7.82 & 3.62 & 2.44 & 0.75 & 13.29 & 1.86 \\
\hline$C(35)$ & 2.16 & 2.27 & 4.44 & 4.23 & 0.1 & 6.64 & 4.71 & 8.57 & 9.2 & 1.17 \\
\hline$C(36)$ & 1.54 & 2.82 & 2.86 & 3.4 & 0.63 & 5.78 & 0.67 & 5.6 & 4 & 0.4 \\
\hline$C(121)$ & 7.79 & 1.93 & 2.43 & 11.19 & 1.91 & 2.08 & 1.71 & 3.83 & 2.75 & 1.33 \\
\hline$C(141)$ & 0.35 & 11.06 & 1.1 & 9 & 7.33 & 5.43 & 1.11 & 6.57 & 10 & 8.29 \\
\hline$C(142)$ & 0.35 & 11.06 & 1.1 & 9 & 7.33 & 5.43 & 1.11 & 6.57 & 10 & 8.29 \\
\hline$C(161)$ & 6.19 & 3.09 & 3 & 4 & 1.22 & 1.91 & 3.33 & 4.8 & 3.25 & 1.6 \\
\hline$C(221)$ & 0.59 & 29.67 & 0.5 & 2.62 & 3.42 & 0.4 & 3 & 3.25 & 5 & 1.17 \\
\hline$C(222)$ & 0.59 & 29.67 & 0.5 & 2.62 & 3.42 & 0.4 & 3 & 3.25 & 5 & 1.17 \\
\hline$C(241)$ & 3.5 & 18.23 & 2.56 & 2.23 & 15.26 & 2.2 & 5.1 & 2.6 & 1.14 & 5.3 \\
\hline$C(242)$ & 3.5 & 18.23 & 2.56 & 2.23 & 15.26 & 2.2 & 5.1 & 2.6 & 1.14 & 5.3 \\
\hline$C(261)$ & 0.65 & 3.57 & 1 & 3.92 & 11.1 & 1.89 & 4.67 & 2.67 & 3.25 & 0.6 \\
\hline
\end{tabular}


Table S6.5: A nalysis of the Probability Density Function.

\begin{tabular}{|c|c|c|c|c|c|c|}
\hline A tom & $\begin{array}{l}\text { Minimu } \\
m \text { PDF } \\
\text { value }\end{array}$ & $\begin{array}{l}\text { Maximum } \\
\text { PDF } \\
\text { Value }\end{array}$ & $\begin{array}{l}\text { Total integrated } \\
\text { negative } \\
\text { probability [\%] }\end{array}$ & $\begin{array}{l}\text { Integrated } \\
\text { volume for } \\
\text { negative } \\
\text { probability }\left[\AA^{-3}\right]\end{array}$ & $\begin{array}{l}\text { Total integrated } \\
\text { positive } \\
\text { probability [\%] }\end{array}$ & $\begin{array}{l}\text { Integrated } \\
\text { volume for } \\
\text { positive } \\
\text { probability }\left[\AA^{-3}\right.\end{array}$ \\
\hline$F(32)$ & -31.06 & 38895.01 & -0.019 & 1.036 & 100.008 & 3.238 \\
\hline$F(33)$ & -95.47 & 28423.35 & -0.07 & 1.008 & 99.956 & 3.266 \\
\hline$F(34)$ & -75.18 & 33492.84 & -0.059 & 1.196 & 100.002 & 3.078 \\
\hline$F(35)$ & -35.38 & 36177.28 & -0.035 & 1.165 & 100.03 & 3.11 \\
\hline$F(36)$ & -15.19 & 54740.83 & -0.005 & 0.885 & 100.005 & 3.389 \\
\hline$F(42)$ & -27.08 & 53401.06 & -0.015 & 1.008 & 100.015 & 3.266 \\
\hline$F(43)$ & -15.27 & 40260 & -0.009 & 0.938 & 99.995 & 3.336 \\
\hline$F(44)$ & -32.77 & 41178.04 & -0.023 & 1.059 & 100.018 & 3.215 \\
\hline$F(45)$ & -53.38 & 44513.91 & -0.038 & 1.155 & 100.037 & 3.119 \\
\hline$F(46)$ & -22.36 & 65527.33 & -0.011 & 1.093 & 100.011 & 3.181 \\
\hline$C(31)$ & -1.46 & 97262.35 & 0 & 0.848 & 100 & 3.426 \\
\hline$C(32)$ & -22.75 & 62234.8 & -0.008 & 0.883 & 100.008 & 3.391 \\
\hline$C(33)$ & -42.06 & 48496.73 & -0.02 & 0.807 & 100.016 & 3.467 \\
\hline$C(34)$ & -9.74 & 52135.86 & -0.004 & 0.841 & 100.001 & 3.433 \\
\hline$C(35)$ & -4.98 & 61567.62 & -0.003 & 1.017 & 100.003 & 3.257 \\
\hline$C(36)$ & -2.62 & 83657.68 & -0.001 & 1.139 & 100.001 & 3.135 \\
\hline$C(121)$ & -2.97 & 60655.93 & -0.001 & 0.902 & 100.001 & 3.373 \\
\hline$C(141)$ & -10.46 & 48264.81 & -0.011 & 1.21 & 100.01 & 3.065 \\
\hline$C(142)$ & -10.46 & 48264.81 & -0.011 & 1.21 & 100.01 & 3.065 \\
\hline$C(161)$ & -1.35 & 74740.6 & -0.001 & 1.24 & 100.001 & 3.034 \\
\hline$C(221)$ & -57.76 & 59340.57 & -0.03 & 0.81 & 100.028 & 3.464 \\
\hline$C(222)$ & -57.76 & 59340.57 & -0.03 & 0.81 & 100.028 & 3.464 \\
\hline$C(241)$ & -31.43 & 37168.65 & -0.022 & 0.744 & 100.01 & 3.53 \\
\hline$C(242)$ & -31.43 & 37168.65 & -0.022 & 0.744 & 100.01 & 3.53 \\
\hline$C(261)$ & -3.3 & 68442.03 & -0.001 & 1.107 & 100.001 & 3.167 \\
\hline
\end{tabular}


Table S6.6: Integrated Charges.

\begin{tabular}{|c|c|c|c|c|c|}
\hline A tom & Charge & Atom & Charge & A tom(second moiety) & Charge \\
\hline $\mathrm{B}(1)$ & 2.04 & $P(1)$ & 2.10 & & \\
\hline$C(31)$ & -0.46 & $C(11)$ & -0.39 & & \\
\hline$C(32)$ & 0.53 & $C(12)$ & -0.02 & & \\
\hline$C(33)$ & 0.56 & $C(121)$ & -0.09 & & \\
\hline$C(34)$ & 0.56 & $H(12 A)$ & 0.05 & & \\
\hline$C(35)$ & 0.56 & $H(12 B)$ & 0.06 & & \\
\hline$C(36)$ & 0.54 & $H(12 C)$ & 0.06 & & \\
\hline$F(32)$ & -0.60 & $C(13)$ & -0.10 & & \\
\hline$F(33)$ & -0.60 & $H(13)$ & 0.03 & & \\
\hline$F(34)$ & -0.60 & $C(14)$ & -0.02 & & \\
\hline$F(35)$ & -0.60 & $C(141)$ & -0.07 & $C(142)$ & -0.06 \\
\hline$F(36)$ & -0.61 & $H(14 A)$ & 0.06 & $H(14 D)$ & 0.06 \\
\hline$C(41)$ & -0.46 & $H(14 B)$ & 0.06 & $H(14 E)$ & 0.06 \\
\hline$C(42)$ & 0.54 & $H(14 C)$ & 0.06 & $H(14 F)$ & 0.06 \\
\hline$C(43)$ & 0.55 & $C(15)$ & -0.10 & & \\
\hline$C(44)$ & 0.55 & $H(15)$ & 0.03 & & \\
\hline$C(45)$ & 0.54 & $C(16)$ & -0.02 & & \\
\hline$C(46)$ & 0.52 & $C(161)$ & -0.08 & & \\
\hline$F(42)$ & -0.60 & $H(16 A)$ & 0.05 & & \\
\hline$F(43)$ & -0.59 & $H(16 B)$ & 0.06 & & \\
\hline$F(44)$ & -0.60 & $H(16 C)$ & 0.05 & & \\
\hline$F(45)$ & -0.60 & $C(21)$ & -0.41 & & \\
\hline$F(46)$ & -0.58 & $C(22)$ & -0.03 & & \\
\hline $\mathrm{N}(1)$ & -1.84 & $C(221)$ & -0.07 & $C(222)$ & -0.05 \\
\hline$H(1 N)$ & 0.49 & $H(22 A)$ & 0.06 & $H(22 D)$ & 0.06 \\
\hline$C(1)$ & -0.37 & $H(22 B)$ & 0.07 & $H(22 E)$ & 0.06 \\
\hline$H(1 A)$ & 0.01 & $H(22 C)$ & 0.05 & $H(22 F)$ & 0.06 \\
\hline$H(1 B)$ & 0.02 & $C(23)$ & -0.10 & $C(242)$ & -0.07 \\
\hline$C(2)$ & -0.47 & $\mathrm{H}(23)$ & 0.03 & $H(24 D)$ & 0.06 \\
\hline$H(2 A)$ & 0.02 & $C(24)$ & -0.02 & $H(24 E)$ & 0.06 \\
\hline \multirow[t]{11}{*}{$\mathrm{H}(2 \mathrm{~B})$} & 0.02 & $C(241)$ & -0.04 & $\mathrm{H}(24 \mathrm{~F})$ & 0.06 \\
\hline & & $H(24 A)$ & 0.06 & & \\
\hline & & $H(24 B)$ & 0.06 & & \\
\hline & & $H(24 C)$ & 0.06 & & \\
\hline & & $C(25)$ & -0.10 & & \\
\hline & & $H(25)$ & 0.03 & & \\
\hline & & $C(26)$ & -0.02 & & \\
\hline & & $C(261)$ & -0.08 & & \\
\hline & & $H(26 \mathrm{~A})$ & 0.05 & & \\
\hline & & $H(26 B)$ & 0.06 & & \\
\hline & & $H(26 C)$ & 0.06 & & \\
\hline
\end{tabular}


Table S6.7: BCP Analysis.

\begin{tabular}{|c|c|c|c|c|c|c|c|}
\hline A tom 1 & Atom2 & $\begin{array}{l}\rho\left(\mathbf{r}_{\mathrm{bcp}}\right) \\
{\left[\mathrm{e} \AA^{-3}\right]}\end{array}$ & $\begin{array}{l}\nabla^{2} \rho\left(\mathbf{r}_{b c p}\right) \\
{\left[e \AA^{-5}\right]}\end{array}$ & $\begin{array}{l}\text { Bond Path } \\
\text { Length }[\AA]\end{array}$ & $\mathbf{r}_{\mathrm{Al-BCP}}[\AA]$ & $\mathbf{r}_{\mathrm{A} 2-\mathrm{BCP}}[\AA]$ & $\varepsilon\left(\mathbf{r}_{\mathrm{bcp}}\right)$ \\
\hline B1 & $\mathrm{C} 2$ & $1.161(13)$ & $-8.9(4) *$ & $1.6390(2)^{*}$ & $0.525(2) *$ & $1.114(2)^{*}$ & $0.09(2) *$ \\
\hline B1 & C31 & $1.017(8)$ & $-7.1(3)^{*}$ & $1.66420(19) *$ & $0.540(3) *$ & $1.124(3) *$ & $0.19(2) *$ \\
\hline B1 & C41 & $1.028(10)$ & $-6.4(3)^{*}$ & $1.65080(19)^{*}$ & $0.533(2)^{*}$ & $1.118(2)^{*}$ & $0.13(2)^{*}$ \\
\hline B1 & N 1 & $1.012(12)$ & $1.4(3)^{*}$ & $1.5662(2)^{*}$ & $0.4980(9)^{*}$ & $1.0683(9)^{*}$ & $0.010(13) *$ \\
\hline $\mathrm{C} 1$ & $\mathrm{C} 2$ & $1.548(12)$ & $-12.4(2)^{*}$ & $1.55090(9)^{*}$ & $0.807(2)^{*}$ & $0.744(2)^{*}$ & $0.010(7)^{*}$ \\
\hline $\mathrm{C} 1$ & $\mathrm{H} 1 \mathrm{~A}$ & $1.778(10)$ & $-19.46(17)^{*}$ & $1.09230(9)^{*}$ & $0.7163(18)^{*}$ & $0.3760(18)^{*}$ & $0.010(6) *$ \\
\hline $\mathrm{C} 1$ & $\mathrm{H} 1 \mathrm{~B}$ & $1.766(8)$ & $-19.01(16)^{*}$ & $1.09340(10)^{*}$ & $0.7176(17)^{*}$ & $0.3758(18)^{*}$ & $0.010(6) *$ \\
\hline $\mathrm{C} 1$ & P1 & $1.172(9)$ & $-8.53(13)^{*}$ & $1.8182(4) *$ & $0.997(4)^{*}$ & $0.821(4)^{*}$ & $0.030(11)^{*}$ \\
\hline C11 & $\mathrm{C} 12$ & $2.020(6)$ & $-15.11(9)^{*}$ & $1.41810(7)^{*}$ & $0.7083(5)^{*}$ & $0.7099(5) *$ & $0.210(5) *$ \\
\hline $\mathrm{C} 11$ & $\mathrm{C} 16$ & $2.031(7)$ & $-15.45(9) *$ & $1.41880(8)^{*}$ & $0.7093(5)^{*}$ & $0.7095(5) *$ & $0.220(6) *$ \\
\hline C11 & P1 & $1.185(7)$ & $-8.49(10)^{*}$ & $1.82560(13)^{*}$ & $0.990(2)^{*}$ & $0.836(2) *$ & $0.090(9) *$ \\
\hline $\mathrm{C} 12$ & C121 & $1.723(7)$ & $-13.51(8)^{*}$ & $1.5071(2)^{*}$ & $0.7641(14)^{*}$ & $0.7430(14)^{*}$ & $0.040(4) *$ \\
\hline $\mathrm{C} 12$ & C13 & $2.134(5)$ & $-17.96(9)^{*}$ & $1.39640(9)^{*}$ & $0.6928(7)^{*}$ & $0.7036(7)^{*}$ & $0.200(2) *$ \\
\hline C121 & $\mathrm{H} 12 \mathrm{~A}$ & $1.791(7)$ & $-19.95(10)^{*}$ & $1.07710(5)^{*}$ & $0.7202(15)^{*}$ & $0.3569(15)^{*}$ & $0.030(2) *$ \\
\hline C121 & $\mathrm{H} 12 \mathrm{~B}$ & $1.781(4)$ & $-19.71(10)^{*}$ & $1.07780(7)^{*}$ & $0.7208(15)^{*}$ & $0.3570(15)^{*}$ & $0.020(2) *$ \\
\hline C121 & $\mathrm{H} 12 \mathrm{C}$ & $1.791(5)$ & $-19.97(10)^{*}$ & $1.077(0)^{*}$ & $0.7202(15)^{*}$ & $0.3568(15)^{*}$ & $0.03(0) *$ \\
\hline $\mathrm{C} 13$ & C14 & $2.171(6)$ & $-18.32(9)^{*}$ & $1.39020(8)^{*}$ & $0.6961(7)^{*}$ & $0.6940(7)^{*}$ & $0.190(3) *$ \\
\hline $\mathrm{C} 13$ & H 13 & $1.833(10)$ & $-18.23(13)^{*}$ & $1.083(0)^{*}$ & $0.7177(9)^{*}$ & $0.3653(9)^{*}$ & $0.030(5)^{*}$ \\
\hline C14 & C141 & $2.489(5)^{*}$ & $-26.0(2)^{*}$ & $1.49760(17)^{*}$ & $0.568(2) *$ & $0.929(2) *$ & $0.030(5) *$ \\
\hline C14 & C15 & $2.169(2) *$ & $-18.28(10)^{*}$ & $1.39070(8) *$ & $0.6942(7)^{*}$ & $0.6965(7)^{*}$ & $0.190(3) *$ \\
\hline C141 & $\mathrm{H} 14 \mathrm{~A}$ & $2.459(4) *$ & $-28.73(18)^{*}$ & $1.07760(4)^{*}$ & $0.8385(10)^{*}$ & $0.2392(10) *$ & $0.26(0)^{*}$ \\
\hline C141 & H 14B & $2.460(4) *$ & $-28.68(18)^{*}$ & $1.0777(0)^{*}$ & $0.8385(10)^{*}$ & $0.2392(10)^{*}$ & $0.260(2)^{*}$ \\
\hline C141 & $\mathrm{H} 14 \mathrm{C}$ & $2.459(4) *$ & $-28.71(18)^{*}$ & $1.07770(5)^{*}$ & $0.8385(10) *$ & $0.2392(10) *$ & $0.26(0) *$ \\
\hline C141 & H 14D & $2.460(4) *$ & $-28.7(17)^{*}$ & $1.07770(2)^{*}$ & $0.8385(10)^{*}$ & $0.2392(10)^{*}$ & $0.260(2) *$ \\
\hline C141 & H 14E & $2.459(4) *$ & $-28.75(17)^{*}$ & $1.07760(4)^{*}$ & $0.8385(10)^{*}$ & $0.2391(10)^{*}$ & $0.26(0) *$ \\
\hline C141 & $\mathrm{H} 14 \mathrm{~F}$ & $2.460(4)^{*}$ & $-28.71(17)^{*}$ & $1.07770(5)^{*}$ & $0.8385(10)^{*}$ & $0.2392(10) *$ & $0.260(2) *$ \\
\hline C15 & $\mathrm{C} 16$ & $2.141(2) *$ & $-18.1(9)^{*}$ & $1.39460(6)^{*}$ & $0.7027(7)^{*}$ & $0.6919(7)^{*}$ & $0.200(4)^{*}$ \\
\hline C15 & H 15 & $1.832(4) *$ & $-18.24(13)^{*}$ & $1.083(0)^{*}$ & $0.7176(9)^{*}$ & $0.3654(9)^{*}$ & $0.030(5) *$ \\
\hline $\mathrm{C} 16$ & C161 & $1.720(4)^{*}$ & $-13.45(8)^{*}$ & $1.50930(8)^{*}$ & $0.7653(14)^{*}$ & $0.7440(14)^{*}$ & $0.040(4) *$ \\
\hline C161 & $\mathrm{H} 16 \mathrm{~A}$ & $1.790(2) *$ & $-19.95(10)^{*}$ & $1.07710(4)^{*}$ & $0.7202(15)^{*}$ & $0.3569(15)^{*}$ & $0.03(0)^{*}$ \\
\hline C161 & H 16B & $1.784(2) *$ & $-19.82(10)^{*}$ & $1.07750(7)^{*}$ & $0.7205(15)^{*}$ & $0.3571(15)^{*}$ & $0.020(4) *$ \\
\hline C161 & $\mathrm{H} 16 \mathrm{C}$ & $1.791(2) *$ & $-19.95(10)^{*}$ & $1.07700(5)^{*}$ & $0.7202(15)^{*}$ & $0.3569(15)^{*}$ & $0.03(0) *$ \\
\hline C2 & $\mathrm{H} 2 \mathrm{~A}$ & $1.786(9)$ & $-19.27(15)^{*}$ & $1.09210(5)^{*}$ & $0.7191(19)^{*}$ & $0.3730(19)^{*}$ & $0.040(5)^{*}$ \\
\hline $\mathrm{C} 2$ & $\mathrm{H} 2 \mathrm{~B}$ & $1.784(8)$ & $-19.16(15)^{*}$ & $1.09230(5)^{*}$ & $0.7193(18)^{*}$ & $0.3730(18)^{*}$ & $0.050(5) *$ \\
\hline $\mathrm{C} 21$ & $\mathrm{C} 22$ & $2.049(2) *$ & $-15.5(9)^{*}$ & $1.41760(9) *$ & $0.7056(5)^{*}$ & $0.7119(5)^{*}$ & $0.220(7)^{*}$ \\
\hline C21 & $\mathrm{C} 26$ & $2.026(2) *$ & $-15.19(10)^{*}$ & $1.41540(7)^{*}$ & $0.7068(5)^{*}$ & $0.7085(5) *$ & $0.210(6)^{*}$ \\
\hline C21 & P1 & $1.192(7)$ & $-8.72(10)^{*}$ & $1.82000(11)^{*}$ & $0.991(2) *$ & $0.830(2) *$ & $0.170(8) *$ \\
\hline $\mathrm{C} 22$ & C221 & $2.458(5) *$ & $-24.9(2)^{*}$ & $1.5059(2)^{*}$ & $0.575(2)^{*}$ & $0.930(2)^{*}$ & $0.030(5) *$ \\
\hline C22 & $\mathrm{C} 23$ & $2.157(2) *$ & $-17.93(9)^{*}$ & $1.39490(8)^{*}$ & $0.6959(7)^{*}$ & $0.6990(7)^{*}$ & $0.190(2)^{*}$ \\
\hline C221 & $\mathrm{H} 22 \mathrm{~A}$ & $2.458(4)^{*}$ & $-28.69(17)^{*}$ & $1.07770(3)^{*}$ & $0.8384(10)^{*}$ & $0.2393(10)^{*}$ & $0.26(0) *$ \\
\hline C221 & $\mathrm{H} 22 \mathrm{~B}$ & $2.459(4) *$ & $-28.61(17)^{*}$ & $1.07780(5)^{*}$ & $0.8384(10)^{*}$ & $0.2393(10)^{*}$ & $0.260(3) *$ \\
\hline C221 & $\mathrm{H} 22 \mathrm{C}$ & $2.458(4) *$ & $-28.67(17)^{*}$ & $1.07760(4)^{*}$ & $0.8383(10)^{*}$ & $0.2393(10)^{*}$ & $0.26(0) *$ \\
\hline C221 & $\mathrm{H} 22 \mathrm{D}$ & $2.461(4) *$ & $-28.64(17)^{*}$ & $1.0777(0)^{*}$ & $0.8383(10)^{*}$ & $0.2394(10)^{*}$ & $0.26(0) *$ \\
\hline C221 & $\mathrm{H} 22 \mathrm{E}$ & $2.457(4)^{*}$ & $-28.75(17)^{*}$ & $1.07760(3)^{*}$ & $0.8384(10)^{*}$ & $0.2392(10) *$ & $0.260(3) *$ \\
\hline C221 & $\mathrm{H} 22 \mathrm{~F}$ & $2.458(4)^{*}$ & $-28.65(17)^{*}$ & $1.07780(5)^{*}$ & $0.8384(10)^{*}$ & $0.2393(10)^{*}$ & $0.260(3)^{*}$ \\
\hline $\mathrm{C} 23$ & C24 & $2.170(2) *$ & $-18.29(9)^{*}$ & $1.39050(9)^{*}$ & $0.6964(7)^{*}$ & $0.6942(7)^{*}$ & $0.190(3) *$ \\
\hline $\mathrm{C} 23$ & $\mathrm{H} 23$ & $1.833(4)^{*}$ & $-18.22(13)^{*}$ & $1.083(0)^{*}$ & $0.7177(9)^{*}$ & $0.3654(9)^{*}$ & $0.020(5) *$ \\
\hline C24 & C241 & $2.498(5) *$ & $-26.3(2)^{*}$ & $1.4948(3)^{*}$ & $0.565(2)^{*}$ & $0.929(2)^{*}$ & $0.030(5)^{*}$ \\
\hline C24 & $\mathrm{C} 25$ & $2.165(2) *$ & $-18.16(9)^{*}$ & $1.39210(11)^{*}$ & $0.6951(7)^{*}$ & $0.6971(7)^{*}$ & $0.190(3) *$ \\
\hline C241 & $\mathrm{H} 24 \mathrm{~A}$ & $2.459(4) *$ & $-28.72(17)^{*}$ & $1.07770(4)^{*}$ & $0.8385(10) *$ & $0.2392(10)^{*}$ & $0.26(0) *$ \\
\hline C241 & $\mathrm{H} 24 \mathrm{~B}$ & $2.458(4) *$ & $-28.74(17)^{*}$ & $1.07760(2)^{*}$ & $0.8385(10)^{*}$ & $0.2391(10)^{*}$ & $0.26(0) *$ \\
\hline C241 & $\mathrm{H} 24 \mathrm{C}$ & $2.460(4) *$ & $-28.66(17)^{*}$ & $1.07770(4)^{*}$ & $0.8385(10)^{*}$ & $0.2392(10)^{*}$ & $0.260(2) *$ \\
\hline C241 & $H 24 D$ & $2.460(4) *$ & $-28.71(18)^{*}$ & $1.07770(4)^{*}$ & $0.8385(10)^{*}$ & $0.2392(10)^{*}$ & $0.260(2) *$ \\
\hline C241 & H 24E & $2.460(4) *$ & $-28.67(18)^{*}$ & $1.07770(2)^{*}$ & $0.8385(10) *$ & $0.2392(10)^{*}$ & $0.260(2) *$ \\
\hline C241 & $\mathrm{H} 24 \mathrm{~F}$ & $2.458(4)^{*}$ & $-28.75(18)^{*}$ & $1.0776(0)^{*}$ & $0.8385(10)^{*}$ & $0.2391(10)^{*}$ & $0.26(0) *$ \\
\hline
\end{tabular}




\begin{tabular}{|c|c|c|c|c|c|c|c|}
\hline C25 & $\mathrm{C} 26$ & $2.138(2)^{*}$ & $-18.05(9)^{*}$ & $1.39520(9)^{*}$ & $0.7031(7)^{*}$ & $0.6921(7)^{*}$ & $0.200(3)^{*}$ \\
\hline C25 & H 25 & $1.833(3)^{*}$ & $-18.24(13)^{*}$ & $1.083(0) *$ & $0.7176(9) *$ & $0.3653(9) *$ & $0.030(5) *$ \\
\hline C26 & C261 & $1.729(4) *$ & $-13.65(9)^{*}$ & $1.5050(2) *$ & $0.7636(14) *$ & $0.7414(14) *$ & $0.040(4)^{*}$ \\
\hline C261 & $\mathrm{H} 26 \mathrm{~A}$ & $1.791(2)^{*}$ & $-19.96(10) *$ & $1.077(0) *$ & $0.7201(15) *$ & 0.3569 (15)* & $0.03(0) *$ \\
\hline C261 & $\mathrm{H} 26 \mathrm{~B}$ & $1.790(2)^{*}$ & $-19.92(10) *$ & $1.07720(6) *$ & $0.7205(15) *$ & $0.3567(15) *$ & $0.03(0) *$ \\
\hline C261 & $\mathrm{H} 26 \mathrm{C}$ & $1.787(2)^{*}$ & $-19.88(10) *$ & $1.07730(6) *$ & $0.7205(15) *$ & $0.3568(15) *$ & $0.030(5) *$ \\
\hline C31 & C32 & $2.116(6)$ & $-18.14(9)^{*}$ & $1.39470(18) *$ & $0.6829(8) *$ & $0.7118(8) *$ & $0.190(5)^{*}$ \\
\hline C31 & C36 & $2.115(7)$ & $-18.01(9)^{*}$ & $1.39330(8)^{*}$ & $0.6824(7)^{*}$ & $0.7110(8) *$ & $0.180(5)^{*}$ \\
\hline C32 & C33 & $2.200(3)$ & $-19.89(7)^{*}$ & $1.3880(3)^{*}$ & $0.69410(16) *$ & $0.69390(17)^{*}$ & $0.320(5)^{*}$ \\
\hline C32 & F32 & $1.915(7)$ & $-15.8(2)^{*}$ & $1.3517(3) *$ & $0.5000(10) *$ & $0.8517(9)^{*}$ & $0.020(6)^{*}$ \\
\hline C33 & C34 & $2.219(3)^{*}$ & $-20.34(8)^{*}$ & $1.3830(5) *$ & $0.6917(2) *$ & $0.6913(2) *$ & $0.320(5)^{*}$ \\
\hline C33 & F33 & $1.938(4)^{*}$ & $-16.1(2)^{*}$ & $1.3430(3) *$ & $0.4901(9) *$ & $0.8529(8) *$ & $0.020(7)^{*}$ \\
\hline C34 & C35 & $2.216(2) *$ & $-20.27(6)^{*}$ & $1.3839(4) *$ & $0.69180(19)^{*}$ & $0.69200(19)^{*}$ & $0.320(5) *$ \\
\hline C34 & F34 & $1.939(4) *$ & $-16.2(2)^{*}$ & $1.3424(4) *$ & $0.4894(10) *$ & $0.8530(8)^{*}$ & $0.020(6)^{*}$ \\
\hline C35 & C36 & $2.186(2)^{*}$ & $-19.59(8) *$ & $1.3920(2) *$ & $0.69600(12) *$ & $0.69590(14) *$ & $0.320(5) *$ \\
\hline C35 & F35 & $1.943(4) *$ & $-16.2(2) *$ & $1.3412(4) *$ & $0.4880(8)^{*}$ & $0.8532(8) *$ & $0.020(7)^{*}$ \\
\hline C36 & F36 & $1.918(4)^{*}$ & $-15.7(2)^{*}$ & $1.3506(2) *$ & $0.4990(9) *$ & $0.8516(9) *$ & $0.020(6)^{*}$ \\
\hline C41 & C42 & $2.123(2)^{*}$ & $-18.18(10) *$ & $1.39160(8)^{*}$ & $0.6815(8) *$ & $0.7101(8) *$ & $0.180(5)^{*}$ \\
\hline C41 & C46 & $2.130(3)^{*}$ & $-18.47(9)^{*}$ & $1.39070(8) *$ & $0.6809(8) *$ & $0.7098(8) *$ & $0.190(5)^{*}$ \\
\hline C42 & C43 & $2.188(2) *$ & $-19.63(7)^{*}$ & $1.39150(10) *$ & $0.69580(6) *$ & $0.69570(7)^{*}$ & $0.320(5) *$ \\
\hline C42 & F42 & $1.927(4) *$ & $-15.9(2)^{*}$ & $1.3477(2)^{*}$ & $0.4955(9)^{*}$ & $0.8522(9) *$ & $0.020(6) *$ \\
\hline C43 & C44 & $2.208(2)^{*}$ & $-20.08(7)^{*}$ & $1.38620(8) *$ & $0.69320(6) *$ & $0.69290(6) *$ & $0.320(5)^{*}$ \\
\hline C43 & F43 & $1.933(4)^{*}$ & $-16.1(2)^{*}$ & $1.34480(18) *$ & $0.4920(9) *$ & $0.8528(8) *$ & $0.020(6)^{*}$ \\
\hline C44 & C45 & $2.208(2) *$ & $-20.06(7)^{*}$ & $1.38640(12) *$ & $0.69300(7)^{*}$ & $0.69340(6) *$ & $0.320(6) *$ \\
\hline C44 & F44 & $1.938(4)^{*}$ & $-16.2(2)^{*}$ & $1.3426(2)^{*}$ & $0.4895(8) *$ & $0.8531(8)^{*}$ & $0.020(6)^{*}$ \\
\hline C45 & C46 & $2.200(2) *$ & $-19.91(7)^{*}$ & $1.38790(6) *$ & $0.69370(5) *$ & $0.69420(6) *$ & $0.320(5) *$ \\
\hline C45 & F45 & $1.934(4) *$ & $-16.1(2)^{*}$ & $1.3443(3) *$ & $0.4915(9) *$ & $0.8529(8) *$ & $0.020(6) *$ \\
\hline C46 & F46 & $1.898(4) *$ & $-15.5(2)^{*}$ & $1.35800(16) *$ & $0.5072(9) *$ & $0.8508(9) *$ & $0.020(6) *$ \\
\hline H $1 N$ & N1 & $2.076(18)$ & $-28.2(4) *$ & $1.02720(4) *$ & $0.2584(14) *$ & $0.7688(14)^{*}$ & $0.080(4) *$ \\
\hline N1 & P1 & $1.397(11)$ & $2.2(2)^{*}$ & $1.62030(7)^{*}$ & $0.9567(6)^{*}$ & $0.6636(5) *$ & 0.040 (14)* \\
\hline
\end{tabular}




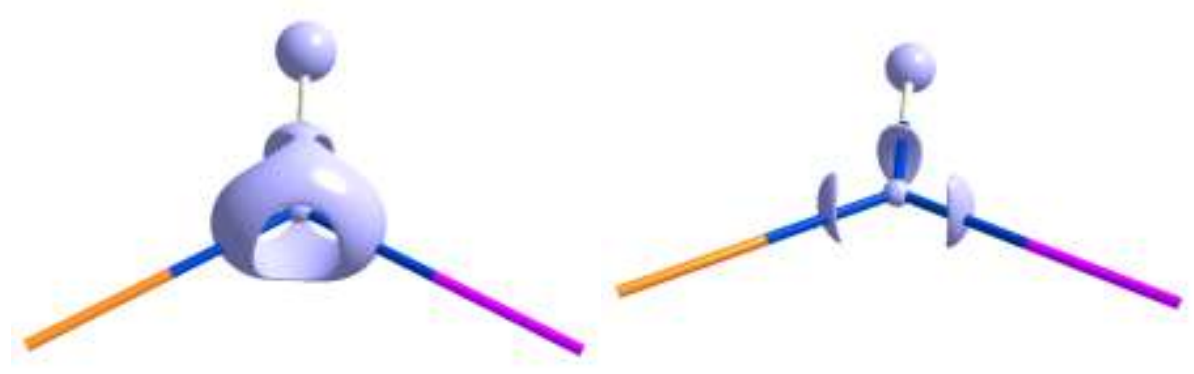

Figure S6.5: I sosurface representation of the VSCCs around $N$ at the Isolevels of- 37 and $-50 \mathrm{e}^{-5}$.

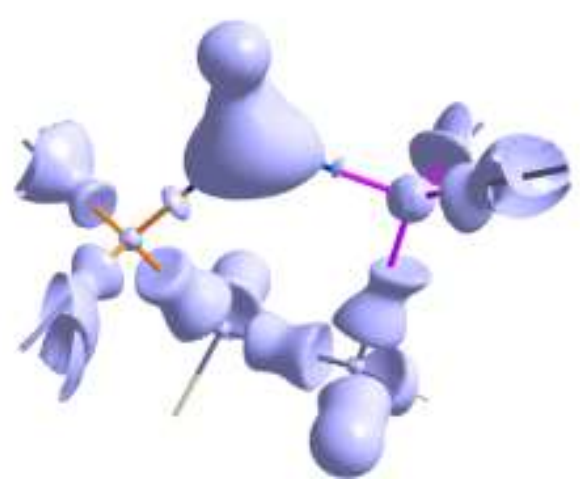

Figure S6.6: I sosurface representation of the VSCCs in the central ring. Isolevel: $-5 \mathrm{e}^{-5}$.
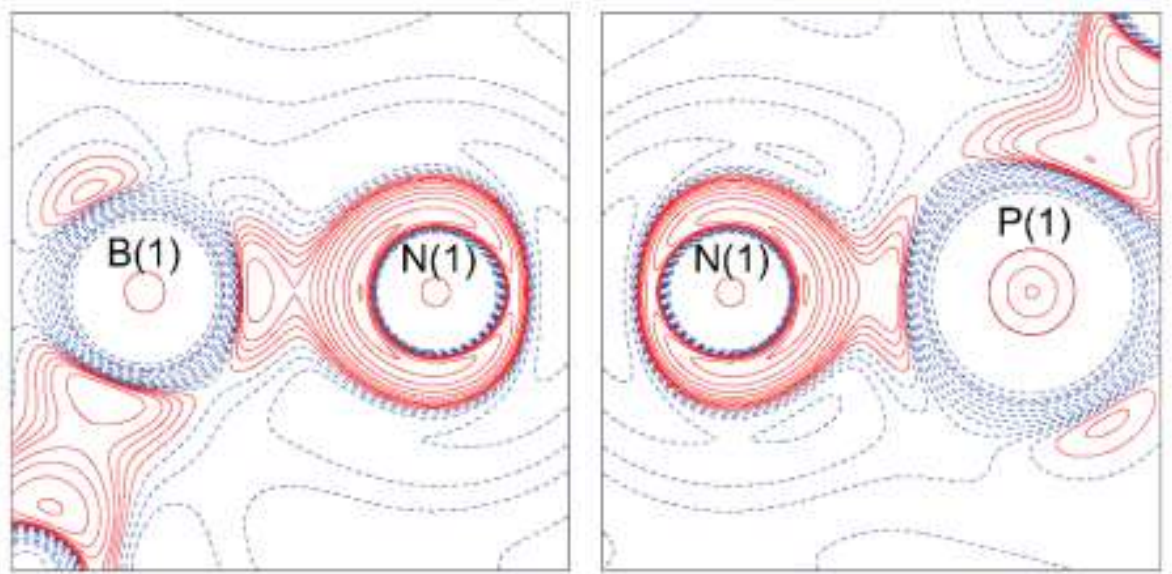

Figure S6.7: Laplacian distributions of perpendicular to the $B(1)-N(1)-P(1)$ plane along the $B(1)-N(1)$ and $B(1)$ $P(1)$ bond. I solevels +(blue)/-(red) $0,1,2,3,5,8,13,21,34,43,55$ and $89 \mathrm{e}^{-5}$. 


\section{Supplementary Information to Chapter 7}

Table S7.1: Data quality statistics from XPREP.

\begin{tabular}{|c|c|c|c|c|c|c|c|c|c|c|c|c|}
\hline \multicolumn{3}{|c|}{ Resolution } & \multirow{2}{*}{$\begin{array}{l}\text { Number } \\
\text { of Data } \\
141\end{array}$} & \multirow{2}{*}{$\begin{array}{l}\text { Theory } \\
141\end{array}$} & \multirow{2}{*}{$\begin{array}{l}\text { Complete- } \\
\text { ness [\%] } \\
100\end{array}$} & \multirow{2}{*}{$\begin{array}{l}\text { Multiplicity } \\
39.21\end{array}$} & \multirow{2}{*}{$\begin{array}{r}\langle I\rangle \\
54.5\end{array}$} & \multirow{2}{*}{$\begin{array}{c}\langle I / \sigma\rangle \\
173.56\end{array}$} & \multirow{2}{*}{$\begin{array}{r}R_{\text {merge }} \\
0.0187\end{array}$} & \multirow{2}{*}{$\begin{array}{c}\text { Rsigma } \\
0.0037\end{array}$} & \multirow{2}{*}{$\begin{array}{c}\text { Rrim } \\
0.0191\end{array}$} & \multirow{2}{*}{$\begin{array}{c}\mathrm{R}_{\text {pim }} \\
0.0038\end{array}$} \\
\hline Inf & - & 2 & & & & & & & & & & \\
\hline 2 & - & 1.2 & 407 & 407 & 100 & 36.63 & 26.06 & 145.69 & 0.0217 & 0.0041 & 0.0221 & 0.0038 \\
\hline 1.2 & - & 0.9 & 690 & 690 & 100 & 29.13 & 12.63 & 82.74 & 0.0318 & 0.0067 & 0.0323 & 0.006 \\
\hline 0.9 & - & 0.76 & 769 & 769 & 100 & 24.68 & 6.48 & 62.51 & 0.0476 & 0.0101 & 0.0486 & 0.0095 \\
\hline 0.76 & - & 0.7 & 529 & 529 & 100 & 18.59 & 3.87 & 58.91 & 0.05 & 0.0119 & 0.0514 & 0.0115 \\
\hline 0.7 & - & 0.64 & 736 & 736 & 100 & 11.88 & 2.9 & 49.02 & 0.0288 & 0.0141 & 0.0301 & 0.0083 \\
\hline 0.64 & - & 0.6 & 662 & 664 & 99.7 & 11.01 & 1.69 & 32.82 & 0.0373 & 0.0224 & 0.039 & 0.0113 \\
\hline 0.6 & & 0.56 & 874 & 879 & 99.4 & 10.6 & 1.28 & 25.25 & 0.0471 & 0.0301 & 0.0494 & 0.0145 \\
\hline 0.56 & - & 0.54 & 540 & 542 & 99.6 & 9.94 & 0.96 & 19.36 & 0.0575 & 0.0409 & 0.0605 & 0.0182 \\
\hline 0.54 & - & 0.52 & 599 & 608 & 98.5 & 9.85 & 0.73 & 15.15 & 0.0718 & 0.0524 & 0.0757 & 0.0231 \\
\hline 0.52 & - & 0.5 & 711 & 717 & 99.2 & 9.67 & 0.63 & 13.19 & 0.0864 & 0.0616 & 0.0912 & 0.0283 \\
\hline 0.5 & - & 0.49 & 404 & 411 & 98.3 & 9 & 0.49 & 10.35 & 0.1012 & 0.0812 & 0.1071 & 0.0338 \\
\hline 0.49 & - & 0.48 & 429 & 429 & 100 & 9.14 & 0.36 & 8.37 & 0.1349 & 0.1053 & 0.1431 & 0.046 \\
\hline 0.48 & - & 0.47 & 461 & 480 & 96 & 7.84 & 0.3 & 7.17 & 0.1429 & 0.126 & 0.1528 & 0.0523 \\
\hline 0.47 & - & 0.46 & 528 & 531 & 99.4 & 7.8 & 0.26 & 6.21 & 0.149 & 0.1484 & 0.1595 & 0.0548 \\
\hline 0.46 & - & 0.45 & 587 & 627 & 93.6 & 6.87 & 0.25 & 5.77 & 0.1636 & 0.1588 & 0.1761 & 0.0625 \\
\hline 0.55 & - & 0.45 & 3996 & 4080 & 97.9 & 8.72 & 0.48 & 10.4 & 0.0965 & 0.0809 & 0.1023 & 0.0329 \\
\hline Inf & & 0.45 & 9067 & 9160 & 99 & 14.5 & 4.48 & 38.23 & 0.0306 & 0.0113 & 0.0313 & 0.0064 \\
\hline
\end{tabular}

Table 57.2: Local coordinate systems, $\kappa$-set assignment and chemical constraints for the refinements. The indicated Gram-Charlier-level corresponds to the final refinement. DUM 0 is located in the M iddle of the heterocycle; DUM 1 to 3 are located in the middle of the atoms neighboring $C / S$ atoms.

\begin{tabular}{|c|c|c|c|c|c|c|c|c|c|}
\hline ATOM & ATOM 0 & AX1 & ATOM 1 & ATOM 2 & AX2 & GC-level & $\kappa$-set & SITESYM & CHEMCON \\
\hline$S(1)$ & $C(2)$ & Z & $S(1)$ & DUM 4 & Y & 3 & 1 & $\mathrm{~m}$ & \\
\hline$S(6)$ & $C(5)$ & Z & $S(6)$ & $C(7)$ & Y & 3 & 1 & $\mathrm{~m}$ & $S(1)$ \\
\hline$C(2)$ & $C(3)$ & Z & $C(2)$ & DUM 0 & Y & 2 & 2 & сус & \\
\hline$C(3)$ & $C(2)$ & Z & $C(3)$ & DUM 0 & Y & 2 & 2 & сус & \\
\hline$C(4)$ & $C(5)$ & Z & $C(4)$ & DUM 0 & Y & 2 & 2 & сус & $C(3)$ \\
\hline$C(5)$ & $C(4)$ & Z & $C(5)$ & DUM 0 & Y & 2 & 2 & сус & $C(2)$ \\
\hline$C(7)$ & DUM 2 & Z & $C(7)$ & $C(8)$ & Y & 2 & 3 & $\mathrm{~mm} 2$ & \\
\hline$C(8)$ & DUM 1 & Z & $C(8)$ & $C(7)$ & Y & 2 & 3 & $\mathrm{~mm} 2$ & \\
\hline$C(9)$ & DUM 3 & Z & $C(9)$ & $C(8)$ & $Y$ & 2 & 3 & $\mathrm{~mm} 2$ & $C(7)$ \\
\hline$H(7 A)$ & $C(7)$ & Z & $H(7 A)$ & $H(7 B)$ & Y & 1 & 4 & сус & \\
\hline$H(7 B)$ & $C(7)$ & Z & $H(7 B)$ & $H(7 A)$ & Y & 1 & 4 & сус & $H(7 A)$ \\
\hline$H(8 A)$ & $C(8)$ & Z & $H(8 A)$ & $H(8 B)$ & Y & 1 & 4 & сус & $H(7 A)$ \\
\hline$H(8 B)$ & $C(8)$ & Z & $H(8 B)$ & $H(8 A)$ & Y & 1 & 4 & сус & $H(7 A)$ \\
\hline$H(9 A)$ & $C(9)$ & Z & $H(9 A)$ & $H(9 B)$ & Y & 1 & 4 & сус & $H(7 A)$ \\
\hline$H(9 B)$ & $C(9)$ & Z & $H(9 B)$ & $H(9 A)$ & Y & 1 & 4 & сус & $H(7 A)$ \\
\hline
\end{tabular}


Table S7.3: XD refinement strategy. A bbreviations: M P: M ultipole, M : monopoles; D: dipoles; Q: quadrupoles; O: octupoles; $\mathrm{H}$ : hexadecapoles, $\mathrm{K}: \mathrm{K}, \mathrm{U} 2$, U3: Gram Charlier $2^{\text {nd }}$ and $3^{\text {rd }}$ order, $\mathrm{HXYZ}$ : hydrogen position against data up to $0.5 \sin (\theta) / \lambda$. Local

\begin{tabular}{llllllllll}
\hline Step & New Para. & Data $<\mathbf{0 . 5} \sin (\boldsymbol{\theta}) / \boldsymbol{\lambda}$ & MP-Para & Data & Para & Data/Para & $\mathbf{R}\left(\mathbf{F}^{2}\right)$ & $\mathbf{W R}\left(\mathbf{F}^{2}\right)$ & $\mathbf{G O F}$ \\
1 & scalefactor & 729 & 0 & 6571 & 1 & 6571 & 0.0371 & 0.064 & 5.327 \\
2 & M & 729 & 6 & 6571 & 6 & 1095.2 & 0.0344 & 0.0565 & 4.754 \\
3 & DQOH & 729 & 50 & 6571 & 50 & 131.4 & 0.0248 & 0.0311 & 2.639 \\
4 & XYZ & 729 & 50 & 6571 & 104 & 63.2 & 0.0224 & 0.0279 & 2.372 \\
5 & U2 & 729 & 50 & 6571 & 131 & 50.2 & 0.0215 & 0.0259 & 2.204 \\
6 & K & 729 & 50 & 6571 & 134 & 49 & 0.0203 & 0.0232 & 1.979 \\
7 & H-XYZ & 729 & 0 & 729 & 19 & 38.4 & 0.0207 & 0.0184 & 3.817 \\
8 & All previous & 729 & 50 & 6571 & 134 & 49 & 0.0203 & 0.023 & 1.959 \\
9 & U3 & 729 & 50 & 6571 & 154 & 42.7 & 0.0188 & 0.0205 & 1.752 \\
10 & K' & 729 & 0 & 6571 & 4 & 1642.8 & 0.0185 & 0.0202 & 1.704 \\
11 & All previous & 729 & 50 & 6571 & 154 & 42.7 & 0.0185 & 0.02 & 1.705 \\
\hline
\end{tabular}

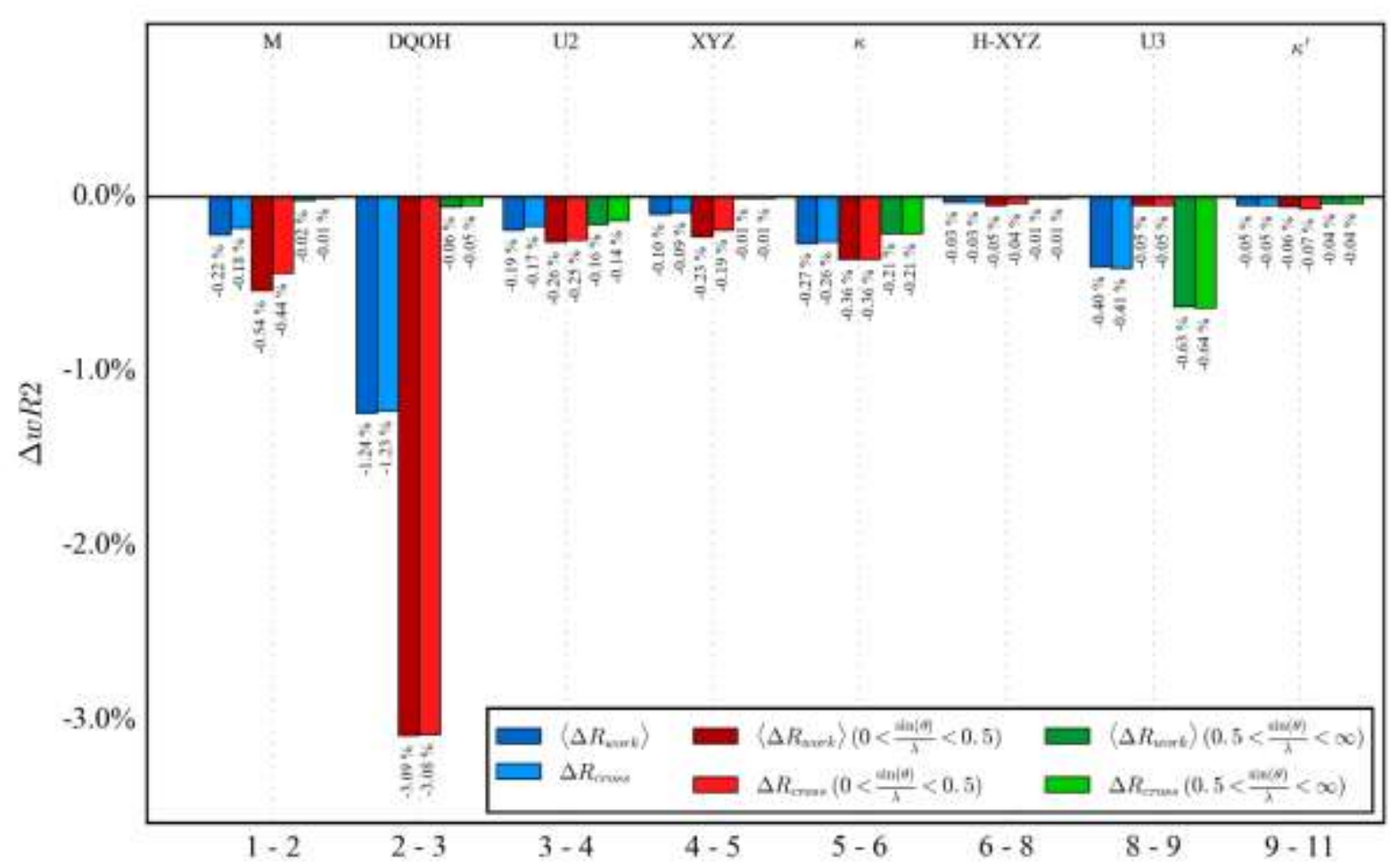

Figure S7.1: $\Delta \mathrm{R}_{\text {cross }}$ values for the XD refinement. A bbreviations: M : monopoles; D: dipoles; Q: quadrupoles; $\mathrm{O}$ : octupoles; K: $\kappa, \mathrm{U} 2$, U3: Gram Charlier $2^{\text {nd }}$ and $3^{\text {rd }}$ order, $\mathrm{H}-\mathrm{XYZ}$ : only hydrogen position against data up to $0.5 \sin (\theta) / \lambda$. 

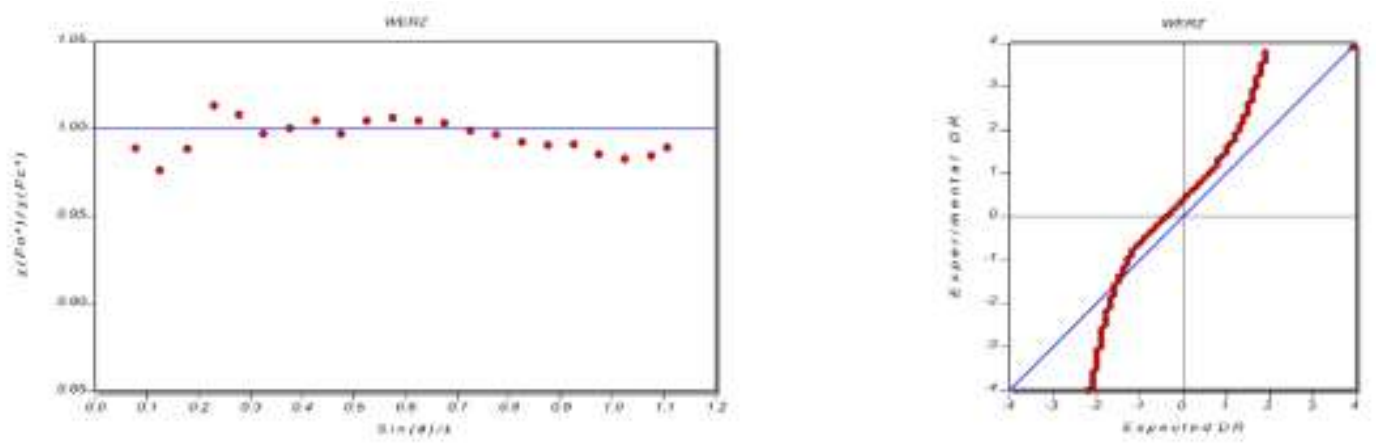

Figure S7.2: DRK-Plots(A dam Stash, 2007; Zhurov et al., 2008; Zavodnik et al., 1999) on F².

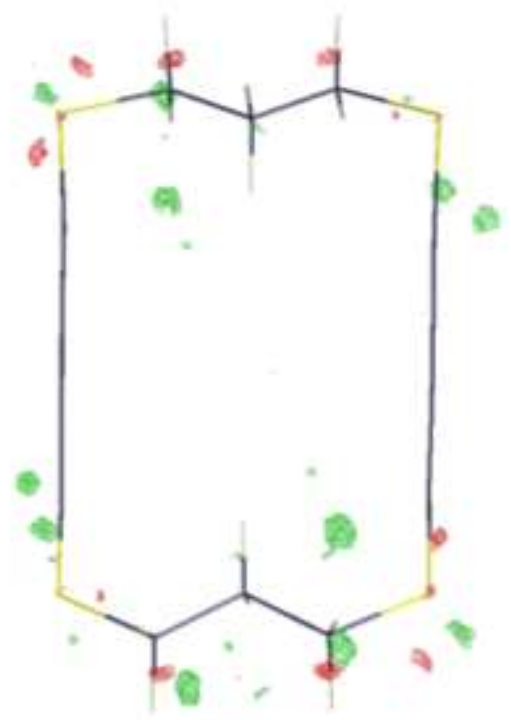

FigureS7.3: Difference electron density after the final refinement at the $+/-0.1 \mathrm{e}^{-3}$-level.

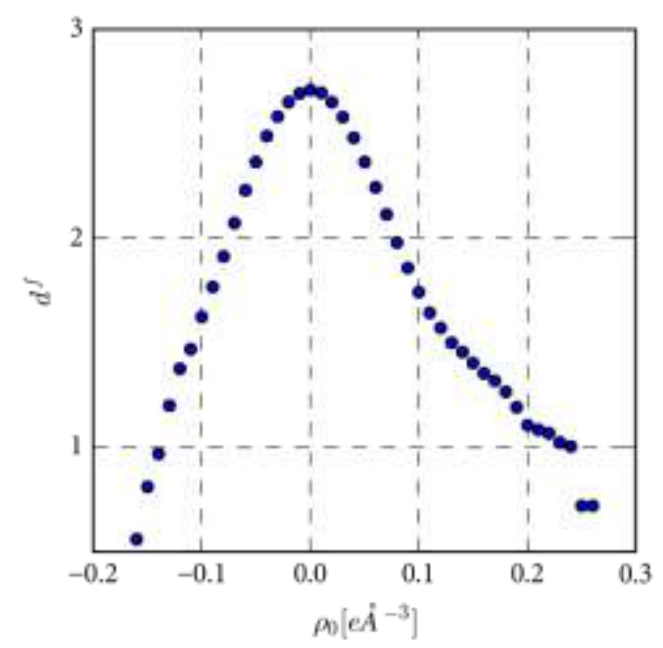

Figure S7.4: Fractal dimension analysis (M eindl $\&$ Henn, 2008)

Table S7.4: Kuhs's Rule

\begin{tabular}{llllll}
\hline A tom & \multicolumn{2}{l}{ Principal M.D.A's $[\AA]$} & $\begin{array}{l}\text { M in. resolution }\left[\AA^{-1}\right] \\
n=3\end{array}$ & $n=4$ \\
$S(1)$ & 0.167 & 0.136 & 0.121 & 0.93 & 1.07 \\
$S(6)$ & 0.196 & 0.136 & 0.12 & 0.88 & 1.01 \\
\hline
\end{tabular}

Table S7.5: Significance test for Gram-Charlier parameters.

\begin{tabular}{lllllllllll}
\hline A tom & $\mathrm{C} 111 / \sigma$ & $\mathrm{C} 222 / \sigma$ & $\mathrm{C} 333 / \sigma$ & $\mathrm{C} 112 / \sigma$ & $\mathrm{C} 122 / \sigma$ & $\mathrm{C} 113 / \sigma$ & $\mathrm{C} 133 / \sigma$ & $\mathrm{C} 223 / \sigma$ & $\mathrm{C} 233 / \sigma$ & $\mathrm{C} 123 / \sigma$ \\
$\mathrm{S}(1)$ & 4.88 & 1.89 & 6.00 & 1.90 & 3.83 & 0.20 & 2.33 & 13.57 & 2.67 & 6.00 \\
$\mathrm{~S}(6)$ & 6.26 & 0.57 & 1.67 & 16.33 & 19.80 & 9.80 & 4.67 & 19.56 & 3.25 & 22.00 \\
\hline
\end{tabular}

Table S7.6: A nalysis of the Probability Density Function.

\begin{tabular}{|c|c|c|c|c|c|c|}
\hline Atom & $\begin{array}{l}\text { Minimu } \\
\text { m PDF } \\
\text { value }\end{array}$ & $\begin{array}{l}\text { Maximum } \\
\text { PDF } \\
\text { Value }\end{array}$ & $\begin{array}{l}\text { Total integrated } \\
\text { negative } \\
\text { probability [\%] }\end{array}$ & $\begin{array}{l}\text { Integrated } \\
\text { volume for } \\
\text { negative } \\
\text { probability }\left[\AA^{-3}\right]\end{array}$ & $\begin{array}{l}\text { Total integrated } \\
\text { positive } \\
\text { probability [\%] }\end{array}$ & $\begin{array}{l}\text { Integrated } \\
\text { volume for } \\
\text { positive } \\
\text { probability }\left[\AA^{-3}\right]\end{array}$ \\
\hline$S(1)$ & -0.01 & 33453.51 & 0.000 & 0.679 & 100 & 3.659 \\
\hline$S(6)$ & -1.68 & 28645.03 & -0.001 & 0.559 & 100 & 3.779 \\
\hline
\end{tabular}


Table S7.7: BCP Analysis. * marked value errors are determined by cross-validation. In any case the larger error value was chosen.

\begin{tabular}{|c|c|c|c|c|c|c|c|}
\hline A tom 1 & Atom2 & $\begin{array}{l}\rho\left(\mathbf{r}_{\mathrm{bcp}}\right) \\
{\left[\mathrm{e} \AA^{-3}\right]}\end{array}$ & $\begin{array}{l}\nabla^{2} \rho\left(\mathbf{r}_{\text {bc }}\right) \\
{\left[\mathrm{e} \AA^{-5}\right]}\end{array}$ & $\begin{array}{l}\text { Bond Path } \\
\text { Length }[\AA]\end{array}$ & $\mathbf{r}_{\mathrm{A} 1-\mathrm{BCP}}[\AA]$ & $\mathbf{r}_{\mathrm{A}-\mathrm{BCP}}[\AA]$ & $\varepsilon\left(\mathbf{r}_{\text {bcp }}\right)$ \\
\hline C2 & S1 & $1.438(8)$ & $-8.11(16)^{*}$ & $1.67070(11)^{*}$ & $0.890(5)^{*}$ & $0.781(5)^{*}$ & $0.240(15)^{*}$ \\
\hline C3 & C4 & $2.169(9) *$ & $-18.6(4) *$ & $1.35520(12) *$ & $0.67760(7)^{*}$ & $0.67760(5)^{*}$ & $0.0(0) *$ \\
\hline C4 & C5 & $2.695(7)^{*}$ & $-25.1(3) *$ & $1.22030(16) *$ & $0.624(7) *$ & $0.596(7)^{*}$ & $0.0(0) *$ \\
\hline C5 & S6 & $1.444(4)^{*}$ & $-8.27(17)^{*}$ & $1.66800(15) *$ & $0.891(5)^{*}$ & $0.777(5)^{*}$ & $0.250(17) *$ \\
\hline C7 & $\mathrm{C} 8$ & $1.724(8)$ & $-19.25(18)^{*}$ & $1.51560(15) *$ & $0.768(7) *$ & $0.748(7)^{*}$ & $0.010(4) *$ \\
\hline C7 & H7A & $1.606(6) *$ & $-16.3(2) *$ & $1.09240(15)^{*}$ & $0.8176(17)^{*}$ & $0.2748(17)^{*}$ & $0.060(5) *$ \\
\hline C7 & $H 7 B$ & $1.607(5)$ & $-16.41(17)^{*}$ & $1.09230(14) *$ & $0.8175(17) *$ & $0.2748(16)^{*}$ & $0.050(5)^{*}$ \\
\hline C7 & 56 & $1.187(8)$ & $-6.17(13)^{*}$ & $1.82370(13)^{*}$ & $0.867(3)^{*}$ & $0.957(3)^{*}$ & $0.080(10)^{*}$ \\
\hline C8 & C9 & $1.717(12)$ & $-19.06(17)^{*}$ & $1.51900(17)^{*}$ & $0.750(7)^{*}$ & $0.769(7) *$ & $0.010(4) *$ \\
\hline C8 & $\mathrm{H} 8 \mathrm{~A}$ & $1.628(7)$ & $-17.1(2)^{*}$ & $1.09200(3)^{*}$ & $0.8184(14) *$ & $0.2736(14)^{*}$ & $0.030(6) *$ \\
\hline $\mathrm{C} 8$ & $\mathrm{H} 8 \mathrm{~B}$ & $1.628(7)$ & $-17.1(2)^{*}$ & $1.09200(5) *$ & $0.8184(14) *$ & $0.2736(14) *$ & $0.030(6) *$ \\
\hline C9 & $\mathrm{H} 9 \mathrm{~A}$ & $1.604(4)^{*}$ & $-16.32(16) *$ & $1.0926(2)^{*}$ & $0.8177(17) *$ & $0.2749(16) *$ & $0.050(5)^{*}$ \\
\hline C9 & $\mathrm{H} 9 \mathrm{~B}$ & $1.605(6) *$ & $-16.3(2) *$ & $1.09240(15) *$ & $0.8176(17) *$ & $0.2748(17) *$ & $0.060(4) *$ \\
\hline S1 & X5_S1 & $0.0620(4)^{*}$ & $0.589(3)^{*}$ & $3.54850(13) *$ & $1.77430(8)^{*}$ & $1.77430(8)^{*}$ & $0.440(13)^{*}$ \\
\hline
\end{tabular}

Table S7.8: Integrated Charges.

\begin{tabular}{llllllllll}
\hline A tom & Charge & Atom & Charge & Atom & Charge & Atom & Charge & Atom & Charge \\
$\mathrm{S}(1)$ & 0.3567 & $\mathrm{C}(4)$ & -0.0851 & $\mathrm{C}(7)$ & -0.4191 & $\mathrm{C}(8)$ & 0.3068 & $\mathrm{C}(9)$ & 0.4423 \\
$\mathrm{C}(2)$ & -0.269 & $\mathrm{C}(5)$ & -0.273 & $\mathrm{H}(7 \mathrm{~A})$ & 0.2027 & $\mathrm{H}(8 \mathrm{~A})$ & 0.1907 & $\mathrm{H}(9 \mathrm{~A})$ & 0.2017 \\
$\mathrm{C}(3)$ & -0.0848 & $\mathrm{~S}(6)$ & 0.352 & $\mathrm{H}(7 \mathrm{~B})$ & 0.1996 & $\mathrm{H}(8 \mathrm{~B})$ & 0.1899 & $\mathrm{H}(9 \mathrm{~B})$ & 0.2032 \\
\hline
\end{tabular}





\section{BIBLIOGRAPHY}

\section{Software}

Adam Stash 2007. DRK plot. M oscow.

Bruker AXS Inc. 2012. APEX2 v2012/2. M adison.

Bruker AXS Inc. 2016. SAINT. M adison.

Cheeseman, J., Keith, T. A. \& Bader, R. F. W . 1992. AIM PAC Program Package. M cM aster U niversity, Hamilton, Ontario.

Guido van Rossum. Python: Python Software Foundation.

Jelsch, C., Guillot, B., Pascale, F., Lagoutte, A., Fournier, B., D omagala, S., Alexandre, S. \& I ordache, C. 2018. M oPro1805. Lorraine.

Keith, T. A. 2017. AIM ALL. Overland Park KS: TK Gristmill Software.

Sheldrick, G. M. 2014a. XPREP in SHELXTL 2014/2. Göttingen.

Sheldrick, G. M. 2016. SH ELXL. Göttingen.

Turner, M. J., M cKinnon, J. J., W olff, S. K., Grimwood, D. J., Spackman, P. R., Jayatilaka, D. \& Spackman, M. A. 2018. CrystalExplorer 17.5: University of W estern Australia.

Volkov, A., King, H. F. \& Koritsanszky, T. 2016a. DenProp version 04/01/16.

Volkov, A., M acchi, P., Farrugia, L. J., Gatti, C., M allinson, P. R., Richter, T. \& Koritsanszky, T. 2006. XD2006 - A Computer Program Package for M ultipole Refinement, Topological A nalysis of Charge Densities and Evaluation of Intermolecular Energies from Experimental and Theoretical Structure Factors.

\section{Articles, books, and thesis}

Aida, M. \& Nagata, C. 1986. Theoret. Chim. Acta. 70, 73-80.

Alexandropoulos, N. G., Cooper, M. J., Suortti, P. \& W illis, B.T.M . in International Tables for Crystallography, M athematical, Physical and Chemical Tables, edited by E. Prince: online edition 2006, pp. 653-665.

Arndt, U. 1990. J. A ppl. Crystallogr. 23, 161-168.

Axenov, K. V., M ömming, C. M., Kehr, G., Fröhlich, R. \& Erker, G. 2010. Chem. Eur. J. 16, 14069_ 14073.

Bader, R. F. W . 1975. Acc. Chem. Res. 8, 34-40.

Bader, R. F. W. 1990. A toms in M olecules. Oxford, N ew York: Clarendon Press.

Bader, R. F. W. 1991. Chem. Rev. 91, 893-928.

Bader, R. F. W . 2009. J. Phys. Chem. A. 113, 10391-10396.

Bader, R. F. W . \& Beddall, P. M. 1972. J. Chem. Phys. 56, 3320-3329.

Bader, R. F. W. \& Beddall, P. M. 1973. J. A m. Chem. Soc. 95, 305-315.

Bader, R. F. W., Beddall, P. M. \& Cade, P. E. 1971. J. Am. Chem. Soc. 93, 3095-3107.

Banumathi, S., Zwart, P. H., Ramagopal, U. A., Dauter, M . \& Dauter, Z. 2004. Acta Crystallogr. A. D 60, 1085-1093. 
Beno, B. R., Y eung, K.-S., Bartberger, M . D., Pennington, L. D. \& M eanwell, N . A. 2015. J. M ed. Chem.

58, 4383-4438.

Bleiholder, C., Gleiter, R., W erz, D. B. \& Köppel, H. 2007. Inorg. Chem. 46, 2249-2260.

Boag, J. W . 1973. Phys. M ed. Biol. 18, 3-37.

Bragg, W . H . \& Bragg, W . L. 1913. Proceedings of the Royal Society A: M athematical, Physical and Engineering Sciences. 88, 428-438.

Bragg, W. L. 1913. Proceedings of the Royal Society of London A: M athematical, Physical and Engineering Sciences. 89, 248-277.

Brezgunova, M. E., Lieffrig, J., A ubert, E., Dahaoui, S., Fertey, P., Lebegue, S., A ngyan, J. G.,

Fourmigue, M . \& Espinosa, E. 2013. Cryst. Growth Des. 13, 3283-3289.

Bridge, N. K. 1960. Nature. 185, 30 EP -.

Brönnimann, C., Eikenberry, E. F., H enrich, B., H orisberger, R., H uelsen, G., Pohl, E., Schmitt, B.,

Schulze-Briese, C., Suzuki, M ., Tomizaki, T., Toyokawa, H. \& W agner, A. 2006. J. Synchrotron

Radiat. 13, 120-130.

Bruker AXS GmbH 2015. Product Sheet SC-XRD 54. Karlsruhe.

Bruker AXS Inc. 2011. Product Sheet SC-XRD 31 - APEX II CCD Detector. Madison.

Brünger, A. T. 1992. Nature. 355, 472-475.

Brünger, A. T. Cross-validation in crystallography. in M ethods in Enzymology, edited by Charles W.

Carter Jr, Robert M. Sweet: A cademic Press 1997, pp. 366-396.

Buhl, H., Verlinden, K., Ganter, C., Novaković, S. B. \& Bogdanović, G. A. 2016. Ber. Dtsch. Chem. Ges. 2016, 3389-3395.

Caputo, C. B. \& Stephan, D. W . in The Chemical Bond III: 100 years old and getting stronger, edited by

D. M ingos \& P. M ichael: Springer. Cham 2017, pp. 1-29.

Chu, J. Y. C., M arsh, D. G. \& Guenther, W. H. H. 1975. J. Am. Chem. Soc. 97, 4905-4908.

Collard, K. \& Hall, G. G. 1977. Int. J. Quantum Chem. 12, 623-637.

DECTRIS Ltd. 2015. PILATUS3 X CdTe. Baden.

Deryagina, E. N., Voronkov, M. G. \& Korchevin, N. A. 1993. Russ. Chem. Rev. 62, 1107-1117.

Destro, R., Loconte, L., Lo Presti, L., Roversi, P. \& Soave, R. 2004. Acta Crystallogr. A. 60, 365-370.

Devillanova, F. A. 2007. Handbook of chalcogen chemistry. London: Royal Society of Chemistry.

Diederichs, K. 2010. Acta Crystallogr. D. 66, 733-740.

Diederichs, K. \& Karplus, P. A . 1997. Nat. Struct. Biol. 4, 269275.

Dittrich, B., Koritsánszky, T. \& Luger, P. 2004. Angew. Chem., Int. Ed. 43, 2718-2721.

Dittrich, B., Schürmann, C. \& Hübschle, C. B. 2016. Z. Kristallogr. 231, 1769.

Engelhardt, F. 2017. Dissertation, Georg-A ugust-U niversität. Göttingen.

Farrugia, L. J., Evans, C. \& Tegel, M. 2006. J. Phys. Chem. A. 110, 7952-7961.

Flierler, U., Leusser, D., Ott, H., Kehr, G., Erker, G., Grimme, S. \& Stalke, D. 2009. Chem. Eur. J. 15, 4595-4601.

Fourier, J.B.J. 1822. Théorie analytique de la chaleur: Chez Firmin Didot, père et fils.

Frenking, G. 2014. Angew. Chem., Int. Ed. 53, 6040-6046. 
Frenking, G. \& Shaik, S. 2014. The Chemical Bond: Wiley.

Friedrich, W., Knipping, P. \& Laue, M . v. 1912. Interferenz-Erscheinungen bei Röntgenstrahlen, von W . Friedrich, P. Knipping und M . Lane. M ünchen: V erlag der Königlich-Bayerischen A kademie der Wissenschaften.

Gatti, C. 2005. Z. Kristallogr. 220, 399_457.

Gatti, C. \& M acchi, P. 2012. M odern Charge Density A nalysis. H eidelberg, London, N ew York: Springer.

Ghadwal, R. S., Lamm, J.-H ., Rottschäfer, D., Schürmann, C. J. \& Demeshko, S. 2017. Dalton Trans. 46, 7664-7667.

Ghadwal, R. S., Rottschäfer, D., A ndrada, D. M ., Frenking, G., Schürmann, C. J. \& Stammler, H .-G. 2017. Dalton Trans. 46, 7791-7799.

Ghadwal, R. S., Schürmann, C. J., Andrada, D. M . \& Frenking, G. 2015. Dalton Trans. 44, 14359_ 14367.

Ghadwal, R. S., Schürmann, C. J., Engelhardt, F. \& Steinmetzger, C. 2014. Eur. J. Inorg. Chem. 2014, 4908.

Gleiter, R., Werz, D. B. \& Rausch, B. J. 2003. Chem. Eur. J. 9, 2676-2683.

Granitzka, M. 2013. Dissertation, Georg-A ugust-U niversität. Göttingen.

Gruner, S. M ., Tate, M. W . \& Eikenberry, E. F. 2002. Rev Sci Instrum. 73, 2815-2842.

Guillot, B., V iry, L., Guillot, R., Lecomte, C. \& J elsch, C. 2001. J. A ppl. Crystallogr. 34, 214-223.

H aaland, A., Shorokhov, D. J. \& Tverdova, N. V. 2004. Chem. Eur. J. 10, 4416-4421.

Hahn, T. 2005. International Tables for Crystallography, V olume A: W iley.

Hansen, N. K. \& Coppens, P. 1978. Acta Crystallogr. A. 34, 909-921.

Hendrickson, W. A. 1991. Science. 254, 51-58.

Hendrickson, W. A., H orton, J. R. \& LeM aster, D. M. 1990. The EM BO Journal. 9, 1665-1672.

Herbst-Irmer, R. \& Stalke, D. 2017. A cta Crystallogr. B. 73, 531-543.

H immel, D., Krossing, I. \& Schnepf, A. 2014a. Angew. Chem. 126, 378-382.

Himmel, D., Krossing, I. \& Schnepf, A. 2014b. Angew. Chem., Int. Ed. 53, 370-374.

Hirshfeld, F. L. 1977. Theoret. Chim. Acta. 44, 129-138.

Hohenberg, P. \& Kohn, W. 1964. Physical Review. B136, 864-871.

H olleman, A. F., W iberg, N.\& W iberg, E. 2007. Lehrbuch der Anorganischen Chemie. 102nd ed.

Berlin, N ew York: Walter de Gruyter.

Hopf, H. 1927. M athematische Annalen. 96, 209-224.

Hübschle, C. B., Sheldrick, G. M. \& Dittrich, B. 2011. J. A ppl. Crystallogr. 44, 1281-1284.

Jackson, C. L. 1875. Ann. Chem. Pharm. 179, 1-20.

Jarzembska, K. N., Kaminski, R., Dobrzycki, L. \& Cyranski, M. K. 2015. Acta Crystallogr. B. 71, 241243.

Jayatilaka, D . \& Grimwood, D. J. 2003. Computational Science - ICCS 2003. 2660, 142-151.

Jelsch, C., Guillot, B., Lagoutte, A. \& Lecomte, C. 2001. J. Appl. Crystallogr. 38, 38-54. 
Johnson, C. K. \& Levy, H. A . in International Tables for X-ray Crystallography: Kynoch Press.

Birmingham 1974, pp. 311-336.

Kabsch, W. 2010. Acta Crystallogr. A. D66, 125-132.

Kehr, G. \& Erker, G. 2017. Chem. Rec. 17, 803-815.

Kocher, N., Leusser, D., M urso, A \& Stalke, D. 2004. Chem. Eur. J. 10, 3622-3631.

Köhler, C. 2017. Bachelor-A rbeit, Georg-August-U niversität. Göttingen.

Koritsanszky, T. S. \& Coppens, P. 2001. Chem. Rev. 101, 1583-1627.

Kottke, T. \& Stalke, D. 1993. J. A ppl. Crystallogr. 26, 615-619.

Kraft, P., Bergamaschi, A., Broennimann, C., Dinapoli, R., Eikenberry, E. F., H enrich, B., Johnson, I., M ozzanica, A., Schlepütz, C. M ., W illmott, P. R. \& Schmitt, B. 2009. J. Synchrotron Radiat. 16, 368375.

Krause, L. 2017. D issertation, Georg-August-U niversität. Göttingen.

Krause, L., H erbst-Irmer, R., Sheldrick, G. M . \& Stalke, D. 2015a. J. A ppl. Crystallogr. 48, 3-10.

Krause, L., H erbst-Irmer, R. \& Stalke, D. 2015b. J. A ppl. Crystallogr. 48, 1907-1913.

Krause, L., N iepötter, B., Schürmann, C. J., Stalke, D. \& Herbst-Irmer, R. 2017. IU Cr] . 4, 420-430.

Kuhs, W. F. 1992. A cta Crystallogr. A. 48, 80-98.

Lederer, C. M., Shirley, V. S. \& Browne, E. 1978. Table of isotopes. 7th ed. N ew York: W iley.

Leiros, H. K. S., Timmins, J., Ravelli, R. B. G. \& M cSweeney, S. M . 2006. Acta Crystallogr. D. 62, 125132.

Lin, L., Sheng, J. \& H uang, Z. 2011. Chem. Soc. Rev. 40, 4591-4602.

Lübben, J. \& Grüne, T. 2015. Proc. N atl. A cad. Sci. USA. 112, 8999-9003.

M acchi, P., Garlaschelli, L. \& Sironi, A. 2002. J. Am. Chem. Soc. 124, 14173-14184.

M ackenzie, C. F., Spackman, P. R., Jayatilaka, D. \& Spackman, M . A. 2017. IU CrJ , 575-587.

M aslen, E. N. in International tables for crystallography. 1st ed., edited by H. Fueß: Springer. Berlin 2006, pp. 599-608.

M atussek, J. 2014. Dissertation, Georg-August-Universität. Göttingen.

M cKinnon, J. J., M itchell, A. S. \& Spackman, M . A. 1998. Chem. Eur. J. 4, 2136-2141.

McKinnon, J. J., Spackman, M. A . \& M itchell, A. S. 2004. Acta Crystallogr. B. 60, 627-668.

M eindl, K. \& Henn, J. 2008. Acta Crystallogr. A. A64, 404-418.

M erkel, S. 2009. Dissertation, Georg-A ugust-U niversität. Göttingen.

M etanis, N. \& Hilvert, D. 2014. Current opinion in chemical biology. 22, 27-34.

M iller, W. H. 1839. A Treatise on Crystallography: For J. \& J. J. Deighton.

M ohapatra, C., Samuel, P. P., Li, B., N iepötter, B., Schürmann, C. J., H erbst-Irmer, R., Stalke, D.,

M aity, B., Koley, D. \& Roesky, H. W. 2016. Inorg. Chem. 55, 1953-1955.

M ömming, C. M ., Frömel, S., K ehr, G., Fröhlich, R., Grimme, S. \& Erker, G. 2009a. J. Am. Chem. Soc. 131, 12280-12289.

M ömming, C. M ., Otten, E., Kehr, G., Fröhlich, R., Grimme, S., Stephan, D. W . \& Erker, G. 2009b. Angew. Chem., Int. Ed. 48, 6643-6646.

M urray, J. S., Lane, P., Clark, T. \& Politzer, P. 2007. J M ol M odel. 13, 1033-1038. 
M urray, J. S., Lane, P. \& Politzer, P. 2008. Int. J. Quantum Chem. 108, 2770-2781.

M urray, J. W., Garman, E. F. \& Ravelli, R. B. G. 2004. J. A ppl. Crystallogr. 37, 513-522.

Nam, Y.-W ., Orfali, R., Liu, T., Yu, K., Cui, M., W ulff, H . \& Zhang, M . 2017. Scientific reports. 7, 17178.

Nave, C. \& Garman, E. F. 2005. J. Synchrotron Radiat. 12, 257-260.

Niepötter, B., H erbst-Irmer, R. \& Stalke, D. 2015. J. A ppl. Crystallogr. 48, 1485-1497.

Ohnishi, S. - i., Tanei, T. \& Nitta, I. 1962. J. Chem. Phys. 37, 2402-2407.

Ortgies, S. \& Breder, A. 2017. ACS Catal. 7, 5828-5840.

Özgün, T., Bergander, K., Liu, L., D aniliuc, C. G., Grimme, S., Kehr, G. \& Erker, G. 2016. Chem. Eur. J. 22, 11958-11961.

Pascoe, D. J., Ling, K. B. \& Cockroft, S. L. 2017. J. Am. Chem. Soc. 139, 15160-15167.

Paul, A., Kubicki, M., Jelsch, C., Durand, P. \& Lecomte, C. 2011. Acta Crystallogr. B. 67, 365-378.

Piers, W. E. \& Chivers, T. 1997. Chem. Soc. Rev. 26, 345-354.

Pike, A. C. W ., Garman, E. F., Krojer, T., D elft, F. von \& Carpenter, E. P. 2016. Acta Crystallogr. D. 72, 303-318.

Pratt, V. 1987. SIGGRAPH Comput. Graph. 21, 145-152.

Rademacher, P. in Strukturen organischer M oleküle. 1st ed., edited by M. Kiessinger: W iley-VCH. W einheim 1987, pp. 55-88.

Romell, J., V ågberg, W ., Romell, M ., H äggman, S., I kram, S. \& H ertz, H. M . 2018. Radiology. 289, 670_ 676.

Rottschäfer, D., Schürmann, C. J., Lamm, J.-H ., Paesch, A. N., N eumann, B. \& Ghadwal, R. S. 2016. Organometallics. 35, 3421-3429.

Roy, S., M ondal, K. C., Kundu, S., Li, B., Schürmann, C. J., Dutta, S., Koley, D., H erbst-Irmer, R., Stalke, D. \& Roesky, H. W. 2017. Chem. Eur. J. 23, 12153-12157.

Roy, S., Schürmann, C. J., M ondal, T., Koley, D., Herbst-Irmer, R., Stalke, D. \& Roesky, H. W . 2016. Chem. Eur. J. 22, 12629-12633.

Roy, S., Schürmann, C. J., M ondal, T., Koley, D., Herbst-Irmer, R., Stalke, D. \& Roesky, H. W . 2016.

Chem. Eur. J. 22, 12944.

Ruth, P. N . 2017. M aster Thesis, Georg-A ugust U niversität Göttingen. Göttingen.

Sampath, P. I. 1966. J. Chem. Phys. 45, 3519-3525.

Sanctis, D. de, Tucker, P. A . \& Panjikar, S. 2011. J. Synchrotron Radiat. 18, 374-380.

Sanctis, D. de, Zubieta, C., Felisaz, F., Caserotto, H. \& Nanao, M . H. 2016. A cta Crystallogr. D. 72, 395402.

Schulte, J. H., W erz, D. B., Rominger, F. \& Gleiter, R. 2003. Org. Biomol. Chem. 1, 2788-2794.

Schulz, T., M eindl, K., Leusser, D., Stern, D., Graf, J., M ichaelsen, C., Ruf, M ., Sheldrick, G. M . \&

Stalke, D. 2009. J. A ppl. Crystallogr. 42, 885-891.

Schürmann, C. J., Pröpper, K., W agner, T. \& Dittrich, B. 2012. A cta Crystallogr. B. 68, 313-317.

Schwendemann, S., Fröhlich, R., Kehr, G. \& Erker, G. 2011. Chem. Sci. 2, 1842-1849.

Shahbazian, S. 2018. Chem. Eur. J. 24, 5401-5405. 
Sheldrick, G. M. 2014b. A cta Crystallogr. A . 70, C1437.

Sheldrick, G. M. 2015. Acta Crystallogr. C. 71, 3-8.

Shmueli, U. 2010. Editor. International Tables for Crystallography Volume B: Reciprocal space. second online edition: Springer N etherlands.

Smith, J. L., H endrickson, W . A., Terwilliger, T. C.\& Berendzen, J. in International tables for crystallography. 1st ed., edited by H. Fueß: Springer. Berlin 2006, pp. 299-309.

Spackman, M. A. \& Byrom, P. G. 1997. Chem Phys Lett. 267, 215-220.

Spies, P., Erker, G., Kehr, G., Bergander, K., Frohlich, R., Grimme, S. \& Stephan, D. W. 2007. Chem. Commun., 5072-5074.

Spies, P., Kehr, G., Bergander, K., W ibbeling, B., Frohlich, R. \& Erker, G. 2009. Dalton Trans., 15341541.

Stalke, D. 1998. Chem. Soc. Rev. 27, 171-178.

Stalke, D. 2014. Acta Crystallogr. B. 70, 781-782.

Stanley, W ., Van De M ark, M. R. \& Kumler, P. L. 1974. J. Chem. Soc., Chem. Commun., 700.

Stephan, D. W. \& Erker, G. 2010. Angew. Chem., Int. Ed. 49, 46-76.

Steudel, R. 1975a. Angew. Chem. 87, 683-692.

Steudel, R. 1975b. Angew. Chem., Int. Ed. 14, 655-664.

Stewart, R. F. 1972. J. Chem. Phys. 57, 1664-1668.

Stewart, R. F. 1973. J. Chem. Phys. 58, 1668-1676.

Stewart, R. F. 1977. I srael Journal of Chemistry. 16, 124-131.

Storm, A. B., M ichaelsen, C., O ehr, A. \& H offmann, C. 2004. Proc. SPIE. 5537, 177-181.

Stute, A., H eletta, L., Fröhlich, R., D aniliuc, C. G., Kehr, G. \& Erker, G. 2012. Chem. Commun. 48, 11739-11741.

Sultana, A., Karim, K. S. \& Rowlands, J. A. 2008. Can. J. Electr. Comput. Eng. 33, 139-143.

Teuteberg, T., Eckhoff, M . \& M ata, R. A. 2018. A Full Additive QM / M M Scheme for the Computation of M olecular Crystals with Extension to M any-Body Expansions, doi:10.26434/chemrxiv.7326491.v1.

Töpperwien, M ., van der M eer, F., Stadelmann, C. \& Salditt, T. 2018. Proc. Natl. A cad. Sci. USA. 115, 6940-6945.

Turner, M. J., Thomas, S. P., Shi, M. W ., Jayatilaka, D . \& Spackman, M . A . 2015. Chem. Commun., 3735-3738.

Volkov, A. \& Coppens, P. 2001. Acta Crystallogr. A . 57, 395-405.

Volkov, A., M acchi, P., Farrugia, L. J., Gatti, C., M allinson, P. R., Richter, T. \& Koritsanszky, T. 2007. XD2006 - M anual.

Volkov, A., M acchi, P., Farrugia, L. J., Gatti, C., M allinson, P. R., Richter, T. \& Koritsanszky, T. 2016b. XD2016- Manual.

Walden, H. 2010. A cta Crystallogr. D. 66, 352-357.

Weiss, M. S. 2001. J. A ppl. Crystallogr. 34, 130-135.

Werz, D. B., Gleiter, R. \& Rominger, F. 2002a. J. Am. Chem. Soc. 124, 10638-10639.

Werz, D. B., Gleiter, R. \& Rominger, F. 2003. Organometallics. 22, 843-849. 
Werz, D. B., Gleiter, R. \& Rominger, F. 2004. J. Org. Chem. 69, 2945-2952.

Werz, D. B., Staeb, T. H., Benisch, C., Rausch, B. J., Rominger, F. \& Gleiter, R. 2002b. Org. Lett. 4, 339_ 342.

Willis, B.T.M . in International Tables for Crystallography, Reciprocal Space, edited by U. Shmueli: online edition 2001, pp. 400-406.

Windle, J. J., W iersema, A. K. \& Tappel, A. L. 1964. J. Chem. Phys. 41, 1996-2002.

W olf, H. 2014. Dissertation, Georg-August-Universität. Göttingen.

W olf, H., Jørgensen, M. R. V., Chen, Y.-S., H erbst-Irmer, R. \& Stalke, D. 2015a. Acta Crystallogr. B. 71, $10-19$.

W olf, H., Lock, N ., Parker, S. F. \& Stalke, D. 2015b. Chem. Eur. J. 21, 4556-4560.

Zaccaria, F., W olters, L. P., Fonseca Guerra, C. \& O rian, L. 2016. J. Comput. Chem. 37, 1672-1680.

Zarychta, B., Pichon-Pesme, V., Guillot, B., Lecomte, C. \& Jelsch, C. 2007. Acta Crystallogr. A. 63, 108125.

Zarychta, B., Zaleski, J., Kyzioł, J., Daszkiewicz, Z. \& Jelsch, C. 2011. Acta Crystallogr. B. 67, 250-262.

Zavodnik, V., Stash, A., Tsirelson, V., V ries, R. de \& Feil, D. 1999. A cta Crystallogr. B. 55, 45-54.

Zhurov, V. V., Zhurova, E. A. \& Pinkerton, A. A. 2008. J. A ppl. Crystallogr. 41, 340-349.

Zwart, P. H., Banumathi, S., Dauter, M . \& Dauter, Z. 2004. Acta Crystallogr. A. D60, 1958-1963. 


\section{Danksagung}

Zunächst einmal möchte ich mich bei meinem Doktorvater Prof. Dr. Dietmar Stalke für das in mich gesetzte Vertrauen bedanken, für die große Freiheit bei der Ausgestaltung meiner Forschung, die Unterstützung bei der Verfolgung selbst gesteckter Projekte und die einmaligen technischen Möglichkeiten zur Erforschung kristallographischer Probleme, welche er mir zu V erfügung gestellt hat.

Ich danke allen M itglieder der Prüfungskommission dafür, dass sie diese Arbeit auf sich nehmen, dass sie diese Promotion mit zu einem erfolgreichen Ende führen.

I would like to thank all members of the Center for M aterial Crystallography (CM C). It has al ways been a pleasure to confer with you guys and to have a glimpse at the sciences, adjacent to chemical crystallography.

Ich danke Prof. George Sheldrick, Dr. Birger Dittrich, Julian, Andrea, Tim, Claudia und Jens dafür, dass sie mich vor ein paar Jahren, als es darum ging eine Bachelorarbeit zu schreiben, vollends für die Kristallographie begeisterten.

Ich bedanke mich bei allen Kooperationspartnern: Prof. Dr. M ata, Dr. Daniel Stern, Dr. Holger Ott, Dr. A. Claudia Stückl, Jasper und insbesondere Thorsten - die interdisziplinäre Zusammenarbeit hat meine Promotion zu Erfolg geführt. Vielen Dank auch an meine Service-Partner, Chandrajeet, Sudipta und insbesondere Rajendra.

M einen Freunden und Kollegen im A rbeitskreis Stalke (aktiven wieehemaligen) dankeich für dieschöne Zeit. Der Zusammenhalt unter euch ist großartig und ich hoffe, dass euch dieser erhalten bleibt. Vielen Dank für entspannte M inuten am Kicker, tolle Konferenzen, informative Seminare und manch einen angenehmen A bend. Ich danke meinen Altvorderen, Hilke, Benedikt, Felix und dem „Commander“ Lennard, die mir vieles über Kristallographie, Python, die M aschinen und das Leben im Allgemeinen beigebracht haben. Die jüngere Generation an Kristallographen ist einfach fabelhaft. Vielen Dank also an Helena, Annika und Christian für ihre stets zuvorkommende Zusammenarbeit, informative ED -Seminare und das Korrekturlesen meiner Diss. Niklas - die besten Diskussionen werden immer noch über einem Kaffee oder einem Bier geführt. Es hat mich sehr gefreut, dass du in den AK gekommen bist und du bist eine große Bereicherung für mich und den ganzen AK. Agnes, M olly, M onique und Inga, ihr seid ein Haufen verdammter Zicken und es war eine Genugtuung, immer wieder mit Schraubenzieher und Inbusschlüssel in euren Eingeweiden herumzufuhrwerken. Vielen Dank an Jochen, Alex, Johannes, Christian S., Timo, Anne und die anderen Synthetiker, die mir das G efühl gaben noch was mit richtiger Chemie zu tun zu haben. Vielen Dank an die Grand Dame der Ladungsdichte, Regine, die akkurat auch noch die letzte Ungereimtheit aufspüren und uns allen ein V orbild an Exaktheit sein kann.

Einige Leute haben mich seit nunmehr zehn Jahren durch das gesamte Studium begleitet. Katha und Christoph - wer hätte gedacht, dass sich unsere kleine Dreiergruppe aus dem AC0 nach all der Zeit immer noch so nahesteht. Jochen, Fabian, Christian, Jan, M ark und $M$ arcel - danke, dass ihr mich über den langen W eg begleitet habt.

Ich dankemeinen Eltern M onika und Bernd für ihr unerschöpfliches Vertrauen. Trotz aller W idrigkeiten habt ihr mir ein entspanntes Studium ermöglicht, nie gemeckert und mich machen lassen. Vielen Dank 
meinem Brüdern Stephan und Thomas, die immer für mich da waren. Vielen Dank an meine Verwandten in Göttingen, Andreas, Kathrin, Sophie und Luise, für Kost und Logis in den ersten M onaten meines Studiums, für so manches W eizen und viele schöne Grillabende. Auch vielen Dank an den Rest der Familie: Hans-Josef, Doro, M athias, Benni, Philip und Gabi.

Zu guter Letzt Malin. Du bist die Beste. Vielen Dank, dass du mich seit fast elf Jahren begleitest und schöne, wie schwierige Zeiten mit mir durchlebt hast. Nicht zuletzt liegt es an dir, dass diese Arbeit gelingen konnte. 